\title{
A STUDY OF OCEAN WAVE STATISTICAL PROPERTIES USING NONLINEAR, DIRECTIONAL, PHASE-RESOLVED OCEAN WAVE-FIELD SIMULATIONS
}

\author{
By \\ Legena Albertha Henry \\ B.Sc., Howard University, 2005 \\ Submitted in partial fulfillment of the requirements for the degree of \\ Master of Science in Mechanical Engineering \\ at the \\ MASSACHUSETTS INSTITUTE OF TECHNOLOGY \\ and the \\ WOODS HOLE OCEANOGRAPHIC INSTITUTION \\ February 2010 \\ (C) 2009 Legena Albertha Henry \\ All rights reserved.
}

The author hereby grants to MIT and WHOI permission to reproduce and to distribute publicly paper and electronic copies of this thesis document in whole or in part in any medium now known or hereafter created.

Signature of Author

Joint Program in Oceanography/Applied Ocean Science and Engineering Massachusetts Institute of Technology and Woods Hole Oceanographic Institution

November 23, 2009

Certified by

Dick K. P. Yue

Philip J. Solondz Professor of Engineering, Professor of Mechanical and Ocean Engineering

Thesis Supervisor

Accepted by

James C. Preisig

Chair, Joint Committee for Applied Ocean Science and Engineering Massachusetts Institute of Technology/ Woods Hole Oceanographic Institution

David E. Hardt

Ralph E. and Eloise F. Cross Professor of Mechanical Engineering Chair, Department of Mechanical Engineering , Massachusetts Institute of Technology 


\title{
A study of ocean wave statistical properties using nonlinear, directional, phase-resolved ocean wave-field simulations
}

\author{
by \\ Legena Albertha Henry
Submitted to the Department of Mechanical Engineering on November 23, 2009, in partial fulfillment of the requirements for the degree of Master of Science in Mechanical Engineering

\begin{abstract}
In the present work, we study the statistics of wavefields obtained from non-linear phase-resolved simulations. The numerical model used to generate the waves models wave-wave interactions based on the fully non-linear Zakharov equations. We vary the simulated wavefield's input spectral properties: directional spreading function, Phillips parameter and peak shape parameter. We then investigate the relationships between a wavefield's input spectral properties and its output physical properties via statistical analysis. We investigate surface elevation distribution, wave definition methods in a nonlinear wavefield with a two-dimensional wavenumber, defined waves' distributions, and the occurrence and spacing of large wave events.
\end{abstract}

Thesis Supervisor: Dick K. P. Yue

Title: Philip J. Solondz Professor of Engineering, Professor of Mechanical and Ocean Engineering 


\section{Acknowledgments}

I would like to acknowledge the useful guidance of my research advisor, Professor D.K.P. Yue and VFRL Research Scientist, Dr. Kelli Hendrickson. I'd also like to acknowledge the gracious assistance with the use of SNOW simulations from my colleagues, Guangyu Wu and Wenting Xaio in MIT's Vortical Flow Research Lab. I'd be wrong not to thank Ronni Schwartz, my family members, and my close friends who offered support, practice talks, proof reading, words of encouragement and free food along the way to this accomplishment. Finally, Nigel - thank you. This project was supported by the US Office of Naval Research and the Joint Program between MIT and Woods Hole Oceanographic Institute.

Je n'ai fait celle-ci plus longue que parce que je n'ai pas eu le loisir de la faire plus courte.

- Blaise Pascal 


\section{Contents}

1 Introduction $\quad 49$

1.1 Present Contribution . . . . . . . . . . . . . . . . 49

1.2 The statistical approach to ocean waves. . . . . . . . . . . . . . 50

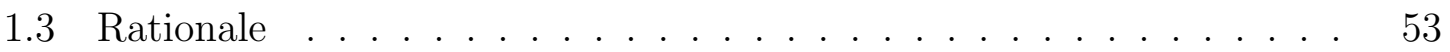

1.4 Thesis Scope. . . . . . . . . . . . . . . . . 55

2 Methods $\quad 57$

2.1 JONSWAP spectrum . . . . . . . . . . . . . . . . 58

2.2 JONSWAP spectral parameters . . . . . . . . . . . . . . . 59

2.3 Simulations of Nonlinear Ocean Wavefields (SNOW) . . . . . . . . . 59

2.4 Statistical Measures . . . . . . . . . . . . . . . . . . 62

3 Ocean surface elevation and spectral parameters. $\quad 65$

3.1 Peak shape parameter . . . . . . . . . . . . . 65

3.1.1 Peak shape parameter and surface elevation statistical moments 66

3.1.2 Peak shape parameter and surface elevation statistical distribution .......................... 69

3.1.3 Peak shape parameter and surface elevation wavenumber spec-

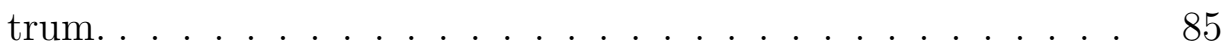

3.1.4 Peak shape parameter and surface elevation Benjamin-Feir Index 88

3.1.5 Peak shape parameter and surface elevation slope distribution 98

3.1.6 Conclusion: Peak shape parameter and surface elevation characteristics . . . . . . . . . . . . . . 103 
3.2 Phillips' parameter . . . . . . . . . . . . . . . . . . . . . . . . . 104

3.2.1 Impact of input Phillips' parameter on surface elevation distribution . . . . . . . . . . . . . . . . . 104

3.2.2 Impact of input Phillips' parameter on surface elevation wavenum-

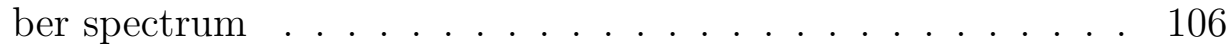

3.2.3 Impact of input Phillips' parameter on surface elevation BFI . 117

3.2.4 Impact of input Phillips' parameter on surface elevation-slope kurtosis . . . . . . . . . . . . . . . . . . 125

3.2.5 Conclusions: Phillips' parameter and surface elevation characteristics. . . . . . . . . . . . . . . . . 125

3.3 Directional spreading function . . . . . . . . . . . . . . . 127

3.3.1 Surface elevation distribution and input spectral directional spreading function. . . . . . . . . . . . . . 128

3.3.2 Input spectral directional spreading function and surface elevation wavenumber spectrum . . . . . . . . . . . . . . . 142

3.3.3 Input spectral directional spreading function and surface elevation Benjamin-Feir Index . . . . . . . . . . . . . . . 145

3.3.4 Input spectral directional spreading function and surface slope kurtosis . . . . . . . . . . . . . . 150

3.3.5 Conclusions: Directional spreading function and surface elevation characteristics . . . . . . . . . . . . . 158

4 Definition of wave height and wavelength 161

4.1 Introduction . . . . . . . . . . . . . . . . . . . . . . . 162

4.2 Zero-crossing method . . . . . . . . . . . . . . . . . . . 162

4.3 Lowest local minimum method . . . . . . . . . . . . . . . . . . . . 164

4.4 Nearest local minimum method . . . . . . . . . . . . . . . . 166

4.5 Comparison between wave definition methods . . . . . . . . . . 169

5 Wave height distributions and spectral parameters 193

5.1 Peak shape parameter . . . . . . . . . . . . . . . . . . . . 193 
5.1.1 Peak shape parameter and wave height distribution deviation from linear theory . . . . . . . . . . . . . . . . . 194

5.1.2 Peak shape parameter and the joint distribution between wave height and wavelength .............. 206

5.1.3 Peak shape parameter and wave length distribution . . . . . . 207

5.1.4 Peak shape parameter and wave height directionality . . . . . 221

5.1.5 Peak shape parameter and the spacing of large waves . . . . . 221

5.1.6 Conclusion: Peak shape parameter and wave height characteristics . . . . . . . . . . . . . . . . . 221

5.2 Effect of input Phillips' parameter on wave height characteristics . . . 235

5.2.1 Wave height distribution in wavefields of various Phillips parameters .......................... 235

5.2.2 Wave height directionality in wavefields of various Phillips parameters ...................... 236

5.2.3 The joint distribution between wave height and wavelength in wavefields of various Phillips parameters . . . . . . . . 236

5.2.4 Wavelength distribution in wavefields of various Phillips parameters .......................... 253

5.2.5 The spacing of large waves in wavefields of various Phillips pa-

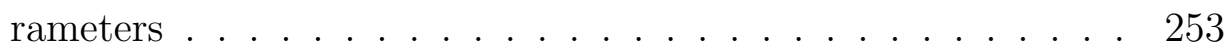

5.2.6 Conclusion: Phillips' parameter and wave height characteristics 253

5.3 Wave height characteristics and input spectral spreading angle . . . . 262

5.3.1 Wave height distribution and input spectral spreading angle . 262

5.3.2 Effect of input spectral directional spreading function on wave height exceedance probability . . . . . . . . . . . 262

5.3.3 Effect of input spectral directional spreading function on the joint distribution between wave height and wave length . . . . 267

5.3.4 Effect of input spectral directional spreading function on wave length distribution . . . . . . . . . . . . 267 
5.3.5 Effect of input spectral directional spreading function on large waves' spacing . . . . . . . . . . . . . . . . . . 280

5.3.6 Input spectral directional spreading function and observed wave travel directions. . . . . . . . . . . . . . . . . . . . . . . . 291

5.3.7 Conclusion: Input spectral directional spreading and wave height characteristics . . . . . . . . . . . . . . . . 291

6 Conclusion 299

6.1 Contributions . . . . . . . . . . . . . . . . . . . . . 299

6.2 Recommendations for further study . . . . . . . . . . . . . 304

6.2.1 Analytical investigation of the evolution of kurtosis . . . . . 305

6.2 .2 Experimental investigations . . . . . . . . . . . . 306

A Linear statistical theory of ocean surface elevation 309

B Non-linear statistical theory of ocean surface elevation 313

C Wave height distribution - linear statistical theory of wave height, $\begin{array}{ll}H \text { in a narrow band } & 319\end{array}$

D Non-linear statistical theory of wave height, H 323 


\section{List of Figures}

1-1 Structure of a random sea. [52] Linear theory stipulates that many plane progressive waves, (each moving in a unique direction, at a unique

phase and frequency), are superimposed to produce a random sea surface. 52

2-1 Orientation of axes in SNOW simulation realizations at every time step. The mean direction of wavefield propagation is the $x$-direction, the $y$-direction is perpendicular to the $x$-direction and the surface elevation height is measured in the $z$-direction. $[71] \ldots \ldots \ldots \ldots \ldots$

3-1 (In Color) Plot of surface elevation moments, variance, skewness and kurtosis, against time, $\left(t / T_{P}\right)$ in three nonlinear simulations of Cases $\mathbf{I}($ where $\gamma=1.0), \mathbf{B}$ (where $\gamma=3.3)$ and $\mathbf{H}($ where $\gamma=5.0) \ldots \ldots$.

3-2 (In Color) Horizontal axis shows non-dimensional surface elevation and vertical axis shows the cumulative distribution of such an elevation in the wavefield. Time evolution of theoretical (- - -) surface elevation distribution, and observed surface elevation distribution data $(++++)$ in a nonlinear simulation of Case $\mathbf{I}$ (where $\gamma=1.0)$. Each line pair represents one time step. Earlier times (from $t=0 T_{p}$ ) are the darkest lines. Later times (up to $t=100 T_{p}$ ) are the brightest lines. . 
3-3 (In Color) Horizontal axis shows non-dimensional surface elevation and vertical axis shows the cumulative distribution of such an elevation in the wavefield. Time evolution of theoretical (- - - ) surface elevation distribution, and observed surface elevation distribution data $(++++)$ in nonlinear simulations of Case $\mathbf{B}($ where $\gamma=3.3)$. Each line pair represents one time step. Earlier times (from $t=0 T_{p}$ ) are the darkest lines. Later times (up to $t=100 T_{p}$ ) are the brightest lines. .

3-4 (In Color) Horizontal axis shows non-dimensional surface elevation and vertical axis shows the cumulative distribution of such an elevation in the wavefield. Time evolution of theoretical (- - - ) surface elevation distribution, and observed surface elevation distribution data $(++++)$ in nonlinear simulations of Case $\mathbf{H}($ where $\gamma=5.0)$. Each line pair represents one time step. Earlier times (from $t=0 T_{p}$ ) are the darkest lines. Later times (up to $t=100 T_{p}$ ) are the brightest lines. .

3-5 (In Color) Horizontal axis shows non-dimensional surface elevation and vertical axis shows the cumulative distribution of such an elevation in the wavefield. Cases I, B and H surface elevation distribution at time, $t=20 T_{p}$. Comparison between three cases in nonlinear simulations of cases where $\gamma=1.0$ (magenta), $\gamma=3.3$ (green), and $\gamma=5.0$ (orange) between theoretical (- - - ) surface elevation distribution, and observed surface elevation distribution data $(++++)$. Each line pair represents one case. . . . . . . . . . . . . . . . .

3-6 (In Color) Horizontal axis shows non-dimensional surface elevation and vertical axis shows the cumulative distribution of such an elevation in the wavefield. Cases I, B and H surface elevation distribution at time, $t=50 T_{p}$. Comparison between three cases in nonlinear simulations of cases where $\gamma=1.0$ (magenta), $\gamma=3.3$ (green), and $\gamma=5.0$ (orange) between theoretical (- - - ) surface elevation distribution, and observed surface elevation distribution data $(++++)$. Each line pair represents one case. . . . . . . . . . . . . . . . . . 
3-7 (In Color) Horizontal axis shows non-dimensional surface elevation and vertical axis shows the cumulative distribution of such an elevation in the wavefield. Cases I, B and H surface elevation distribution at time, $t=100 T_{p}$. Comparison between three cases in nonlinear simulations of cases where $\gamma=1.0$ (magenta), $\gamma=3.3$ (green), and $\gamma=5.0$ (orange) between theoretical (- - - ) surface elevation distribution, and observed surface elevation distribution data $(++++)$ at times $t=20 T_{p}, 50 T_{p}$, and $100 T_{p}$. Each line pair represents one case. . . . . . . . . 76

3-8 Horizontal axis shows non-dimensional surface elevation and vertical axis shows the cumulative distribution of such an elevation in the wavefield where $\gamma=3.3$ ar $t=100 T_{p}$. Kurtosis is more apparent than skewness for the large values of surface elevation (seen on the far right of the graphic) as the observed data series $(+++)$ falls below the Gaussian theoretical line(- - ). . . . . . . . . . . . . . .

3-9 Horizontal axis shows non-dimensional surface elevation and vertical axis shows the cumulative distribution of such an elevation in the wavefield where $\gamma=5.0$ at time $t=100 T_{p}$. Kurtosis is more apparent than skewness for the large values of surface elevation (seen on the far right of the graphic) as the observed data series $(+++)$ falls below the Gaussian theoretical line(- - ). . . . . . . . . . . . . . .

3-10 Horizontal axis shows non-dimensional surface elevation and vertical axis shows the cumulative distribution of such an elevation in the wavefield where $\gamma=1.0$ at time $t=100 T_{p}$. Kurtosis is more apparent than skewness for the large values of surface elevation (seen on the far right of the graphic) as the observed data series $(+++)$ falls below the

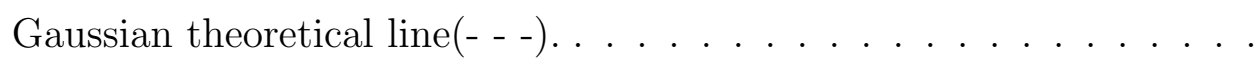


3-11 (In Color) Horizontal axis shows non-dimensional surface elevation and vertical axis shows the cumulative distribution of such an elevation in the wavefield. We compare a linear followed by a non-linear simulation of Case $\mathbf{H}$ (where $\gamma=5.0$ ) observed surface elevation distribution data $(++++)$. The order of nonlinearity has measurable effects on surface elevation wavenumber spectrum. . . . . . . . . . . . .

3-12 (In Color) Horizontal axis depicts non-dimensional surface elevation and vertical axis depicts probability. Nonlinear simulation of Case I (where $\gamma=1.0$ ), observed surface elevation distribution data at time, $t=0 T_{p}$ compared to Gram Charlier (G-C) distributions Order 1, 2

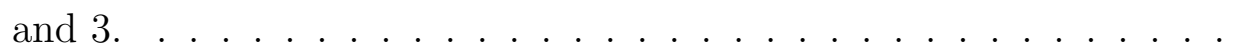

3-13 (In Color) Horizontal axis depicts non-dimensional surface elevation and vertical axis depicts probability. Nonlinear simulation of Case I (where $\gamma=1.0$ ), observed surface elevation distribution data at time, $t=50 T_{p}$ compared to Gram Charlier (G-C) distributions Order 1, 2

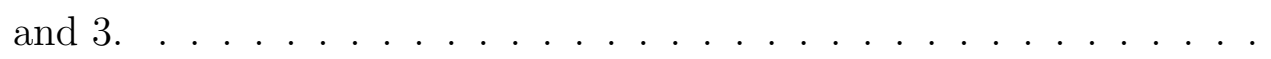

3-14 (In Color) Horizontal axis depicts non-dimensional surface elevation and vertical axis depicts probability. Nonlinear simulation of Case I (where $\gamma=1.0$ ), observed surface elevation distribution data at time, $t=100 T_{p}$ compared to Gram Charlier (G-C) distributions Order 1, 2

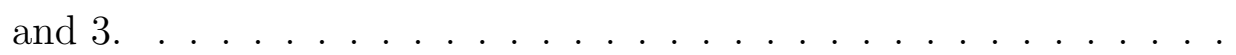

3-15 (In Color) Horizontal axis depicts non-dimensional surface elevation and vertical axis depicts probability. Case $\mathbf{B}$ (where $\gamma=3.3$ ), observed surface elevation distribution data at time, $t=50 T_{p}$ compared to Gram Charlier (G-C) distributions Order 1, 2 and 3.. . . . . . . . . 86

3-16 (In Color) Horizontal axis depicts non-dimensional surface elevation and vertical axis depicts probability. Case $\mathbf{H}$ (where $\gamma=5.0$ ), observed surface elevation distribution data at time, $t=50 T_{p}$ compared to Gram Charlier (G-C) distributions Order 1, 2 and 3. . . . . . . . 
3-17 (In Color) Wavenumber spectrum time evolution in a nonlinear wavefield simulation of Case $\mathbf{I}$ (where $\gamma=1.0$ ). Time $\left(t / T_{p}\right)$ is on the horizontal axis, wavenumber $\lambda_{p} k$ on the vertical axis and spectral energy level is represented in the color bar. . . . . . . . . . . . .

3-18 (In Color) Wavenumber spectrum time evolution in a nonlinear wavefield simulation of Case $\mathbf{B}$ (where $\gamma=3.3$ ). Time $\left(t / T_{p}\right)$ is on the horizontal axis, wavenumber $\lambda_{p} k$ on the vertical axis and spectral energy level is represented in the color bar.. . . . . . . . . . . . .

3-19 (In Color) Wavenumber spectrum time evolution in a nonlinear wavefield simulation of Case $\mathbf{H}$ (where $\gamma=5.0$ ). Time $\left(t / T_{p}\right)$ is on the horizontal axis, wavenumber $\lambda_{p} k$ on the vertical axis and spectral energy level is represented in the color bar. . . . . . . . . . . . .

3-20 (In Color) Wavenumber spectrum time evolution in linear and nonlinear wavefield simulation of Case $\mathbf{I}$ where $\gamma=1.0$. Time $\left(t / T_{p}\right)$ on the horizontal axis, wavenumber $\lambda_{p} k$ on the vertical axis and spectral energy level represented in color bar. The order of nonlinearity has measurable effects on surface elevation wavenumber spectrum. . . . .

3-21 (In Color) Wavenumber spectrum time evolution in linear and nonlinear wavefield simulation of Case $\mathbf{H}$ where $\gamma=5.0$. Time $\left(t / T_{p}\right)$ on the horizontal axis, wavenumber $\lambda_{p} k$ on the vertical axis and spectral energy level represented in color bar. The order of nonlinearity has measurable effects on surface elevation wavenumber spectrum. . . .

3-22 (In Color)Plot of surface elevation spectral moments against time $\left(t / T_{p}\right)$ where $m_{0}$ is the first spectral moment, $m_{1}$ is the second spectral moment, $m_{2}$ is the third spectral moment, and BFI is a scaled version of the BFI defined in Section 3.1.4. We use non-linear simulations of Cases $\mathbf{I}($ where $\gamma=1.0), \mathbf{B}($ where $\gamma=3.3)$ and $\mathbf{H}($ where $\gamma=5.0)$. 
3-23 (In Color) Plot of surface elevation spectral moments against time $\left(t / T_{p}\right)$ where $m_{0}$ is the first spectral moment, $m_{1}$ is the second spectral moment, $m_{2}$ is the third spectral moment, and BFI is a scaled version of the BFI defined in Section 3.1.4. We use linear simulations of Cases I where $\gamma=1.0$, and $\mathbf{H}$ where $\gamma=5.0 \ldots \ldots \ldots$

3-24 (In Color) Surface elevation slope in the x-direction is defined as: $\eta-$

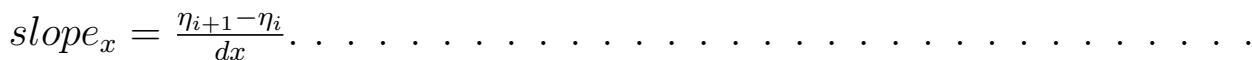

3-25 (In Color) Plot of kurtosis (on vertical axis) against time (on horizontal axis), $\left(t / T_{p}\right)$ in non-linear simulations of Cases $\mathbf{I}$ (where $\left.\gamma=1.0\right), \mathbf{B}$ (where $\gamma=3.3$ ) and $\mathbf{H}$ (where $\gamma=5.0$ ). We see the distinct impact of peak shape parameter on the kurtosis of surface elevation and the kurtosis of surface elevation slope in a nonlinear wavefield. . . . . . .

3-26 (In Color) Plot of kurtosis (on vertical axis) against time (on horizontal axis), $\left(t / T_{p}\right)$ in linear simulations of Cases $\mathbf{I}$ (where $\left.\gamma=1.0\right)$ and $\mathbf{H}$ (where $\gamma=5.0$ ). We see the negligible impact of peak shape parameter on the kurtosis of surface elevation and the kurtosis of surface elevation slope in a linear wavefield. . . . . . . . . . . . . . . . .

3-27 (In Color) Plots of the statistical moments (kurtosis, skewness and variance) of surface elevation against time $\left(t / T_{p}\right)$ in nonlinear simulations of Cases $\mathbf{E}$, where $\alpha=0.0032$, Case $\mathbf{B}$ where $\alpha=0.0160$ and

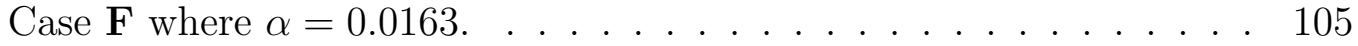

3-28 (In Color) Horizontal axis shows non-dimensional surface elevation and vertical axis shows the cumulative distribution of such an elevation in the wavefield. Time evolution of theoretical (- - - ) surface elevation distribution, and observed surface elevation distribution data $(++++)$ in a nonlinear simulation of Case $\mathbf{E}($ where $\alpha=0.0032)$. Each line pair represents one time step. Earlier times (from $t=0 T_{p}$ ) are the darkest lines. Later times (up to $t=100 T_{p}$ ) are the brightest lines. . . . . . . . . . . . . . . . . . . . 107 
3-29 (In Color) Horizontal axis shows non-dimensional surface elevation and vertical axis shows the cumulative distribution of such an elevation in the wavefield. Time evolution of theoretical (- - - ) surface elevation distribution, and observed surface elevation distribution data $(++++)$ in a nonlinear simulation of Case B (where $\alpha=0.0160)$. Each line pair represents one time step. Earlier times (from $t=0 T_{p}$ ) are the darkest lines. Later times (up to $t=100 T_{p}$ ) are the brightest lines. . . . . . . . . . . . . . . . . . . 108

3-30 (In Color) Horizontal axis shows non-dimensional surface elevation and vertical axis shows the cumulative distribution of such an elevation in the wavefield. Time evolution of theoretical (- - - ) surface elevation distribution, and observed surface elevation distribution data $(++++)$ in a nonlinear simulation of Case $\mathbf{F}$ (where $\alpha=0.0163)$. Each line pair represents one time step. Earlier times (from $t=0 T_{p}$ ) are the darkest lines. Later times (up to $t=100 T_{p}$ ) are the brightest

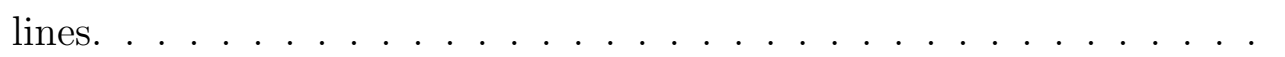

3-31 (In Color) Horizontal axis depicts non-dimensional surface elevation and vertical axis depicts probability. Observed data from nonlinear simulation of Case $\mathbf{E}$ (where $\alpha=0.0032$ ) at time, $t=0 T_{p}$ compared to Gram Charlier distributions Order 1, 2 and 3. . . . . . . . . . . 110

3-32 (In Color) Horizontal axis depicts non-dimensional surface elevation and vertical axis depicts probability. Observed data from nonlinear simulation of Case $\mathbf{E}$ (where $\alpha=0.0032$ ) at time, $t=50 T_{p}$ compared to Gram Charlier distributions Order 1, 2 and 3. . . . . . . .

3-33 (In Color) Horizontal axis depicts non-dimensional surface elevation and vertical axis depicts probability. Observed data from nonlinear simulation of Case $\mathbf{E}$ (where $\alpha=0.0032$ ) at time, $t=100 T_{p}$ compared to Gram Charlier distributions Order 1, 2 and 3. . . . . . . . . 
3-34 (In Color) Horizontal axis depicts non-dimensional surface elevation and vertical axis depicts probability. Observed data from nonlinear simulation of Case $\mathbf{F}$ (where $\alpha=0.0163$ ) at time, $t=0 T_{p}$ compared to Gram Charlier distributions Order 1, 2 and 3. . . . . . . . .

3-35 (In Color) Horizontal axis depicts non-dimensional surface elevation and vertical axis depicts probability. Observed data from nonlinear simulation of Case $\mathbf{F}$ (where $\alpha=0.0163$ ) at time, $t=50 T_{p}$ compared to Gram Charlier distributions Order 1, 2 and 3.. . . . . . . . .

3-36 (In Color) Horizontal axis depicts non-dimensional surface elevation and vertical axis depicts probability. Observed data from nonlinear simulation of Case $\mathbf{F}$ (where $\alpha=0.0163$ ) at time, $t=100 T_{p}$ compared to Gram Charlier distributions Order 1, 2 and 3. . . . . . . .

3-37 (In Color) Wavenumber spectrum time evolution in a nonlinear wavefield simulation of Case $\mathbf{E}$ (where $\alpha=0.0032$ ). Time $\left(t / T_{p}\right)$ is on the horizontal axis, wavenumber $\lambda_{p} k$ on the vertical axis and spectral energy level is represented in the color bar. . . . . . . . . . . . . . 118

3-38 (In Color) Wavenumber spectrum time evolution in a nonlinear wavefield simulation of Case $\mathbf{B}$ (where $\alpha=0.0160$ ). Time $\left(t / T_{p}\right)$ is on the horizontal axis, wavenumber $\lambda_{p} k$ on the vertical axis and spectral energy level is represented in the color bar. . . . . . . . . . . . .

3-39 (In Color) Wavenumber spectrum time evolution in a nonlinear wavefield simulation of Case $\mathbf{F}$ (where $\alpha=0.0163$ ). Time $\left(t / T_{p}\right)$ is on the horizontal axis, wavenumber $\lambda_{p} k$ on the vertical axis and spectral energy level is represented in the color bar. . . . . . . . . . . . . . . 120

3-40 (In Color) Wavenumber spectrum time evolution in a linear equivalent simulation of Case $\mathbf{E}$ where $\alpha=0.0032$. We plot time $\left(t / T_{p}\right)$ on the horizontal axis, wavenumber $k$ on the vertical axis and we represent spectral energy levels in color (see color bars). There is very little difference between the wavenumber spectrum of the linear and nonlinear Case E. . . . . . . . . . . . . . . . . . . . 
3-41 (In Color) For nonlinear simulations of Case $\mathbf{E}$ where $\alpha=0.0032$, Case B where $\alpha=0.0160$ and Case $\mathbf{F}$ where $\alpha=0.0163$, we plot surface elevation spectral moments against time $\left(t / T_{p}\right)$, where $m_{0}$ is the first spectral moment, $m_{1}$ is the second spectral moment, $m_{2}$ is the third spectral moment, and BFI is a scaled version of the BFI defined in Section 3.1.4 . . . . . . . . . . . . . . . . . . .

3-42 (In Color) For a linear simulation of Case $\mathbf{E}$ where $\alpha=0.0032$, we plot surface elevation spectral moments against time $\left(t / T_{p}\right)$ where $m_{0}$ is the first spectral moment, $m_{1}$ is the second spectral moment, $m_{2}$ is the third spectral moment, and BFI is a scaled version of the BFI defined in Section 3.1.4. . . . . . . . . . . . . . . . . 123

3-43 (In Color) Plot of kurtosis (on vertical axis) against time (on horizontal axis), $\left(t / T_{p}\right)$ in non-linear simulations of Case $\mathbf{E}$ where $\alpha=0.0032$, Case $\mathbf{B}$ where $\alpha=0.0160$ and Case $\mathbf{F}$ where $\alpha=0.0163$. Case $\mathbf{E}$ where $\alpha=0.0032$, has the appearance of a linear wavefield (see Figure 3-26) while Cases $\mathbf{B}$ and $\mathbf{F}$ with higher $\alpha$-values display typical nonlinear properties. . . . . . . . . . . . . . . . . . 124

3-44 (In Color) Spectral moments are displayed on the vertical axes while time $\left(t / T_{p}\right)$ is displayed on the horizontal axes. Variance, skewness and kurtosis of surface elevation is plotted against time $\left(t / T_{p}\right)$ in nonlinear simulations of Cases $\mathbf{J}$ where $\theta=180^{\circ}, \mathbf{A}$ where $\theta=80^{\circ}, \mathbf{B}$ where $\theta=40^{\circ}, \mathbf{M}$ where $\theta=30^{\circ}, \mathbf{K}$ where $\theta=20^{\circ}, \mathbf{L}$ where $\theta=10^{\circ}$ and $\mathbf{G}$ where $\theta=1^{o}$. . . . . . . . . . . . . . . . . . . . . . 131

3-45 (In Color) Horizontal axis shows non-dimensional surface elevation and vertical axis shows the cumulative distribution of such an elevation in the wavefield. Theoretical surface elevation distribution (- - - ), and observed surface elevation distribution data $(++++)$ in nonlinear simulations of Cases $\mathbf{J}$ where $\theta=180^{\circ}$, A where $\theta=80^{\circ}, \mathbf{B}$ where $\theta=40^{\circ}, \mathbf{M}$ where $\theta=30^{\circ}, \mathbf{K}$ where $\theta=20^{\circ}, \mathbf{L}$ where $\theta=10^{\circ}$ and $\mathbf{G}$ where $\theta=1^{o}$ at time $t=0 T_{p}$. Each line pair represents one case. . . . 
3-46 (In Color) Horizontal axis shows non-dimensional surface elevation and vertical axis shows the cumulative distribution of such an elevation in the wavefield. Theoretical surface elevation distribution (- - - ), and observed surface elevation distribution data $(++++)$ in nonlinear simulations of Cases $\mathbf{J}$ where $\theta=180^{\circ}$, A where $\theta=80^{\circ}, \mathbf{B}$ where $\theta=40^{\circ}, \mathbf{M}$ where $\theta=30^{\circ}, \mathbf{K}$ where $\theta=20^{\circ}, \mathbf{L}$ where $\theta=10^{\circ}$ and $\mathbf{G}$ where $\theta=1^{o}$ at time $t=50 T_{p}$. Each line pair represents one case. . .

3-47 (In Color) Horizontal axis shows non-dimensional surface elevation and vertical axis shows the cumulative distribution of such an elevation in the wavefield. Theoretical surface elevation distribution (- - - ), and observed surface elevation distribution data $(++++)$ in nonlinear simulations of Cases $\mathbf{J}$ where $\theta=180^{\circ}$, A where $\theta=80^{\circ}$, B where $\theta=40^{\circ}, \mathbf{M}$ where $\theta=30^{\circ}, \mathbf{K}$ where $\theta=20^{\circ}, \mathbf{L}$ where $\theta=10^{\circ}$ and $\mathbf{G}$ where $\theta=1^{o}$ at time $t=100 T_{p}$. Each line pair represents one case. .

3-48 Horizontal axis shows non-dimensional surface elevation and vertical axis shows the cumulative distribution of such an elevation in the unidirectional case (with $\theta=1^{o}$ ) at $t=20 T_{p}$. Trough occurrences on the far left of the graphic far exceed linear theory for this low value of directional spreading. Observed data series $(+++)$; Theoretical line $(--) . \ldots \ldots \ldots \ldots \ldots$

3-49 Horizontal axis shows non-dimensional surface elevation and vertical axis shows the cumulative distribution of such an elevation in the directional case (with $\theta=10^{\circ}$ ) at $t=20 T_{p}$. Kurtosis is more apparent than skewness for the large values of surface elevation on the far right of the graphic. Trough occurrences on the far left of the graphic far exceed linear theory for these fairly low values of directional spreading. Observed data series $(+++)$; Theoretical line $\left(--_{-}\right)$. . . . . . . . . 136 
3-50 Horizontal axis shows non-dimensional surface elevation and vertical axis shows the cumulative distribution of such an elevation in the directional case (with $\theta=20^{\circ}$ ) at $t=20 T_{p}$. Kurtosis is more apparent than skewness for the large values of surface elevation on the far right of the graphic. Trough occurrences on the far left of the graphic far exceed linear theory for these fairly low values of directional spreading. Observed data series $(+++)$; Theoretical line(- - $)$. . . . . . . . . .

3-51 Horizontal axis shows non-dimensional surface elevation and vertical axis shows the cumulative distribution of such an elevation in the directional case (with $\theta=30^{\circ}$ ) at $t=20 T_{p}$. Trough occurrences on the far left of the graphic are far less than linear theory in these examples with fairly high directional spreading values. Observed data series $(+++)$; Theoretical line(- - ). . . . . . . . . . . . . . . . .

3-52 Horizontal axis shows non-dimensional surface elevation and vertical axis shows the cumulative distribution of such an elevation in the directional case (with $\theta=40^{\circ}$ ) at $t=20 T_{p}$. Kurtosis is more apparent than skewness for the large values of surface elevation on the far right of the graphic. Trough occurrences on the far left of the graphic are far less than linear theory in these examples with fairly high directional spreading values. Observed data series $(+++)$; Theoretical line $(---)$.

3-53 Horizontal axis shows non-dimensional surface elevation and vertical axis shows the cumulative distribution of such an elevation in the directional case (with $\theta=80^{\circ}$ ) at $t=20 T_{p}$. Kurtosis is less apparent than skewness for the large values of surface elevation on the far right of the graphic. Trough occurrences on the far left of the graphic are far less than linear theory in these examples with fairly high directional spreading values. Observed data series $(+++)$; Theoretical line $(---)$. 
3-54 Horizontal axis shows non-dimensional surface elevation and vertical axis shows the cumulative distribution of such an elevation in the directional case (with $\theta=180^{\circ}$ ) at $t=20 T_{p}$. Kurtosis is less apparent than skewness for the large values of surface elevation on the far right of the graphic. Trough occurrences on the far left of the graphic are far less than linear theory in these examples with fairly high directional spreading values. Observed data series $(+++)$; Theoretical line $(---)$.

3-55 (In Color) Horizontal axis depicts non-dimensional surface elevation and vertical axis depicts probability. Nonlinear simulation of Cases with input spectral directional spreading function $D(\theta)$ having $\theta=1^{o}$ and $\theta=180^{\circ}$, at time $t=50 T_{p}$. Gram Charlier distributions Order 1, 2 and 3 compared with observed SNOW nonlinear surface elevation data distribution. . . . . . . . . . . . . . . . . . .

3-56 (In Color) Horizontal axis depicts non-dimensional surface elevation and vertical axis depicts probability. Nonlinear simulation of Cases with input spectral directional spreading function $D(\theta)$ having $\theta=1^{o}$ and $\theta=180^{\circ}$, at time $t=100 T_{p}$. Gram Charlier distributions Order 1, 2 and 3 compared with observed SNOW nonlinear surface elevation data distribution. . . . . . . . . . . . . . . . . . . . . . . 144

3-57 (In Color) Wavenumber spectrum time evolution in nonlinear wavefield simulation Cases $\mathbf{J}$ where $\theta=180^{\circ}$ and Case $\mathbf{A}$ where $\theta=80^{\circ}$. Time $\left(t / T_{p}\right)$ is on the horizontal axis, wavenumber $\lambda_{p} k$ on the vertical axis and spectral energy level is represented in the color bar. . . . . . . . . 146

3-58 (In Color) Wavenumber spectrum time evolution in nonlinear wavefield simulation Cases $\mathbf{B}$ where $\theta=40^{\circ}$, and $\mathbf{M}$ where $\theta=30^{\circ}$. Time $\left(t / T_{p}\right)$ is on the horizontal axis, wavenumber $\lambda_{p} k$ on the vertical axis and spectral energy level is represented in the color bar. . . . . . . . . . . 147 
3-59 (In Color) Wavenumber spectrum time evolution in nonlinear wavefield simulation in Case $\mathbf{K}$ where $\theta=20^{\circ}$, and Case $\mathbf{L}$ where $\theta=10^{\circ}$. Time $\left(t / T_{p}\right)$ is on the horizontal axis, wavenumber $\lambda_{p} k$ on the vertical axis and spectral energy level is represented in the color bar. . . . . . . .

3-60 (In Color) Wavenumber spectrum time evolution in nonlinear wavefield simulation in Case $\mathbf{G}$ where $\theta=1^{o}$. Time $\left(t / T_{p}\right)$ is on the horizontal axis, wavenumber $\lambda_{p} k$ on the vertical axis and spectral energy level is represented in the color bar. . . . . . . . . . . . . . . . .

3-61 (In Color) Plot of surface elevation spectral moments against time $\left(t / T_{p}\right)$ where $m_{0}$ is the first spectral moment, $m_{1}$ is the second spectral moment, $m_{2}$ is the third spectral moment, and BFI is a scaled version of the BFI defined in Section 3.1.4. We use nonlinear simulations of Cases $\mathbf{J}$ where $\theta=180^{\circ}, \mathbf{A}$ where $\theta=80^{\circ}, \mathbf{B}$ where $\theta=40^{\circ}, \mathbf{M}$ where $\theta=30^{\circ}, \mathbf{K}$ where $\theta=20^{\circ}, \mathbf{L}$ where $\theta=10^{\circ}$ and $\mathbf{G}$ where $\theta=1^{\circ}$.

3-62 (In Color) Plot of surface elevation spectral moments against time $\left(t / T_{p}\right)$ where $m_{0}$ is the first spectral moment, $m_{1}$ is the second spectral moment, $m_{2}$ is the third spectral moment, and BFI is a scaled version of the BFI defined in Section 3.1.4. We use nonlinear simulations of Cases $\mathbf{J}$ where $\theta=180^{\circ}$, A where $\theta=80^{\circ}$, $\mathbf{B}$ where $\theta=40^{\circ}$ and $\mathbf{M}$ where $\theta=30^{\circ} \ldots \ldots \ldots \ldots \ldots \ldots \ldots$

3-63 (In Color) Plot of surface elevation spectral moments against time $\left(t / T_{p}\right)$ where $m_{0}$ is the first spectral moment, $m_{1}$ is the second spectral moment, $m_{2}$ is the third spectral moment, and BFI is a scaled version of the BFI defined in Section 3.1.4. We use nonlinear simulations of Cases $\mathbf{K}$ where $\theta=20^{\circ}, \mathbf{L}$ where $\theta=10^{\circ}$, and $\mathbf{G}$ where $\theta=1^{\circ} . \quad \ldots 153$

3-64 (In Color) Plot of surface elevation kurtosis, surface elevation x-slope kurtosis and surface elevation y-slope kurtosis (on vertical axis) against time (on horizontal axis), $\left(t / T_{p}\right)$ in non-linear simulations of Cases $\mathbf{J}$ where $\theta=180^{\circ}, \mathbf{A}$ where $\theta=80^{\circ}, \mathbf{B}$ where $\theta=40^{\circ}$ and $\mathbf{M}$ where $\theta=30^{\circ} \ldots \ldots \ldots \ldots \ldots \ldots \ldots$ 
3-65 (In Color) Plot of surface elevation kurtosis, surface elevation x-slope kurtosis and surface elevation y-slope kurtosis (on vertical axis) against time (on horizontal axis), $\left(t / T_{p}\right)$ in non-linear simulations of Cases $\mathbf{K}$ where $\theta=20^{\circ}, \mathbf{L}$ where $\theta=10^{\circ}$ and $\mathbf{G}$ where $\theta=1^{\circ} \ldots \ldots \ldots 157$

4-1 Wave definitions are simple with point records. . . . . . . . . . . 163

4-2 (In Color) Zero crossing method of wave height definition and wave length definition involves taking the wave field cross-sections in the mean direction of propagation spread one peak wavelength apart from each other, and finding zero-crossing minima and maxima along that cross section. [12] [4]. . . . . . . . . . . . . . . . . . 165

4-3 All local maxima $\left(\frac{\partial \eta}{\partial x}=0, \frac{\partial \eta}{\partial y}=0, \frac{\partial^{2} \eta}{\partial x^{2}}<0\right.$ and $\left.\frac{\partial^{2} \eta}{\partial y^{2}}<0\right)$ and all the local minima $\left(\frac{\partial \eta}{\partial x}=0, \frac{\partial \eta}{\partial y}=0, \frac{\partial^{2} \eta}{\partial x^{2}}>0\right.$ and $\left.\frac{\partial^{2} \eta}{\partial y^{2}}>0\right)$ are defined in the

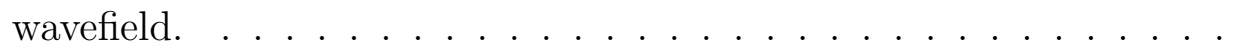

4-4 Surface elevation is depicted on the vertical axes and time is depicted on the horizontal axes. Non-narrow-band wave heights are captured in the half-cycle excursion method of wave height definition [41]. . . . .

4-5 Probability is depicted on the vertical axis and wave height is depicted on the horizontal axis. Case $\mathbf{J}$ with peak shape parameter $\gamma=3.3$, Phillips' parameter, $\alpha=0.0160$ and input spreading angle, $\theta=180^{\circ}$ wave height probability distribution when $t=0 T_{p}$ where wave heights are defined by the zero-crossing method. . . . . . . . . . . .

4-6 Probability is depicted on the vertical axis and wave height is depicted on the horizontal axis. Case $\mathbf{J}$ with peak shape parameter $\gamma=3.3$, Phillips' parameter, $\alpha=0.0160$ and input spreading angle, $\theta=180^{\circ}$ wave height probability distribution when $t=0 T_{p}$ where wave heights are defined by the local-maximum-to-lowest-local-minimum method. 
4-7 Probability is depicted on the vertical axis and wave height is depicted on the horizontal axis. Case $\mathbf{J}$ with peak shape parameter $\gamma=3.3$, Phillips' parameter, $\alpha=0.0160$ and input spreading angle, $\theta=180^{\circ}$ wave height probability distribution when $t=0 T_{p}$ where wave heights are defined by the local-maximum-to-nearest-local-minimum method.

4-8 Probability is depicted on the vertical axis and wave height is depicted on the horizontal axis. Case $\mathbf{K}$ with peak shape parameter $\gamma=3.3$, Phillips' parameter, $\alpha=0.0160$ and input spreading angle, $\theta=20^{\circ}$ wave height probability distribution when $t=0 T_{p}$ where wave heights are defined by the zero-crossing method. . . . . . . . . . . .

4-9 Probability is depicted on the vertical axis and wave height is depicted on the horizontal axis. Case $\mathbf{K}$ with peak shape parameter $\gamma=3.3$, Phillips' parameter, $\alpha=0.0160$ and input spreading angle, $\theta=20^{\circ}$ wave height probability distribution when $t=0 T_{p}$ where wave heights are defined by the local-maximum-to-lowest-local-minimum method. .

4-10 Probability is depicted on the vertical axis and wave height is depicted on the horizontal axis. Case $\mathbf{K}$ with peak shape parameter $\gamma=3.3$, Phillips' parameter, $\alpha=0.0160$ and input spreading angle, $\theta=20^{\circ}$ wave height probability distribution when $t=0 T_{p}$ where wave heights are defined by the local-maximum-to-nearest-local-minimum method.

4-11 Polar coordinates are used to represent wave height, $H$ in the length of the vector and the wave travel direction, $\Theta$ in the tilt of the vector around the origin. Case $\mathbf{J}$ with peak shape parameter $\gamma=3.3$, Phillips' parameter, $\alpha=0.0160$ and input spreading angle, $\theta=180^{\circ}$ at time, $t=0 T_{p}$ large wave heights' directionality where wave heights are defined by the zero-crossing method when $t=0 T_{p} \ldots \ldots 178$ 
4-12 Polar coordinates represent wave height, $H$ in the length of the vector and the wave travel direction, $\Theta$ in the tilt of the vector around the origin. Case $\mathbf{J}$ with peak shape parameter $\gamma=3.3$, Phillips' parameter, $\alpha=0.0160$ and input spreading angle, $\theta=180^{\circ}$ at time, $t=0 T_{p}$ large wave heights' directionality where wave heights are defined by the lowest-local-minimum method when $t=0 T_{p} \ldots \ldots$.

4-13 Polar coordinates represent wave height, $H$ in the length of the vector and the wave travel direction, $\Theta$ in the tilt of the vector around the origin. Case $\mathbf{J}$ with peak shape parameter $\gamma=3.3$, Phillips' parameter, $\alpha=0.0160$ and input spreading angle, $\theta=180^{\circ}$ at time, $t=0 T_{p}$ large wave heights' directionality where wave heights are defined by the nearest-local-minimum method when $t=0 T_{p} \ldots . .$.

4-14 Polar coordinates represent wave height, $H$ in the length of the vector and the wave travel direction, $\Theta$ in the tilt of the vector around the origin. Case $\mathbf{K}$ with peak shape parameter $\gamma=3.3$, Phillips' parameter, $\alpha=0.0160$ and input spreading angle, $\theta=20^{\circ}$ at time, $t=0 T_{p}$ large wave directionality where waves are defined by the zero-crossing

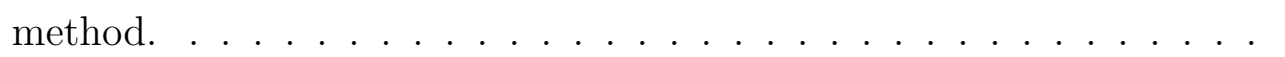

4-15 Polar coordinates represent wave height, $H$ in the length of the vector and the wave travel direction, $\Theta$ in the tilt of the vector around the origin. Case $\mathbf{K}$ with peak shape parameter $\gamma=3.3$, Phillips' parameter, $\alpha=0.0160$ and input spreading angle, $\theta=20^{\circ}$ at time, $t=0 T_{p}$ large wave directionality where waves are defined by the lowest-localminimum method. . . . . . . . . . . . . . .

4-16 Polar coordinates represent wave height, $H$ in the length of the vector and the wave travel direction, $\Theta$ in the tilt of the vector around the origin. Case $\mathbf{K}$ with peak shape parameter $\gamma=3.3$, Phillips' parameter, $\alpha=0.0160$ and input spreading angle, $\theta=20^{\circ}$ at time, $t=0 T_{p}$ large wave directionality where waves are defined by the nearest-localminimum method. . . . . . . . . . . . . . . 
4-17 Probability is depicted on the vertical axis and wave height is depicted on the horizontal axis. Case $\mathbf{J}$ with peak shape parameter $\gamma=3.3$, Phillips' parameter, $\alpha=0.0160$ and input spreading angle, $\theta=180^{\circ}$ wave height probability distribution from $t=0 T_{p}$ to $t=100 T_{p}$ where wave heights are defined by two 3D methods. . . . . . . . . . .

4-18 Probability is depicted on the vertical axis and wave height is depicted on the horizontal axis in Case $\mathbf{K}$ with peak shape parameter $\gamma=3.3$, Phillips' parameter, $\alpha=0.0160$ and input spreading angle, $\theta=20^{\circ}$ wave height probability distribution from $t=0 T_{p}$ to $t=100 T_{p}$ where wave heights are defined by two 3D methods. . . . . . . . . . . . .

4-19 Probability is depicted on the vertical axis and wave length is depicted on the horizontal axis in Case $\mathbf{J}$ with peak shape parameter $\gamma=3.3$, Phillips' parameter, $\alpha=0.0160$ and input spreading angle, $\theta=180^{\circ}$ from $t=0 T_{p}$ to $t=100 T_{p}$ where wave lengths are defined by three methods. . . . . . . . . . . . . . . . . .

4-20 Probability is depicted on the vertical axis and wave length is depicted on the horizontal axis in Case $\mathbf{K}$ with peak shape parameter $\gamma=3.3$, Phillips' parameter, $\alpha=0.0160$ and input spreading angle, $\theta=20^{\circ}$ from $t=0 T_{p}$ to $t=100 T_{p}$ where wave lengths are defined by three methods. . . . . . . . . . . . . . . . . . . . . 188

4-21 Probability is depicted on the vertical axis and wave length is depicted on the horizontal axis. Lowest minimum method wave lengths compared for Cases $\mathbf{K}$ and $\mathbf{J} \ldots \ldots \ldots \ldots . . \ldots . . . \ldots$

4-22 Probability is depicted on the vertical axis and wave length is depicted on the horizontal axis. Nearest minimum method wavelengths (3D analogue of non-narrow-band half-cycle excursion method) compared for Cases $\mathbf{K}$ and $\mathbf{J} . \ldots \ldots \ldots \ldots \ldots$ 
5-1 (In Color) (Left) Horizontal axis shows non-dimensional wave height and vertical axis shows the probability of occurrence of heights in the wavefield. Thick, brown line represents Rayleigh distribution. Other lines represent Case $\mathbf{I}$ where $\gamma=1$.0, each such line representing one time step. Earlier times (from $t=0 T_{p}$ ) are the darkest lines. Later times (up to $t=100 T_{p}$ ) are the brightest lines. (Right) Horizontal axis shows non-dimensional wave height and vertical axis shows a probability ratio. Each line represents the ratio between a Case I time-step line and the Rayleigh distribution. . . . . . . . . . . . . . . . . 195

5-2 (In Color) (Left) Horizontal axis shows non-dimensional wave height and vertical axis shows the probability of occurrence of heights in the wavefield. Thick, brown line represents Rayleigh distribution. Other lines represent Case $\mathbf{B}$ where $\gamma=3.3$, each such line representing one time step. Earlier times (from $t=0 T_{p}$ ) are the darkest lines. Later times (up to $t=100 T_{p}$ ) are the brightest lines. (Right) Horizontal axis shows non-dimensional wave height and vertical axis shows a probability ratio. Each line represents the ratio between a Case $\mathbf{B}$ time-step line and the Rayleigh distribution. . . . . . . . . . . . . . 196

5-3 (In Color) (Left) Horizontal axis shows non-dimensional wave height and vertical axis shows the probability of occurrence of heights in the wavefield. Thick, brown line represents Rayleigh distribution. Other lines represent Case $\mathbf{H}$ where $\gamma=5.0$, each such line representing one time step. Earlier times (from $t=0 T_{p}$ ) are the darkest lines. Later times (up to $t=100 T_{p}$ ) are the brightest lines. (Right) Horizontal axis shows non-dimensional wave height and vertical axis shows a probability ratio. Each line represents the ratio between a Case $\mathbf{H}$ time-step line and the Rayleigh distribution. . . . . . . . . . . . . . . . 197 
5-4 (In Color) Vertical axis represents correlation coefficient and horizontal axis represents time. Correlation coefficient between simulated wave height distribution data and theoretical Rayleigh Distribution are shown. A correlation coefficient, $r^{2}$ can take values between 0 and +1 where $r^{2}=1$ indicates that the wave height distribution correlates well with the Rayleigh Distribution, and $r^{2}=0$ means wave height distribution and the Rayleigh distribution are uncorrelated. We observe the effect of peak shape parameter on this correlation for three different wavefields: Cases $\mathbf{I}$ (where $\gamma=1.0$ ), $\mathbf{B}$ (where $\gamma=3.3$ ) and

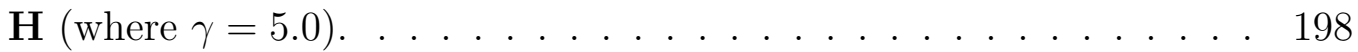

5-5 (In Color) Left: Horizontal axis shows non-dimensional wave height and vertical axis shows the probability of exceeding each height in the wavefield. Thick, brown line represents linear narrow band theory for the distribution of wave height exceedance probability. Other lines represent Case $\mathbf{I}$ where $\gamma=1.0$, each such line representing one time step. Earlier times (from $t=0 T_{p}$ ) are the darkest lines. Later times (up to $\left.t=100 T_{p}\right)$ are the brightest lines. Right: Horizontal axis shows nondimensional wave height and vertical axis shows a probability ratio. Each line represents the ratio between a Case I time-step line and the linear narrow band theory for the wave height exceedance probability distribution. . . . . . . . . . . . . . . . . 200 
5-6 (In Color) Left: Horizontal axis shows non-dimensional wave height and vertical axis shows the probability of exceeding each height in the wavefield. Thick, brown line represents linear narrow band theory for the distribution of wave height exceedance probability. Other lines represent Case $\mathbf{B}$ where $\gamma=3.3$, each such line representing one time step. Earlier times (from $t=0 T_{p}$ ) are the darkest lines. Later times (up to $\left.t=100 T_{p}\right)$ are the brightest lines. Right: Horizontal axis shows nondimensional wave height and vertical axis shows a probability ratio. Each line represents the ratio between a Case $\mathbf{B}$ time-step line and the linear narrow band theory for the wave height exceedance probability distribution. . . . . . . . . . . . . . . . . . . . . 201

5-7 (In Color) Left: Horizontal axis shows non-dimensional wave height and vertical axis shows the probability of exceeding each height in the wavefield. Thick, brown line represents linear narrow band theory for the distribution of wave height exceedance probability. Other lines represent Case $\mathbf{H}$ where $\gamma=5$.0, each such line representing one time step. Earlier times (from $t=0 T_{p}$ ) are the darkest lines. Later times (up to $t=100 T_{p}$ ) are the brightest lines. Right: Horizontal axis shows nondimensional wave height and vertical axis shows a probability ratio. Each line represents the ratio between a Case $\mathbf{H}$ time-step line and the linear narrow band theory for the wave height exceedance probability distributions in the Left graphic. . . . . . . . . . . . . . . . . 202 
5-8 (In Color) Left: Horizontal axis shows probability, $p$ where $0<p<1$ and vertical axis shows the mean of the highest $p$ waves, $H^{(p)}$ in the wavefield. Each line represents a time in the time evolution of firstmoment distribution in Case $\mathbf{I}$ (where $\gamma=1.0$ ). Graphics depict the mean of the highest $p$ waves, $H^{(p)}$, v.s. $p$, where $0<p<1$. Earlier times are the darkest lines. Later times are the brightest lines. Right: Horizontal axis shows probability, $p$ where $0<p<1$ and vertical axis shows a height ratio. Each line represents the ratio between a Case $\mathbf{I}$ observed time-step line in the Left graphic and the linear narrow band theory for the mean of the highest $p$ waves, $H^{(p)}$ v.s. $p$ in the wavefield. 203

5-9 (In Color) Left: Horizontal axis shows probability, $p$ where $0<p<1$ and vertical axis shows the mean of the highest $p$ waves, $H^{(p)}$ in the wavefield. Each line represents a time in the time evolution of firstmoment distribution in Case $\mathbf{B}$ (where $\gamma=3.3$ ). Graphics depict the mean of the highest $p$ waves, $H^{(p)}$, v.s. $p$, where $0<p<1$. Earlier times are the darkest lines. Later times are the brightest lines. Right: Horizontal axis shows probability, $p$ where $0<p<1$ and vertical axis shows a height ratio. Each line represents the ratio between a Case $\mathbf{B}$ observed time-step line in the Left graphic and the linear narrow band theory for the mean of the highest $p$ waves, $H^{(p)}$ v.s. $p$ in the wavefield. 204

5-10 (In Color) Left: Horizontal axis shows probability, $p$ where $0<p<1$ and vertical axis shows the mean of the highest $p$ waves, $H^{(p)}$ in the wavefield. Each line represents a time in the time evolution of firstmoment distribution in Case $\mathbf{H}$ (where $\gamma=5.0$ ). Graphics depict the mean of the highest $p$ waves, $H^{(p)}$, v.s. $p$, where $0<p<1$. Earlier times are the darkest lines. Later times are the brightest lines. Right: Horizontal axis shows probability, $p$ where $0<p<1$ and vertical axis shows a height ratio. Each line represents the ratio between a Case $\mathbf{H}$ observed time-step line in the Left graphic and the linear narrow band theory for the mean of the highest $p$ waves, $H^{(p)}$ v.s. $p$ in the wavefield. 205 
5-11 Theoretical contours of dimensionless joint probability density function of wave amplitude and wave period for spectral width parameters, $(\mathrm{a}) \nu=0.3$ and $(\mathrm{b}) \nu=0.4[34] \ldots \ldots \ldots \ldots$

5-12 Scatter plot of dimensionless wave height vs. dimensionless wavelength in Case $\mathbf{I}$ (where $\gamma=1.0$ ) at time, $t=0 T_{p} \ldots \ldots$. . . . . . . 209

5-13 Scatter plot of dimensionless wave height vs. dimensionless wavelength in Case $\mathbf{B}$ (where $\gamma=3.3$ ) at time, $t=0 T_{p} . \ldots . . . . .210$

5-14 Scatter plot of dimensionless wave height vs. dimensionless wavelength in Case $\mathbf{H}($ where $\gamma=5.0)$ at time, $t=0 T_{p} \ldots \ldots$

5-15 Scatter plot of dimensionless wave height vs. dimensionless wavelength in Case $\mathbf{I}$ (where $\gamma=1.0$ ) at time, $t=50 T_{p}$. . . . . . . . . . . . 212

5-16 Scatter plot of dimensionless wave height vs. dimensionless wavelength in Case $\mathbf{B}($ where $\gamma=3.3)$ at time, $t=50 T_{p} \ldots \ldots . . . .$.

5-17 Scatter plot of dimensionless wave height vs. dimensionless wavelength in Case $\mathbf{H}($ where $\gamma=5.0)$ at time, $t=50 T_{p} \ldots \ldots . . . . . .214$

5-18 Scatter plot of dimensionless wave height vs. dimensionless wavelength in Case $\mathbf{I}($ where $\gamma=1.0)$ at time, $t=100 T_{p} \ldots \ldots . . . .$.

5-19 Scatter plot of dimensionless wave height vs. dimensionless wavelength in Case $\mathbf{B}$ (where $\gamma=3.3$ ) at time, $t=100 T_{p} \ldots \ldots . . \ldots 215$

5-20 Scatter plot of dimensionless wave height vs. dimensionless wavelength in Case $\mathbf{H}($ where $\gamma=5.0)$ at time, $t=100 T_{p} \ldots \ldots . . . . .216$ 
5-21 (In Color) (Left) Horizontal axis shows non-dimensional wavelength and vertical axis shows the probability of occurrence of wavelength in the wavefield. Thick, brown line represents Rayleigh distribution. Other lines represent observed wavelength distribution of Case I where $\gamma=1.0$, each such line representing all wavelengths in one time step. Earlier times (from $t=0 T_{p}$ ) are the darkest lines. Later times (up to $t=100 T_{p}$ ) are the brightest lines. (Right) Horizontal axis shows non-dimensional wavelength and vertical axis shows a probability ratio. Each line represents the ratio between a Case I time-step line and the Rayleigh distribution. . . . . . . . . . . . . . . 217

5-22 (In Color) (Left) Horizontal axis shows non-dimensional wavelength and vertical axis shows the probability of occurrence of wavelength in the wavefield. Thick, brown line represents Rayleigh distribution. Other lines represent observed wavelength distribution of Case $\mathbf{B}$ where $\gamma=3.3$, each such line representing all wavelengths in one time step. Earlier times (from $t=0 T_{p}$ ) are the darkest lines. Later times (up to $t=100 T_{p}$ ) are the brightest lines. (Right) Horizontal axis shows non-dimensional wavelength and vertical axis shows a probability ratio. Each line represents the ratio between a Case $\mathbf{B}$ time-step line and the Rayleigh distribution. . . . . . . . . . . . . . . . . 218

5-23 (In Color) (Left) Horizontal axis shows non-dimensional wavelength and vertical axis shows the probability of occurrence of wavelength in the wavefield. Thick, brown line represents Rayleigh distribution. Other lines represent observed wavelength distribution of Case $\mathbf{H}$ where $\gamma=5.0$, each such line representing all wavelengths in one time step. Earlier times (from $t=0 T_{p}$ ) are the darkest lines. Later times (up to $t=100 T_{p}$ ) are the brightest lines. (Right) Horizontal axis shows non-dimensional wavelength and vertical axis shows a probability ratio. Each line represents the ratio between a Case $\mathbf{H}$ time-step line and the Rayleigh distribution. . . . . . . . . . . . . . . . . 219 
5-24 (In Color). Correlation coefficient, $r^{2}$-values between the simulated wavefields' wavelength distribution and the Rayleigh distribution. Correlation coefficient, $r^{2}$ takes values between 0 and 1 where $r^{2}=1$ indicates that the simulated wavefields' wavelength distribution and the Rayleigh distribution are highly correlated and $r^{2}=0$ indicates that they are not correlated at all. We observe wavelength distribution correlation with the Rayleigh distribution in cases with $\gamma=1.0, \gamma=3.3$ and $\gamma=5.0$, for all times, $t=0 T_{p}$ to $t=100 T_{p}$. . . . . . . . . 220

5-25 (In Color) Wave height and travel direction in Case $\mathbf{I}$ (where $\gamma=1.0$ ) at time, $t=50 T_{p}$. Graphics depict wave height $H / H_{r m s}$ as the length of the vector and the direction in which the wave travels $\Theta$ as the angle of the vector for $H>1.0 * H_{s}$ at time, $t=50 T_{p} \ldots \ldots . .$.

5-26 (In Color) Wave height and travel direction in Case $\mathbf{B}$ (where $\gamma=3.3$ ) at time, $t=50 T_{p}$. Graphics depict wave height $H / H_{r m s}$ as the length of the vector and the direction in which the wave travels $\Theta$ as the angle of the vector for $H>1.0 * H_{s}$ at time, $t=50 T_{p} \ldots \ldots 223$

5-27 (In Color) Wave height and travel direction in Case $\mathbf{H}$ (where $\gamma=5.0$ ) at time, $t=50 T_{p}$. Graphics depict wave height $H / H_{r m s}$ as the length of the vector and the direction in which the wave travels $\Theta$ as the angle of the vector for $H>1.0 * H_{s}$ at time, $t=50 T_{p} \ldots \ldots . .$.

5-28 (In Color) Wave height and travel direction in Case $\mathbf{I}$ (where $\gamma=1.0$ ) at time, $t=100 T_{p}$. Graphics depict wave height $H / H_{r m s}$ as the length of the vector and the direction in which the wave travels $\Theta$ as the angle of the vector for $H>1.0 * H_{s}$ at time, $t=100 T_{p} \ldots \ldots . . . .225$

5-29 (In Color) Wave height and travel direction in Case $\mathbf{B}$ (where $\gamma=3.3$ ) at time, $t=100 T_{p}$. Graphics depict wave height $H / H_{r m s}$ as the length of the vector and the direction in which the wave travels $\Theta$ as the angle of the vector for $H>1.0 * H_{s}$ at time, $t=100 T_{p} \ldots \ldots 226$ 
5-30 (In Color) Wave height and travel direction in Case $\mathbf{H}$ (where $\gamma=5.0$ ) at time, $t=100 T_{p}$. Graphics depict wave height $H / H_{r m s}$ as the length of the vector and the direction in which the wave travels $\Theta$ as the angle of the vector for $H>1.0 * H_{s}$ at time, $t=100 T_{p} . \ldots . . . . .227$

5-31 (In Color)Large wave spacing is seen as a plot of the number of waves found within a specified radius (for e.g. the pink lines marking $r_{0}, r_{1}$, $r_{2}$ and $r_{3}$ in the graphic) around the largest wave (red tip at the center of the pink rings) at each time step. (Image modified from [73]) . . . 228

5-32 (In Color) Vertical axis depicts number of waves and horizontal axis depicts radius. Large wave spacing in Case $\mathbf{I}$ (where $\gamma=1.0$ ) from times, $t=0 T_{p}$ (darkest line) to $t=100 T_{p}$ (brightest line). Spacing in waves with height $H>1.0 * H_{s}$ from times, $t=0 T_{p}$ (darkest lines) to $t=100 T_{p}$ (brightest lines). Where a line ends the largest wave has struck the perimeter of the wavefield. . . . . . . . . . . . . .

5-33 (In Color) Vertical axis depicts number of waves and horizontal axis depicts radius. Large wave spacing in Case $\mathbf{B}$ (where $\gamma=3.3$ ) from times, $t=0 T_{p}$ (darkest line) to $t=100 T_{p}$ (brightest line). Spacing in waves with height $H>1.0 * H_{s}$ from times, $t=0 T_{p}$ (darkest lines) to $t=100 T_{p}$ (brightest lines). Where a line ends the largest wave has struck the perimeter of the wavefield. . . . . . . . . . . . . . . 230

5-34 (In Color) Vertical axis depicts number of waves and horizontal axis depicts radius. Large wave spacing in Case $\mathbf{H}$ (where $\gamma=5.0$ ) from times, $t=0 T_{p}$ (darkest line) to $t=100 T_{p}$ (brightest line). Spacing in waves with height $H>1.0 * H_{s}$ from times, $t=0 T_{p}$ (darkest lines) to $t=100 T_{p}$ (brightest lines). Where a line ends the largest wave has struck the perimeter of the wavefield. . . . . . . . . . . . . . 231

5-35 (In Color) Vertical axis depicts number of waves and horizontal axis depicts radius. Large wave spacing in Case $\mathbf{I}$ (where $\gamma=1.0$ ), Case B (where $\gamma=3.3$ ), and Case $\mathbf{H}$ (where $\gamma=5.0$ ) at time, $t=20 T_{p}$. Spacing in waves with height $H>1.0 * H_{s} \ldots \ldots . . . . . . . .232$ 
5-36 (In Color) Vertical axis depicts number of waves and horizontal axis depicts radius. Large wave spacing in Case $\mathbf{I}$ (where $\gamma=1.0$ ), Case B (where $\gamma=3.3$ ), and Case $\mathbf{H}$ (where $\gamma=5.0$ ) at time, $t=50 T_{p}$. Spacing in waves with height $H>1.0 * H_{s} \ldots \ldots \ldots 233$

5-37 (In Color) Vertical axis depicts number of waves and horizontal axis depicts radius. Large wave spacing in Case $\mathbf{I}$ (where $\gamma=1.0$ ), Case $\mathbf{B}$ (where $\gamma=3.3$ ), and Case $\mathbf{H}$ (where $\gamma=5.0$ ) at time, $t=100 T_{p}$. Spacing in waves with height $H>1.0 * H_{s} \ldots \ldots \ldots \ldots \ldots$

5-38 (In Color) (Left) Horizontal axis shows non-dimensional wave height and vertical axis shows the probability of occurrence of heights in the wavefield. Thick, brown line represents Rayleigh distribution. Other lines represent Case $\mathbf{E}$ where $\alpha=0.0032$, each such line representing one time step. Earlier times (from $t=0 T_{p}$ ) are the darkest lines. Later times (up to $t=100 T_{p}$ ) are the brightest lines. (Right) Horizontal axis shows non-dimensional wave height and vertical axis shows a probability ratio. Each line represents the ratio between a Case $\mathbf{E}$ time-step line and the Rayleigh distribution. . . . . . . . . . . . . . . . . 237

5-39 (In Color) (In Color) (Left) Horizontal axis shows non-dimensional wave height and vertical axis shows the probability of occurrence of heights in the wavefield. Thick, brown line represents Rayleigh distribution. Other lines represent Case $\mathbf{B}$ where $\alpha=0.0160$, each such line representing one time step. Earlier times (from $t=0 T_{p}$ ) are the darkest lines. Later times (up to $t=100 T_{p}$ ) are the brightest lines. (Right) Horizontal axis shows non-dimensional wave height and vertical axis shows a probability ratio. Each line represents the ratio between a Case $\mathbf{B}$ time-step line and the Rayleigh distribution. . . . . . . . . 238 
5-40 (In Color) (In Color) (Left) Horizontal axis shows non-dimensional wave height and vertical axis shows the probability of occurrence of heights in the wavefield. Thick, brown line represents Rayleigh distribution. Other lines represent Case $\mathbf{F}$ where $\alpha=0.0163$, each such line representing one time step. Earlier times (from $t=0 T_{p}$ ) are the darkest lines. Later times (up to $t=100 T_{p}$ ) are the brightest lines. (Right) Horizontal axis shows non-dimensional wave height and vertical axis shows a probability ratio. Each line represents the ratio between a Case F time-step line and the Rayleigh distribution. . . . . . . . . . . 239

5-41 (In Color) Correlation coefficient, $r^{2}$-values between the simulated wavefields' wave height probability distribution and the Rayleigh distribution. Correlation coefficient, $r^{2}$ takes values between 0 and 1 where $r^{2}=1$ indicates that the simulated wavefields' wave height probability distribution and the Rayleigh distribution are highly correlated and $r^{2}=0$ indicates that they are not correlated at all. We observe this correlation for three simulated nonlinear wavefields: (a)Case $\mathbf{E}, \alpha=$ 0.0032, (b)Case B, $\alpha=0.0160$ and (c)Case F, $\alpha=0.0163$. . . . . . 240

5-42 Wave height and travel direction in Case $\mathbf{E}$ (where $\alpha=0.0032$ ) at time, $t=50 T_{p}$. Graphics depict wave height $H / H_{r m s}$ as the length of the vector and the direction in which the wave travels $\Theta$ as the angle of the vector for $H>1.0 * H_{s}$ at time, $t=50 T_{p} \ldots \ldots . . . . .241$

5-43 Wave height and travel direction in Case $\mathbf{B}$ (where $\alpha=0.0160$ ) at time, $t=50 T_{p}$. Graphics depict wave height $H / H_{r m s}$ as the length of the vector and the direction in which the wave travels $\Theta$ as the angle of the vector for $H>1.0 * H_{s}$ at time, $t=50 T_{p} \ldots \ldots . . . . .242$

5-44 Wave height and travel direction in Case $\mathbf{F}$ (where $\alpha=0.0163$ ) at time, $t=50 T_{p}$. Graphics depict wave height $H / H_{r m s}$ as the length of the vector and the direction in which the wave travels $\Theta$ as the angle of the vector for $H>1.0 * H_{s}$ at time, $t=50 T_{p} \ldots \ldots . . . . .243$ 
5-45 Wave height and travel direction in Case $\mathbf{E}$ (where $\alpha=0.0032$ ) at time, $t=100 T_{p}$. Graphics depict wave height $H / H_{r m s}$ as the length of the vector and the direction in which the wave travels $\Theta$ as the angle of the vector for $H>1.0 * H_{s}$ at time, $t=100 T_{p} . \ldots \ldots 24$

5-46 Wave height and travel direction in Case $\mathbf{B}$ (where $\alpha=0.0160$ ) at time, $t=100 T_{p}$. Graphics depict wave height $H / H_{r m s}$ as the length of the vector and the direction in which the wave travels $\Theta$ as the angle of the vector for $H>1.0 * H_{s}$ at time, $t=100 T_{p} \ldots \ldots . . . . .245$

5-47 Wave height and travel direction in Case $\mathbf{F}$ (where $\alpha=0.0163$ ) at time, $t=100 T_{p}$. Graphics depict wave height $H / H_{r m s}$ as the length of the vector and the direction in which the wave travels $\Theta$ as the angle of the vector for $H>1.0 * H_{s}$ at time, $t=100 T_{p} \ldots \ldots . . . .246$

5-48 Scatter plot of dimensionless wave height vs. dimensionless wavelength in Case $\mathbf{E}$ (where $\alpha=0.0032$ ) at time, $t=50 T_{p} \ldots \ldots . . . . .247$

5-49 Scatter plot of dimensionless wave height vs. dimensionless wavelength in Case $\mathbf{B}$ (where $\alpha=0.0160$ ) at time, $t=50 T_{p} \ldots \ldots . . . . .248$

5-50 Scatter plot of dimensionless wave height vs. dimensionless wavelength in Case $\mathbf{F}$ (where $\alpha=0.0163$ ) at time, $t=50 T_{p} \ldots \ldots$. . . . . . 249

5-51 Scatter plot of dimensionless wave height vs. dimensionless wavelength in Case $\mathbf{E}\left(\right.$ where $\alpha=0.0032$ ) at time, $t=100 T_{p} \ldots \ldots$. . . . . . 250

5-52 Scatter plot of dimensionless wave height vs. dimensionless wavelength in Case $\mathbf{B}$ (where $\alpha=0.0160$ ) at time, $t=100 T_{p} . \ldots . . . . .251$

5-53 Scatter plot of dimensionless wave height vs. dimensionless wavelength in Case $\mathbf{F}$ (where $\alpha=0.0163$ ) at time, $t=100 T_{p} . \ldots . . . . .252$ 
5-54 (Left) Horizontal axis shows non-dimensional wavelength and vertical axis shows the probability of occurrence of wavelength in the wavefield. Thick, brown line represents Rayleigh distribution. Other lines represent observed wavelength distribution of Case $\mathbf{E}$ where $\alpha=0.0032$, each such line representing all wavelengths in one time step. Earlier times (from $t=0 T_{p}$ ) are the darkest lines. Later times (up to $t=100 T_{p}$ ) are the brightest lines. (Right) Horizontal axis shows nondimensional wavelength and vertical axis shows a probability ratio. Each line represents the ratio between a Case $\mathbf{E}$ time-step line and the Rayleigh distribution. . . . . . . . . . . . . . . . . 254

5-55 (Left) Horizontal axis shows non-dimensional wavelength and vertical axis shows the probability of occurrence of wavelength in the wavefield. Thick, brown line represents Rayleigh distribution. Other lines represent observed wavelength distribution of Case B where $\alpha=0.0160$, each such line representing all wavelengths in one time step. Earlier times (from $t=0 T_{p}$ ) are the darkest lines. Later times (up to $\left.t=100 T_{p}\right)$ are the brightest lines. (Right) Horizontal axis shows nondimensional wavelength and vertical axis shows a probability ratio. Each line represents the ratio between a Case $\mathbf{B}$ time-step line and the Rayleigh distribution. . . . . . . . . . . . . . . . . . . . . . 255

5-56 (Left) Horizontal axis shows non-dimensional wavelength and vertical axis shows the probability of occurrence of wavelength in the wavefield. Thick, brown line represents Rayleigh distribution. Other lines represent observed wavelength distribution of Case $\mathbf{F}$ where $\alpha=0.0163$, each such line representing all wavelengths in one time step. Earlier times (from $t=0 T_{p}$ ) are the darkest lines. Later times (up to $t=100 T_{p}$ ) are the brightest lines. (Right) Horizontal axis shows nondimensional wavelength and vertical axis shows a probability ratio. Each line represents the ratio between a Case $\mathbf{F}$ time-step line and the Rayleigh distribution. . . . . . . . . . . . . . . 256 
5-57 (In Color) Correlation coefficient, $r^{2}$-values between the simulated wavefields' wave length distribution and the Rayleigh distribution. Correlation coefficient, $r^{2}$ takes values between 0 and 1 where $r^{2}=1$ indicates that the simulated wavefields' wavelength distribution and the Rayleigh distribution are highly correlated and $r^{2}=0$ indicates that they are not correlated at all. We observe the time evolution of the correlation between wavelength distribution and the Rayleigh Distribution for three different non-linear simulated wavefields: (a)Case $\mathbf{E}$, $\alpha=0.0032$, (b)Case B, $\alpha=0.0160$ and (c)Case $\mathbf{F}, \alpha=0.0163 \ldots . .257$

5-58 (In Color) Vertical axis depicts number of waves and horizontal axis depicts radius. Large wave spacing in Case $\mathbf{E}$ (where $\alpha=0.0032$ ), Case $\mathbf{B}$ (where $\alpha=0.0160$ ), and Case $\mathbf{F}$ (where $\alpha=0.0163$ ) at initial time, $t=0 T_{p}$. Spacing in waves with height $H>1.5 * H_{s}$ at times, $t=20 T_{p}, t=50 T_{p}$, and $t=100 T_{p} \ldots \ldots \ldots \ldots$

5-59 (In Color) Vertical axis depicts number of waves and horizontal axis depicts radius. Large wave spacing in Case $\mathbf{E}$ (where $\alpha=0.0032$ ), Case $\mathbf{B}$ (where $\alpha=0.0160$ ), and Case $\mathbf{F}$ (where $\alpha=0.0163$ ) at time, $t=50 T_{p}$. Spacing in waves with height $H>1.5 * H_{s}$ at times, $t=20 T_{p}, t=50 T_{p}$, and $t=100 T_{p} \ldots \ldots \ldots \ldots . \ldots \ldots$

5-60 (In Color) Vertical axis depicts number of waves and horizontal axis depicts radius. Large wave spacing in Case $\mathbf{E}$ (where $\alpha=0.0032$ ), Case $\mathbf{B}$ (where $\alpha=0.0160$ ), and Case $\mathbf{F}$ (where $\alpha=0.0163$ ) at time, $t=$ $100 T_{p}$. Spacing in waves with height $H>1.5 * H_{s}$ at times, $t=20 T_{p}$, $t=50 T_{p}$, and $t=100 T_{p} \ldots \ldots \ldots \ldots \ldots$ 
5-61 (In Color) (Left) Horizontal axis shows non-dimensional wave height and vertical axis shows the probability of occurrence of heights in the wavefield. Thick, brown line represents Rayleigh distribution. Other lines represent Case $\mathbf{J}$ (where $\theta=180^{\circ}$ ), each such line representing one time step. Earlier times (from $t=0 T_{p}$ ) are the darkest lines. Later times (up to $t=100 T_{p}$ ) are the brightest lines. (Right) Horizontal axis shows non-dimensional wave height and vertical axis shows a probability ratio. Each line represents the ratio between a Case $\mathbf{J}$ time-step line and the Rayleigh distribution. . . . . . . . . . . . . . 263

5-62 (In Color) (Left) Horizontal axis shows non-dimensional wave height and vertical axis shows the probability of occurrence of heights in the wavefield. Thick, brown line represents Rayleigh distribution. Other lines represent Case $\mathbf{A}$ (where $\theta=80^{\circ}$ ), each such line representing one time step. Earlier times (from $t=0 T_{p}$ ) are the darkest lines. Later times (up to $t=100 T_{p}$ ) are the brightest lines. (Right) Horizontal axis shows non-dimensional wave height and vertical axis shows a probability ratio. Each line represents the ratio between a Case $\mathbf{A}$ time-step line and the Rayleigh distribution. . . . . . . . . . . . . . . . . . 264

5-63 (In Color) (Left) Horizontal axis shows non-dimensional wave height and vertical axis shows the probability of occurrence of heights in the wavefield. Thick, brown line represents Rayleigh distribution. Other lines represent Case $\mathbf{K}$ (where $\theta=20^{\circ}$ ), each such line representing one time step. Earlier times (from $t=0 T_{p}$ ) are the darkest lines. Later times (up to $t=100 T_{p}$ ) are the brightest lines. (Right) Horizontal axis shows non-dimensional wave height and vertical axis shows a probability ratio. Each line represents the ratio between a Case $\mathbf{K}$ time-step line and the Rayleigh distribution. . . . . . . . . . . . . . . 265 
5-64 (In Color) Correlation coefficient, $r^{2}$-values between the simulated wavefields' wave height distribution and the Rayleigh distribution. Correlation coefficient, $r^{2}$ takes values between 0 and 1 where $r^{2}=1$ indicates that the simulated wavefields' wave height distribution and the Rayleigh distribution are highly correlated and $r^{2}=0$ indicates that they are not correlated at all. We observe wave height distribution correlation with the Rayleigh distribution in Case $\mathbf{J}$ (where $\theta=180^{\circ}$ ), Case $\mathbf{A}$ (where $\theta=80^{\circ}$ ), Case $\mathbf{B}$ (where $\theta=40$ ), Case $\mathbf{M}$ (where $\theta=30$ ), and Case $\mathbf{K}\left(\right.$ where $\left.\theta=20^{\circ}\right)$. . . . . . . . . . 266

5-65 (In Color) Wave height exceedance probability in Case $\mathbf{J}$ (where $\theta=$ $180^{\circ}$ ) from times, $t=0 T_{p}$ (darkest line) to $t=100 T_{p}$ (brightest line) compared to the wave height exceedance probability in linear narrowband theory (thick, red line). . . . . . . . . . . . . . 268

5-66 (In Color) Wave height exceedance probability in Case $\mathbf{A}$ (where $\theta=$ $80^{\circ}$ ) from times, $t=0 T_{p}$ (darkest line) to $t=100 T_{p}$ (brightest line) compared to the wave height exceedance probability in linear narrowband theory (thick, red line). . . . . . . . . . . . . . . 269

5-67 (In Color) Wave height exceedance probability in Case $\mathbf{K}$ (where $\theta=$ $20^{\circ}$ ) from times, $t=0 T_{p}$ (darkest line) to $t=100 T_{p}$ (brightest line) compared to the wave height exceedance probability in linear narrowband theory (thick, red line). . . . . . . . . . . . . . . . 270

5-68 Scatter plot of dimensionless wave height vs. dimensionless wavelength in Case $\mathbf{J}$ (where $\theta=180^{\circ}$ ) at time, $t=0 T_{p} \ldots \ldots$. . . . . . . 271

5-69 Scatter plot of dimensionless wave height vs. dimensionless wavelength in Case $\mathbf{A}\left(\right.$ where $\theta=80^{\circ}$ ) at time, $t=0 T_{p} \ldots \ldots . . .$.

5-70 Scatter plot of dimensionless wave height vs. dimensionless wavelength in Case $\mathbf{K}\left(\right.$ where $\theta=20^{\circ}$ ) at time, $t=0 T_{p} \ldots \ldots$. . . . . . 273

5-71 Scatter plot of dimensionless wave height vs. dimensionless wavelength in Case $\mathbf{J}\left(\right.$ where $\theta=180^{\circ}$ ) at time, $t=50 T_{p} \ldots \ldots$. . . . . . . . 274 
5-72 Scatter plot of dimensionless wave height vs. dimensionless wavelength in Case $\mathbf{A}\left(\right.$ where $\left.\theta=80^{\circ}\right)$ at time, $t=50 T_{p} \ldots \ldots 275$

5-73 Scatter plot of dimensionless wave height vs. dimensionless wavelength in Case $\mathbf{K}$ (where $\theta=20^{\circ}$ ) at time, $t=50 T_{p} \ldots \ldots \ldots 276$

5-74 Scatter plot of dimensionless wave height vs. dimensionless wavelength in Case $\mathbf{J}$ (where $\theta=180^{\circ}$ ) at time, $t=100 T_{p} \ldots \ldots \ldots \ldots . \ldots 277$

5-75 Scatter plot of dimensionless wave height vs. dimensionless wavelength in Case $\mathbf{A}\left(\right.$ where $\left.\theta=80^{\circ}\right)$ at time, $t=100 T_{p} \ldots \ldots 278$

5-76 Scatter plot of dimensionless wave height vs. dimensionless wavelength in Case $\mathbf{K}$ (where $\theta=20^{\circ}$ ) at time, $t=100 T_{p} \ldots \ldots \ldots 279$

5-77 (In Color) Time evolution of the wave length distribution in Case $\mathbf{J}$ where $\theta=180^{\circ}$. Earlier times (from $t=0 T_{p}$ ) are the darkest lines. Later times (up to $t=100 T_{p}$ ) are the brightest lines, compared to Rayleigh distribution, (solid, red line). . . . . . . . . . . . . .

5-78 (In Color) Time evolution of the wave length distribution in Case $\mathbf{A}$ where $\theta=80^{\circ}$. Earlier times (from $t=0 T_{p}$ ) are the darkest lines. Later times (up to $t=100 T_{p}$ ) are the brightest lines, compared to Rayleigh distribution, (solid, red line). . . . . . . . . . . . 282

5-79 (In Color) Time evolution of the wave length distribution in Case Kwhere $\theta=20^{\circ}$. Earlier times (from $t=0 T_{p}$ ) are the darkest lines. Later times (up to $t=100 T_{p}$ ) are the brightest lines, compared to Rayleigh distribution, (solid, red line). . . . . . . . . . . . 283 
5-80 (In Color) Correlation coefficient, $r^{2}$-values between the simulated wavefields' wavelength distribution and the Rayleigh distribution. Correlation coefficient, $r^{2}$ takes values between 0 and 1 where $r^{2}=1$ indicates that the simulated wavefields' wavelength distribution and the Rayleigh distribution are highly correlated and $r^{2}=0$ indicates that they are not correlated at all. We observe wavelength distribution correlation with the Rayleigh distribution in three different nonlinear simulated wavefields: (a)Case $\mathbf{J}, \theta=180^{\circ}$, (b)Case $\mathbf{A}, \theta=80^{\circ}$ and (c) Case $\mathbf{K}, \theta=20^{\circ} \ldots \ldots \ldots \ldots \ldots$

5-81 (In Color) Large wave spacing in Case $\mathbf{J}$ (where $\theta=180^{\circ}$ ), Case $\mathbf{A}$ (where $\theta=80^{\circ}$ ), Case $\mathbf{B}($ where $\theta=40$ ), Case $\mathbf{M}$ (where $\theta=30$ ), and Case $\mathbf{K}\left(\right.$ where $\theta=20^{\circ}$ ) at time, $t=20 T_{p}$. Spacing in waves with five different spreading angles with height $H>1.5 * H_{s}$ at time $t=20 T_{p} .285$

5-82 (In Color) Large wave spacing in Case $\mathbf{J}$ (where $\theta=180^{\circ}$ ), Case A (where $\theta=80^{\circ}$ ), Case $\mathbf{B}$ (where $\theta=40$ ), Case $\mathbf{M}$ (where $\theta=30$ ), and Case $\mathbf{K}\left(\right.$ where $\left.\theta=20^{\circ}\right)$ at time, $t=50 T_{p}$. Spacing in waves with five different spreading angles with height $H>1.5 * H_{s}$ at time $t=50 T_{p} .286$

5-83 (In Color) Large wave spacing in Case $\mathbf{J}$ (where $\theta=180^{\circ}$ ), Case $\mathbf{A}$ (where $\theta=80^{\circ}$ ), Case $\mathbf{B}$ (where $\theta=40$ ), Case $\mathbf{M}$ (where $\theta=30$ ), and Case $\mathbf{K}\left(\right.$ where $\left.\theta=20^{\circ}\right)$ at time, $t=100 T_{p}$. Spacing in waves with five different spreading angles with height $H>1.5 * H_{s}$ at time $t=100 T_{p} .287$

5-84 (In Color) Large wave spacing in Case $\mathbf{J}$ (where $\theta=180^{\circ}$ ) from times, $t=0 T_{p}$ (darkest line) to $t=100 T_{p}$ (brightest line) . . . . . . . 288

5-85 (In Color) Large wave spacing in Case $\mathbf{A}\left(\right.$ where $\theta=80^{\circ}$ ) from times, $t=0 T_{p}$ (darkest line) to $t=100 T_{p}$ (brightest line) . . . . . . . . 289

5-86 (In Color) Large wave spacing in Case $\mathbf{K}\left(\right.$ where $\theta=20^{\circ}$ ) from times, $t=0 T_{p}$ (darkest line) to $t=100 T_{p}$ (brightest line) . . . . . . . . 290 
5-87 (In Color) Wave height and travel direction in Case $\mathbf{J}\left(\right.$ where $\theta=180^{\circ}$ ) at time, $t=50 T_{p}$. Graphics depict wave height $H / H_{r m s}$ as the length of the vector and the direction in which the wave travels $\Theta$ as the angle of the vector for $H>1.0 * H_{s}$ at time, $t=50 T_{p} \ldots \ldots \ldots \ldots$

5-88 (In Color) Wave height and travel direction in Case $\mathbf{A}$ (where $\theta=80^{\circ}$ ) at time, $t=50 T_{p}$. Graphics depict wave height $H / H_{r m s}$ as the length of the vector and the direction in which the wave travels $\Theta$ as the angle of the vector for $H>1.0 * H_{s}$ at time, $t=50 T_{p} \ldots \ldots \ldots \ldots$

5-89 (In Color) Wave height and travel direction in Case $\mathbf{K}\left(\right.$ where $\theta=20^{\circ}$ ) at time, $t=50 T_{p}$. Graphics depict wave height $H / H_{r m s}$ as the length of the vector and the direction in which the wave travels $\Theta$ as the angle of the vector for $H>1.0 * H_{s}$ at time, $t=50 T_{p} \ldots \ldots \ldots 294$

5-90 (In Color) Wave height and travel direction in Case $\mathbf{J}$ (where $\theta=180^{\circ}$ ) at time, $t=100 T_{p}$. Graphics depict wave height $H / H_{r m s}$ as the length of the vector and the direction in which the wave travels $\Theta$ as the angle of the vector for $H>1.0 * H_{s}$ at time, $t=100 T_{p} \ldots \ldots \ldots \ldots 295$

5-91 (In Color) Wave height and travel direction in Case $\mathbf{A}$ (where $\theta=80^{\circ}$ ) at time, $t=100 T_{p}$. Graphics depict wave height $H / H_{r m s}$ as the length of the vector and the direction in which the wave travels $\Theta$ as the angle of the vector for $H>1.0 * H_{s}$ at time, $t=100 T_{p} \ldots \ldots \ldots 296$

5-92 (In Color) Wave height and travel direction in Case $\mathbf{K}\left(\right.$ where $\theta=20^{\circ}$ ) at time, $t=100 T_{p}$. Graphics depict wave height $H / H_{r m s}$ as the length of the vector and the direction in which the wave travels $\Theta$ as the angle of the vector for $H>1.0 * H_{s}$ at time, $t=100 T_{p} \ldots \ldots \ldots \ldots$

C-1 This figure, taken from On the Statistical Distribution of the Heights of Sea Waves, M.S. Longuet-Higgins, 1952, shows a narrow frequency band disturbance, $\zeta(t)$, and its envelope $|B(t)| \ldots \ldots \ldots \ldots$ 


\section{List of Tables}

2.1 SNOW simulations used for investigating wavefield statistical properties. Note that for every simulated nonlinear wavefield (i.e. of nonlinearity order, $O(3)$ in surface potential), we simulated a linear equivalent wavefield, (i.e. of nonlinearity order, $O(1)$ in surface potential) to isolate the effect of non-linearity order from the effects of spectral parameters. . . . . . . . . . . . . . . . . . . 6 62

2.2 Symbols used in the present work . . . . . . . . . . 63

3.1 Cases used in testing the effects of peak shape parameter, $\gamma \ldots \ldots 68$

3.2 Cases used in testing the effects of steepness via Phillips' parameter, $\alpha$, with directional spreading angle, $\theta=40$ and peak shape parameter,

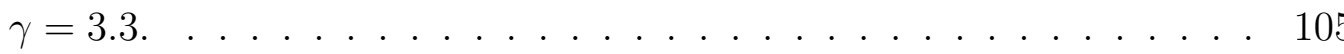

3.3 Simulated wavefield cases used in testing the effects of directional spreading with peak shape parameter, $\gamma=3.3$ and Phillips' parameter $\alpha=0.0160 \ldots \ldots \ldots \ldots \ldots \ldots \ldots \ldots$

6.1 Methods/approaches used in our work to build on what has been done by prior authors . . . . . . . . . . . . . . . . . 307

A.1 Symbols used in Appendix A . . . . . . . . . . . . . . 309

B.1 Symbols used in Appendix B . . . . . . . . . . . . . . . . 313

C.1 Symbols used in Appendix C . . . . . . . . . . . . . . . . . . . 319

D.1 Symbols used in Appendix D . . . . . . . . . . . . . . 323 


\section{Chapter 1}

\section{Introduction}

The present work focuses primarily on the response of surface elevation statistics, and ocean wave statistics to initial spectral parameters, namely directional spreading function, peak shape parameter and Phillips parameter. We do so via numerical simulations of nonlinear, directional ocean wavefields, varying the input spectral parameters. These directional ocean wavefields are modeled as random nonlinear wave-wave interactions in a field with a two-dimensional wavenumber.

\subsection{Present Contribution}

We simulate wavefields initialized with nonlinear wave-wave interactions under a specified JONSWAP spectrum in a Gaussian wavefield. We then define exactly how nonlinear the wavefield is, given specific, quantifiable changes in directional spreading, average wavefield steepness (from input spectral Phillips parameter) and wavefield spectral peak shape parameter. The insight drawn from the wavefields in this study is statistical in nature, owing to the fact that the modern stochastic approach to wavefields is effective in delineating the physical characteristics of a wavefield. Further insight is found in the present work by investigating the details of the sensitivity of nonlinear wavefield surface elevation kurtosis (and therefore, the formation of large waves) to finite, quantifiable changes in spectral properties: directional spreading,

peak shape parameter and Phillips parameter. In response to allusions [45] [65] [48] 
[45] that the effect of directionality on ocean wavefield probability distribution has been poorly addressed in the past, this work presents a detailed investigation of the statistical effect of directionality, across a spread of directional angles, alongside the additional effects of wavefield spectral Phillips parameter and spectral peak shape parameter.

Ideally, a realistic simulation of ocean waves is a helpful tool for understanding ocean wave statistics, as the difficulty of gathering accurate and detailed field and experimental readings is circumvented. In order to perform detailed statistical analysis of a realistic simulated wavefield, a detailed simulated surface elevation is needed. This is an insurmountable problem when wavefield simulations are not detailed enough, and it presents a limitation in many studies prior to the present work, [67] [44] [16] where models like SWAN [9] (based on a phase-averaged approximate ocean energy equation) and WAM [9] are used. In the present work a phase-resolved simulation of ocean surface elevation, called Simulations of Nonlinear Ocean Wavefields (SNOW) is used. SNOW [71] provides sufficient simulated details for investigating the impact of input spectral properties on wavefield physical properties via a statistical approach.

\subsection{The statistical approach to ocean waves.}

The problem of analytically capturing ocean waves seems to be an impossible task when facing the dauntingly untidy ocean surface with only the relative simplicity of classical wave theory. A brave effort to understand wave generation appears with Jeffrey's [26] early attempt to define ocean waves as instabilities, primarily driven by normal pressure, on the interface between the air/atmosphere and the sea. Another monumental effort comes in response to the second world war, when Sverdrup and Munk [43] [63] produce an inadequate but ingenious application of the classical theory of waves to the complex surface of the open sea. This theory is produced under a great sense of haste, as pressures around the second world war (the need for military vessels to better out-maneuver large, dangerous ocean waves) necessitate an attempt 
to predict ocean waves. This rough theory, including only cursory statistical elements proves to be enough of an approximation to save many lives [30].

Subsequent to this, statistical elements take center stage in ocean surface studies as surface elevation is eventually defined primarily as a random process in the modern stochastic approach to ocean waves. This approach clearly delineates a rigorous underlying order in ocean surface elevation's seeming chaos [57]. In the wake of prior work [26], [63], the modern stochastic approach to ocean surface elevation is remarkably well aligned with reality. In contrast to the difficult comparison between classical wave theory and the messy ocean surface, the statistical analysis of all the waves in a common wavefield reveals a striking order, producing great insight about the wavefield's physical properties.

This modern probabilistic approach to ocean surface elevation becomes interesting in the main stream of ocean science in the early 1950's, with the seminal work of Rudnick, [57] who derives an exponential distribution law for the probability distribution of ocean waves, equivalent to the Gaussian distribution. This is soon followed by the work of Pierson et al. [52] where a link between Rudnick's [57] exponential distribution and the ocean surface elevation, via central limit theorem (see Figure 1-1) can be seen. They conclude that the ocean, consisting of waves that are made up of large numbers of super-imposed simple sinusoidal signals of comparable height, different frequencies, different directional angles, and different phases, must follow the central limit theorem in its statistical properties (see Appendix A). Similar conclusions on ocean surface elevation statistics are published by Neumann [40], St. Denis [62] and Pierson [51] [52] around the same time that Longuet-Higgins [33] analytically derives the probability distribution of wave heights in a wavefield with a narrow frequency band (see Appendix C). He concludes a distinct exponential function for the distribution of wave heights in an ocean wavefield that follows the central limit theorem, building on the earlier work of Lord Rayleigh [55] in the field of vibrations, and Rice [56] who works with random noise . Returning to the original intent of the stochastic description of ocean waves, the prediction of ocean surface properties, Pierson and Neumann [53] consequently revise the Sverdrup and Munk attempts at ocean surface 
prediction.

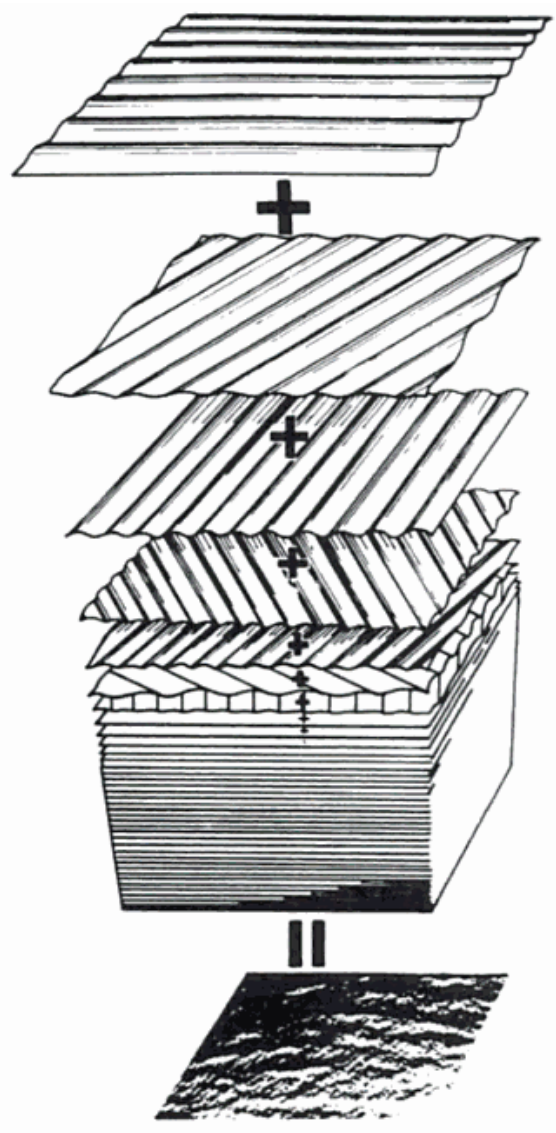

Figure 1-1: Structure of a random sea. [52] Linear theory stipulates that many plane progressive waves, (each moving in a unique direction, at a unique phase and frequency), are superimposed to produce a random sea surface.

Beyond producing the possibility of predicting the ocean surface, many physical characteristics of ocean wavefields can be drawn from the wavefield's statistical properties. Alber [1] shows that wavefield instability and the subsequent formation of large waves can be understood using statistically derived insight. He demonstrates that the amplification rate of wave trains, associated with the Benjamin-Feir type instability [5], diminishes and then vanishes as the stochastically defined correlation length scale of the random wavefield is reduced to the order of the characteristic length scale for modulational instability of the wave system. It is also shown that for random deep water waves, the instability of the wave train can exist, as in the corresponding deterministic Benjamin-Feir problem, provided that the normalized 
spectral bandwidth is less than twice the stochastically defined root mean square wave slope, scaled by the perturbation wave angle [5]. The Benjamin-Feir Index [48], a quantitative measure of wavefield stability, will be referred to later in this work, as one of the physical characteristics of ocean wavefields which can be understood using the wavefield's statistical properties. In addition air-sea interaction among investigations such as Sobey's [60] study of wind-wave prediction, Phillips' [50] study of the equilibrium range of wind-generated gravity waves and Janssen's [22] study of the interaction of ocean waves and wind, is built on ocean surface statistical theory. Further, studies of highly probabilistic wave breaking phenomena such as Melville's [39] study of the distribution of breaking waves on the ocean surface, Phillips' [49] study of spectral and statistical properties of the equilibrium range in wind-generated gravity waves, Makin's [38] study of the effect of dominant wave breaking on the drag of the sea surface and Phillips' [50] study of the attenuation of long gravity waves by short breaking waves require the use of surface wave statistical behavior. Even in understanding non-linear interactions and transfers between waves at the sea surface [69] and wave-current interaction [31] ocean wave statistics are an indispensable source of insight.

\subsection{Rationale}

In the present work we seek to understand the effect of spectral properties: peak shape parameter, Phillips parameter and directional spreading function on a wavefields' physics, using the tool of statistical analysis.

Firstly, the present work is a useful source of scientific insight to the link between an ocean wavefield's initial spectrum and the resulting surface elevation statistical properties, including large wave formation [45], [65], [67].

Secondly, we provide empirical evidence of the theoretical assumption that large non-Gaussian traits in ocean wavefields can sometimes be formed just by wave-wave interactions [35] in directional seas [48] sometimes in the absence of explanatory weather conditions. Ships and offshore structures are designed for $10 m-15 m$ wave 
heights in bad weather [14], whereas observed wave heights far exceed such heights in many cases of severe and sometimes not-so-severe weather [10], [8], [21], [32]. Large waves on the order of near $30 \mathrm{~m}$ are observed at sea, and continue to be observed with increasing intensity and frequency of occurrence every year [27]. Forty percent of the ship-related casualties occurring in the last ten years are related to sunken vessels falling prey to such large waves [66]. Such large waves (on the order of twice the size of the expected large waves) are frequently observed in the absence of explanatory weather conditions, and we similarly observe them in the upper tails of the statistical distributions of the simulated stormy, directional ocean wavefields studied within the present work.

Additionally, there are also engineering applications to the present work, since the present study of directional ocean wavefields can be helpful in the analysis of oceanbased stresses on structures caused by wave-body interactions in (common, naturally occurring) directional ocean wavefields. The stresses on an offshore structure can be over-accounted for in purely uni-directional analysis, whereas certain modes of coupling due to directionality in the typical 3D wavefield can be completely overlooked. Capturing nonlinear wave loads on a slender vertical cylinder [13], computing largescale phase-resolved wavefield simulations towards the improvement of ship models [73], measuring and analyzing the mechanics of wave-induced forces on cylinders [20], developing slender-body expressions for the wave load on offshore structures [54], experimentally studying nonlinear loads on vertical cylinders in steep random waves [61], calculating forces produced by irregular waves [70], and even capturing the features of nonlinear wave-body interactions [75] are all dependent on an accurate understanding of sea severity under directionality.

Finally, our study of wave height definition methods in a 3D wavefield with a $2 D$ wavenumber adds useful insight to common field practices in methods of defining wave heights from knowledge of a wavefield's ocean surface elevation values. We study the non-directional, narrowband zero-crossing method of wave-height definition alongside a $3 D$, directional narrow-band approach and we also study a $3 D$ analogue of the $2 D$ half-cycle excursion method. In so doing, we systematically observe the effect of non- 
directional approaches on wave height values in directional wavefields, and the effect of narrow-band approaches to wave heights in non-narrow-band wavefields.

\subsection{Thesis Scope}

The present thesis reports findings on the investigation of the impact of input spectral properties on ocean wavefield statistics, obtained through statistical analyses of simulated ocean wavefields' surface elevation records. Each wavefield simulation is individually defined by its unique input spectral density function parameters: Peak shape parameter, Phillips parameter, and directional spreading function.

The thesis is organized as follows: Chapter 1 begins with an introduction which includes a short outline of the present contribution, followed by a historic outline of the stochastic approach to ocean waves followed by field-related motivations for the present work, after which the chapter is brought to a close with a description of the thesis scope.

Chapter 2 describes the methods involved in this investigation. It begins with a description of the simulation tool used to generate wavefields for the present investigation, and then describes the quantities investigated by statistical measures.

Chapter 3 follows with an investigation of the effect of peak shape parameter, $\gamma$, Phillips parameter, $\alpha$ and directional spreading, $D(\theta)$ on ocean surface elevation statistics in nonlinear wavefields.

Next is Chapter 4 in which we study three methods of defining wave height and wavelength in a random, nonlinear wavefield with a two-dimensional wavenumber.

Chapter 5 follows, where we implement one definition method from Chapter 4 to observe the impact of peak shape parameter, $\gamma$, Phillips parameter, $\alpha$ and directional spreading, $D(\theta)$ on wave height and wave length statistics in a $3 D$, directional, nonlinear wavefield.

Chapter 6 then summarizes the contributions of the present work within the current literature-related context, and then outlines the future directions possible in wake of the present work. The appendices follow, in which Appendix A outlines linear the- 
ory for surface elevation distribution, Appendix B outlines a weakly nonlinear theory of ocean surface elevation distribution, Appendix $\mathrm{C}$ outlines the linear narrow-band theory of ocean wave height distributions, and Appendix D outlines a nonlinear wave height distribution theory.

While we do not close the problem of the effect of spectral parameters on ocean surface statistics, the present work is an important step forward as we demonstrate definite characteristics in the effects of peak shape parameter and Phillips' parameter on ocean surface elevation statistics, and circumvent the bias of non-directional approaches to wave height values in directional wavefields, and the bias of narrow-band approaches to wave heights in non-narrow-band wavefields in order to observe wave height and wavelength distributions. 


\section{Chapter 2}

\section{Methods}

We investigate an ocean wavefield's statistical properties in response to its "initial" spectral parameters. To do so we use phase-resolved, non-linear ocean wavefield simulations. In these simulations we vary the input spectral parameters: Phillips parameter, peak shape parameter and directional spreading in the wavefields' inital JONSWAP spectrum.

The simulated ocean surface evolves in time according to the potential equations for irrotational, incompressible water flow under the influence of gravity with infinite depth and periodic side boundaries. We observe ocean surface elevation as a simulated product of wave-wave interactions within wavefields that are initialized as random Gaussian surfaces using Simulations of Nonlinear Ocean Wavefields (SNOW) [71]. Such simulations provide realistic statistical insight, as these simulated wavewave interactions accurately stand alone in representing the physical properties of ocean surface waves. Among the wavefield properties investigated are surface elevation distribution, surface elevation spectrum, surface elevation statistical moments, surface slope statistical moments, wave definition methods, defined waves' distributions, and occurrence and spacing of large wave events. The adherence of these simulated wavefields' statistical distributions to standard linear and weakly non-linear statistical theory is investigated where possible and in some cases quantified. Since we adjust spectral parameters of a simulated wavefield initialized with the JONSWAP energy spectrum we now introduce this spectral formulation. 


\subsection{JONSWAP spectrum}

The fourier transform of the autocorrelation of a surface elevation record is the called its spectral density function [41]. In such a function we express the energy components of the surface elevation in the wavenumber domain, and we use this function in our initial conditions for SNOW. The particular spectrum we use is called the JONSWAP spectrum, and the shape of this spectrum varies considerably depending on the peak shape parameter, Phillips' parameter and directional spreading function in the wavefield. The JONSWAP spectrum is generated from a 1968 field survey called the Joint North Sea Wave Project [19] starting from Sylt Island and extending over $160 \mathrm{~km}$ into the North Sea. The shape of the wave spectrum during the growing stages of a hurricane-generated sea is well represented by the JONSWAP spectral formulation. This spectrum represents growing wind-generated seas with fetch limitation (i.e. with finite reach of wind influence). Its input parameters in this study are Phillips' parameter, $\alpha$, peak shape parameter, $\gamma$, and directional spreading function, $D(\theta)$, where $\theta$ represents the spreading angle in the angular spreading function:

$$
S(k, \theta)=\frac{2 \omega}{g} \alpha \frac{g^{2}}{k^{5}} e^{-1.25\left(k_{m} / k\right)^{4}} \gamma^{e x p-\left(k-k_{m}\right)^{2} / 2\left(\sigma k_{m}\right)^{2}} D(\theta),
$$

where the following definitions apply:

$D(\theta)=\left|\cos \frac{1}{2} \theta\right|^{2 s}$, where $s=1$ (See Section 3.3.)

$\theta=$ spreading angle i.e. angular input for $D(\theta)$ where $-\pi<\theta<\pi$

$\gamma=$ peak shape parameter (see Section 3.1)

$\alpha=5.061\left(\frac{H_{s}^{2}}{T_{p}^{4}}\right)(1-0.287 \ln (\gamma))($ see Section 3.2)

$\sigma=0.07$ for $f \leq f_{m}$ and $\sigma=0.09$ for $f>f_{m}$

$\lambda=$ wavelength

$k=2 \pi / \lambda$

$k_{m}=\frac{2 \pi f_{m}^{2}}{g}$

$f_{m}=3.5(g / \bar{U}) \bar{x}^{-0.33}$ where $\bar{U}$ is mean wind speed and $\bar{x}$ is fetch length

$g=$ constant gravitational acceleration. 


\subsection{JONSWAP spectral parameters}

The simulations used to compare across different independent variables, peak shape parameter, $\gamma$, Phillips' parameter, $\alpha$, and spreading angle, $\theta$, are listed in Tables 3.3, 3.1 and 3.2 .

JONSWAP spectral peak shape parameter, $\gamma$, represents the ratio of the maximum spectral energy density to the maximum of the equivalent average, unchanging spectrum. Spectral peak shape parameter values of 1.0, 3.3 and 5.0 (See Table 3.1) are used to test the effect of spectral peak shape parameter on simulated linear and non-linear, directional wavefields. Usually spectral peak shape parameter value is normally distributed, resting at a mean value 3.3 and going from 1.0 to 6.0 at the extremes [41].

Phillips' parameter, $\alpha$ is a spectral parameter associated with wave steepness, in physical space. Its definition in the present work can be seen in Equation 3.6. The $\alpha$ values, $0.0032,0.0160$, and 0.0163 (See Table 3.2) are used to test the effect of this spectral parameter, and therefore wave steepness on non-linear, directional wavefields.

Spectral spreading angle, $\theta$ (see Section 3.3) can range from $0^{\circ}$ in a unidirectional/longcrested sea surface to a large value of spreading occurring at $180^{\circ}$ in a short-crested sea. Investigations of the effect of the spectral directional spreading function, $D(\theta)$, are executed by adjusting the value of spreading angle, $\theta$, to the angles $180^{\circ}, 80^{\circ}, 40^{\circ}$, $30^{\circ}, 20^{\circ}, 10^{\circ}$ and $1^{\circ}$ (See Table 3.3) in the wave spectrum. The spectral spreading function is related to actual physical energy spreading in wavefields in Section 5.3.6 where we relate the input $\theta$ value with polar coordinates of energy spreading.

\subsection{Simulations of Nonlinear Ocean Wavefields (SNOW)}

This is a study of ocean wave statistical properties using Simulations of Nonlinear Ocean Wavefields (SNOW) [71]. SNOW is a simulation tool used to generate detailed three-dimensional, nonlinear, ocean wavefields. These wavefields have varying initial input spectra. In the present work, variations are input via three spectral parameters, 
namely directional spreading function, $D(\theta)$, Phillips parameter, $\alpha$ and peak shape parameter, $\gamma$. SNOW simulates nonlinear gravity wave interactions under adherence to the Zakharov [74] equations for a fluid in an inviscid, irrotational flow. The axes, $x-y-z$ are oriented as shown in Figure 2-1.

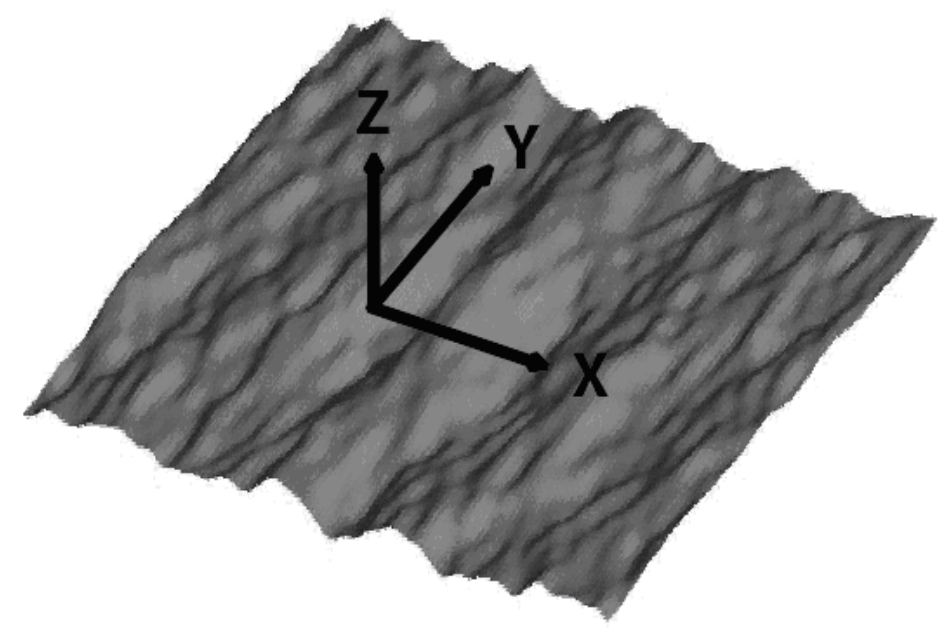

Figure 2-1: Orientation of axes in SNOW simulation realizations at every time step. The mean direction of wavefield propagation is the $x$-direction, the $y$-direction is perpendicular to the $x$-direction and the surface elevation height is measured in the $z$-direction. [71]

Wu's [71] first challenge in building SNOW is to properly specify the initial conditions and boundary conditions. His computation of the initial surface is based on a consideration of the higher-order non-linear wave-wave interactions of an ocean wavefield, and takes physical processes such as energy transfer due to resonant and nonresonant wave-wave interactions, and energy dissipation due to wave breaking into account [39]). This is achieved with a multi-level iterative wave reconstruction tool using both analytic low-order Stokes solutions and the High-Order-Spectral (HOS) nonlinear wave model, in order to deterministically reconstruct a nonlinear ocean wavefield which adheres to the Gaussian distribution and the JONSWAP spectrum (see Section 2.1).

The initial conditions are defined by a detailed, simulated wavefield constructed under three constraints: initial wave components are physically sound under the potential flow equations for surface gravity waves, input wave spectrum is completely 
specified, and wavefields are initially forced to be Gaussian, regardless of their spectral properties.

With the reconstructed wavefield as the initial condition, future time steps of the ocean wavefield are then forecasted deterministically with the physics-based HOS wave model which is applied to study the three-dimensional ocean wavefield evolutions for realistic scales in space and time. Let $\phi$ be the potential function representing the fluid flow, $\eta$ the free surface elevation, $t$ the time, and $g$ the acceleration due to gravity. Assume the mean direction of wavefield propagation is the $x$-direction, the $y$-direction is perpendicular to the $x$-direction and the surface elevation height is measured in the $z$-direction. The fully non-linear equations integrated using pseudo-spectral methods in SNOW are as follows:

Conservation of mass for an incompressible fluid:

$$
\nabla^{2} \phi=0 \quad \text { for }-\infty \leq z \leq \eta
$$

Dynamic boundary condition:

$$
\frac{\partial \phi}{\partial t}+\frac{1}{2} \nabla \phi \cdot \nabla \phi+\frac{P_{a}}{\rho}+g z=0 \quad \text { on } z=\eta
$$

Kinematic boundary conditions:

$$
\begin{array}{cc}
\frac{\partial \eta}{\partial t}+\frac{\partial \phi}{\partial x} \frac{\partial \eta}{\partial x}+\frac{\partial \phi}{\partial y} \frac{\partial \eta}{\partial y}-\frac{\partial \phi}{\partial z}=0 & \text { on } z=\eta \\
\nabla \phi \rightarrow 0 \text { as } z \rightarrow-\infty &
\end{array}
$$

The resulting surface elevations in the simulated wavefields are solved up to an arbitrary order in nonlinear expansions of the surface elevation associated with $\phi$. In our case we solve up to third order in surface potential for non-linear simulations and up to first order for linear simulations.

We investigate the relationships between the simulated wavefield's input spectral parameters and its resulting surface elevation statistical properties. These spec- 
tral parameters are specifically peak shape parameter, $\gamma$, Phillips parameter, $\alpha$ and spreading angle, $\theta$ (see Table 6.1). Among the resulting wavefield statistical properties observed after simulating are surface elevation distribution, surface elevation spectrum, surface elevation statistical moments, surface slope kurtosis, wave definitions, wave distributions, and occurrence and spacing of large wave events.

Table 2.1: SNOW simulations used for investigating wavefield statistical properties. Note that for every simulated nonlinear wavefield (i.e. of nonlinearity order, $O(3)$ in surface potential), we simulated a linear equivalent wavefield, (i.e. of nonlinearity order, $O(1)$ in surface potential) to isolate the effect of non-linearity order from the effects of spectral parameters.

\begin{tabular}{lllll}
\hline \hline Case & $\theta$, Deg & $\gamma$ & $\alpha$ & Comment \\
\hline A & 80 & 3.3 & 0.01604 & violent waves \\
B & 40 & 3.3 & 0.01604 & \\
E & 40 & 3.3 & 0.0032 & \\
F & 40 & 3.3 & 0.01630 & \\
G & 1 & 3.3 & 0.01604 & almost uni-directional sea \\
H & 40 & 5.0 & 0.0131 & extremely violent waves \\
I & 40 & 1.0 & 0.0244 & calm waves \\
J & 180 & 3.3 & 0.01604 & \\
K & 20 & 3.3 & 0.01604 & \\
L & 10 & 3.3 & 0.01604 & \\
M & 30 & 3.3 & 0.01604 & \\
\hline
\end{tabular}

N.B.

\subsection{Statistical Measures}

In our investigation we use detailed observed surface elevation space-time values from SNOW's simulated wavefields, $\eta\left(x_{i}, y_{j}, t\right)$, at every $(x, y)$-pair for $i, j=1,2,3, \ldots, 4096$, in the equivalent of a $30 \mathrm{~km} X 30 \mathrm{~km}$ space. We observe such a surface at each time step, $t=n T_{p}$, where $n=0,1, \ldots 10$. Statistically, we observe ocean surface elevation statistical distribution, statistical moments, and wavenumber spectrum.

We also observe surface elevation slope, which we calculate in the $x$-direction (mean direction of wavefield propagation) using $\frac{d \eta}{d x}$ between two spatially adjacent points, as well as in the $y$-direction (direction perpendicular to the mean direction of 
wavefield propagation) by using $\frac{d \eta}{d y}$ between two spatially adjacent points. Statistically, we quantify wavefield nonlinearity by observing surface elevation slope statistical moments.

We approach the unanswered [58] [41] [30] question of wave height definitions in a $3 D$ wave field with two-dimensional wave numbers carefully in Chapter 4 where we speak of definitions for wave height and wavelength and investigate statistical properties among our definitions. Investigations are performed on wave heights defined by two methods (two narrow-band approaches and one non-narrow-band approach) and the results of the two kinds of approaches are compared. The wave height probability distribution, the wave length probability distribution, the joint distribution between wave height and wave length, the wave height exceedance probability, the wave height first moment distribution, the direction of energy travel, large wave occurrences as a function of wavefield properties, and the spacing of large waves are all observed after waves are defined in the simulated wavefields.

\section{List of Symbols in the present work.}

Table 2.2: Symbols used in the present work

\begin{tabular}{|r||l|}
\hline \hline Symbol & Meaning \\
\hline$\alpha$ & Phillips parameter in JONSWAP spectrum. \\
$D(\theta)$ & Wavefield surface elevation. \\
$\epsilon$ & Spectral directional spreading function. \\
$\gamma$ & Average initial wave field steepness, $H_{s 0} / \lambda_{p}$. \\
$H$ & Spectral peak shape parameter. \\
$H_{s 0}$ & Wave height. \\
$H_{s}$ & Initial significant wave height. \\
$\lambda$ & Significant wave height. Mean of the highest $\frac{1}{3}$ waves. \\
$\lambda_{p}$ & Wavelength. \\
$\mu$ & Input peak wave length. \\
$\nu$ & Wavefield surface elevation mean elevation. \\
$\sigma^{2}$ & Wavenumber Spectrum width. \\
$\theta$ & Savefield surface elevation variance. \\
$T_{p}$ & Peak wave period; from peak wavelength via dispersion. \\
\hline
\end{tabular}




\section{Chapter 3}

\section{Ocean surface elevation and spectral parameters.}

In this chapter we examine the distribution of ocean surface elevation based on input spectral parameters. Changing the input spectral peak shape parameter, is equivalent to investigating impact of wind fetch on wavefield statistics. Changing the input spectral Phillips Parameter, $\alpha$ is equivalent to investigating the effect of average wavefield steepness on the statistics of the wavefield. Changing the input spectral directional spreading function $D(\theta)$ is equivalent to investigating impact of directional spreading in physical space on wavefield statistics.

\subsection{Peak shape parameter}

In the JONSWAP spectrum formulation (see Section 2.1) the peak shape parameter, $\gamma$, represents the ratio of the maximum spectral energy density to the maximum of the equivalent spectrum in a fully developed sea [19]. As found in the Joint North Sea Wave Project (JONSWAP) [41], the value of $\gamma$ may vary between 1.0 and 6.0, being normally distributed with mean value 3.3 and variance 0.6 [42]. Physically, the value of $\gamma$ is based on dimensionless fetch, $\bar{x}$, defined physically as the uninterrupted distance over which the effect of wind is void of any significant change in direction. The cases used to test the effect of peak shape parameter, $\gamma$, on a wave-field's physical 
characteristics are Cases $\mathbf{I}($ where $\gamma=1.0), \mathbf{B}($ where $\gamma=3.3)$ and $\mathbf{H}$ (where $\gamma=5.0)$ with directional spreading angle of $\theta=40^{\circ}$, and initial average steepness $\epsilon=0.04444$ which gives different $\alpha^{\prime} s$ only due to the effect of changing $\gamma$ seen in Equation 3.6 (see Table 3.1).

\subsubsection{Peak shape parameter and surface elevation statistical moments}

In this section we look at the effect of peak shape parameter, $\gamma$, on the statistical moments of ocean surface elevation by comparing the moments of the theoretical distribution in Equation A.9 to data derived in wavefield Cases I (where $\gamma=1.0$ ), B (where $\gamma=3.3$ ) and $\mathbf{H}$ (where $\gamma=5.0$ ). If the distribution of the surface elevation is compliant with Equation A.9, then we have specific expectations on its statistical moments. Let the first moment of surface elevation be represented by its mean value, $\mu$ and let the second moment of surface elevation be represented by its variance, $\sigma^{2}$. Skewness or third moment, $\lambda_{3}$ of surface elevation is a property which quantifies the asymmetry in a statistical distribution. For a Gaussian distribution, positive skewness indicates a bias in the distribution above the mean, negative skewness indicates a bias in the distribution below the mean, and zero skewness indicates a mean-centered distribution.

$$
\lambda_{3}=E\left[(x-\mu)^{3}\right] / \sigma^{3}=0,
$$

The kurtosis or fourth moment in a Gaussian distribution is a symmetrical property which quantifies the fatness of the two tails of the bell-shaped Gaussian distribution. For Gaussian distributions, the kurtosis of surface elevation should be:

$$
\lambda_{4}=E\left[(x-\mu)^{4}\right] / \sigma^{4}=3 .
$$

A precise measure of the linearity of a wave-field's surface elevation distribution is how the actual statistical third and fourth moments vary from their linear theoretical 
values, 0 and 3, respectively [41]. Evidence of the impact of $\gamma$ on these deviations is presented in Figure 3-1.

In Figure 3-1 the evolution of kurtosis shows that higher spectral peak shape parameter produces higher kurtosis, and also that the non-Gaussian traits in kurtosis swiftly grow in the first $50 T_{p}$ of time and then gradually fall from $t=50 T_{p}$ to $t=$ $100 T_{p}$. Time evolution of variance shows that higher spectral peak shape parameter produces higher variance, and also that variance gradually falls in the first $100 T_{p}$ of time. Time evolution of skewness shows that spectral peak shape parameter produces no effect on skewness and also that non-Gaussian traits in skewness remain stagnant and slightly oscillating around a fixed value in the first $100 T_{p}$ of time in a directional wavefield for all peak shape parameters.

In this section we investigate the effect of peak shape parameter, $\gamma$, on the statistical moments of ocean surface elevation in a directional wavefield with initial average wavefield steepness, $\epsilon=0.04444$, and an initial spreading angle, $\theta=40^{\circ}$. We see that a larger peak shape parameter systematically produces larger statistical moments, comparing Cases $\mathbf{I}$ (where $\gamma=1.0), \mathbf{B}$ (where $\gamma=3.3$ ) and $\mathbf{H}$ (where $\gamma=5.0$ ). We find that statistical moments evolve in the case of non-linear surface elevation away from the Gaussian values where they begin. It is found that a greater peak shape parameter produces greater kurtosis and also greater variance, but that it does not influence surface elevation skewness, as the wavefields' skewness hold values that do not correspond with their different peak shape parameters. In light of Section 3.1.2 which follows, it can also be concluded that the skewness, which quantifies the entire wavefield does not necessarily tell us the details of what happens to surface elevation distribution at the tails of the distribution, distribution of the tails, where the most potent nonlinearities reside, (see Figures 3-2, 3-3 and 3-4.) 
Table 3.1: Cases used in testing the effects of peak shape parameter, $\gamma$.

\begin{tabular}{llll}
\hline \hline Case & $\mathrm{B}$ & $\mathrm{H}$ & $\mathrm{I}$ \\
\hline peak shape parameter, $\gamma$ & 1.0 & 3.3 & 5.0 \\
\hline
\end{tabular}
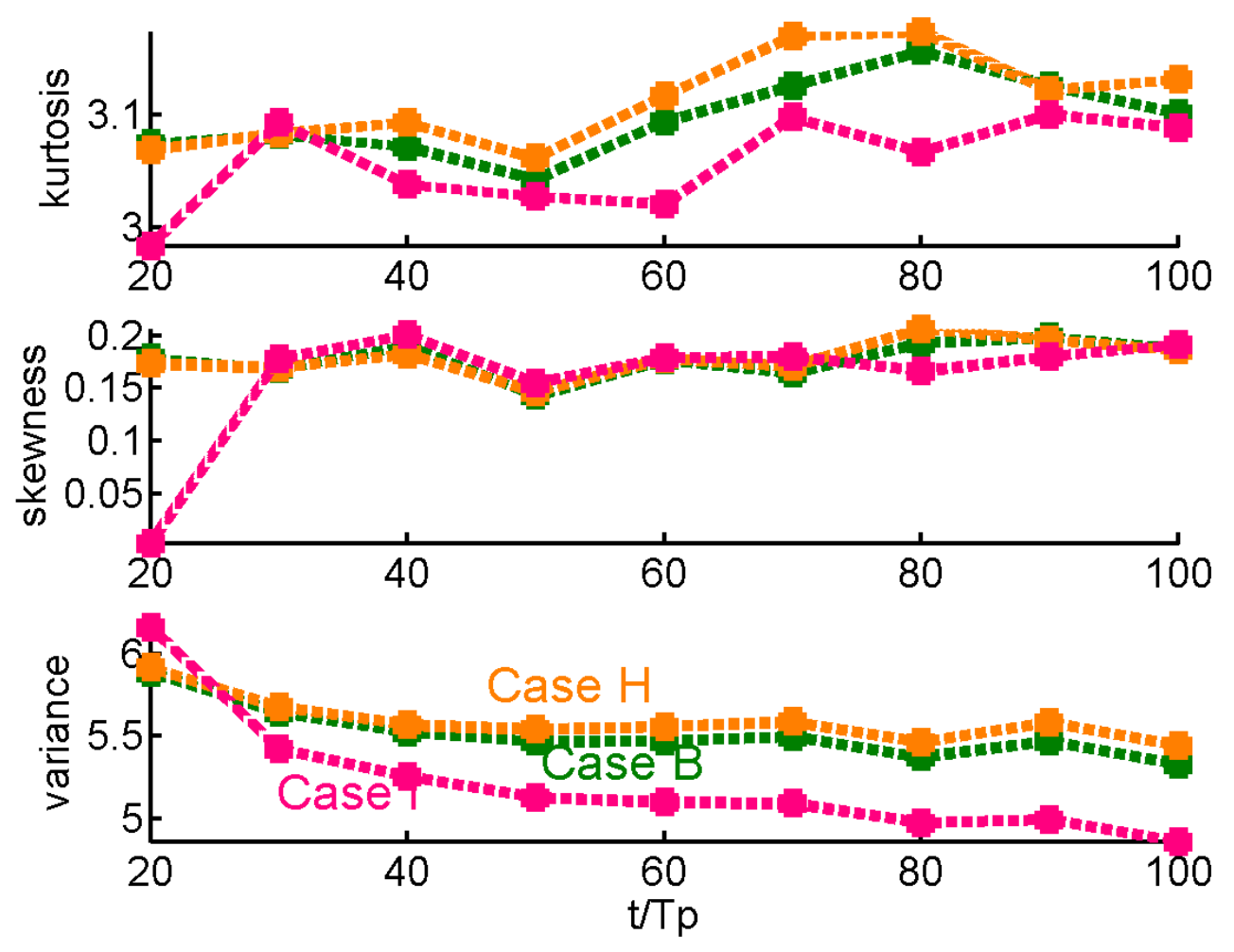

Figure 3-1: (In Color) Plot of surface elevation moments, variance, skewness and kurtosis, against time, $\left(t / T_{P}\right)$ in three nonlinear simulations of Cases $\mathbf{I}$ (where $\gamma=$ 1.0), $\mathbf{B}$ (where $\gamma=3.3$ ) and $\mathbf{H}$ (where $\gamma=5.0$ ). 


\subsubsection{Peak shape parameter and surface elevation statistical distribution}

In this section we look at the effect of peak shape parameter, $\gamma$, on the statistical distribution of ocean surface elevation. In Figures 3-5, 3-6 and 3-7 the straight lines represent the linear theoretical probability distribution function, or the Gaussian Distribution seen in Appendix A, Equation A.9. In this type of plot, curvature of observed data away from the straight theoretical line is an indication of non-linearity in the data. The consistent drop of the data series below the linear theoretical lines seen on the left of the image at the lower values of surface elevation indicates that low troughs in the wavefield surface elevation are less probable than linear theory predicts. The consistent curvature of the data series away from the linear theoretical lines at the far right of the data series is an indication of higher probabilities of larger elevations than the linear theory predicts. When the data series at the far right curves below the linear theoretical line, this indicates even greater probability of the extremely high surface elevations, hence larger kurtosis than the Gaussian. When the data series at the far right curves above the linear theory, this indicates that all of the large surface elevations (extreme and not extreme) display higher probabilities than predicted by linear theory, hence larger skewness than the Gaussian.

We expect a spectral peak shape parameter of approximately $\gamma \geq 3.3$ to produce violent surface wave characteristics [18], moving the wavefield furthest from linear theory and the following data confirms this. This data also shows that for directional seas, a higher value of spectral peak shape parameter $\gamma=5.0$ produces a more narrowband wavefield, allowing for greater superposition effects and eventually causing the wavefield to move further away from linear theory than in the case where $\gamma=1.0$. In Figure 3-3 we see that after one hundred peak periods, $t=100 T_{p}$, Case $\mathbf{B}$ has the largest expected skew, by linear theory, and a far larger apparent skew, rendering it the most non-linear wavefield. Case $B$ where $\gamma=3.3$, shows the smallest elevations are far less present than predicted by linear theory, further indicating that the distribution is skewed towards larger surface elevations. Case $\mathbf{H}$, in Figure 3-4 which has 
the highest spectral peak shape parameter, $\gamma=5.0$, has a narrower bandwidth. As a result, Case $\mathbf{H}$ persistently shows higher values for positive elevations than linear theory predicts, therefore showing great skewness towards higher surface elevations after $t=50 T_{p}$. This cases evolves to show a distribution with a large kurtosis indicating largest surface elevations become more probable around $t=100 T_{p}$. Case $\mathbf{I}$, which has the lowest spectral peak shape parameter, $\gamma=1.0$, shows the lowest expected skew, and also the smallest apparent skew towards higher values of surface elevation. In this case we confirm that directional wavefields with lower $\gamma$-values produce less kurtosis, less skew, and more near-linear random surfaces. We also note that in the absence of a weather condition, $\gamma=3.3$ is associated with the most apparent nonlinearity. Note that in Figure 3-3 we see that for peak shape parameter value $\gamma=3.3$ which usually coincide with highly nonlinear waves [18], the most apparent non-linear characteristics appear at the distribution tails, and increase with time.

In Figures 3-5, 3-6, and 3-7,each value of peak shape parameter, $\gamma$ produces different types of changes in the surface elevation distribution over time and this can be observed in the changing non-linearity in these plots where non-linearity is seen as data series $(+++)$ curvature away from the theoretical(- - -) straight line. Different slopes correspond to different values of surface elevation skewness in the wavefields. Arbitrary changes in slopes comply with the arbitrary changes seen in surface skewness in Figure 3-1, and this is consistent with unstable spectral energy at the highest spectral peaks. We confirm this in Section 3.1.3.

We see in comparing Figures 3-8, 3-9 and 3-10 that peak shape parameter value $\gamma=3.3$ most coincides with the violent types of waves associated with storms at the distribution tails. We wonder how much of the dynamics we observe are due to the order of nonlinearity, so we compare the linear and nonlinear versions of Case $\mathbf{H}$ (see Figure 3-11), finding that

As surface elevation statistical moments change, the nonlinearities in the data change in nature. Appendix B outlines the weakly non-linear statistical theory of ocean surface elevation as a product of statistical moments. We take the results of the previous section dealing with statistical moments and apply them to observing the 


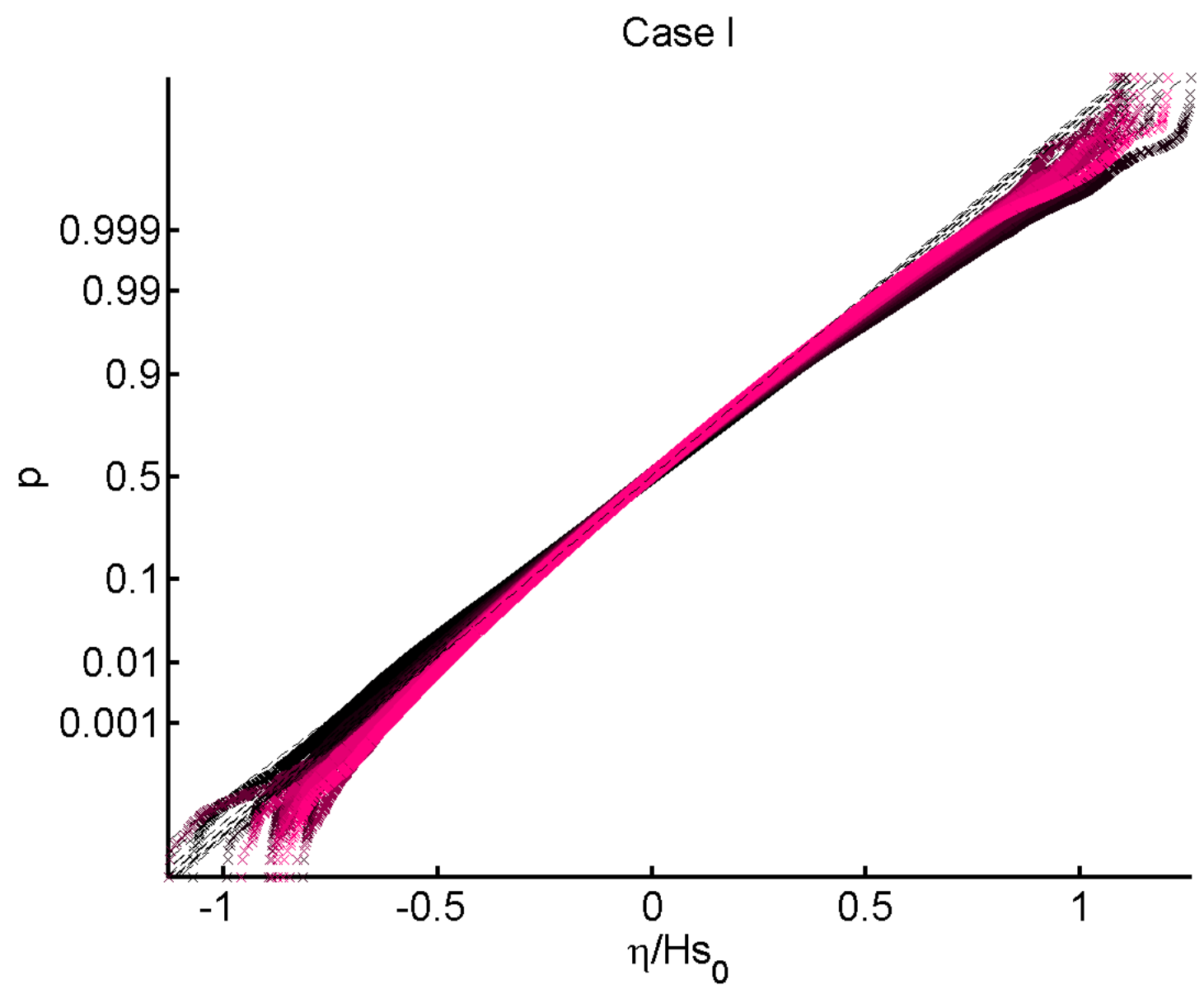

Figure 3-2: (In Color) Horizontal axis shows non-dimensional surface elevation and vertical axis shows the cumulative distribution of such an elevation in the wavefield. Time evolution of theoretical (- - -) surface elevation distribution, and observed surface elevation distribution data $(++++)$ in a nonlinear simulation of Case I (where $\gamma=1.0$ ). Each line pair represents one time step. Earlier times (from $t=0 T_{p}$ ) are the darkest lines. Later times (up to $t=100 T_{p}$ ) are the brightest lines. 


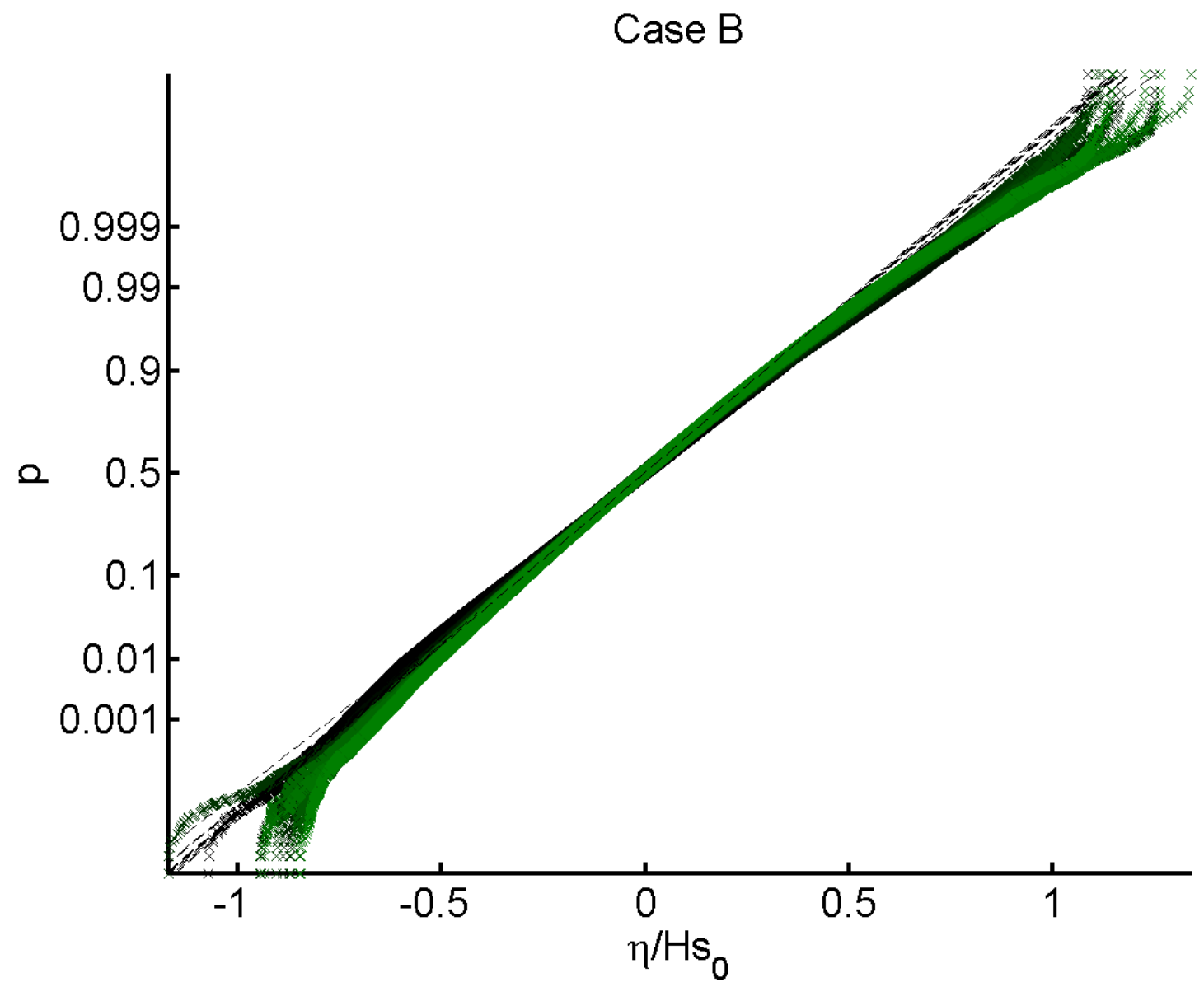

Figure 3-3: (In Color) Horizontal axis shows non-dimensional surface elevation and vertical axis shows the cumulative distribution of such an elevation in the wavefield. Time evolution of theoretical (- - -) surface elevation distribution, and observed surface elevation distribution data $(++++)$ in nonlinear simulations of Case $\mathbf{B}$ (where $\gamma=3.3$ ). Each line pair represents one time step. Earlier times (from $t=0 T_{p}$ ) are the darkest lines. Later times (up to $t=100 T_{p}$ ) are the brightest lines. 


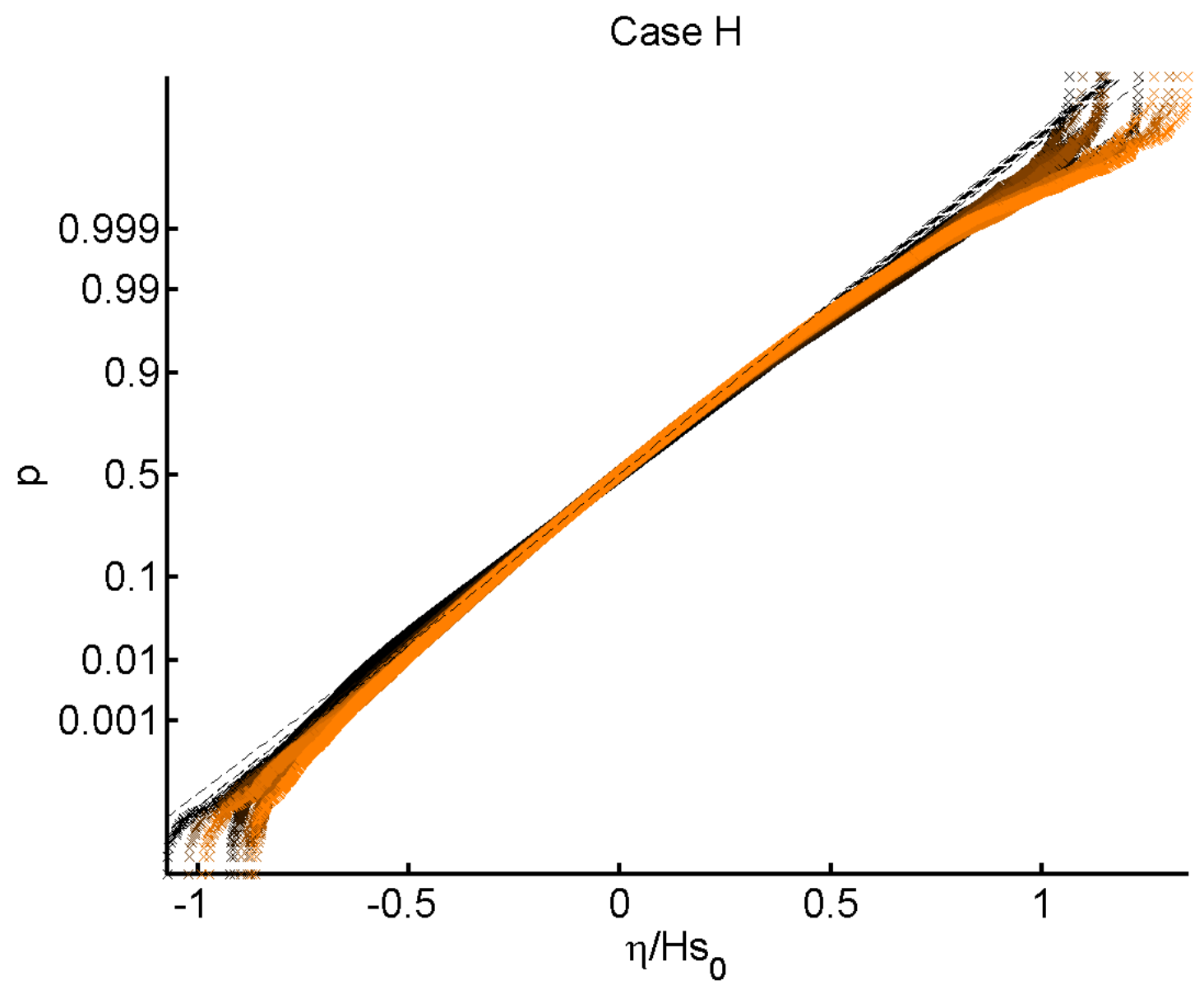

Figure 3-4: (In Color) Horizontal axis shows non-dimensional surface elevation and vertical axis shows the cumulative distribution of such an elevation in the wavefield. Time evolution of theoretical (- - -) surface elevation distribution, and observed surface elevation distribution data $(++++)$ in nonlinear simulations of Case $\mathbf{H}$ (where $\gamma=5.0$ ). Each line pair represents one time step. Earlier times (from $t=0 T_{p}$ ) are the darkest lines. Later times (up to $t=100 T_{p}$ ) are the brightest lines. 


\section{Time 20Tp}

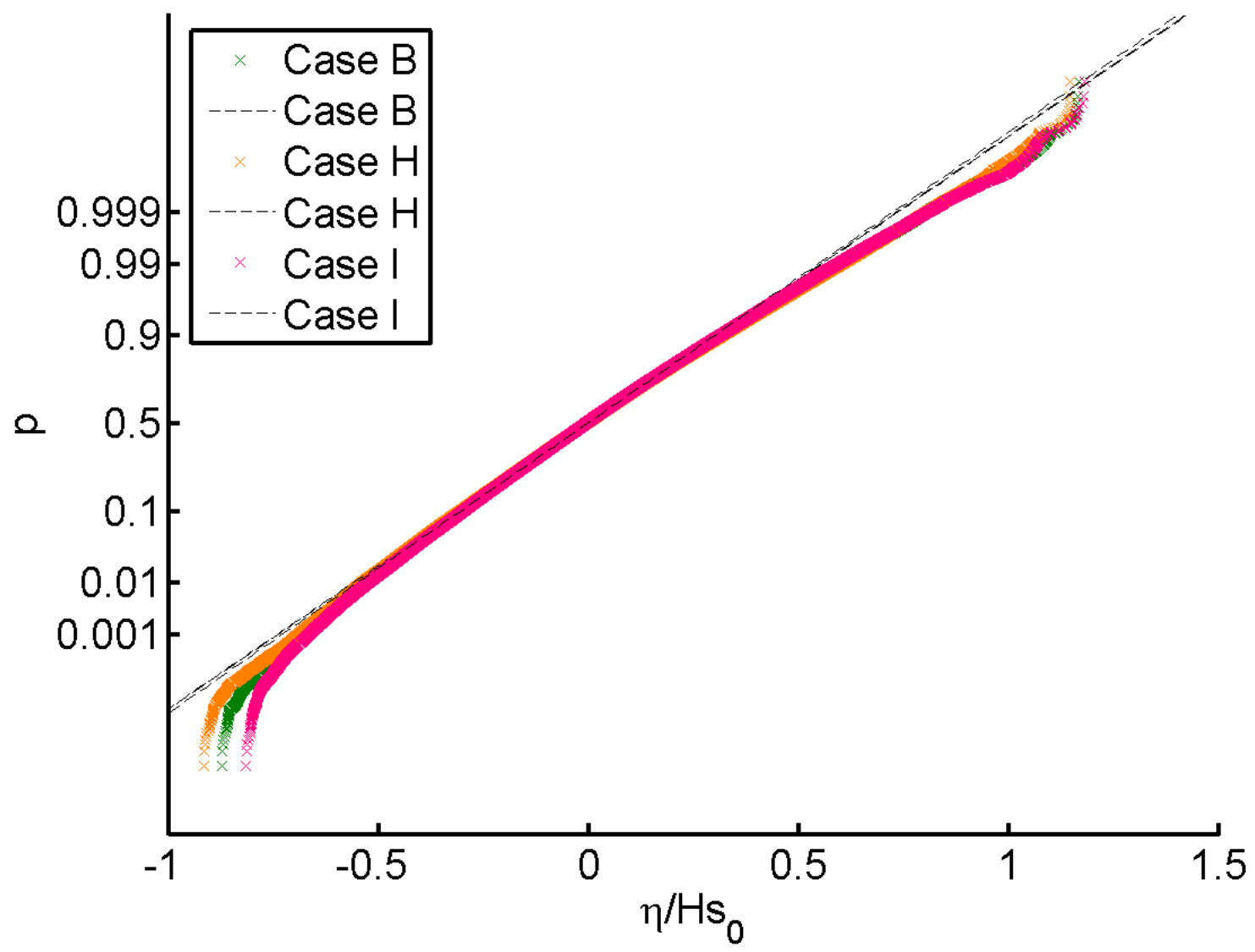

Figure 3-5: (In Color) Horizontal axis shows non-dimensional surface elevation and vertical axis shows the cumulative distribution of such an elevation in the wavefield. Cases I, B and $\mathrm{H}$ surface elevation distribution at time, $t=20 T_{p}$. Comparison between three cases in nonlinear simulations of cases where $\gamma=1.0$ (magenta), $\gamma=3.3$ (green), and $\gamma=5.0$ (orange) between theoretical (- - -) surface elevation distribution, and observed surface elevation distribution data $(++++)$. Each line pair represents one case. 


\section{Time 50Tp}

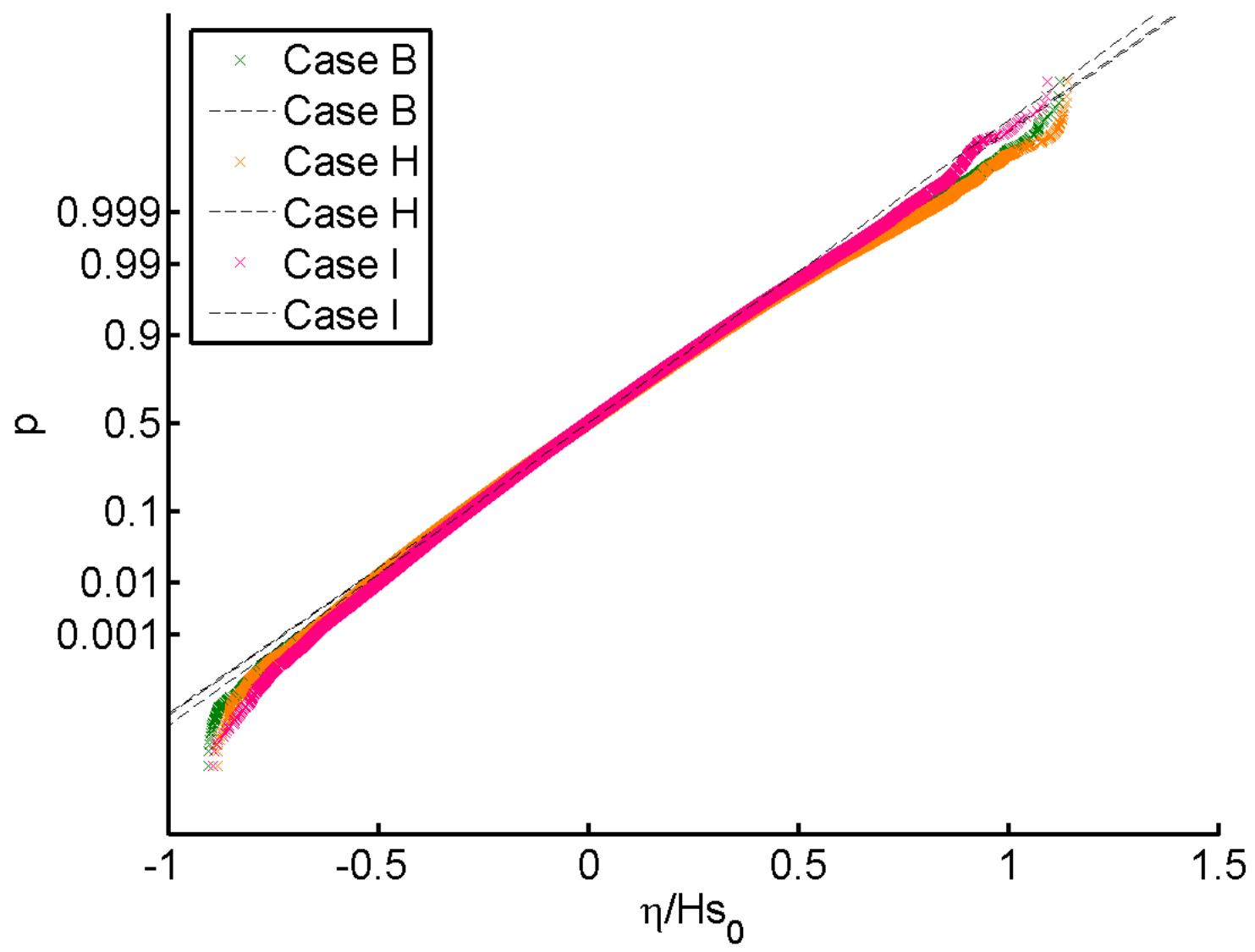

Figure 3-6: (In Color) Horizontal axis shows non-dimensional surface elevation and vertical axis shows the cumulative distribution of such an elevation in the wavefield. Cases I, B and $\mathrm{H}$ surface elevation distribution at time, $t=50 T_{p}$. Comparison between three cases in nonlinear simulations of cases where $\gamma=1.0$ (magenta), $\gamma=3.3$ (green), and $\gamma=5.0$ (orange) between theoretical (- - -) surface elevation distribution, and observed surface elevation distribution data $(++++)$. Each line pair represents one case. 


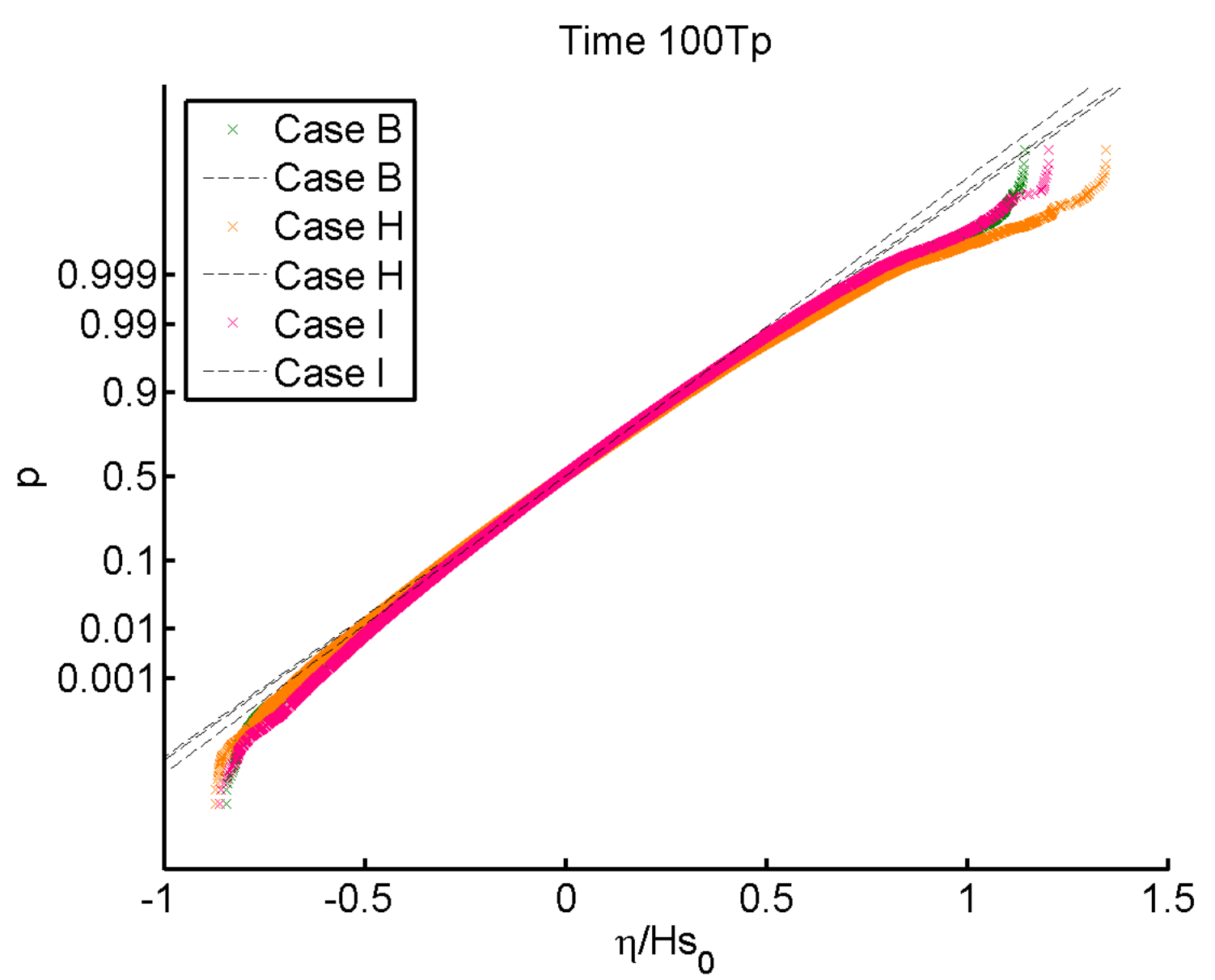

Figure 3-7: (In Color) Horizontal axis shows non-dimensional surface elevation and vertical axis shows the cumulative distribution of such an elevation in the wavefield. Cases I, B and $\mathrm{H}$ surface elevation distribution at time, $t=100 T_{p}$. Comparison between three cases in nonlinear simulations of cases where $\gamma=1.0$ (magenta), $\gamma=3.3$ (green), and $\gamma=5.0$ (orange) between theoretical (- - - ) surface elevation distribution, and observed surface elevation distribution data $(++++)$ at times $t=20 T_{p}, 50 T_{p}$, and $100 T_{p}$. Each line pair represents one case. 


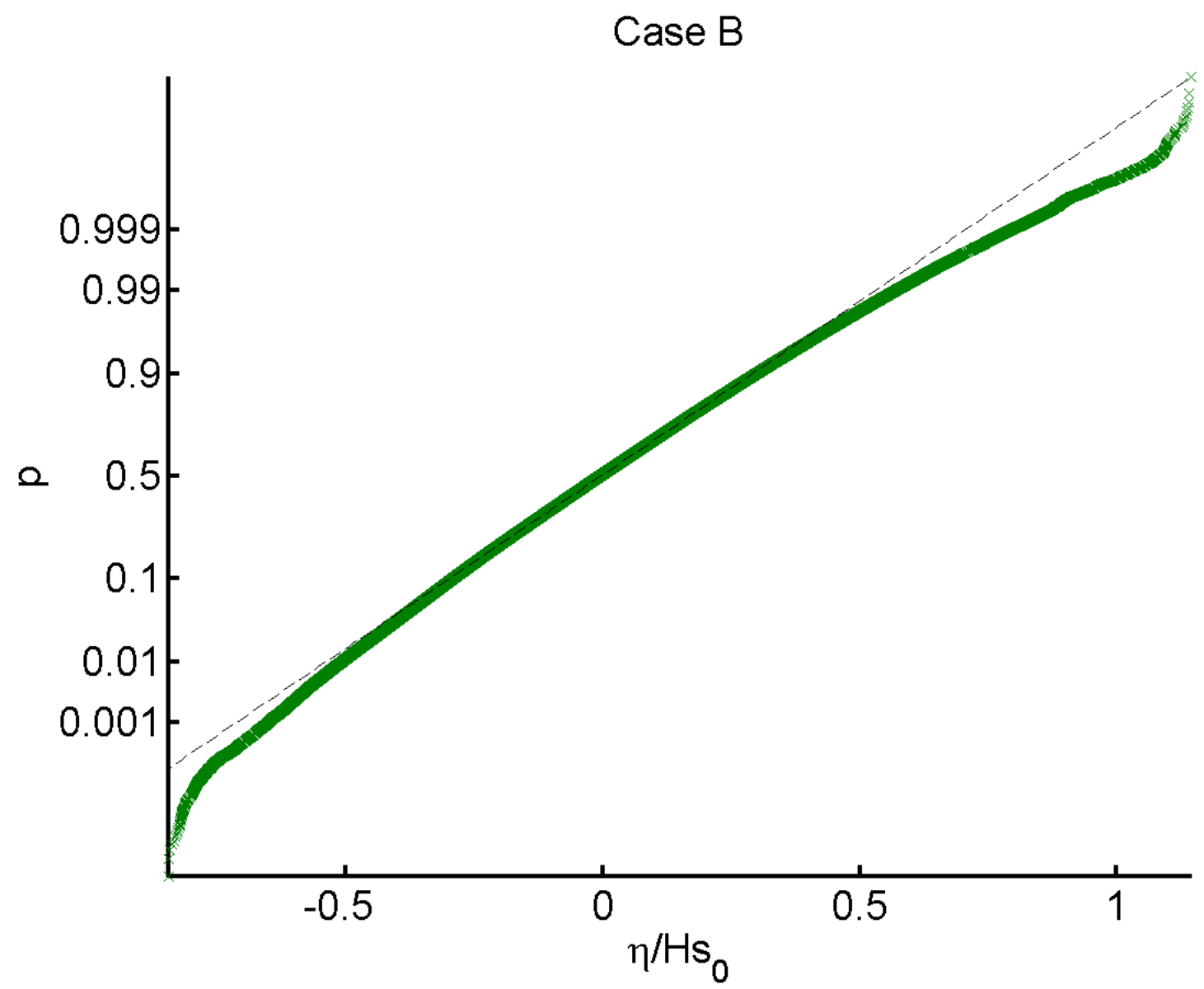

Figure 3-8: Horizontal axis shows non-dimensional surface elevation and vertical axis shows the cumulative distribution of such an elevation in the wavefield where $\gamma=3.3$ ar $t=100 T_{p}$. Kurtosis is more apparent than skewness for the large values of surface elevation (seen on the far right of the graphic) as the observed data series $(+++)$ falls below the Gaussian theoretical line(- - -). 


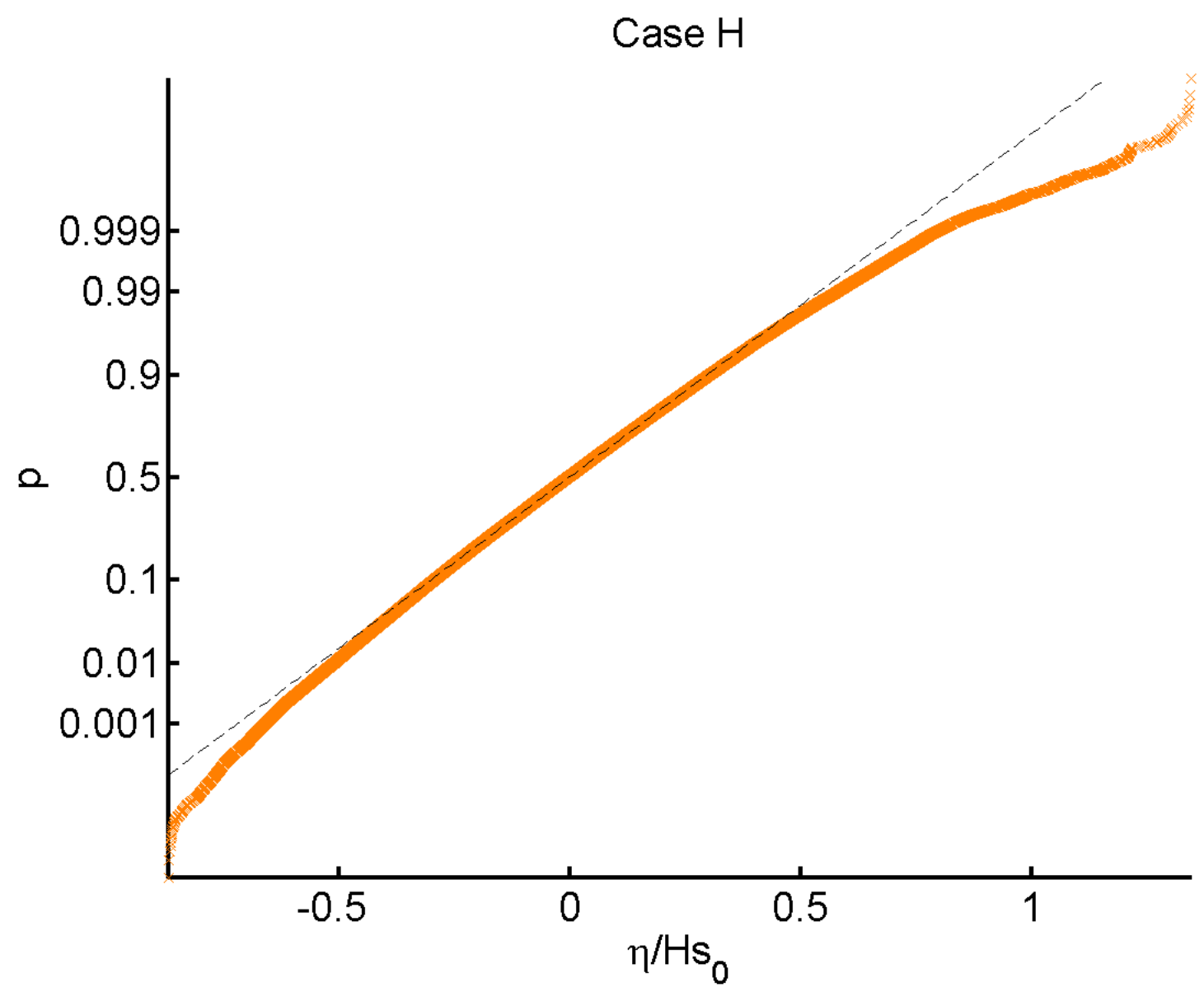

Figure 3-9: Horizontal axis shows non-dimensional surface elevation and vertical axis shows the cumulative distribution of such an elevation in the wavefield where $\gamma=$ 5.0 at time $t=100 T_{p}$. Kurtosis is more apparent than skewness for the large values of surface elevation (seen on the far right of the graphic) as the observed data series $(+++)$ falls below the Gaussian theoretical line(- - -). 


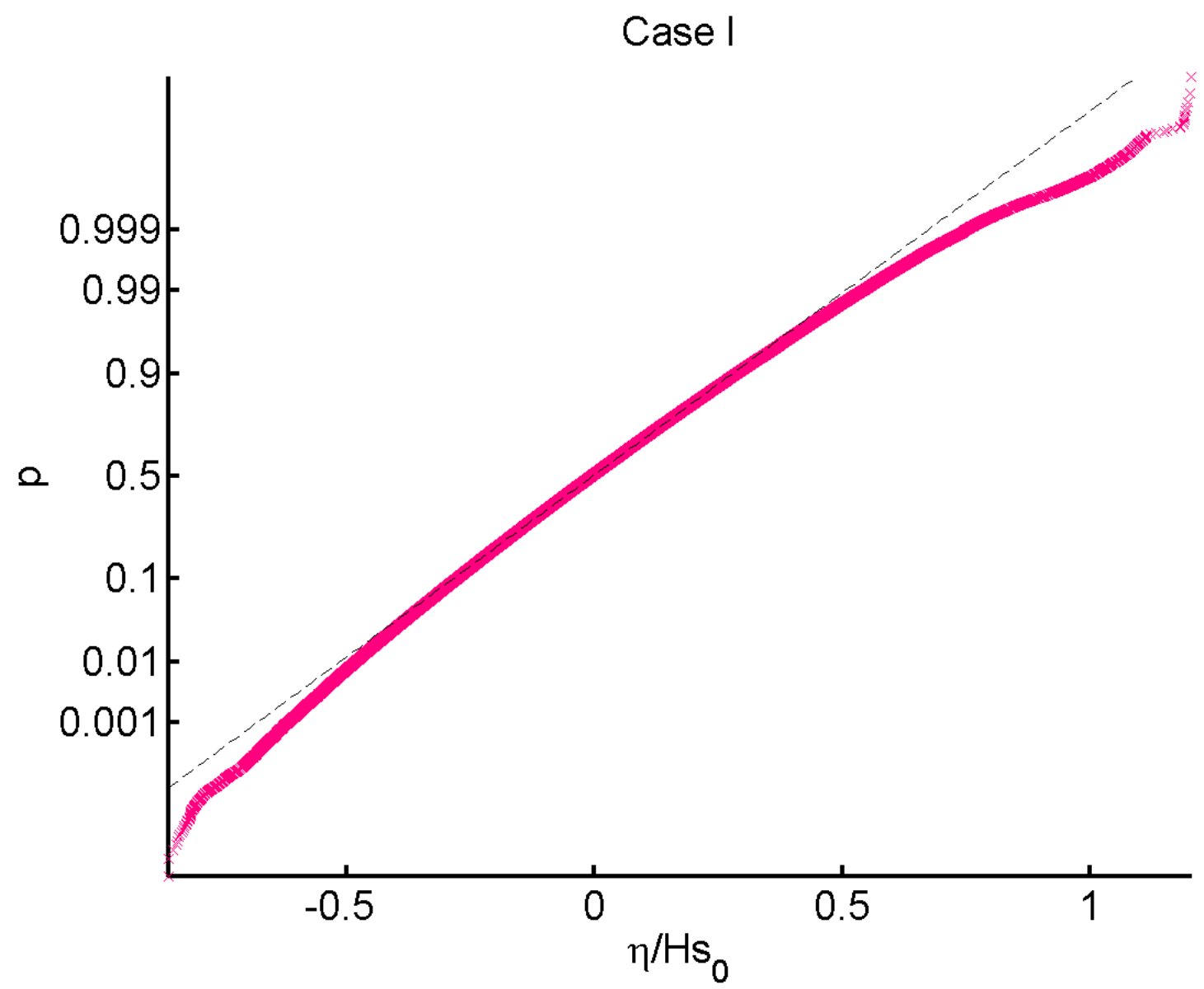

Figure 3-10: Horizontal axis shows non-dimensional surface elevation and vertical axis shows the cumulative distribution of such an elevation in the wavefield where $\gamma=1.0$ at time $t=100 T_{p}$. Kurtosis is more apparent than skewness for the large values of surface elevation (seen on the far right of the graphic) as the observed data series $(+++)$ falls below the Gaussian theoretical line(- - -). 


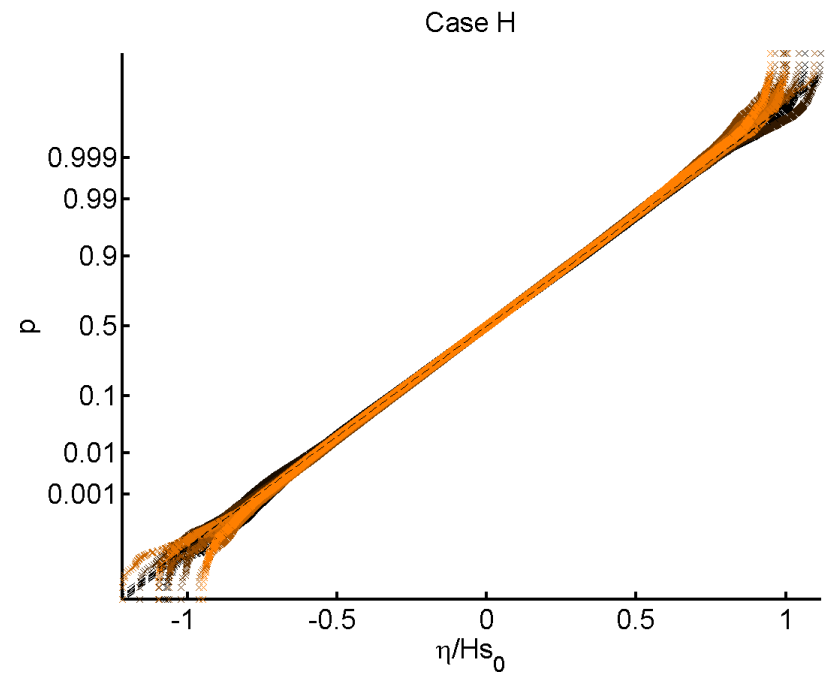

(a) Linear simulation of Case $\mathbf{H}$ (where $\gamma=5.0$ ).

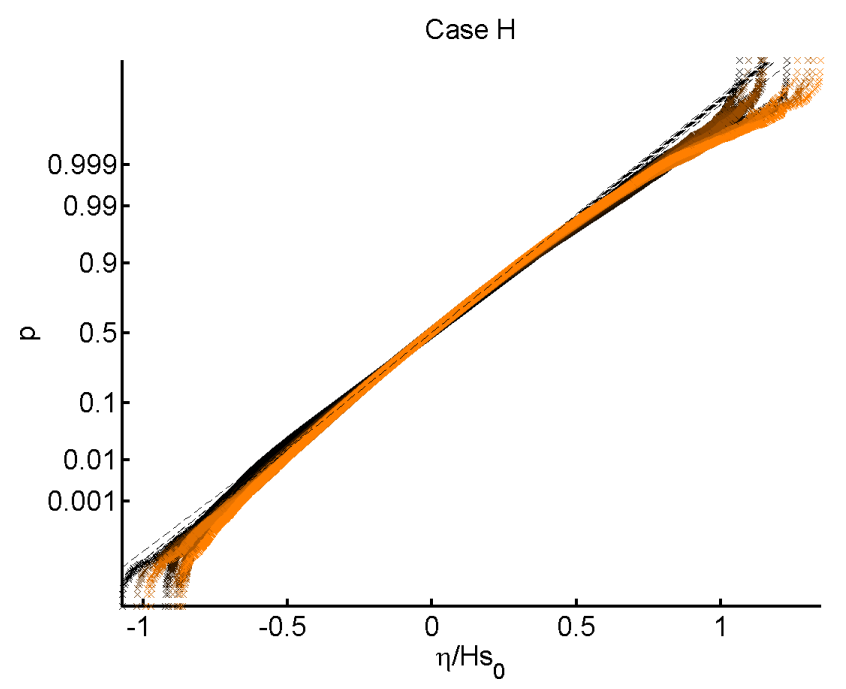

(b) Non-linear simulation of Case $\mathbf{H}$ (where $\gamma=5.0$ ).

Figure 3-11: (In Color) Horizontal axis shows non-dimensional surface elevation and vertical axis shows the cumulative distribution of such an elevation in the wavefield. We compare a linear followed by a non-linear simulation of Case $\mathbf{H}$ (where $\gamma=5.0$ ) observed surface elevation distribution data $(++++)$. The order of nonlinearity has measurable effects on surface elevation wavenumber spectrum. 
effect of the peak shape parameter, $\gamma$, on the nonlinear qualities in the distribution of the surface elevation in a simulated wavefield (modeled up to nonlinearity order $M=3$ ), using the Gram-Charlier system of nonlinear theoretical distributions up to the $3^{\text {rd }}$ order in nonlinearity shown in Equation B.26.

Three basic approaches to the non-Gaussian nature in ocean surface elevation have been developed, namely the application of the Hermite polynomial, which is orthogonal to the Gaussian distribution, the assumption that the wave profile can be represented by Stokes second- and third-order expansions, and finally, the application of the stochastic solution of a nonlinear system to a random input [41].

We now refer to the application of the Hermite polynomial as we observe the observed surface elevation data's statistical adherence to theoretical surface elevation distribution from the first, second and third order Gram Charlier distribution series. The Gram-Charlier system of distributions is a weakly non-linear statistical distribution for ocean surface elevation, generated from using the linear theory as a first approximation and successively adding higher-order terms that are functions of the Hermite polynomial and surface elevation statistical moments. We observe surface elevation statistical moments in Section 3.1.1. Our wavefield simulations are thirdorder nonlinear, and for this reason we compare surface elevation distribution to the first-, second- and third-order Gram Charlier distributions (recall that the first order of the Gram-Charlier distribution is the Gaussian distribution, as stated in Appendix B), studying the effect of the input nonlinear characteristics on the surface elevation properties and also assessing the accuracy of the Gram-Charlier distribution in capturing our nonlinear wavefields' surface elevations.

Gram-Charlier series are based on the statistical moments of surface elevation. On observing Figures 3-12, 3-13 and 3-14 we see that the time evolution of the wavefield produces different surface elevation Gram-Charlier distributions. We see as time proceeds, the lower elevation tails become more aligned with the first order and higher elevation tails have higher deviations in probability distribution from the first, second and third order theoretical distributions in Case $\mathbf{I}$ where $\gamma=1.0$. This indicates that a low peak shape parameter $(\gamma=1.0)$ which is expected to produce 


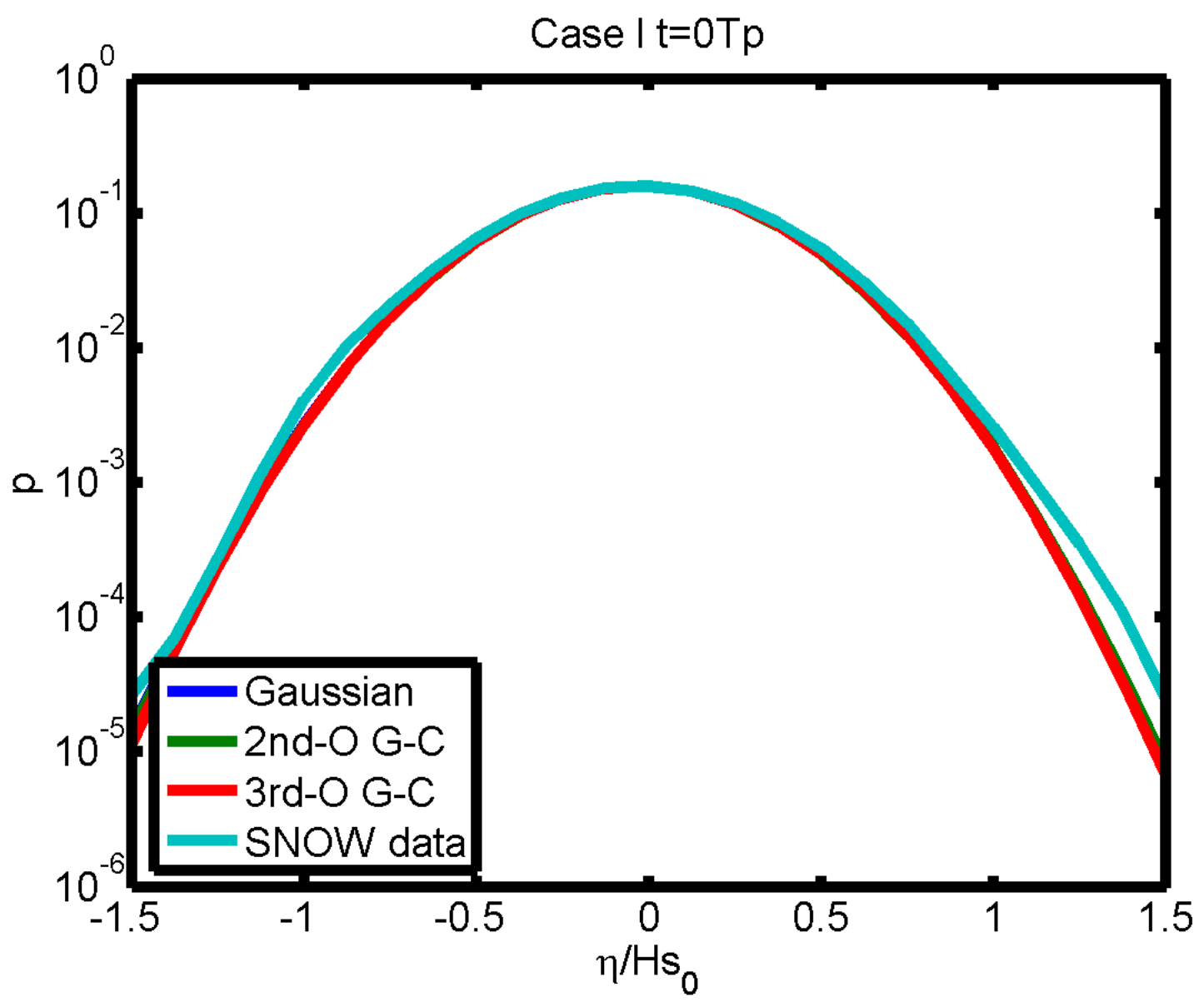

Figure 3-12: (In Color) Horizontal axis depicts non-dimensional surface elevation and vertical axis depicts probability. Nonlinear simulation of Case $\mathbf{I}$ (where $\gamma=1.0$ ), observed surface elevation distribution data at time, $t=0 T_{p}$ compared to Gram Charlier (G-C) distributions Order 1, 2 and 3. 


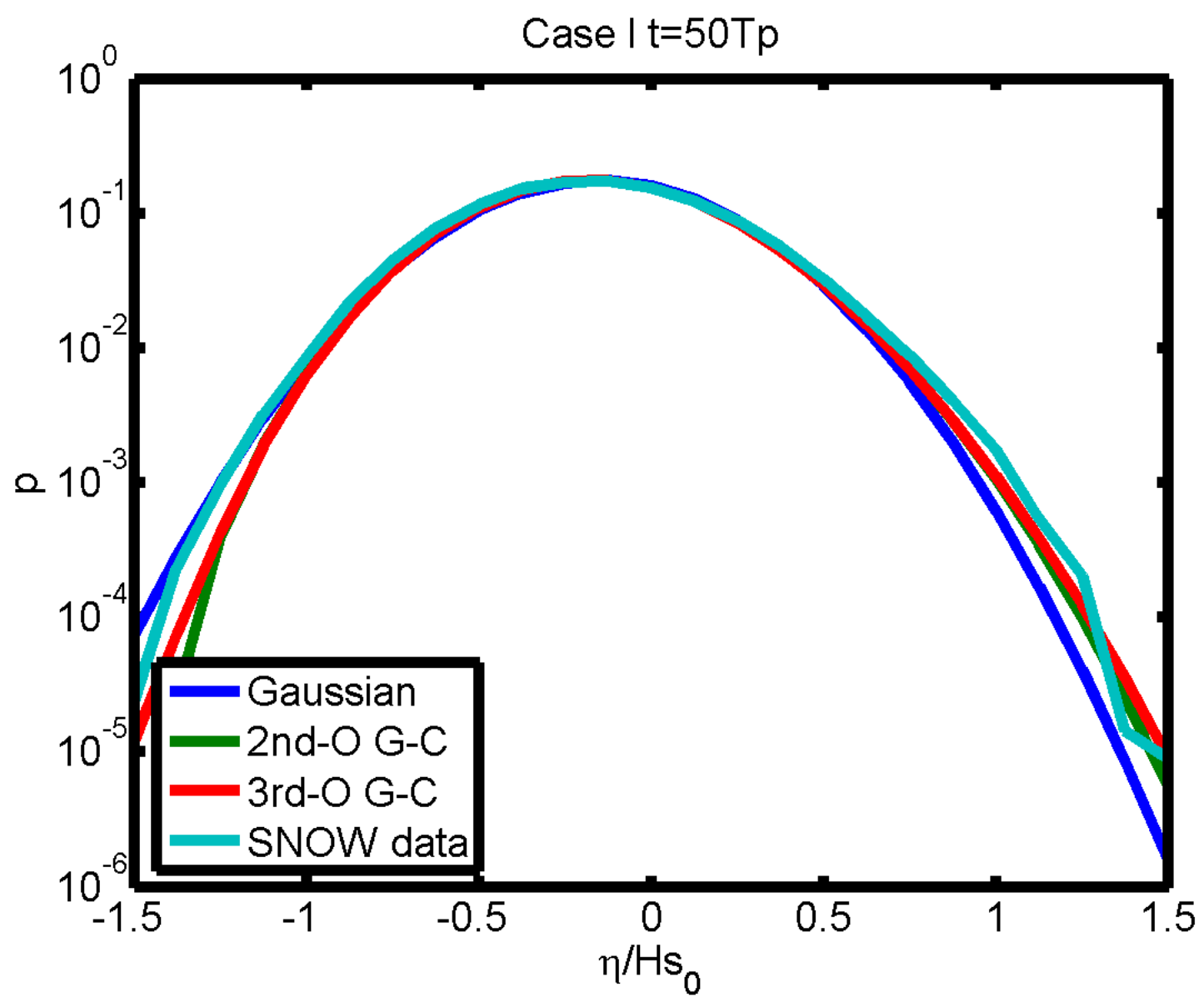

Figure 3-13: (In Color) Horizontal axis depicts non-dimensional surface elevation and vertical axis depicts probability. Nonlinear simulation of Case $\mathbf{I}$ (where $\gamma=1.0$ ), observed surface elevation distribution data at time, $t=50 T_{p}$ compared to Gram Charlier (G-C) distributions Order 1, 2 and 3. 


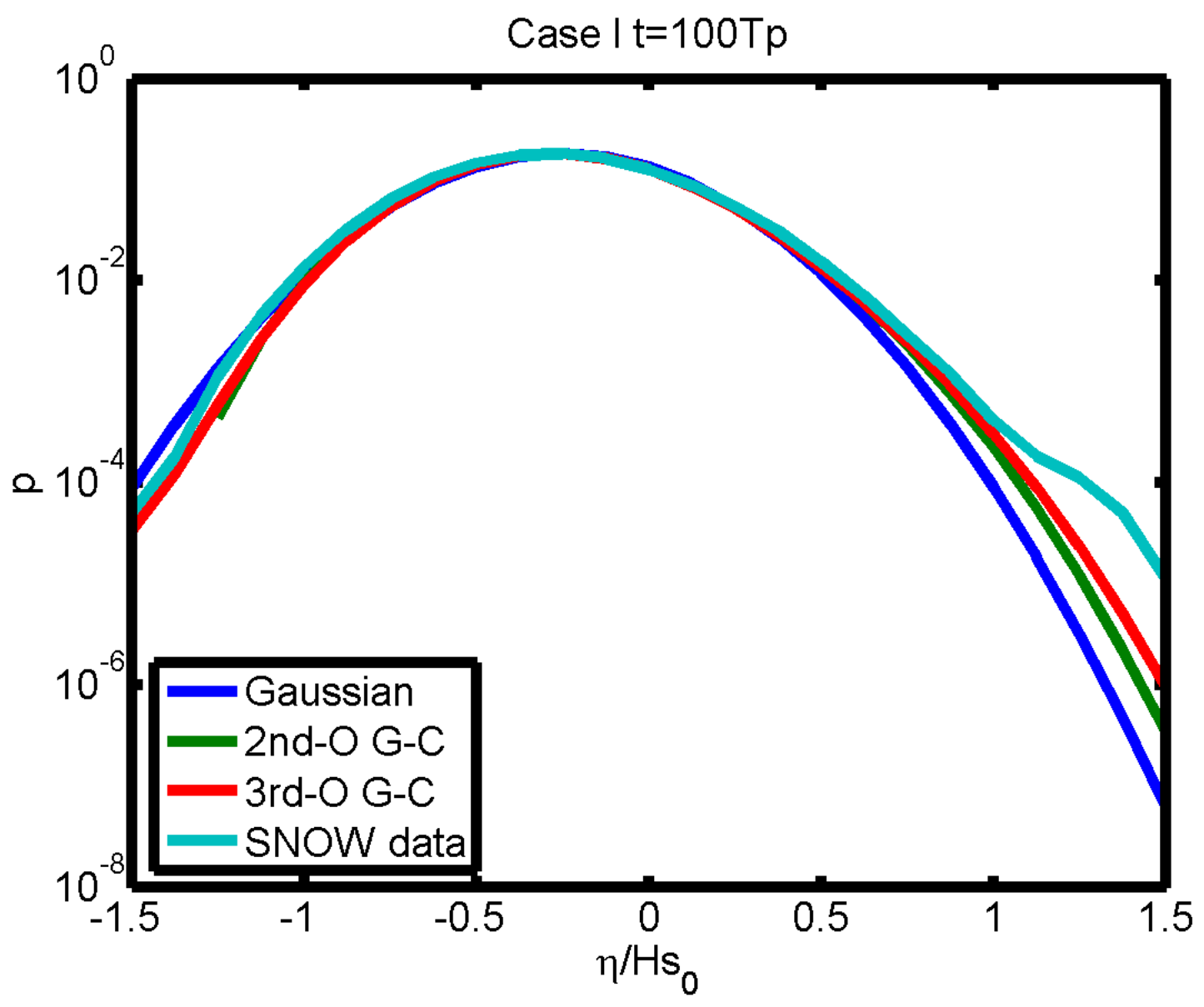

Figure 3-14: (In Color) Horizontal axis depicts non-dimensional surface elevation and vertical axis depicts probability. Nonlinear simulation of Case $\mathbf{I}$ (where $\gamma=1.0$ ), observed surface elevation distribution data at time, $t=100 T_{p}$ compared to Gram Charlier (G-C) distributions Order 1, 2 and 3. 
near-linear wavefields, does not mask the effects of nonlinearity in the wavefield.

In Figures 3-13, 3.2.1 and 3-16 we observe surface elevation distribution in three cases at time $50 T_{p}$. We see the effect of peak shape parameter, $\gamma$, on the statistical distribution of ocean surface elevation, as it compares to nonlinear theoretical distributions up to the $3^{\text {rd }}$ order in nonlinearity. It is confirmed here that a higher value of peak shape parameter, producing higher statistical moments, produces greater spreading between the $1^{\text {st }}, 2^{\text {nd }}$, and $3^{\text {rd }}$ order plots. Results show that for Case $\mathbf{I}$, where $\gamma=1.0$, the upper tail of the data distribution is nearest the theoretical $3^{\text {rd }}$ order distribution. In Case $\mathbf{B}$, where $\gamma=3.3$ results show the upper tail of the data distribution is further from the theoretical $3^{\text {rd }}$ order distribution. We also see in Case $\mathbf{H}$ (where $\gamma=5.0$ ) that the upper tail of the data distribution is furthest from the theoretical $3^{\text {rd }}$ order distribution. In all three cases, the lower tails of the distributions, where the troughs are distributed, we find that the $1^{\text {st }}$ and $3^{\text {rd }}$ order theoretical distributions are exactly aligned with the data, while the $2^{\text {nd }}$ order distribution veers away from the data and the other theoretical distributions. This shows that peak shape parameter has a greater effect on the higher surface elevations' nonlinear traits than it does on the lowest surface elevations' nonlinear traits.

In this section we look at the effect of peak shape parameter, $\gamma$, on the statistical distribution of ocean surface elevation, paying particular attention to distribution tails. It is found that for peak shape parameter value $\gamma=3.3$ which produces waves that are consistent with sea waves in stormy conditions, the most non-linear characteristics appear in surface elevation distribution tails.

\subsubsection{Peak shape parameter and surface elevation wavenum- ber spectrum.}

In this section we test the effect of peak shape parameter on surface elevation wavenumber spectrum by comparing results derived between Cases $\mathbf{I}$ (where $\gamma=1.0$ ), B (where $\gamma=3.3$ ) and $\mathbf{H}$ (where $\gamma=5.0$ ). We also test the importance of nonlinearity in such

effects by comparing the results in the nonlinear wavefields with results from linear 


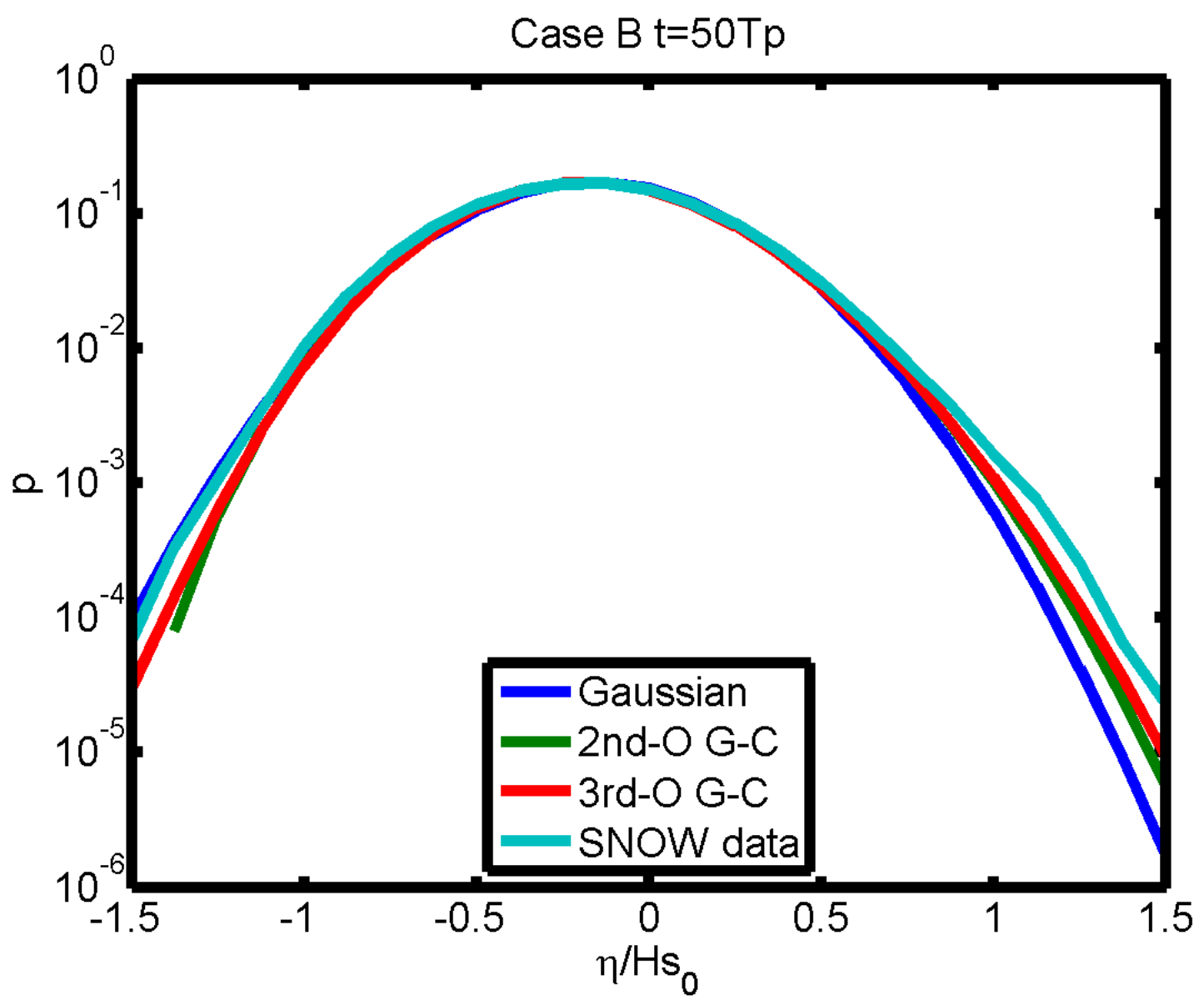

Figure 3-15: (In Color) Horizontal axis depicts non-dimensional surface elevation and vertical axis depicts probability. Case $\mathbf{B}$ (where $\gamma=3.3$ ), observed surface elevation distribution data at time, $t=50 T_{p}$ compared to Gram Charlier (G-C) distributions Order 1,2 and $3 .$. 


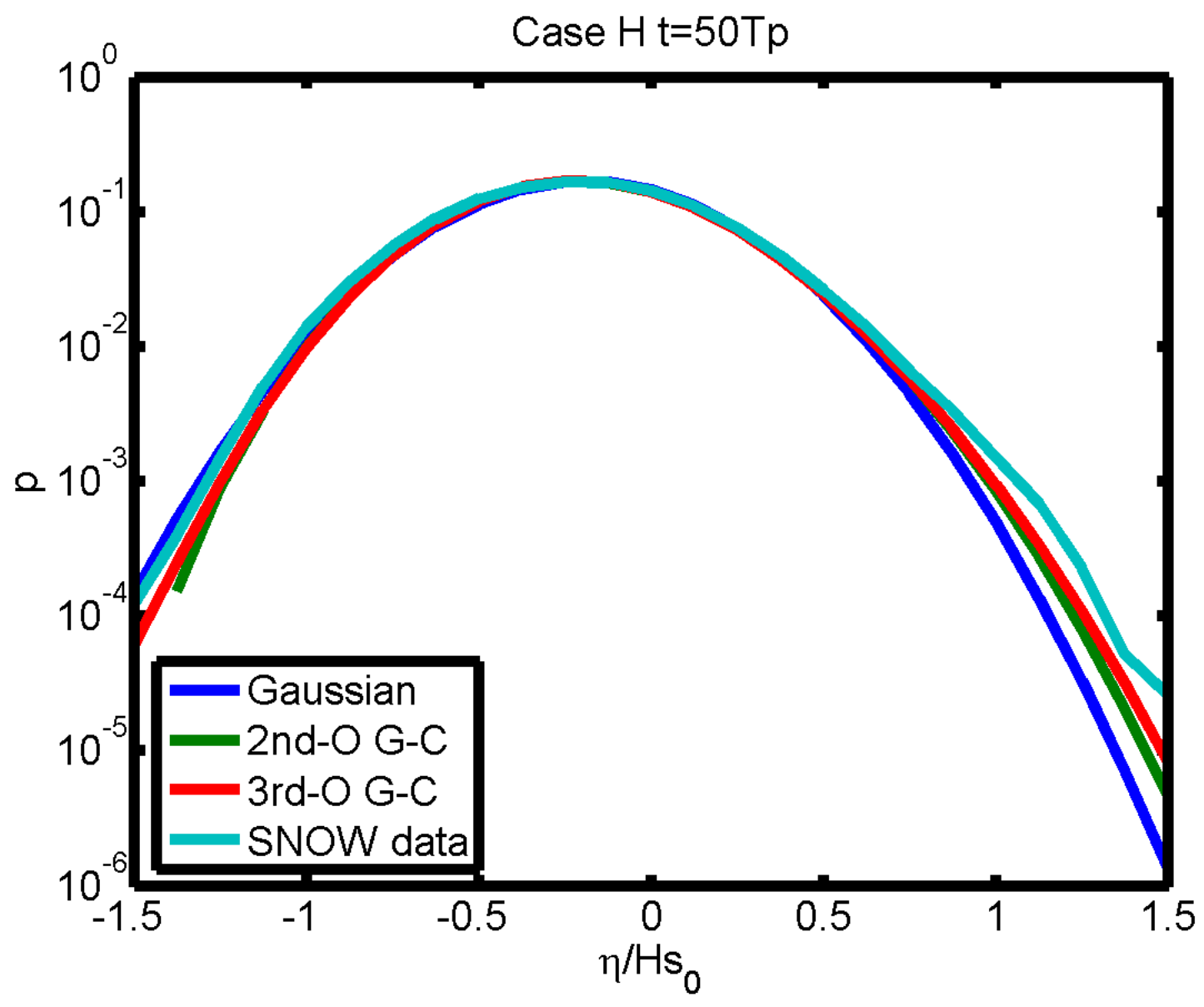

Figure 3-16: (In Color) Horizontal axis depicts non-dimensional surface elevation and vertical axis depicts probability. Case $\mathbf{H}$ (where $\gamma=5.0$ ), observed surface elevation distribution data at time, $t=50 T_{p}$ compared to Gram Charlier (G-C) distributions Order 1,2 and 3. 
simulations.

From Figures 3-17, 3-18 and 3-19 we observe that a higher value of peak shape parameter produces a higher value of the maximum spectral energy in surface elevation wavenumber spectrum in a nonlinear wavefield. We also see that the maximum peak spectral energy is most stable near $\gamma=3.3$ and less stable when $\gamma=1.0$ or $\gamma=5.0$. We also see the spectral energy peaks at dimensionless wavenumber, 8 in the cases when $\gamma=1.0$ and $\gamma=3.3$, but the maximum spectral energy occurs at dimensionless wavenumber, 9 when $\gamma=5$.0. In all three nonlinear cases we see a downshift in the wavenumber spectral energy where the wavefield carries the peak energy in longer wavelengths after the first $t=100 T_{p}$ of wavefield evolution.

We now examine the importance of non-linearity order in determining the effect of $\gamma$ on surface elevation wavenumber spectrum by comparing results derived in a non-linear simulated wavefield, with non-linearity order 3 , to results derived from a linear simulated wavefield with non-linearity order 1 (see Figures 3-20 and 3-21).

In the linear wavefield simulations (see Figures 3-20 and 3-21), Case $\mathbf{I}$ and $\mathbf{H}$ appear to be almost identical to their equivalent non-linear case, apart from two notable differences. In a linear wavefield there is no spectral downshift during the first $t=100 T_{p}$ in time evolution, and in a linear wavefield the maximum spectral energy in each wavefield is lower than in the nonlinear equivalent case.

\subsubsection{Peak shape parameter and surface elevation Benjamin- Feir Index}

In this section we observe the effect of spectral peak shape parameter on the BenjaminFeir Index (BFI), by comparing the time-evolution of the BFI between Cases I (where $\gamma=1.0$ ), $\mathbf{B}$ (where $\gamma=3.3$ ) and $\mathbf{H}$ (where $\gamma=5.0$ ). The Benjamin-Feir index is a non-dimensional quantifier of non-linearity in a wavefield.

The Benjamin-Feir instability [5] can occur in a deep-water periodic, progressive wave-train with fundamental frequency $\omega$ interacting with residual periodic motions at adjacent side-band frequencies $\omega(1+\delta)$. This can be made possible, if for example, 


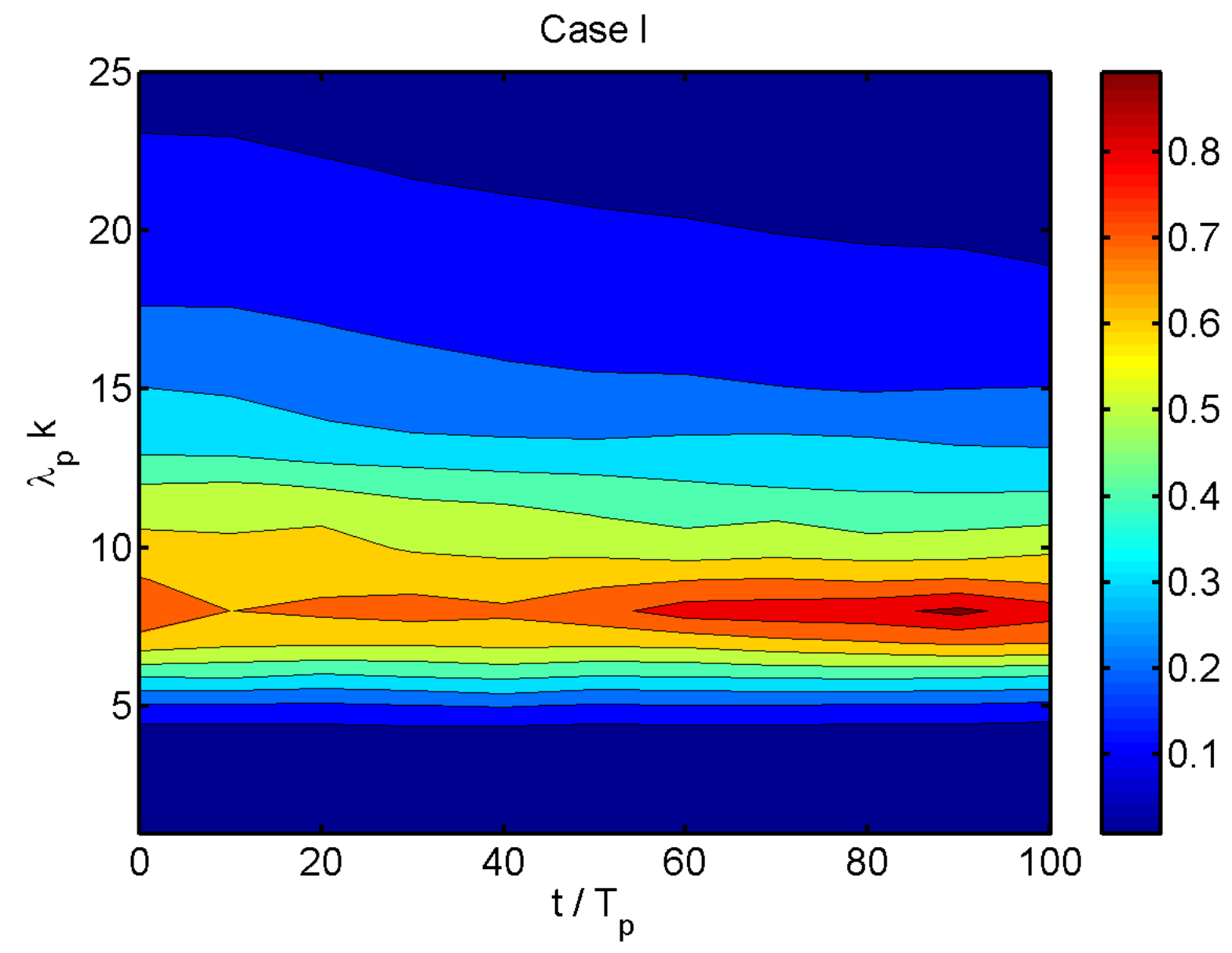

Figure 3-17: (In Color) Wavenumber spectrum time evolution in a nonlinear wavefield simulation of Case $\mathbf{I}$ (where $\gamma=1.0$ ). Time $\left(t / T_{p}\right)$ is on the horizontal axis, wavenumber $\lambda_{p} k$ on the vertical axis and spectral energy level is represented in the color bar. 
Case B

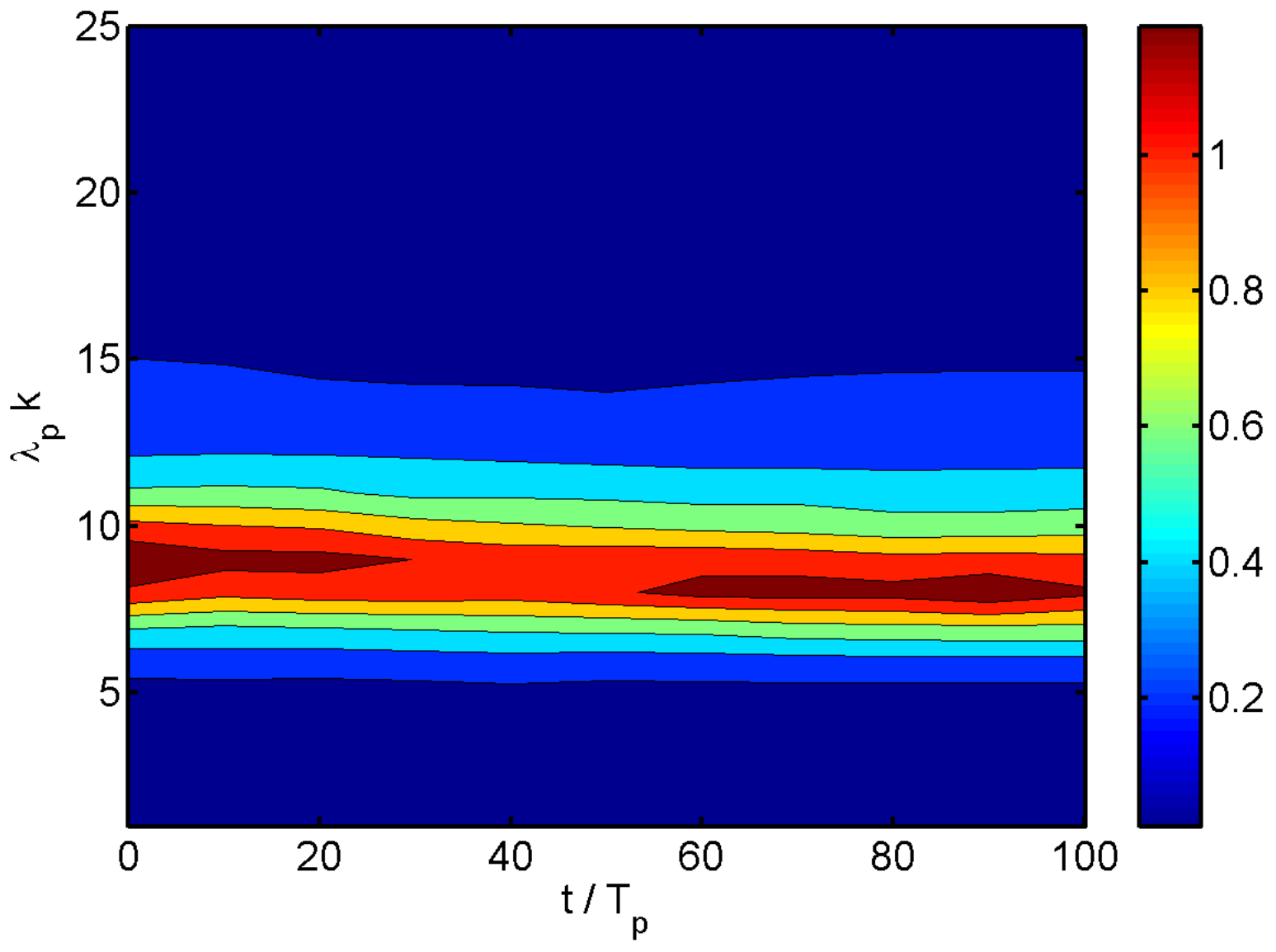

Figure 3-18: (In Color) Wavenumber spectrum time evolution in a nonlinear wavefield simulation of Case $\mathbf{B}$ (where $\gamma=3.3$ ). Time $\left(t / T_{p}\right)$ is on the horizontal axis, wavenumber $\lambda_{p} k$ on the vertical axis and spectral energy level is represented in the color bar.. 


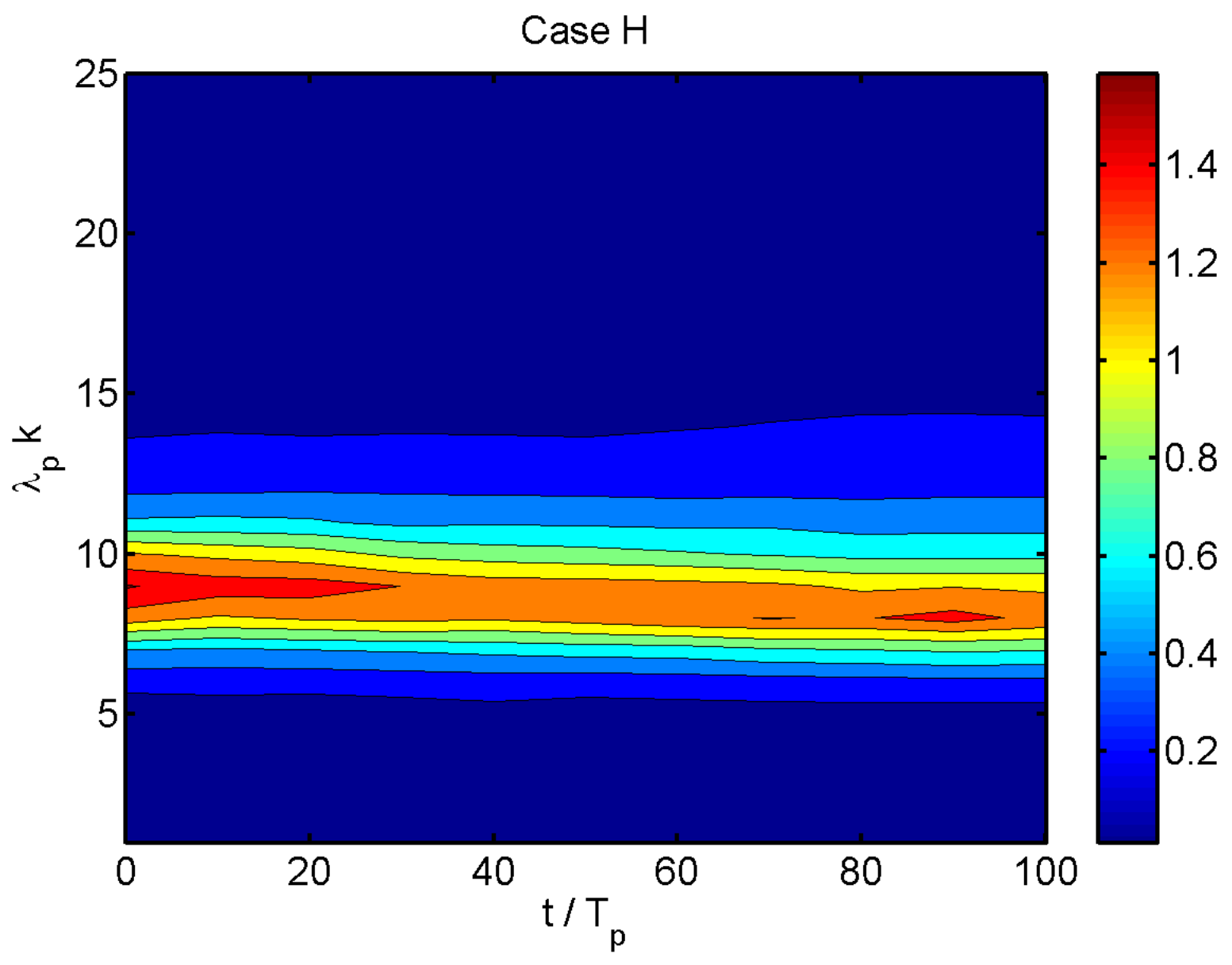

Figure 3-19: (In Color) Wavenumber spectrum time evolution in a nonlinear wavefield simulation of Case $\mathbf{H}$ (where $\gamma=5.0$ ). Time $\left(t / T_{p}\right)$ is on the horizontal axis, wavenumber $\lambda_{p} k$ on the vertical axis and spectral energy level is represented in the color bar. 


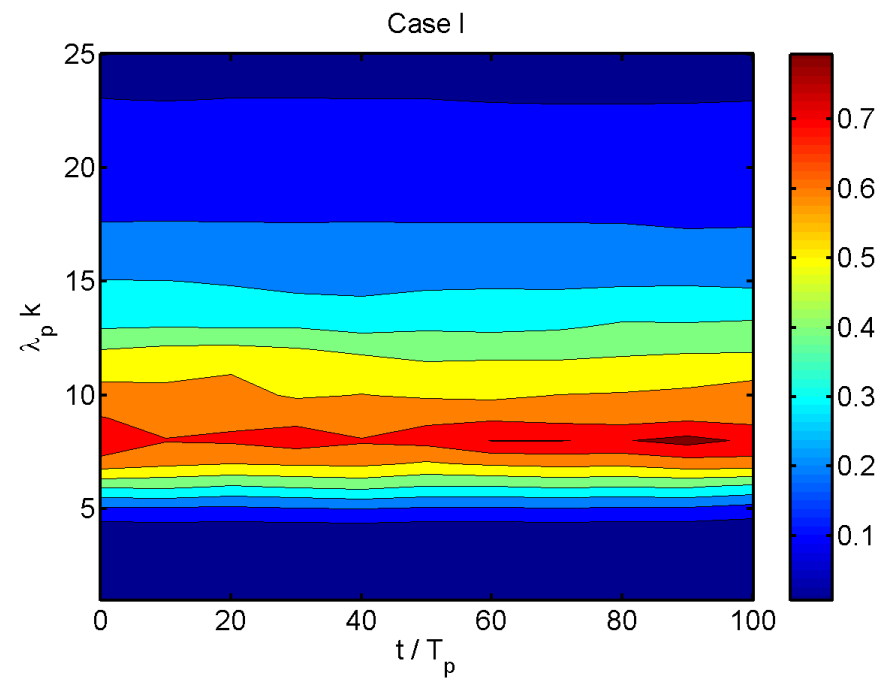

(a) Linear simulation of Case $\mathbf{I}$ (where $\gamma=1.0$ ).

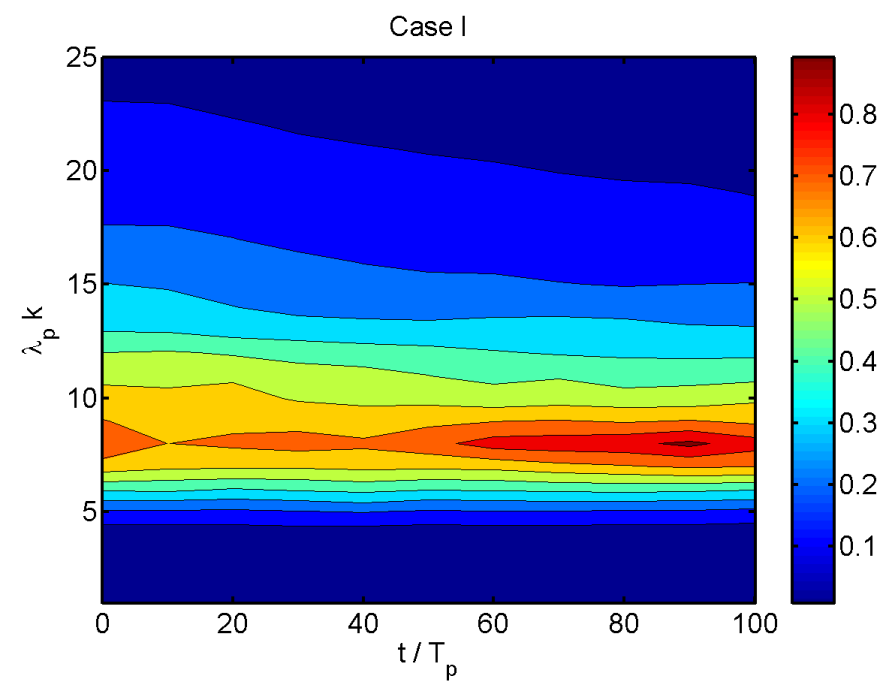

(b) Non-linear simulation of Case $\mathbf{I}$ (where $\gamma=1.0$ ).

Figure 3-20: (In Color) Wavenumber spectrum time evolution in linear and nonlinear wavefield simulation of Case $\mathbf{I}$ where $\gamma=1.0$. Time $\left(t / T_{p}\right)$ on the horizontal axis, wavenumber $\lambda_{p} k$ on the vertical axis and spectral energy level represented in color bar. The order of nonlinearity has measurable effects on surface elevation wavenumber spectrum. 


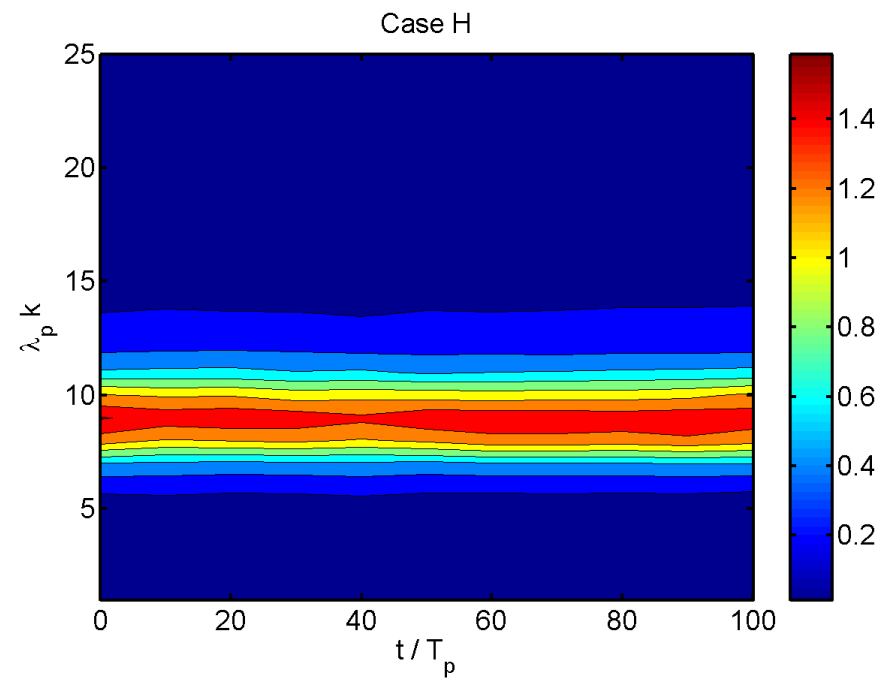

(a) Linear simulation of Case $\mathbf{H}$ (where $\gamma=5.0$ ).

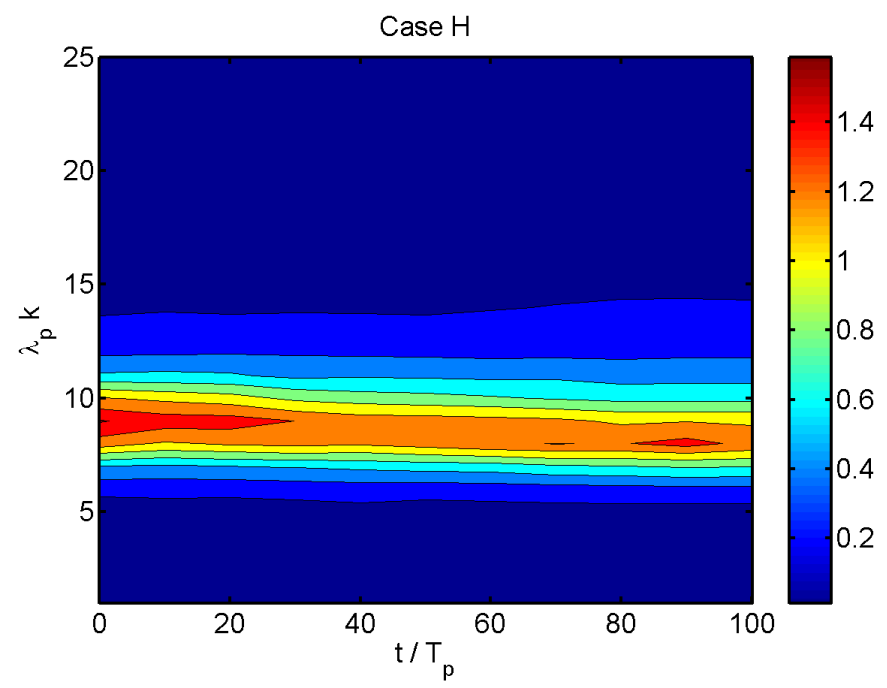

(b) Non-linear simulation of Case $\mathbf{H}$ (where $\gamma=5.0$ ).

Figure 3-21: (In Color) Wavenumber spectrum time evolution in linear and nonlinear wavefield simulation of Case $\mathbf{H}$ where $\gamma=5.0$. Time $\left(t / T_{p}\right)$ on the horizontal axis, wavenumber $\lambda_{p} k$ on the vertical axis and spectral energy level represented in color bar. The order of nonlinearity has measurable effects on surface elevation wavenumber spectrum. 
the movement of the wave source (e.g. a wave-maker on the side of a tank) had a slight low-frequency modulation perhaps caused by a mechanical fault in the instrumentation. Benjamin and Feir [5] show the analytic and experimental proof that in the presence of coupling via non-linear boundary conditions at the free surface boundary, energy can be transferred from the primary motion to the side bands, at an exponentially increasing rate. The main conclusion of this theory is that infinitesimal disturbances undergo unbounded magnification if $0<\delta<\sqrt{2}(k a)$, where $k$ is the wavenumber of the wave train and $a$ is the amplitude of the wave train. Janssen [24] then defines a non-linearity quantifier, the Benjamin-Feir Index, which is associated with the conditions under which the The Benjamin-Feir instability occurs.

In studying nonlinear wave interactions and freak waves, Janssen [24] uses the Benjamin-Feir Index (BFI) to study the likelihood of large wave formation. Prior to this, Onorato et al [47] use dimensional arguments to derive the square of the BFI, referring to it as an Ursell number. In the present work we use a nonlinear model derived from the Zakharov [48] to define a scaled BFI. This definition holds in arbitrary depth and in a frame of reference moving with the group velocity. When the Zakharov equation describes the evolution of free waves under the narrow-band approximation [74], the BenjaminFeir index (associated with the Benjamin-Feir non-resonant wavefield modulational instability where wave energy at the peak wavenumber is fed to side-bands) is defined as the square root of the coefficient that multiplies the nonlinear term in the non-dimensional Zakharov equation:

$$
B F I=\frac{2 \epsilon}{\nu / K_{0}} \sqrt{\frac{|\beta|}{\sigma}}
$$

where the peak spectral width parameter, $\nu$ is defined as:

$$
\nu=\sqrt{\frac{m_{0} m_{2}}{m_{1}^{2}}-1}
$$

Spectral moments, $m_{0}, m_{1}$ and $m_{2}$ are defined as :

$$
m_{j}=\int_{0}^{\infty} \omega^{j} S(\omega) d \omega
$$


The depth-scaling term $\sqrt{\frac{|\beta|}{\sigma}}$ on the right hand side includes the influence of the water depth. In the case of the simulations used in this work, where we use infinite depth, we approximate that $\sqrt{\frac{|\beta|}{\sigma}}=1$. Hence the BFI is simply the ratio between the average wave steepness and the peak spectral width. As the BFI increases (i.e. the average steepness increases, or the spectral width decreases or both) the nonlinearity increases; therefore we expect the number of freak waves to increase [48].

In Figures 3-22 and 3-23, results depicting the effects of peak shape parameter, $\gamma$ on the BFI of non-linear and linear directional ocean wavefields are shown for Cases $\mathbf{I}($ where $\gamma=1.0), \mathbf{B}($ where $\gamma=3.3)$ and $\mathbf{H}($ where $\gamma=5.0)$.

The spectral peak shape parameter has a visible impact on the BFI in Figures 3-22 and 3-23. The higher the peak shape parameter, the higher the BFI, and therefore the lower the stability of a wavefield. For both linear simulations tested (see Figure 3-23) for Cases $\mathbf{I}$ where $\gamma=1.0$, and $\mathbf{H}$ where $\gamma=5.0$ ), there is less decay of spectral moments and BFI in time, while for non-linear cases (see Figure 3-22) we see a reduction in all of the spectral moments and the Benjamin-Feir index in time. This shows that there is an evolution towards stability in non-linear wavefield simulations but none in linear wavefield simulations.

Peak shape parameter 3.3 and 5.0 have very close first spectral moments, but peak shape parameter 1.0 has a much lower first spectral moment than these. A similar distance is seen in the second and third spectral moments, where peak shape parameter 3.3 and 5.0 have very close second and third spectral moments, but peak shape parameter 1.0 has much lower second and third spectral moments than these. The behavior of the first spectral moment in the linear cases is quite similar to its behavior in the non-linear cases, although it takes on a higher absolute value in the linear cases than in the non-linear cases. The second and third spectral moments appear to be slightly oscillatory and swiftly decaying in the non-linear case and highly oscillatory with no decay and higher absolute values in the linear case.

In this section we observed the effect of spectral peak shape parameter, $\gamma$ on spectral moments and the Benjamin-Feir Index (BFI), by comparing the time-evolution of the BFI between Cases $\mathbf{I}$ (where $\gamma=1.0), \mathbf{B}($ where $\gamma=3.3)$ and $\mathbf{H}$ (where $\gamma=5.0$ ). 


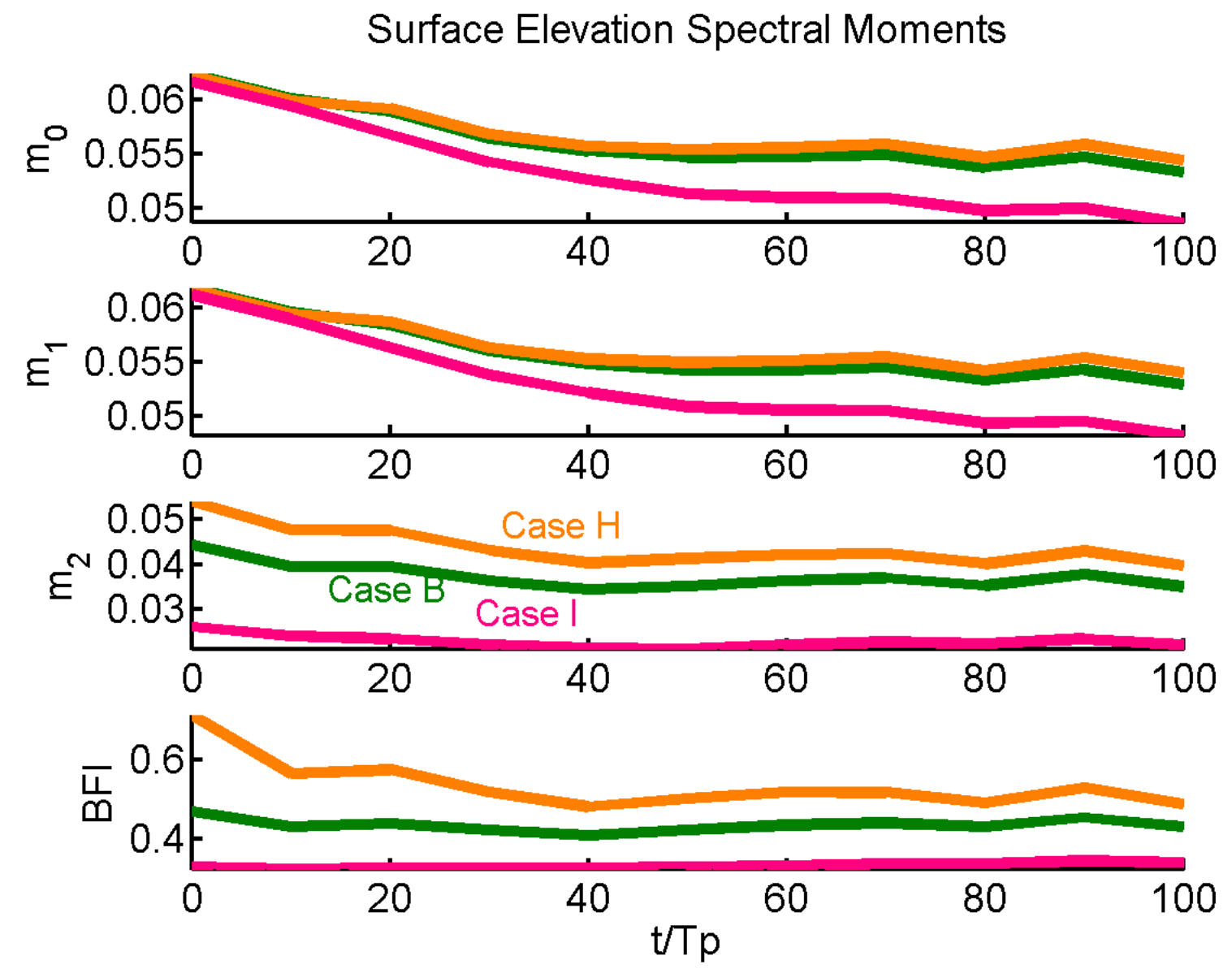

Figure 3-22: (In Color)Plot of surface elevation spectral moments against time $\left(t / T_{p}\right)$ where $m_{0}$ is the first spectral moment, $m_{1}$ is the second spectral moment, $m_{2}$ is the third spectral moment, and BFI is a scaled version of the BFI defined in Section 3.1.4. We use non-linear simulations of Cases $\mathbf{I}$ (where $\gamma=1.0$ ), $\mathbf{B}$ (where $\gamma=3.3$ ) and $\mathbf{H}$ (where $\gamma=5.0$ ). 


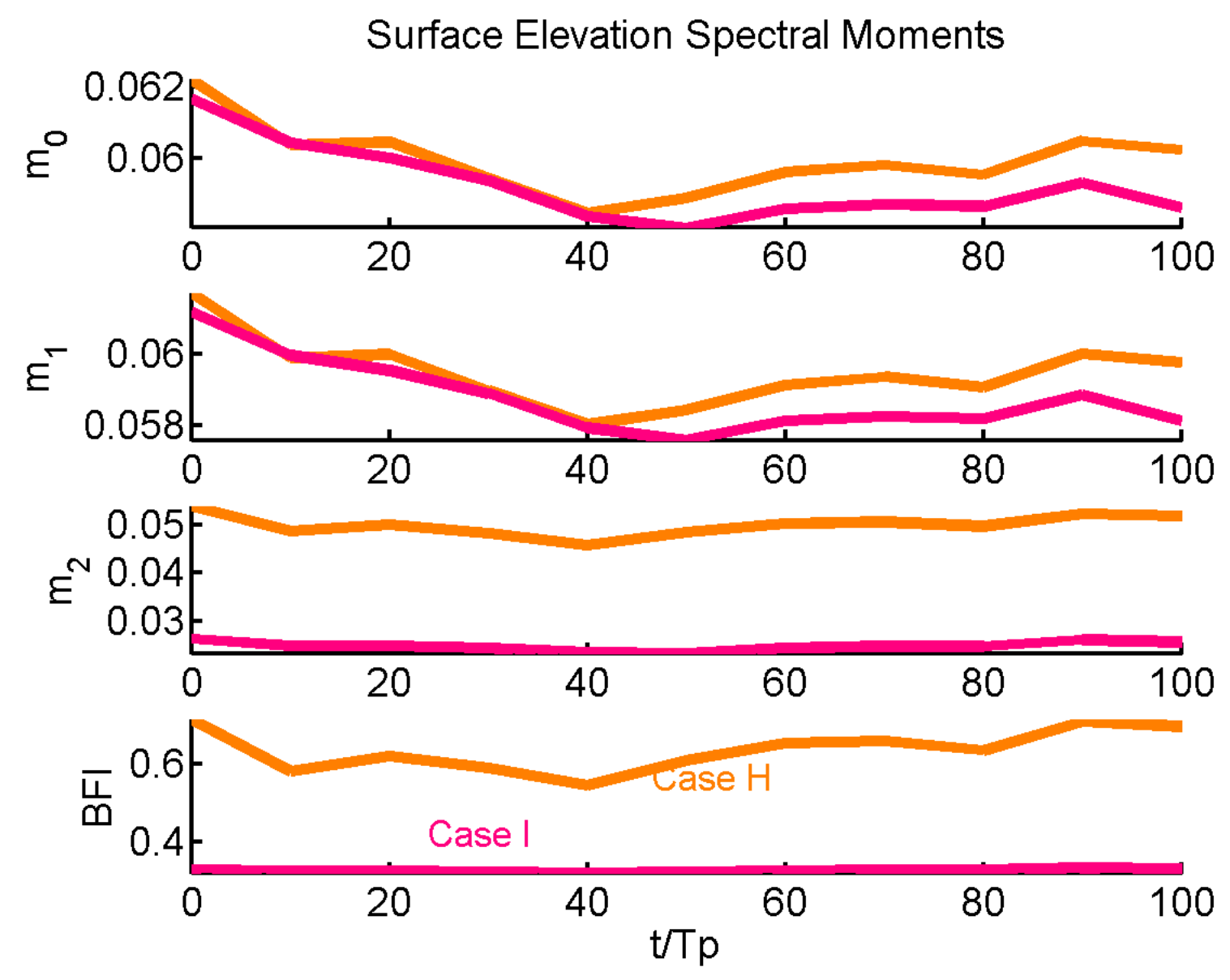

Figure 3-23: (In Color) Plot of surface elevation spectral moments against time $\left(t / T_{p}\right)$ where $m_{0}$ is the first spectral moment, $m_{1}$ is the second spectral moment, $m_{2}$ is the third spectral moment, and BFI is a scaled version of the BFI defined in Section 3.1.4. We use linear simulations of Cases $\mathbf{I}$ where $\gamma=1.0$, and $\mathbf{H}$ where $\gamma=5.0$. 
The Benjamin-Feir index is a non-dimensional quantifier of non-linearity. Further, in the next section we observe spectral peak shape parameter, $\gamma$, and surface elevation slope distribution, as another quantification of wavefield non-linearity.

\subsubsection{Peak shape parameter and surface elevation slope dis- tribution}

Let $x$ be defined as the mean wavefield propagation direction and $y$ be defined as the direction perpendicular to wavefield propagation direction. To see how we define surface elevation slope, see Figure 3-24.

In this section we investigate the distribution of surface slope, $d(\eta) / d x$ and $d(\eta) / d y$, which can be used as a quantitative metric for wavefield linearity. This is true since the length scale of the kurtosis of surface elevation slope in the direction of wave propagation, $d(\eta) / d x$, compared to the length scale of the kurtosis of surface elevation, $\eta$ tells us about the degree of non-linearity of a wave field. Such a comparison is presented in Figure 3-25 and Figure 3-26, as we compare the scales of the kurtosis of surface elevation, $\eta$, surface elevation slope in the mean direction of wavefield propagation, $d(\eta) / d x$ and surface slope perpendicular to the mean direction of wavefield propagation $(d(\eta) / d y)$, for linear and non-linear wavefields. It is expected that surface elevation slope adheres closely to the Gaussian distribution in a linear wave field. A surface elevation slope kurtosis of higher order of magnitude than a surface elevation kurtosis is an indicator of non-linearity [36]. We observe the distribution of the surface slope in non-linear Cases $\mathbf{I}$ (where $\gamma=1.0$ ), $\mathbf{B}$ (where $\gamma=3.3$ ) and $\mathbf{H}$ (where $\gamma=5.0$ ) compared to linear Cases $\mathbf{I}$ (where $\gamma=1.0$ ) and $\mathbf{H}$ (where $\gamma=5.0$ ) in Figures 3-25 and 3-26. 

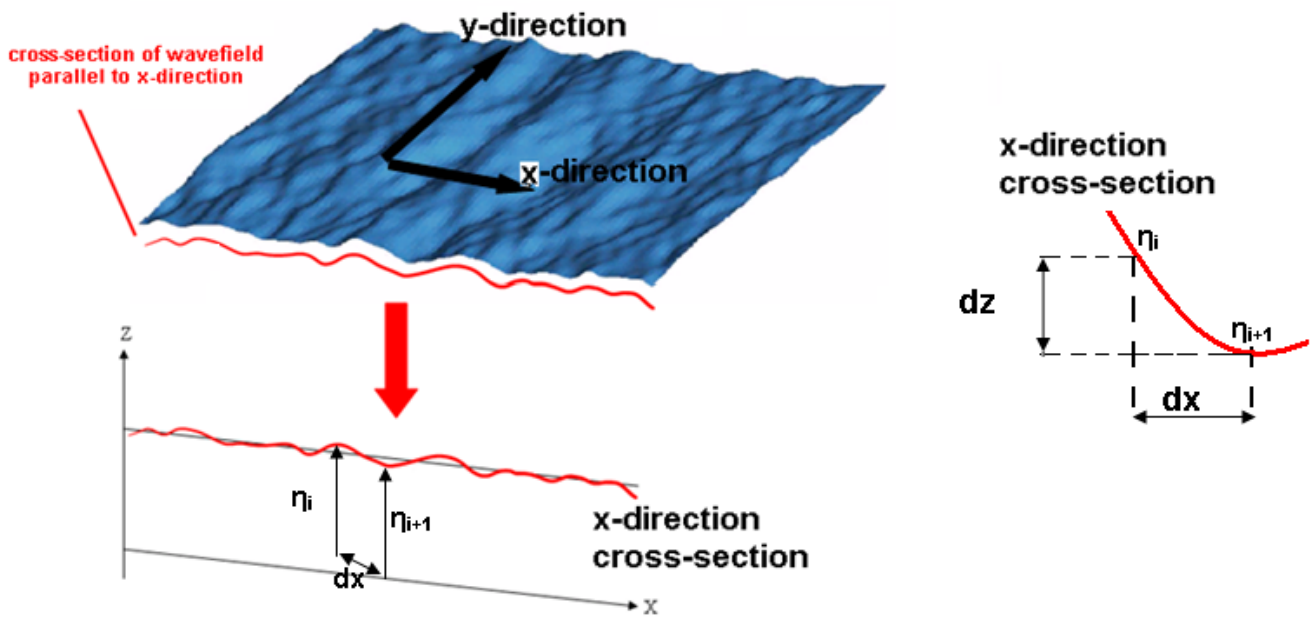

Figure 3-24: (In Color) Surface elevation slope in the x-direction is defined as: $\eta-$ slope $_{x}=\frac{\eta_{i+1}-\eta_{i}}{d x}$. 
From our observations, (see Figures 3-25 and 3-26) we learn that all the values of kurtosis in our non-linear wavefields are highly non-Gaussian, although the most violent departure from the Gaussian is seen in the distribution of surface slope in the direction of propagation, $d(\eta) / d x$ for Case $\mathbf{H}$ where $\gamma=5.0$.

Higher the peak shape parameter produces higher kurtosis values in surface elevation, $\eta$. The value of the kurtosis in a Gaussian distribution is 3, whereas the kurtosis for the expectedly Gaussian distribution of $\eta$ in Case $\mathbf{H}$ with peak shape parameter 5.0 is greater than 3. This proves the wavefield is quantitatively non-Gaussian throughout time with a maximum kurtosis value slightly less than 3.2. Further, the maximum value of kurtosis for the expectedly Gaussian distribution of surface slope in the direction of propagation, $d(\eta) / d x$ is 4.2 , occurring as expected in the wavefield with peak shape parameter 5.0. In cases with peak shape parameter, $\gamma=3.3$ and $\gamma=5.0$, kurtosis grows for the first $50 T_{p}$ of time and decays after that point, but there's less decay in the near-linear $\eta$-kurtosis in the wavefield with $\gamma=1.0$.

In non-linear wavefields as time proceeds the non-Gaussian kurtosis values decay. For non-linear wavefields, (see Figure 3-25) we see lower values of $d(\eta) / d y$ kurtosis but they are still non-Gaussian, and grow gradually and consistently with time until $t=80 T_{p}$. At any point in time, with all other spectral parameters fixed a higher $\gamma$-value produces higher $d(\eta) / d y$ kurtosis.

For linear simulated wavefields, (see Figure 3-26) peak shape parameter has no impact on $\eta$-kurtosis, $d(\eta) / d x$ kurtosis, or $d(\eta) / d y$ kurtosis. In all these measures at all times for all values of $\gamma$, kurtosis remains distinctly close to the Gaussian value of 3.0. In our simulated linear cases, the random rules in kurtosis, but for nonlinear cases, there is a distinct predictable order that emerges in the kurtosis values.

In this section we observe the scale of the kurtosis of surface elevation slope compared to the scale of the kurtosis of surface elevation, and we connect these to wavefield nonlinearity. In the next section we draw general conclusions about peak shape parameter and surface elevation characteristics. 


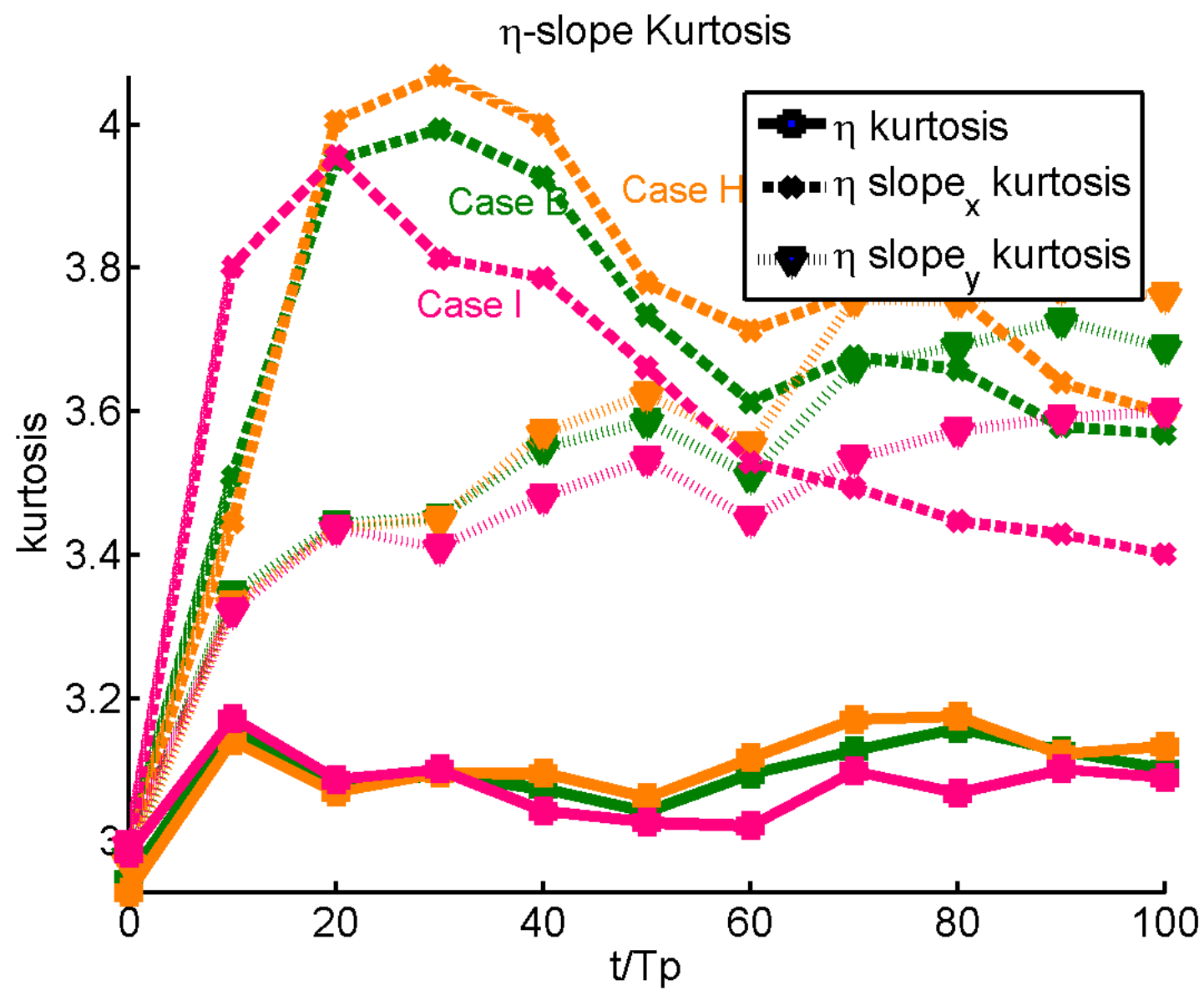

Figure 3-25: (In Color) Plot of kurtosis (on vertical axis) against time (on horizontal axis), $\left(t / T_{p}\right)$ in non-linear simulations of Cases $\mathbf{I}$ (where $\left.\gamma=1.0\right)$, $\mathbf{B}$ (where $\gamma=3.3$ ) and $\mathbf{H}$ (where $\gamma=5.0$ ). We see the distinct impact of peak shape parameter on the kurtosis of surface elevation and the kurtosis of surface elevation slope in a nonlinear wavefield. 


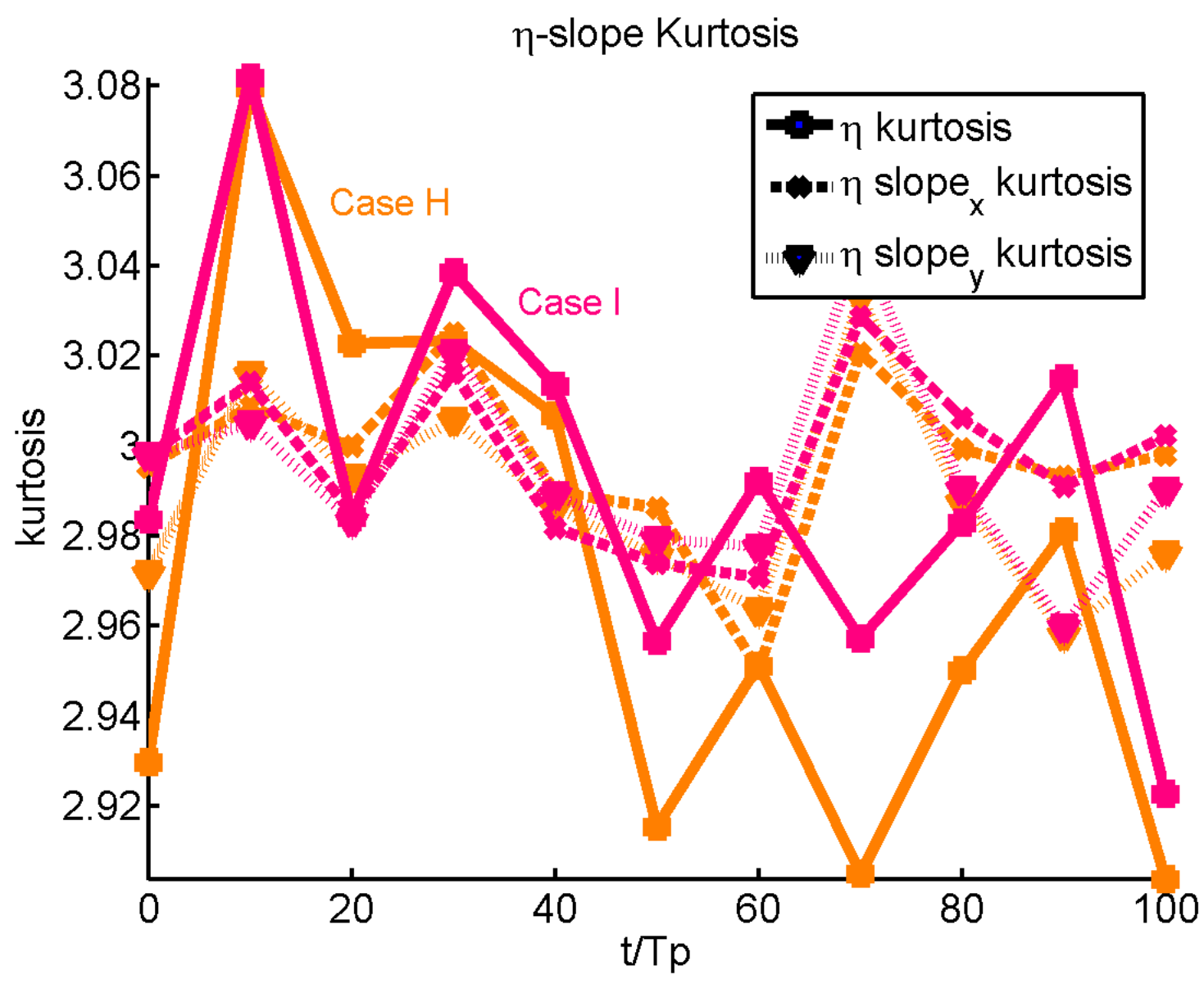

Figure 3-26: (In Color) Plot of kurtosis (on vertical axis) against time (on horizontal axis), $\left(t / T_{p}\right)$ in linear simulations of Cases $\mathbf{I}$ (where $\gamma=1.0$ ) and $\mathbf{H}$ (where $\gamma=5.0$ ). We see the negligible impact of peak shape parameter on the kurtosis of surface elevation and the kurtosis of surface elevation slope in a linear wavefield. 


\subsubsection{Conclusion: Peak shape parameter and surface eleva- tion characteristics}

In this section we look at peak shape parameter, $\gamma$, and surface elevation characteristics, finding that:

- Higher peak shape parameter produces higher surface elevation kurtosis and variance but produces no effect on surface elevation skewness. We therefore conclude that $\gamma$ does not impact surface elevation skewness

- Observed surface elevation data distributions move further away from the first, second and third order theoretical statistical distributions in a nonlinear wavefield as time proceeds, due to its non-stationary statistical moments. We find a higher peak shape parameter produces higher even and this impacts surface elevation moments such that it moves the higher-order distributions apart similar to the spread seen in the time evolution of nonlinear wavefields.

- The highest elevations in a wavefield with $\gamma=3.3$ (which is the mean peak shape parameter of wave in a storm where peak shape parameter is normally distributed) are stabler than those in wavefields with $\gamma=1.0$, and 5.0 (which are usually the tails of the peak shape parameter distribution).

- The random rules in surface elevation kurtosis and surface slope kurtosis in linear wavefields. Kurtosis values are all very near the Gaussian value 3.0. We note that this is quite unlike what is seen in non-linear wavefields. We find that in non-linear wavefields, surface elevation kurtosis is much smaller than surface elevation slope kurtosis and we find that higher values of peak shape parameter, (see Figure 3-25) produce higher kurtosis of surface slope in the mean direction of propagation. 


\subsection{Phillips' parameter}

The input spectrum in the simulated SNOW wavefields can be seen in Equation 2.1. The input Phillips' parameter, $\alpha$ is dependent on average wavefield steepness in physical space.

$$
\alpha=5.061\left(\frac{H_{s}^{2}}{T_{p}^{4}}\right)(1-0.287 \ln (\gamma))
$$

Phillips' parameter is generally a measure of steepness of high-frequency waves, and for short fetches (young wind sea, before $t=40 T_{p}$ ) the steepness of the waves is much larger than for long fetches (or in old wind seas, after $t=100 T_{p}$ ) [23]. We therefore expect that Phillips' parameter's effects will become less dominant in the wavefield as time passes. As seen in Equation 3.6 Phillips' parameter, $\alpha$ is also dependent on peak shape parameter, $\gamma$ in the wave spectrum [41]. A smaller fetch and a greater average wind speed usually produce larger Phillips' parameter. We study the effect of Phillips' parameter, $\alpha$, on a wavefield's physical properties in this section. Cases used in testing the effects of Phillips' parameter all have directional spreading angle, $\theta=40$ and peak shape parameter, $\gamma=3.3$, (see Table 3.2).

\subsubsection{Impact of input Phillips' parameter on surface eleva- tion distribution}

In Figure 3-27 the time evolution of kurtosis suggests that a higher Phillips' parameter produces a higher kurtosis. For a Phillips' parameter near 0.0160 we see nonlinear values for statistical moments persist for $100 T_{p}$, while for Phillips' parameter near 0.0032, the surface elevation statistical moments' values remain near-linear holding a randomly fluctuating kurtosis near 3.0 for $100 T_{p}$. The time evolution of kurtosis shows that the non-Gaussian traits in kurtosis oscillate over time. The time evolution of skewness shows that Phillips' parameter produces a large effect on skewness having almost identical skewness in cases B and F, while having less skewness in Case E. The time evolution of variance shows that Phillips' parameter produces a very visible, 
Table 3.2: Cases used in testing the effects of steepness via Phillips' parameter, $\alpha$, with directional spreading angle, $\theta=40$ and peak shape parameter, $\gamma=3.3$.

\begin{tabular}{llll}
\hline \hline Case & $\mathrm{E}$ & $\mathrm{B}$ & $\mathrm{F}$ \\
\hline Phillips' parameter, $\alpha$ & 0.0032 & 0.0160 & 0.0163
\end{tabular}
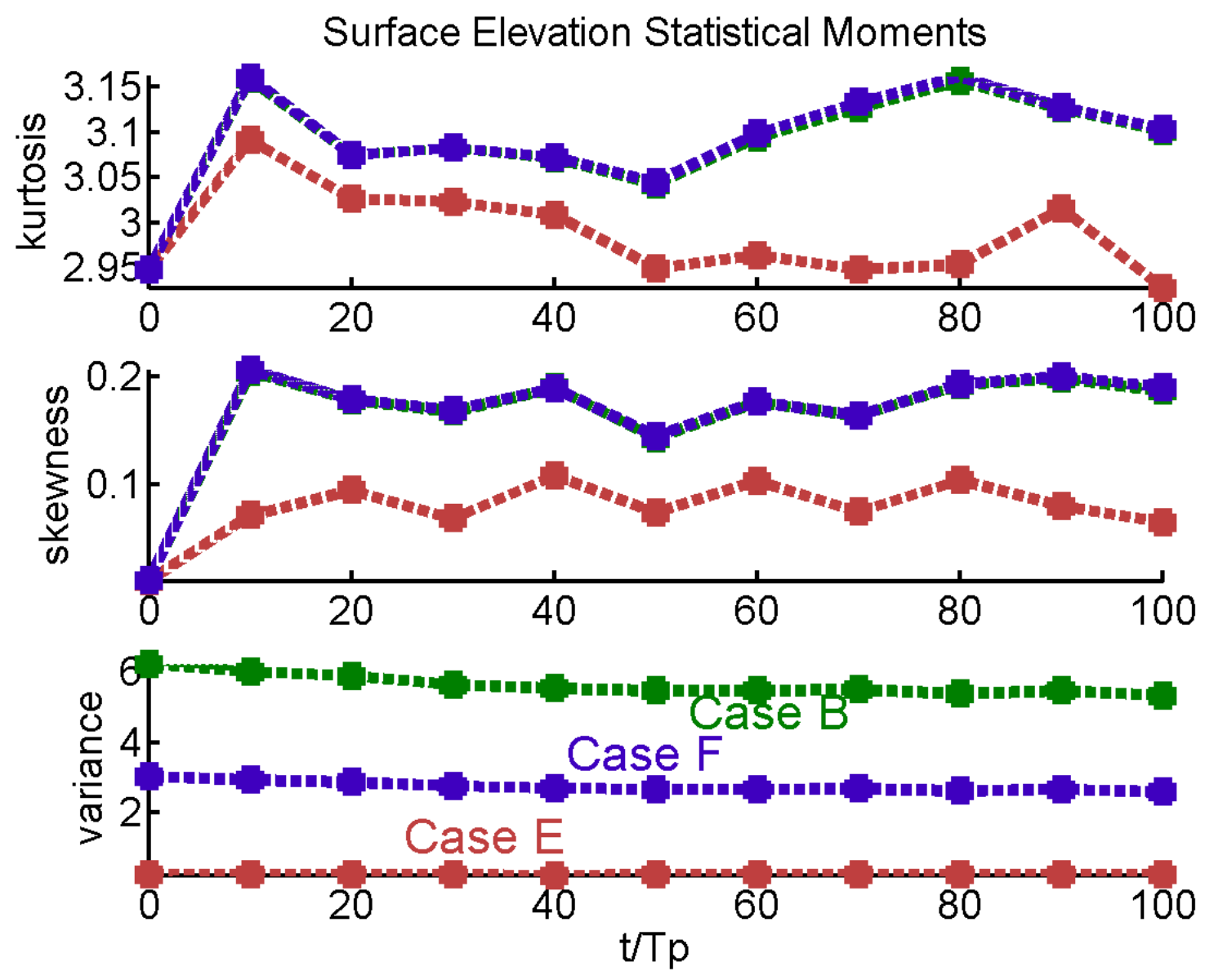

Figure 3-27: (In Color) Plots of the statistical moments (kurtosis, skewness and variance) of surface elevation against time $\left(t / T_{p}\right)$ in nonlinear simulations of Cases $\mathbf{E}$, where $\alpha=0.0032$, Case $\mathbf{B}$ where $\alpha=0.0160$ and Case $\mathbf{F}$ where $\alpha=0.0163$. 
non-linear impact on variance, as we see a near-zero variance in the most near-linear wavefield, the highest variance in case B which has a lower Phillips' parameter than case F, which carries the lower variance throughout time. This indicates that a higher Phillips' parameter does not necessarily produce a larger variance.

The results in Figures 3-37, 3-38 and 3-39 show the time evolution of surface elevation distribution where $\alpha=0.0032, \alpha=0.0160$, and $\alpha=0.0163$ respectively. Earlier times (from $t=0 T_{p}$ ) are depicted in the darkest $(+++)$ series, while data observed at later times are depicted in the brighter $(+++)$ series. We compare results in these figures, finding greater stability in the highest values of surface elevation in Case E where the value of Phillips' parameter is lowest. This greater stability is manifest in near constant slopes in theoretical straight line (- - ) in Case $\mathbf{E}$ compared to Case $\mathbf{B}$ and Case F.

We note that a low value of Phillips' parameter (in Case $\mathbf{E}$ where $\alpha=0.0032$ ) has the effect of producing a near-linear wave-field throughout time evolution, as non-linear distribution traits in the wavefield appear to be very small.

Further, we observe Case F from Figures 3-34, 3-35 and 3-36, concluding that a higher Phillips' parameter produces a more visible non-linear effect in a wavefield.

At $t=50 T_{p}$, we see changes in the statistical moments in all the wavefields, due to the presence of nonlinear wave-wave interactions. Figures 3-32 and 3-35 depict that a higher Phillips' parameter produces greater spread between higher-order distributions and observed data distribution.

\subsubsection{Impact of input Phillips' parameter on surface eleva- tion wavenumber spectrum}

In Figures 3-37, 3-38 and 3-39 we observe the wavenumber spectra of Case $\mathbf{E}$ where $\alpha=0.0032$, Case $\mathbf{B}$ where $\alpha=0.0160$ and Case $\mathbf{F}$ where $\alpha=0.0163$. We find that they all display narrow-band, uni-modal wavenumber spectra evolving in time, peaking around the same wavenumber. Case $\mathbf{E}$, however, has the most stable highest spectral energy range fixed at dimensionless wavenumber, 9 , between $t=30 T_{p}$ and 


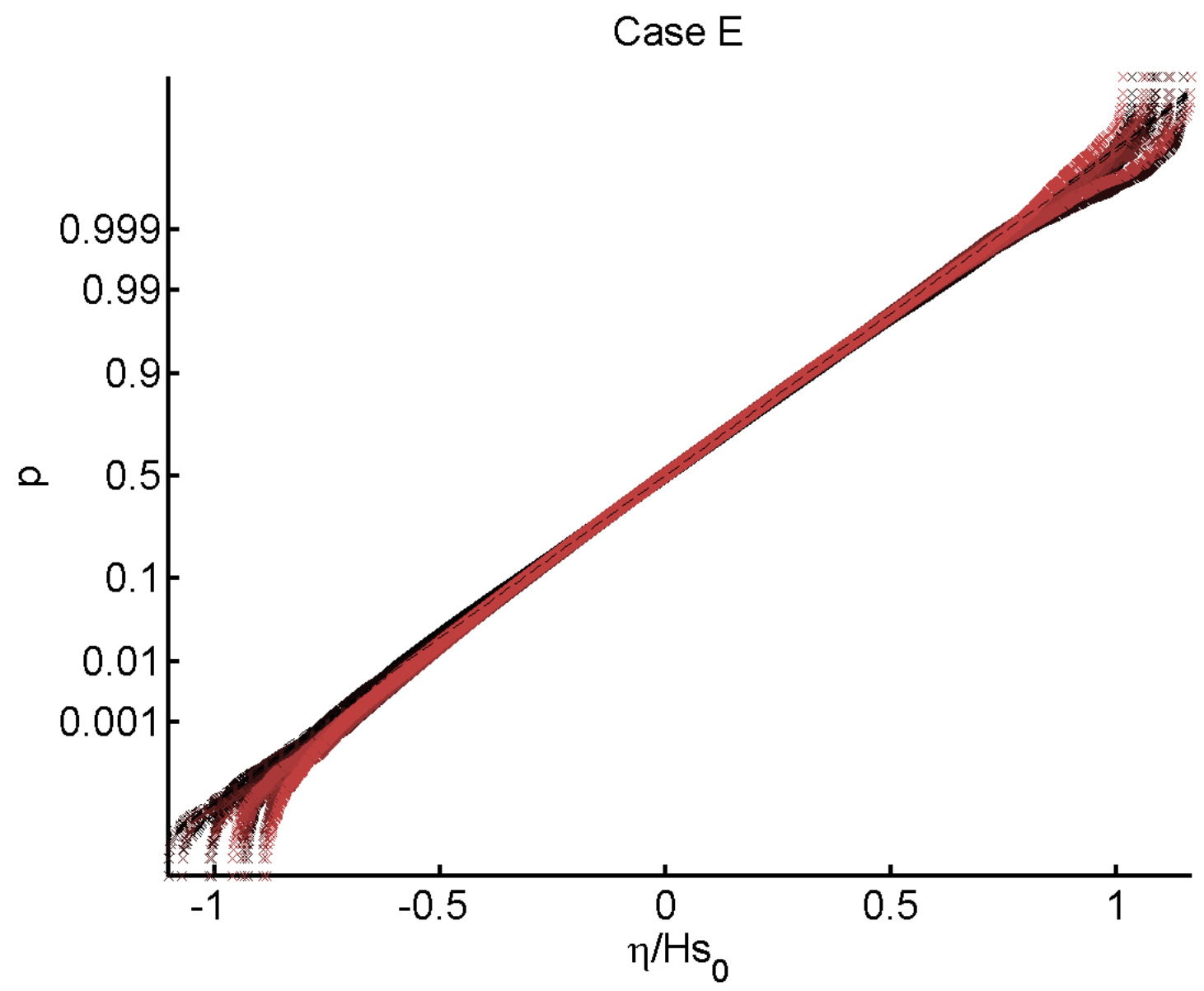

Figure 3-28: (In Color) Horizontal axis shows non-dimensional surface elevation and vertical axis shows the cumulative distribution of such an elevation in the wavefield. Time evolution of theoretical (- - -) surface elevation distribution, and observed surface elevation distribution data $(++++)$ in a nonlinear simulation of Case $\mathbf{E}$ (where $\alpha=0.0032$ ). Each line pair represents one time step. Earlier times (from $t=0 T_{p}$ ) are the darkest lines. Later times (up to $t=100 T_{p}$ ) are the brightest lines. 


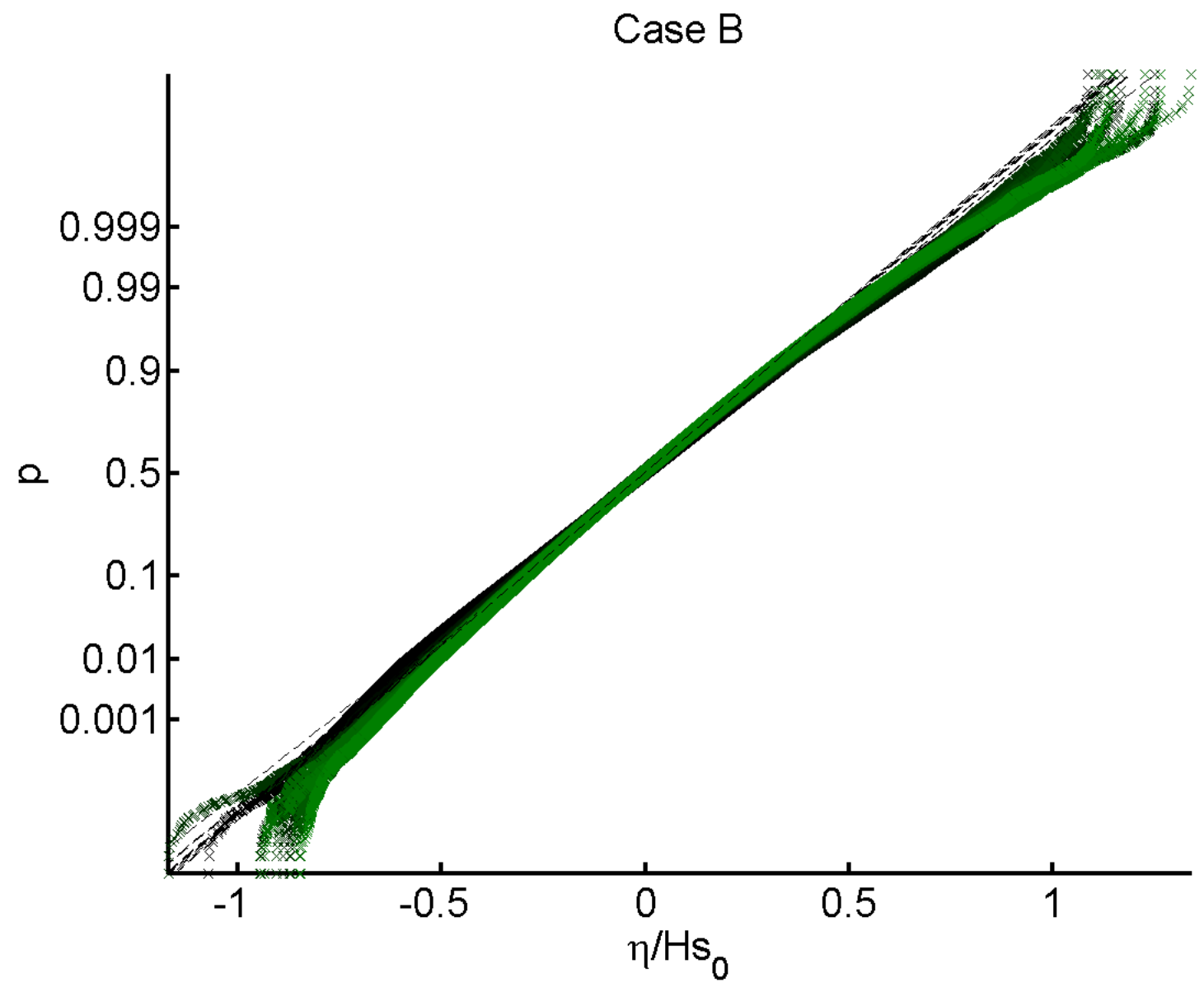

Figure 3-29: (In Color) Horizontal axis shows non-dimensional surface elevation and vertical axis shows the cumulative distribution of such an elevation in the wavefield. Time evolution of theoretical (- - -) surface elevation distribution, and observed surface elevation distribution data $(++++)$ in a nonlinear simulation of Case $\mathbf{B}$ (where $\alpha=0.0160$ ). Each line pair represents one time step. Earlier times (from $t=0 T_{p}$ ) are the darkest lines. Later times (up to $t=100 T_{p}$ ) are the brightest lines. 


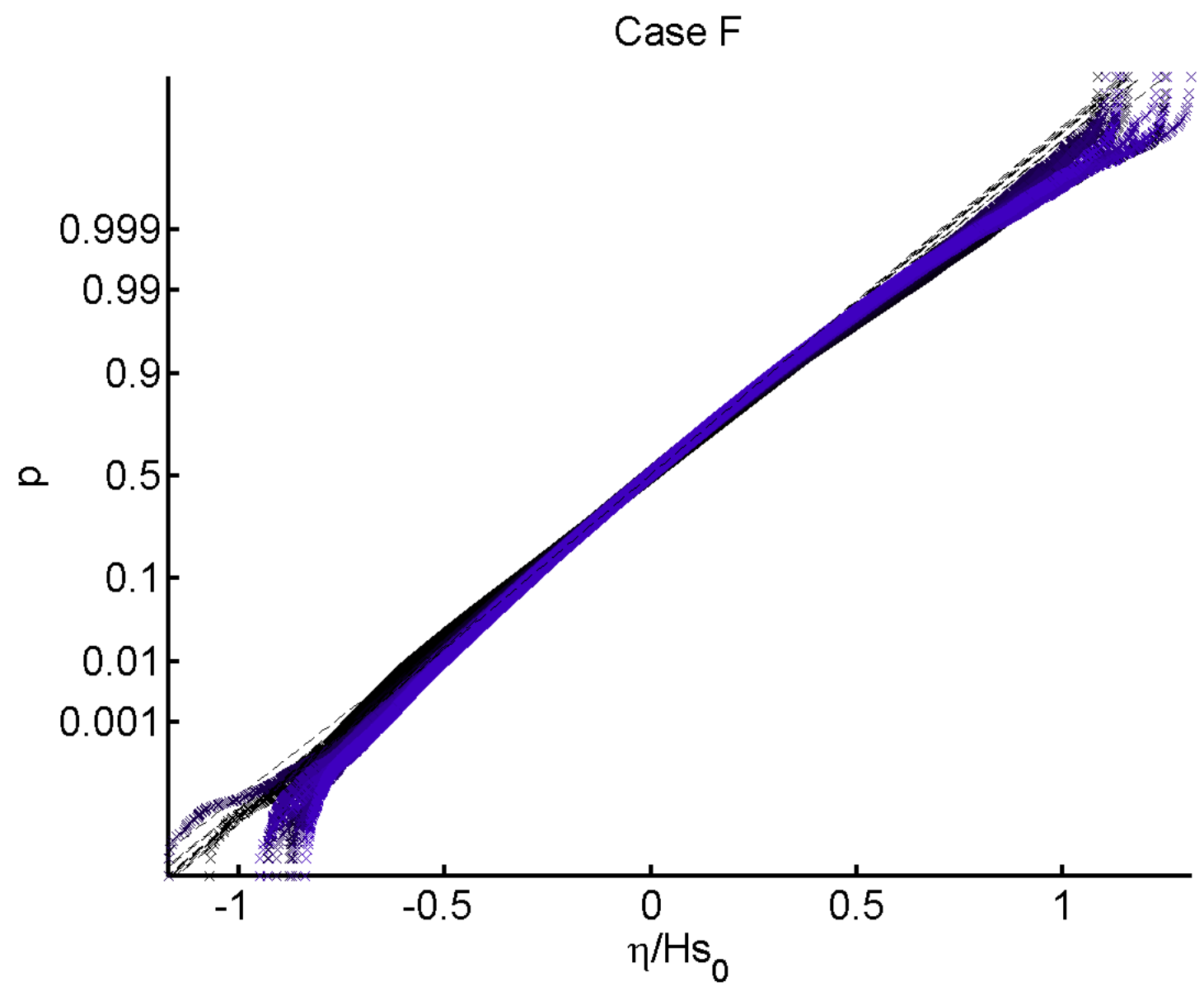

Figure 3-30: (In Color) Horizontal axis shows non-dimensional surface elevation and vertical axis shows the cumulative distribution of such an elevation in the wavefield. Time evolution of theoretical (- - -) surface elevation distribution, and observed surface elevation distribution data $(++++)$ in a nonlinear simulation of Case $\mathbf{F}$ (where $\alpha=0.0163$ ). Each line pair represents one time step. Earlier times (from $t=0 T_{p}$ ) are the darkest lines. Later times (up to $t=100 T_{p}$ ) are the brightest lines. 


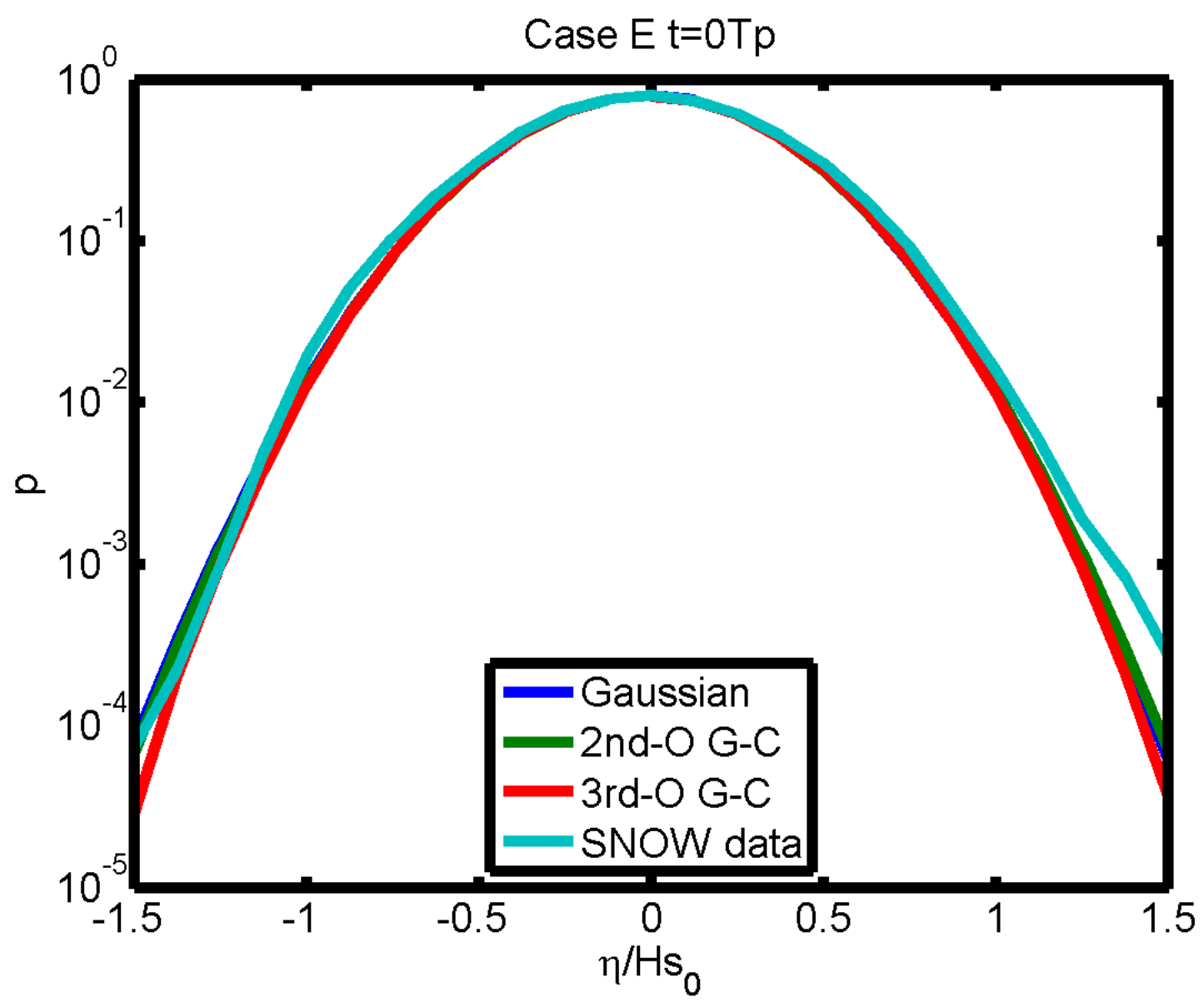

Figure 3-31: (In Color) Horizontal axis depicts non-dimensional surface elevation and vertical axis depicts probability. Observed data from nonlinear simulation of Case $\mathbf{E}$ (where $\alpha=0.0032$ ) at time, $t=0 T_{p}$ compared to Gram Charlier distributions Order 1,2 and 3 . 


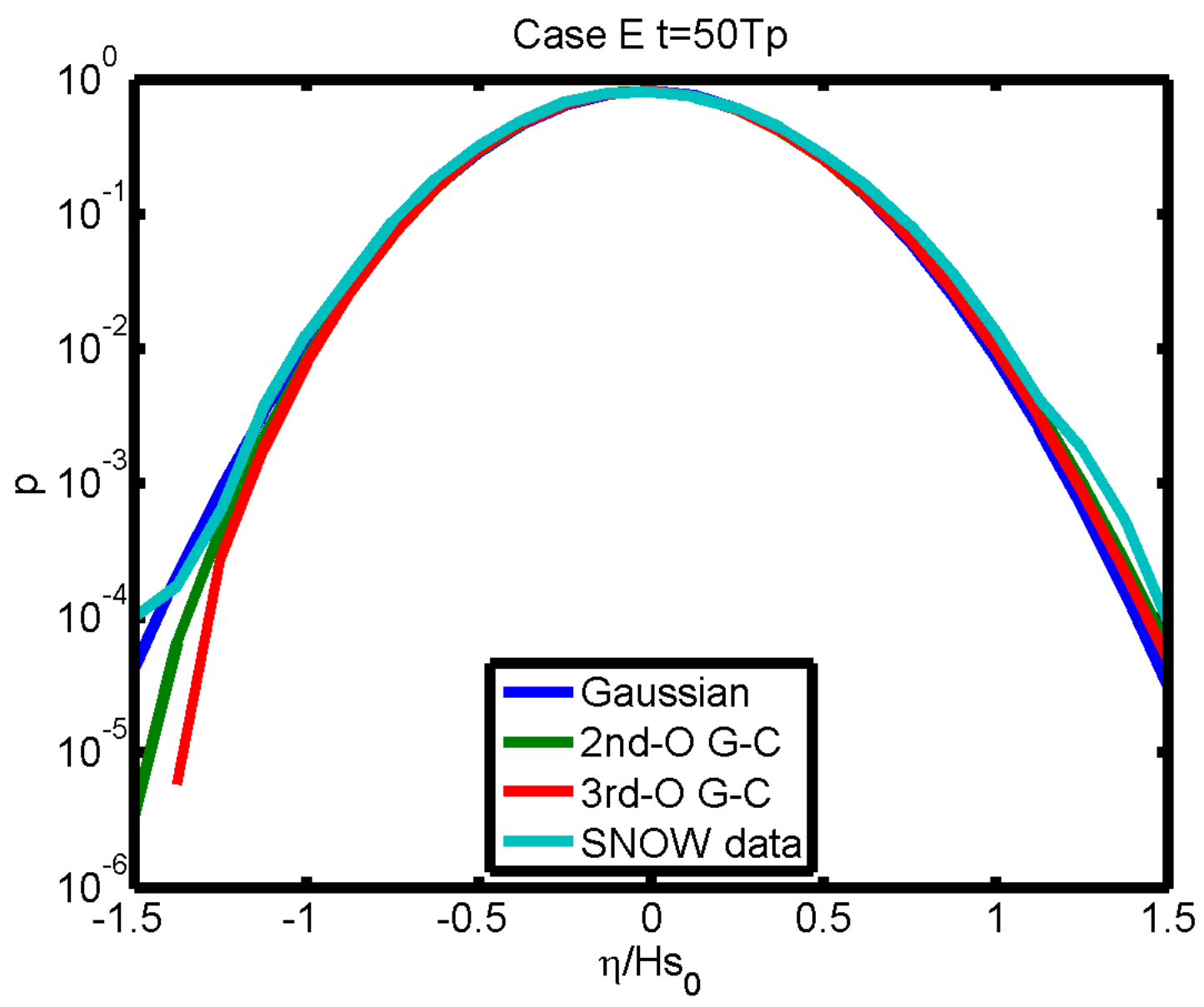

Figure 3-32: (In Color) Horizontal axis depicts non-dimensional surface elevation and vertical axis depicts probability. Observed data from nonlinear simulation of Case E (where $\alpha=0.0032$ ) at time, $t=50 T_{p}$ compared to Gram Charlier distributions Order 1,2 and 3. 


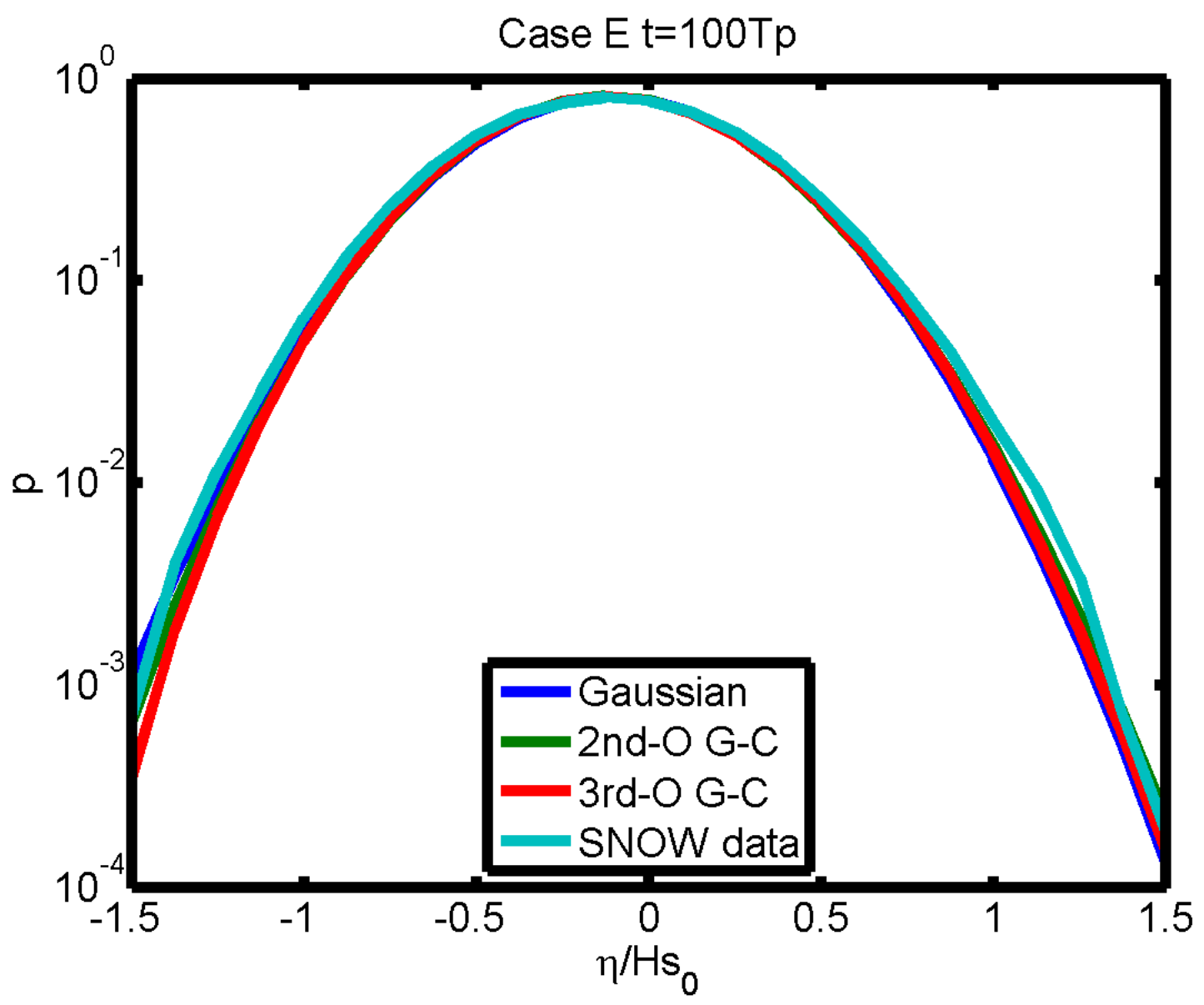

Figure 3-33: (In Color) Horizontal axis depicts non-dimensional surface elevation and vertical axis depicts probability. Observed data from nonlinear simulation of Case $\mathbf{E}$ (where $\alpha=0.0032$ ) at time, $t=100 T_{p}$ compared to Gram Charlier distributions Order 1,2 and 3. 


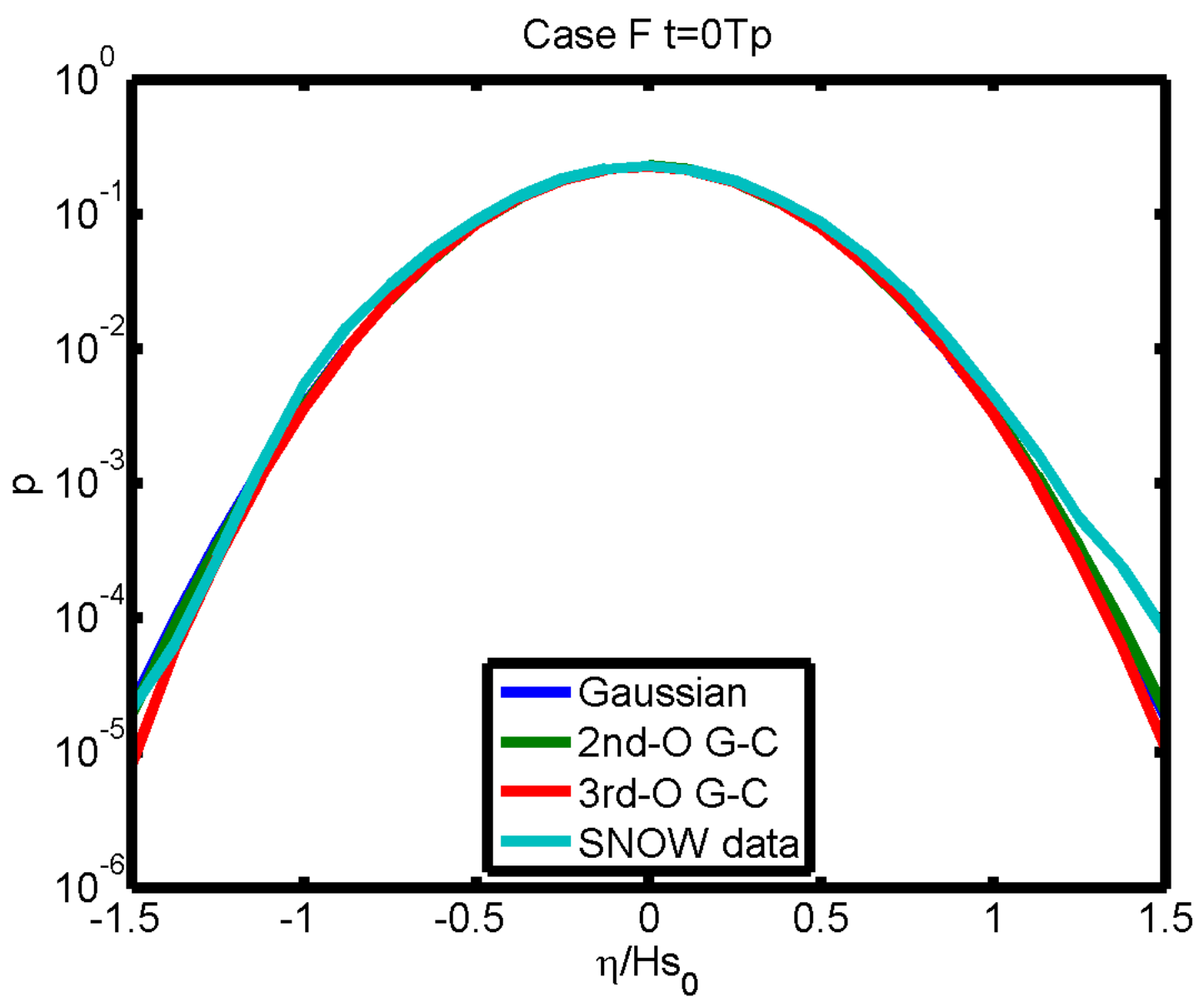

Figure 3-34: (In Color) Horizontal axis depicts non-dimensional surface elevation and vertical axis depicts probability. Observed data from nonlinear simulation of Case $\mathbf{F}$ (where $\alpha=0.0163$ ) at time, $t=0 T_{p}$ compared to Gram Charlier distributions Order 1,2 and 3 . 


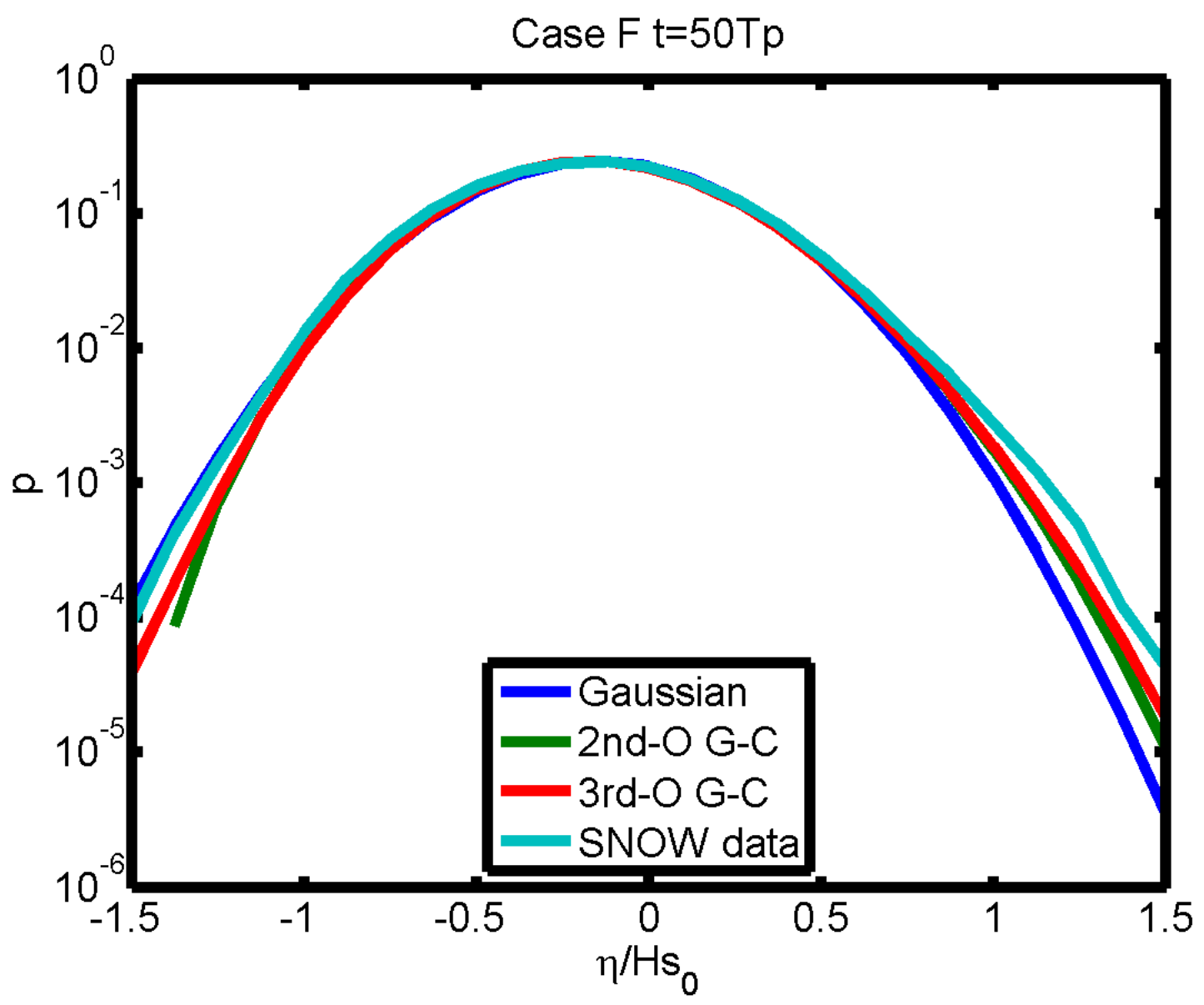

Figure 3-35: (In Color) Horizontal axis depicts non-dimensional surface elevation and vertical axis depicts probability. Observed data from nonlinear simulation of Case $\mathbf{F}$ (where $\alpha=0.0163$ ) at time, $t=50 T_{p}$ compared to Gram Charlier distributions Order 1,2 and 3. . 


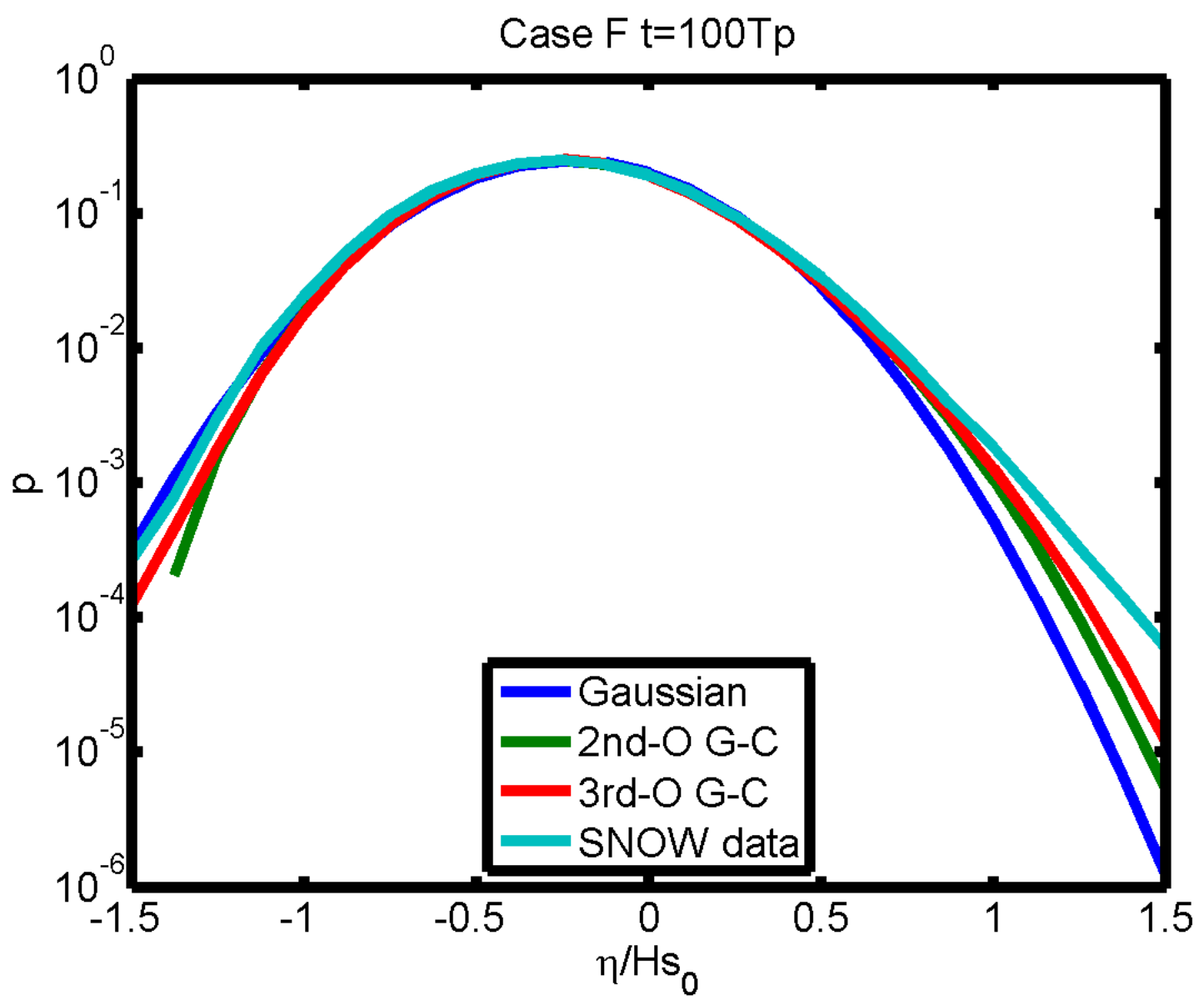

Figure 3-36: (In Color) Horizontal axis depicts non-dimensional surface elevation and vertical axis depicts probability. Observed data from nonlinear simulation of Case $\mathbf{F}$ (where $\alpha=0.0163$ ) at time, $t=100 T_{p}$ compared to Gram Charlier distributions Order 1,2 and 3. 


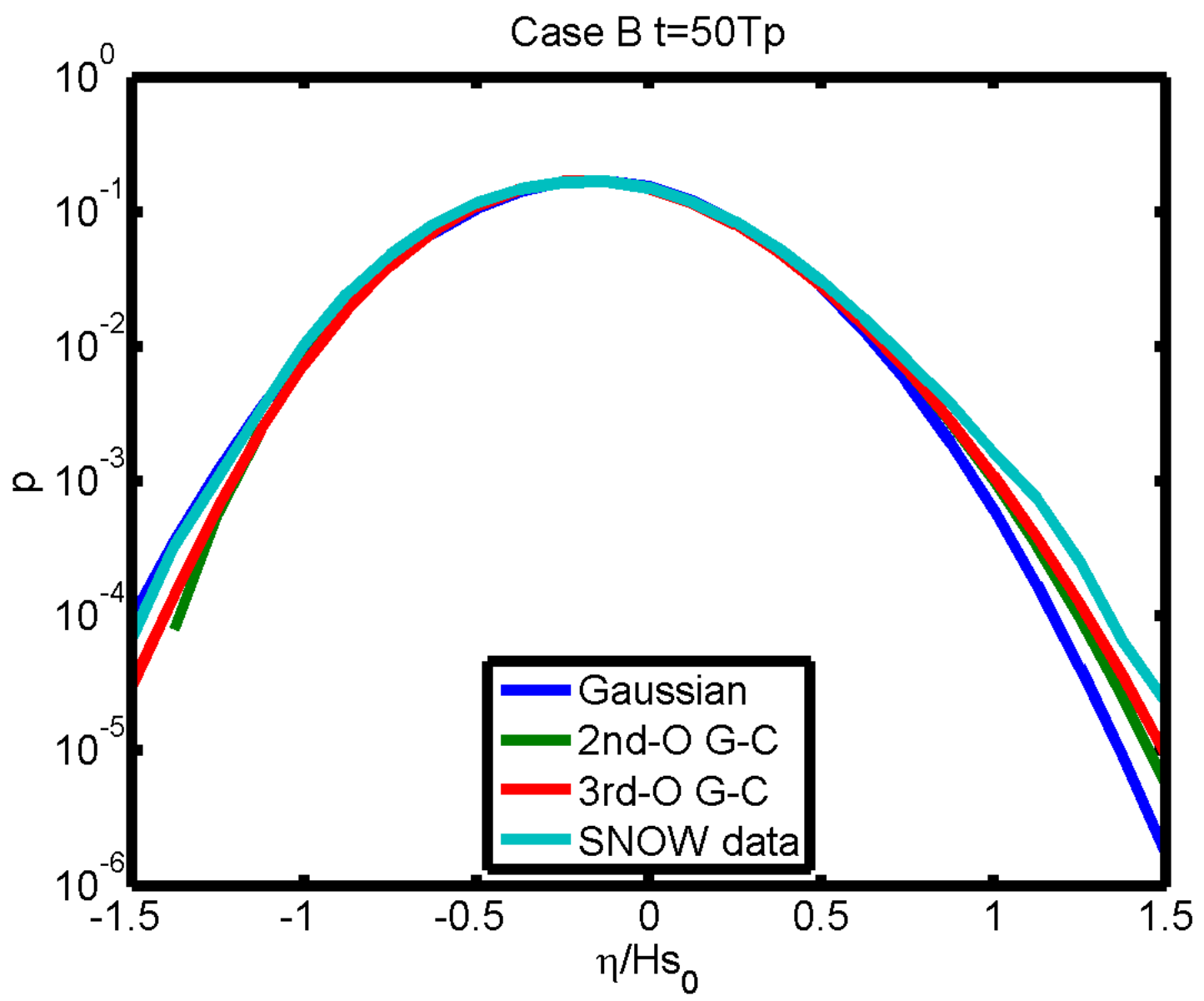

caption(In Color) Horizontal axis depicts non-dimensional surface elevation and vertical axis depicts probability. Observed data from nonlinear simulation of Case $\mathbf{B}$ (where $\alpha=0.0160$ ) at time, $t=50 T_{p}$ compared to Gram Charlier distributions Order 1, 2 and 3. 
$t=50 T_{p}$ when the highest spectral energy in Cases $\mathbf{B}$ and $\mathbf{F}$ are seen to fall.

In Figure 3-40 we see that the linear simulation of Case $\mathbf{E}$ is not very different from its non-linear equivalent. In a case with a very low Phillips' parameter, the observed effect of the nonlinearity order in the simulation is negligible. A low Phillips' parameter reduces the appearance of nonlinearity in the wavefield.

\subsubsection{Impact of input Phillips' parameter on surface eleva- tion BFI}

In Figure 3-41 we observe the impact of Phillips' parameter on the spectral moments in nonlinear simulations of wavefields. Note that $m_{0}$ is the first spectral moment, $m_{1}$ is the second spectral moment, $m_{2}$ is the third spectral moment, and BFI is a scaled version of the BFI defined in Section 3.1.4. We use Case $\mathbf{E}$ where $\alpha=0.0032$, Case B where $\alpha=0.0160$ and Case $\mathbf{F}$ where $\alpha=0.0163$. The time evolution of the first three spectral moments displays the fact that Phillips' parameter influences spectral moments in a wavefield. The spectral moments of Case $\mathbf{E}$ where $\alpha=0.0160$ move further from those of Case $\mathbf{B}$ where $\alpha=0.0160$, and $\mathrm{F}$ where $\alpha=0.0163$, as time proceeds. However, when we look at the ratio of spectral moments, the BFI, it becomes clear that Phillips' parameter does not dictate wavefield stability, as the BFI values that emerge are inconsistent with Phillips' parameter values.

We investigate whether the order of non-linearity impact the effect of input Phillips' parameter on surface elevation BFI. We achieve this (see Figure 3-42) by observing the impact of Phillips' parameter on the spectral moments in a linear simulation of Case $\mathbf{E}$ where $\alpha=0.0032$. We find that the order of nonlinearity has a very negligible effect on the spectral moments and the BFI in Case E, proving that a small Phillips' parameter, $\alpha=0.0032$ produces a near-linear wavefield. 


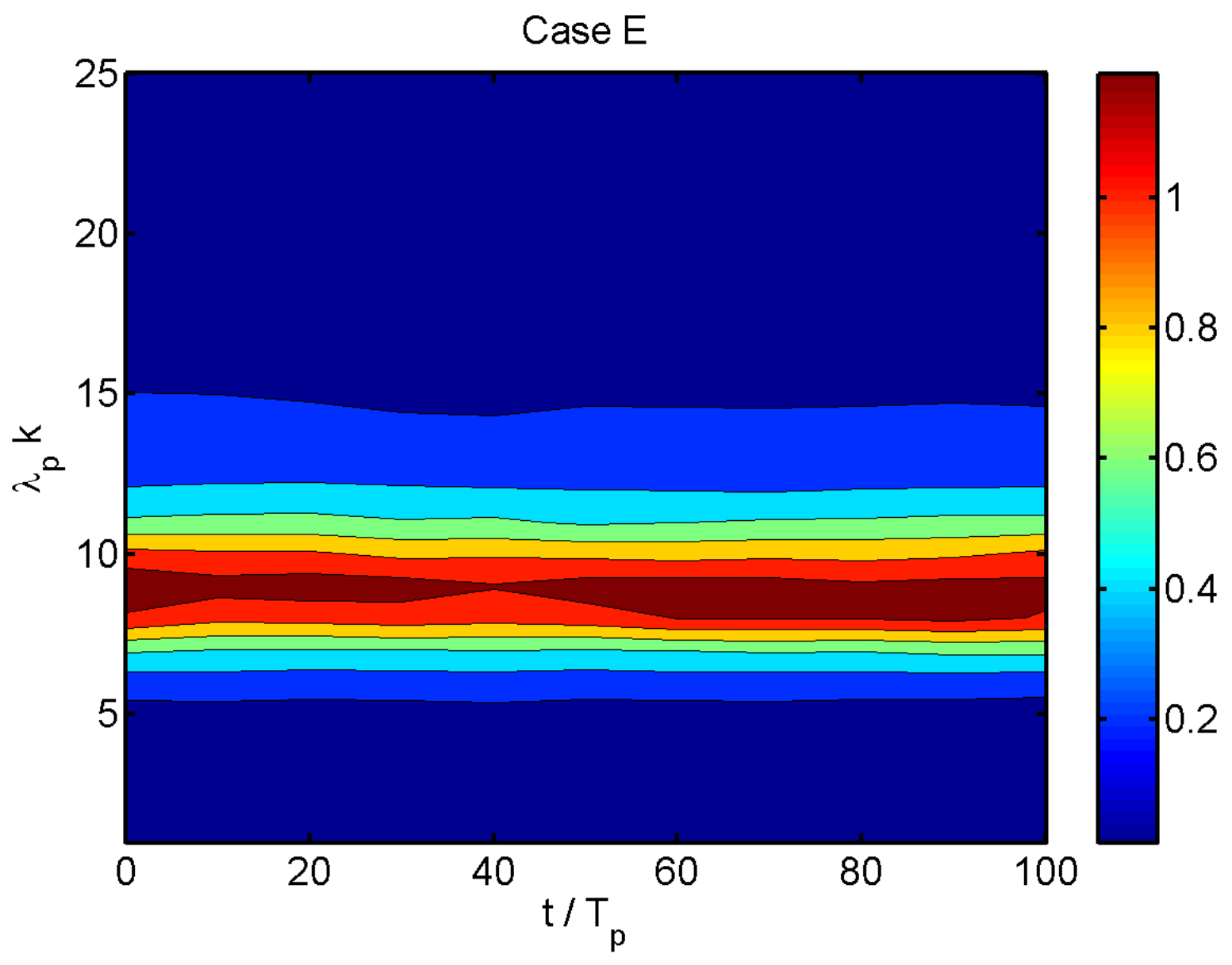

Figure 3-37: (In Color) Wavenumber spectrum time evolution in a nonlinear wavefield simulation of Case $\mathbf{E}$ (where $\alpha=0.0032$ ). Time $\left(t / T_{p}\right)$ is on the horizontal axis, wavenumber $\lambda_{p} k$ on the vertical axis and spectral energy level is represented in the color bar. 
Case B

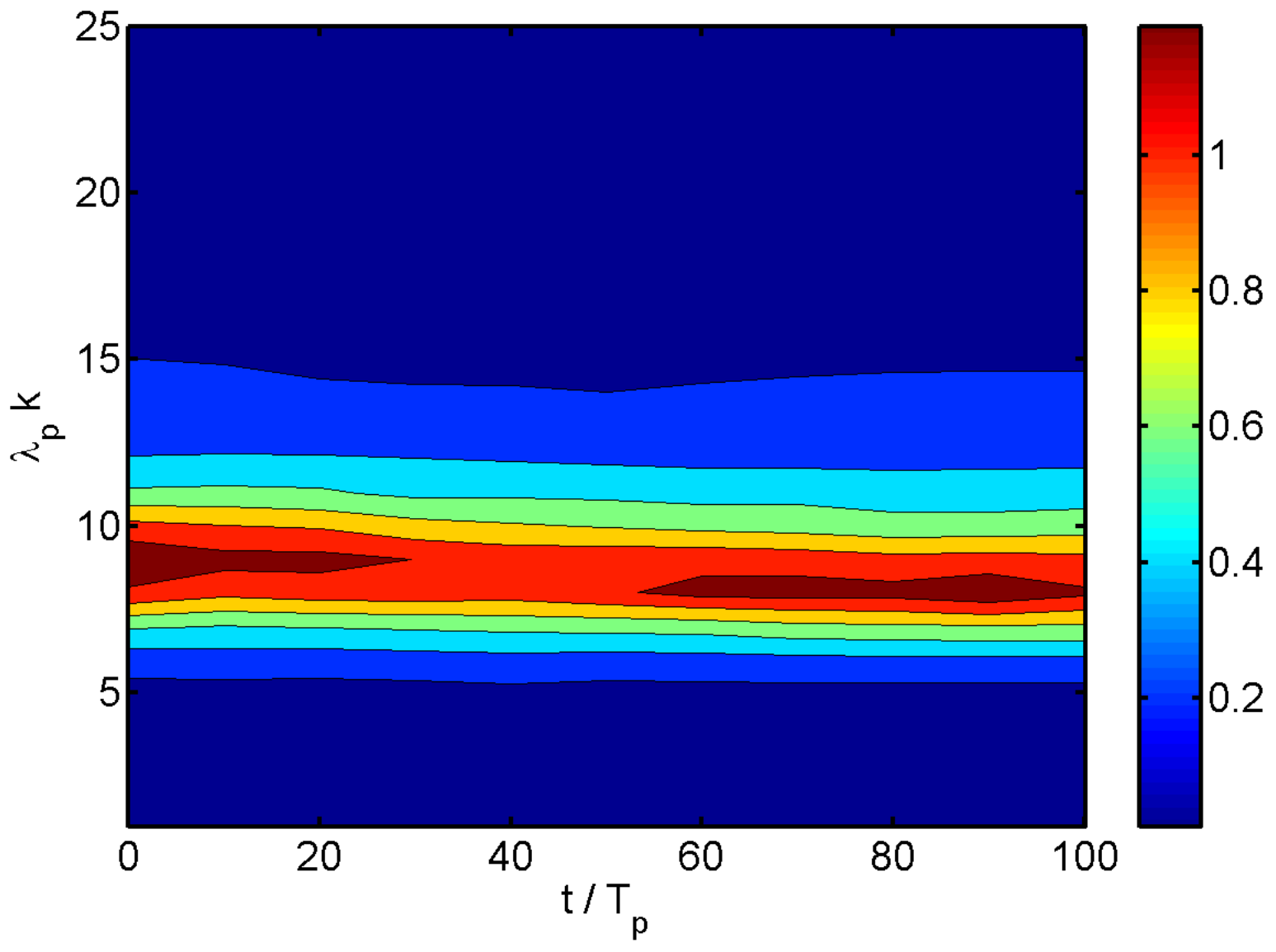

Figure 3-38: (In Color) Wavenumber spectrum time evolution in a nonlinear wavefield simulation of Case $\mathbf{B}$ (where $\alpha=0.0160$ ). Time $\left(t / T_{p}\right)$ is on the horizontal axis, wavenumber $\lambda_{p} k$ on the vertical axis and spectral energy level is represented in the color bar. 


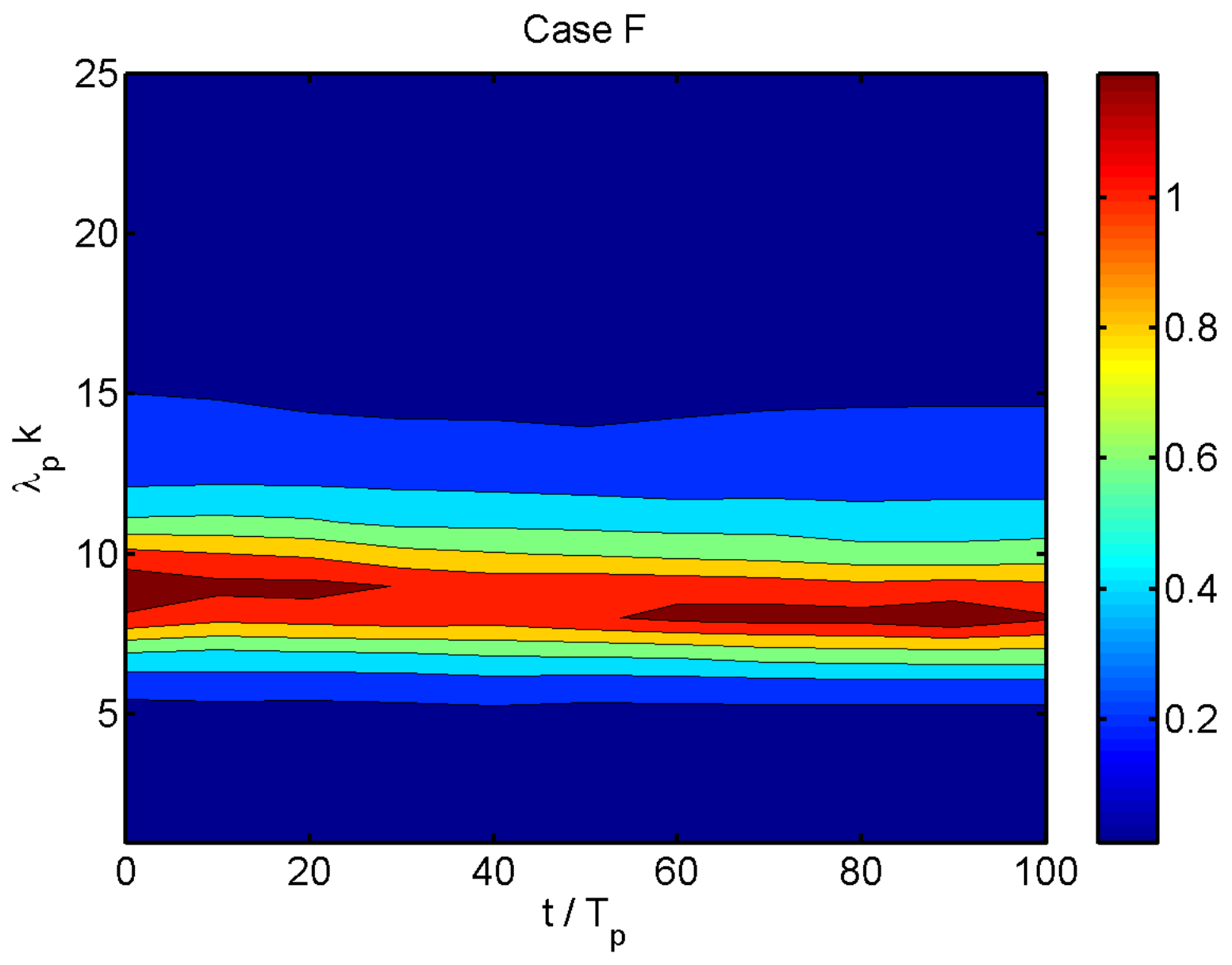

Figure 3-39: (In Color) Wavenumber spectrum time evolution in a nonlinear wavefield simulation of Case $\mathbf{F}$ (where $\alpha=0.0163$ ). Time $\left(t / T_{p}\right)$ is on the horizontal axis, wavenumber $\lambda_{p} k$ on the vertical axis and spectral energy level is represented in the color bar. 


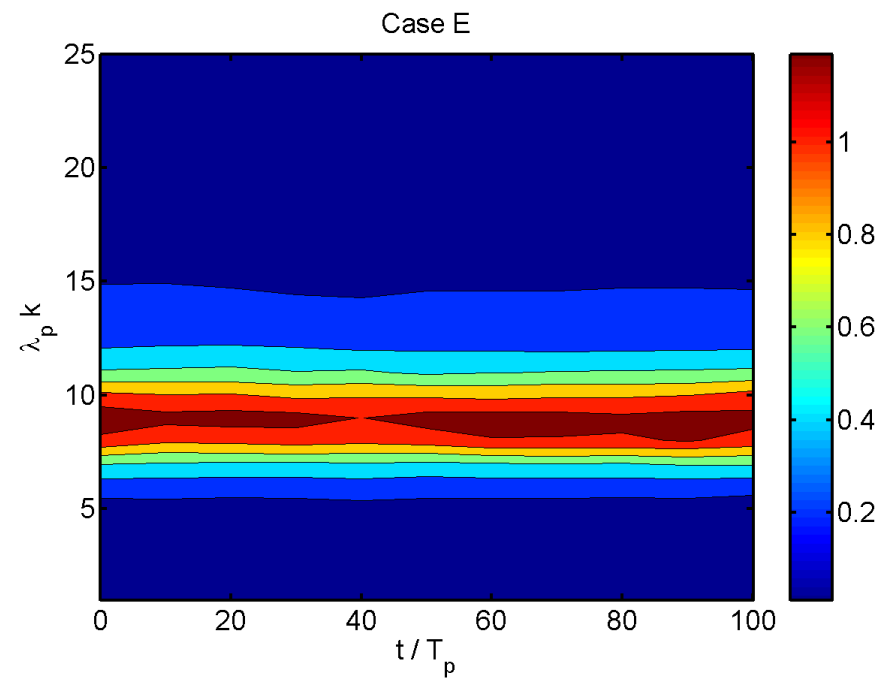

(a) Linear simulation of Case $\mathbf{E}$ where $\alpha=0.0032$.

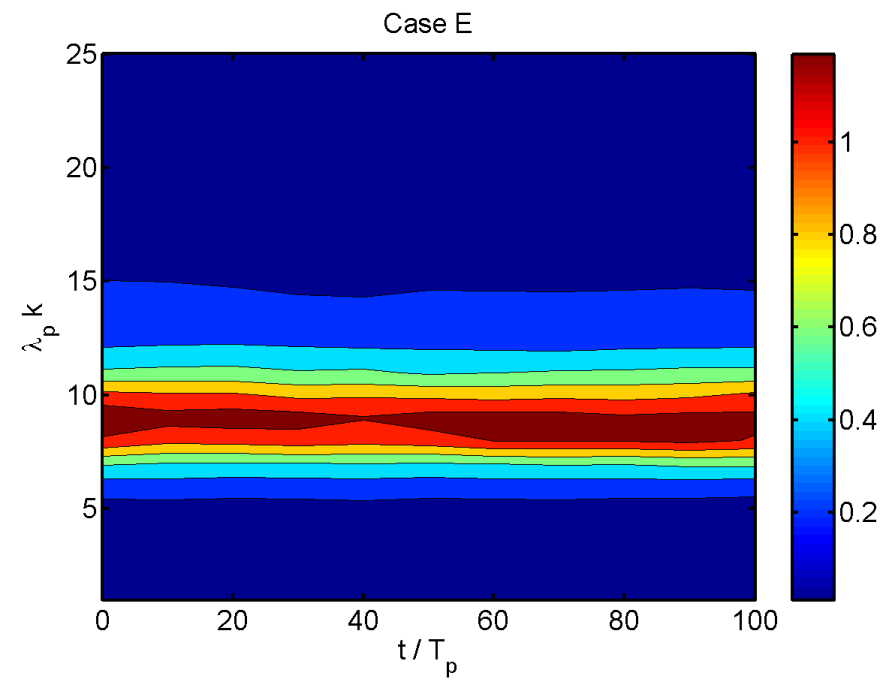

(b) Non-linear simulation of Case $\mathbf{E}$ where $\alpha=0.0032$.

Figure 3-40: (In Color) Wavenumber spectrum time evolution in a linear equivalent simulation of Case $\mathbf{E}$ where $\alpha=0.0032$. We plot time $\left(t / T_{p}\right)$ on the horizontal axis, wavenumber $k$ on the vertical axis and we represent spectral energy levels in color (see color bars). There is very little difference between the wavenumber spectrum of the linear and nonlinear Case $\mathbf{E}$. 


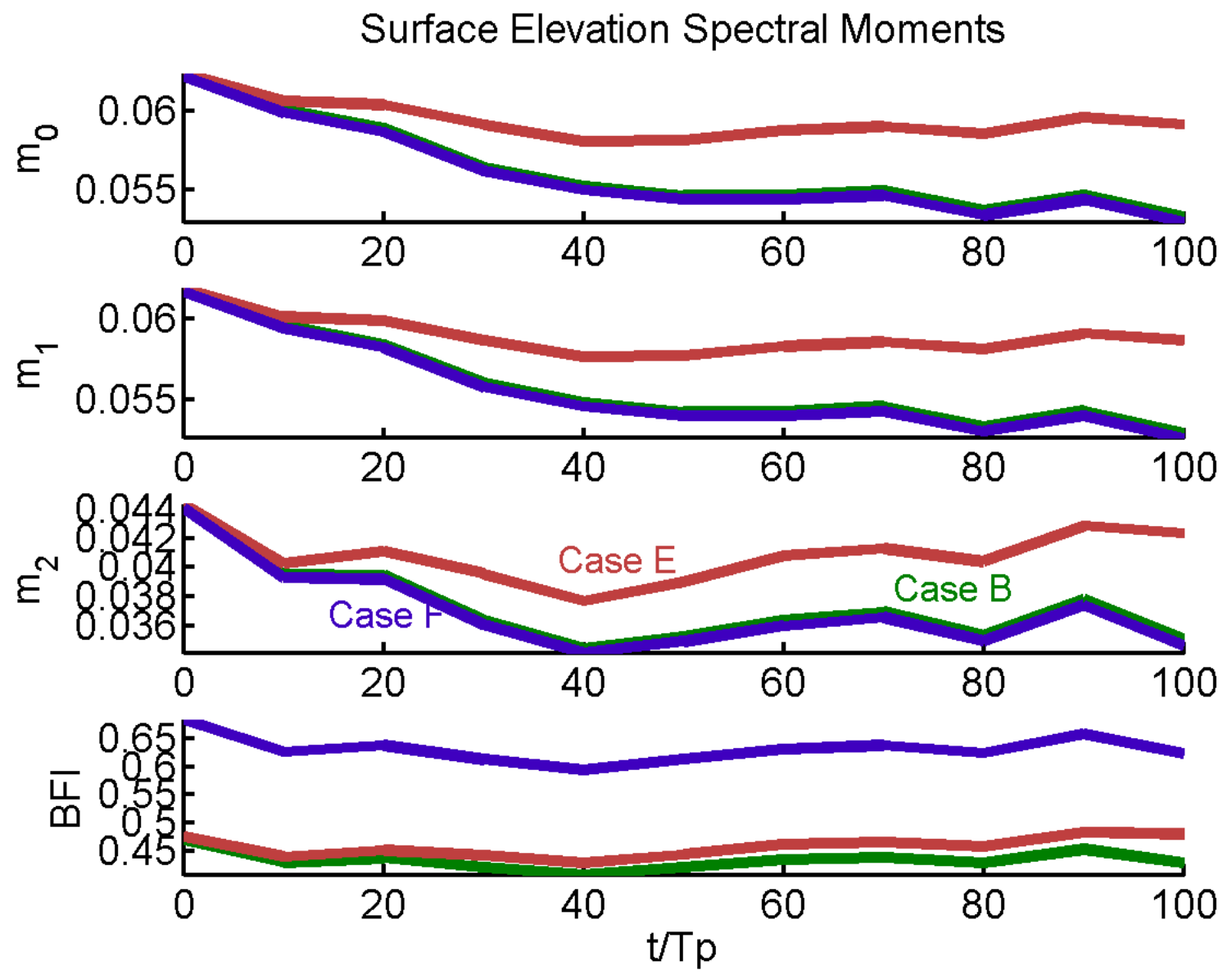

Figure 3-41: (In Color) For nonlinear simulations of Case $\mathbf{E}$ where $\alpha=0.0032$, Case B where $\alpha=0.0160$ and Case $\mathbf{F}$ where $\alpha=0.0163$, we plot surface elevation spectral moments against time $\left(t / T_{p}\right)$, where $m_{0}$ is the first spectral moment, $m_{1}$ is the second spectral moment, $m_{2}$ is the third spectral moment, and BFI is a scaled version of the BFI defined in Section 3.1.4 


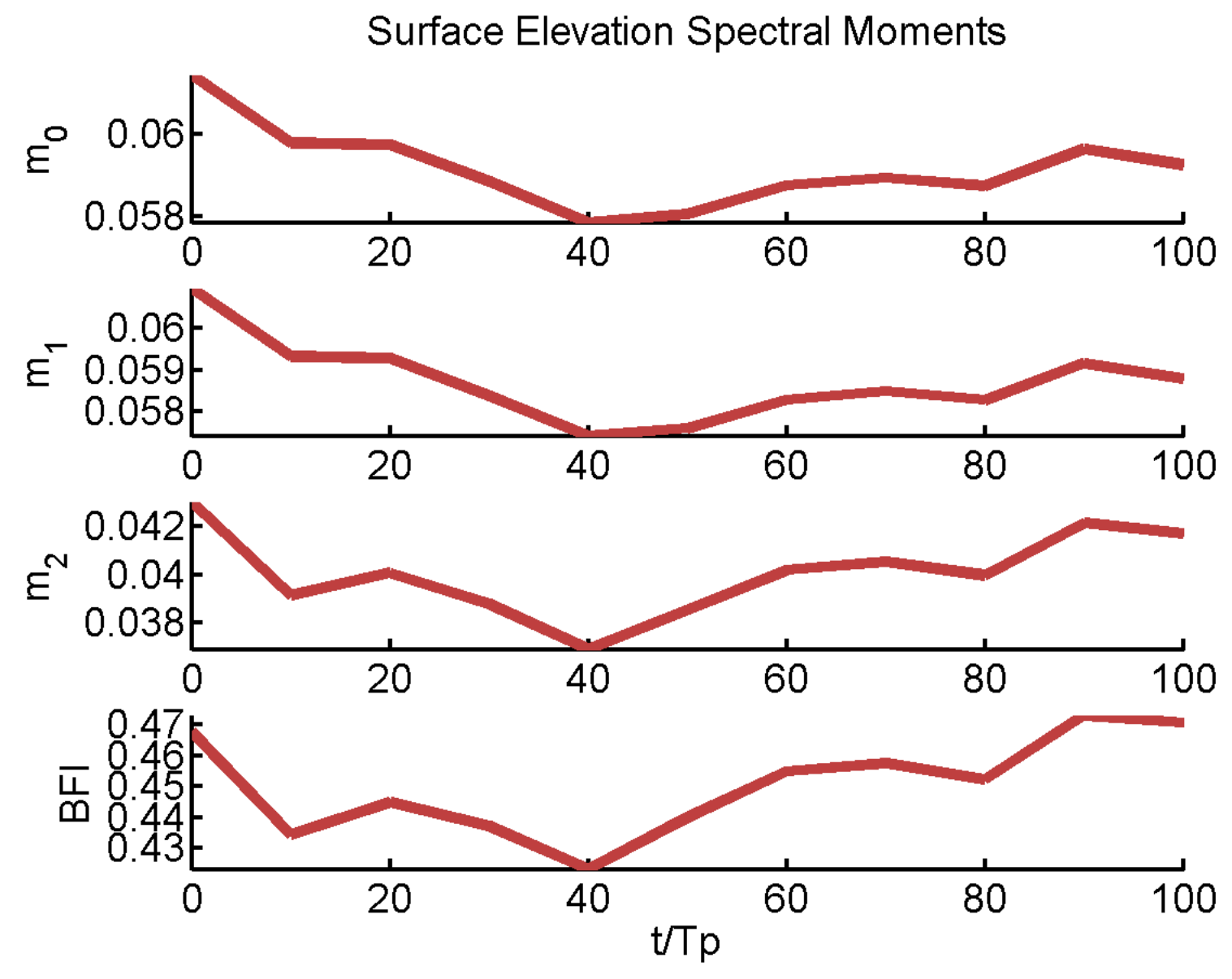

Figure 3-42: (In Color) For a linear simulation of Case $\mathbf{E}$ where $\alpha=0.0032$, we plot surface elevation spectral moments against time $\left(t / T_{p}\right)$ where $m_{0}$ is the first spectral moment, $m_{1}$ is the second spectral moment, $m_{2}$ is the third spectral moment, and BFI is a scaled version of the BFI defined in Section 3.1.4. 


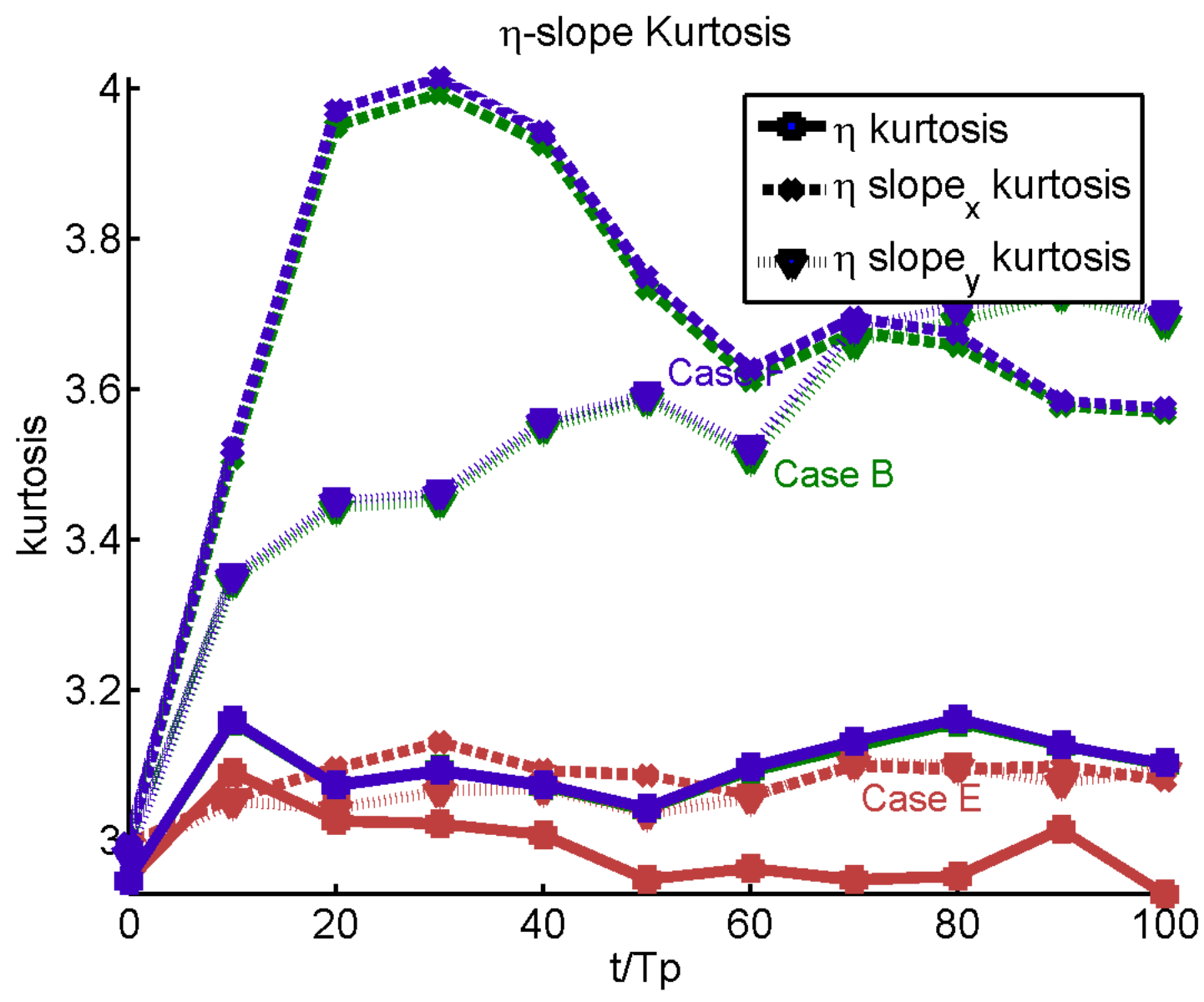

Figure 3-43: (In Color) Plot of kurtosis (on vertical axis) against time (on horizontal axis), $\left(t / T_{p}\right)$ in non-linear simulations of Case $\mathbf{E}$ where $\alpha=0.0032$, Case $\mathbf{B}$ where $\alpha=0.0160$ and Case $\mathbf{F}$ where $\alpha=0.0163$. Case $\mathbf{E}$ where $\alpha=0.0032$, has the appearance of a linear wavefield (see Figure 3-26) while Cases B and F with higher $\alpha$-values display typical nonlinear properties. 


\subsubsection{Impact of input Phillips' parameter on surface elevation- slope kurtosis}

Figure 3-43 shows the impact of peak shape parameter on the kurtosis of surface elevation $\eta$ and surface elevation slope in a non-linear simulation. The lowest kurtosis is seen in surface elevation, the second level of kurtosis is seen in surface slope in the direction perpendicular to propagation, $d(\eta) / d y$, and the highest kurtosis is seen in surface slope in the direction of wave propagation, $d(\eta) / d x$. Lower Phillips' parameter produces lower kurtosis in surface elevation and surface elevation slope. Lower Phillips' parameter produces smaller deviations between the three observed kurtosis evolutions.

\subsubsection{Conclusions: Phillips' parameter and surface elevation characteristics.}

In this section we look at Phillips' parameter, $\alpha$, and surface elevation characteristics, finding that:

- A higher Phillips' parameter produces higher kurtosis, and higher skewness in nonlinear simulations of surface elevation, although the influence of Phillips' parameter on surface elevation variance is unapparent, if at all existent.

- The maximum spectral energy in the nonlinear wavefield's wavenumber spectrum where $\alpha=0.0032$, is much more stable than maximum energy in the wavenumber spectrum of cases where $\alpha=0.0160$ and $\alpha=0.0163$.

- With all other spectral parameters equal, Benjamin Feir Index is independent of Phillips' parameter.

- Phillips' parameter is intimately connected to surface slope kurtosis values. A lower Phillips' parameter produces smaller deviations between the three kurtosis evolutions: surface elevation kurtosis, surface elevation slope in the $x$-direction 
kurtosis, and surface elevation slope in the $y$-direction kurtosis. If Phillips' parameter is small enough, it produces kurtosis evolutions that are so close that they resemble linear simulations.

- In a case with a very low Phillips' parameter, the observed effect of the nonlinearity order in the simulation is negligible. i.e. A low Phillips' parameter reduces the appearance of nonlinearity in the wavefield. 


\subsection{Directional spreading function}

Wind-generated wave energy does not necessarily propagate in the same direction as the wind. Energy usually spreads over various directions, although the major part of the energy may propagate in the wind direction. The way in which wave energy spreads in a wavefield is crucial in accurately representing wavefield statistics. Only a fixed average input wave travel direction is present in the SNOW simulated wavefields. We use a standard simplification [37] to define the spreading aspect of the spectral density function in SNOW:

$$
S(\omega, \theta)=S(\omega) D(\theta)
$$

The input spectral directional spreading function, $D(\theta)$ has a spreading angle, $\theta$, and the theoretical impact of these on wavefield physics is that a larger $\theta$ produces a wavefield with greater directional spreading, and therefore more Gaussian physical traits. $D(\theta)$ is combined in Equation 2.1 with the surface wave spectral density function to completely define the nature of the energy distribution in the wave field. According to Longuet-Higgins' 1961 study, $D(\theta)$ can take the form [37]:

$$
D(\theta)=\left|\cos \frac{1}{2}(\theta-\bar{\theta})\right|^{2 s}
$$

where in the present study, $s=1$.

Onorato et al, [45] investigate the effect of directional spreading on a wavefield's statistical properties, particularly the maximum wave height, using experimental investigations, and Toffoli [65] investigates the same question using a simulation of a the Euler equations. These studies conclude that large waves in directional seas are not as probable as large waves in uni-directional seas. In this section we take this investigation a step further by manipulating the value of directional spreading and observing the occurrence of large waves with height, $\mathrm{H} / \mathrm{Hs}>1.5$ as a function of directional spreading. Later, we also look at directional spreading as it acts alongside 
initial spectral Phillips' parameter and spectral peak shape parameter in linear and non-linear seas. In addition to the fact that experimental data indicates that large directional spreading moves a wavefield's distribution closer to linear theory [45], and unidirectional wavefields show statistical trends far from the linear theory [59], we investigate the details of the divergence of directional wavefields and near-unidirectional wavefields from linear theory.

Table 3.3 shows a list of the cases being used in testing the effects of directional spreading.

In this section we introduce the concept of directionality, and in the following section we observe the impact of directionality on surface elevation statistical distribution.

\subsubsection{Surface elevation distribution and input spectral direc- tional spreading function.}

In section 3.3 we are introduced to the concept of the spectral directional spreading function, $D(\theta)$, and the spreading angle, $\theta$. In this section we observe the impact of directionality on surface elevation statistical distribution, comparing between simulated wavefields of different directional spreading functions. We compare these wavefields to the linear theoretical distribution.

Figure 3-44 shows the statistical moments of various directional wavefields' surface elevation. These wavefields all have different values of $\theta$. Given constant $\gamma$ and $\alpha$, we see all the wavefields' surface elevation showing a growth and a subsequent fall in kurtosis. We see much higher values of surface elevation kurtosis among the wavefields with $\theta$-values $1^{\circ}, 10^{\circ}$, and $20^{\circ}$ than in the wavefields with $\theta$-values around $30^{\circ}, 40^{\circ}, 80^{\circ}$, and $180^{\circ}$. Surface elevation skewness appears to be oscillatory in these wavefields, but smaller spreading angles among the wavefields with $\theta$-values $1^{\circ}, 10^{\circ}$, and $20^{\circ}$ produce greater amplitudes of surface elevation skewness oscillations. Cases with $\theta$-values around $30^{\circ}, 40^{\circ}, 80^{\circ}$, and $180^{\circ}$ have more stable surface elevation skewness, with smaller variations in surface elevation skewness values. Sur- 
face elevation variance changes most in the unidirectional wavefield whose variance oscillates between 5 and 7 during the first $100 T_{p}$ whereas in the directional wavefields we find that surface elevation variance stays between 5.5 and 6.0.

We find that the impact of directional spreading is far greater than the impact of peak shape parameter (compare Figures 3-1 and 3-44) on surface elevation kurtosis, but that surface elevation variance and skewness have comparable values in both variations.

Figures 3-45, 3-46 and 3-47 show a comparison of all the cases used in testing the effects of directional spreading at three different times, $t=0 T_{p}, t=50 T_{p}$ and $t=100 T_{p}$

Various tilts in the lines indicate various ranges of $\eta$ values and therefore different types of stability in the energy at the highest surface elevations. Note that cases with $\theta$-values around $30^{\circ}, 40^{\circ}, 80^{\circ}$, and $180^{\circ}$ which have more stable surface elevation skewness have almost constant tilts throughout time, whereas wavefields with $\theta$-values $1^{\circ}, 10^{\circ}$, and $20^{\circ}$ appear to be less stable in maintaining their surface elevation distribution tilt.

More interesting is the curvature of the data series on the far right or the far left of the graphic away from linear theory. Comparing the data series in more detail in Figures 3-48, 3-49, 3-50, 3-51, 3-52, 3-53 and 3-54 we find three regimes for the probabilities of the highest wavefield surface elevations and three regimes for probabilities of the lowest wavefield surface elevations.

First we look at regimes for high surface elevation probability. The first regime among high surface elevations consists of the unidirectional case, $\theta=1^{o}$ where in Figure 3-48 skewness overrides kurtosis in the upper tail, so we see the majority of the higher elevations exceeding the linear theory. The second regime among high surface elevation probability distribution tails consists of the directional cases with $\theta=10^{\circ}, 20^{\circ}, 30^{\circ}$, and $40^{\circ}$ in Figures 3-49, 3-50, 3-51, and 3-52 (resp.) where surface elevation kurtosis is more dominant than surface elevation skewness among the highest elevations in the wavefields. We see the effect of high kurtosis in the highest elevations' 
probability exceeding that of the linear theory so drastically that it takes away from the cumulative probability of the less-extreme high surface elevations, causing the data series to drop below the theoretical line among the less-extreme high surface elevations. The third regime among high surface elevation probability distribution tails consists of the highly directional cases with $\theta=80^{\circ}$ and $180^{\circ}$ seen in Figures 3-53 and 3-54 where surface elevation skewness is again more apparent than surface elevation kurtosis, but the curvature away from linear theory is not as pronounced as in the first regime.

Next we look at regimes for lower surface elevation probabilities. The first regime among low surface elevations consists of the unidirectional case, $\theta=1^{o}$ where in Figure 3-48 low surface elevations' probabilities exceed the linear theory. The second regime among low surface elevations consists of directional cases where $\theta=10^{\circ}$ and $20^{\circ}$, where we see continuous curves that exceed linear theory and show a higher probability of the occurrence of low surface elevations. The third regime among low surface elevations consists of directional cases where $\theta=30^{\circ}, 40^{\circ}, 80^{\circ}$ and $180^{\circ}$ where surface elevation distribution is much lower than seen in linear theory and this indicates the presence of Stokes'-like waves, where highest elevations are higher than in linear plane-progressive waves and lowest elevations are not as low as in linear, plane progressive waves.

In Figures 3-48, 3-49 and 3-50 graphics depict the cumulative form of the Gaussian distribution, as a straight line. Different directional spreading functions produce different types of changes in the surface elevation distribution over time and this can be observed in the changing non-linearity (seen as data series curvature away from the theoretical straight line in these plots).

We present results of an investigation of the impact of directionality on surface elevation statistical distribution. In this study we compare observed data from simulated wavefields with different directional spreading functions to each other, and to linear theory. We find that directionality has a quantifiable impact on surface elevation statistical distribution, but that there are different regimes of directionality. Presently, we investigate the impact of directionality on the non-Gaussian character- 
Table 3.3: Simulated wavefield cases used in testing the effects of directional spreading with peak shape parameter, $\gamma=3.3$ and Phillips' parameter, $\alpha=0.0160$

\begin{tabular}{llllllll}
\hline \hline Case & J & A & B & M & K & L & G \\
\hline directional spreading, $\theta$ & 180 & 80 & 40 & 30 & 20 & 10 & 1
\end{tabular}
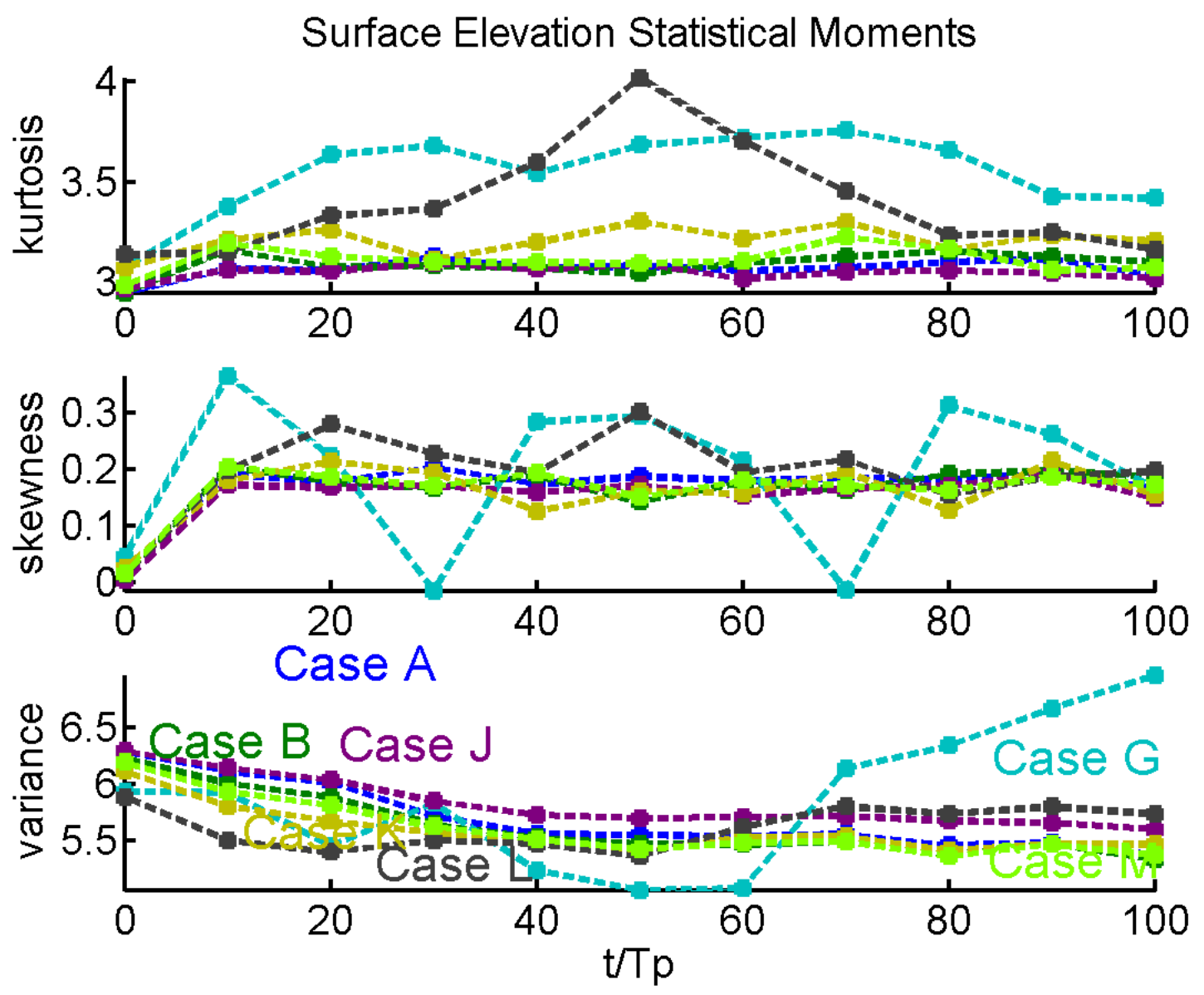

Figure 3-44: (In Color) Spectral moments are displayed on the vertical axes while time $\left(t / T_{p}\right)$ is displayed on the horizontal axes. Variance, skewness and kurtosis of surface elevation is plotted against time $\left(t / T_{p}\right)$ in nonlinear simulations of Cases $\mathbf{J}$ where $\theta=180^{\circ}$, A where $\theta=80^{\circ}, \mathbf{B}$ where $\theta=40^{\circ}, \mathbf{M}$ where $\theta=30^{\circ}, \mathbf{K}$ where $\theta=20^{\circ}, \mathbf{L}$ where $\theta=10^{\circ}$ and $\mathbf{G}$ where $\theta=1^{\circ}$. 


\section{Time 0Tp}

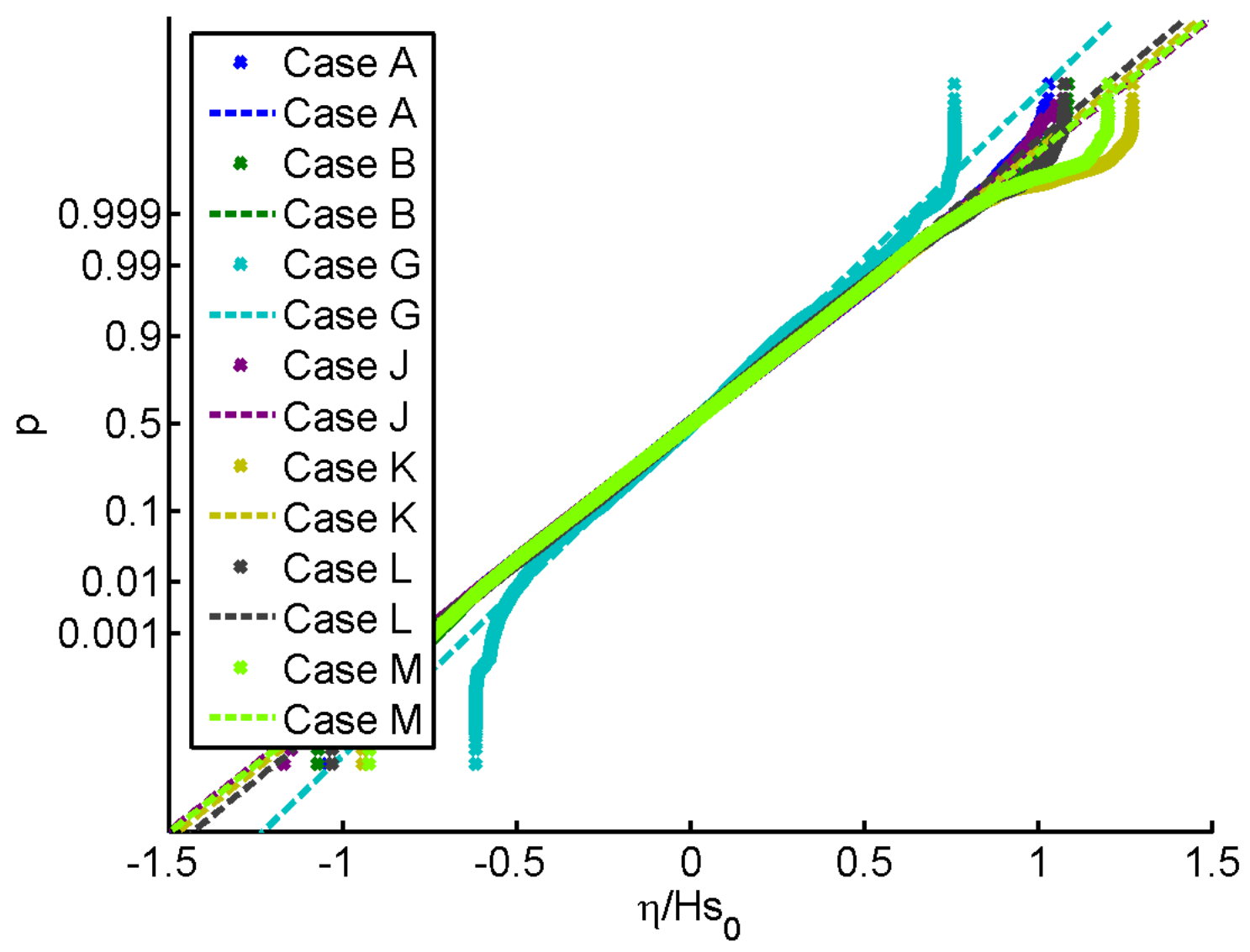

Figure 3-45: (In Color) Horizontal axis shows non-dimensional surface elevation and vertical axis shows the cumulative distribution of such an elevation in the wavefield. Theoretical surface elevation distribution (- - - ), and observed surface elevation distribution data $(++++)$ in nonlinear simulations of Cases $\mathbf{J}$ where $\theta=180^{\circ}$, A where $\theta=80^{\circ}, \mathbf{B}$ where $\theta=40^{\circ}, \mathbf{M}$ where $\theta=30^{\circ}, \mathbf{K}$ where $\theta=20^{\circ}, \mathbf{L}$ where $\theta=10^{\circ}$ and $\mathbf{G}$ where $\theta=1^{\circ}$ at time $t=0 T_{p}$. Each line pair represents one case. 


\section{Time 50Tp}

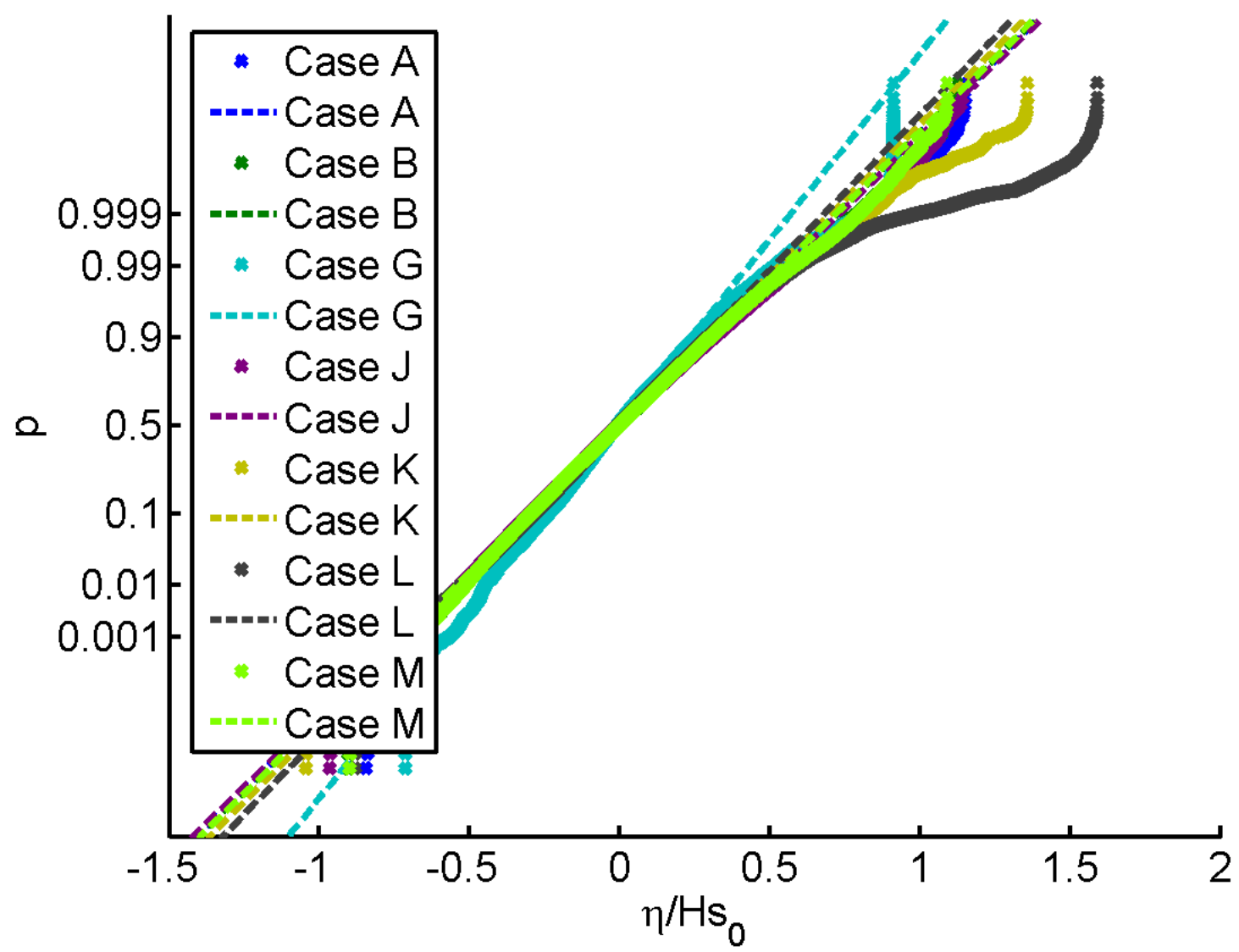

Figure 3-46: (In Color) Horizontal axis shows non-dimensional surface elevation and vertical axis shows the cumulative distribution of such an elevation in the wavefield. Theoretical surface elevation distribution (- - - ), and observed surface elevation distribution data $(++++)$ in nonlinear simulations of Cases $\mathbf{J}$ where $\theta=180^{\circ}$, A where $\theta=80^{\circ}, \mathbf{B}$ where $\theta=40^{\circ}, \mathbf{M}$ where $\theta=30^{\circ}, \mathbf{K}$ where $\theta=20^{\circ}, \mathbf{L}$ where $\theta=10^{\circ}$ and $\mathbf{G}$ where $\theta=1^{\circ}$ at time $t=50 T_{p}$. Each line pair represents one case. 


\section{Time 100Tp}

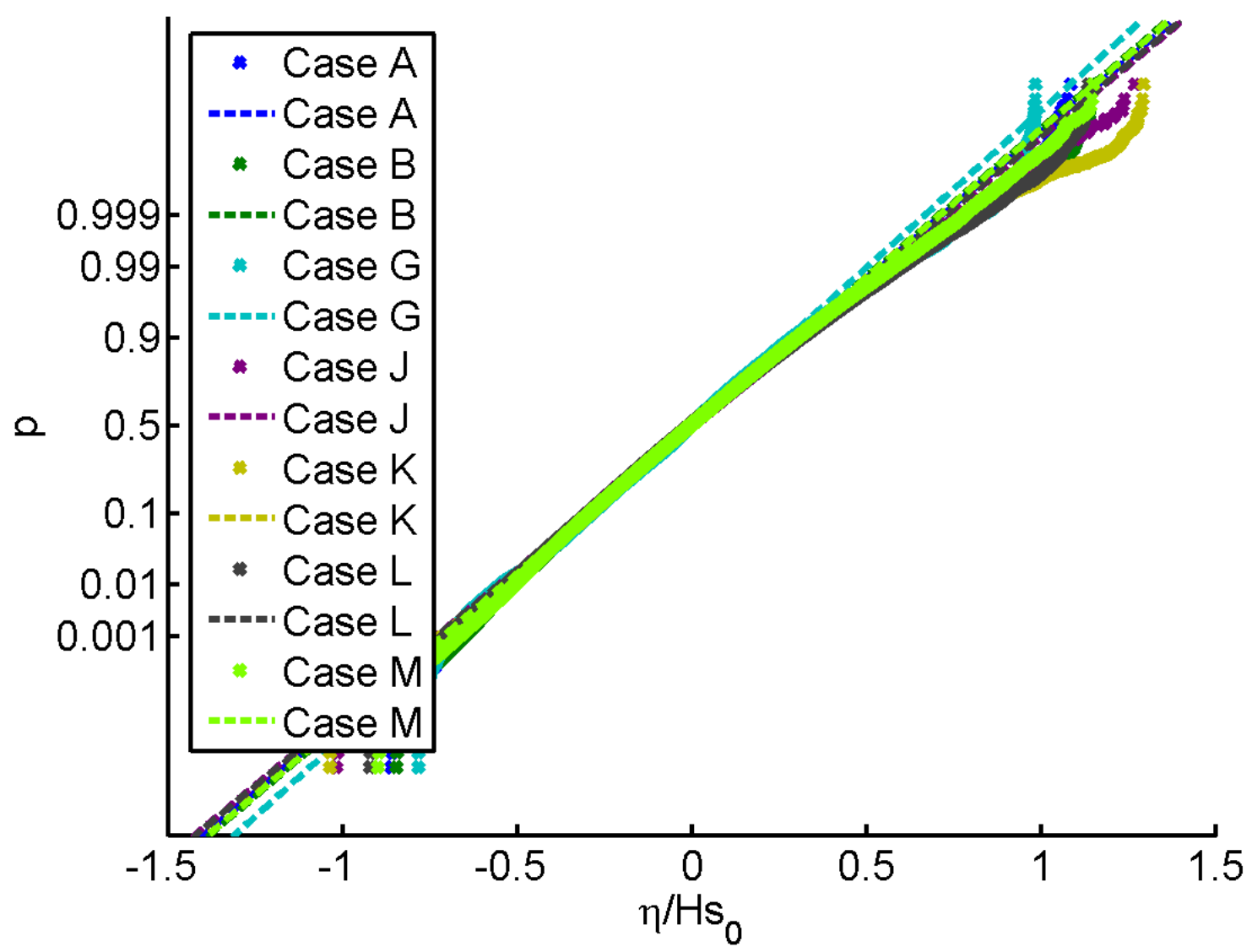

Figure 3-47: (In Color) Horizontal axis shows non-dimensional surface elevation and vertical axis shows the cumulative distribution of such an elevation in the wavefield. Theoretical surface elevation distribution (- - - ), and observed surface elevation distribution data $(++++)$ in nonlinear simulations of Cases $\mathbf{J}$ where $\theta=180^{\circ}$, A where $\theta=80^{\circ}, \mathbf{B}$ where $\theta=40^{\circ}, \mathbf{M}$ where $\theta=30^{\circ}, \mathbf{K}$ where $\theta=20^{\circ}, \mathbf{L}$ where $\theta=10^{\circ}$ and $\mathbf{G}$ where $\theta=1^{\circ}$ at time $t=100 T_{p}$. Each line pair represents one case. 


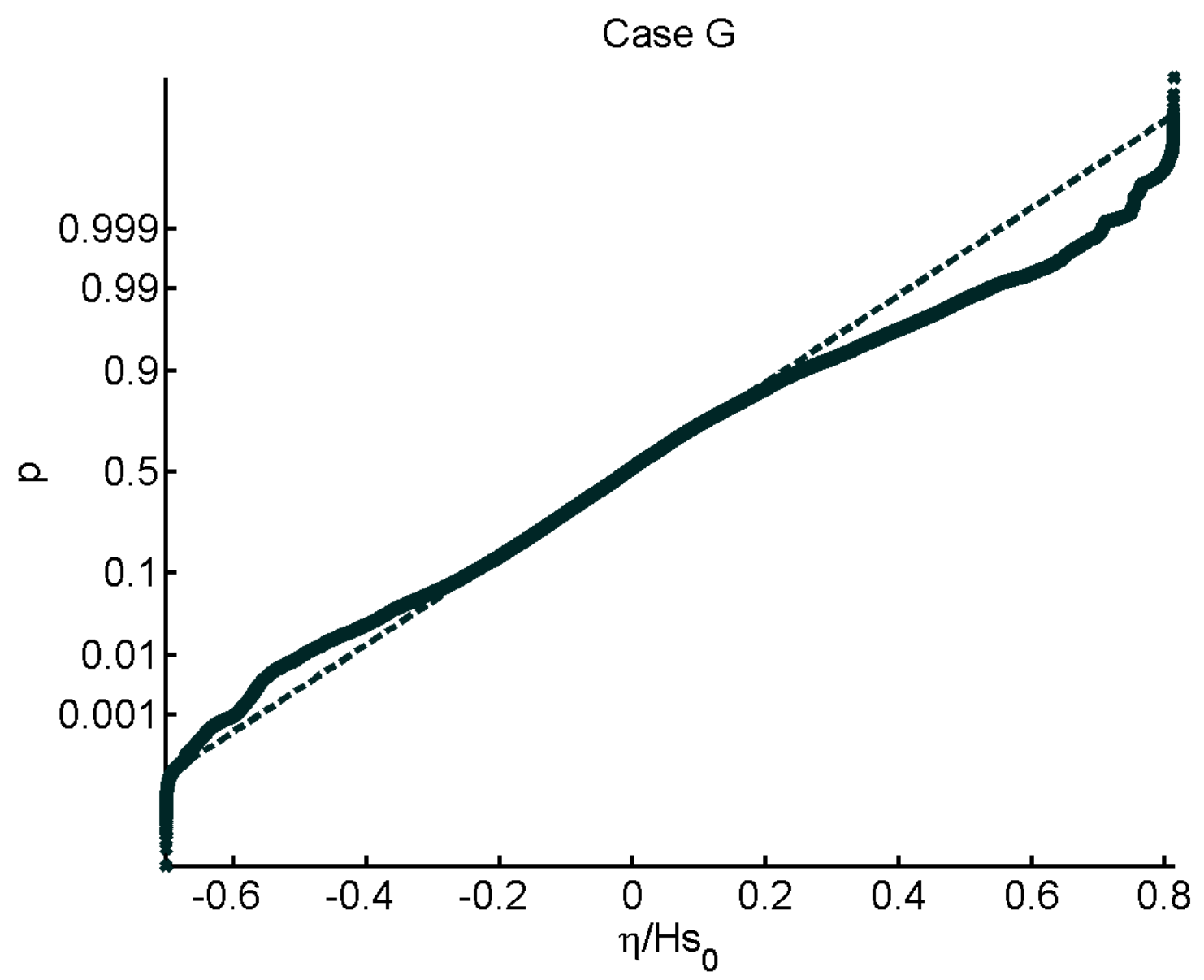

Figure 3-48: Horizontal axis shows non-dimensional surface elevation and vertical axis shows the cumulative distribution of such an elevation in the unidirectional case (with $\theta=1^{o}$ ) at $t=20 T_{p}$. Trough occurrences on the far left of the graphic far exceed linear theory for this low value of directional spreading. Observed data series $(+++)$; Theoretical line(- - -). 


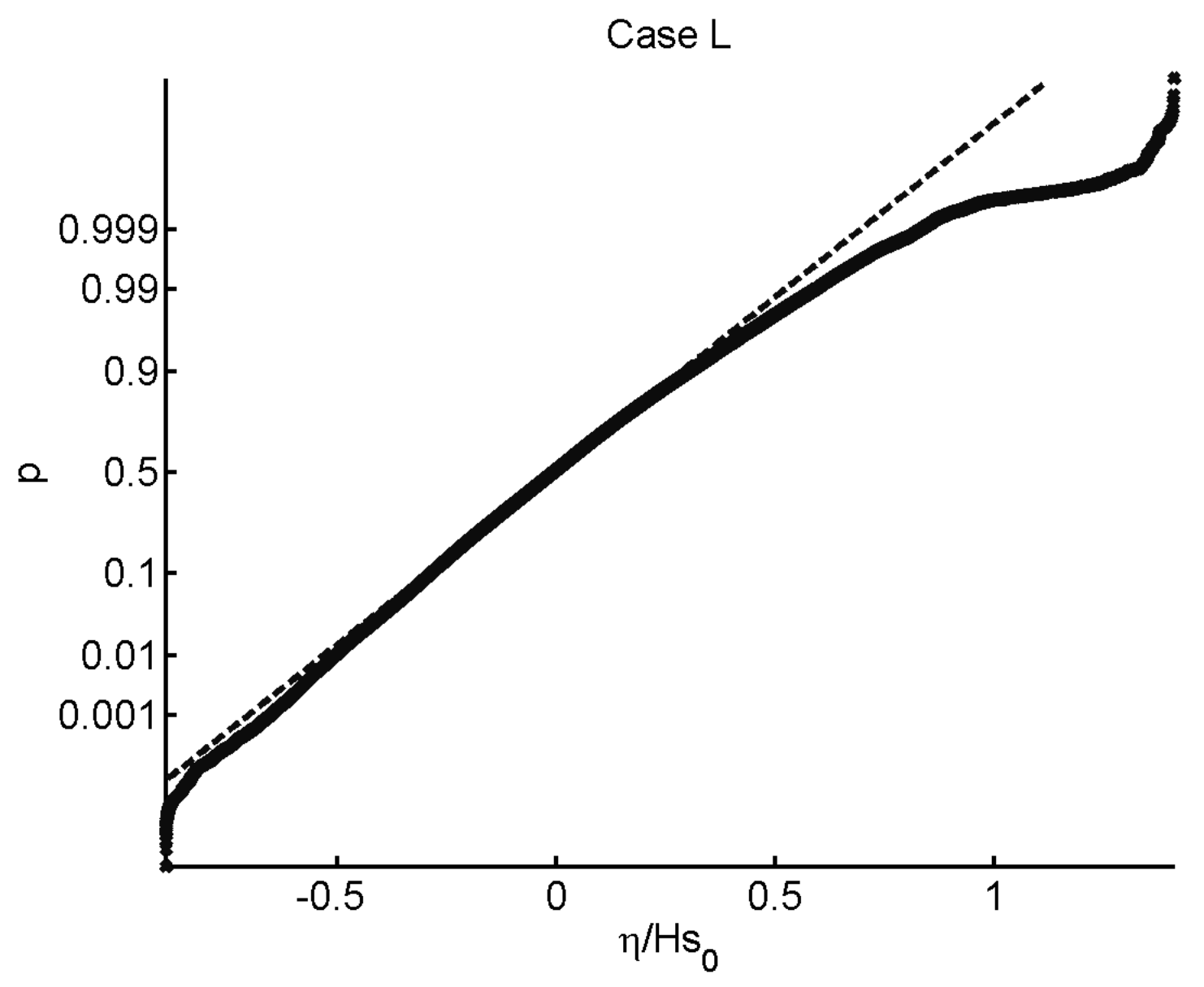

Figure 3-49: Horizontal axis shows non-dimensional surface elevation and vertical axis shows the cumulative distribution of such an elevation in the directional case (with $\theta=10^{\circ}$ ) at $t=20 T_{p}$. Kurtosis is more apparent than skewness for the large values of surface elevation on the far right of the graphic. Trough occurrences on the far left of the graphic far exceed linear theory for these fairly low values of directional spreading. Observed data series $(+++)$; Theoretical line(- - -). 


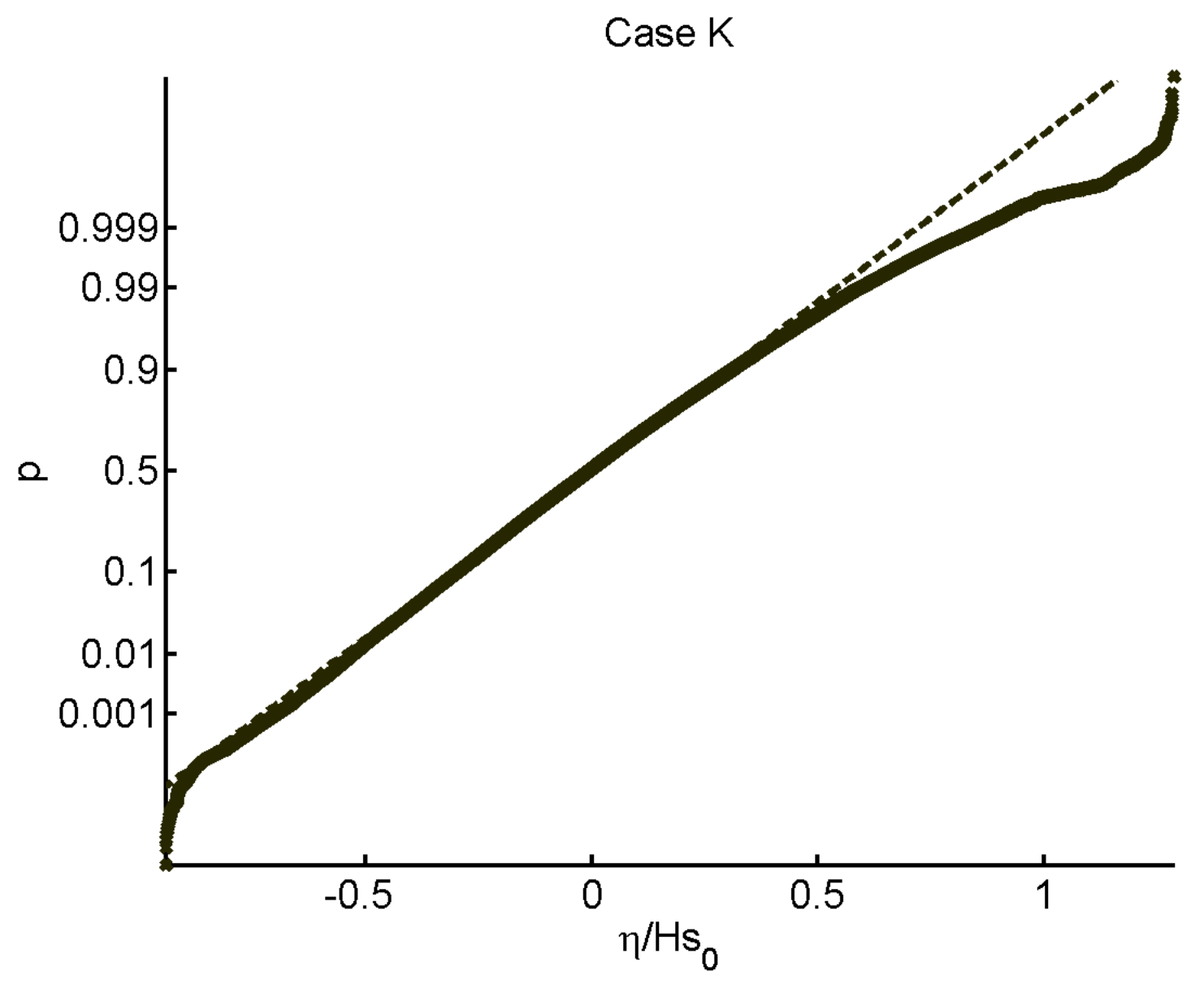

Figure 3-50: Horizontal axis shows non-dimensional surface elevation and vertical axis shows the cumulative distribution of such an elevation in the directional case (with $\theta=20^{\circ}$ ) at $t=20 T_{p}$. Kurtosis is more apparent than skewness for the large values of surface elevation on the far right of the graphic. Trough occurrences on the far left of the graphic far exceed linear theory for these fairly low values of directional spreading. Observed data series $(+++)$; Theoretical line(- - -). 


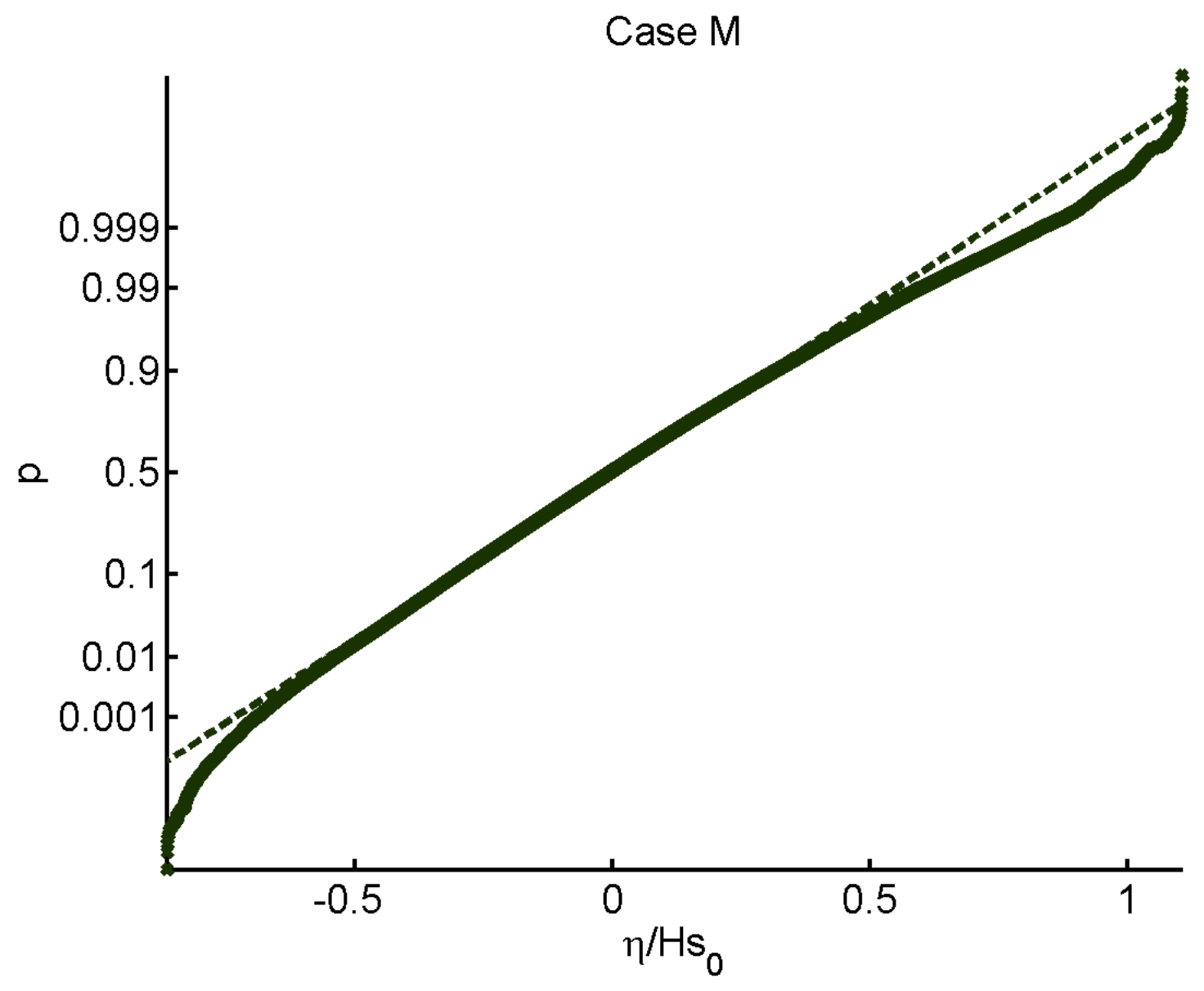

Figure 3-51: Horizontal axis shows non-dimensional surface elevation and vertical axis shows the cumulative distribution of such an elevation in the directional case (with $\theta=30^{\circ}$ ) at $t=20 T_{p}$. Trough occurrences on the far left of the graphic are far less than linear theory in these examples with fairly high directional spreading values. Observed data series $(+++)$; Theoretical line(- - -). 


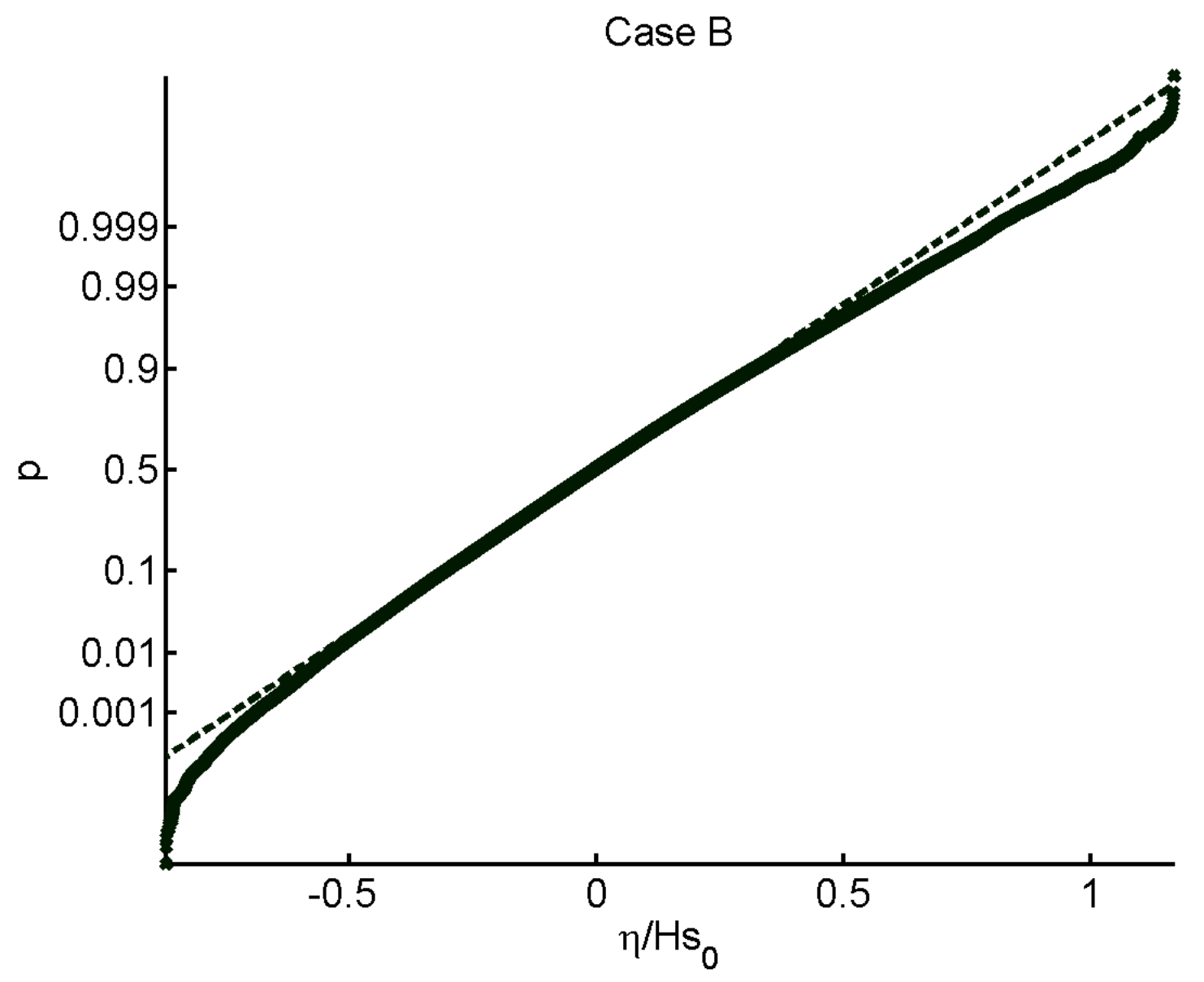

Figure 3-52: Horizontal axis shows non-dimensional surface elevation and vertical axis shows the cumulative distribution of such an elevation in the directional case (with $\theta=40^{\circ}$ ) at $t=20 T_{p}$. Kurtosis is more apparent than skewness for the large values of surface elevation on the far right of the graphic. Trough occurrences on the far left of the graphic are far less than linear theory in these examples with fairly high directional spreading values. Observed data series $(+++)$; Theoretical line $\left(--_{-}\right)$. 


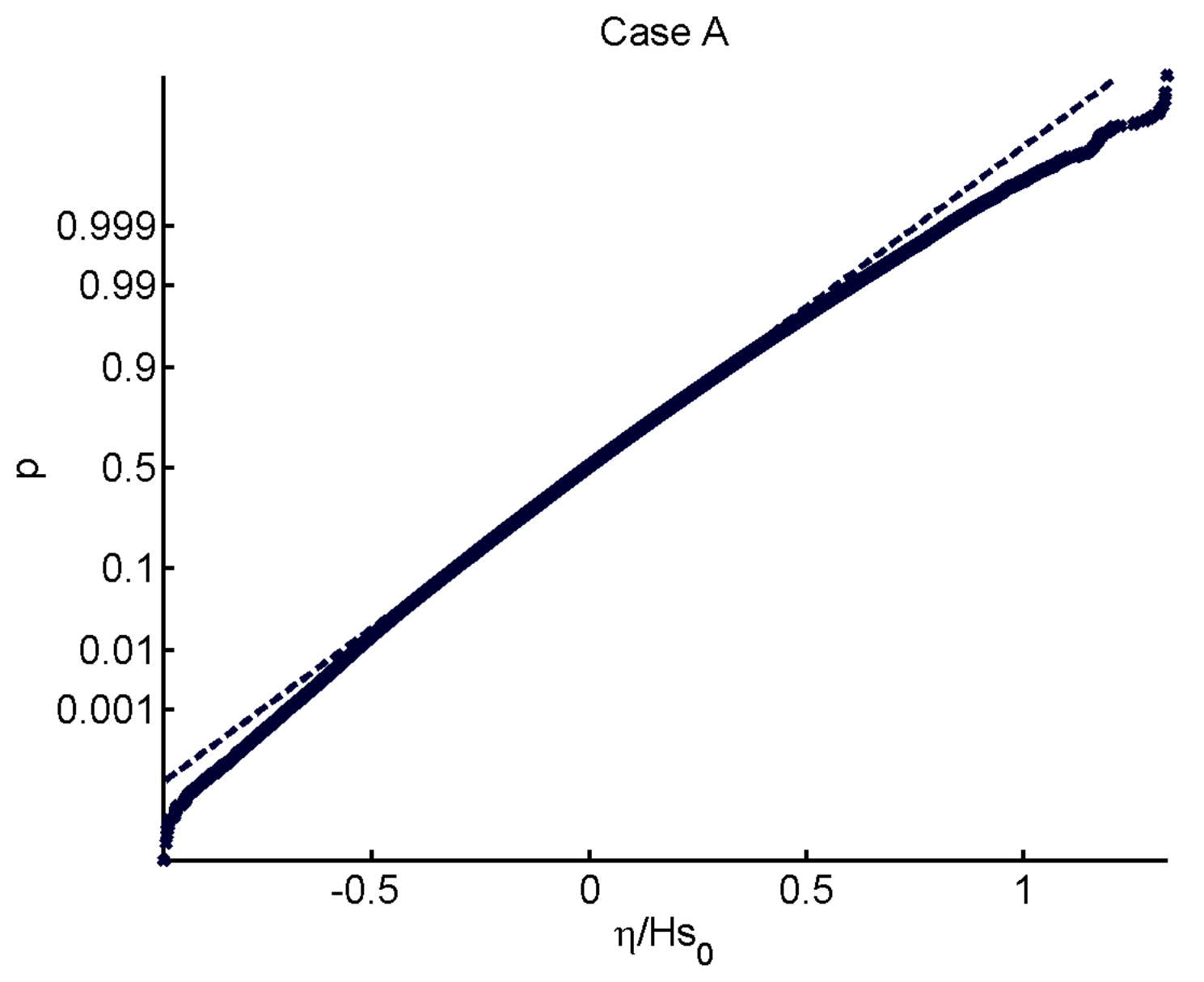

Figure 3-53: Horizontal axis shows non-dimensional surface elevation and vertical axis shows the cumulative distribution of such an elevation in the directional case (with $\theta=80^{\circ}$ ) at $t=20 T_{p}$. Kurtosis is less apparent than skewness for the large values of surface elevation on the far right of the graphic. Trough occurrences on the far left of the graphic are far less than linear theory in these examples with fairly high directional spreading values. Observed data series $(+++)$; Theoretical line $\left(--_{-}\right)$. 


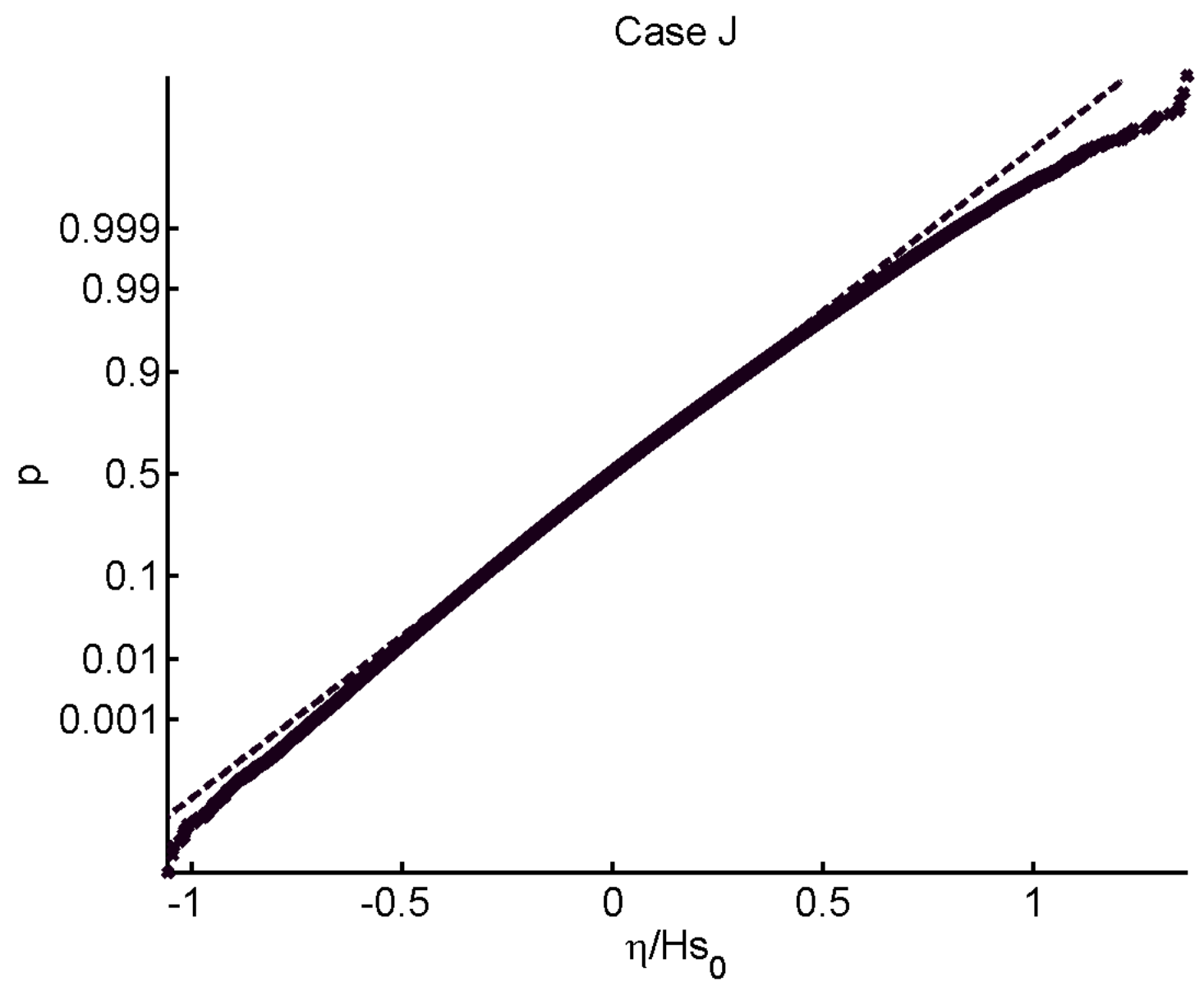

Figure 3-54: Horizontal axis shows non-dimensional surface elevation and vertical axis shows the cumulative distribution of such an elevation in the directional case (with $\left.\theta=180^{\circ}\right)$ at $t=20 T_{p}$. Kurtosis is less apparent than skewness for the large values of surface elevation on the far right of the graphic. Trough occurrences on the far left of the graphic are far less than linear theory in these examples with fairly high directional spreading values. Observed data series $(+++)$; Theoretical line $\left(--_{-}\right)$. 
istics of surface elevation statistics.

At time $t=50 T_{p}$, we see the distribution of surface elevation in Figure 3-55 in cases with $\theta=1^{\circ}$ and $\theta=180^{\circ}$, therefore comparing unidirectional and directional wavefields. The distribution of surface elevation in Figure 3-55 after $t=50 T_{p}$ shows a clear distinction between directional and unidirectional wavefields. This distinction is an indication that unidirectionality magnifies the impact of non-linear constructive wave-wave interactions in a wavefield, producing greater probabilities of high surface elevation than expected in the first-, second- and third-order Gram Charlier distributions. This also shows how the weakly nonlinear Gram-Charlier higher-order distributions can under-estimate surface elevation probabilities even in highly directional wavefields where $\theta=180^{\circ}$.

As time proceeds, we observe the same wavefields at time $t=100 T_{p}$. We see the further evolution of the surface elevation distribution wavefield in Figures 3-55 and 3-56. Consistent with all our previous results, we see the unidirectional wavefield's highest surface elevations having probabilities that far exceed the first-, secondand third-order Gram Charlier distributions for weakly nonlinear wavefields. Studies demonstrate this series reasonably accurately represents the probability distributions of coastal surface waves [6] [41], and we see that it captures our surface elevation displacements fairly well away from distribution tails. At surface elevation extremes for a uni-directional wavefield, the first-, second- and third-order Gram Charlier distributions consistently under-estimate extreme high-elevation probabilities and extreme low-elevation probabilities as well.

In the next section we observe the effect of input directional spreading on surface elevation stability via the wavenumber spectrum.

\subsubsection{Input spectral directional spreading function and sur- face elevation wavenumber spectrum}

In this section, we observe the impact of directional spreading on wavenumber spectrum. The largest value of directional spreading angle, $\theta=180^{\circ}$ produces much 


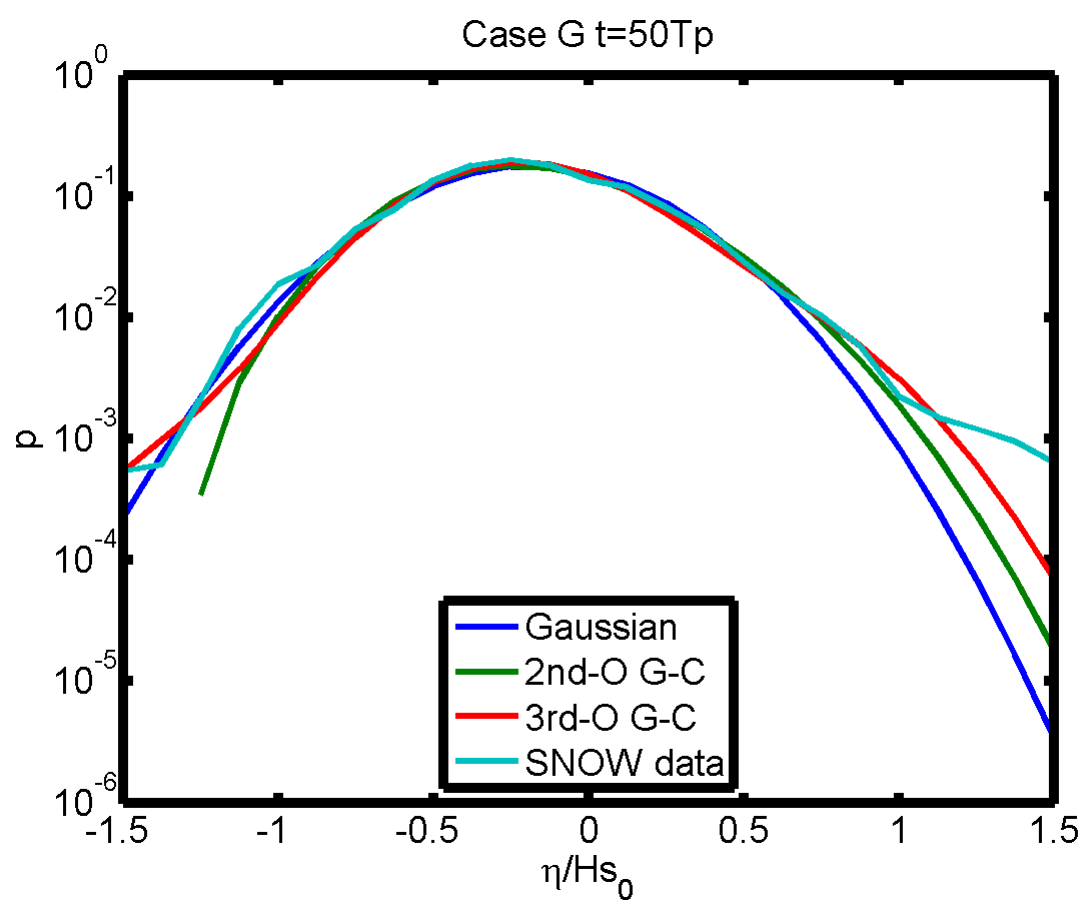

(a) Distribution of surface elevation with $\theta=1^{\circ}$.

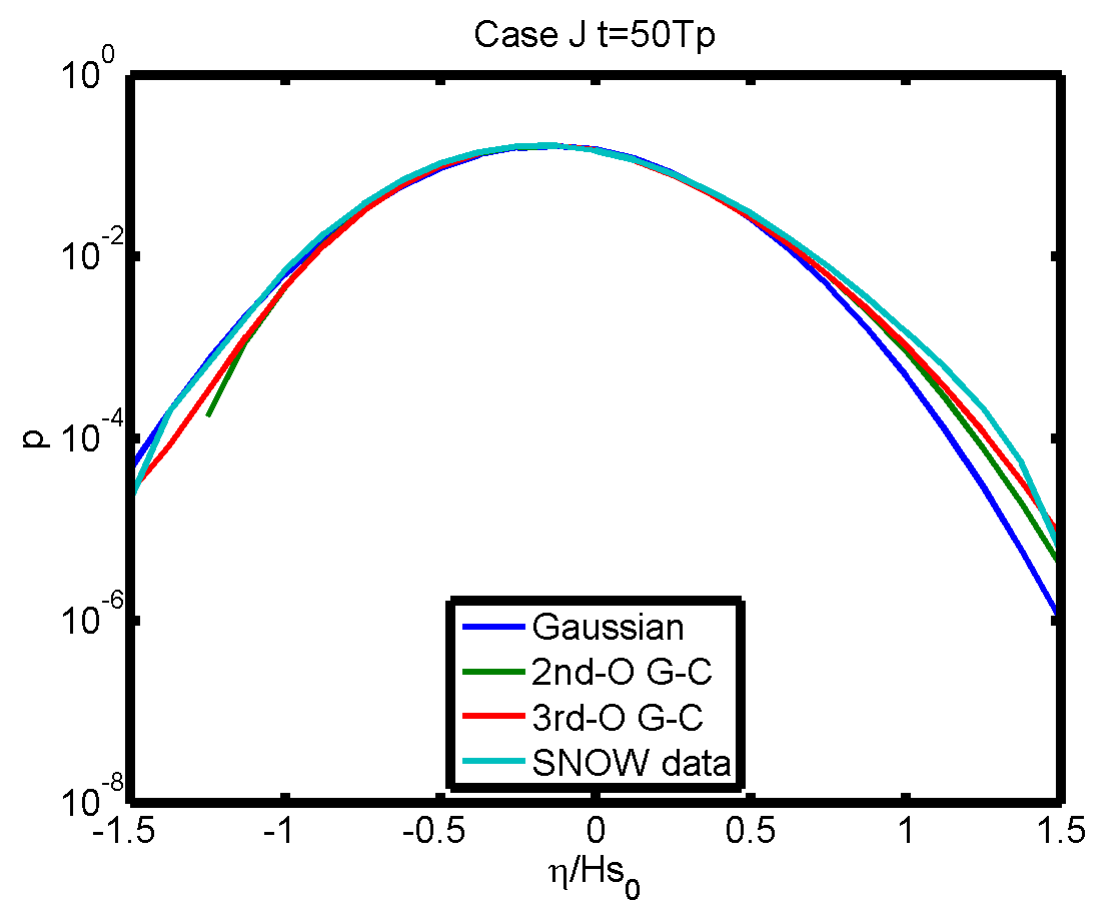

(b) Distribution of surface elevation with $\theta=180^{\circ}$.

Figure 3-55: (In Color) Horizontal axis depicts non-dimensional surface elevation and vertical axis depicts probability. Nonlinear simulation of Cases with input spectral directional spreading function $D(\theta)$ having $\theta=1^{\circ}$ and $\theta=180^{\circ}$, at time $t=50 T_{p}$. Gram Charlier distributions Order 1, 2 and 3 compared with observed SNOW nonlinear surface elevation data distribution. 


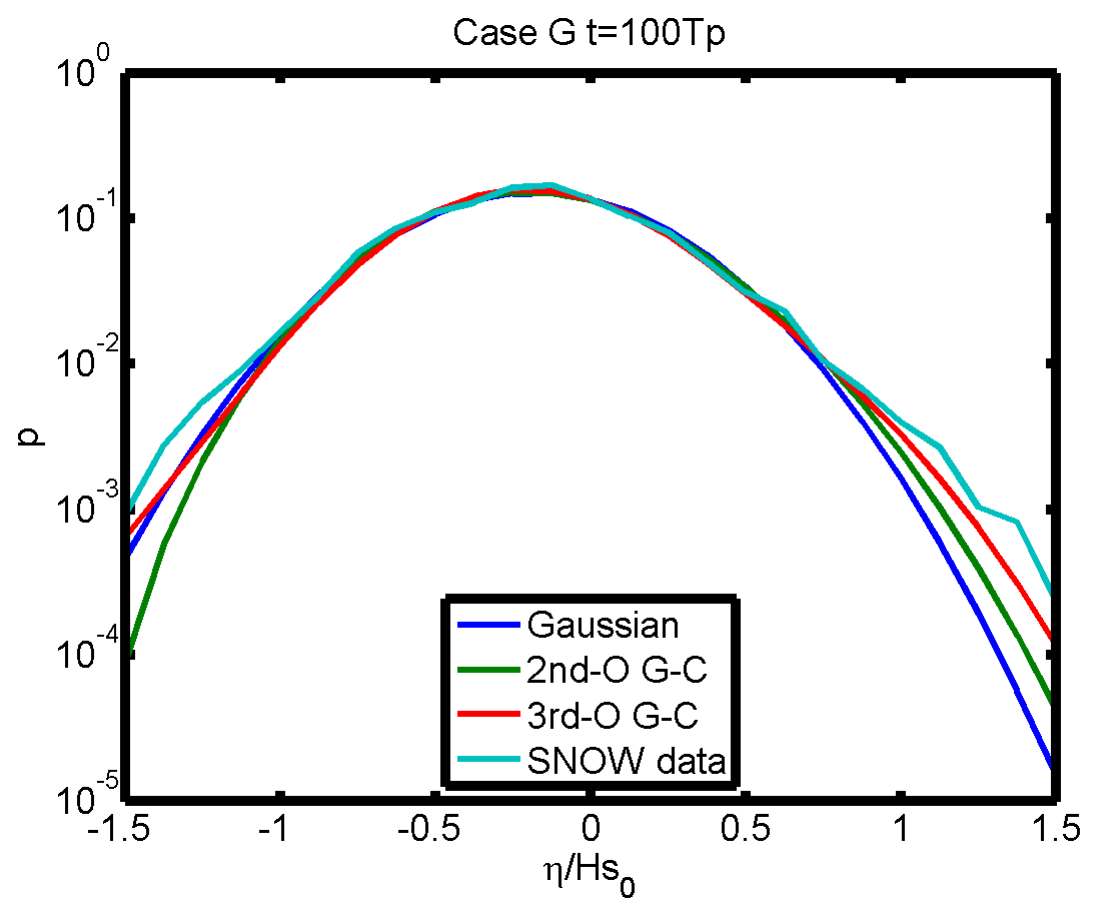

(a) Distribution of surface elevation with $\theta=1^{o}$ at $t=100 T_{p}$.

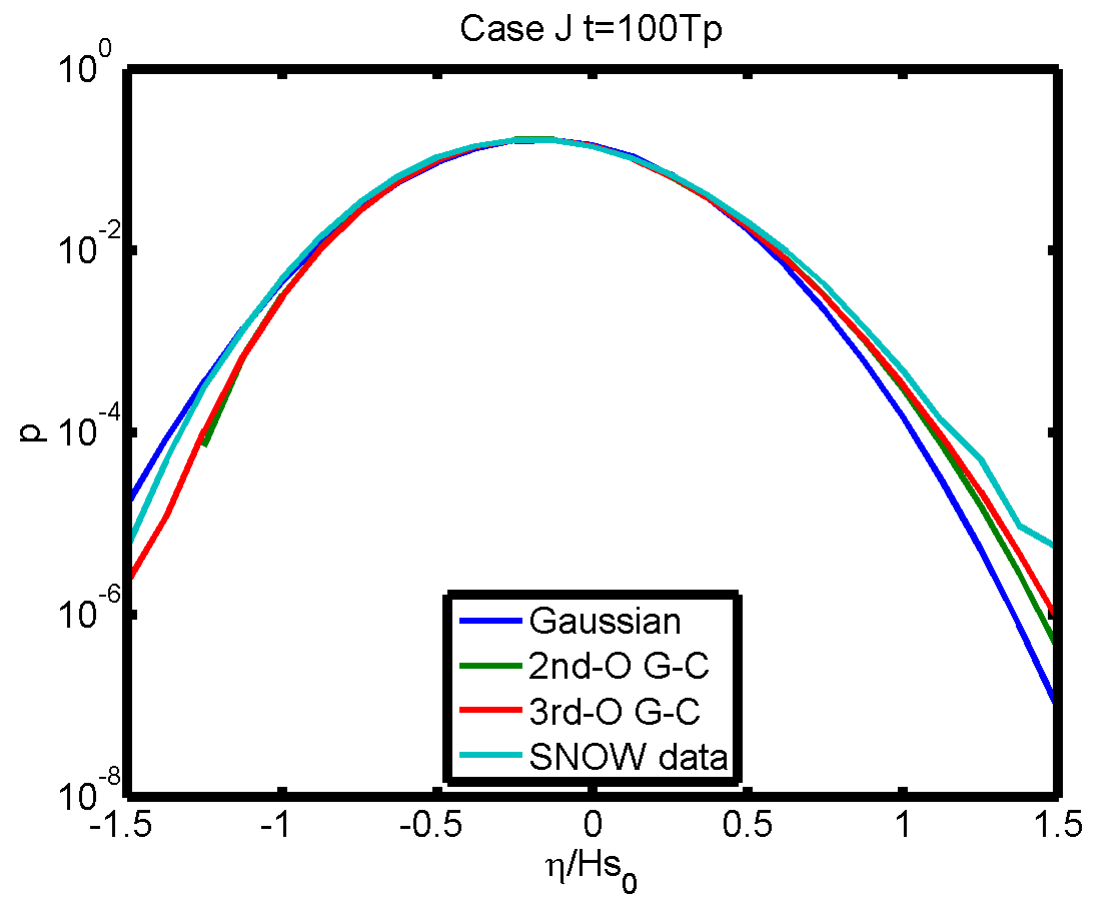

(b) Distribution of surface elevation with $\theta=180^{\circ}$ at $t=100 T_{p}$.

Figure 3-56: (In Color) Horizontal axis depicts non-dimensional surface elevation and vertical axis depicts probability. Nonlinear simulation of Cases with input spectral directional spreading function $D(\theta)$ having $\theta=1^{\circ}$ and $\theta=180^{\circ}$, at time $t=100 T_{p}$. Gram Charlier distributions Order 1, 2 and 3 compared with observed SNOW nonlinear surface elevation data distribution. 
smaller spectral slope and slightly lower maximum spectral energy than in cases with smaller spreading angles (see Figure 3-57). Further, a larger directional spreading angle, $\theta=180^{\circ}$ produces greatest stability in the highest spectral energy in the time evolution of spectrum, compared to all the other equivalent wavefields with smaller $\theta$-values.

In Figure 3-58 we see the surface elevation wavenumber spectrum in Cases B where $\theta=40^{\circ}$, and $\mathbf{M}$ where $\theta=30^{\circ}$. Maximum spectral energy in these cases is higher than that in the case where $\theta=180^{\circ}$, but the maximum energy is less stable, disappearing between $t=30 T_{p}$ and $t=50 T_{p}$ in both cases.

In Figure 3-59 we see the surface elevation wavenumber spectrum in Cases B where $\theta=20^{\circ}$, and $\mathbf{M}$ where $\theta=10^{\circ}$. Maximum spectral energy in these cases is higher than that in the case with much greater directional spreading where $\theta=180^{\circ}$, but the same as in the cases where $\theta=40^{\circ}$, and $\theta=30^{\circ}$. The maximum spectral energy in these cases is far less stable, disappearing completely between $t=10 T_{p}$ and $t=60 T_{p}$ in both cases.

In Figure 3-60 we see the surface elevation wavenumber spectrum in a unidirectional Case $\mathbf{G}$ where $\theta=1^{\circ}$. Maximum spectral energy in these cases is higher than that in all the directional cases, but the maximum spectral energy in this case is far less stable, appearing only at the end of the entire observed surface evolution period at $t=100 T_{p}$.

This association between lower directional spreading and higher spectral energy is consistent with literature where we commonly see the expectation of rogue waves and extreme surface elevations decrease in the presence of increasing .directional spreading $[65],[45]$.

\subsubsection{Input spectral directional spreading function and sur- face elevation Benjamin-Feir Index}

In this section we observe the effect of directional spreading, $D(\theta)$ on the timeevolution of spectral moments and the Benjamin-Feir Index (BFI), by comparing 


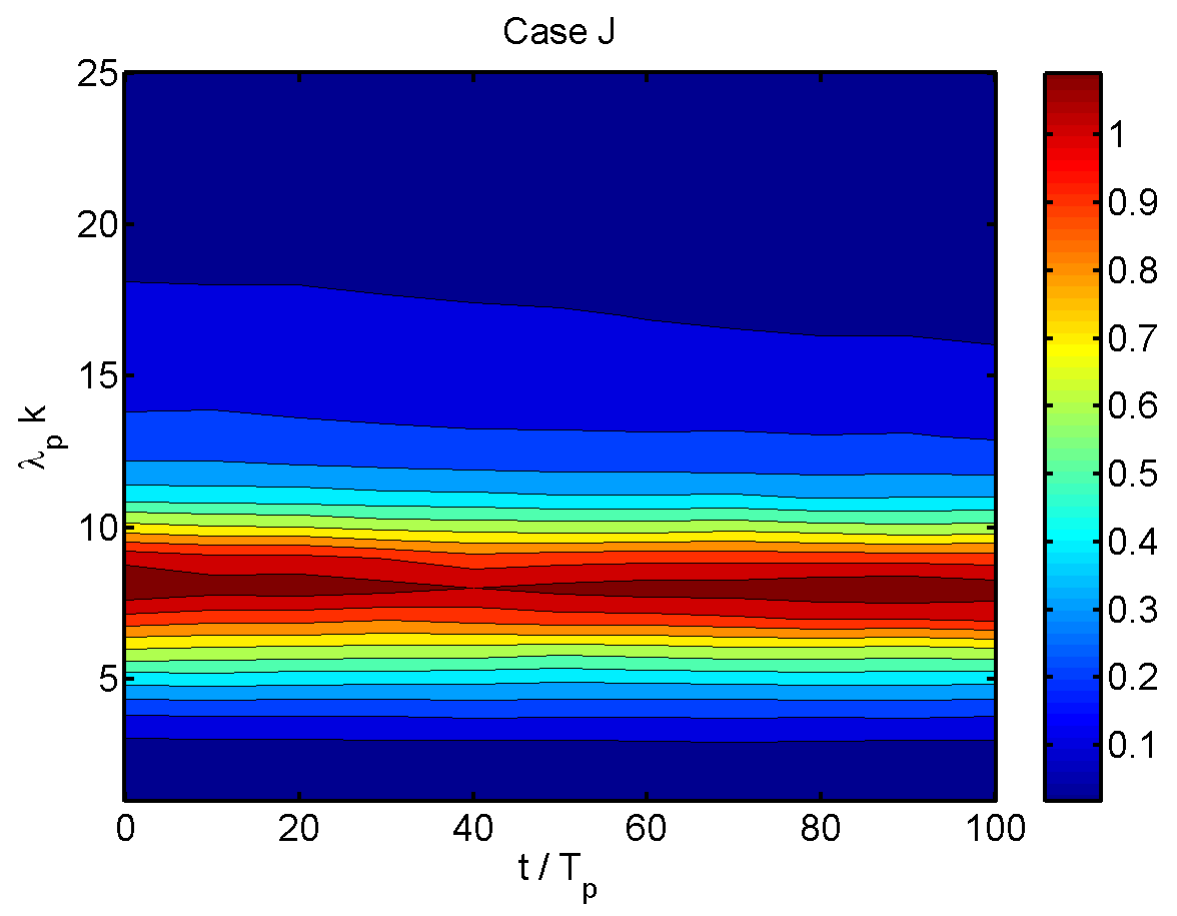

(a) Time evolution of surface elevation wavenumber spectrum for $\theta=180^{\circ}$.

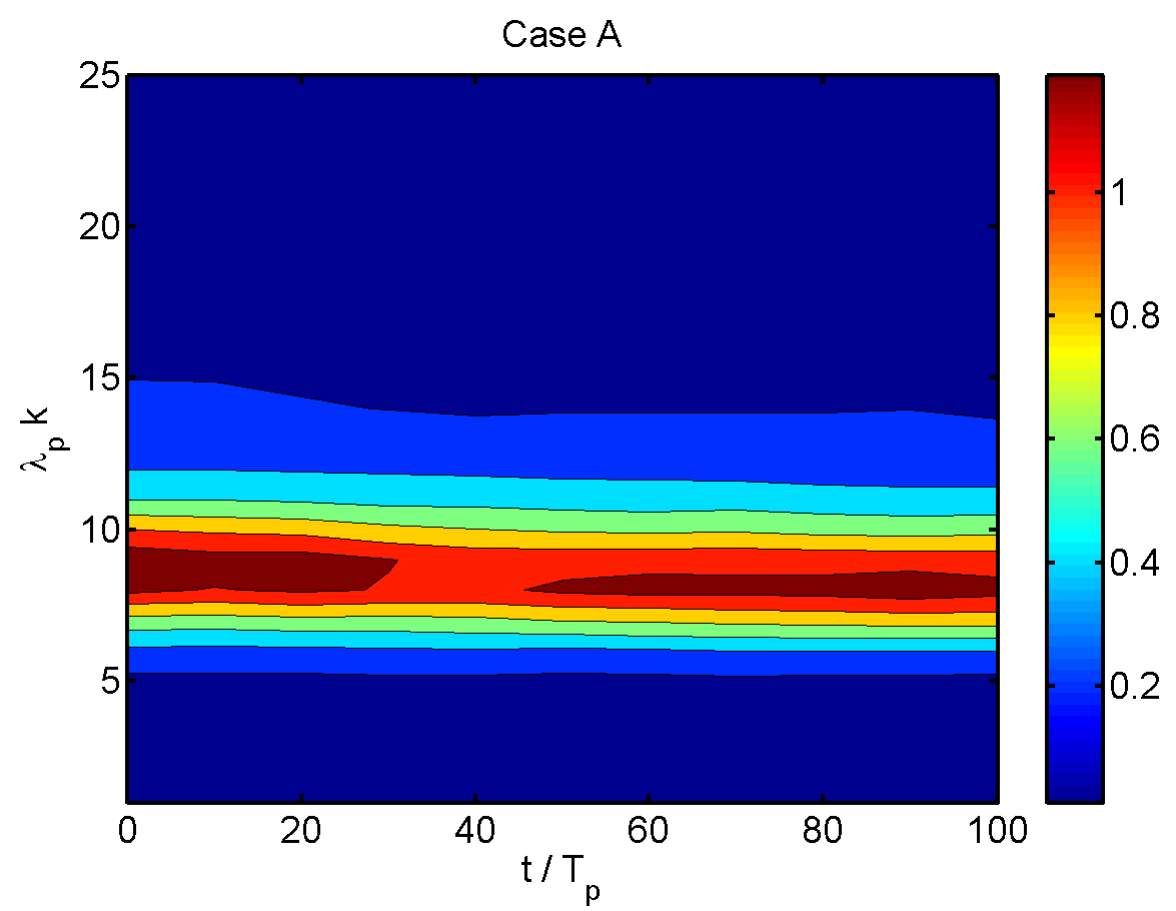

(b) Time evolution of surface elevation wavenumber spectrum for $\theta=80^{\circ}$.

Figure 3-57: (In Color) Wavenumber spectrum time evolution in nonlinear wavefield simulation Cases $\mathbf{J}$ where $\theta=180^{\circ}$ and Case $\mathbf{A}$ where $\theta=80^{\circ}$. Time $\left(t / T_{p}\right)$ is on the horizontal axis, wavenumber $\lambda_{p} k$ on the vertical axis and spectral energy level is represented in the color bar. 


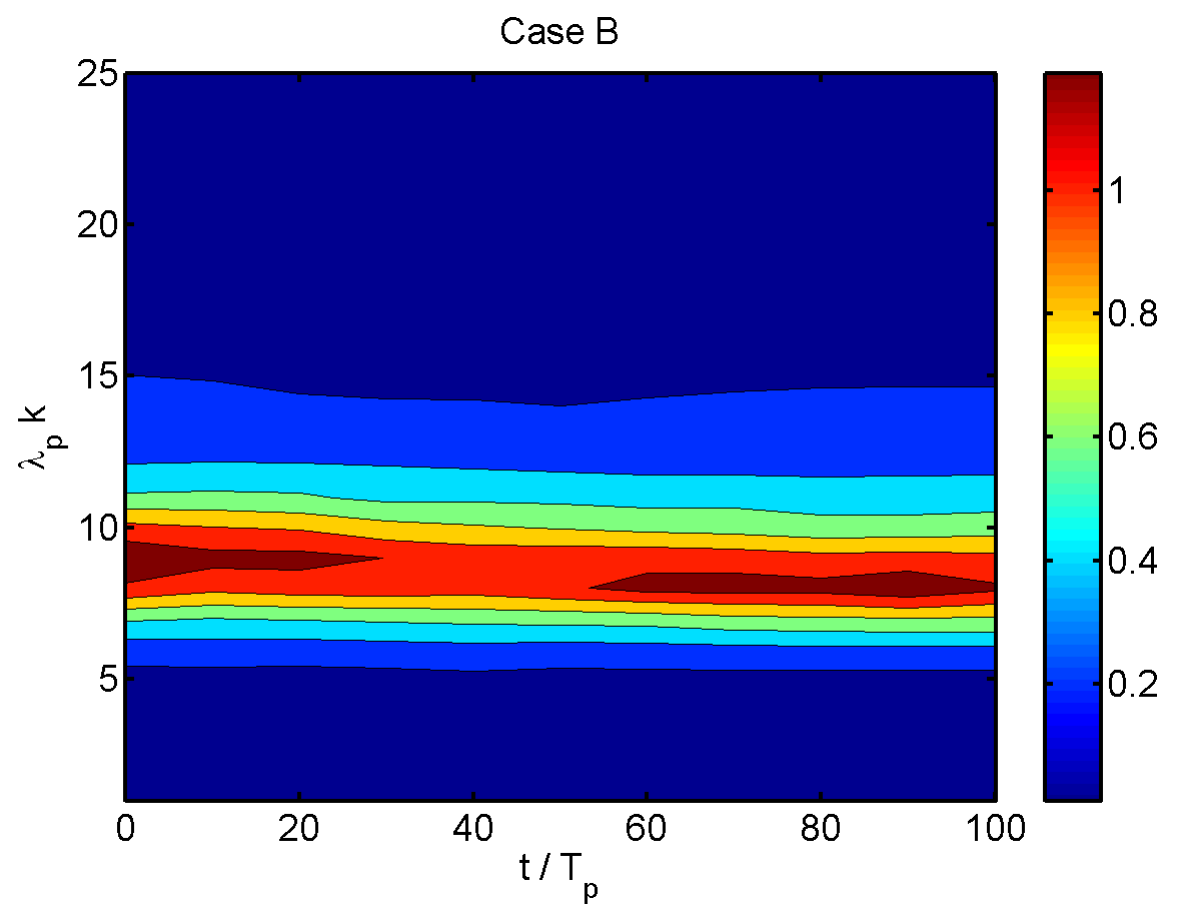

(a) Time evolution of surface elevation wavenumber spectrum for $\theta=40^{\circ}$.

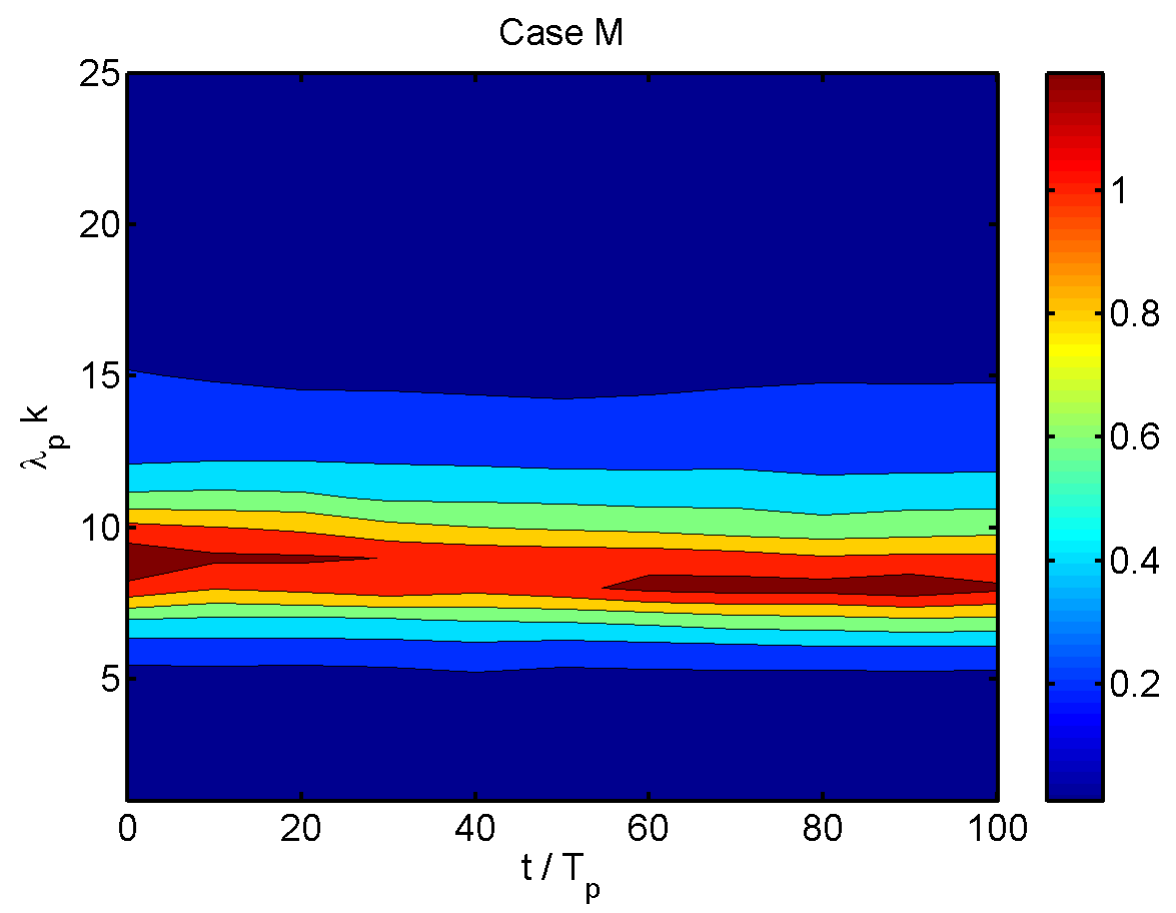

(b) Time evolution of surface elevation wavenumber spectrum for $\theta=30^{\circ}$.

Figure 3-58: (In Color) Wavenumber spectrum time evolution in nonlinear wavefield simulation Cases $\mathbf{B}$ where $\theta=40^{\circ}$, and $\mathbf{M}$ where $\theta=30^{\circ}$. Time $\left(t / T_{p}\right)$ is on the horizontal axis, wavenumber $\lambda_{p} k$ on the vertical axis and spectral energy level is represented in the color bar. 


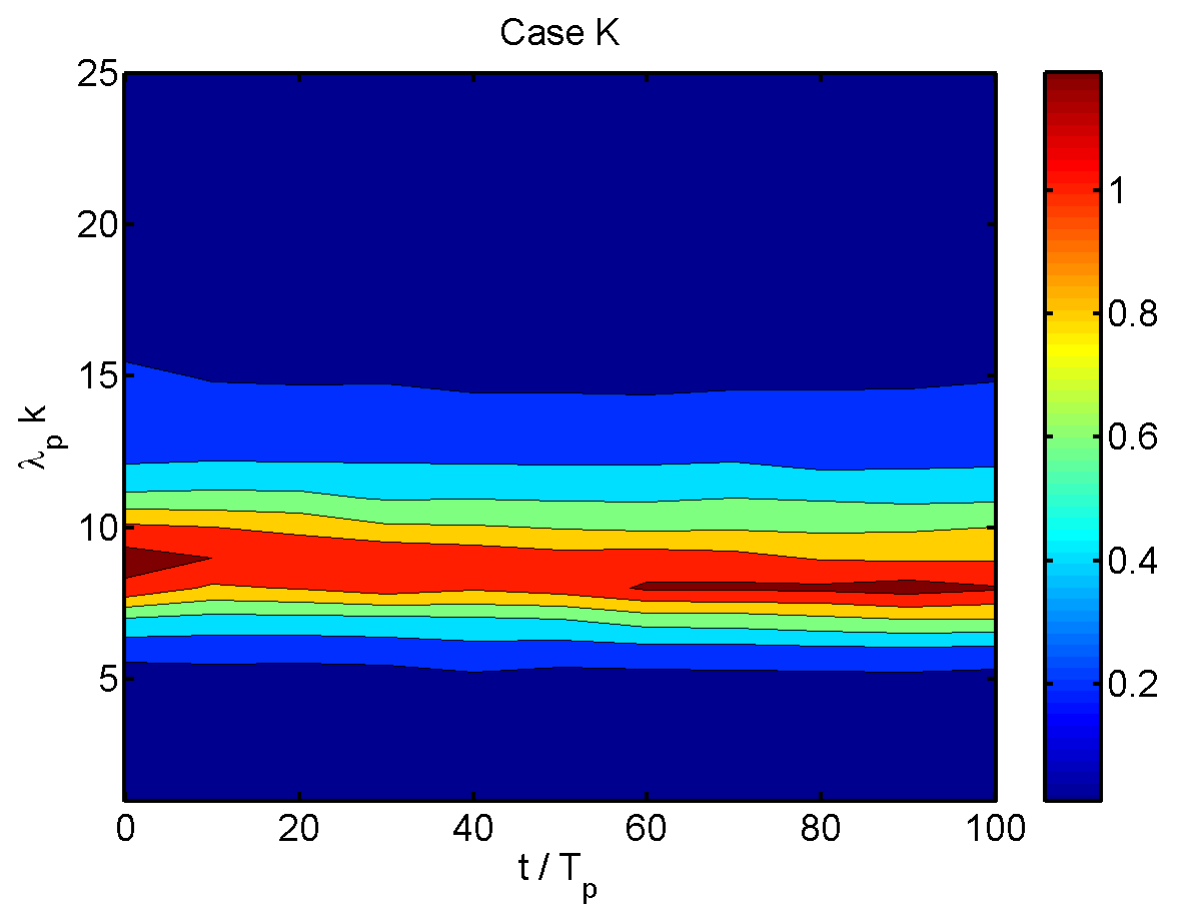

(a) Time evolution of surface elevation wavenumber spectrum for $\theta=20^{\circ}$.

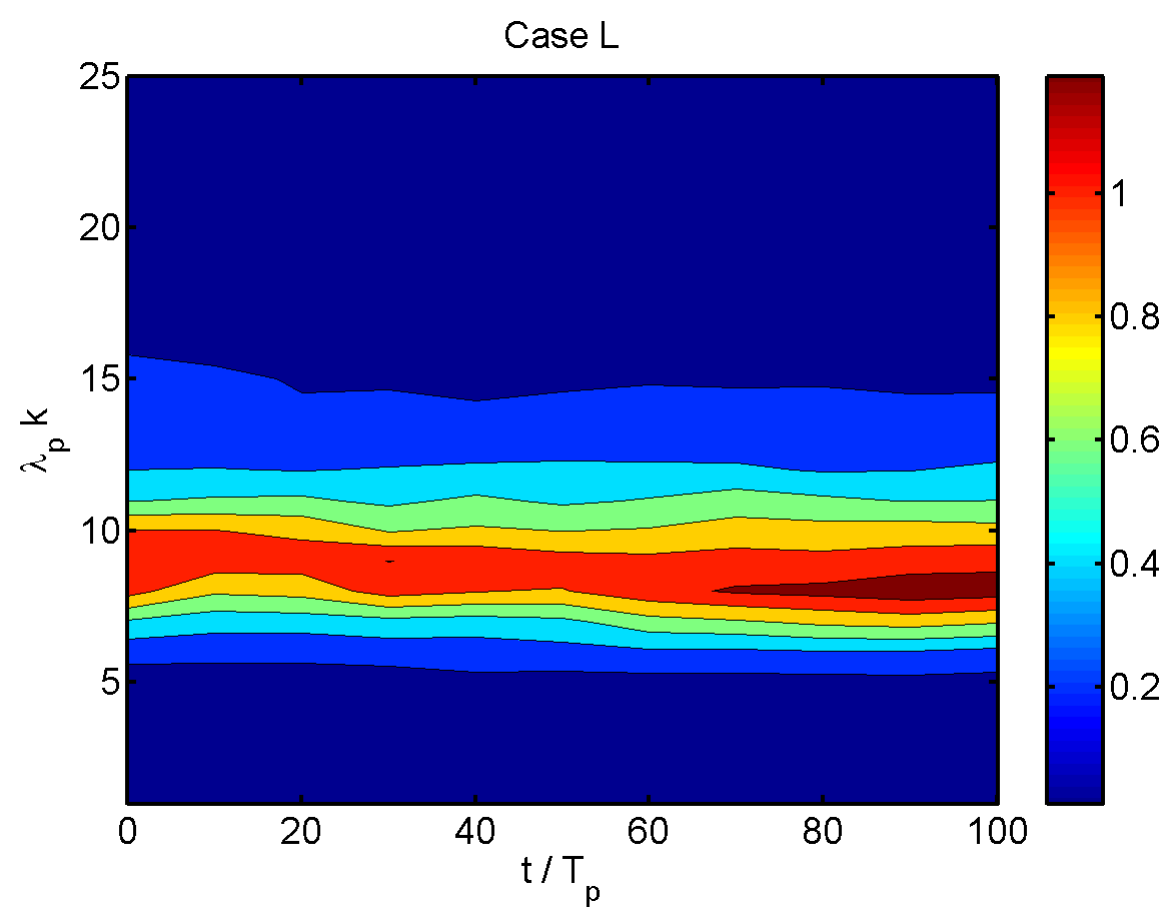

(b) Time evolution of surface elevation wavenumber spectrum for $\theta=10^{\circ}$.

Figure 3-59: (In Color) Wavenumber spectrum time evolution in nonlinear wavefield simulation in Case $\mathbf{K}$ where $\theta=20^{\circ}$, and Case $\mathbf{L}$ where $\theta=10^{\circ}$. Time $\left(t / T_{p}\right)$ is on the horizontal axis, wavenumber $\lambda_{p} k$ on the vertical axis and spectral energy level is represented in the color bar. 


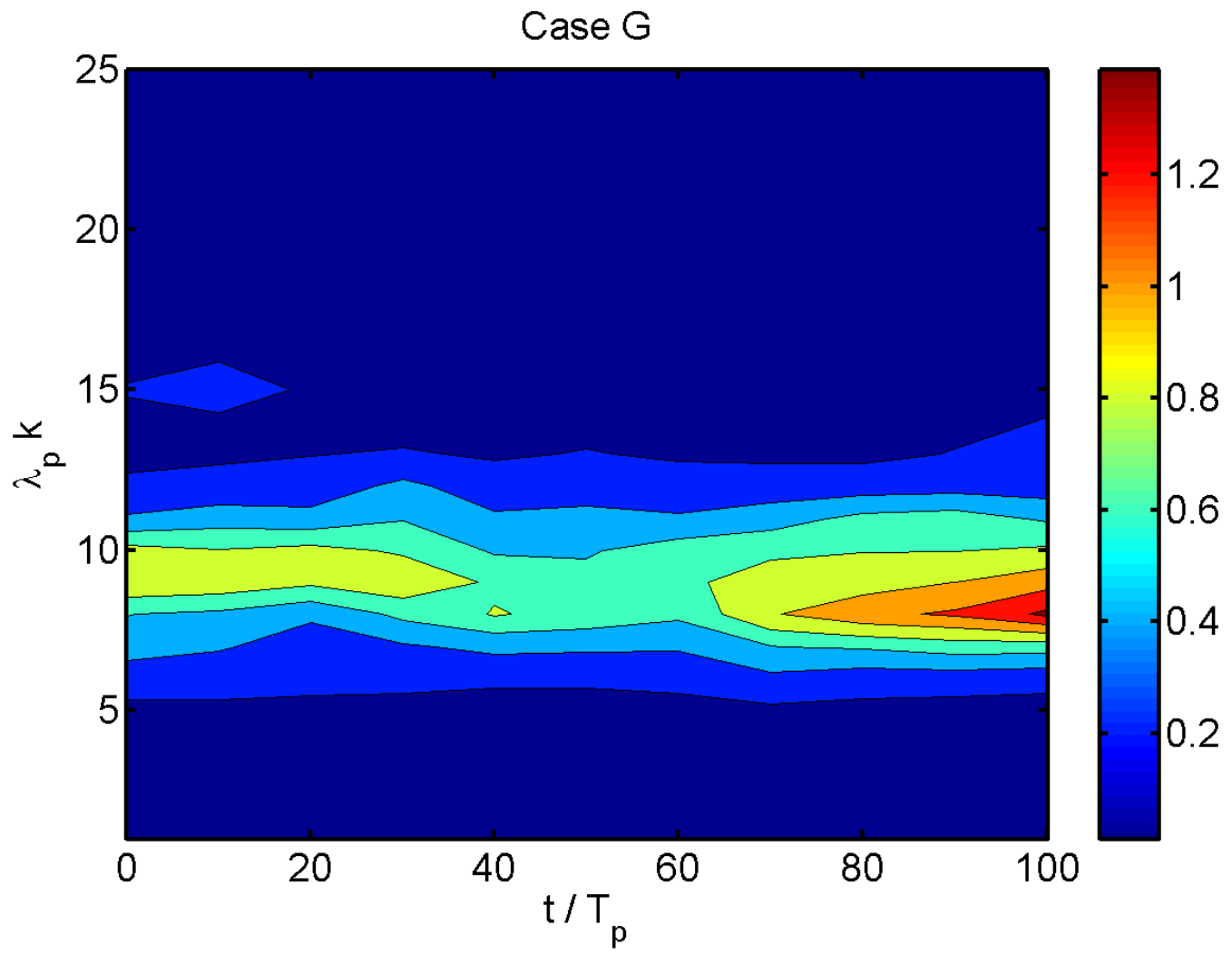

Figure 3-60: (In Color) Wavenumber spectrum time evolution in nonlinear wavefield simulation in Case $\mathbf{G}$ where $\theta=1^{o}$. Time $\left(t / T_{p}\right)$ is on the horizontal axis, wavenumber $\lambda_{p} k$ on the vertical axis and spectral energy level is represented in the color bar. 
the time-evolution of the BFI of cases in Table 3.3.

When we observe all directional spreading angles, i.e. in Cases $\mathbf{J}$ where $\theta=180^{\circ}$, A where $\theta=80^{\circ}, \mathbf{B}$ where $\theta=40^{\circ}, \mathbf{M}$ where $\theta=30^{\circ}, \mathbf{K}$ where $\theta=20^{\circ}, \mathbf{L}$ where $\theta=10^{\circ}$ and $\mathbf{G}$ where $\theta=1^{\circ}$ in Figure 3-61, we find that in general, as $\theta$ increases, the first three spectral moments, $m_{0}, m_{1}$ and $m_{2}$ all increase.

When we observe Cases $\mathbf{J}$ where $\theta=180^{\circ}, \mathbf{A}$ where $\theta=80^{\circ}, \mathbf{B}$ where $\theta=40^{\circ}, \mathbf{M}$ where $\theta=30^{\circ}$, (see Figure 3-62) we find that higher $\theta$-values produce higher $m_{0}$ and $m_{1}$

We find this holds for $m_{2}$, except for the case where $\theta=180^{\circ}$ which seems to be a trend outlier, due to its extreme directional spreading. For BFI values, $\theta=180^{\circ}$ again appears to stay in its own very stable regime, while for the other three $\theta$-values, $30^{\circ} 40^{\circ}$ and $80^{\circ}$, higher $\theta$ is associated with higher BFI, and therefore less stability.

When we observe Cases $\mathbf{K}$ where $\theta=20^{\circ}$, $\mathbf{L}$ where $\theta=10^{\circ}$ and $\mathbf{G}$ where $\theta=1^{\circ}$, we see two major trends. As time proceeds, spectral moments, $m_{0}, m_{1}$ and $m_{2}$ increase in value. As time proceeds, the stability of the spectra stays intact, and the value is conserved, until $t=80 T_{p}$ when we see a sudden spike in all three BFI's, separating according to a trend that associates higher $\theta$ with lower BFI, and therefore greater stability.

We observe the effect of directional spreading angle on spectral moments in this section. In the nest section, we observe the impact of directional spreading angle on surface slope kurtosis.

\subsubsection{Input spectral directional spreading function and sur- face slope kurtosis}

In this section we observe the kurtosis of surface elevation slope distribution compared to the kurtosis of surface elevation distribution. Section 3.1.5 states that a good indication of nonlinearity in a wavefield is if the wavefield has surface elevation slope distribution kurtosis values that are greater than the kurtosis values of surface elevation distribution in the same wavefield. The magnitude of this disparity between 


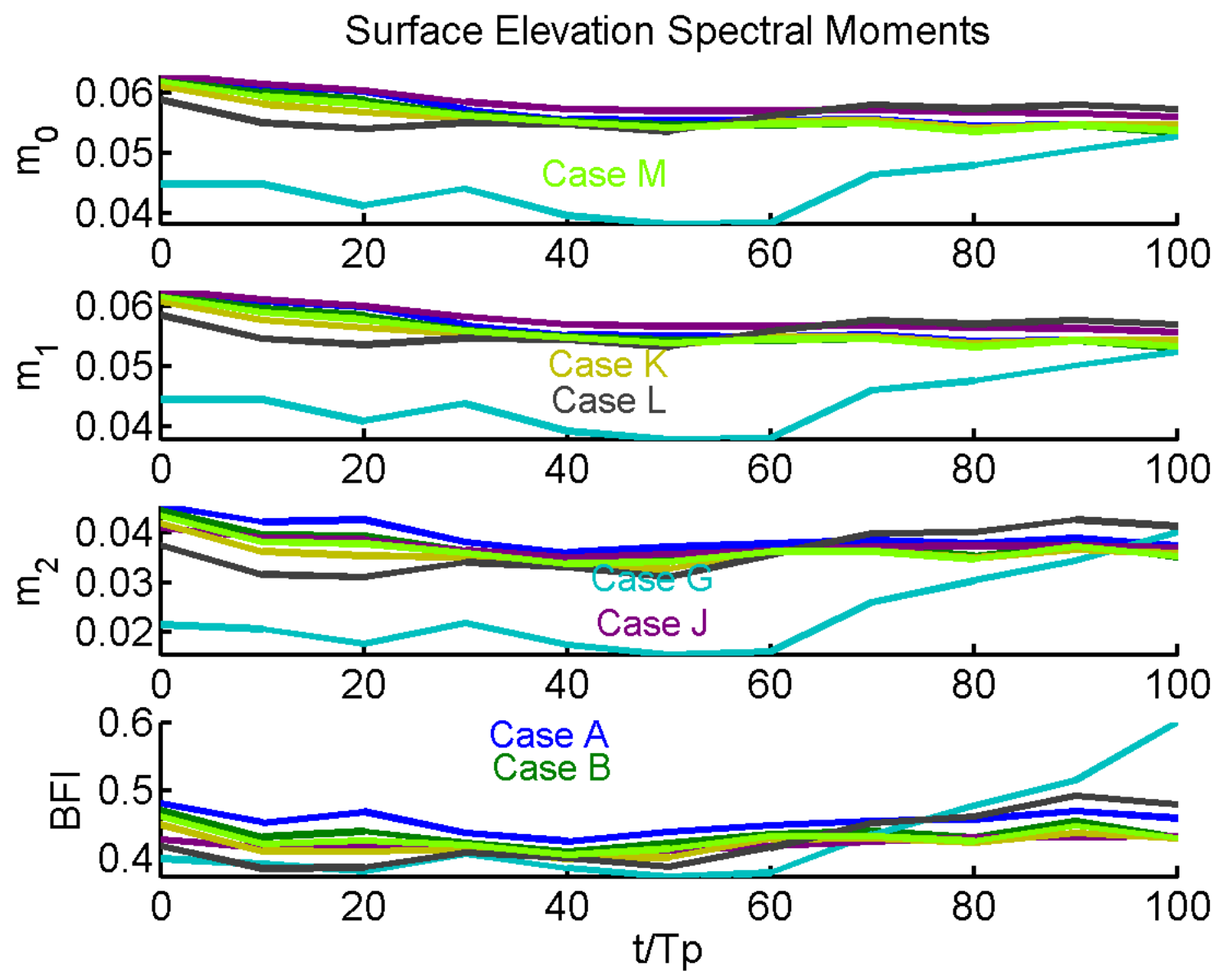

Figure 3-61: (In Color) Plot of surface elevation spectral moments against time $\left(t / T_{p}\right)$ where $m_{0}$ is the first spectral moment, $m_{1}$ is the second spectral moment, $m_{2}$ is the third spectral moment, and BFI is a scaled version of the BFI defined in Section 3.1.4. We use nonlinear simulations of Cases $\mathbf{J}$ where $\theta=180^{\circ}$, A where $\theta=80^{\circ}, \mathbf{B}$ where $\theta=40^{\circ}, \mathbf{M}$ where $\theta=30^{\circ}, \mathbf{K}$ where $\theta=20^{\circ}, \mathbf{L}$ where $\theta=10^{\circ}$ and $\mathbf{G}$ where $\theta=1^{\circ}$. 


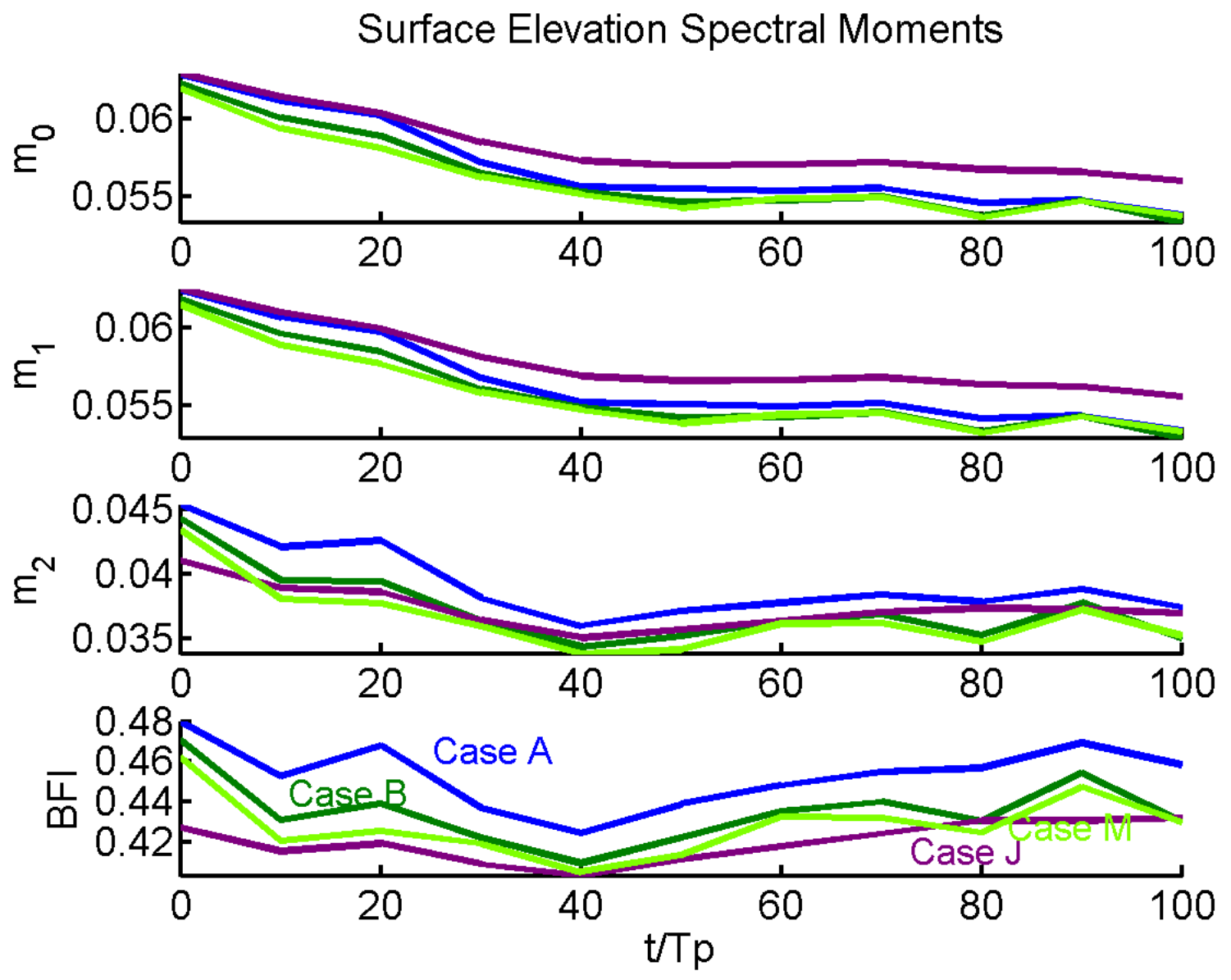

Figure 3-62: (In Color) Plot of surface elevation spectral moments against time $\left(t / T_{p}\right)$ where $m_{0}$ is the first spectral moment, $m_{1}$ is the second spectral moment, $m_{2}$ is the third spectral moment, and BFI is a scaled version of the BFI defined in Section 3.1.4. We use nonlinear simulations of Cases $\mathbf{J}$ where $\theta=180^{\circ}$, A where $\theta=80^{\circ}$, $\mathbf{B}$ where $\theta=40^{\circ}$ and $\mathbf{M}$ where $\theta=30^{\circ}$. 


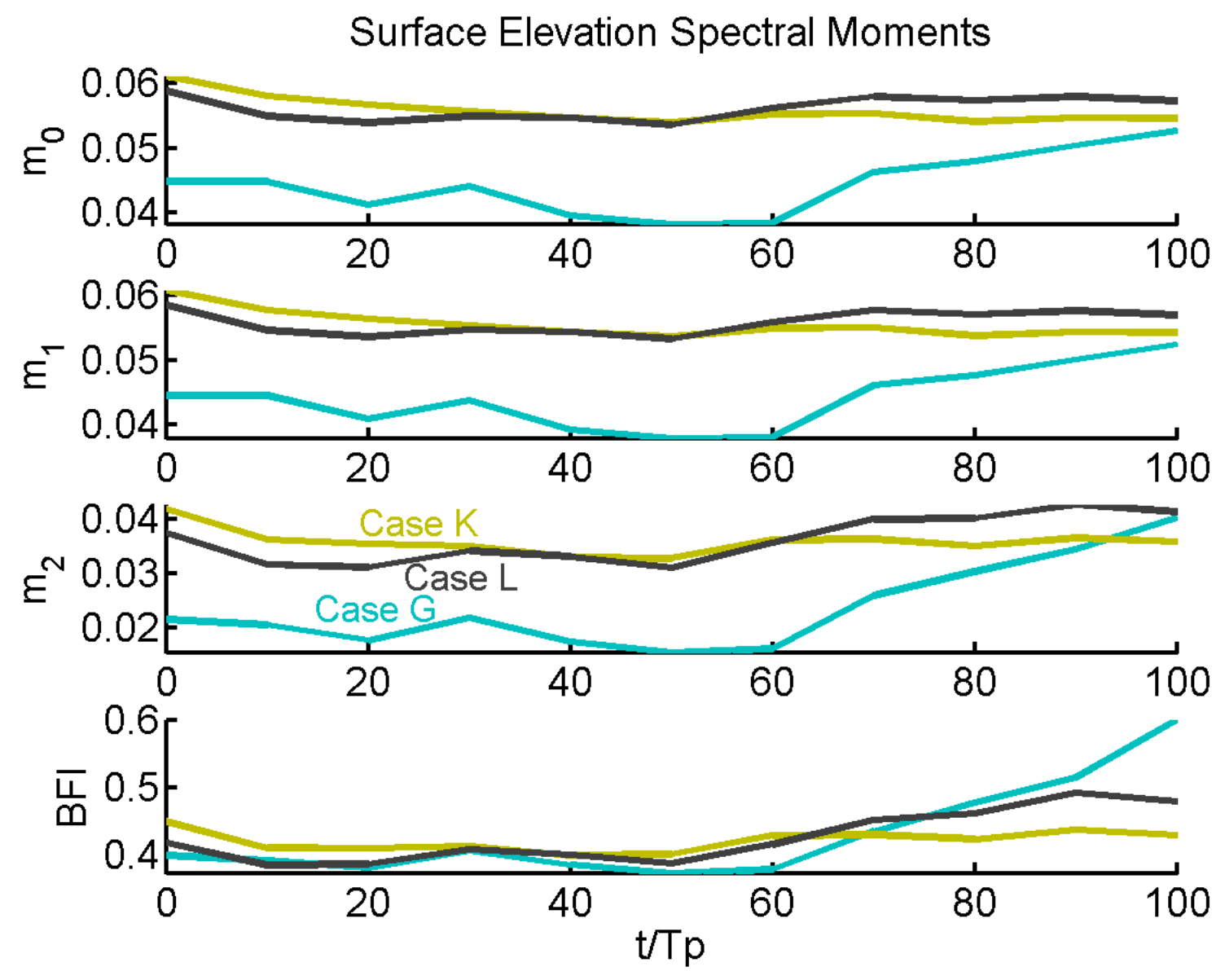

Figure 3-63: (In Color) Plot of surface elevation spectral moments against time $\left(t / T_{p}\right)$ where $m_{0}$ is the first spectral moment, $m_{1}$ is the second spectral moment, $m_{2}$ is the third spectral moment, and BFI is a scaled version of the BFI defined in Section 3.1.4. We use nonlinear simulations of Cases $\mathbf{K}$ where $\theta=20^{\circ}$, $\mathbf{L}$ where $\theta=10^{\circ}$, and $\mathbf{G}$ where $\theta=1^{\circ}$. 
kurtosis values is an indication of the extent of nonlinearity [36].

In Figures 3-64 and 3-65 we see that the unidirectional wavefield, Case $\mathbf{G}$ where $\theta=1^{o}$ shows the strongest disparity between the three time evolutions of kurtosis (surface elevation, surface elevation x-slope, and surface elevation y-slope). A large directional spreading angle such as in Case $\mathbf{J}$, where $\theta=180^{\circ}$ produces a smaller separation between surface elevation distribution kurtosis and surface elevation slope distribution kurtosis. Toffoli [65] says that the modulational instability responsible for high kurtosis values can be tampered with under strong directional conditions, as interactions between otherwise unidirectional wave signals lessen and here we confirm this effect and go further to establish regimes of directional spreading to enhance or weaken such an effect.

In Figure 3-64 we see the time evolution of kurtosis of surface elevation and surface elevation $\mathbf{x}$ - and y-slopes in Cases $\mathbf{J}$ where $\theta=180^{\circ}$, A where $\theta=80^{\circ}$, $\mathbf{B}$ where $\theta=40^{\circ}$, and $\mathbf{M}$ where $\theta=30^{\circ}$. In these cases we observe the effect of strong directional spreading in a wavefield. We see kurtosis values ranging from a highly Gaussian value of 2.95 to a highly non-Gaussian value of 4.25, among directional wavefields. We find in general that smaller $\theta$ produces larger surface elevation slope kurtosis. Two regimes are seen among these highly directional wavefields. The first regime is comprised of Cases $\mathbf{J}$ where $\theta=180^{\circ}$ and $\mathbf{A}$ where $\theta=80^{\circ}$ in which surface elevation slope kurtosis in the $\mathrm{x}$-direction appears to be surprisingly less than surface elevation slope kurtosis in the y-direction. The second regime is comprised of Cases B where $\theta=40^{\circ}$ and $\mathbf{M}$ where $\theta=30^{\circ}$. We observe further, that a maximum value emerges in the time evolution of kurtosis, when $\theta=80^{\circ}, \theta=40^{\circ}$ and $\theta=30^{\circ}$ compared to Case $\mathbf{J}$ where $\theta=180^{\circ}$ where there is constant, unchanging surface elevation $\mathrm{x}$-directional-slope kurtosis. The kurtosis of surface elevation y-directionalslope maximizes in all four cases, although it seems to stay within a $3.2 \pm 0.1$ range for the regime were $\theta=80^{\circ}$, and $\theta=180^{\circ}$ but consistently grows in time for the second regime where $\theta=40^{\circ}$ and $\theta=30^{\circ}$. Surface elevation kurtosis is the lowest absolute value of kurtosis among all the kurtosis values in all the wavefield cases. In the case with the smallest directional effects where we expect the most deviation 


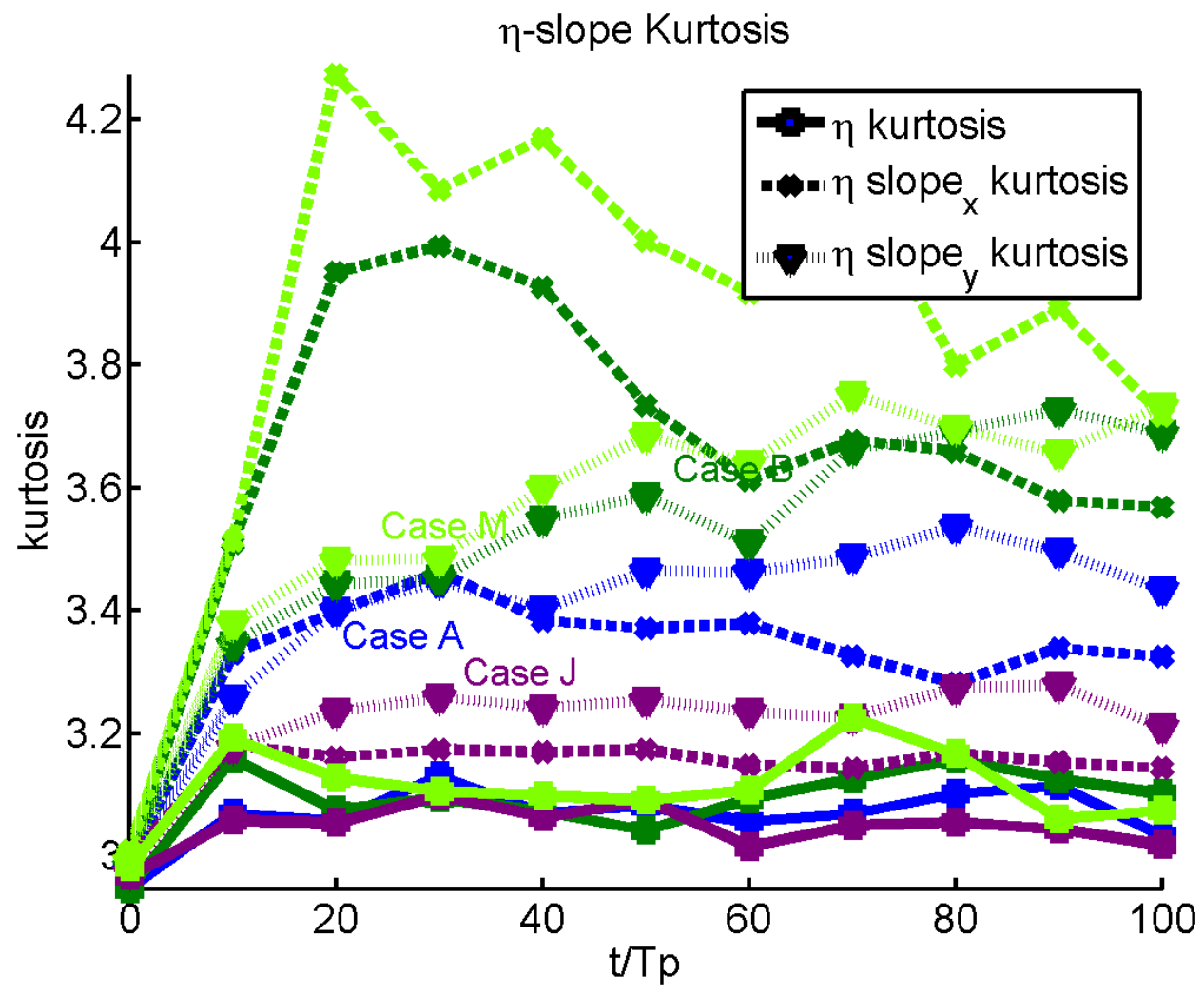

Figure 3-64: (In Color) Plot of surface elevation kurtosis, surface elevation x-slope kurtosis and surface elevation y-slope kurtosis (on vertical axis) against time (on horizontal axis), $\left(t / T_{p}\right)$ in non-linear simulations of Cases $\mathbf{J}$ where $\theta=180^{\circ}, \mathbf{A}$ where $\theta=80^{\circ}, \mathbf{B}$ where $\theta=40^{\circ}$ and $\mathbf{M}$ where $\theta=30^{\circ}$. 
from a kurtosis of 3.0, we find a maximum surface elevation kurtosis of 3.25. After $t=60 T_{p}$ wavefield surface elevation kurtosis aligns itself according to $\theta$-values in all the cases, and this stays true except for Case $\mathbf{M}$, where $\theta=30^{\circ}$ (the least directional case), whose kurtosis falls lower than expected at $t=90 T_{p}$.

In Figure 3-65 we see the time evolution of kurtosis of surface elevation and surface elevation $\mathbf{x}$ - and $\mathbf{y}$-slopes in Cases $\mathbf{K}$ where $\theta=20^{\circ}, \mathbf{L}$ where $\theta=10^{\circ}$ and $\mathbf{G}$ where $\theta=1^{\circ}$. The entire range of kurtosis is from 3 to 15 . The kurtosis of surface elevation $\mathrm{x}$-directional slope in Case $\mathbf{G}$ where $\theta=1^{\circ}$ attains multiple maxima, the first being at $t=70 T_{p}$, and the second being at $t=100 T_{p}$ at its earliest possible value. Comparably, Cases $\mathbf{K}$ where $\theta=20^{\circ}$ and $\mathbf{L}$ where $\theta=10^{\circ}$ attain lower maxima each at an earlier time (Case $\mathbf{K}$ at $t=20 T_{p}$ and Case $\mathbf{L}$ at $t=30 T_{p}$ ) than in Case $\mathbf{G}$. In this group of very weak directionality, larger spreading angles lead to earlier occurrence of maximum kurtosis and subsequent decay in kurtosis.

In comparing Figures 3-65 and 3-64 we see the time evolution of kurtosis of surface elevation and surface elevation x- and y-slopes in Cases $\mathbf{J}$ where $\theta=180^{\circ}, \mathbf{A}$ where $\theta=80^{\circ}, \mathbf{B}$ where $\theta=40^{\circ}, \mathbf{M}$ where $\theta=30^{\circ}, \mathbf{K}$ where $\theta=20^{\circ}, \mathbf{L}$ where $\theta=10^{\circ}$ and $\mathbf{G}$ where $\theta=1^{\circ}$. We see kurtosis values range from the Gaussian value of 3 to an extremely non-Gaussian maximum value, 15 in the case of very low directional spreading. When comparing all the cases, some general trends prevail. The $\mathrm{x}$-directional-slopes (along the mean direction of wave propagation) have the highest set of kurtosis values, and the surface elevation has the lowest set of kurtosis values. Lower directional spreading corresponds to higher kurtosis values for $\mathrm{x}$-directional surface elevation slopes, y-directional surface elevation slopes and surface elevation. In the unidirectional case with $\theta=1^{o}$ the time evolution of kurtosis of the surface elevation $\mathrm{x}$-slope is the highest. In the case where $\theta=10^{\circ}$, the time evolution of surface elevation $\mathrm{x}$-directional-slope kurtosis is also very high, but not as high as when $\theta=1^{\circ}$. In almost unidirectional Case $\mathbf{G}$ where $\theta=1^{\circ}$ there are multiple maxima while in Case $\mathbf{L}$ where $\theta=10^{\circ}$ only one maximum is apparent. The kurtosis of surface elevation $\mathbf{x}$-directional slope in Case $\mathbf{G}$ where $\theta=1^{\circ}$ is the highest and in Case $\mathbf{L}$ where $\theta=10^{\circ}$ is a near second. The kurtosis of surface elevation $\mathrm{x}$-directional slope in Case 


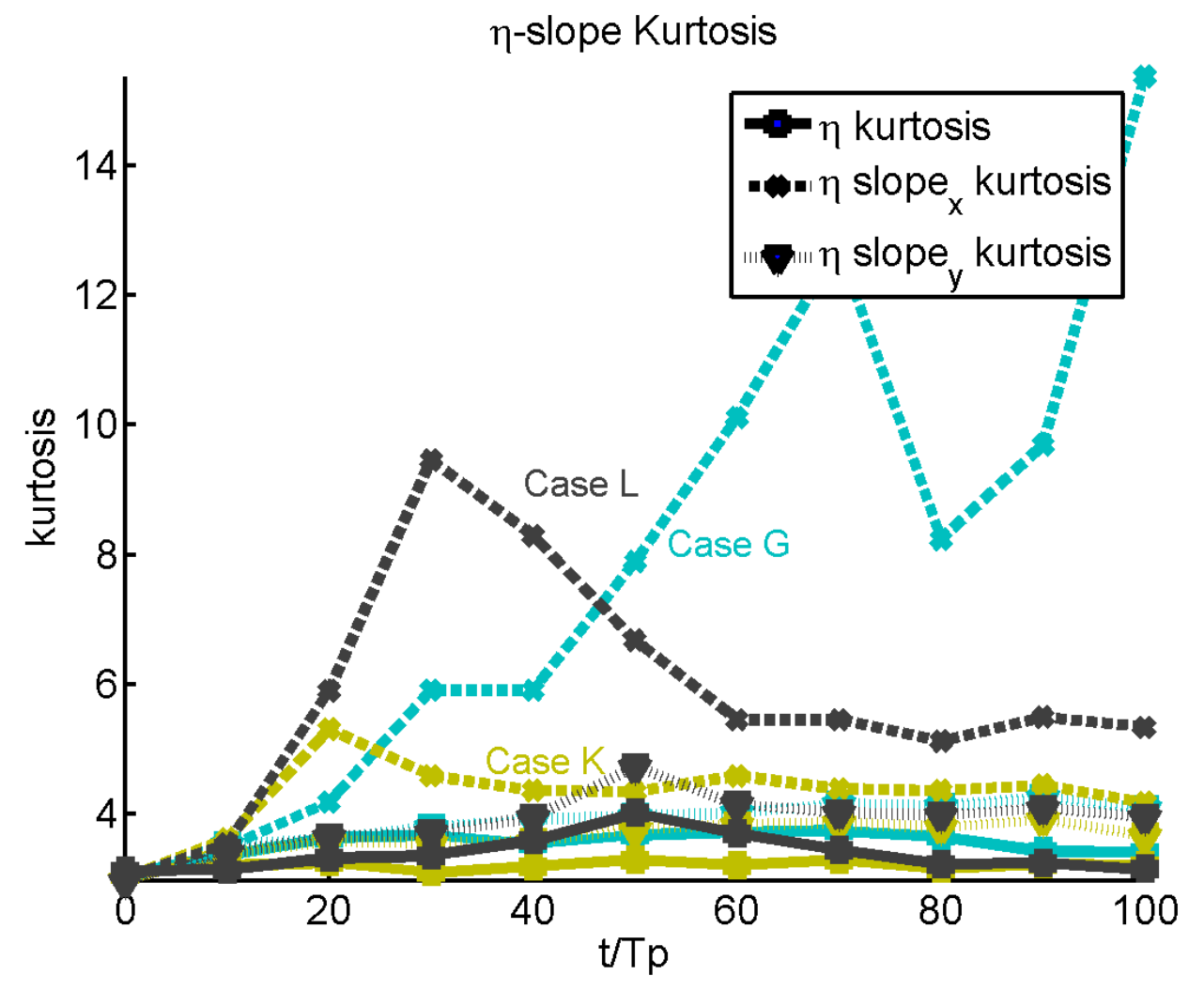

Figure 3-65: (In Color) Plot of surface elevation kurtosis, surface elevation x-slope kurtosis and surface elevation y-slope kurtosis (on vertical axis) against time (on horizontal axis), $\left(t / T_{p}\right)$ in non-linear simulations of Cases $\mathbf{K}$ where $\theta=20^{\circ}, \mathbf{L}$ where $\theta=10^{\circ}$ and $\mathbf{G}$ where $\theta=1^{\circ}$. 
$\mathrm{G}$ has multiple maxima while the kurtosis of surface elevation x-directional slope in Case $\mathbf{L}$ only has one apparent maximum. Recall that kurtosis of surface elevation has the lowest kurtosis trajectory among the three kurtosis trajectories (x-directional surface elevation slopes, y-directional surface elevation slopes and surface elevation) for any case. The kurtosis of surface elevation in Case $\mathbf{L}$ is higher than all the 3 kurtosis values in cases $\mathrm{J}, \mathrm{A}$, and $\mathrm{B}$ at time $t=50 T_{p}$. Different kurtosis values in the same wavefield peak at different times, so for example, in Case L, surface elevation $\mathrm{x}$-directional slope kurtosis peaks at $t=30 T_{p}$, while surface elevation kurtosis and surface elevation y-directional surface elevation slope kurtosis peak at $t=50 T_{p}$ while the surface elevation slope kurtosis in the $\mathrm{x}$-direction is falling.

Conclusively we say that a strong relationship exists between the wavefield's input $\theta$-value and its kurtosis values. This in turn feeds into the large wave formation, as high kurtosis is a reliable indicator of the presence of large waves.

\subsubsection{Conclusions: Directional spreading function and sur- face elevation characteristics}

In this chapter we look at the effect of the directional spreading function on surface elevation characteristics during the first $100 T_{p}$ in surface evolution, finding that:

- Regimes are seen to form as a unidirectional wavefield has kurtosis, skewness and variance which compare unpredictably to those in a directional nonlinear wavefield. We see these regimes when we compare the order of magnitude of kurtosis and skewness and find their order of magnitude is much greater in a unidirectional wavefield than that of kurtosis and skewness in a directional wavefield.

- We see regime-type dependence of spectral stability on the values of directional spreading in wavefields. Unidirectional wavefields depict much lower stability for their highest spectral energy, compared to equivalent directional cases. 
Larger directional spreading is associated with more stable but slightly smaller maximum spectral energy.

- Directional spreading angle has regime-type effects on wavefield stability seen in the BFI. Uni-directional and near uni-directional cases experience a sudden spike in BFI value, and therefore a sudden decrease in wavefield stability after $t=50 T_{p}-60 T_{p}$. The directional cases huddle close in the first $t=100 T_{p}$ of time, showing very little difference or growth in their BFI values.

- Directional spreading angle has a regime-type impact on surface slope kurtosis. Smaller directional spreading, is associated with larger difference between the kurtosis of the surface elevation and the kurtosis of the surface elevation slopes in two perpendicular directions. Directional spreading angle has regime-type effects on the kurtosis of surface elevation x-directional slope, surface elevation y-directional slope and surface elevation. For example, cases with greater directional effects show surface elevation $\mathrm{x}$-directional slope kurtosis less than surface elevation y-directional slope kurtosis.

- Directional spreading angle has regime-type effects on the spectral moments of surface elevation since we see one regime (cases with less directional effects) having growing spectral moments and the other regime (cases with strong directional effects) having falling spectral moments. 


\section{Chapter 4}

\section{Definition of wave height and wavelength}

What is a wave? The answer depends on who is asking. For an oceanographer interested in short waves of wavelength $\lambda$ having a broad range of wave heights and interacting with a floating buoy, of dimension, $L$ where $L / \lambda \approx 1$ the answer is a non-narrow-band, nonlinear one. For a large oil tanker the right answer ignores small ripples and considers only the main, energy-carrying larger waves in the wavefield. We present three solutions to the problem of defining waves on a non-linear, evolving free surface with a $2 \mathrm{D}$ wavenumber ${ }^{2}, k=\left(k_{x}, k_{y}\right)$. Our study of wave height definition methods in a $3 \mathrm{D}$ wavefield with a $2 D$ wavenumber adds useful insight to common field practices. We study the uni-directional, narrow-band zero-crossing method of wave-height definition alongside a $3 D$ directional narrow-band approach to finding the distribution of the largest waves in the wavefield and also a $3 D$ analogue of the $2 D$ half-cycle excursion method. In so doing, we circumvent the bias of non-directional approaches to wave height values in directional wavefields, and the bias of narrowband approaches to wave heights in non-narrow-band wavefields.

\footnotetext{
${ }^{2}$ Wavenumber, $\mathrm{k}=2 \pi / \lambda$, where $\lambda$ is wavelength.
} 


\subsection{Introduction}

It is difficult to define a wave without being vague and inexact [58]. It is even more difficult to point out a wave in an ocean wavefield, when nonlinearities and 3D spatial characteristics move ocean wavefields far away from the classical theory of water wave definitions ([30] pp. $27-28)$. In the following three sections, we define three solutions to the problem of defining waves on a non-linear, evolving, free surface with a $2 \mathrm{D}$ wavenumber, $k=\left(k_{x}, k_{y}\right)$, finding different kinds of insight about our wavefields via the different wave-height or wavelength definition methods. We compare the defined heights' distributions to the theoretical linear narrow band theory [33].

Crests, troughs and individual waves can easily be identified in a narrow-band or non-narrow-band point-record of ocean surface elevation (see Figure 4-1) using several methods. A zero-crossing method, totally accurate in the case of a narrowband wave record, identifies a crest as a maximum point above a defined mean level and a trough as a minimum point below a defined mean level. In this case wave height would be related to the sum of crest height and trough depth defined from the mean level. A half-cycle excursion method (see Figure 4-1(b)), more suitable for non-narrow-band wave records, defines wave height as the vertical distance between a consecutive crest and trough irrespective of the location of a mean height in the record. The answer to the question of wave height definitions in a wave field with two-dimensional wave numbers is left completely open-ended in the modern study of ocean waves [58], [41],[30]. We look at this question carefully in this chapter.

\subsection{Zero-crossing method}

In performing our zero-crossing analysis, we adjust a method used by Ducrozet [12] to define wave heights in simulated wavefields. This method is the most commonly used method to define wave heights from field data and in computational work [12] [59]. Waves are counted by first defining a zero-level within a point-measurement record of surface elevation at a fixed location, or at a computationally derived fixed time in 


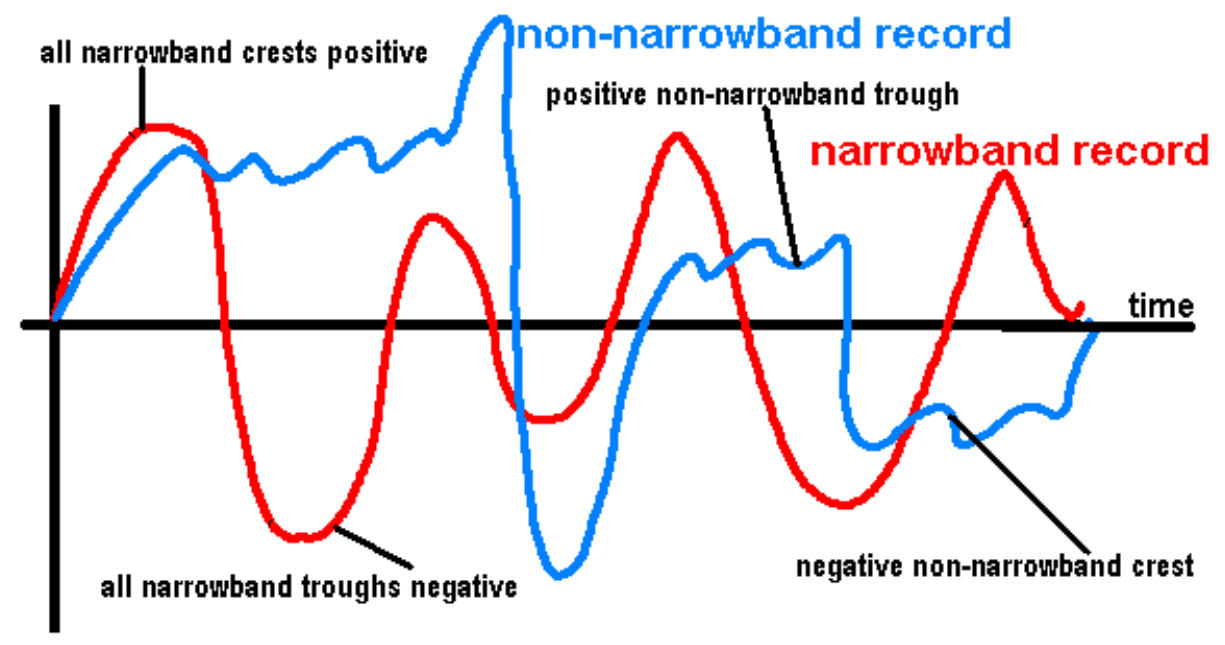

(a) (In color) Narrowband (red) ocean surface point-record in time shows only positive crests and only negative troughs. Non-Narrowband (blue) ocean surface point-record shows positive troughs and negative crests.

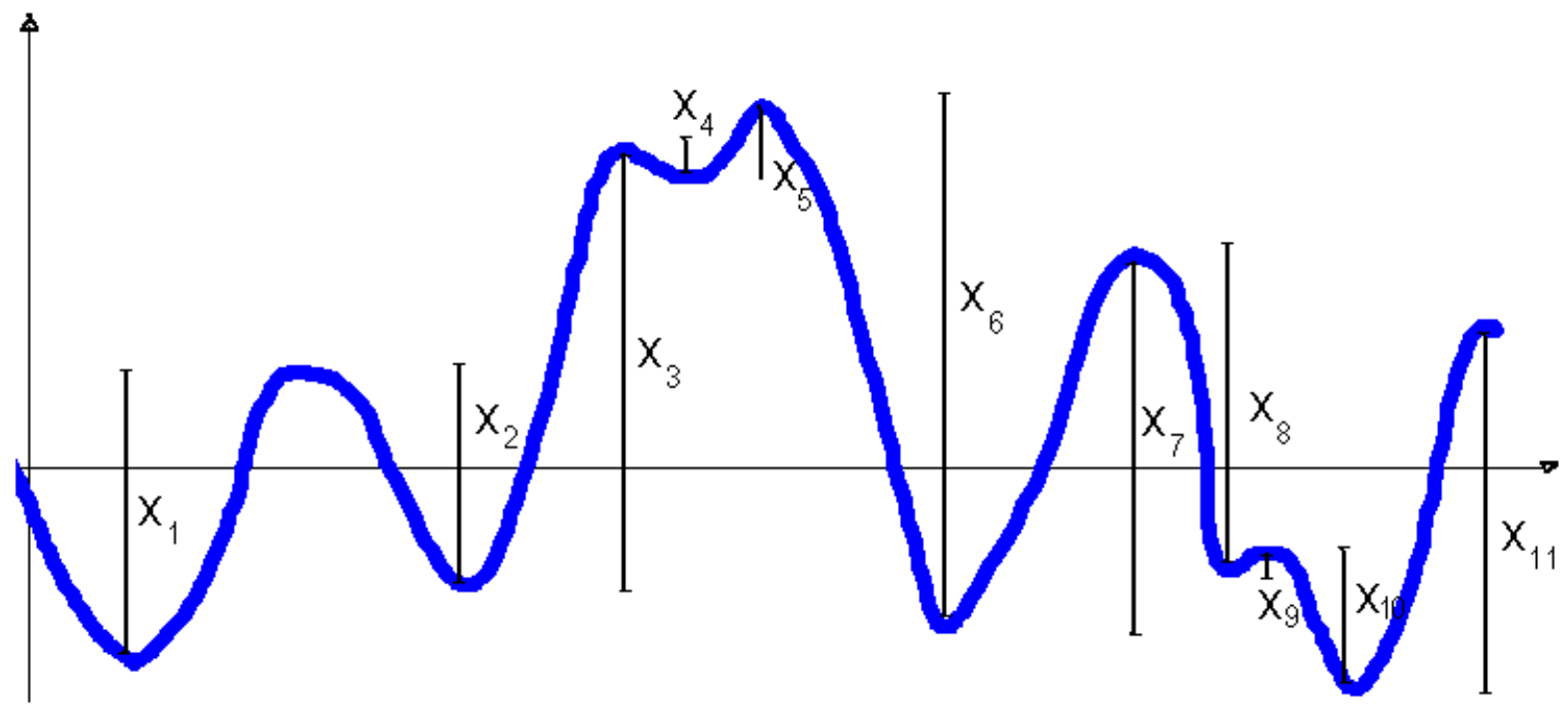

(b) Half cycle excursion definition of wave heights for non-narrow-band records [41]. Each excursion $x_{1}, x_{2}, \ldots, x_{11}$, the vertical distance between every crest and the adjacent trough, represents a wave height.

Figure 4-1: Wave definitions are simple with point records. 
a 2D space. At many times, this is a narrow-band approach to a near-narrow-band wavefield, and a non-directional approach to a highly directional wavefield, so the information it provides is only partial. This approach filters the wavefield as though it is a carrier-frequency signal that is centered at the zero-level and disregards other frequencies that are higher or lower ([41] pp. $60-63)$. Using this method of wave height definition therefore produces narrow-band results.

To define wave heights, we first divide the wavefields into $\lambda_{p}$-wide strips ${ }^{3}$ (see Figure 4-2(a)). Wave heights are then generated simply by counting zero up-crossings along the cross section (see Figure 4-2(a)) at each straight-line cut parallel to $x$, the mean wavefield propagation direction, by counting one wave as the surface elevation between two consecutive up-crossings. Wave height, $H$ is found by computing the difference between the highest and lowest surface elevation value, $H=\eta_{\max }-\eta_{\min }$ between the two consecutive up-crossings. The corresponding wave length, $\lambda$, is found by computing the $\mathrm{x}$-directional distance between the two consecutive up-crossings in Figure 4-2(b).

\subsection{Lowest local minimum method}

We know that the zero-crossing method filters out the full expanse of heights in the wavefield, focusing on carrier wave dynamics, but we expect that an observation of the wavefield with a more 3D approach, without the constriction of defined mean propagation direction or zero-level, will help us to gain more realistic insight of the wavefield's main, energy-carrying wave heights. We therefore introduce the present method of wave height and wave length definitions, in which an individual wave is defined as a connection between the local maximum in surface elevation and its lowest connected minimum. This approach, ignoring the smaller waves and ripples in the wavefield, is narrow-banded, and, as predicted [30] [41] [33], it proves that the narrow band of largest, energy-carrying waves in a wavefield strongly adhere to linear theory.

\footnotetext{
${ }^{3}$ The wavelength associated with the greatest spectral energy in a wavefield's wavenumber spectrum is the peak wavelength, $\lambda_{p}$
} 
Cross section of wave field, parallel to flow direction -

i.e. mean direction of wave

travel

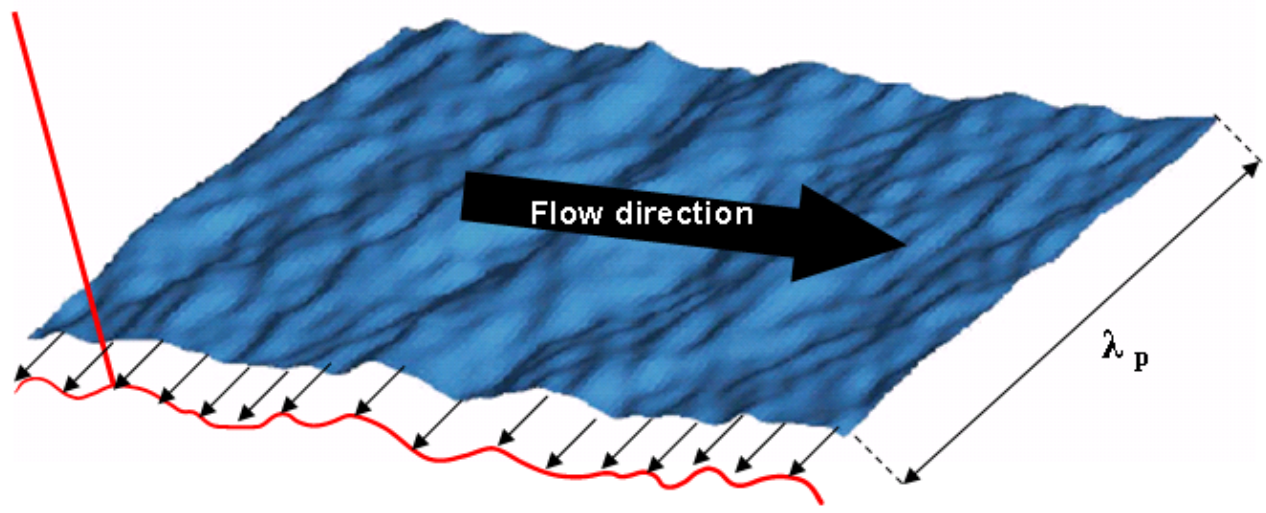

(a) Cross section of wavefield, parallel to mean direction of wave travel for zero-crossing method of wave height, wavelength definition.

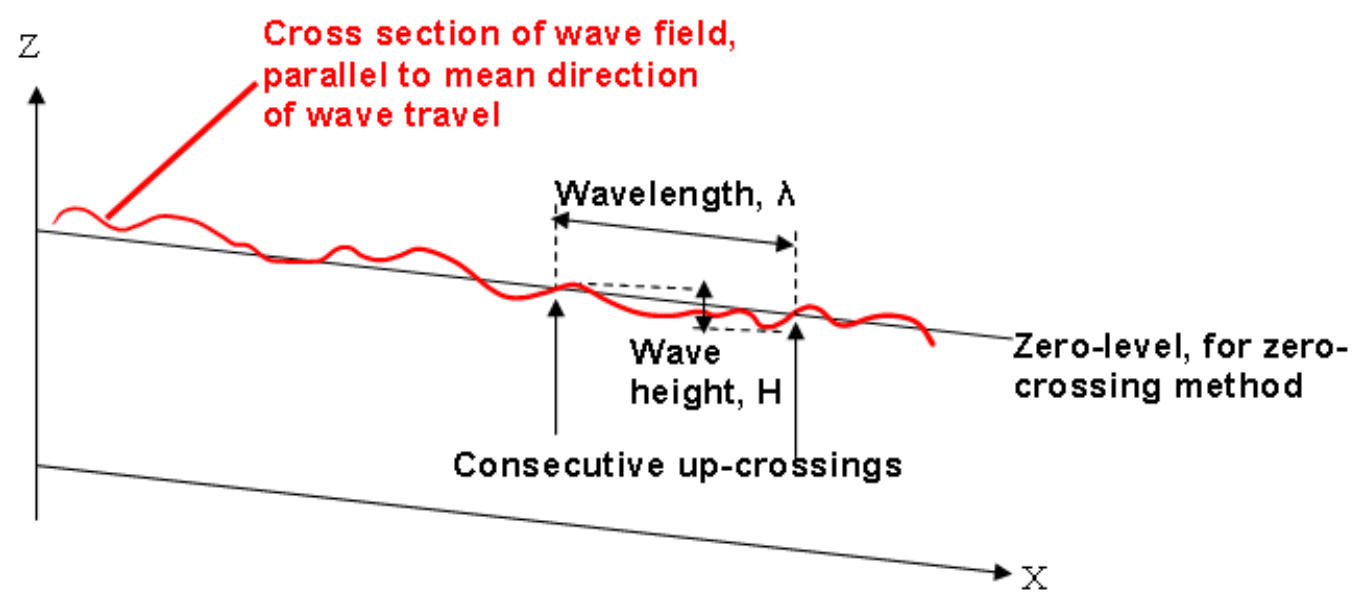

(b) Using the cross section of the wavefield to define finite waves.

Figure 4-2: (In Color) Zero crossing method of wave height definition and wave length definition involves taking the wave field cross-sections in the mean direction of propagation spread one peak wavelength apart from each other, and finding zerocrossing minima and maxima along that cross section. [12] [4]. 
In order to filter out the distribution of the main, we first identify all the wavefield's local maxima where $\frac{\partial \eta}{\partial x}=0, \frac{\partial \eta}{\partial y}=0, \frac{\partial^{2} \eta}{\partial x^{2}}<0$ and $\frac{\partial^{2} \eta}{\partial y^{2}}<0$. We then identify all the wavefield's local minima where $\frac{\partial \eta}{\partial x}=0, \frac{\partial \eta}{\partial y}=0, \frac{\partial^{2} \eta}{\partial x^{2}}>0$ and $\frac{\partial^{2} \eta}{\partial y^{2}}>0$. The next step is finding the lowest local minimum linked to each local maximum. Figure 4-3 shows a local maximum and identifies its lowest linked local minimum. We define a minimum as "linked" to a maximum if at least one continuous, upward-sloping line leads from the minimum to the maximum. The maximum-minimum pair found is then use to define $\eta_{1}$ as surface elevation at the maximum point and $\eta_{3}$ as surface elevation at the lowest linked minimum point, and ultimately, define wave height as $H=\eta_{1}-\eta_{3}$ and wavelength is defined as twice the distance between the $(x, y)$ coordinates of $\eta_{1}$ and $\eta_{3}$.

We find that the waves defined as the main, energy-carrying waves in our simulations of deep ocean wavefields, adhere to linear theory. The largest wave heights in these distributions are even less probable than linear, narrow-band theory predicts. This corresponds to the expectation that deep water waves adhere to linear theory, and also our expectation that narrowing down the wavefield to only consider the largest waves will give us a narrow-band perspective of the non-narrow-band wavefield. However, rogue waves are more frequently sighted than linear theory predicts in the ocean. What accounts for such a phenomenon, if we see less-than-Gaussian probabilities for the occurrence of the largest waves among the large, energy-carrying waves? In the following section, we will observe a half-cycle excursion analysis of our simulated wavefield and find the strongest non-linear effects there.

\subsection{Nearest local minimum method}

A non-narrow-band approach to finding wave height distribution in a non-narrowband wavefield is the half-cycle excursion method [41]. Half-cycle excursion analysis is suitable in wave records as seen in Figure 4-4(a), where positive minima and negative maxima are found with respect to a defined zero-mean surface elevation in the wave record. Half-cycle excursion analysis is the statistical analysis of the vertical 


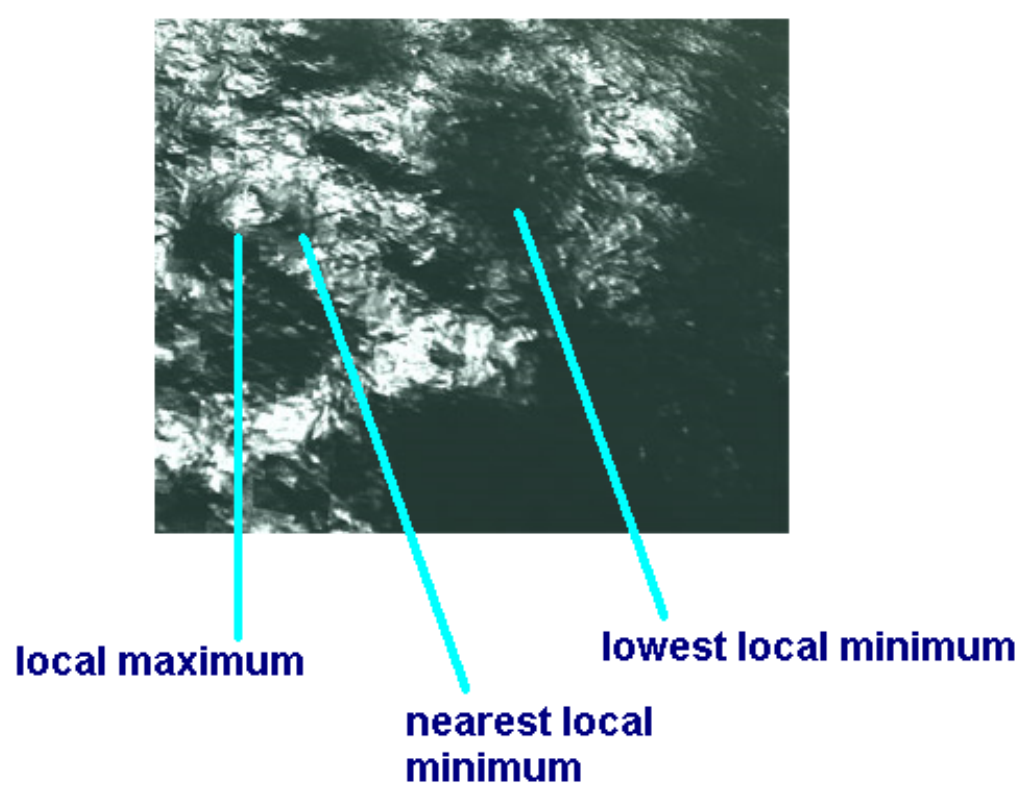

Figure 4-3: All local maxima $\left(\frac{\partial \eta}{\partial x}=0, \frac{\partial \eta}{\partial y}=0, \frac{\partial^{2} \eta}{\partial x^{2}}<0\right.$ and $\left.\frac{\partial^{2} \eta}{\partial y^{2}}<0\right)$ and all the local minima $\left(\frac{\partial \eta}{\partial x}=0, \frac{\partial \eta}{\partial y}=0, \frac{\partial^{2} \eta}{\partial x^{2}}>0\right.$ and $\left.\frac{\partial^{2} \eta}{\partial y^{2}}>0\right)$ are defined in the wavefield. 
distance between adjacent local peak elevation to local trough elevation defined as $x_{1}, x_{2}, \ldots, x_{12}$ in Figure 4-4(b). In this method, we can interpret two types of excursions: Type I, where the crest is above the zero-level and the trough is below the zero level, and Type II, where the excursion between crest and trough does not cross zero-level ([41], pp.84-88). We expect that such a method will consider the range of the broad band of surface elevation wave heights.

We therefore design a 3D analog to this method (see Figures 4-4 and 4-3, counting the distribution of waves' heights defined as the local maximum to its nearest local minimum method. We see the wavefield's non-linearity emerge as the largest and smallest wave heights' probabilities always exceed the linear narrow-band definition of wave height probability distribution.

In this method, waves are defined by first identifying all the wavefield's local maxima where $\frac{\partial \eta}{\partial x}=0, \frac{\partial \eta}{\partial y}=0, \frac{\partial^{2} \eta}{\partial x^{2}}<0$ and $\frac{\partial^{2} \eta}{\partial y^{2}}<0$. We then identify all the wavefield's local minima where $\frac{\partial \eta}{\partial x}=0, \frac{\partial \eta}{\partial y}=0, \frac{\partial^{2} \eta}{\partial x^{2}}>0$ and $\frac{\partial^{2} \eta}{\partial y^{2}}>0$. The next step is finding, for each local maximum, the nearest local minimum "linked" to each local maximum. We define a minimum as "linked" to a maximum if at least one continuously upward-sloping line leads from the minimum to the maximum. The maximum-minimum pair found is then defined as a wave. By defining waves as such, we filter out the distribution of the shortest waves in the wavefield. In each case, where we define $\eta_{1}$ as surface elevation at the local maximum and we define $\eta_{2}$ as surface elevation at the nearest local minimum, wave height is defined as $H=\eta_{1}-\eta_{2}$ and wavelength is defined as twice the distance between the $(x, y)$ coordinates of $\eta_{1}$ and $\eta_{2}$. This stands as out 3D-analog to half-cycle excursion analysis.

This method is highly non-linear, as the Type I distribution acts as the first order in the global distribution and the Type II distribution acts as a strong higher order component of the distribution superimposed on the Type I distribution ([41], pp. 84 - 87). This distribution method is therefore attuned to both the high-frequency characteristics and the carrier-frequency characteristics of the wavefield. By this method we observe a broad range from the smallest gravity waves to the largest, main energy-carrying waves in the wavefield. 
This method tells us that the nonlinear effects in wavefields are most visible when the entire bandwidth of wave heights is considered, and not just a narrow band around the carrier frequency, or the narrow band around the largest waves in the wavefield.

\subsection{Comparison between wave definition methods}

Each wave definition method filters unique insight from the wavefield's physics. We demonstrate this via wave height distribution results and large-wave travel-direction results compared across the three wave definition methods. We compare the three wave height definition methods at $t=0 T_{p}$.

In Figure 4-5, 4-6 and 4-7 the linear distribution theoretical line is brown, and data is black and we're observing the wavefield at $t=0 T_{p}$ when we're certain of its spectral properties, namely peak shape parameter, Phillips' parameter, and directional spreading function. Here we examine a wavefield where directional spreading angle, $\theta=180^{\circ}$. We expect to see very near-linear behavior due to this large directional spreading angle, from our results in Chapter 3. We see in Figure 4-6 that linear theory over-predicts the occurrence of the largest wave heights defined by the zero-crossing method, and this is held true as linear theory over-predicts the occurrence of the largest wave heights defined by the local-maximum-to-lowest-local-minimum method seen in Figure 4-6. In our non-narrow-band approach which mimics a half-cycle method in 3D space, we see linear theory under-predicting the largest and smallest waves in the wavefield (see Figure 4-7). In this method, which best captures the non-linear aspects of the wavefield, we define a wave as the local maximum connected to its nearest-local-minimum, as seen in the $2 \mathrm{D}$ description of the half-cycle method in Section 4.4.

In Figure 4-8, 4-9 and 4-10 the linear distribution theoretical line is brown, and data is black and we're observing the wavefield at $t=0 T_{p}$ when we're certain of its spectral properties. Here we examine a wavefield where directional spreading angle, $\theta=20^{\circ}$. We expect to see highly non-linear behavior due to this small directional spreading angle, from our results in Chapter 3. We see in Figure 4-8 that linear theory 


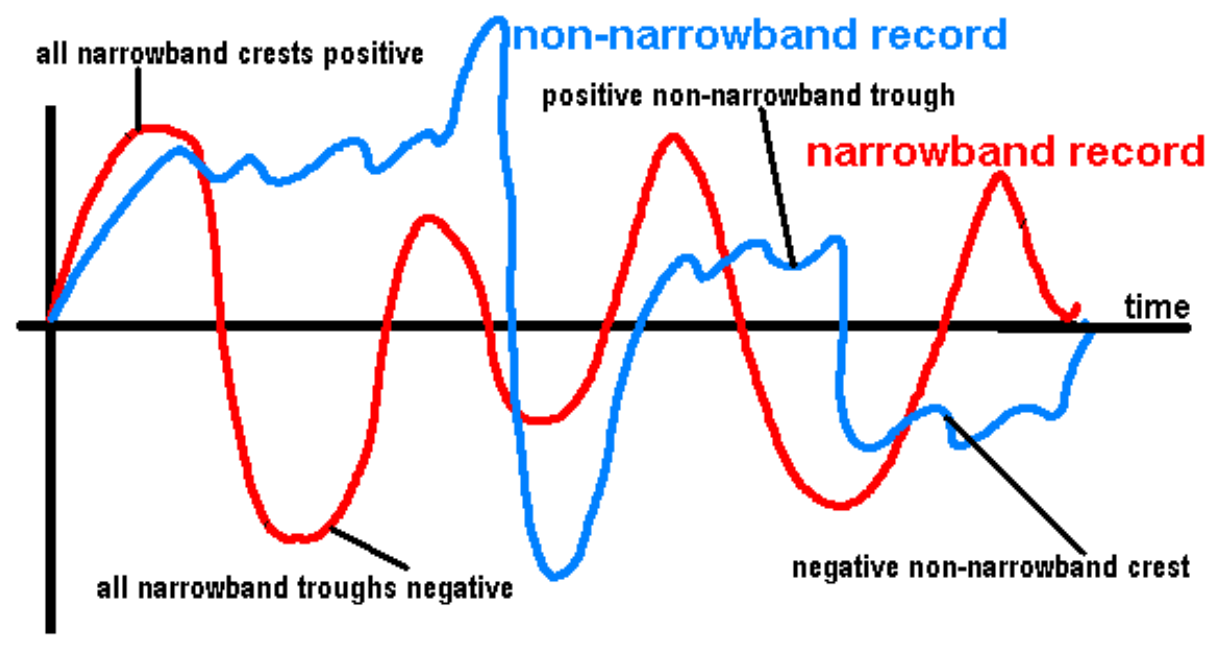

(a) Positive minima and negative maxima are depicted. These characterize non-narrow-band waves.

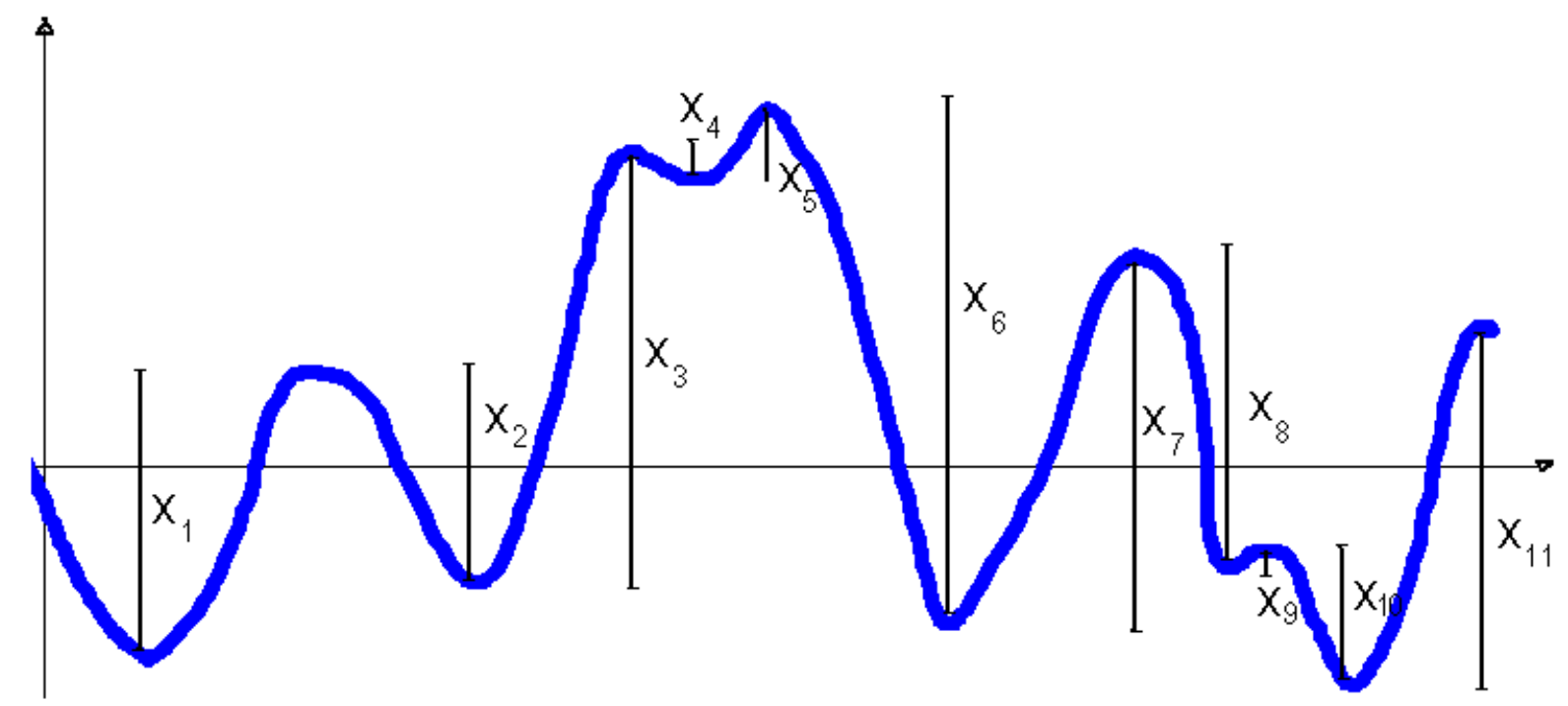

(b) Half-cycle excursions, labeled $x_{1}, x_{2}, \ldots, x_{11}$, are non-narrow-band wave heights. We see examples of Type I excursions in $x_{1}, x_{2}, x_{6}, x_{7}, x_{8}$ and $x_{1} 1$ and examples of Type II excursions in $x_{4}, x_{5}, x_{9}$ and $x_{10}$

Figure 4-4: Surface elevation is depicted on the vertical axes and time is depicted on the horizontal axes. Non-narrow-band wave heights are captured in the half-cycle excursion method of wave height definition [41]. 

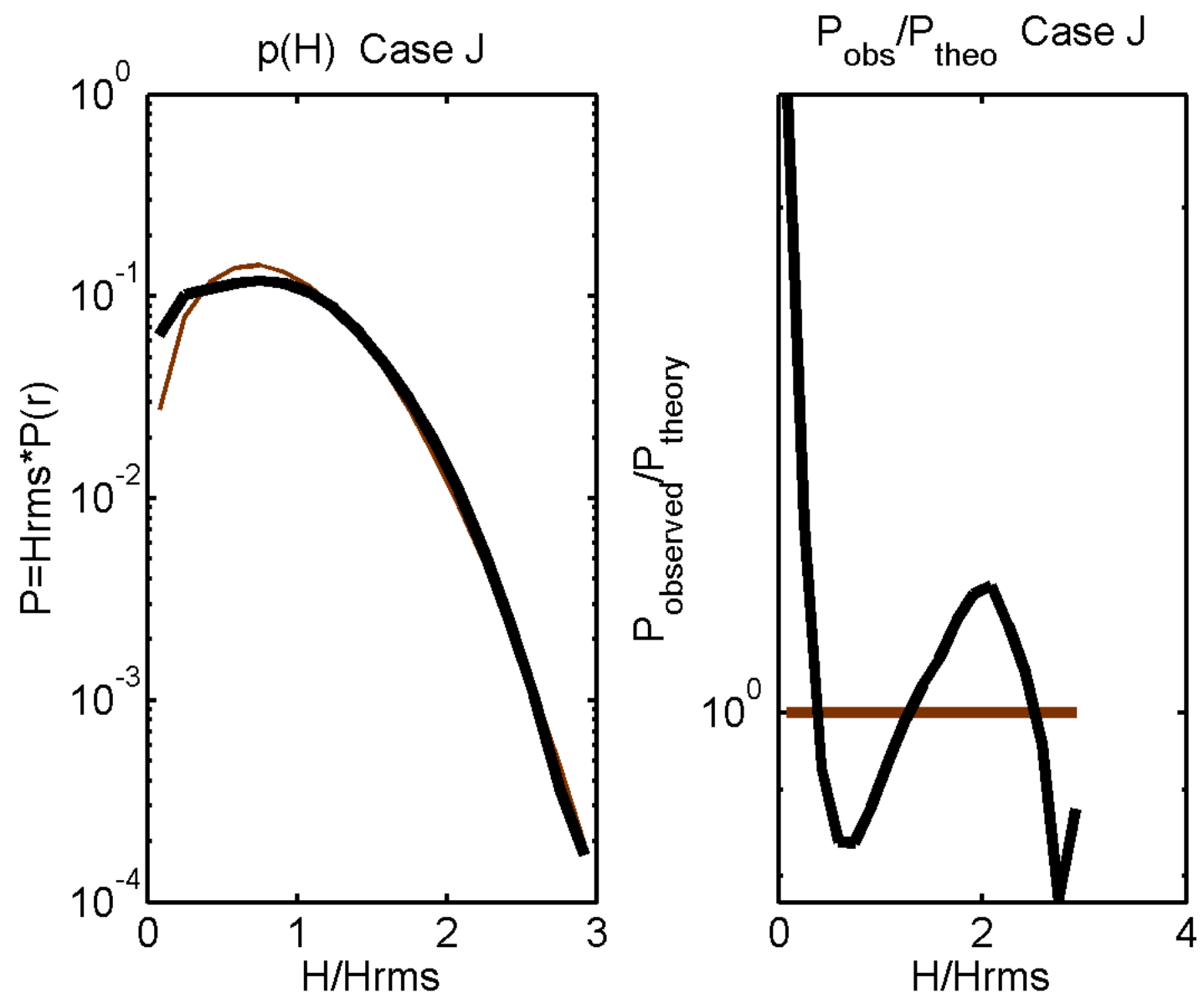

Figure 4-5: Probability is depicted on the vertical axis and wave height is depicted on the horizontal axis. Case $\mathbf{J}$ with peak shape parameter $\gamma=3.3$, Phillips' parameter, $\alpha=0.0160$ and input spreading angle, $\theta=180^{\circ}$ wave height probability distribution when $t=0 T_{p}$ where wave heights are defined by the zero-crossing method. 

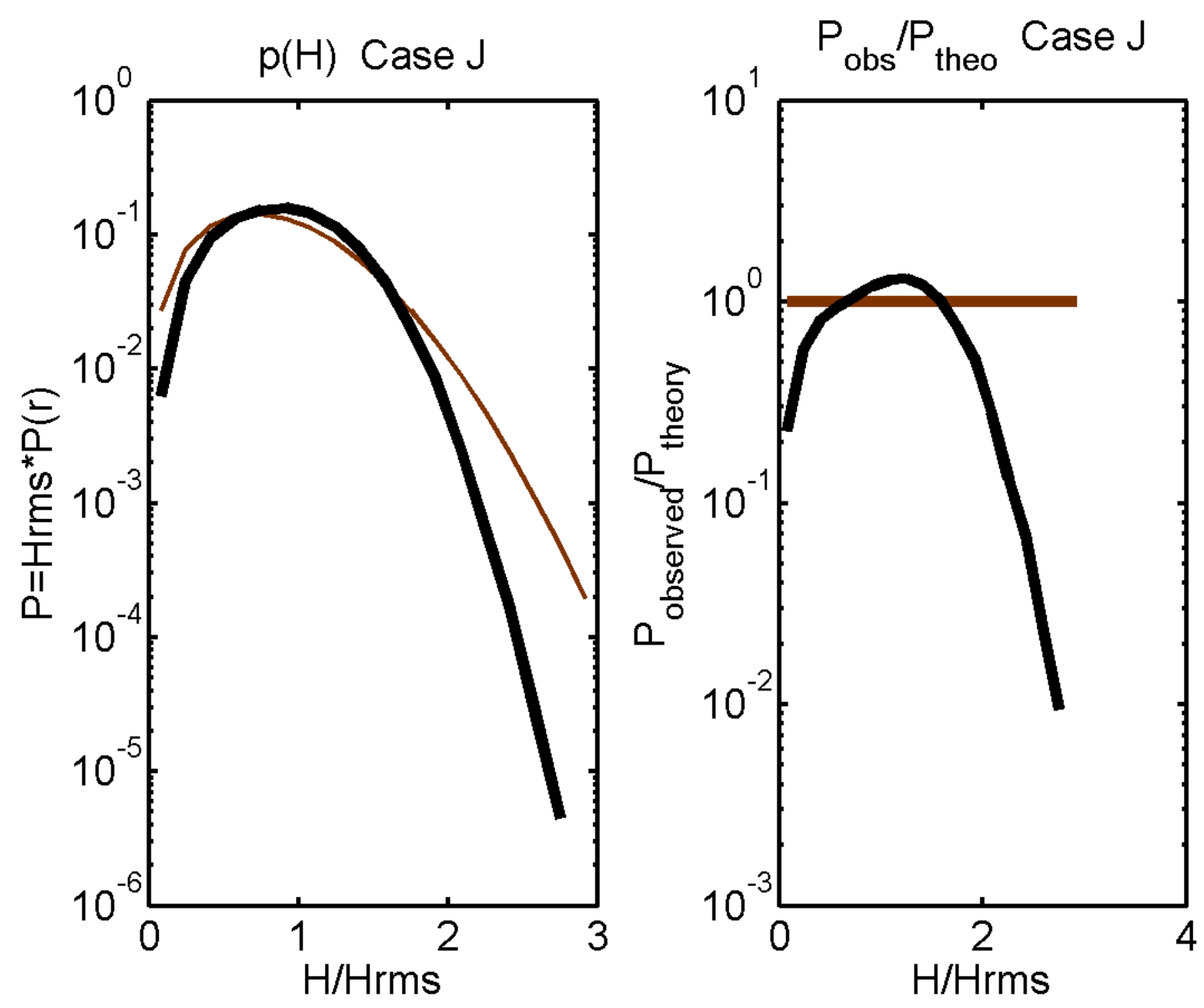

Figure 4-6: Probability is depicted on the vertical axis and wave height is depicted on the horizontal axis. Case $\mathbf{J}$ with peak shape parameter $\gamma=3.3$, Phillips' parameter, $\alpha=0.0160$ and input spreading angle, $\theta=180^{\circ}$ wave height probability distribution when $t=0 T_{p}$ where wave heights are defined by the local-maximum-to-lowest-localminimum method. 

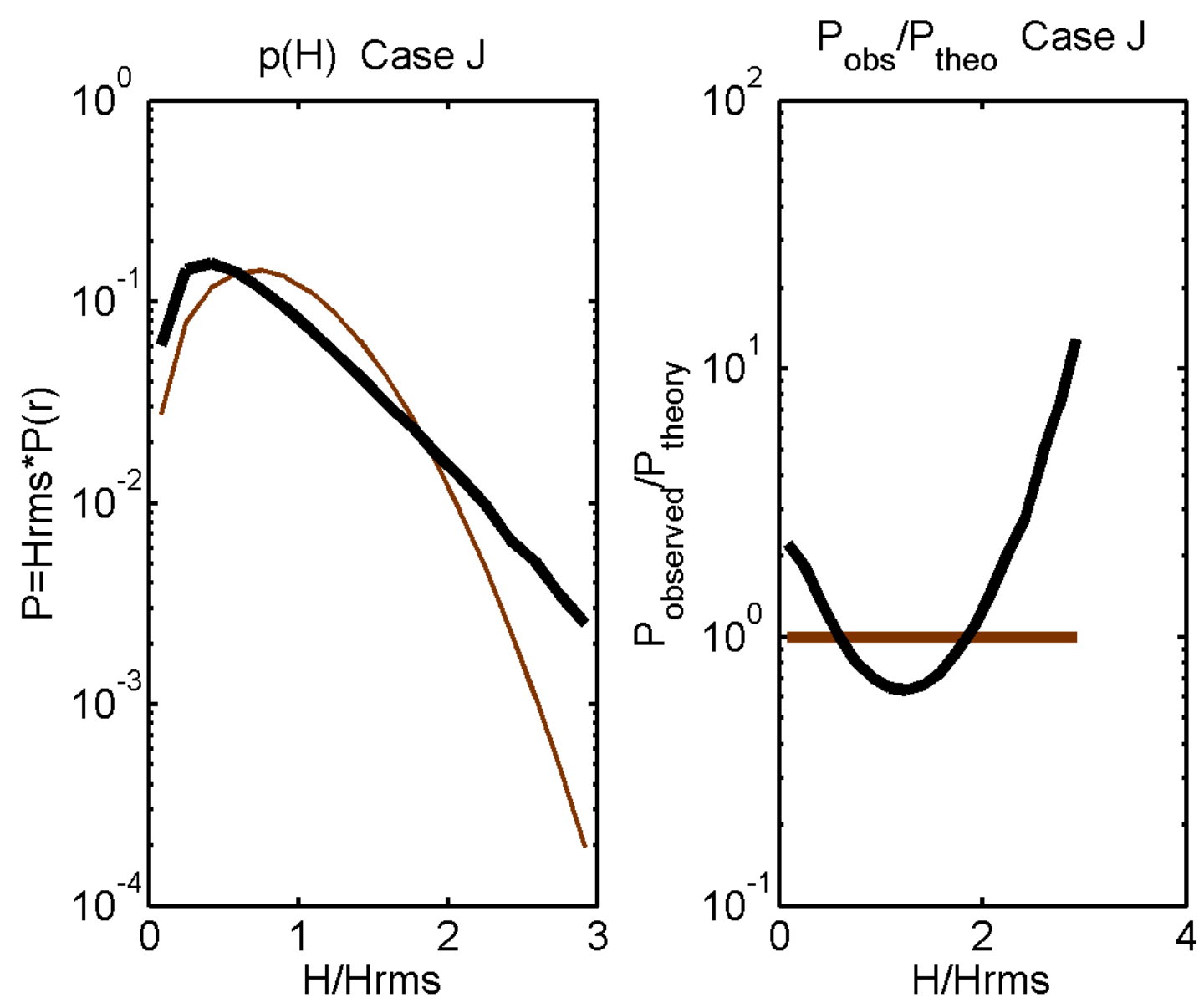

Figure 4-7: Probability is depicted on the vertical axis and wave height is depicted on the horizontal axis. Case $\mathbf{J}$ with peak shape parameter $\gamma=3.3$, Phillips' parameter, $\alpha=0.0160$ and input spreading angle, $\theta=180^{\circ}$ wave height probability distribution when $t=0 T_{p}$ where wave heights are defined by the local-maximum-to-nearest-localminimum method. 

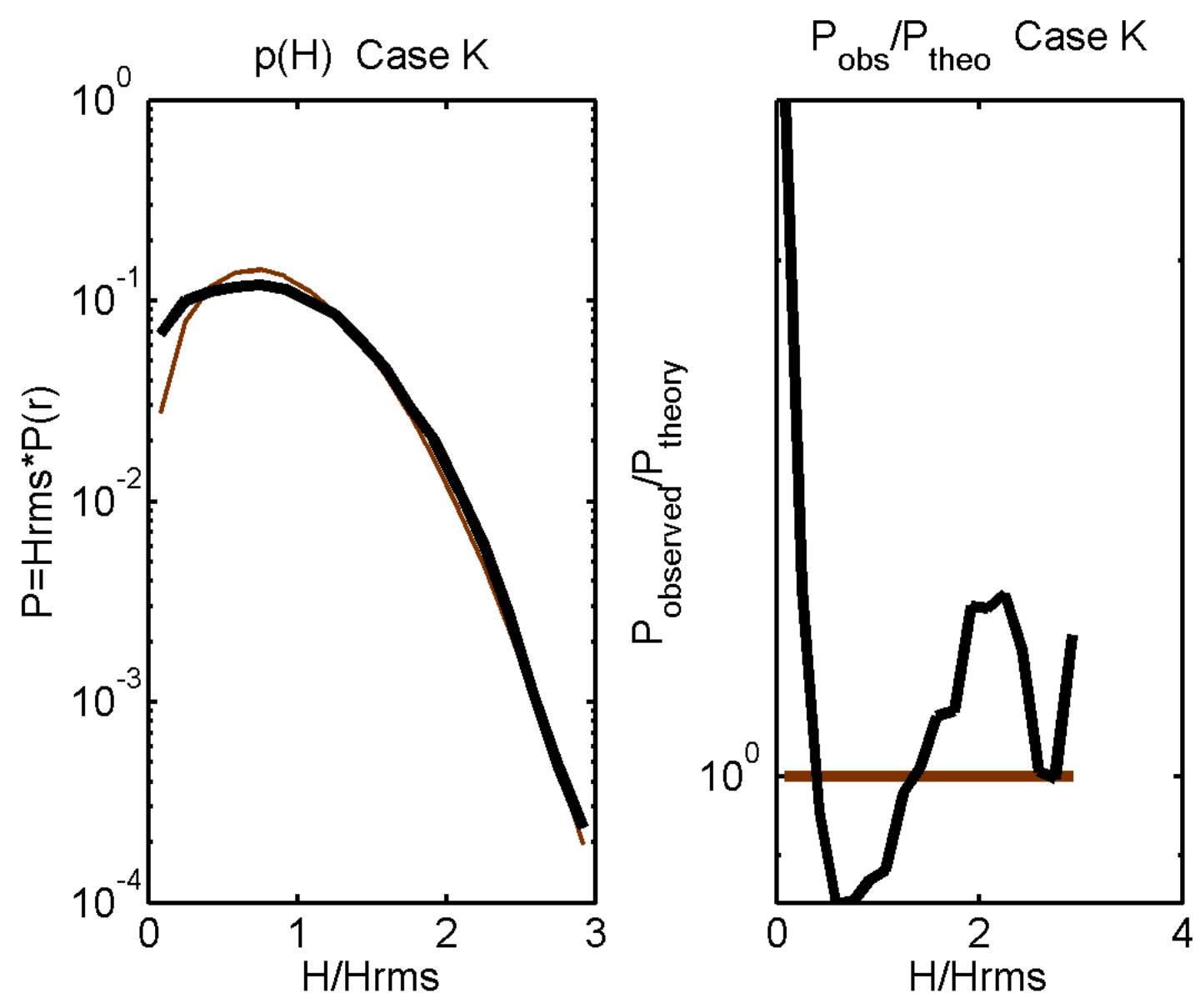

Figure 4-8: Probability is depicted on the vertical axis and wave height is depicted on the horizontal axis. Case $\mathbf{K}$ with peak shape parameter $\gamma=3.3$, Phillips' parameter, $\alpha=0.0160$ and input spreading angle, $\theta=20^{\circ}$ wave height probability distribution when $t=0 T_{p}$ where wave heights are defined by the zero-crossing method. 

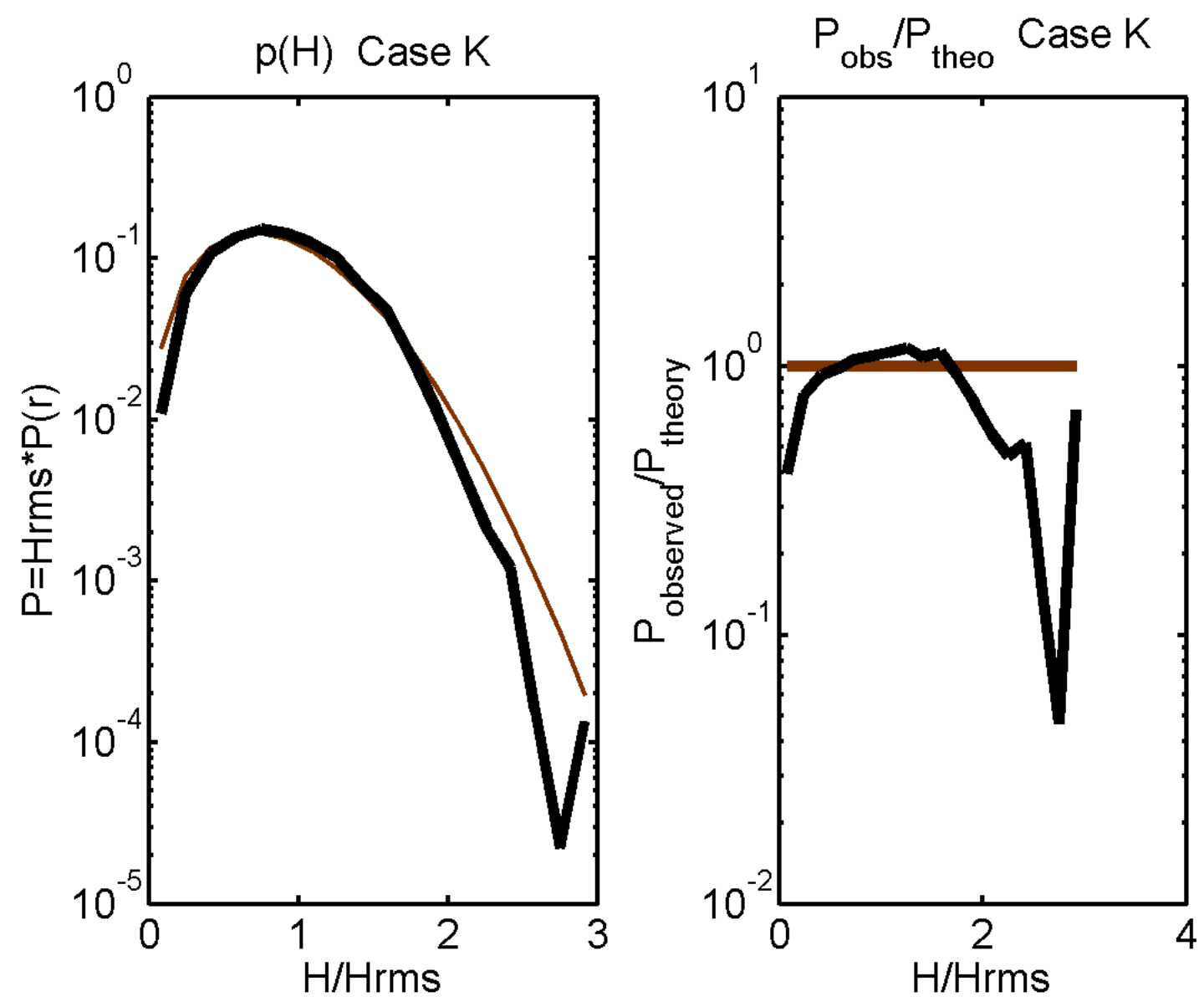

Figure 4-9: Probability is depicted on the vertical axis and wave height is depicted on the horizontal axis. Case $\mathbf{K}$ with peak shape parameter $\gamma=3.3$, Phillips' parameter, $\alpha=0.0160$ and input spreading angle, $\theta=20^{\circ}$ wave height probability distribution when $t=0 T_{p}$ where wave heights are defined by the local-maximum-to-lowest-localminimum method. 

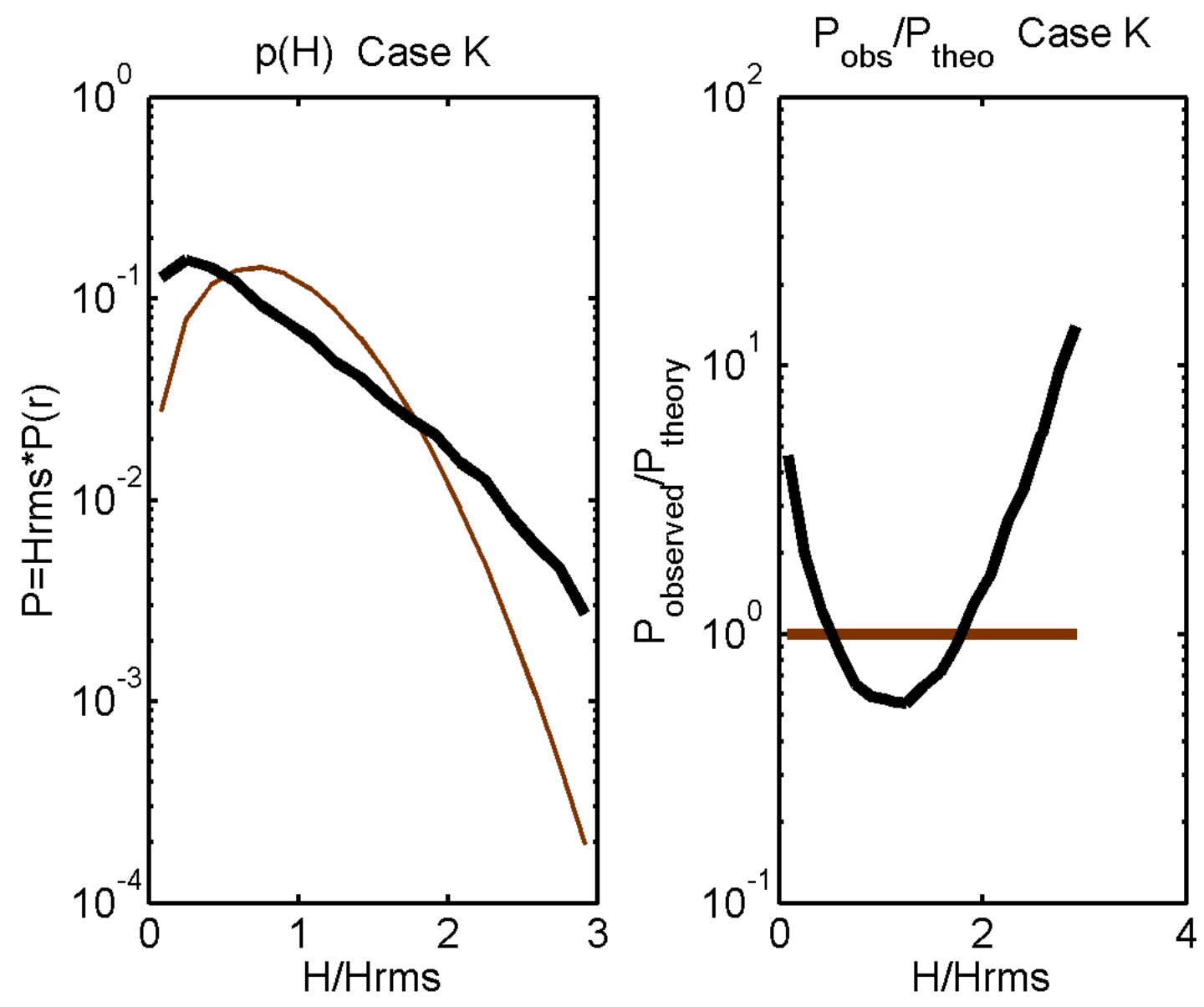

Figure 4-10: Probability is depicted on the vertical axis and wave height is depicted on the horizontal axis. Case $\mathbf{K}$ with peak shape parameter $\gamma=3.3$, Phillips' parameter, $\alpha=0.0160$ and input spreading angle, $\theta=20^{\circ}$ wave height probability distribution when $t=0 T_{p}$ where wave heights are defined by the local-maximum-to-nearest-localminimum method. 
under-predicts the occurrence of the largest wave heights defined by the zero-crossing method in this wavefield. As predicted by Ochi [41], the main, energy-carrying waves in the wavefield, defined by the local-maximum-to-lowest-local-minimum method, adhere to linear theory and this is seen in Figure 4-9. In our non-narrow-band approach which mimics a half-cycle method in 2D space, we see (in Figure 4-10) linear theory under-predicting the largest and smallest waves in the wavefield. In this method, which best captures the non-linear aspects of the wavefield, we see the type of nonGaussian behavior that we expect from a near-unidirectional wave field with directional spreading angle, $\theta=20^{\circ}$.

$\Theta$ and $h$ form the coordinate system used in the directionality plots in Figures 4-11 and 4-14. In the zero-crossing method of defining waves, directional information is lost, as can be seen in Figures 4-11 and 4-14, where we assume one mean wave travel direction. In the other two methods which do not assume a mean direction of wave travel, each wave defined has a direction of travel, $\Theta$, defined as the tilt of the straight line between the crest and the trough away from the x-direction. In Figures 4-15, 4-16, 4-12 and 4-13 we plot wave direction, $\Theta$, and wave height, $h$, as polar coordinates for each of the waves in the wavefield where $H / H_{s}>1.5$ and we term these the largest waves in the wavefield.

Observing the wavefields at $t=0 T_{p}$ when we're certain of their spectral properties, we compare Figures 4-12 and 4-15 which depict results from waves defined according to the local-maximum-to-lowest-local-minimum method which happen to be the main, energy-carrying set of waves in the wavefield. We find that a spreading angle of $\theta=180^{\circ}$ produces a near-uniform distribution of the travel directions among the largest waves in the wavefield. We also see that a spreading angle of $\theta=20^{\circ}$ produces a totally non-uniform distribution of the largest waves in the wavefield's energy-carrying waves, as we see the heaviest concentration of the largest waves traveling in the range $\Theta=0^{\circ} \pm 20^{\circ}$ at $t=0 T_{p}$, in direct response to its input spreading angle.

Figures 4-13 and 4-16 depict results from waves defined according to the localmaximum-to-nearest-local-minimum method at $t=0 T_{p}$ when we're certain of their spectral properties. We find that Figure 4-13 depicts uniformity of spread when input 


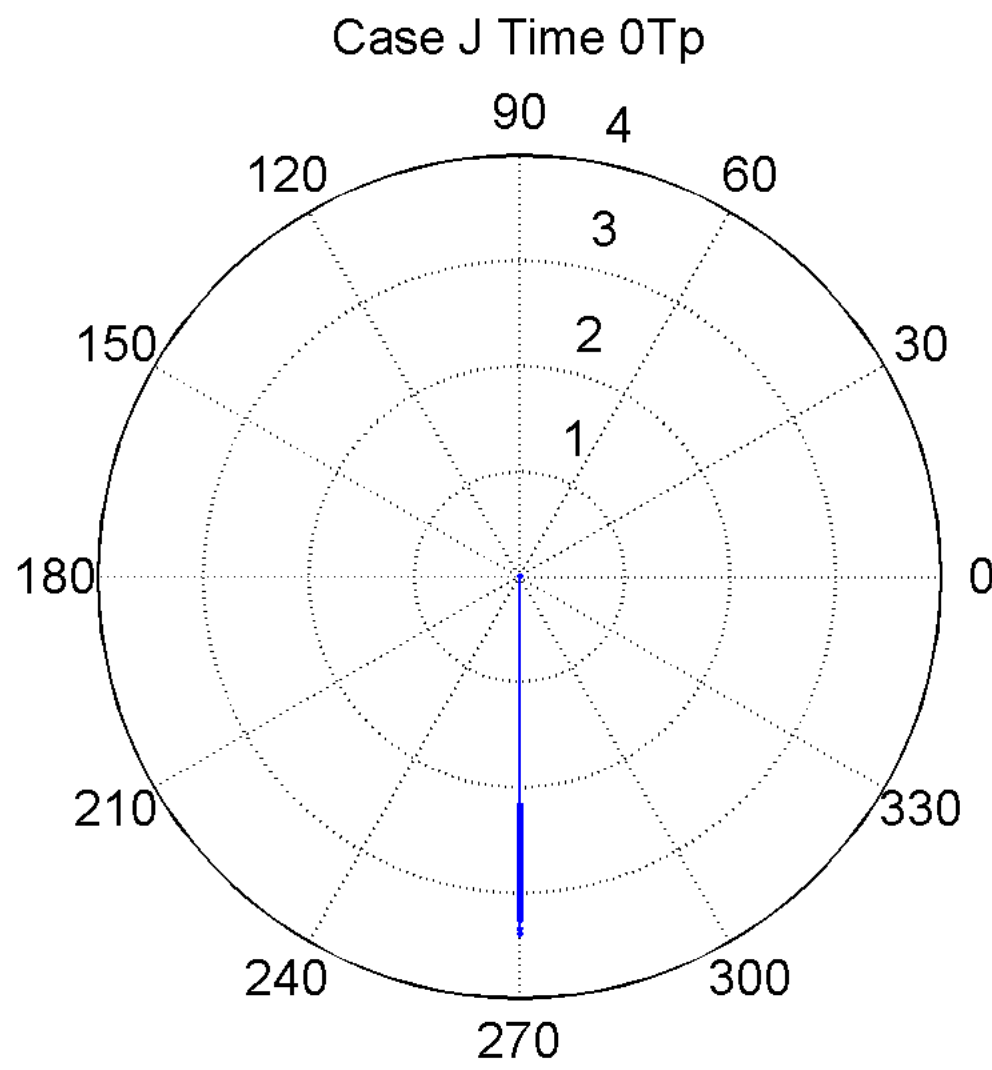

$\mathrm{H} / \mathrm{Hs}_{0}$

Figure 4-11: Polar coordinates are used to represent wave height, $H$ in the length of the vector and the wave travel direction, $\Theta$ in the tilt of the vector around the origin. Case $\mathbf{J}$ with peak shape parameter $\gamma=3.3$, Phillips' parameter, $\alpha=0.0160$ and input spreading angle, $\theta=180^{\circ}$ at time, $t=0 T_{p}$ large wave heights' directionality where wave heights are defined by the zero-crossing method when $t=0 T_{p}$. 


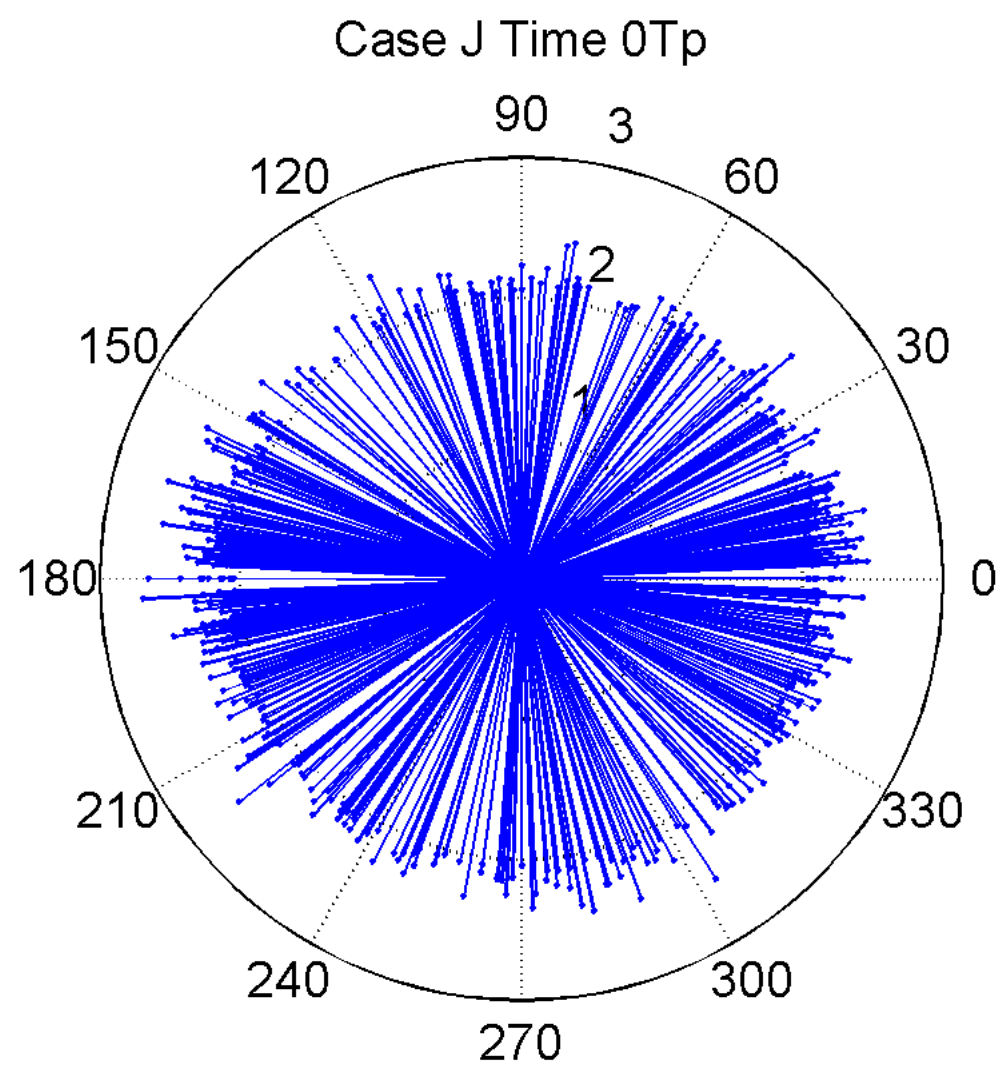

$\mathrm{H} / \mathrm{Hs}_{0}$

Figure 4-12: Polar coordinates represent wave height, $H$ in the length of the vector and the wave travel direction, $\Theta$ in the tilt of the vector around the origin. Case $\mathbf{J}$ with peak shape parameter $\gamma=3.3$, Phillips' parameter, $\alpha=0.0160$ and input spreading angle, $\theta=180^{\circ}$ at time, $t=0 T_{p}$ large wave heights' directionality where wave heights are defined by the lowest-local-minimum method when $t=0 T_{p}$. 


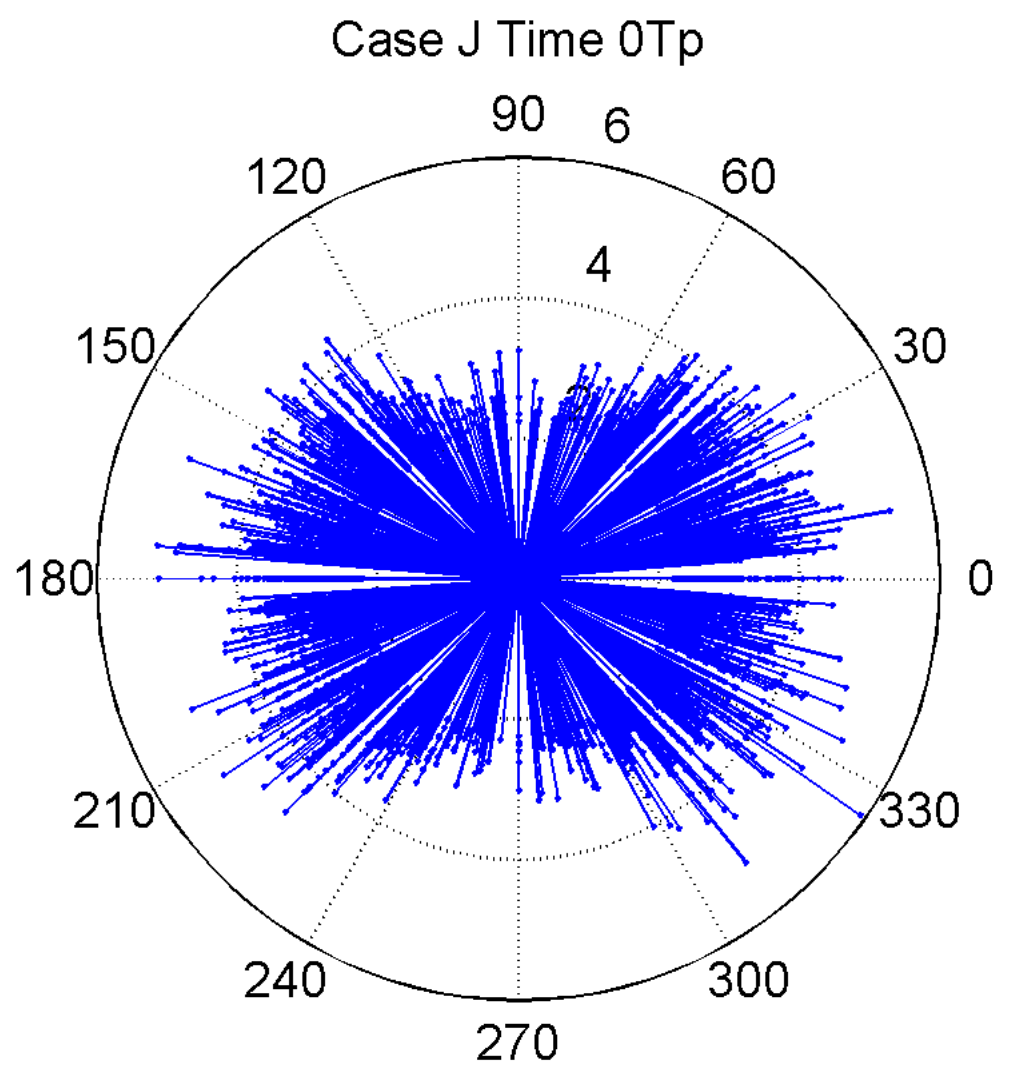

$\mathrm{H} / \mathrm{Hs}_{0}$

Figure 4-13: Polar coordinates represent wave height, $H$ in the length of the vector and the wave travel direction, $\Theta$ in the tilt of the vector around the origin. Case $\mathbf{J}$ with peak shape parameter $\gamma=3.3$, Phillips' parameter, $\alpha=0.0160$ and input spreading angle, $\theta=180^{\circ}$ at time, $t=0 T_{p}$ large wave heights' directionality where wave heights are defined by the nearest-local-minimum method when $t=0 T_{p}$. 


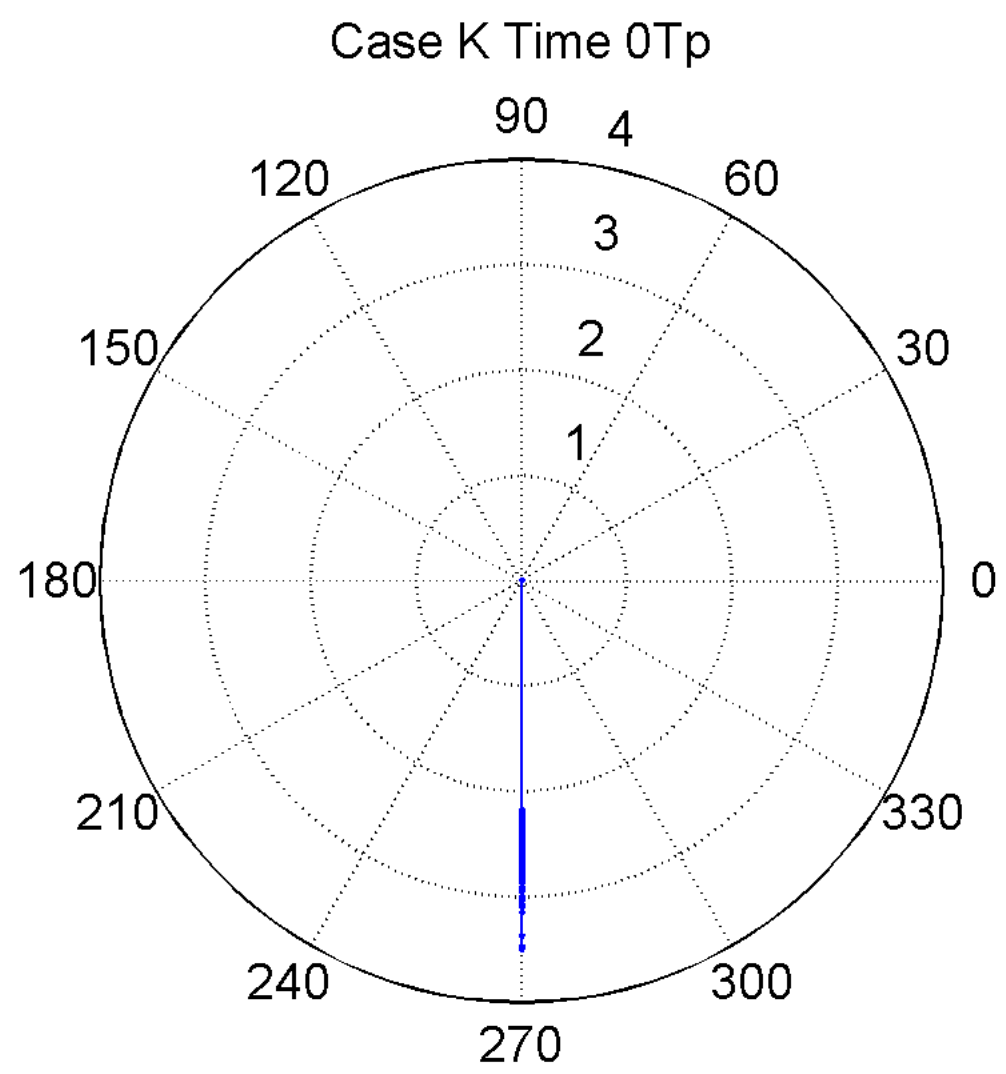

$\mathrm{H} / \mathrm{Hs}_{0}$

Figure 4-14: Polar coordinates represent wave height, $H$ in the length of the vector and the wave travel direction, $\Theta$ in the tilt of the vector around the origin. Case $\mathbf{K}$ with peak shape parameter $\gamma=3.3$, Phillips' parameter, $\alpha=0.0160$ and input spreading angle, $\theta=20^{\circ}$ at time, $t=0 T_{p}$ large wave directionality where waves are defined by the zero-crossing method. 


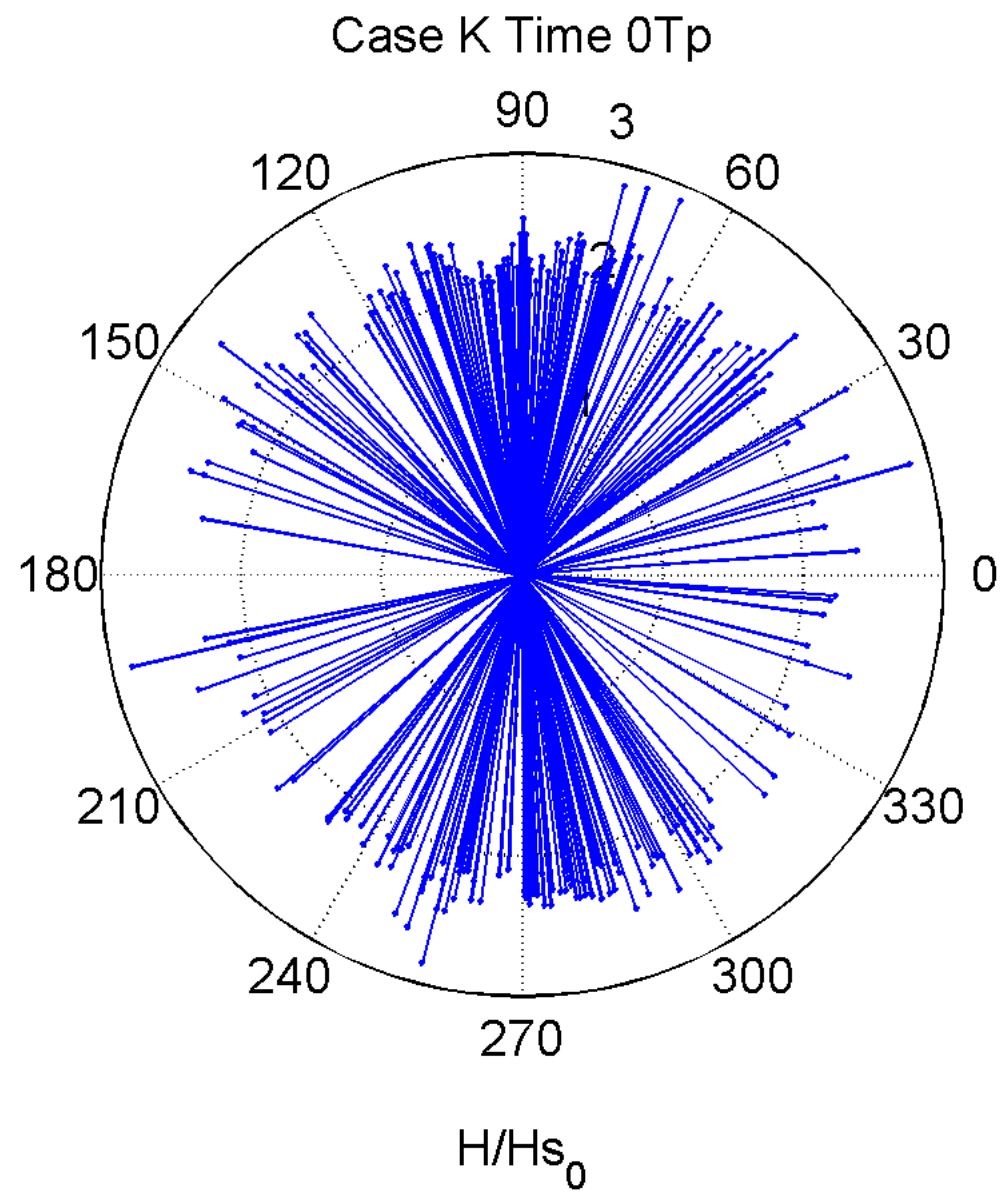

Figure 4-15: Polar coordinates represent wave height, $H$ in the length of the vector and the wave travel direction, $\Theta$ in the tilt of the vector around the origin. Case $\mathbf{K}$ with peak shape parameter $\gamma=3.3$, Phillips' parameter, $\alpha=0.0160$ and input spreading angle, $\theta=20^{\circ}$ at time, $t=0 T_{p}$ large wave directionality where waves are defined by the lowest-local-minimum method. 


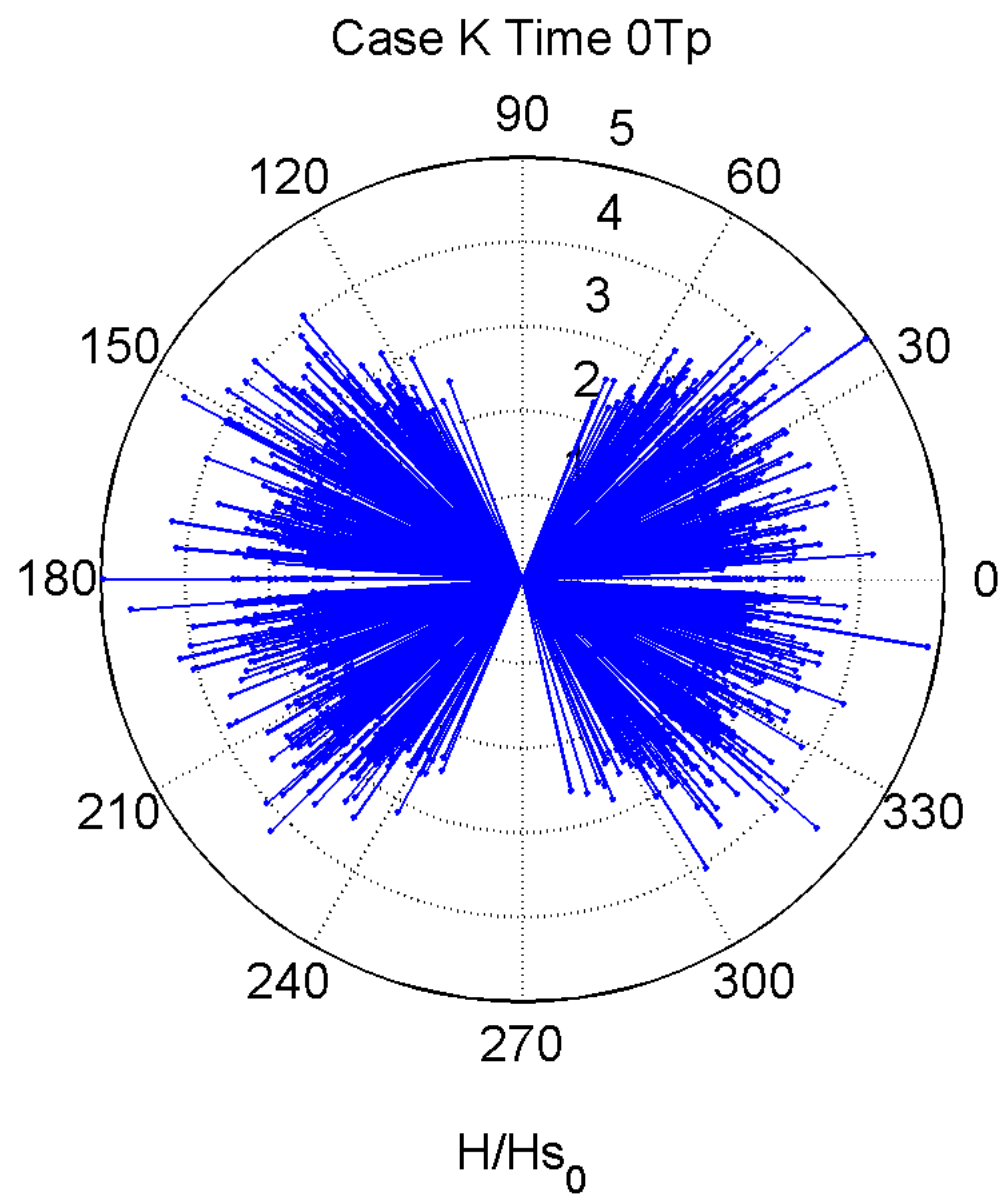

Figure 4-16: Polar coordinates represent wave height, $H$ in the length of the vector and the wave travel direction, $\Theta$ in the tilt of the vector around the origin. Case $\mathbf{K}$ with peak shape parameter $\gamma=3.3$, Phillips' parameter, $\alpha=0.0160$ and input spreading angle, $\theta=20^{\circ}$ at time, $t=0 T_{p}$ large wave directionality where waves are defined by the nearest-local-minimum method. 
$\theta=180^{\circ}$ among the largest waves in the wavefield defined according to this method, although among these waves the highest waves travel in ranges that cluster around four specific values: $\Theta=n \pi \pm \frac{\pi}{4}$ where $n=0,1$. Compared to that, when input $\theta=20^{\circ}$, we see in Figure 4-16 that none of the largest waves defined according this non-narrow-band method travel in the prescribed directionality range, $\Theta=0^{\circ} \pm 20^{\circ}$, seen in the previous wave definition results.

In Figure 4-17 we compare the effect of two 3D wave height definition methods on wave height distributions for a highly directional wavefield, Case $\mathbf{J}$ where $\theta=$ $180^{\circ}$. Two methods of wave height definition produce quite different wave height distributions, one emphasizing the effect of higher order characteristics and the other ignoring them.

In Figure 4-17 we compare the effect of two 3D wave height definition methods on wave height distributions for a near uni-directional wavefield, Case $\mathbf{K}$ where $\theta=$ $20^{\circ}$. Two methods of wave height definition produce quite different wave height distributions, one emphasizing the effect of higher order characteristics and the other ignoring them.

We also see in both cases where $\theta=20^{\circ}$ and where $\theta=180^{\circ}$ (although more so when $\theta=20^{\circ}$ ) that the non-narrow-band definition of wave height in this case produces an unchanging distribution, but that the narrow-band definition of wave height produces an unstable, shifting distribution. This tells us that details of the narrow-band effects around the largest waves in the wavefield change while the broad, non-narrow-band picture stays constant as the wavefield evolves.

In Figure 4-19 we have a comparison of two 3D methods of defining wave heights in Case $\mathbf{J}$. Case $\mathbf{J}$ is expected to display broad-band properties since it is broadly spread across a wide directional spreading range, $\theta=180^{\circ}$ and we see its wavelengths' distributions fall exactly along the rayleigh distribution at every time step. This speaks of the broad spectral bandwidth of Case $\mathbf{J}$ 's half-cycle excursion wavelengths, and of the accuracy in analyzing Case $\mathbf{J}$ using the nearest connected minimum method of wave height and wave length definition.

Additionally we see in Figure 4-20 that the non-narrow-band nearest minimum 

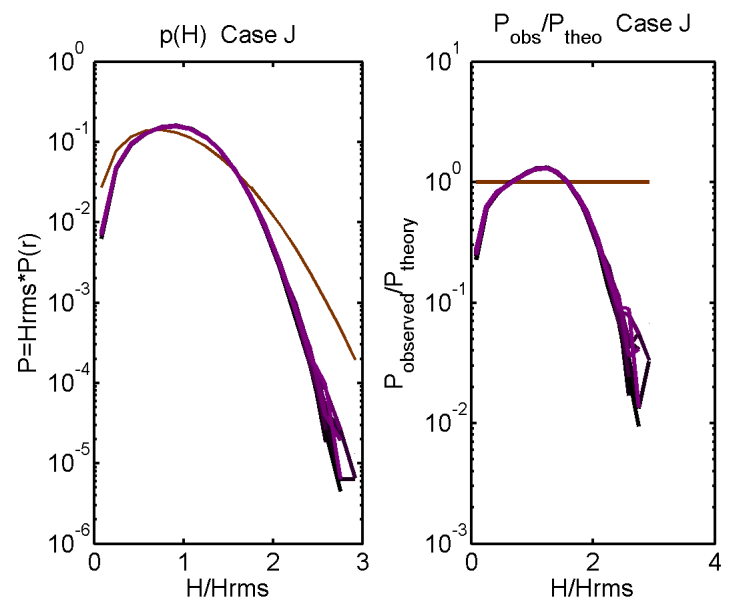

(a) Case $\mathbf{J}$ wave heights are defined by narrow-band local-maximum-to-lowest-local-minimum method.
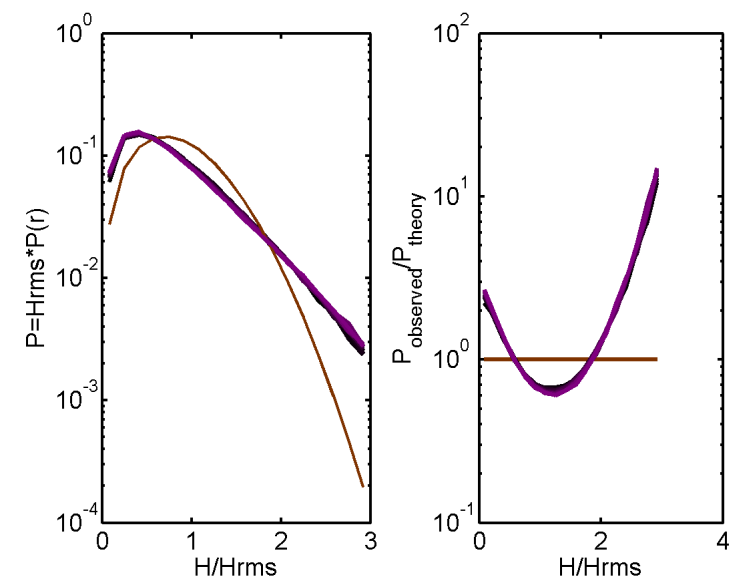

(b) Case $\mathbf{J}$ wave heights are defined by non-narrowband local-maximum-to-nearest-local-minimum method.

Figure 4-17: Probability is depicted on the vertical axis and wave height is depicted on the horizontal axis. Case $\mathbf{J}$ with peak shape parameter $\gamma=3.3$, Phillips' parameter, $\alpha=0.0160$ and input spreading angle, $\theta=180^{\circ}$ wave height probability distribution from $t=0 T_{p}$ to $t=100 T_{p}$ where wave heights are defined by two 3D methods. 

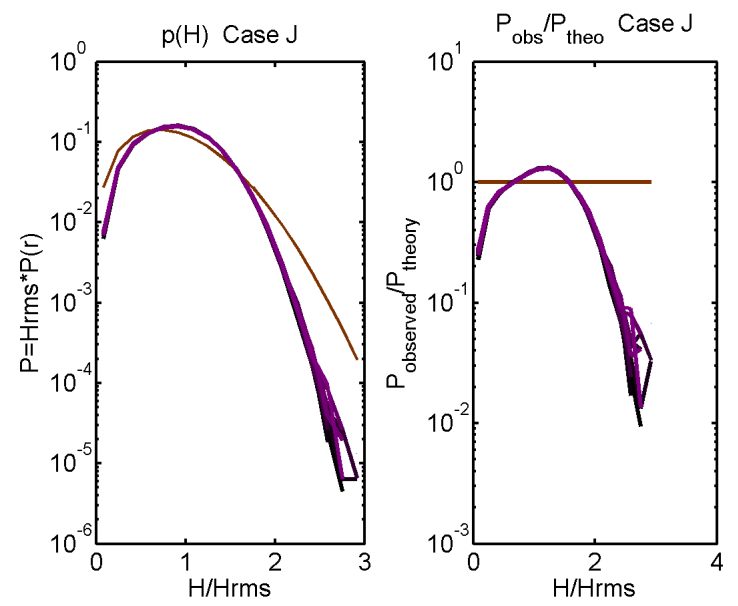

(a) Case $\mathbf{K}$ wave heights are defined by narrow-band local-maximum-to-lowest-local-minimum method.
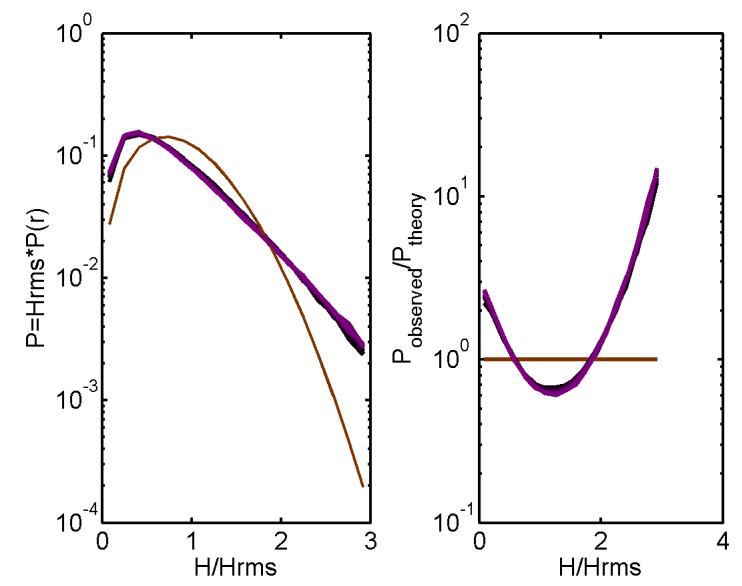

(b) Case $\mathbf{K}$ wave heights are defined by non-narrowband local-maximum-to-nearest-local-minimum method.

Figure 4-18: Probability is depicted on the vertical axis and wave height is depicted on the horizontal axis in Case $\mathbf{K}$ with peak shape parameter $\gamma=3.3$, Phillips' parameter, $\alpha=0.0160$ and input spreading angle, $\theta=20^{\circ}$ wave height probability distribution from $t=0 T_{p}$ to $t=100 T_{p}$ where wave heights are defined by two 3D methods. 

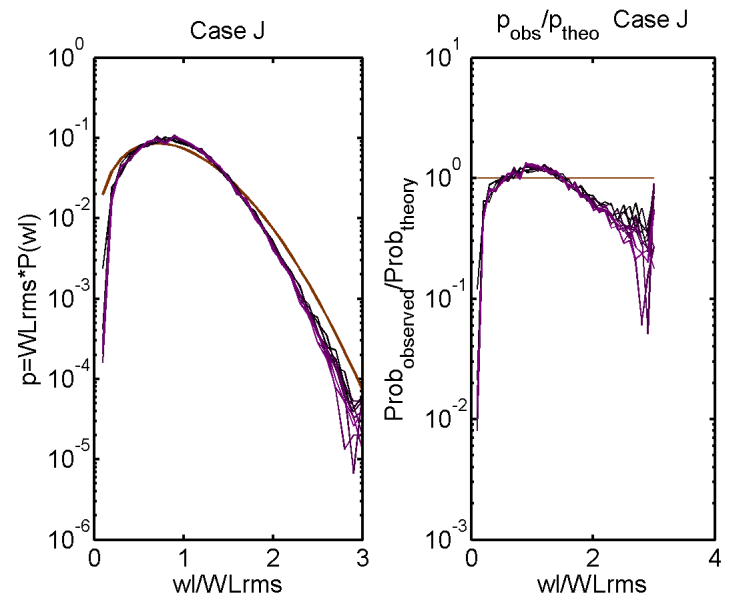

(a) Case $\mathbf{J}$ wave lengths are defined by the narrowband lowest minimum method.
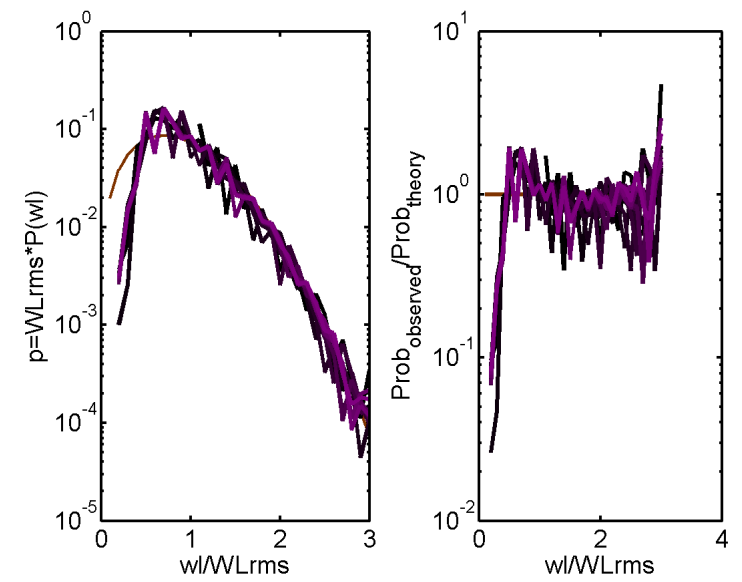

(b) Case $\mathbf{J}$ wave lengths are defined by the nonnarrow-band nearest minimum method.

Figure 4-19: Probability is depicted on the vertical axis and wave length is depicted on the horizontal axis in Case $\mathbf{J}$ with peak shape parameter $\gamma=3.3$, Phillips' parameter, $\alpha=0.0160$ and input spreading angle, $\theta=180^{\circ}$ from $t=0 T_{p}$ to $t=100 T_{p}$ where wave lengths are defined by three methods. 

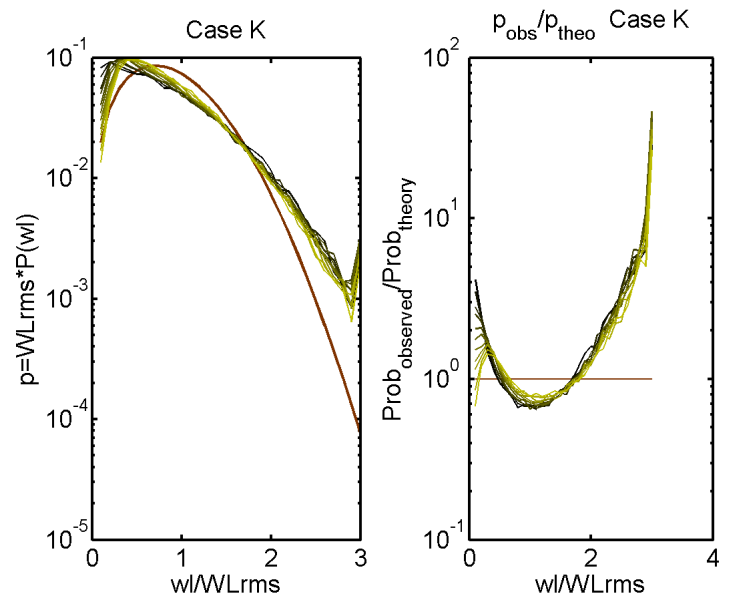

(a) Case $\mathbf{K}$ wave lengths are defined by the narrowband lowest minimum method.
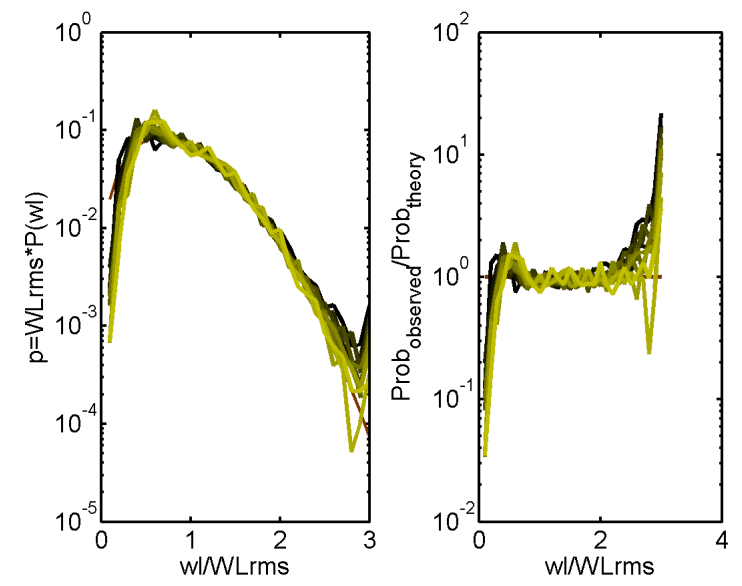

(b) Case $\mathbf{K}$ wave lengths are defined by the nonnarrow-band nearest minimum method.

Figure 4-20: Probability is depicted on the vertical axis and wave length is depicted on the horizontal axis in Case $\mathbf{K}$ with peak shape parameter $\gamma=3.3$, Phillips' parameter, $\alpha=0.0160$ and input spreading angle, $\theta=20^{\circ}$ from $t=0 T_{p}$ to $t=100 T_{p}$ where wave lengths are defined by three methods. 
method accurately matches the theoretical expectation for a broad-band wavelength distribution even in a case of low angular spreading where $\theta=20^{\circ}$.

In Figure 4-21 we examine the sensitivity of the narrow-band 3D method of wavelength definition (lowest min method) to directional spreading. Case K (input spreading angle, $\theta=20^{\circ}$ ) where wave lengths are defined by the narrow-band lowest minimum method, shows wavelength distributions that exceed the Rayleigh distribution at their two tails. Similarly defined Case $\mathbf{J}$ wave length distributions display completely opposite behavior as wavelength distributions fall below the Rayleigh distribution at their two tails.

In Figure 4-22 we examine the sensitivity of the non-narrow-band 3D method of wavelength definition (nearest min method) to directional spreading. Case $\mathbf{K}$ (input spreading angle, $\theta=20^{\circ}$ ) and Case $\mathbf{J}$ (input spreading angle, $\theta=180^{\circ}$ ) are compared where wave lengths are defined by the non-narrow-band nearest minimum method. We see for both spreading angles, wavelength distributions match the Rayleigh distribution very well, although the distributions are more stable in time for the case with the smaller input spreading angle, $\theta=20^{\circ}$.

In Chapter 5, following this section, we look at the statistics and trends of the wave heights defined according to the two 3D methods outlined in the present chapter. 

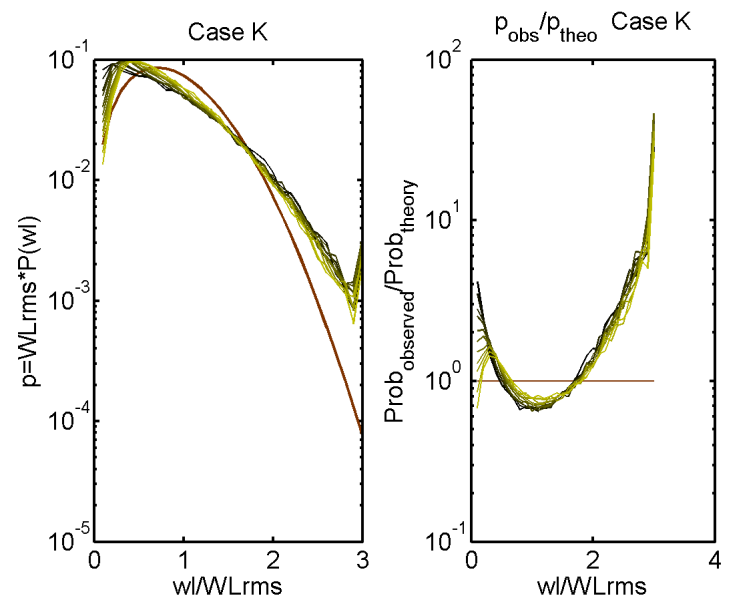

(a) Case $\mathbf{K}$ wave lengths are defined by the lowest min method.
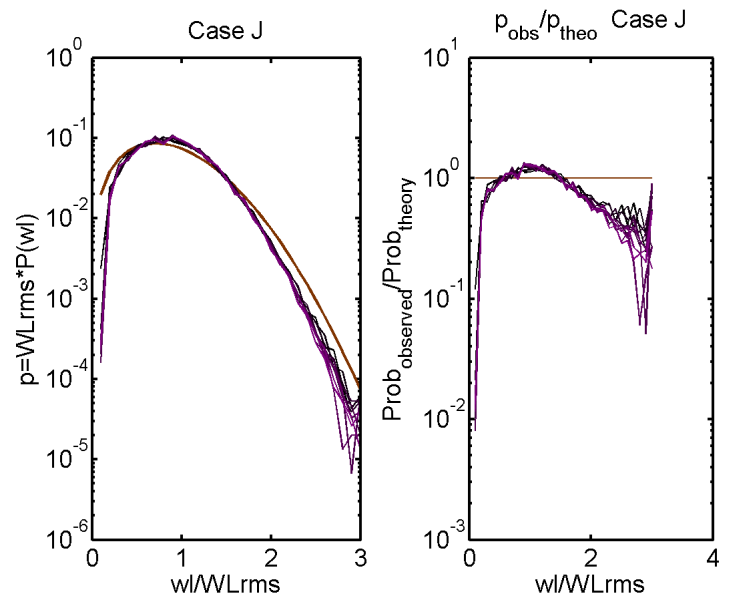

(b) Case $\mathbf{J}$ wave lengths are defined by the lowest min method.

Figure 4-21: Probability is depicted on the vertical axis and wave length is depicted on the horizontal axis. Lowest minimum method wave lengths compared for Cases $\mathbf{K}$ and $\mathbf{J}$. 

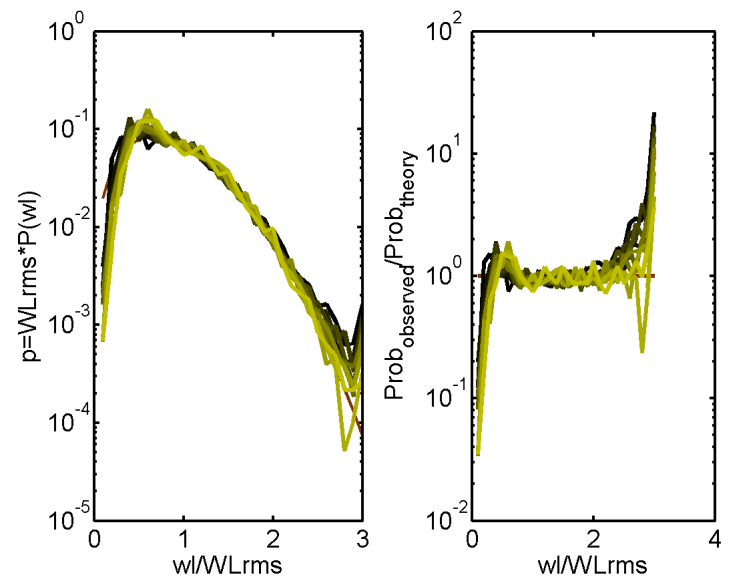

(a) Case $\mathbf{K}$ wave lengths are defined by the nearest min method.
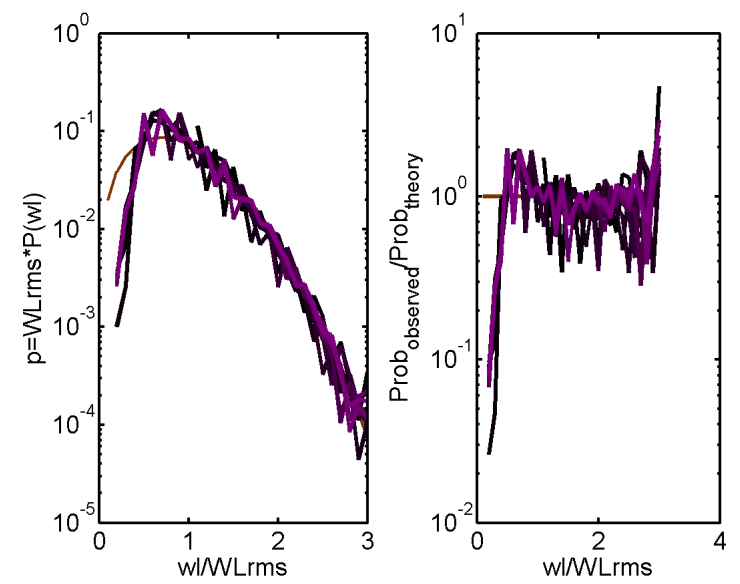

(b) Case $\mathbf{J}$ wave lengths are defined by the nearest min method.

Figure 4-22: Probability is depicted on the vertical axis and wave length is depicted on the horizontal axis. Nearest minimum method wavelengths (3D analogue of nonnarrow-band half-cycle excursion method) compared for Cases $\mathbf{K}$ and $\mathbf{J}$. 


\section{Chapter 5}

\section{Wave height distributions and spectral parameters}

In the present chapter, we study wave height and wavelength distributions utilizing the previously outlined methods of wave height and wavelength definitions (see Chapter 4) in a 3-D, directional wavefield. We vary the input wavefield spectral parameters and investigate the effect of an initialized spectrum on wave height and wavelength probability distributions, travel directions, and the occurrence and spacing of large wave height events among other wavefield properties.

\subsection{Peak shape parameter}

In this section we investigate the impact of initialized spectral peak shape parameter, $\gamma$ on statistical characteristics of ocean wave heights and wavelengths in simulated nonlinear wavefields. We generally use wave height defined according to the threedimensional methods outlined in Chapter 4 unless otherwise stated. 


\subsubsection{Peak shape parameter and wave height distribution de- viation from linear theory}

In this section we compare the theory of wave height distributions in linear, narrowband wavefields to the observed statistics of wave heights (defined in a non-linear, nonnarrow-band 3D analogue to the half-cycle excursion method ) in our simulated linear and non-linear wavefields, observing in all cases the effect of peak shape parameter on statistical deviations from linear, narrow-band theory.

In Table 3.1 we refer to the Cases $\mathbf{I}$ (where $\gamma=1.0$ ), $\mathbf{B}($ where $\gamma=3.3$ ) and $\mathbf{H}$ (where $\gamma=5.0$ ) used in testing the effect of spectral peak shape parameter, $\gamma$. They each have a directional spreading angle of $\theta=40^{\circ}$, and very low initial average steepness $\epsilon=0.04444$, although their three input $\gamma$-values cause them to have three different input $\alpha$-values.

In comparing results in Figures 5-1, 5-2, 5-3 we can conclude that each value of peak shape parameter, $\gamma$ produces visible differences in the ratios between wave height distribution and the Rayleigh distribution over time (the right image on each graphic). Despite vastly different surface elevation properties between these three cases (see Figure 3-1), Cases $\mathbf{I}$ (where $\gamma=1.0$ ), $\mathbf{B}$ (where $\gamma=3.3$ ) and $\mathbf{H}$ (where $\gamma=5.0$ ) display very similar wave height distributions. Correlating the Rayleigh distribution with to the observed statistics of wave heights in our simulated nonlinear wavefields yields interesting finds. Correlation coefficient between simulated wave height distribution data and theoretical Rayleigh Distribution are shown in

Figure 5-4. A correlation coefficient, $r^{2}$ can take values between 0 and +1 where $r^{2}=1$ indicates that the wave height distribution correlates well with the Rayleigh Distribution, and $r^{2}=0$ means wave height distribution and the Rayleigh distribution are uncorrelated. We observe the effect of peak shape parameter on this correlation for three different wavefields: Cases $\mathbf{I}$ (where $\gamma=1.0$ ), $\mathbf{B}$ (where $\gamma=3.3$ ) and $\mathbf{H}$ (where $\gamma=5.0)$. From this result we see that the lowest peak shape parameter produces the greatest correlation coefficient between our observed non-linear, non-narrow-band defined wave heights and the Rayleigh distribution. 

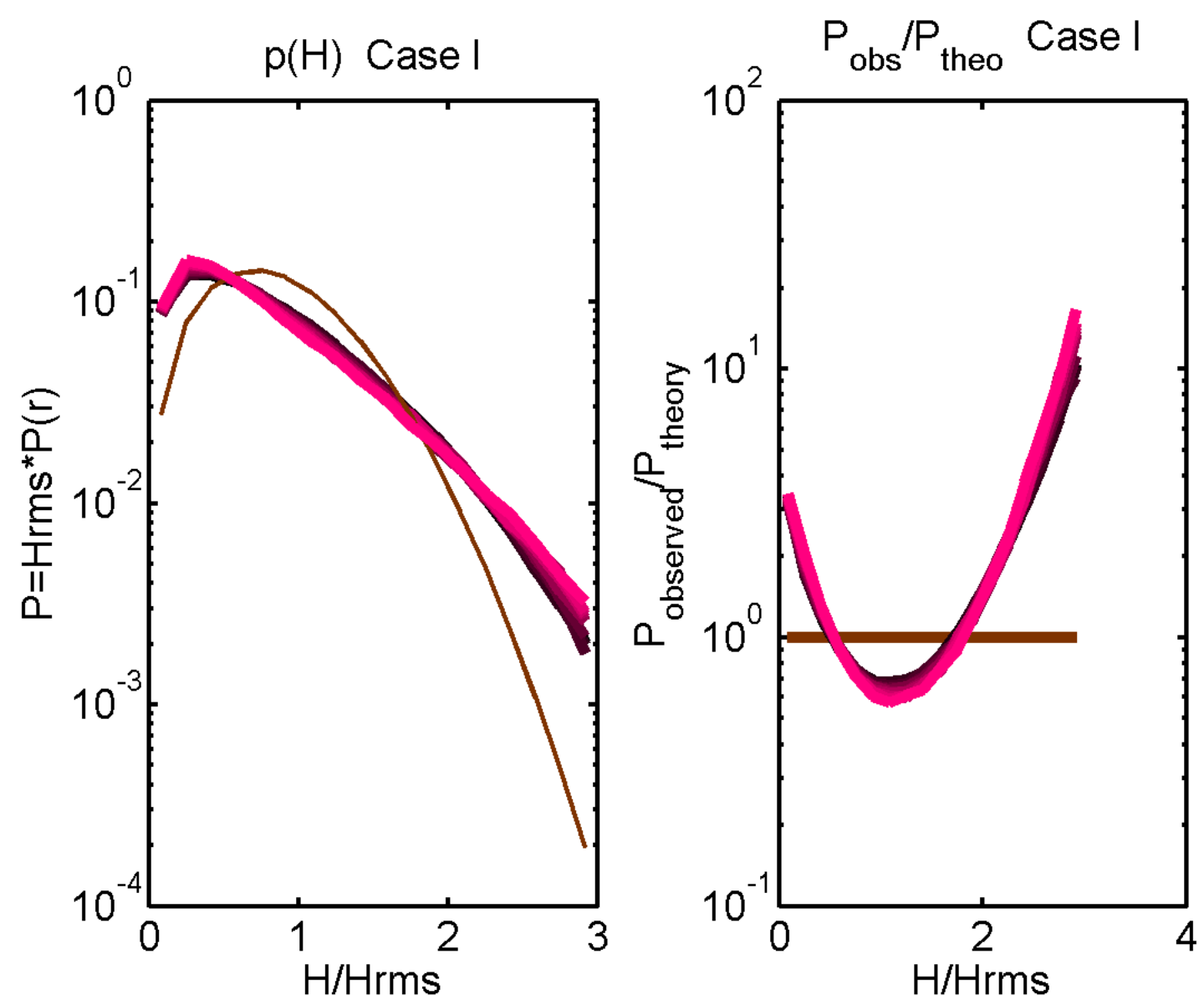

Figure 5-1: (In Color) (Left) Horizontal axis shows non-dimensional wave height and vertical axis shows the probability of occurrence of heights in the wavefield. Thick, brown line represents Rayleigh distribution. Other lines represent Case I where $\gamma=1.0$, each such line representing one time step. Earlier times (from $t=0 T_{p}$ ) are the darkest lines. Later times (up to $t=100 T_{p}$ ) are the brightest lines. (Right) Horizontal axis shows non-dimensional wave height and vertical axis shows a probability ratio. Each line represents the ratio between a Case I time-step line and the Rayleigh distribution. 

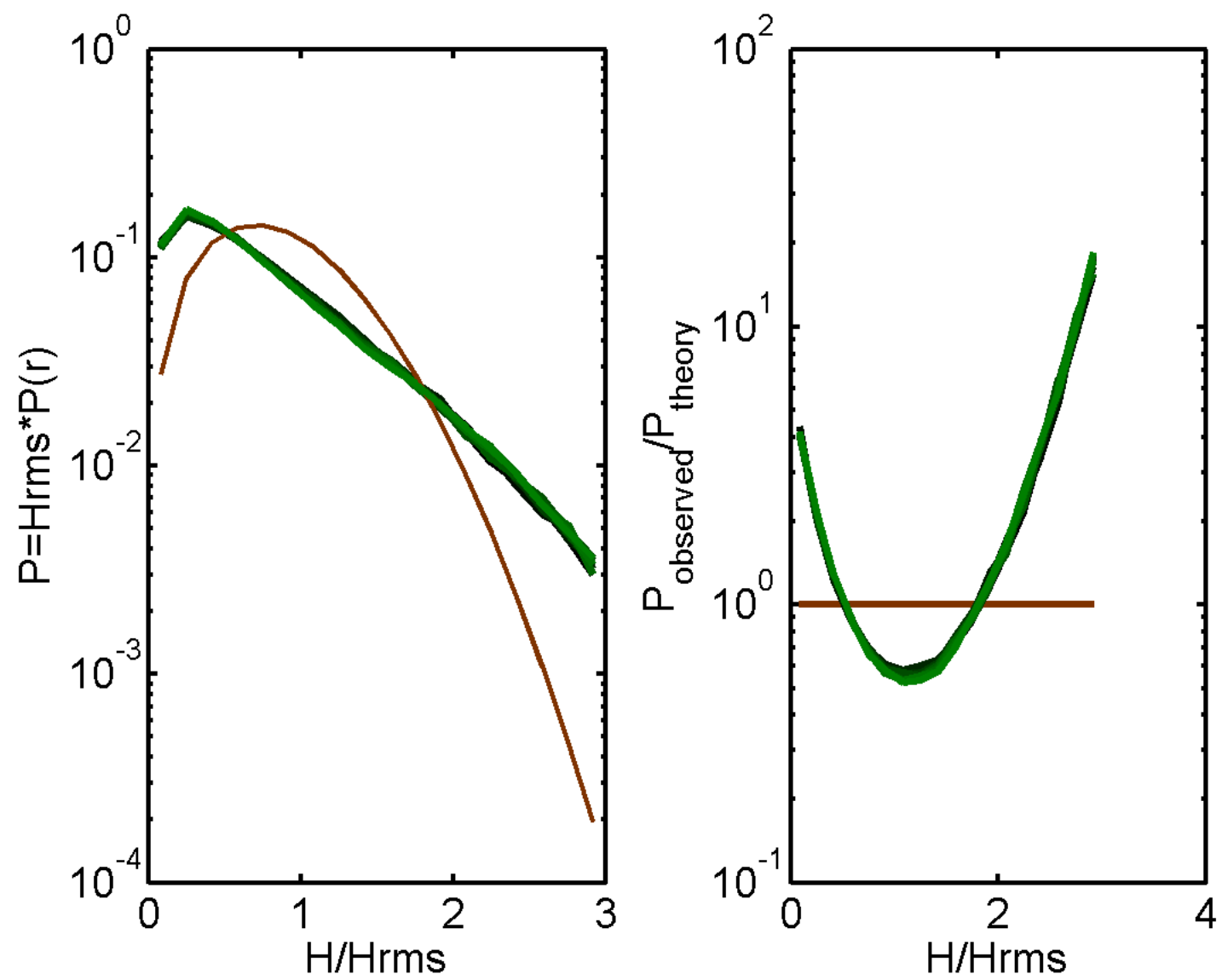

Figure 5-2: (In Color) (Left) Horizontal axis shows non-dimensional wave height and vertical axis shows the probability of occurrence of heights in the wavefield. Thick, brown line represents Rayleigh distribution. Other lines represent Case B where $\gamma=3.3$, each such line representing one time step. Earlier times (from $t=0 T_{p}$ ) are the darkest lines. Later times (up to $t=100 T_{p}$ ) are the brightest lines. (Right) Horizontal axis shows non-dimensional wave height and vertical axis shows a probability ratio. Each line represents the ratio between a Case B time-step line and the Rayleigh distribution. 

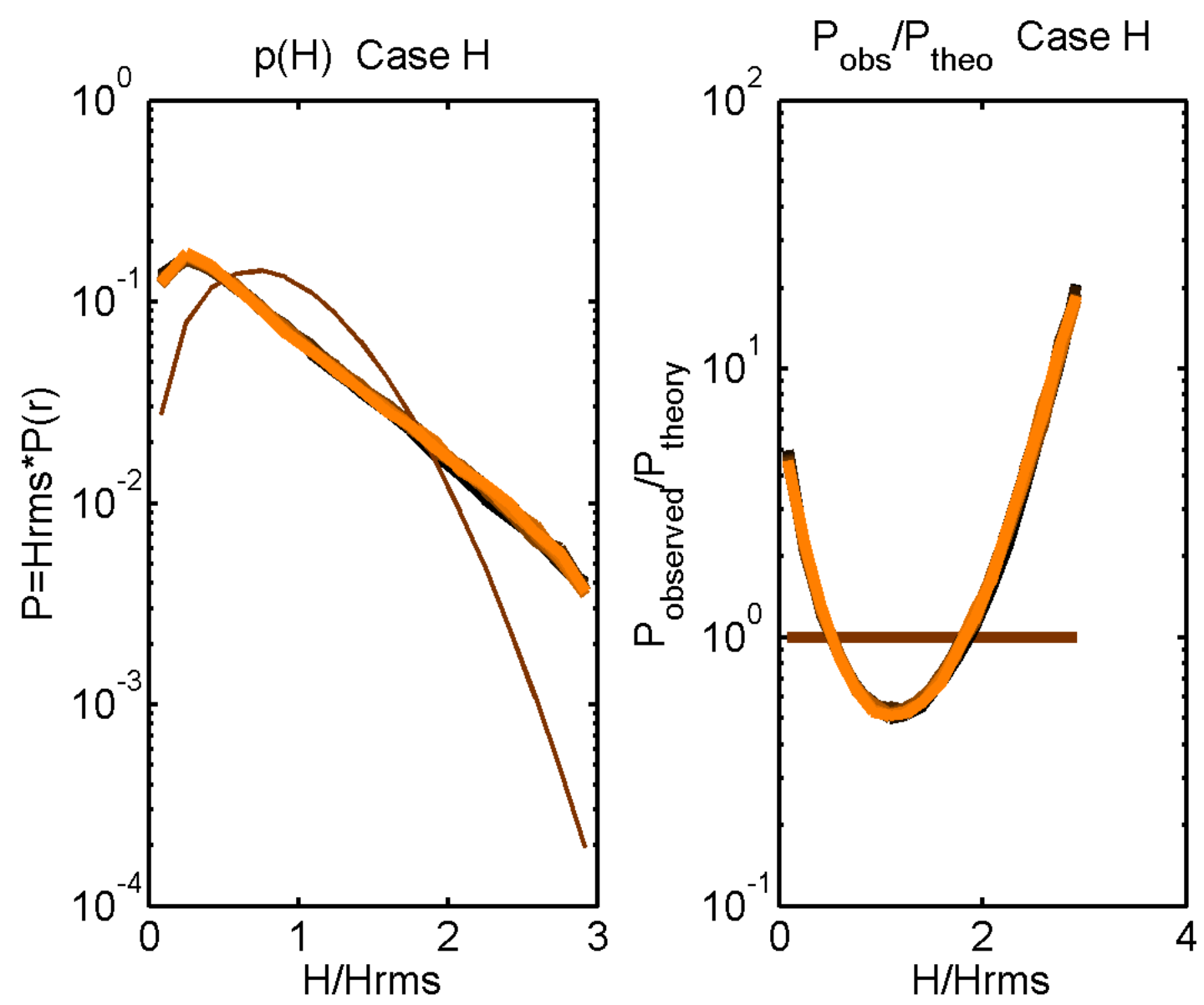

Figure 5-3: (In Color) (Left) Horizontal axis shows non-dimensional wave height and vertical axis shows the probability of occurrence of heights in the wavefield. Thick, brown line represents Rayleigh distribution. Other lines represent Case $\mathbf{H}$ where $\gamma=5.0$, each such line representing one time step. Earlier times (from $t=0 T_{p}$ ) are the darkest lines. Later times (up to $t=100 T_{p}$ ) are the brightest lines. (Right) Horizontal axis shows non-dimensional wave height and vertical axis shows a probability ratio. Each line represents the ratio between a Case $\mathbf{H}$ time-step line and the Rayleigh distribution. 


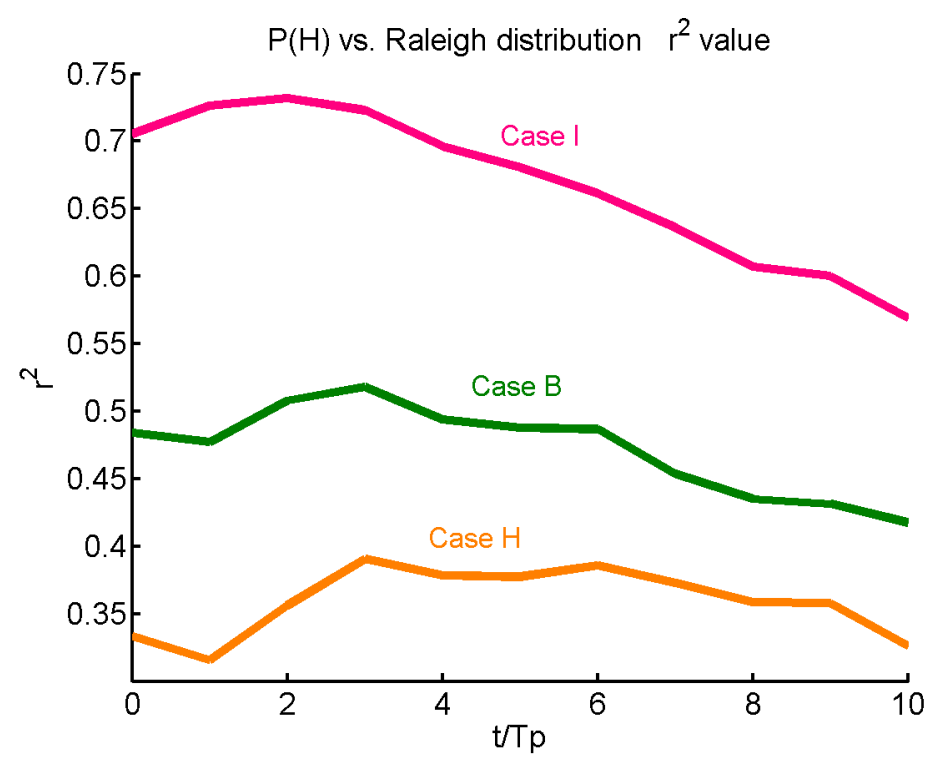

Figure 5-4: (In Color) Vertical axis represents correlation coefficient and horizontal axis represents time. Correlation coefficient between simulated wave height distribution data and theoretical Rayleigh Distribution are shown. A correlation coefficient, $r^{2}$ can take values between 0 and +1 where $r^{2}=1$ indicates that the wave height distribution correlates well with the Rayleigh Distribution, and $r^{2}=0$ means wave height distribution and the Rayleigh distribution are uncorrelated. We observe the effect of peak shape parameter on this correlation for three different wavefields: Cases $\mathbf{I}($ where $\gamma=1.0), \mathbf{B}($ where $\gamma=3.3)$ and $\mathbf{H}$ (where $\gamma=5.0$ ). 
Taking the integral of the wave height distribution between zero and a certain wave height gives the probability of exceeding that wave height [33]. In such a plot we see a different perspective of the previously plotted data in Figures 5-5, 5-6, and 5-7. In this perspective it seems clearer that the passage of time allows for the wave height distributions in Case $\mathbf{I}$ where $\gamma=1.0$ to move away from the linear narrow band theory, but for Cases $\mathbf{B}$ where $\gamma=3.3$ and $\mathbf{H}$ where $\gamma=5.0$ to move closer to the theory. Note from Figure 5-4 that Case I starts and remains nearest the linear narrow band theory while the other two cases are further away for the entire duration of the simulation.

We observe the effect of peak shape parameter, $\gamma$, on the distribution of the first moment of the wave heights. We generate the first moment distribution by observing $p$ (where $0<p<1$ ) v.s. $H^{(p)}$, the mean of the highest $p$ wave heights, $H$ in a wavefield. Longuet-Higgins derives a theoretical distribution for the case of the linear, narrow-band wavefield by integrating the product of the Rayleigh distribution height probability and its corresponding height [33]. We compare this theoretical distribution to our simulated nonlinear wavefields. We conclude from Figures 5-8, 5-9, and 5-10 that a higher $\gamma$ puts the first moment distribution further from the theoretical linear, narrow-band theory at the start of the time evolution, but allows for the wavefield to move towards this theory in time (see Figure 5-10). A lower $\gamma$ puts the first moment distribution nearer to the theoretical linear, narrow-band theory at the start of the time evolution, but allows for the wavefield to move away from theory in time (see Figures 5-8 and 5-9).

In this section we observe the effect of peak shape parameter, $\gamma$ on non-linear, non-narrow-band wave heights by comparing the deviations of simulated wavefields of varying $\gamma$-values to the linear, narrow-band theory of wave height distributions. The linear narrow-band theory under-predicts large wave heights in non-linear, nonnarrow-band wavefields. In our wavefields we confirm this. We learn that peak shape parameter determines how much the linear narrow-band theory under-predicts the occurrence of large waves. Higher $\gamma$ values produce greater under-prediction of large wave occurrence by the linear narrow-band theory. We conclude that non-linear, 

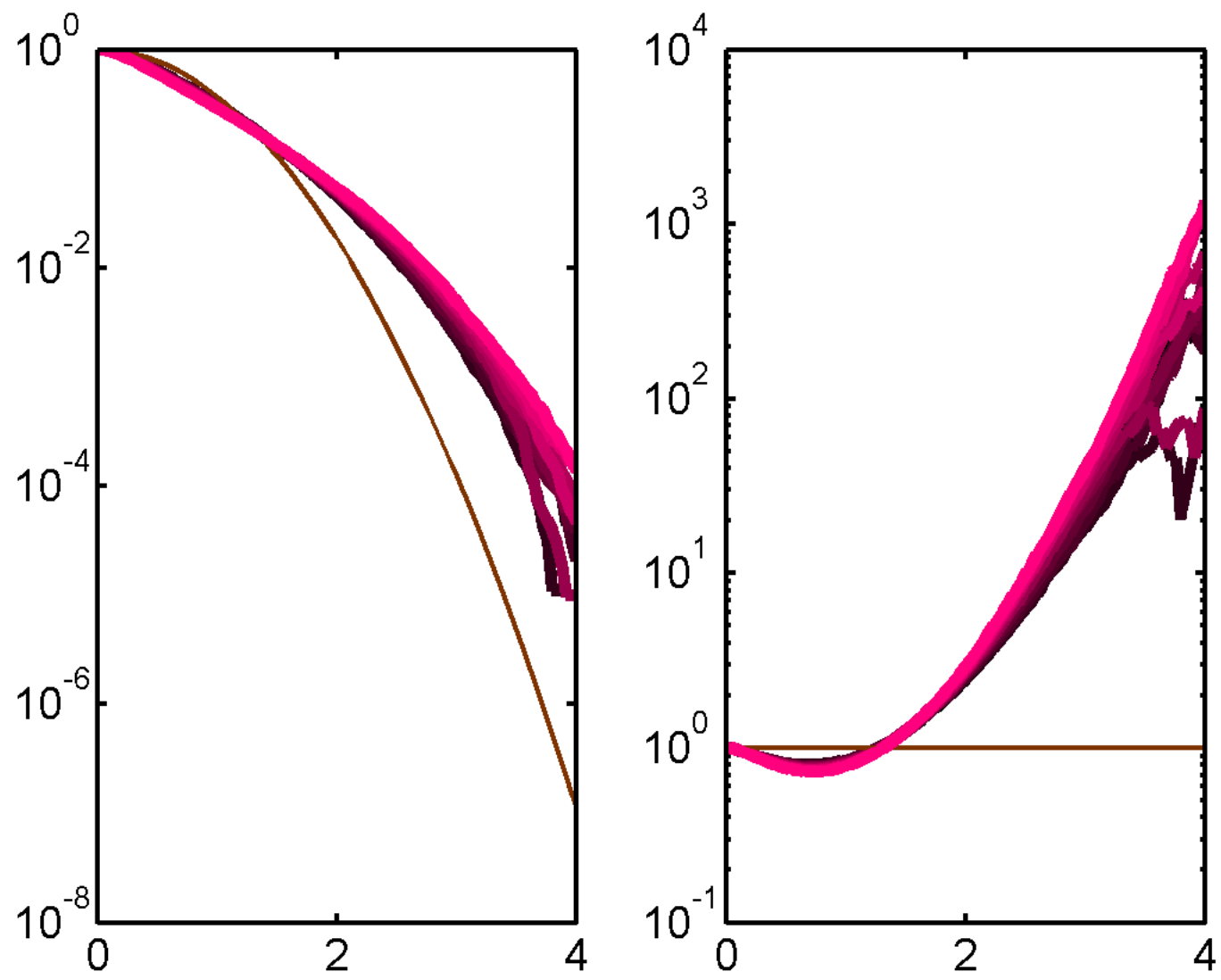

Figure 5-5: (In Color) Left: Horizontal axis shows non-dimensional wave height and vertical axis shows the probability of exceeding each height in the wavefield. Thick, brown line represents linear narrow band theory for the distribution of wave height exceedance probability. Other lines represent Case $\mathbf{I}$ where $\gamma=1.0$, each such line representing one time step. Earlier times (from $t=0 T_{p}$ ) are the darkest lines. Later times (up to $t=100 T_{p}$ ) are the brightest lines. Right: Horizontal axis shows nondimensional wave height and vertical axis shows a probability ratio. Each line represents the ratio between a Case $\mathbf{I}$ time-step line and the linear narrow band theory for the wave height exceedance probability distribution. 

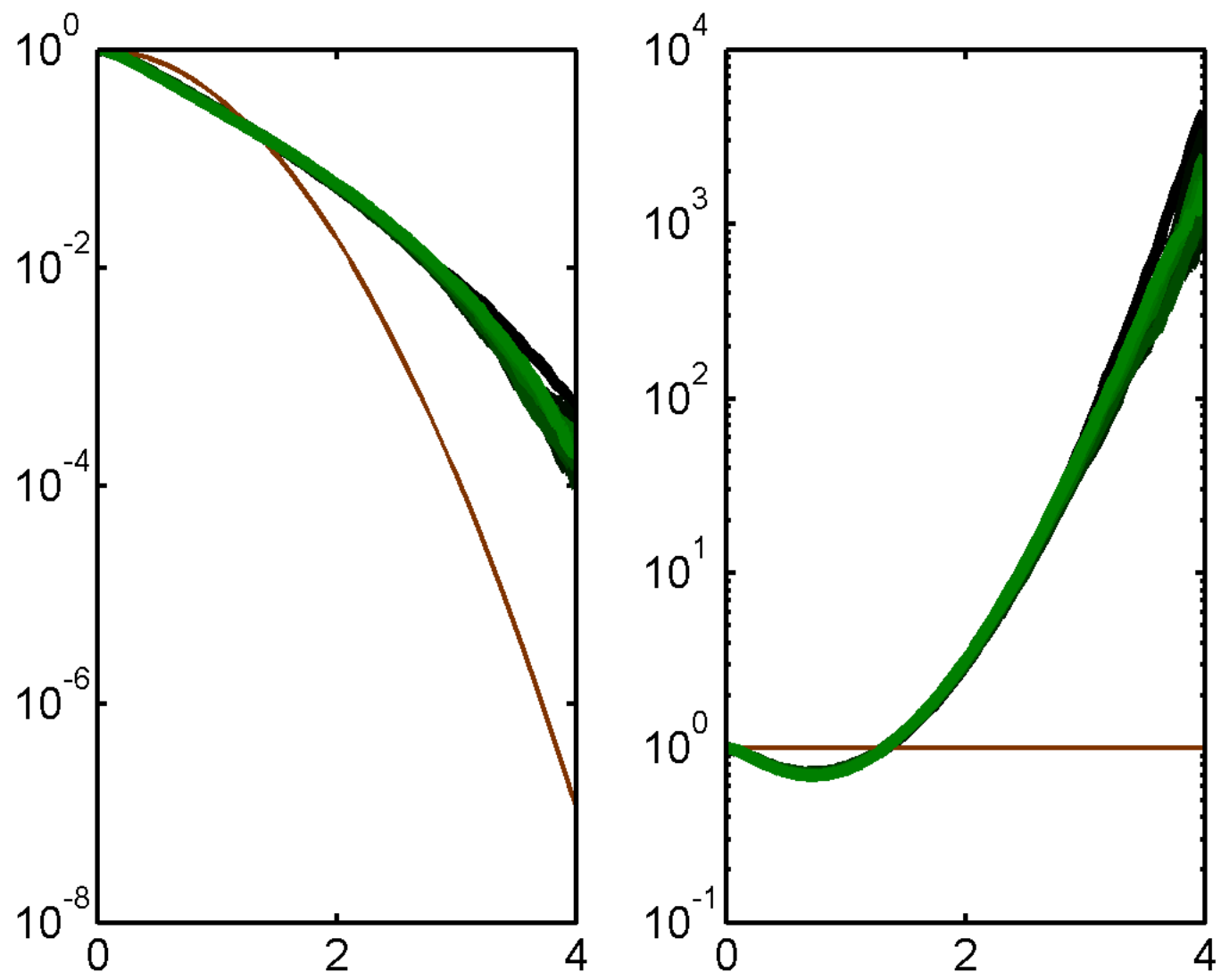

Figure 5-6: (In Color) Left: Horizontal axis shows non-dimensional wave height and vertical axis shows the probability of exceeding each height in the wavefield. Thick, brown line represents linear narrow band theory for the distribution of wave height exceedance probability. Other lines represent Case $\mathbf{B}$ where $\gamma=3.3$, each such line representing one time step. Earlier times (from $t=0 T_{p}$ ) are the darkest lines. Later times (up to $t=100 T_{p}$ ) are the brightest lines. Right: Horizontal axis shows non-dimensional wave height and vertical axis shows a probability ratio. Each line represents the ratio between a Case $\mathbf{B}$ time-step line and the linear narrow band theory for the wave height exceedance probability distribution. 

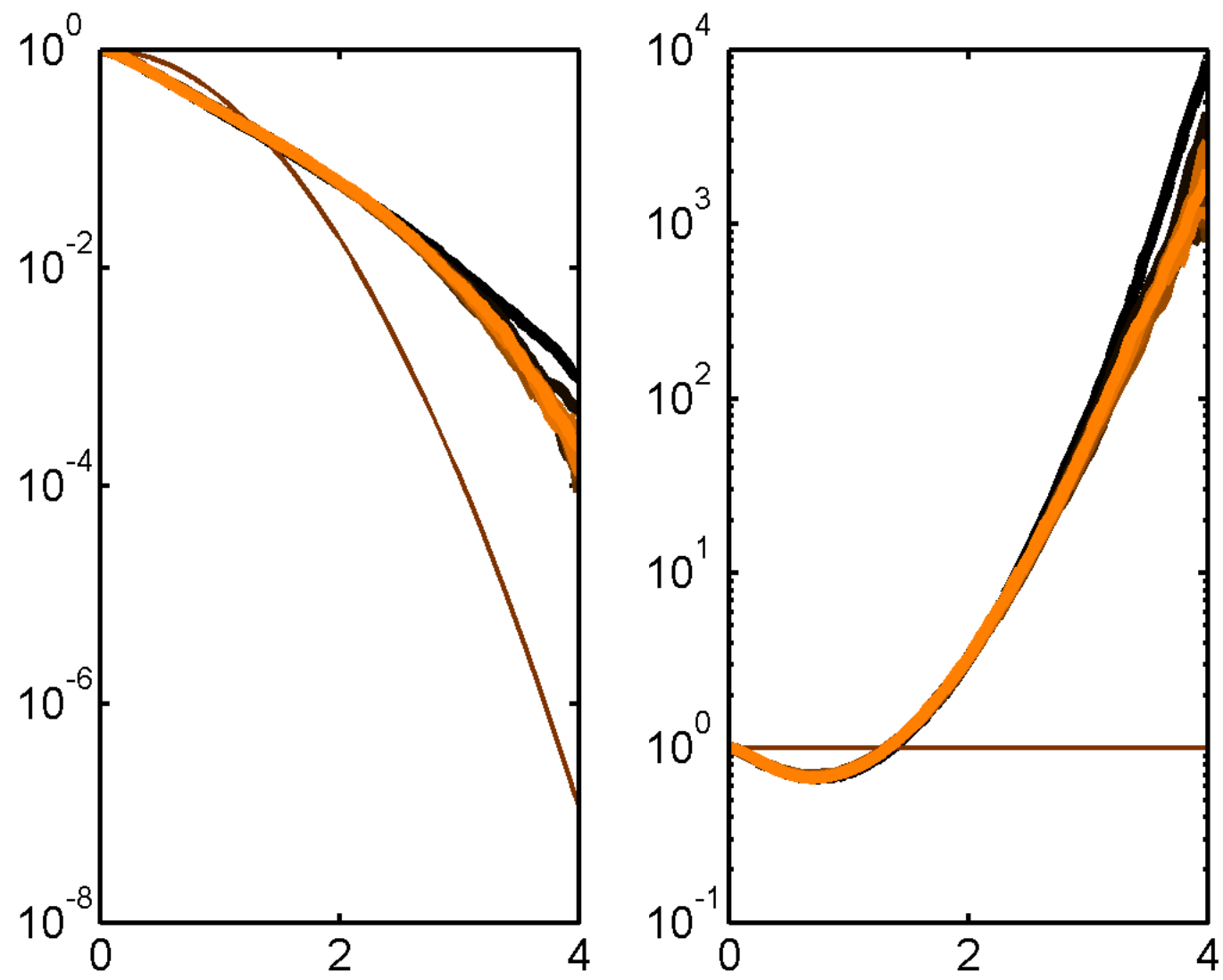

Figure 5-7: (In Color) Left: Horizontal axis shows non-dimensional wave height and vertical axis shows the probability of exceeding each height in the wavefield. Thick, brown line represents linear narrow band theory for the distribution of wave height exceedance probability. Other lines represent Case $\mathbf{H}$ where $\gamma=5.0$, each such line representing one time step. Earlier times (from $t=0 T_{p}$ ) are the darkest lines. Later times (up to $t=100 T_{p}$ ) are the brightest lines. Right: Horizontal axis shows non-dimensional wave height and vertical axis shows a probability ratio. Each line represents the ratio between a Case $\mathbf{H}$ time-step line and the linear narrow band theory for the wave height exceedance probability distributions in the Left graphic. 

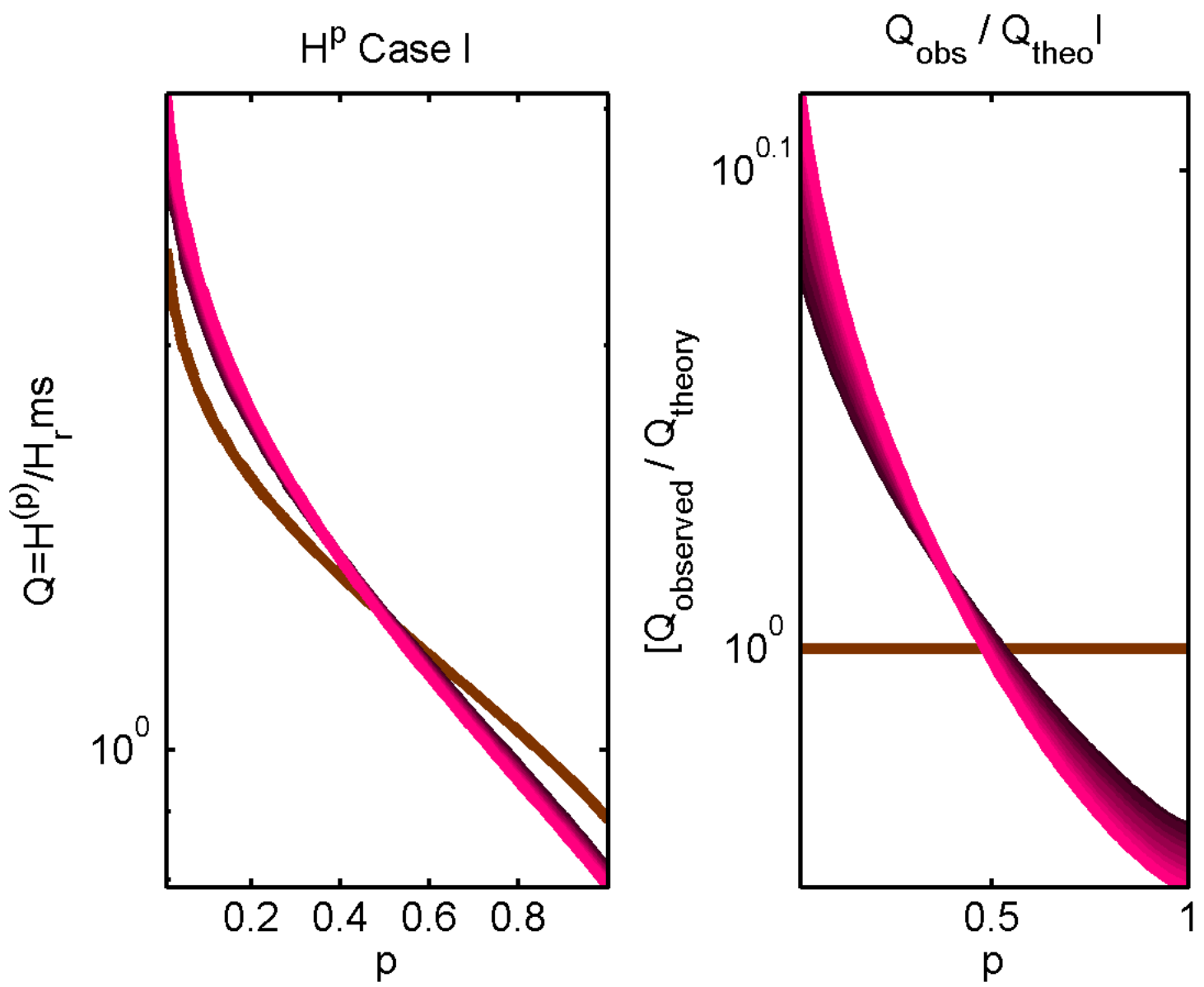

Figure 5-8: (In Color) Left: Horizontal axis shows probability, $p$ where $0<p<1$ and vertical axis shows the mean of the highest $p$ waves, $H^{(p)}$ in the wavefield. Each line represents a time in the time evolution of first-moment distribution in Case $\mathbf{I}$ (where $\gamma=1.0$ ). Graphics depict the mean of the highest $p$ waves, $H^{(p)}$, v.s. $p$, where $0<p<1$. Earlier times are the darkest lines. Later times are the brightest lines. Right: Horizontal axis shows probability, $p$ where $0<p<1$ and vertical axis shows a height ratio. Each line represents the ratio between a Case $\mathbf{I}$ observed time-step line in the Left graphic and the linear narrow band theory for the mean of the highest $p$ waves, $H^{(p)}$ v.s. $p$ in the wavefield. 

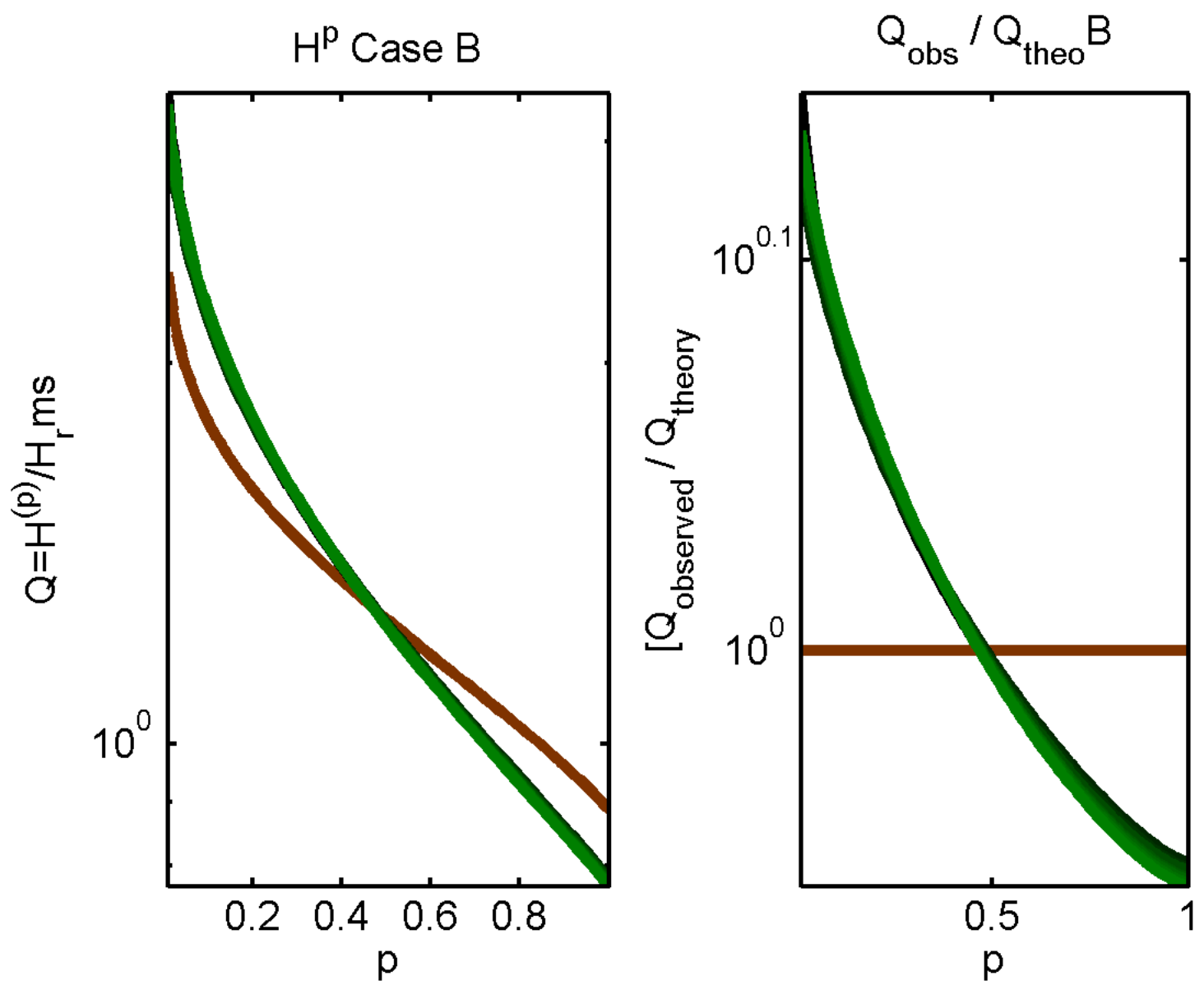

Figure 5-9: (In Color) Left: Horizontal axis shows probability, $p$ where $0<p<1$ and vertical axis shows the mean of the highest $p$ waves, $H^{(p)}$ in the wavefield. Each line represents a time in the time evolution of first-moment distribution in Case $\mathbf{B}$ (where $\gamma=3.3$ ). Graphics depict the mean of the highest $p$ waves, $H^{(p)}$, v.s. $p$, where $0<p<1$. Earlier times are the darkest lines. Later times are the brightest lines. Right: Horizontal axis shows probability, $p$ where $0<p<1$ and vertical axis shows a height ratio. Each line represents the ratio between a Case $\mathbf{B}$ observed time-step line in the Left graphic and the linear narrow band theory for the mean of the highest $p$ waves, $H^{(p)}$ v.s. $p$ in the wavefield. 

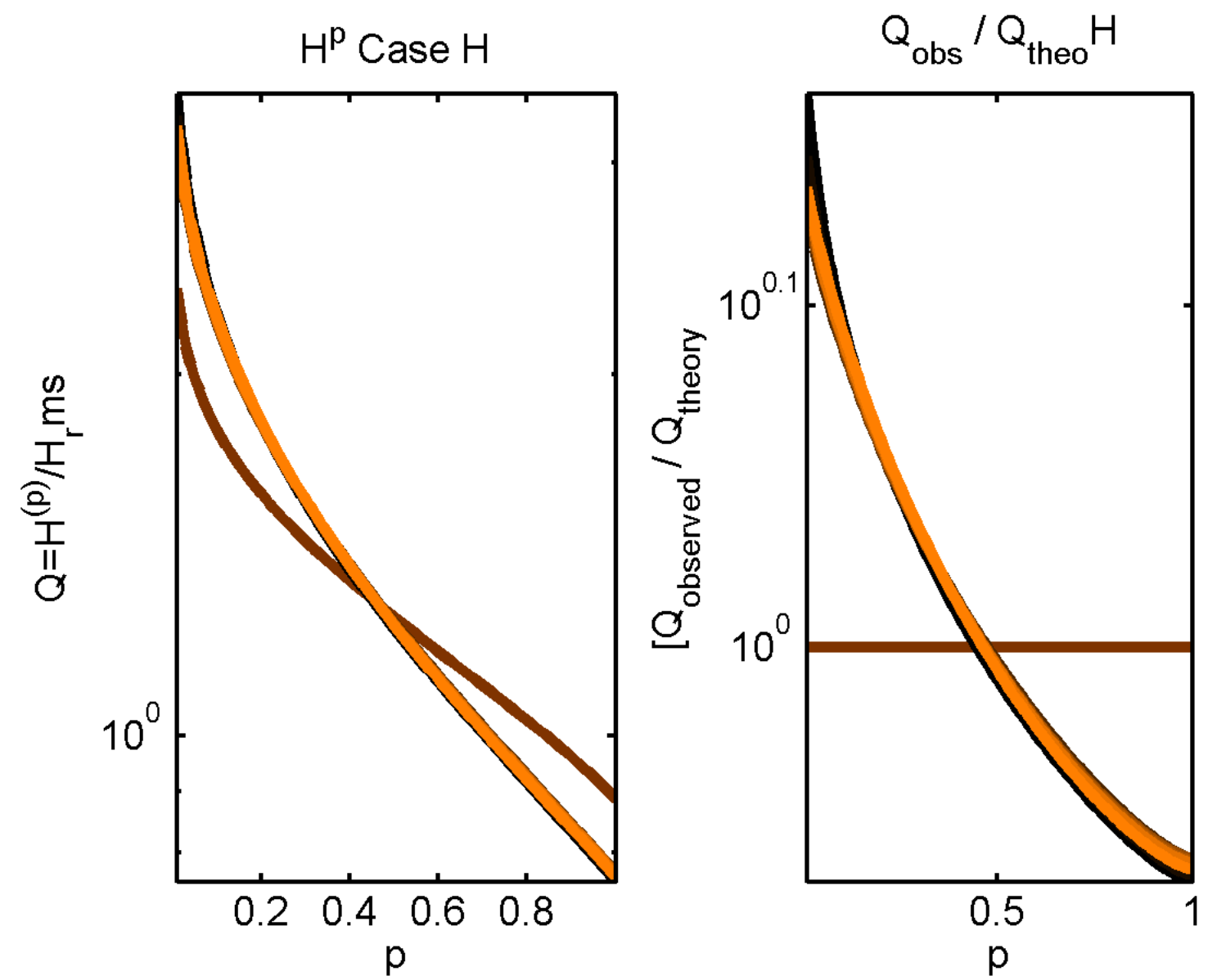

Figure 5-10: (In Color) Left: Horizontal axis shows probability, $p$ where $0<p<1$ and vertical axis shows the mean of the highest $p$ waves, $H^{(p)}$ in the wavefield. Each line represents a time in the time evolution of first-moment distribution in Case $\mathbf{H}$ (where $\gamma=5.0$ ). Graphics depict the mean of the highest $p$ waves, $H^{(p)}$, v.s. $p$, where $0<p<1$. Earlier times are the darkest lines. Later times are the brightest lines. Right: Horizontal axis shows probability, $p$ where $0<p<1$ and vertical axis shows a height ratio. Each line represents the ratio between a Case $\mathbf{H}$ observed time-step line in the Left graphic and the linear narrow band theory for the mean of the highest $p$ waves, $H^{(p)}$ v.s. $p$ in the wavefield. 
non-narrow-band wave height probability distributions are sensitive to peak shape parameter.

\subsubsection{Peak shape parameter and the joint distribution be- tween wave height and wavelength}

In this section we observe the impact of peak shape parameter, $\gamma$ on the joint distribution between wave height and wave length.

Theoretically, Longuet-Higgins [34] derives a joint distribution between wave height and wave period. To derive such a theory, waves must follow a Gaussian, random process (so ergodicity holds in any wavefield for which this theory holds). Considering the wavefield as a sum of cosines with random phase, amplitudes and periods, expressing surface elevation as a sum of Fourier terms in a narrow-band process around a mean frequency, and finally assuming a narrow band wavefield around the mean frequency, Longuet-Higgins develops a joint distribution between the four Fourier transform series of surface elevation position and velocity which are all statistically independent and normally distributed. Expressing this joint distribution in terms of dimensionless period, $L$ and dimensionless amplitude, $H$ gives:

$$
f(H, L)=\frac{1}{8 \sqrt{2 \pi} \nu}\left(1+\frac{\nu^{2}}{4}\right)\left(\frac{H}{L}\right)^{2} \exp \frac{-H^{2}}{8}\left[1+\left(1-\frac{1}{L}\right)^{2} \frac{1}{\nu^{2}}\right]
$$

This joint distribution function for $0<H<\infty$ and $0<L<\infty$ is depicted in Figure 5-11, for different values of spectral width, $\nu$. Since it is taken for granted that the ergodic property holds for random waves [41], we consider the distributions of wavelength in SNOW wavefields (which are initialized as random), to be equivalent to the distribution of wave periods in Longuet-Higgins' theory. We see evidence of the validity of such an assumption in our results.

At initial time, we compare scatter plots in Figures 5-12, 5-13 and 5-14 to observe that initially, the longest, largest non-linear, non-narrow-band waves are associated with the highest peak shape parameter.

At time, $t=50 T_{p}$ we compare scatter plots in Figures 5-15, 5-49 and 5-17 to 
observe that the wave fields are all growing in the direction of longer, higher waves, but that the longest, largest non-linear, non-narrow-band waves are still associated with the highest peak shape parameter. This same trend persists at time, $t=100 T_{p}$ as we confirm in comparing Figures 5-18, 5-52 and 5-20.

Throughout the time evolution of the wavefields we observe the effects of peak shape parameter, $\gamma$ on the joint distribution between wave height and wavelength for non-linear, non-narrow-band waves. Higher spectral peak shape parameters produce more large waves with large wavelengths.

\subsubsection{Peak shape parameter and wave length distribution}

In this section we observe the effect of $\gamma$ on the distribution of wave lengths in a SNOW wavefield. The distribution of wave periods (and equivalently wavelengths in ergodicity) was derived by Longuet-Higgins as the marginal distribution attained in integrating Equation 5.1 over all wave heights. Under a large spectral bandwidth, we can see that this distribution is identical to the theoretical Rayleigh distribution and we compare our non-linear, non-narrow-band wavelength distributions to the Rayleigh distribution in this work. It should be noted that in a directional sea, where we tamper with the randomness of the wavefield [4] ergodicity does not hold. Despite this, in Figures 5-21, 5-22 and 5-23 that the theoretical Rayleigh distribution matches the wavelength in our non-linear non-narrow-band wavelength definitions quite well. Higher peak shape parameter produces a better total correlation between the observed data and the Rayleigh distribution, as seen in Figure 5-24. 

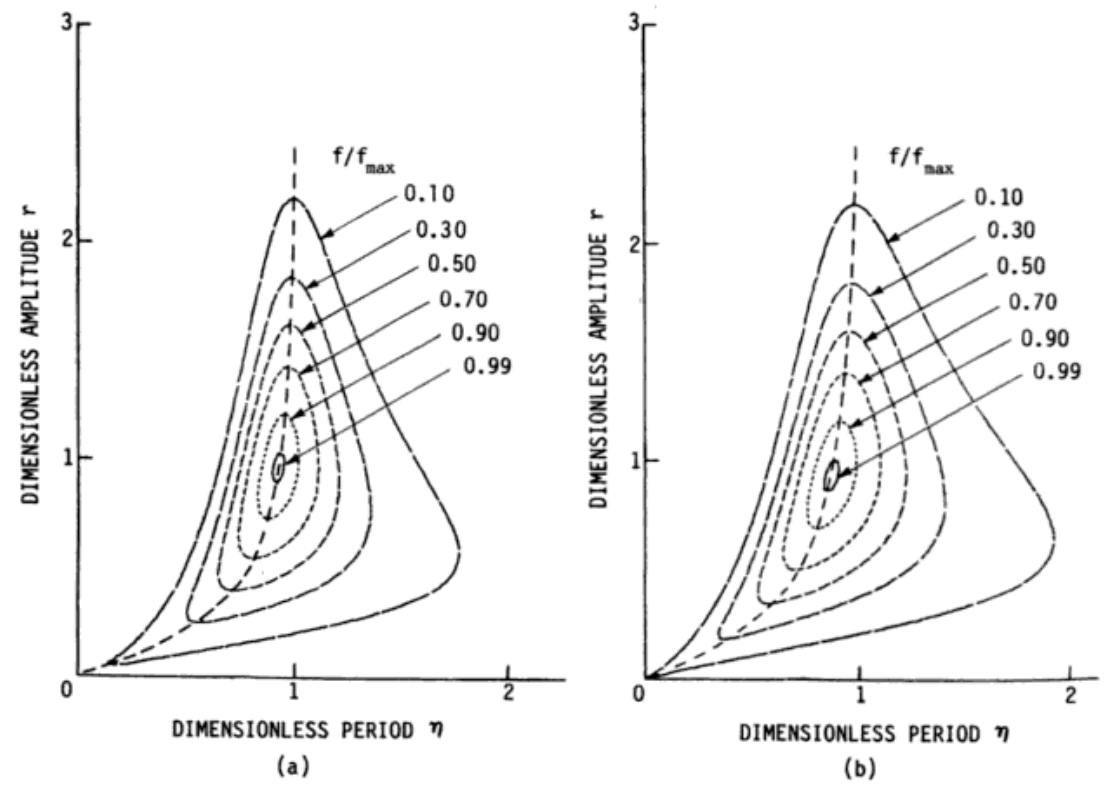

Figure 5-11: Theoretical contours of dimensionless joint probability density function of wave amplitude and wave period for spectral width parameters, (a) $\nu=0.3$ and (b) $\nu=0.4[34]$. 


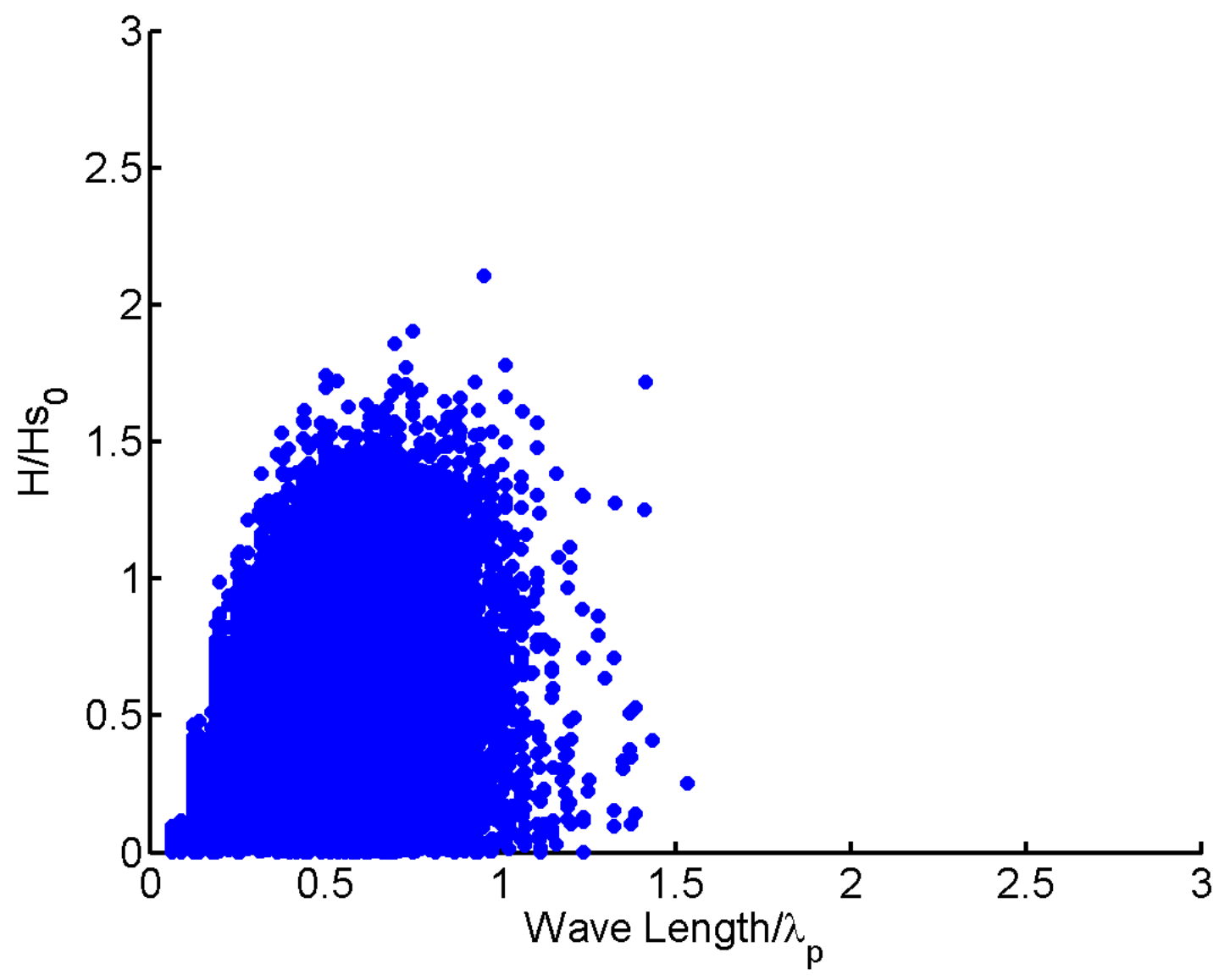

Figure 5-12: Scatter plot of dimensionless wave height vs. dimensionless wavelength in Case $\mathbf{I}$ (where $\gamma=1.0$ ) at time, $t=0 T_{p}$. 


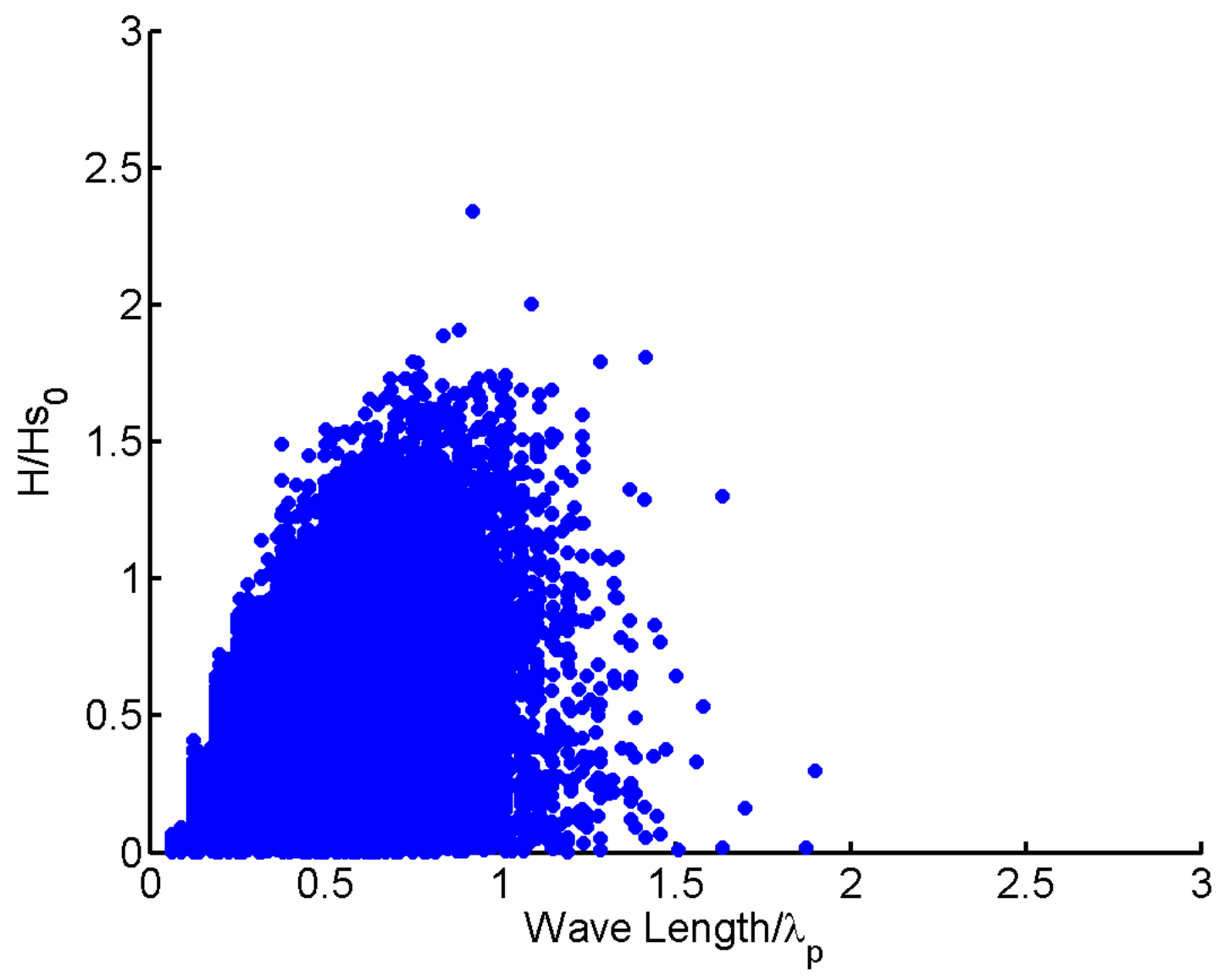

Figure 5-13: Scatter plot of dimensionless wave height vs. dimensionless wavelength in Case $\mathbf{B}$ (where $\gamma=3.3$ ) at time, $t=0 T_{p}$. 


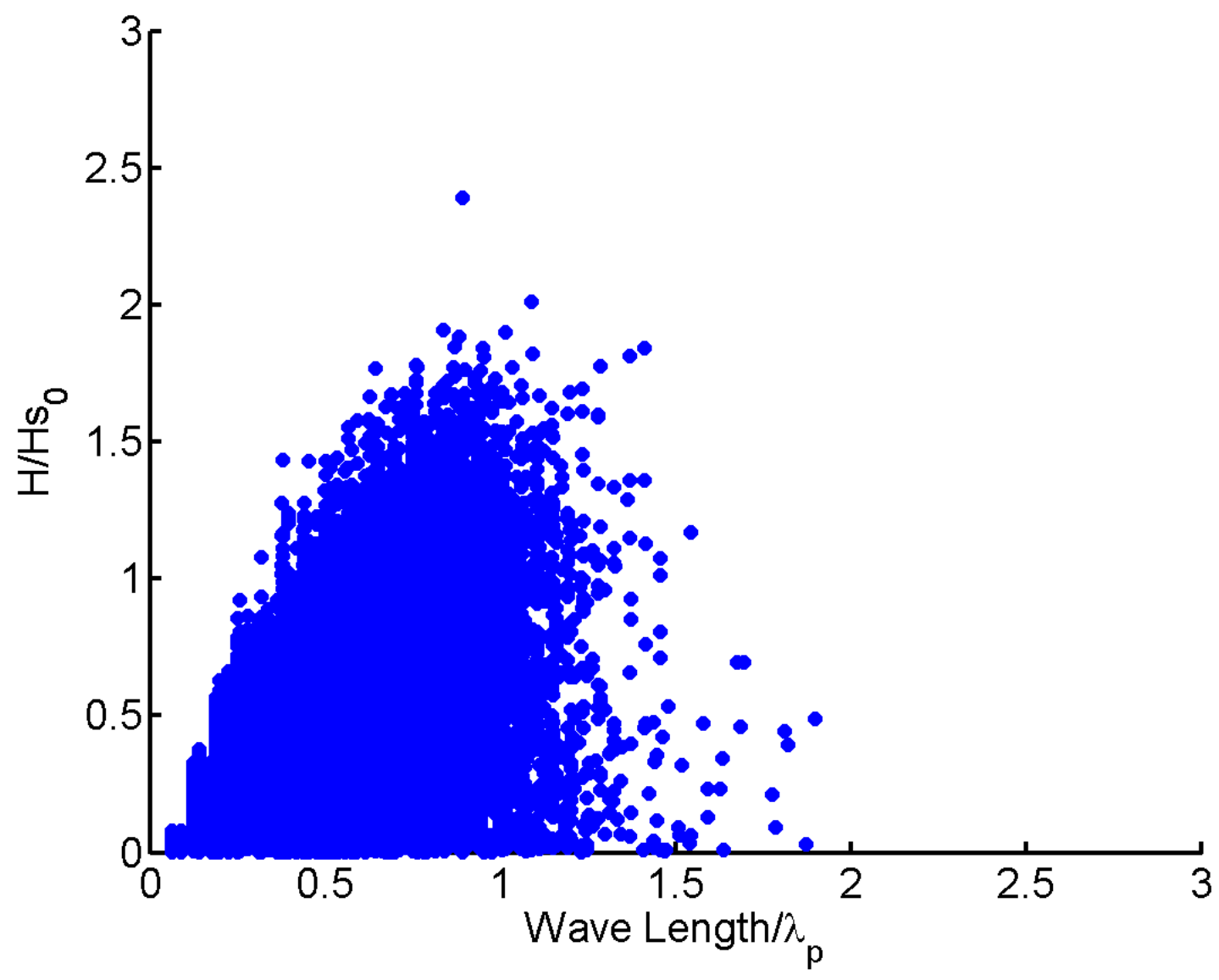

Figure 5-14: Scatter plot of dimensionless wave height vs. dimensionless wavelength in Case $\mathbf{H}$ (where $\gamma=5.0$ ) at time, $t=0 T_{p}$. 


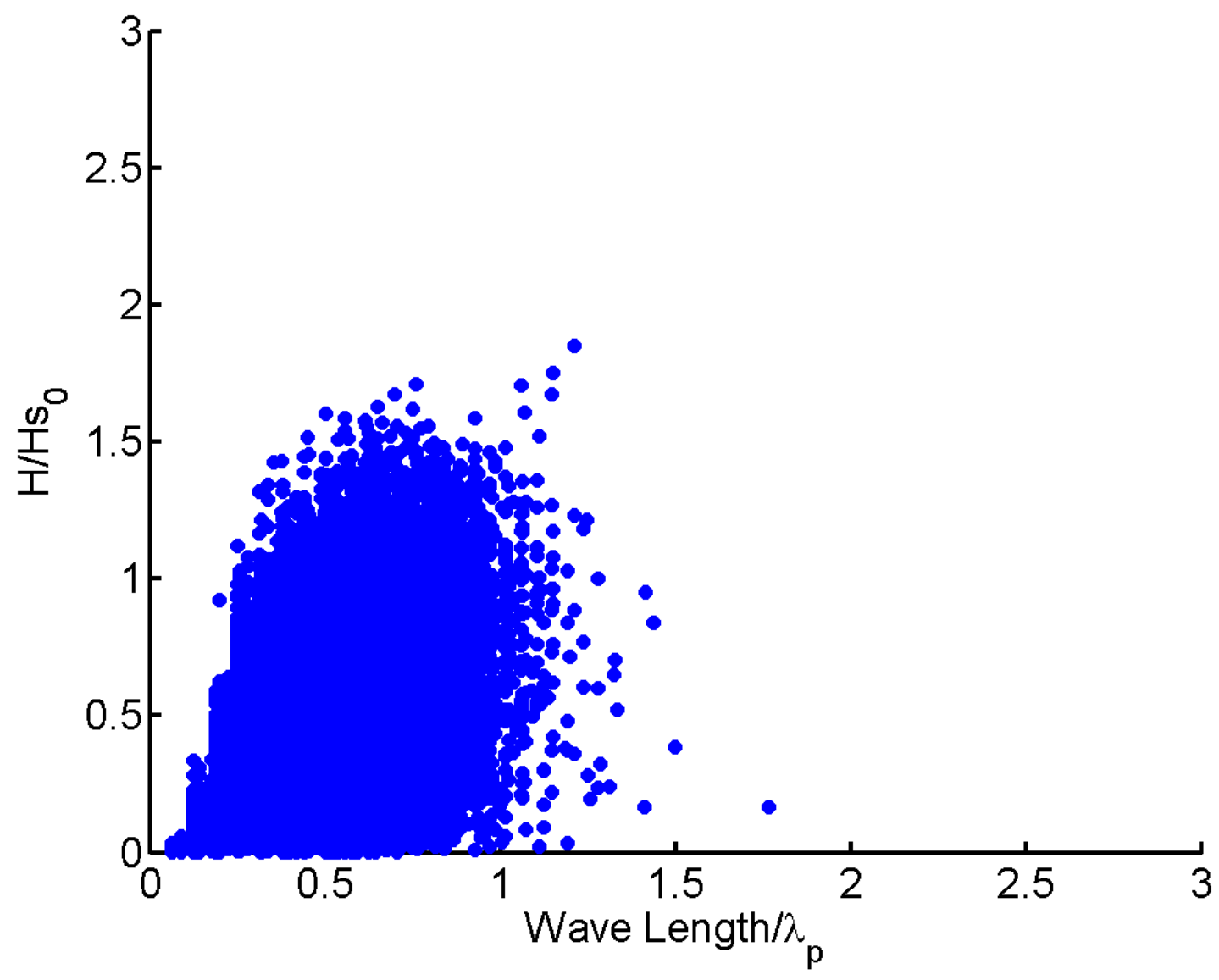

Figure 5-15: Scatter plot of dimensionless wave height vs. dimensionless wavelength in Case $\mathbf{I}$ (where $\gamma=1.0$ ) at time, $t=50 T_{p}$. 


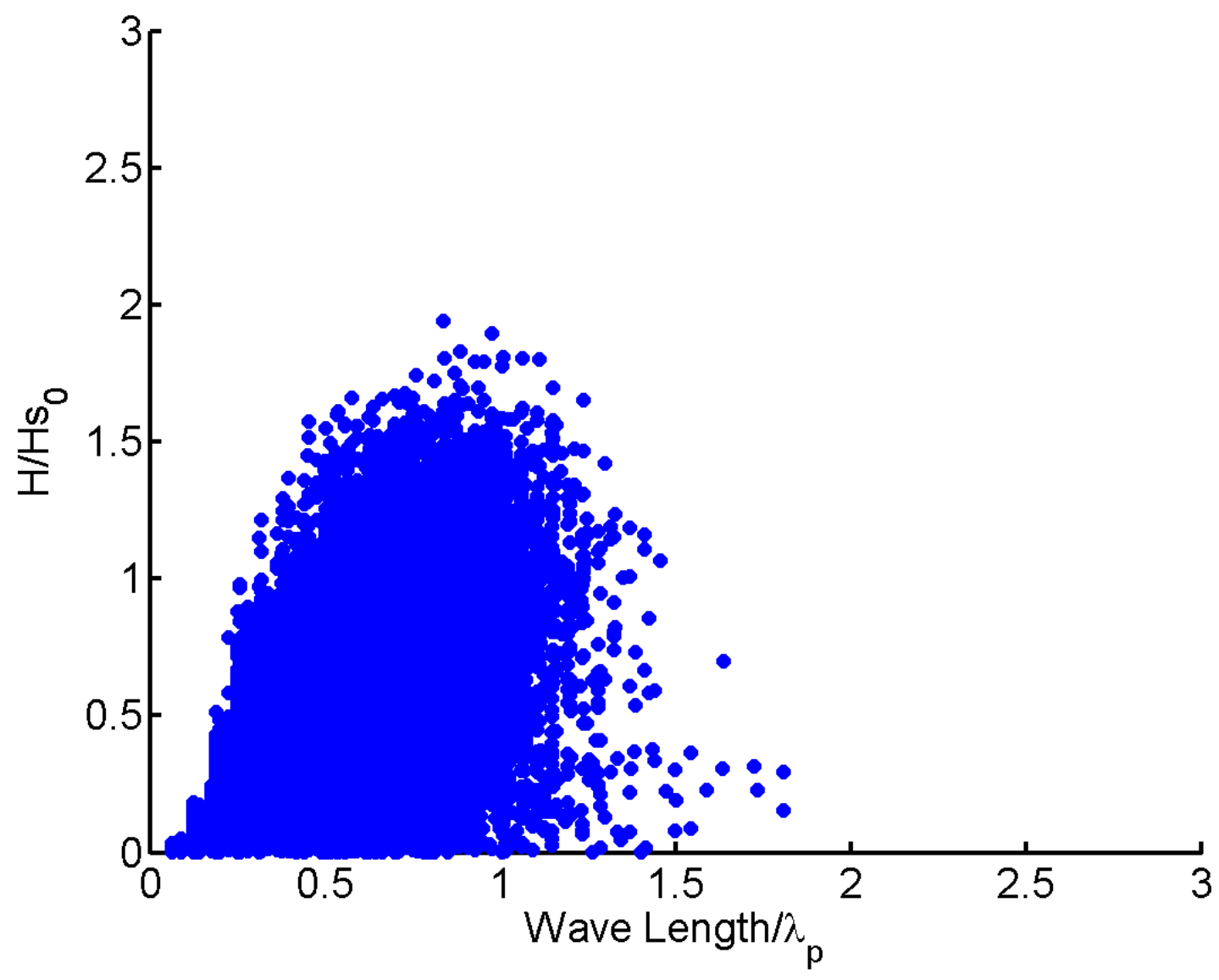

Figure 5-16: Scatter plot of dimensionless wave height vs. dimensionless wavelength in Case $\mathbf{B}$ (where $\gamma=3.3$ ) at time, $t=50 T_{p}$. 


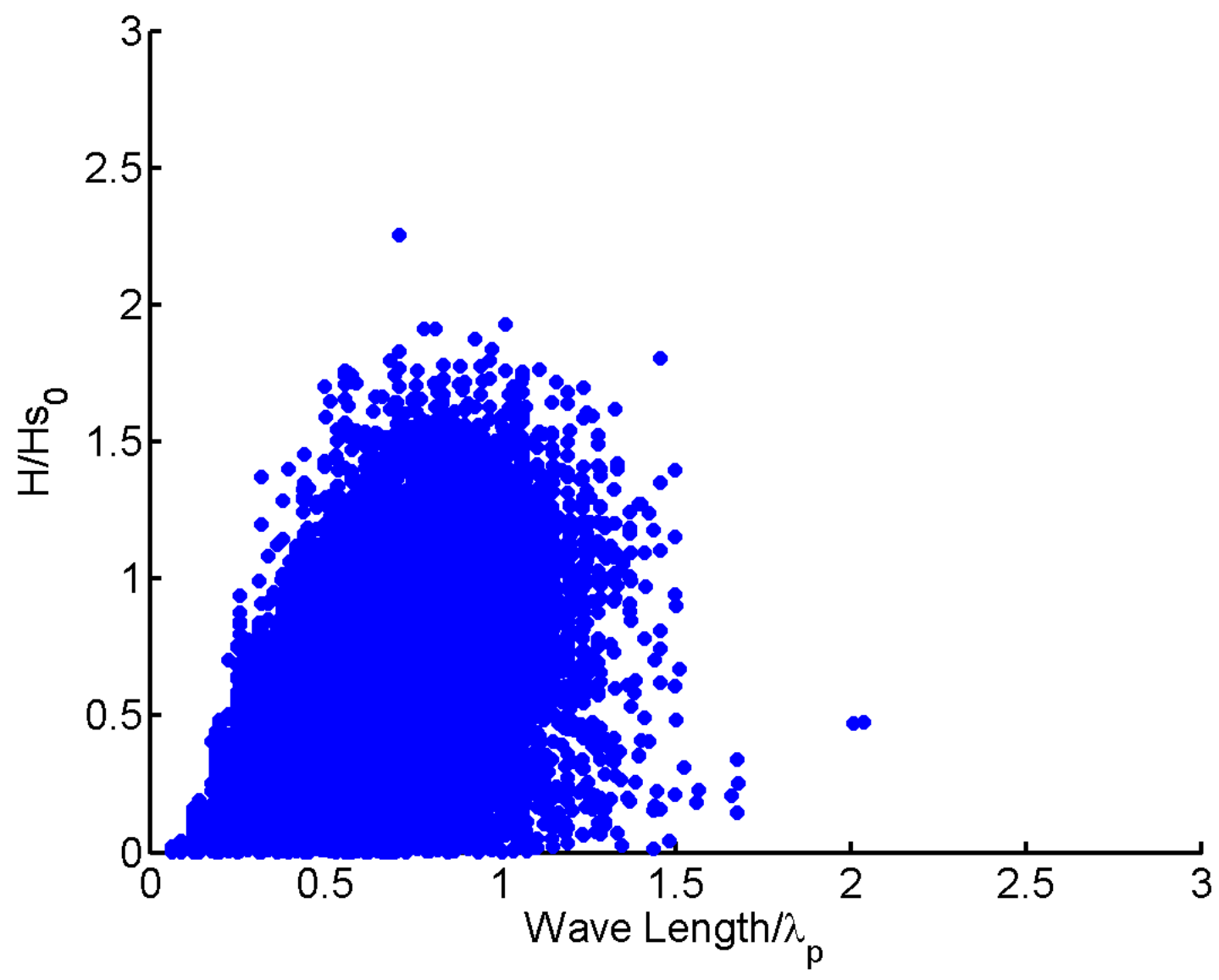

Figure 5-17: Scatter plot of dimensionless wave height vs. dimensionless wavelength in Case $\mathbf{H}$ (where $\gamma=5.0$ ) at time, $t=50 T_{p}$. 


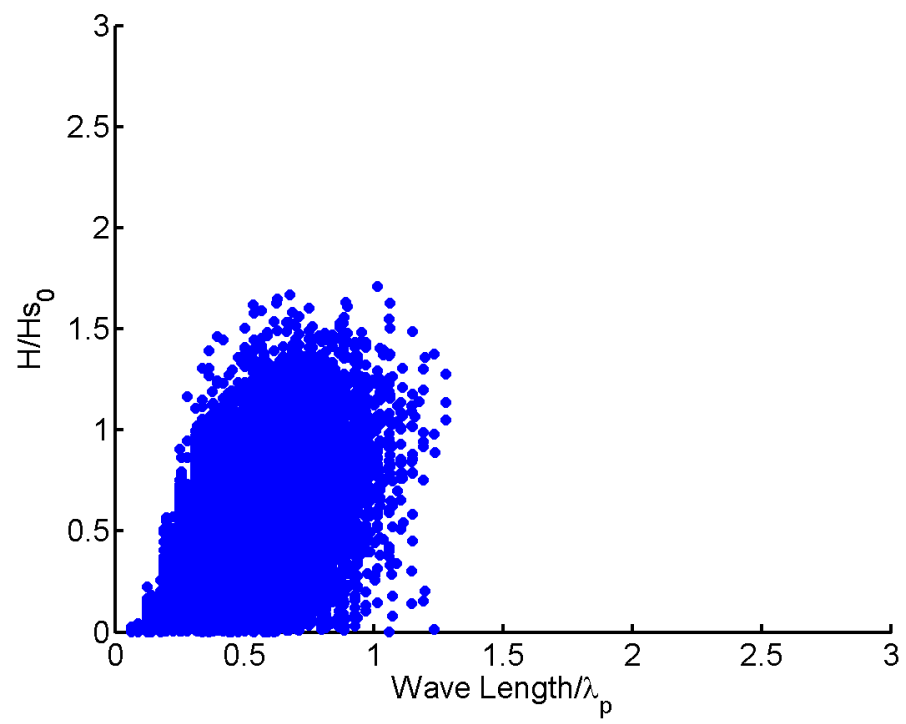

Figure 5-18: Scatter plot of dimensionless wave height vs. dimensionless wavelength in Case $\mathbf{I}$ (where $\gamma=1.0$ ) at time, $t=100 T_{p}$.

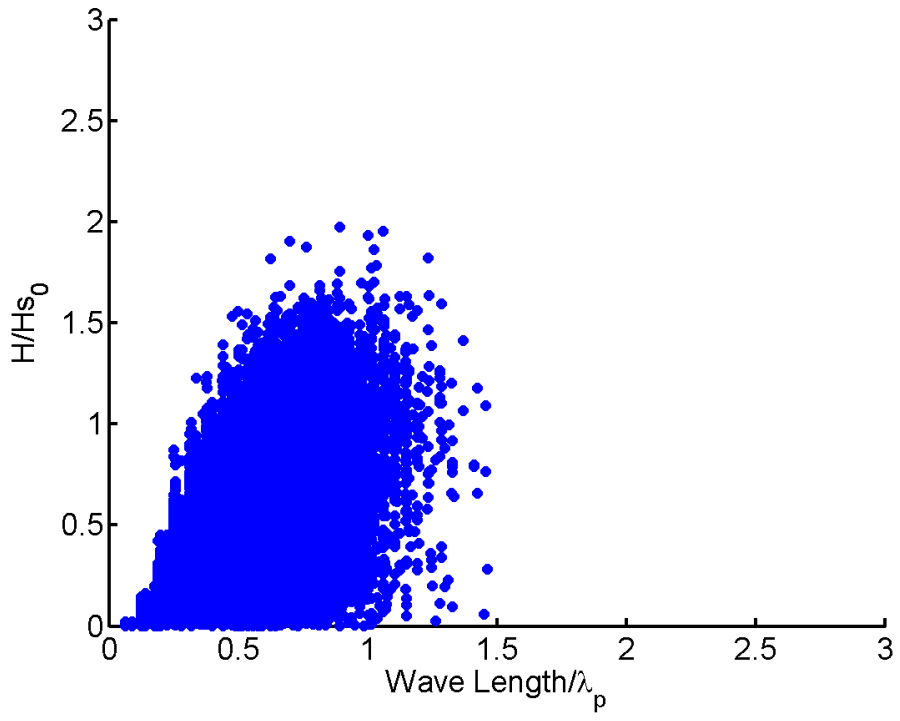

Figure 5-19: Scatter plot of dimensionless wave height vs. dimensionless wavelength in Case $\mathbf{B}$ (where $\gamma=3.3$ ) at time, $t=100 T_{p}$. 


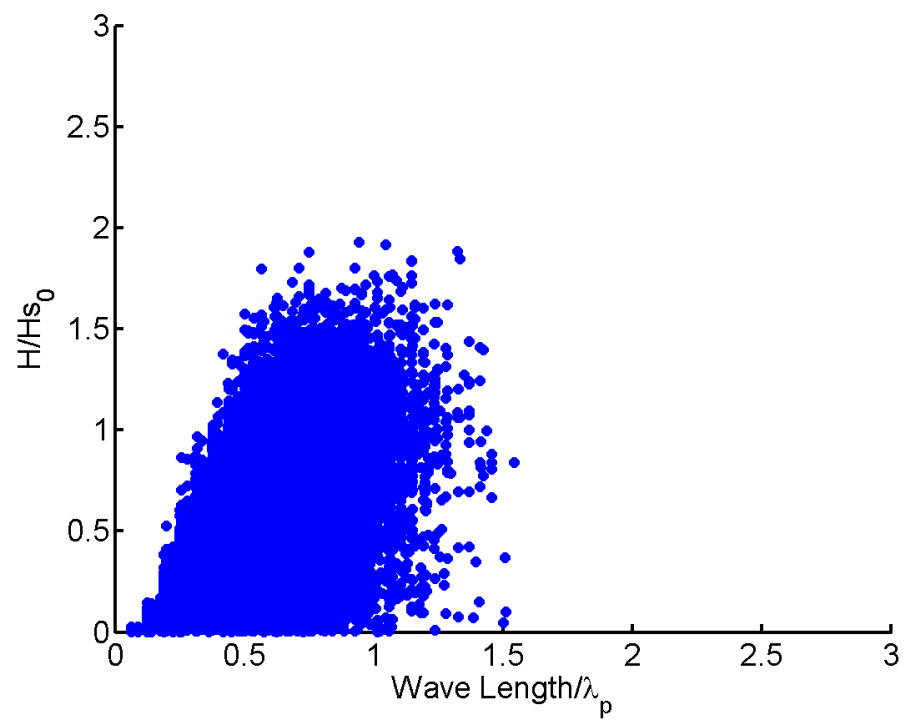

Figure 5-20: Scatter plot of dimensionless wave height vs. dimensionless wavelength in Case $\mathbf{H}$ (where $\gamma=5.0$ ) at time, $t=100 T_{p}$. 

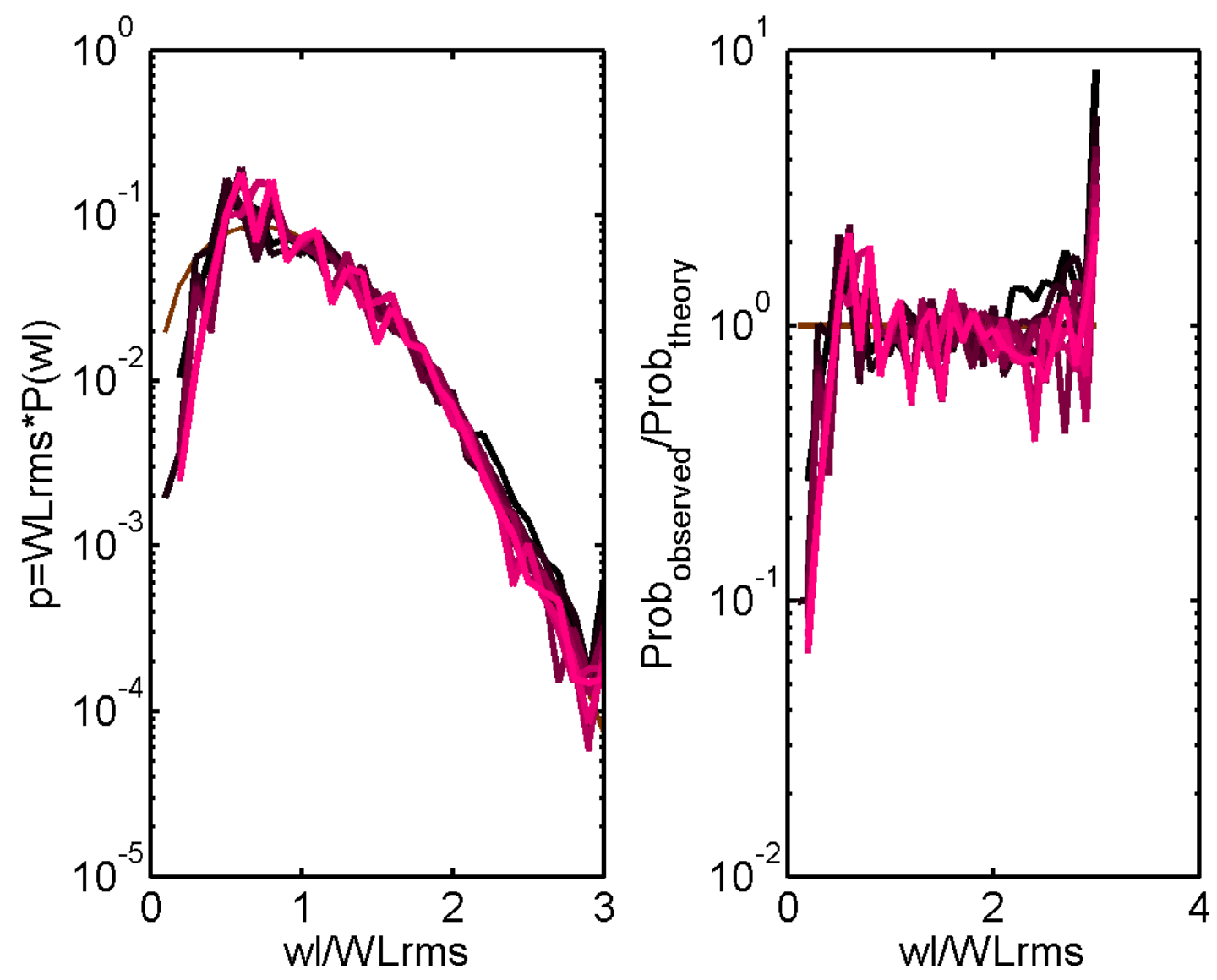

Figure 5-21: (In Color) (Left) Horizontal axis shows non-dimensional wavelength and vertical axis shows the probability of occurrence of wavelength in the wavefield. Thick, brown line represents Rayleigh distribution. Other lines represent observed wavelength distribution of Case $\mathbf{I}$ where $\gamma=1.0$, each such line representing all wavelengths in one time step. Earlier times (from $t=0 T_{p}$ ) are the darkest lines. Later times (up to $t=100 T_{p}$ ) are the brightest lines. (Right) Horizontal axis shows non-dimensional wavelength and vertical axis shows a probability ratio. Each line represents the ratio between a Case I time-step line and the Rayleigh distribution. 

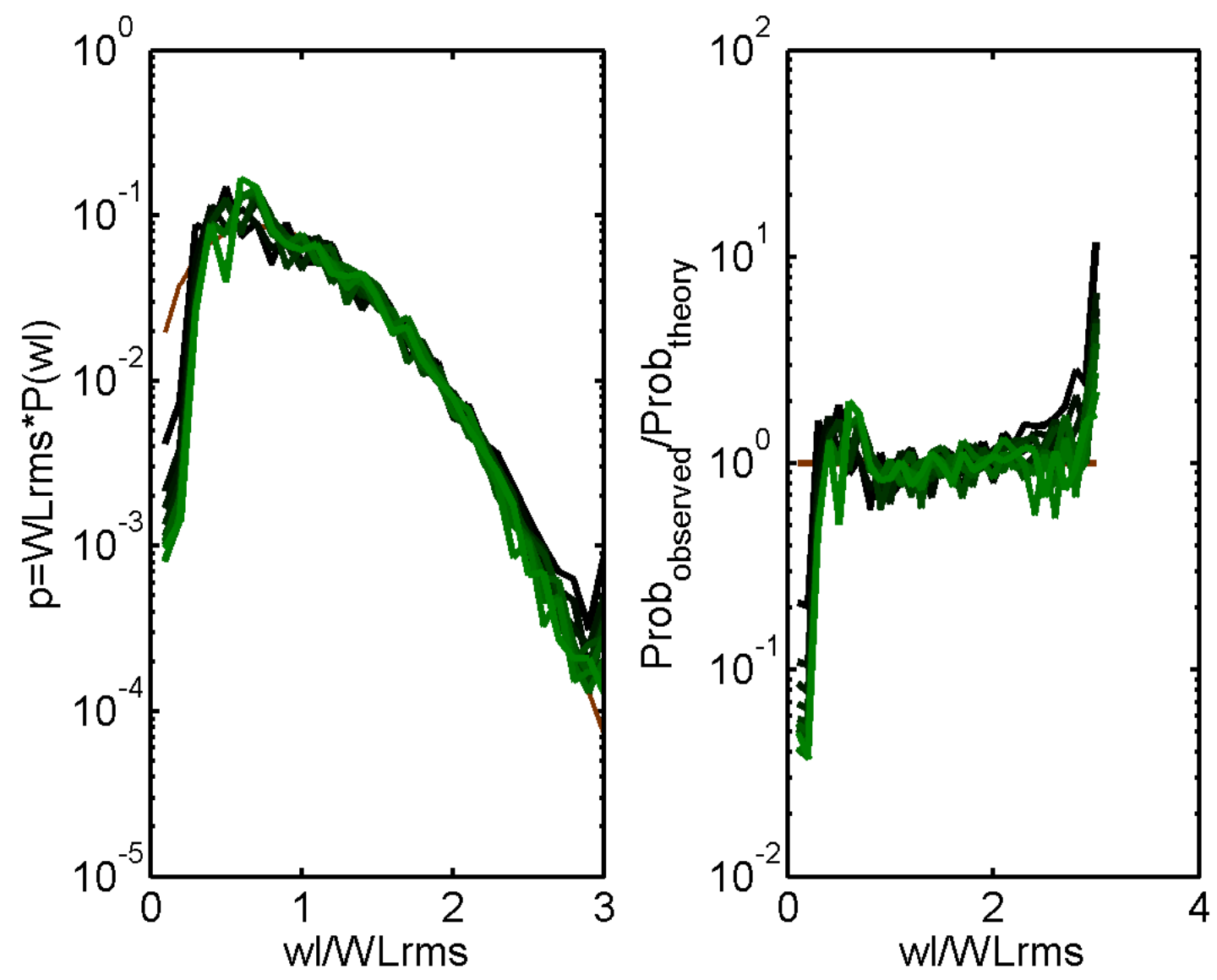

Figure 5-22: (In Color) (Left) Horizontal axis shows non-dimensional wavelength and vertical axis shows the probability of occurrence of wavelength in the wavefield. Thick, brown line represents Rayleigh distribution. Other lines represent observed wavelength distribution of Case $\mathbf{B}$ where $\gamma=3.3$, each such line representing all wavelengths in one time step. Earlier times (from $t=0 T_{p}$ ) are the darkest lines. Later times (up to $t=100 T_{p}$ ) are the brightest lines. (Right) Horizontal axis shows non-dimensional wavelength and vertical axis shows a probability ratio. Each line represents the ratio between a Case $\mathbf{B}$ time-step line and the Rayleigh distribution. 

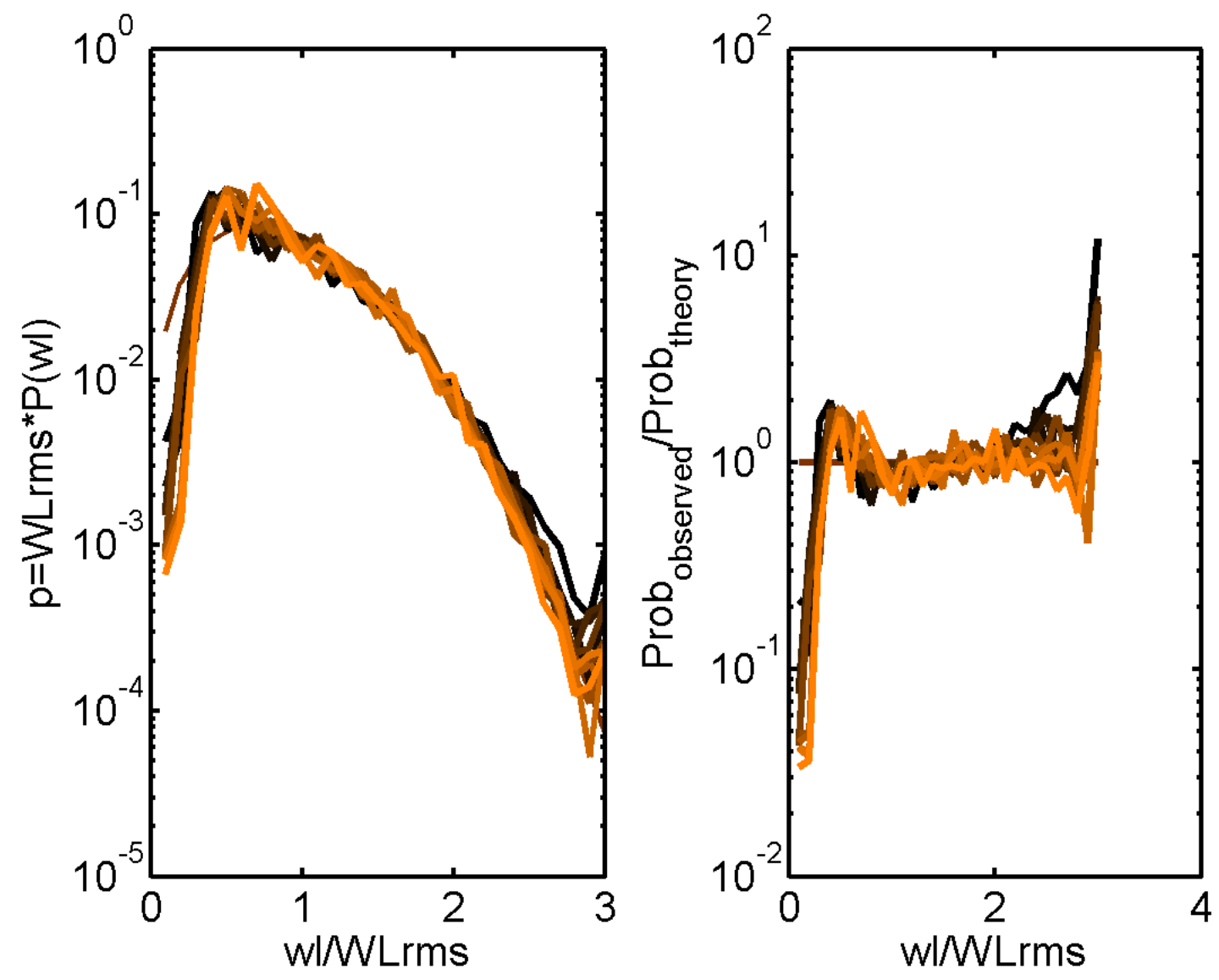

Figure 5-23: (In Color) (Left) Horizontal axis shows non-dimensional wavelength and vertical axis shows the probability of occurrence of wavelength in the wavefield. Thick, brown line represents Rayleigh distribution. Other lines represent observed wavelength distribution of Case $\mathbf{H}$ where $\gamma=5.0$, each such line representing all wavelengths in one time step. Earlier times (from $t=0 T_{p}$ ) are the darkest lines. Later times (up to $t=100 T_{p}$ ) are the brightest lines. (Right) Horizontal axis shows non-dimensional wavelength and vertical axis shows a probability ratio. Each line represents the ratio between a Case $\mathbf{H}$ time-step line and the Rayleigh distribution. 


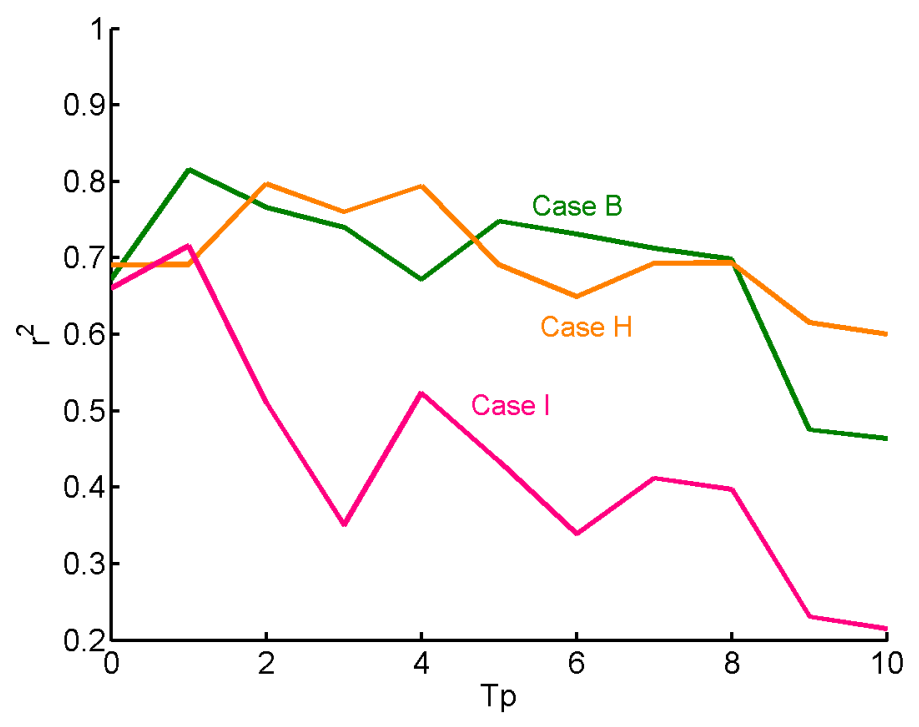

Figure 5-24: (In Color). Correlation coefficient, $r^{2}$-values between the simulated wavefields' wavelength distribution and the Rayleigh distribution. Correlation coefficient, $r^{2}$ takes values between 0 and 1 where $r^{2}=1$ indicates that the simulated wavefields' wavelength distribution and the Rayleigh distribution are highly correlated and $r^{2}=0$ indicates that they are not correlated at all. We observe wavelength distribution correlation with the Rayleigh distribution in cases with $\gamma=1.0, \gamma=3.3$ and $\gamma=5.0$, for all times, $t=0 T_{p}$ to $t=100 T_{p}$. 


\subsubsection{Peak shape parameter and wave height directionality}

In this section section we study the effect of peak shape parameter, $\gamma$ on the directions the waves are moving in. We see from observing the direction of travel of long waves by gathering all the waves in the wavefield where their height, $H / H s_{0}>1.0$ and plotting their height and direction in polar coordinates. Figures 5-25, 5-26, 5-27, 5-28, 5-29, and 5-30 all depict such results. From these images we conclude that peak shape parameter has no effect on the direction of travel of the largest waves

in a wavefield, and that it has an effect on the heights of the waves, showing the longest waves associated with largest peak shape parameter and the shortest waves associated with smallest peak shape parameter.

\subsubsection{Peak shape parameter and the spacing of large waves}

In this section, we define the larger waves in the wavefield as waves where $H / H_{s}>1.0$. As we are focused on the largest waves for this result and not necessarily the shortest waves, we use the narrow-band 3D method of wave definitions in Section 4.3. At each time step, we count the large waves occurring within a fixed radius around the largest wave in the wavefield (see Figure 5-31). This result (number of waves v.s. radius) is plotted in Figures 5-32, 5-33 and 5-34 showing no predictable effect of time on the wave spacing trajectories associated with any peak shape parameter.

We see that neither peak shape parameter nor time have any visible effect on the spacing between the large waves in the wavefield. In Figures 5-35, 5-36 and 5-37 show that the paths move closer together with time but no visible discrimination between different peak shape parameters. i.e. The differences between the paths are not produced by peak shape parameter, but these differences diminish as time proceeds.

\subsubsection{Conclusion: Peak shape parameter and wave height characteristics}

In this section we study the effect of peak shape parameter, $\gamma$ on wave height characteristics, finding that: 


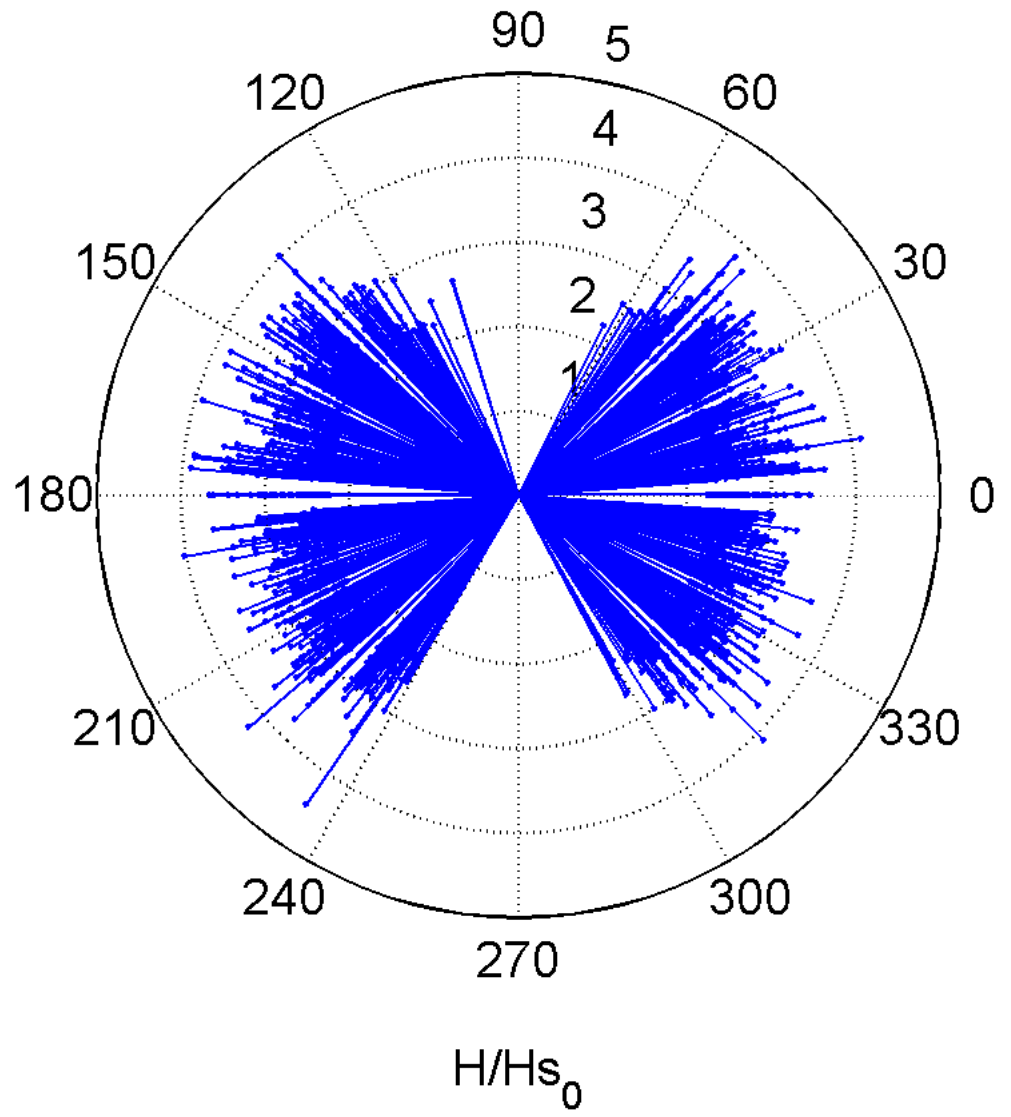

Figure 5-25: (In Color) Wave height and travel direction in Case $\mathbf{I}$ (where $\gamma=1.0$ ) at time, $t=50 T_{p}$. Graphics depict wave height $H / H_{r m s}$ as the length of the vector and the direction in which the wave travels $\Theta$ as the angle of the vector for $H>1.0 * H_{s}$ at time, $t=50 T_{p}$. 


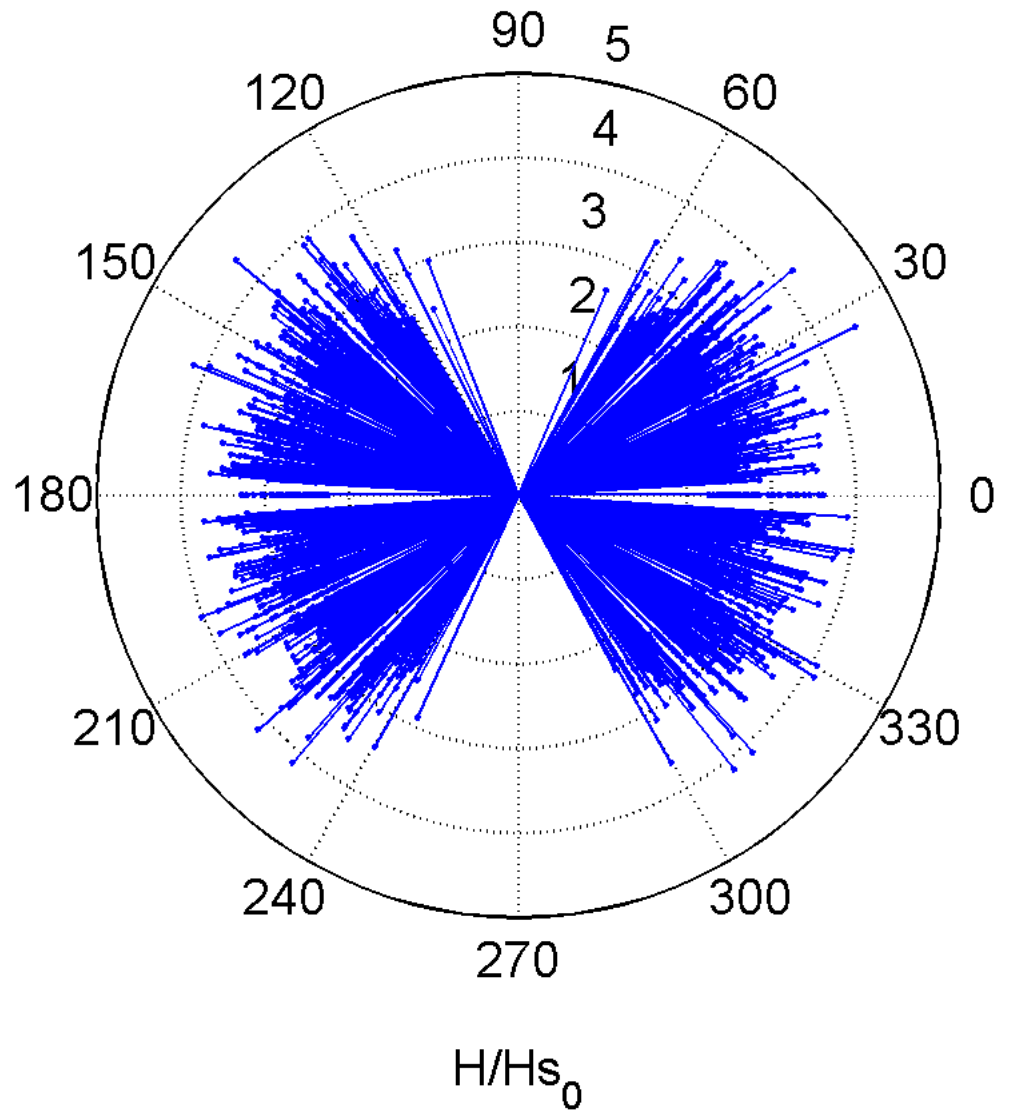

Figure 5-26: (In Color) Wave height and travel direction in Case $\mathbf{B}$ (where $\gamma=3.3$ ) at time, $t=50 T_{p}$. Graphics depict wave height $H / H_{r m s}$ as the length of the vector and the direction in which the wave travels $\Theta$ as the angle of the vector for $H>1.0 * H_{s}$ at time, $t=50 T_{p}$. 


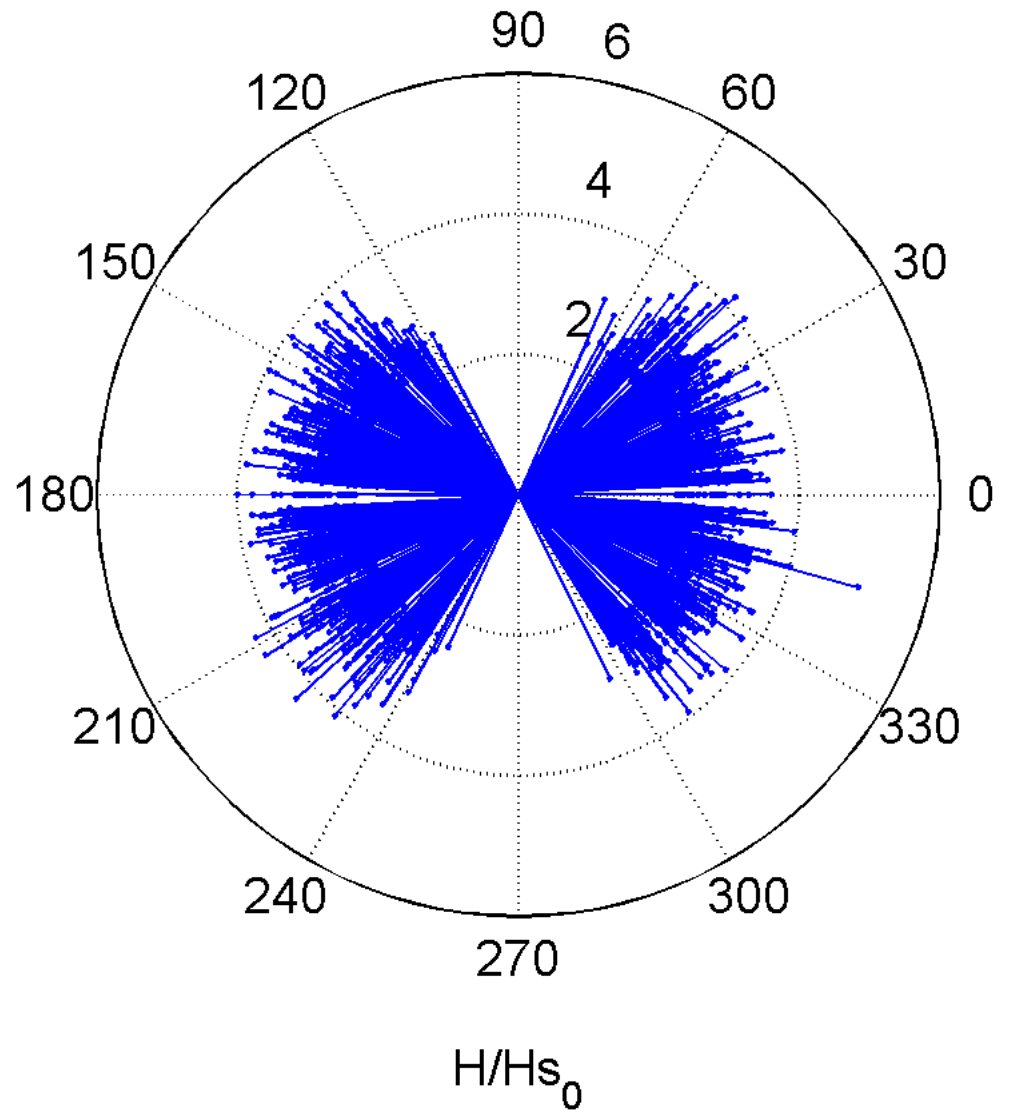

Figure 5-27: (In Color) Wave height and travel direction in Case $\mathbf{H}$ (where $\gamma=5.0$ ) at time, $t=50 T_{p}$. Graphics depict wave height $H / H_{r m s}$ as the length of the vector and the direction in which the wave travels $\Theta$ as the angle of the vector for $H>1.0 * H_{s}$ at time, $t=50 T_{p}$. 


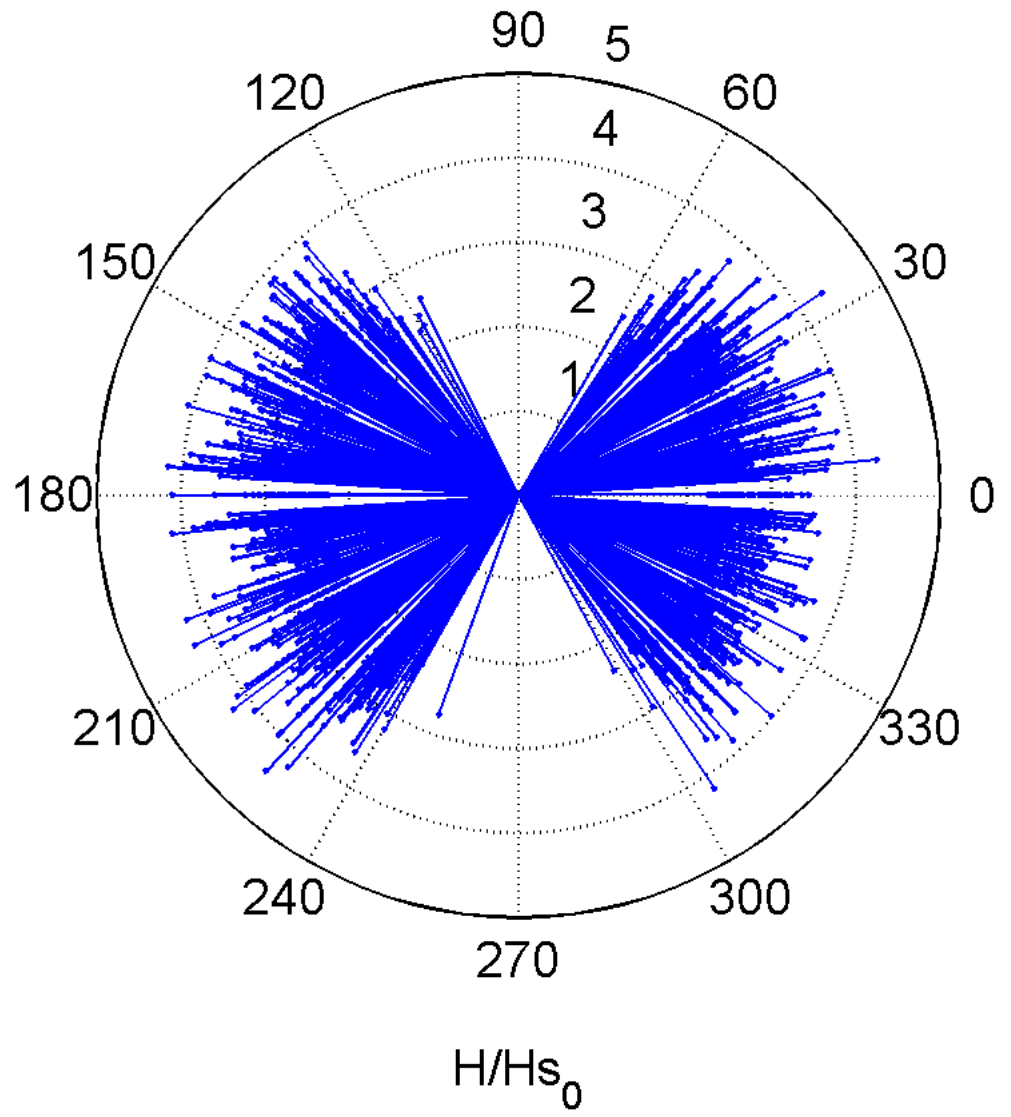

Figure 5-28: (In Color) Wave height and travel direction in Case $\mathbf{I}$ (where $\gamma=1.0$ ) at time, $t=100 T_{p}$. Graphics depict wave height $H / H_{r m s}$ as the length of the vector and the direction in which the wave travels $\Theta$ as the angle of the vector for $H>1.0 * H_{s}$ at time, $t=100 T_{p}$. 


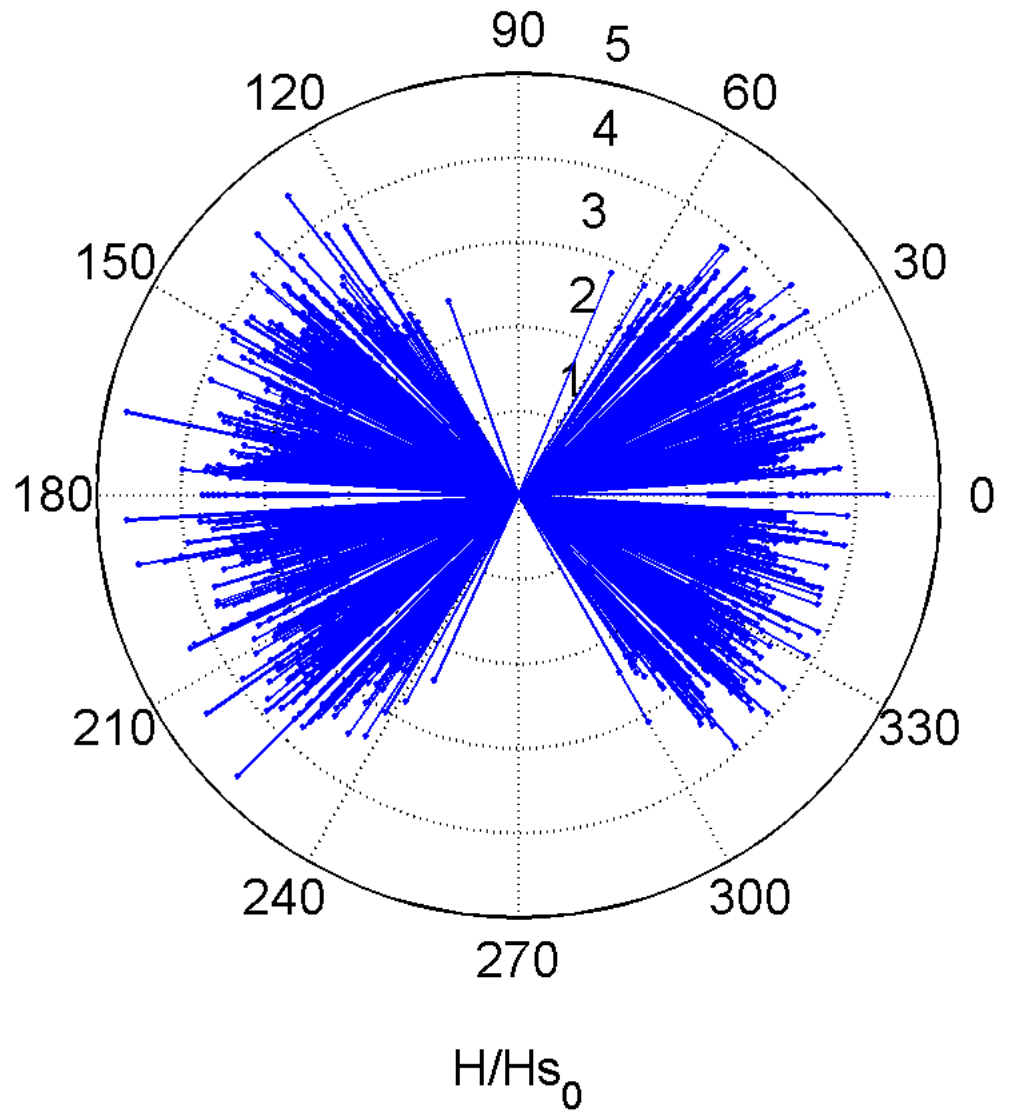

Figure 5-29: (In Color) Wave height and travel direction in Case $\mathbf{B}$ (where $\gamma=3.3$ ) at time, $t=100 T_{p}$. Graphics depict wave height $H / H_{r m s}$ as the length of the vector and the direction in which the wave travels $\Theta$ as the angle of the vector for $H>1.0 * H_{s}$ at time, $t=100 T_{p}$. 


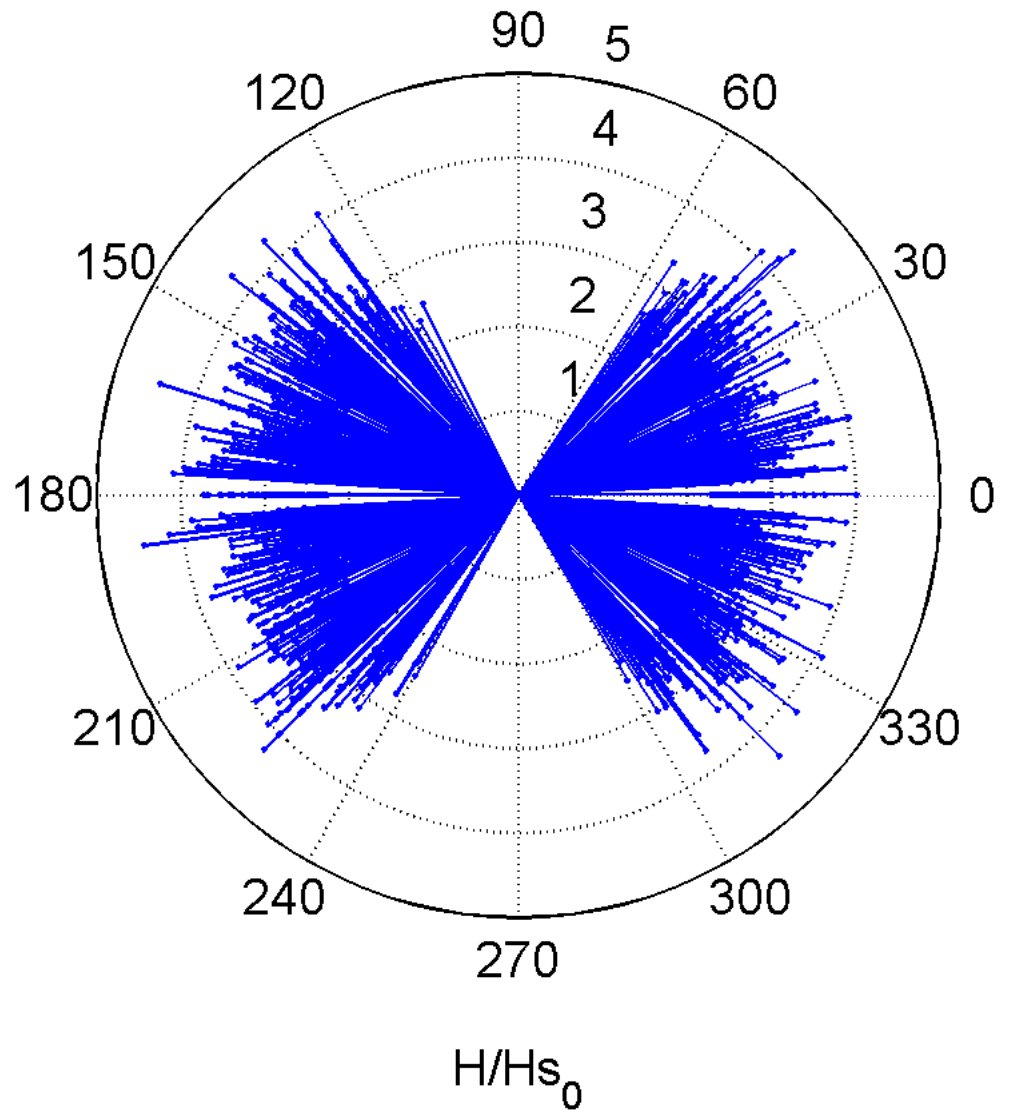

Figure 5-30: (In Color) Wave height and travel direction in Case $\mathbf{H}$ (where $\gamma=5.0$ ) at time, $t=100 T_{p}$. Graphics depict wave height $H / H_{r m s}$ as the length of the vector and the direction in which the wave travels $\Theta$ as the angle of the vector for $H>1.0 * H_{s}$ at time, $t=100 T_{p}$. 


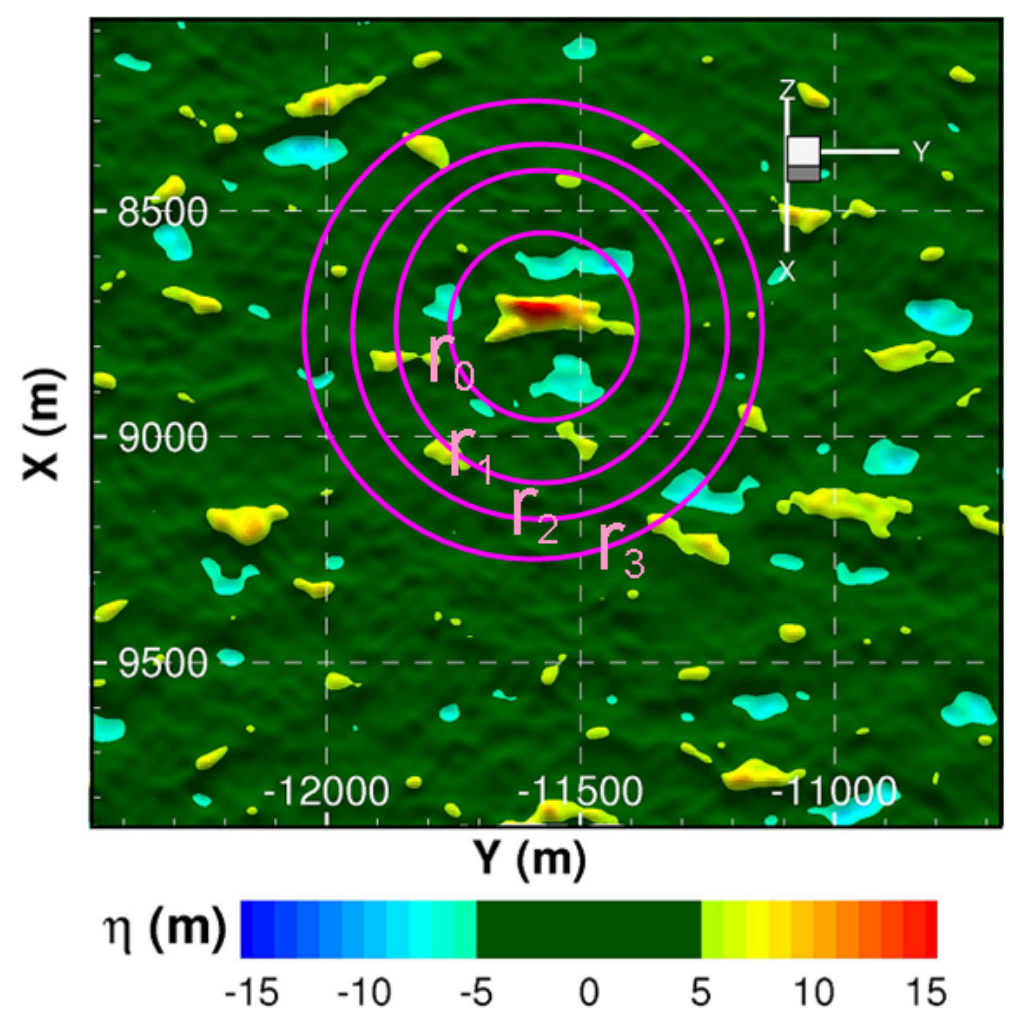

Figure 5-31: (In Color)Large wave spacing is seen as a plot of the number of waves found within a specified radius (for e.g. the pink lines marking $r_{0}, r_{1}, r_{2}$ and $r_{3}$ in the graphic) around the largest wave (red tip at the center of the pink rings) at each time step. (Image modified from [73]) 


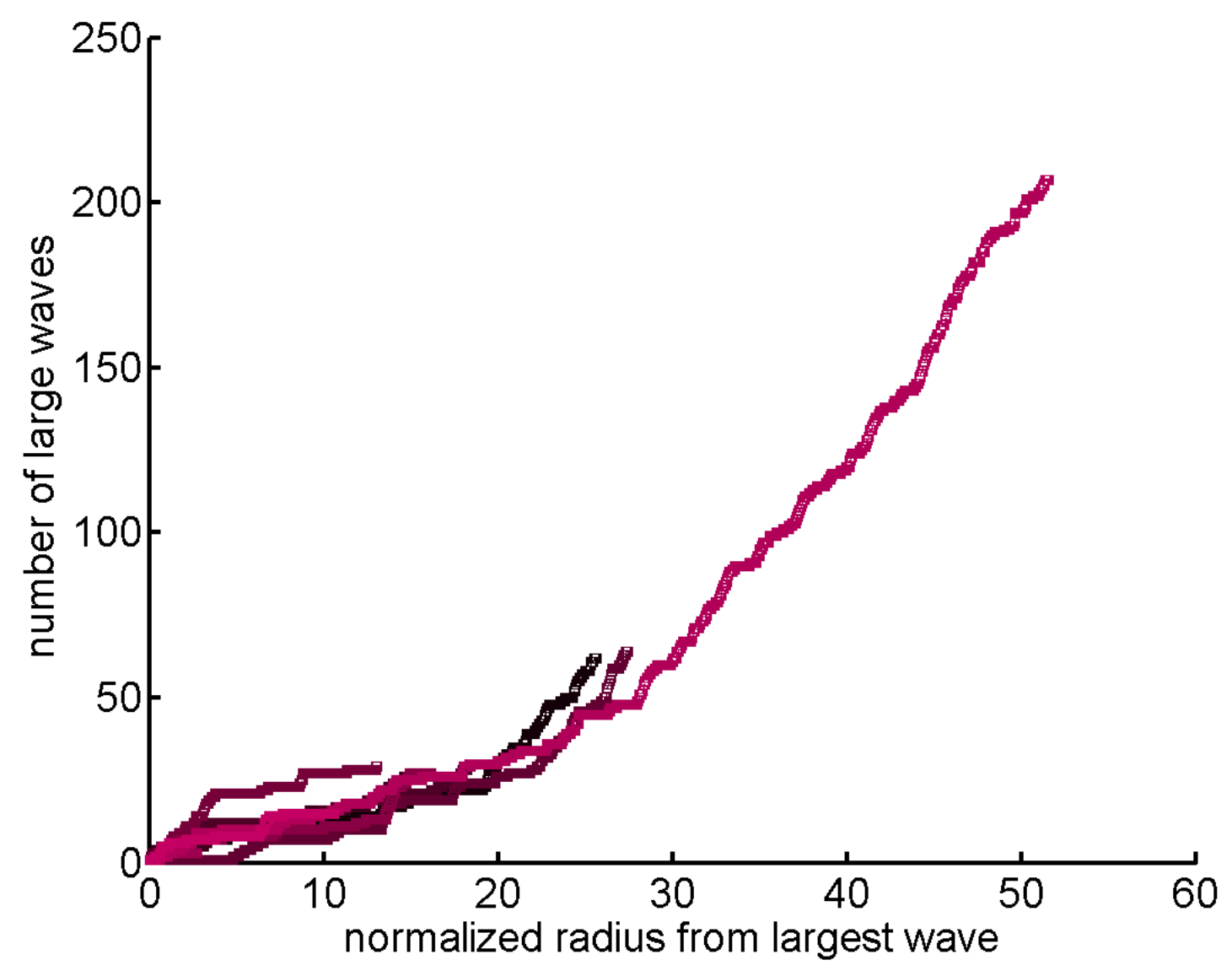

Figure 5-32: (In Color) Vertical axis depicts number of waves and horizontal axis depicts radius. Large wave spacing in Case $\mathbf{I}$ (where $\gamma=1.0$ ) from times, $t=0 T_{p}$ (darkest line) to $t=100 T_{p}$ (brightest line). Spacing in waves with height $H>1.0 * H_{s}$ from times, $t=0 T_{p}$ (darkest lines) to $t=100 T_{p}$ (brightest lines). Where a line ends the largest wave has struck the perimeter of the wavefield. 


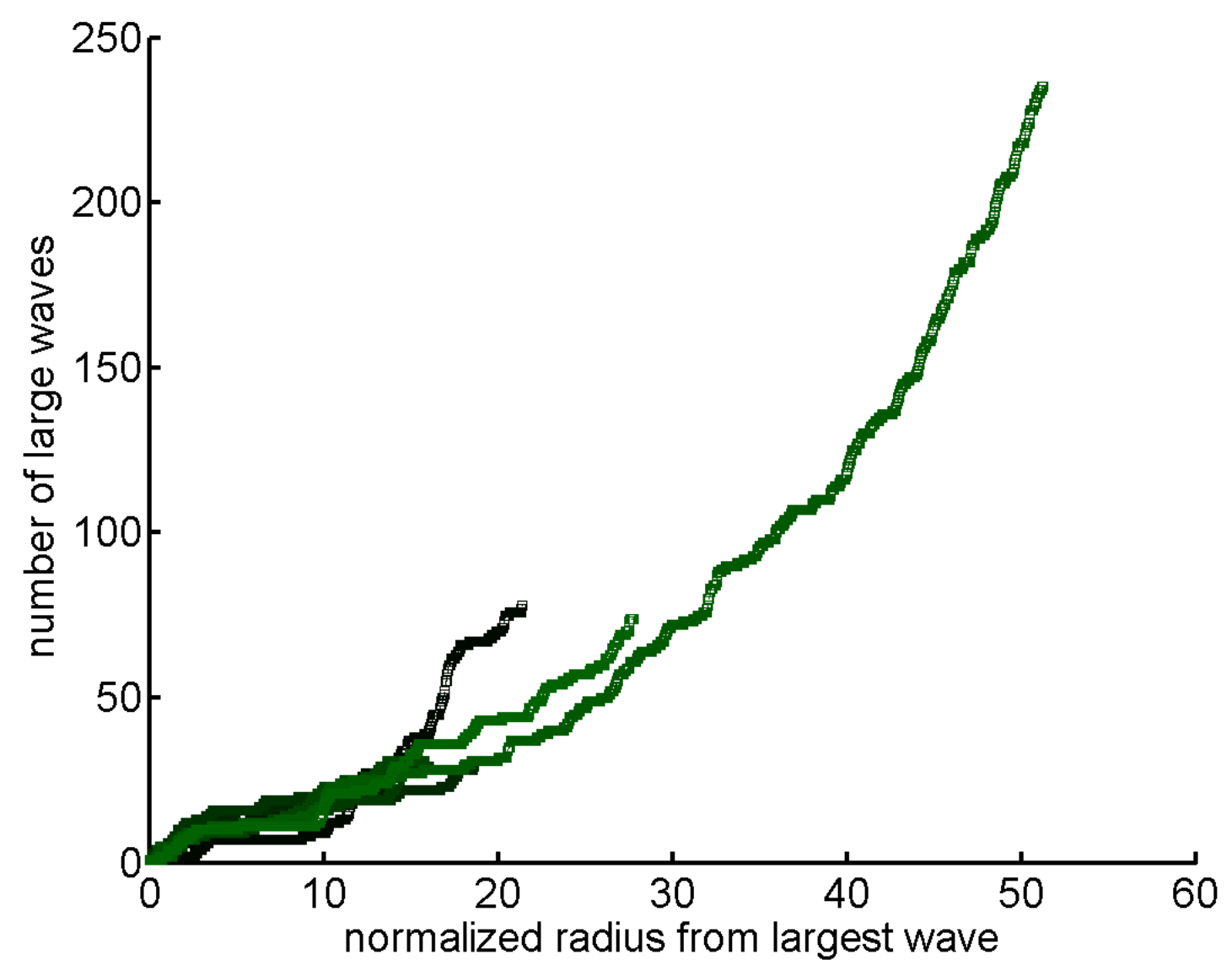

Figure 5-33: (In Color) Vertical axis depicts number of waves and horizontal axis depicts radius. Large wave spacing in Case $\mathbf{B}$ (where $\gamma=3.3$ ) from times, $t=0 T_{p}$ (darkest line) to $t=100 T_{p}$ (brightest line). Spacing in waves with height $H>1.0 * H_{s}$ from times, $t=0 T_{p}$ (darkest lines) to $t=100 T_{p}$ (brightest lines). Where a line ends the largest wave has struck the perimeter of the wavefield. 


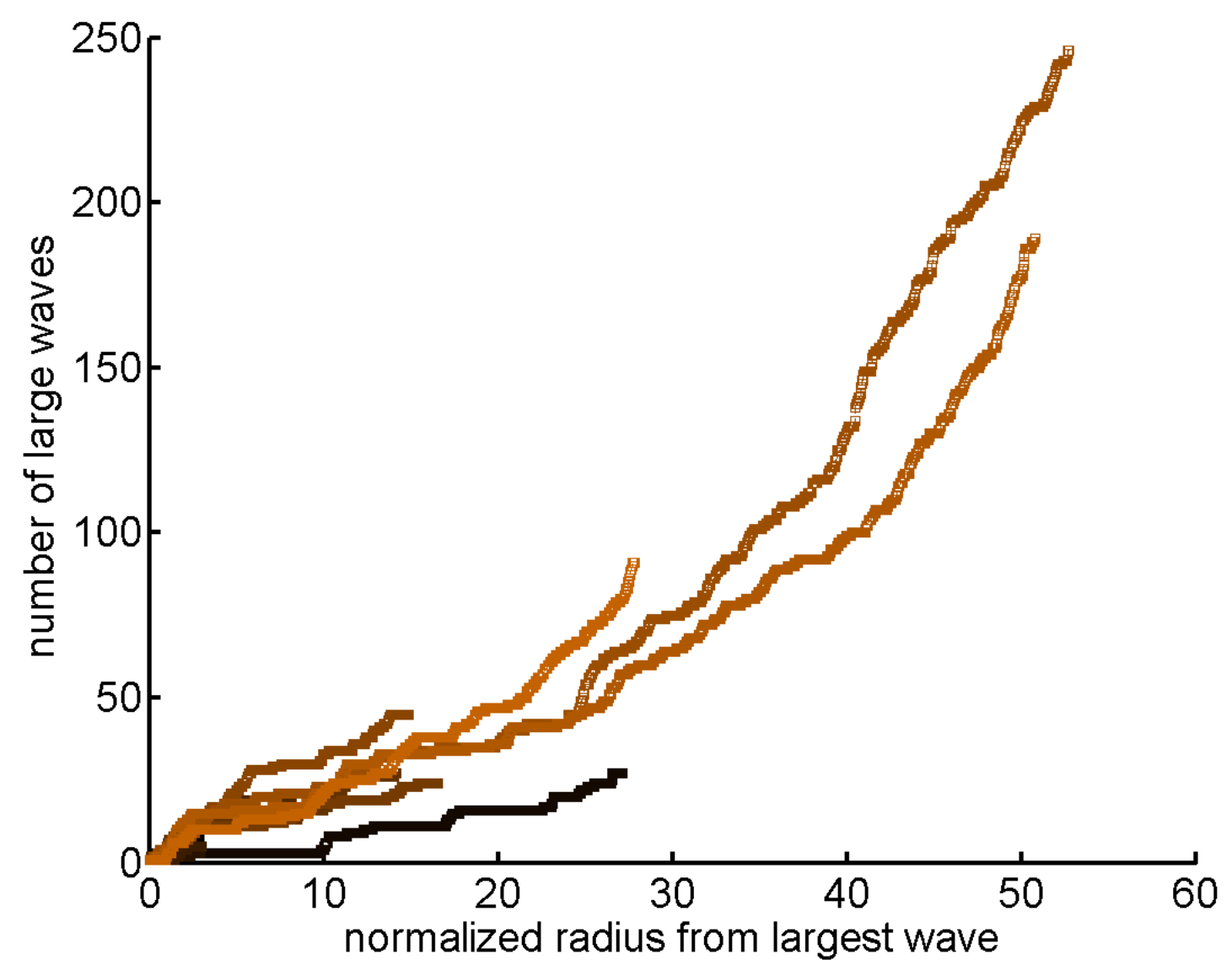

Figure 5-34: (In Color) Vertical axis depicts number of waves and horizontal axis depicts radius. Large wave spacing in Case $\mathbf{H}$ (where $\gamma=5.0$ ) from times, $t=0 T_{p}$ (darkest line) to $t=100 T_{p}$ (brightest line). Spacing in waves with height $H>1.0 * H_{s}$ from times, $t=0 T_{p}$ (darkest lines) to $t=100 T_{p}$ (brightest lines). Where a line ends the largest wave has struck the perimeter of the wavefield. 


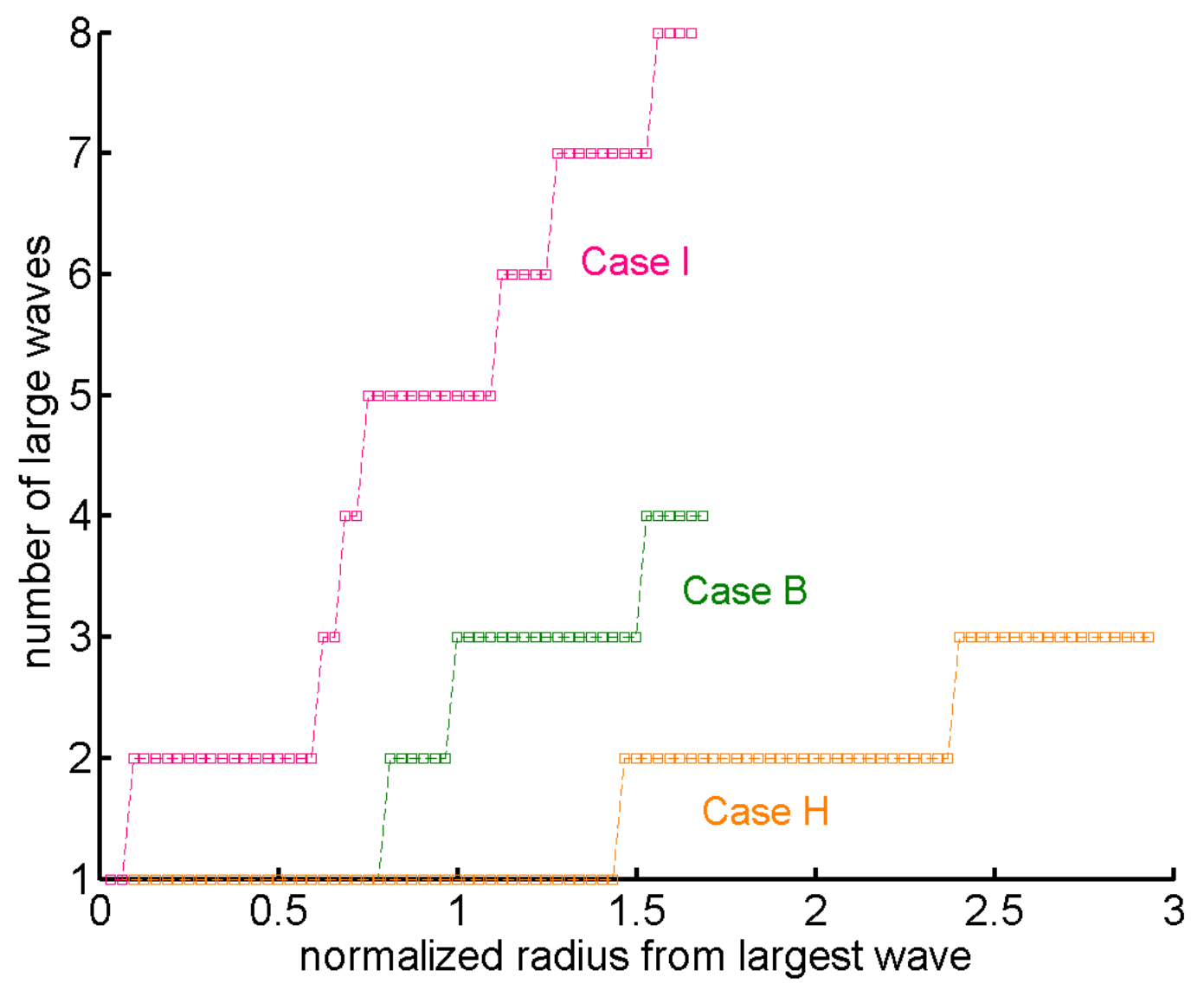

Figure 5-35: (In Color) Vertical axis depicts number of waves and horizontal axis depicts radius. Large wave spacing in Case $\mathbf{I}$ (where $\gamma=1.0$ ), Case $\mathbf{B}$ (where $\gamma=3.3$ ), and Case $\mathbf{H}$ (where $\gamma=5.0$ ) at time, $t=20 T_{p}$. Spacing in waves with height $H>1.0 * H_{s}$. 


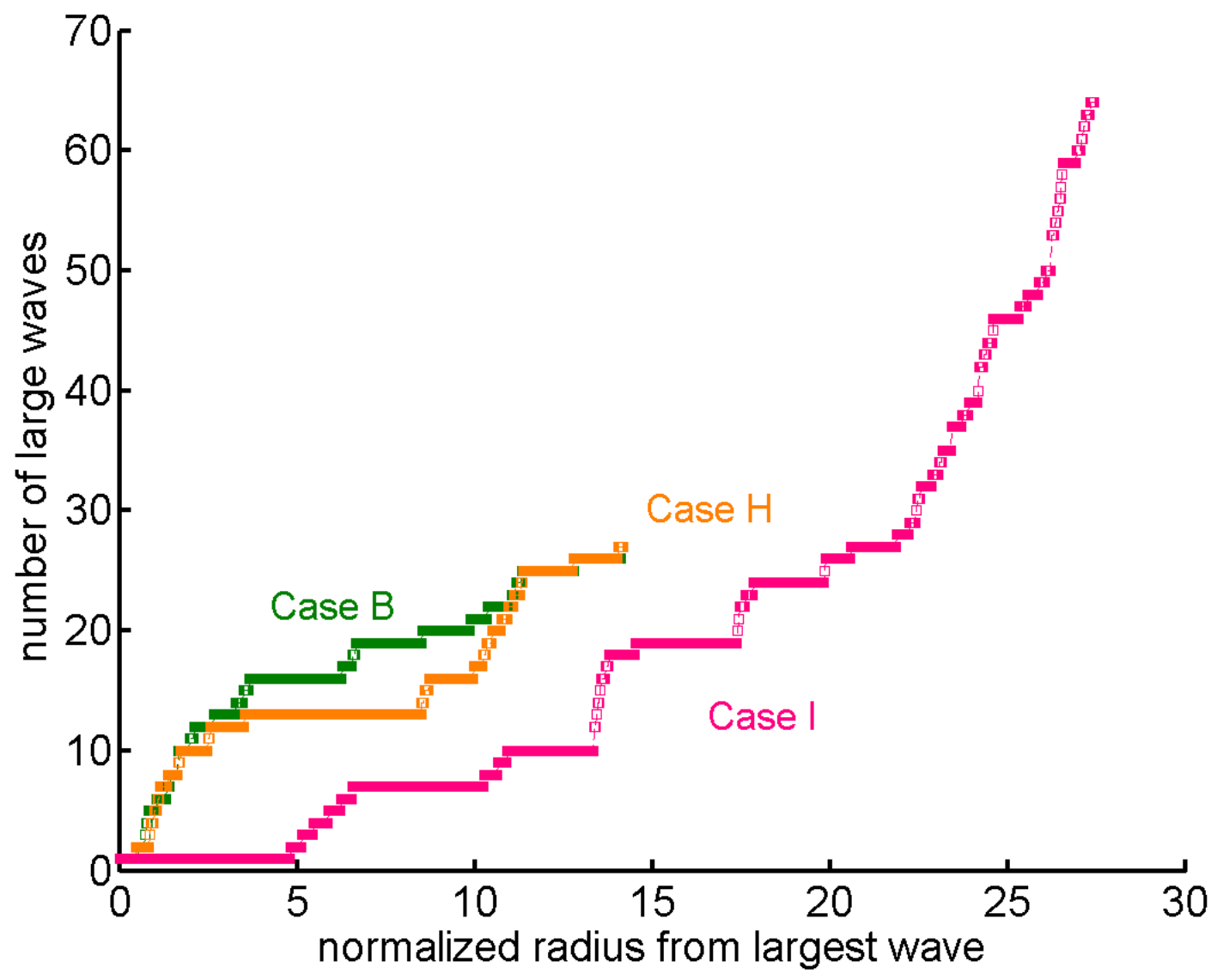

Figure 5-36: (In Color) Vertical axis depicts number of waves and horizontal axis depicts radius. Large wave spacing in Case $\mathbf{I}$ (where $\gamma=1.0$ ), Case $\mathbf{B}$ (where $\gamma=3.3$ ), and Case $\mathbf{H}$ (where $\gamma=5.0$ ) at time, $t=50 T_{p}$. Spacing in waves with height $H>1.0 * H_{s}$. 


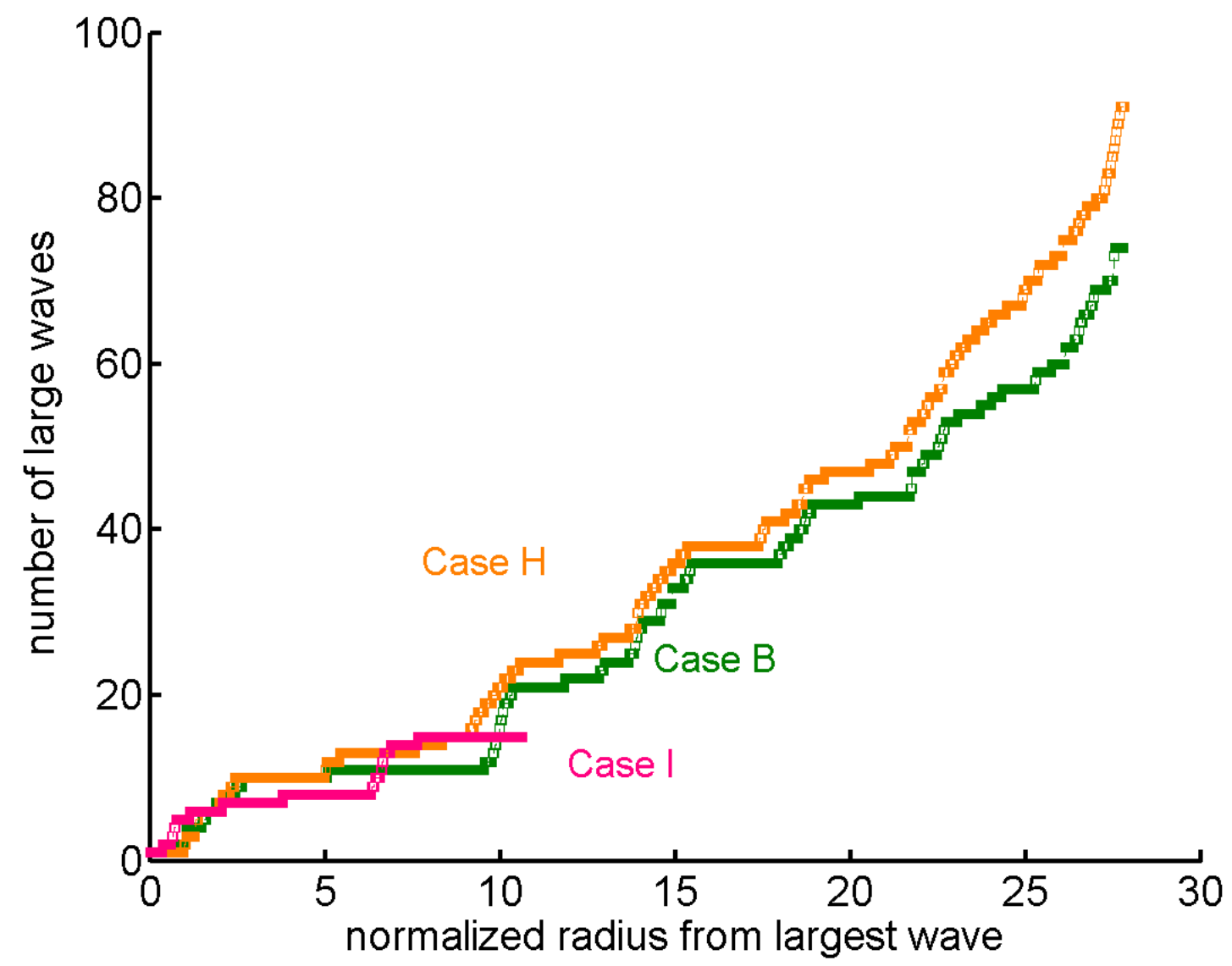

Figure 5-37: (In Color) Vertical axis depicts number of waves and horizontal axis depicts radius. Large wave spacing in Case $\mathbf{I}$ (where $\gamma=1.0$ ), Case $\mathbf{B}$ (where $\gamma=3.3$ ), and Case $\mathbf{H}$ (where $\gamma=5.0$ ) at time, $t=100 T_{p}$. Spacing in waves with height $H>1.0 * H_{s}$. 
- A higher peak shape parameter, $\gamma$ value moves the wave height distribution closer to the linear, narrow-band theory, and exceedance probability is sensitive to higher peak shape parameters.

- In the joint distribution between wave height and wave length we see a higher peak shape parameter, $\gamma$ value producing many more "rogue waves" with large height but short wavelength.

- A lower peak shape parameter $\gamma$ value moves wavelength distribution closer to the Rayleigh distribution which resembles Longuet-Higgins' [34] theoretical wavelength distribution for large spectral width.

- Peak shape parameter has no effect on the direction of travel of the largest waves in a wavefield, or the spacing of the large waves relative to each other in the wavefield

\subsection{Effect of input Phillips' parameter on wave height characteristics}

In this section we investigate the impact of spectral Phillips' parameter, $\alpha$ on statistical characteristics of ocean waves in SNOW wavefields. We generally use the non-narrow-band wave height defined according to the local maximum corresponding with the nearest connected local minimum method in Section 4.4 unless otherwise stated.

\subsubsection{Wave height distribution in wavefields of various Phillips parameters}

From Figures 5-38, 5-39 and 5-40 we see huge similarities among the wave height distributions of wavefields with different Phillips' parameter values. In all three cases 
the observed probability of largest and smallest waves exceed linear theory. In Figure 5-64 we see that a lower Phillips' parameter produces greatest correlation between observed non-linear, non-narrow-band wave height distributions and the Rayleigh distribution.

\subsubsection{Wave height directionality in wavefields of various Phillips parameters}

In Figures 5-42, 5-43, 5-44, 5-45, 5-46 and 5-47 we see a plot of the direction in which all the large waves, where $H / H_{s}>1.5$ in the wavefield are moving. As we see much more large waves in cases $\mathrm{B}$ and $\mathrm{F}$, but no difference in the directionality between cases E, B, and F, we can conclude that the Phillips' parameter has a quantifiable effect on the occurrence of large waves, but little effect on the direction in which these large waves move.

\subsubsection{The joint distribution between wave height and wave- length in wavefields of various Phillips parameters}

In Figures 5-48, 5-49, 5-50, 5-51, 5-52 and 5-53, we see scatter plots of dimensionless wave height vs. dimensionless wavelength in Cases $\mathrm{E}, \mathrm{B}$ and $\mathrm{F}$ at times $t=50 T_{p}$ and $t=100 T_{p}$. We see a prevalence of longer waves resulting from the smallest Phillips parameter and we that higher Phillips parameter produces a higher number of rogue-like waves with height, $H / H_{s} \approx 2$. We can conclude from this result that in a non-linear, non-narrow-band wavefield, Phillips' parameter does not predictably impact the wavelengths, but it does impact the number of large wave heights in the wavefield. 

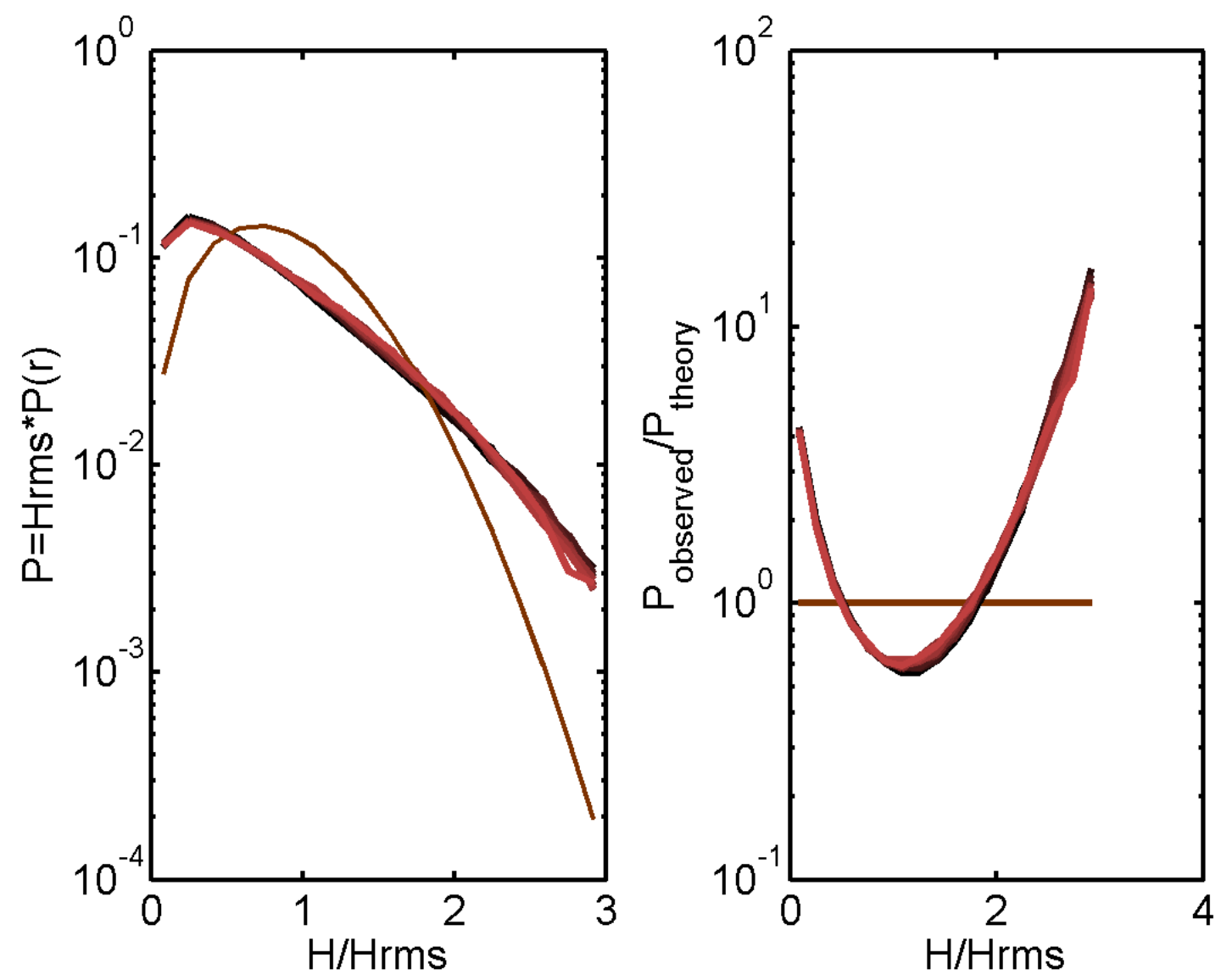

Figure 5-38: (In Color) (Left) Horizontal axis shows non-dimensional wave height and vertical axis shows the probability of occurrence of heights in the wavefield. Thick, brown line represents Rayleigh distribution. Other lines represent Case E where $\alpha=0.0032$, each such line representing one time step. Earlier times (from $t=0 T_{p}$ ) are the darkest lines. Later times (up to $t=100 T_{p}$ ) are the brightest lines. (Right) Horizontal axis shows non-dimensional wave height and vertical axis shows a probability ratio. Each line represents the ratio between a Case $\mathbf{E}$ time-step line and the Rayleigh distribution. 

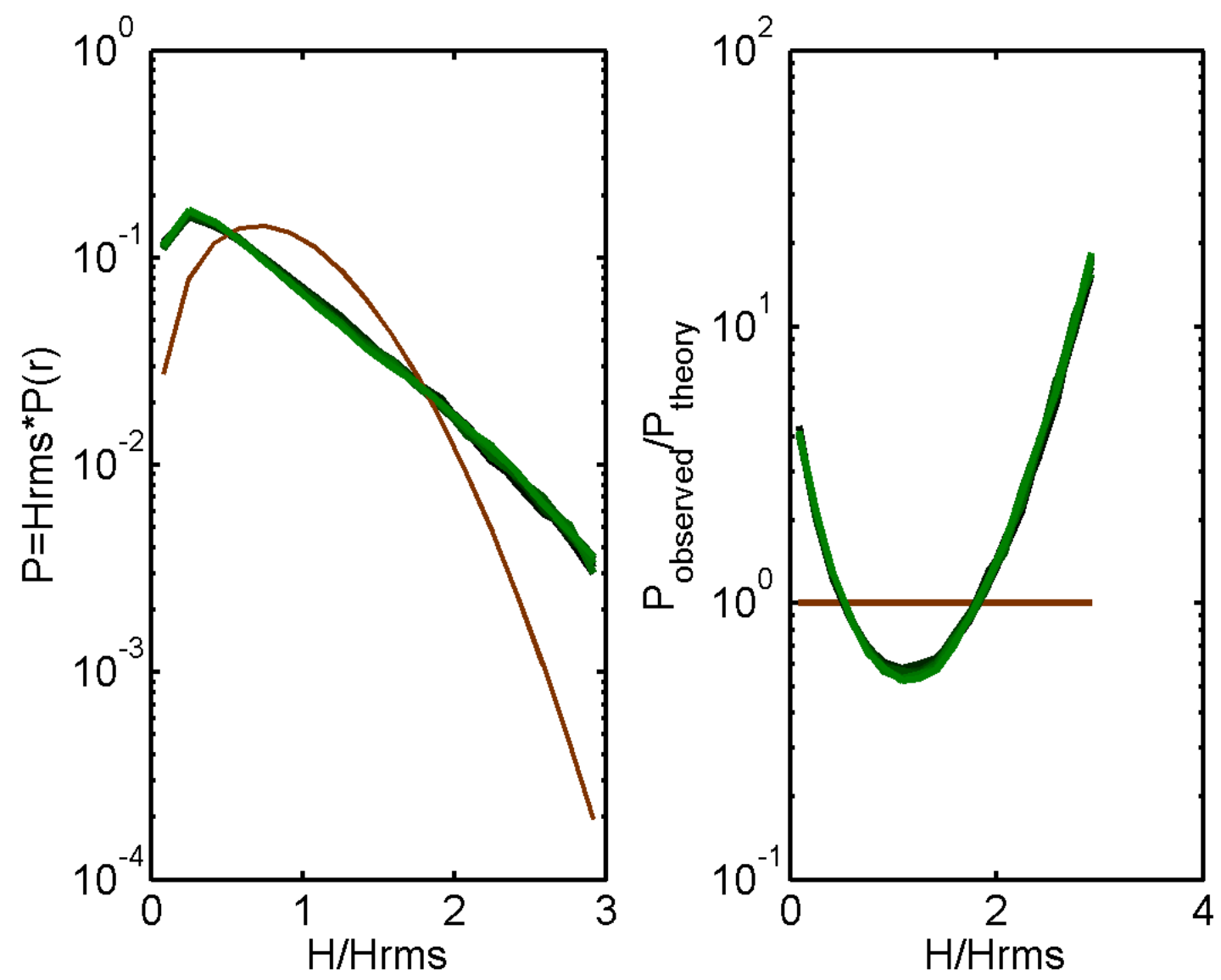

Figure 5-39: (In Color) (In Color) (Left) Horizontal axis shows non-dimensional wave height and vertical axis shows the probability of occurrence of heights in the wavefield. Thick, brown line represents Rayleigh distribution. Other lines represent Case B where $\alpha=0.0160$, each such line representing one time step. Earlier times (from $t=0 T_{p}$ ) are the darkest lines. Later times (up to $t=100 T_{p}$ ) are the brightest lines. (Right) Horizontal axis shows non-dimensional wave height and vertical axis shows a probability ratio. Each line represents the ratio between a Case $\mathbf{B}$ time-step line and the Rayleigh distribution. 

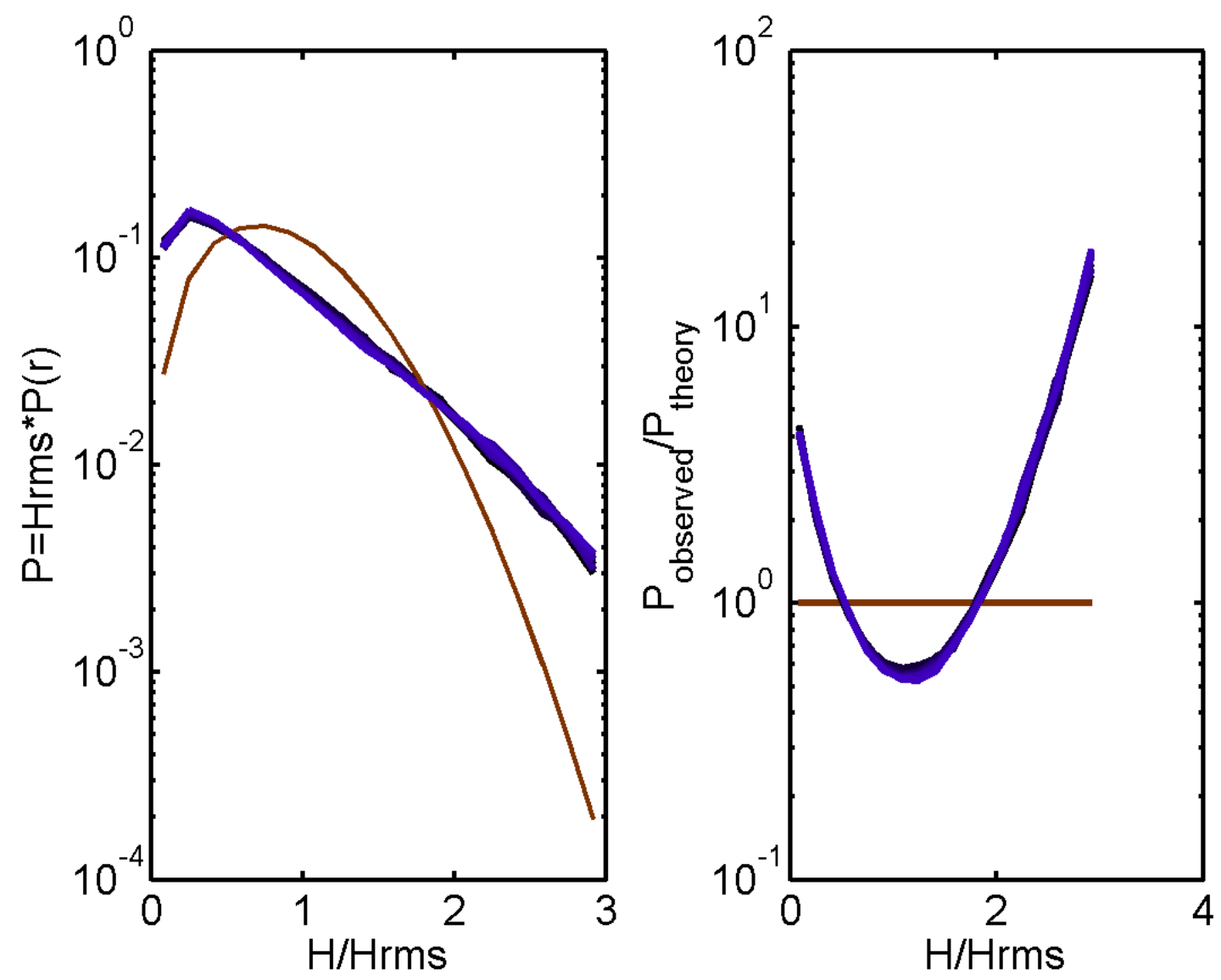

Figure 5-40: (In Color) (In Color) (Left) Horizontal axis shows non-dimensional wave height and vertical axis shows the probability of occurrence of heights in the wavefield. Thick, brown line represents Rayleigh distribution. Other lines represent Case F where $\alpha=0.0163$, each such line representing one time step. Earlier times (from $t=0 T_{p}$ ) are the darkest lines. Later times (up to $t=100 T_{p}$ ) are the brightest lines. (Right) Horizontal axis shows non-dimensional wave height and vertical axis shows a probability ratio. Each line represents the ratio between a Case $\mathbf{F}$ time-step line and the Rayleigh distribution. 


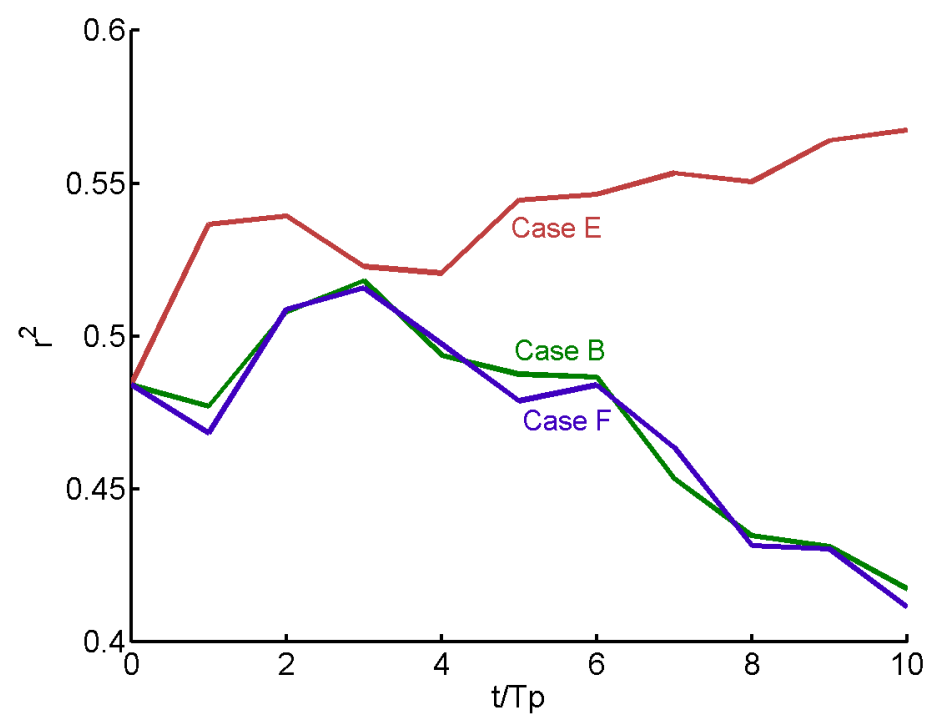

Figure 5-41: (In Color) Correlation coefficient, $r^{2}$-values between the simulated wavefields' wave height probability distribution and the Rayleigh distribution. Correlation coefficient, $r^{2}$ takes values between 0 and 1 where $r^{2}=1$ indicates that the simulated wavefields' wave height probability distribution and the Rayleigh distribution are highly correlated and $r^{2}=0$ indicates that they are not correlated at all. We observe this correlation for three simulated nonlinear wavefields: (a)Case $\mathbf{E}, \alpha=0.0032$, (b)Case B, $\alpha=0.0160$ and (c)Case $\mathbf{F}, \alpha=0.0163$. 


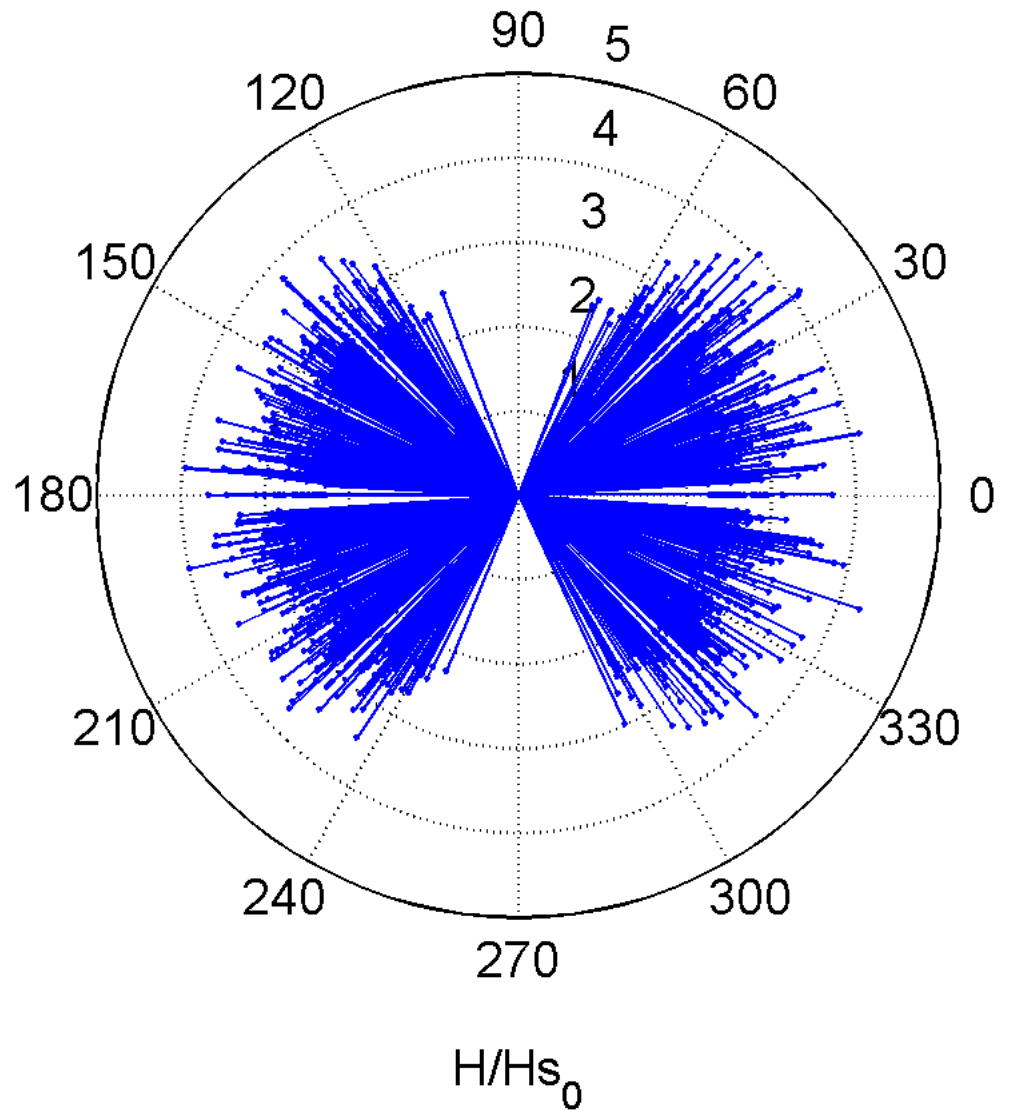

Figure 5-42: Wave height and travel direction in Case $\mathbf{E}$ (where $\alpha=0.0032$ ) at time, $t=50 T_{p}$. Graphics depict wave height $H / H_{r m s}$ as the length of the vector and the direction in which the wave travels $\Theta$ as the angle of the vector for $H>1.0 * H_{s}$ at time, $t=50 T_{p}$. 


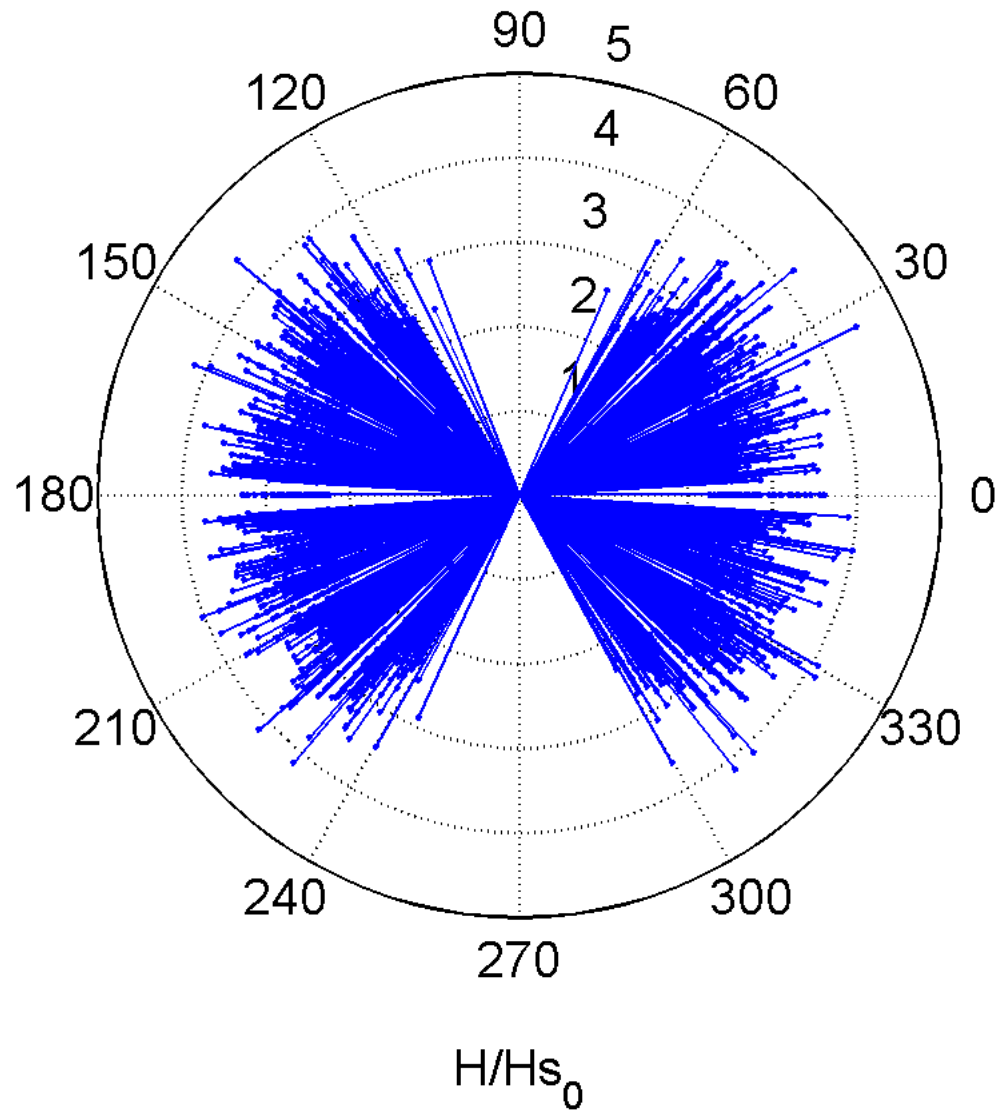

Figure 5-43: Wave height and travel direction in Case $\mathbf{B}$ (where $\alpha=0.0160$ ) at time, $t=50 T_{p}$. Graphics depict wave height $H / H_{r m s}$ as the length of the vector and the direction in which the wave travels $\Theta$ as the angle of the vector for $H>1.0 * H_{s}$ at time, $t=50 T_{p}$. 


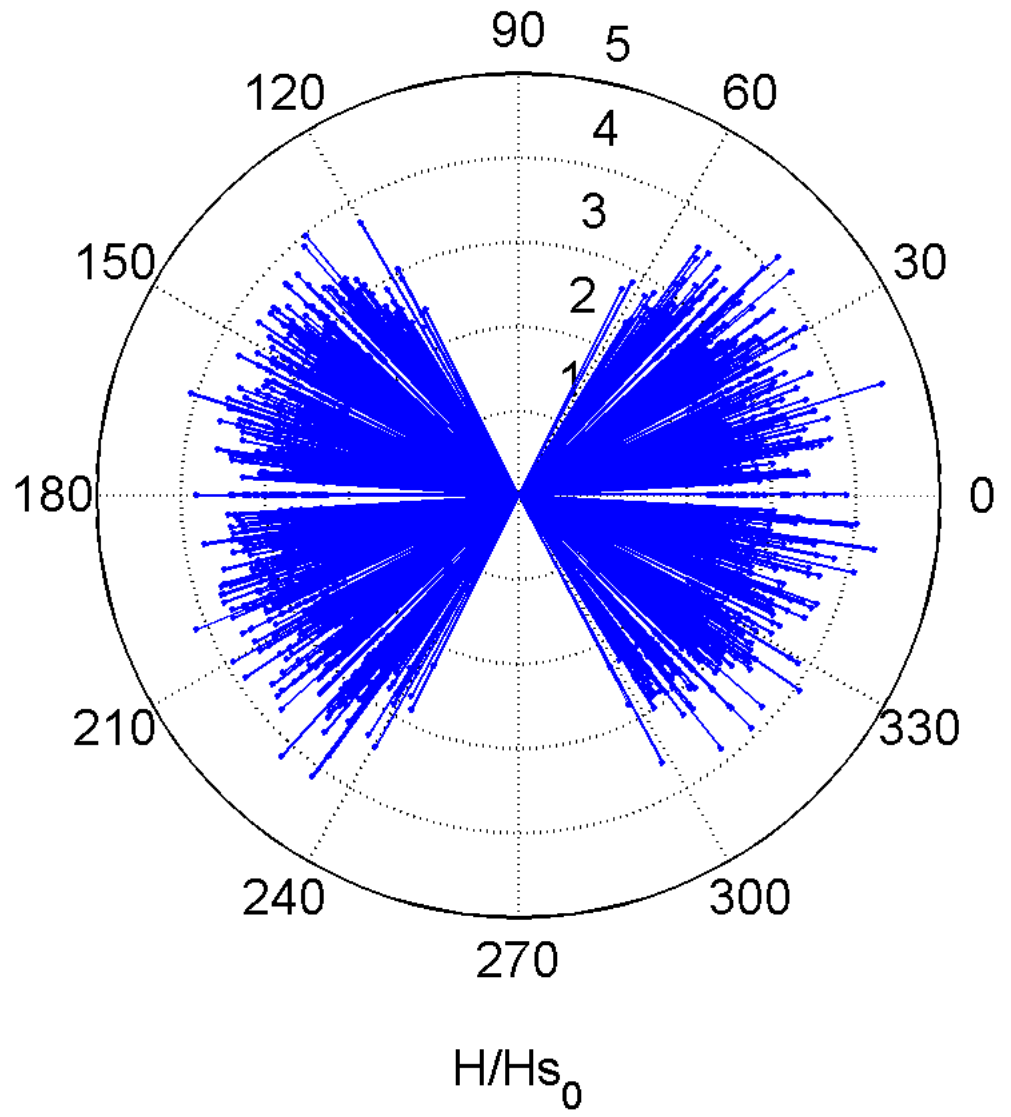

Figure 5-44: Wave height and travel direction in Case $\mathbf{F}$ (where $\alpha=0.0163$ ) at time, $t=50 T_{p}$. Graphics depict wave height $H / H_{r m s}$ as the length of the vector and the direction in which the wave travels $\Theta$ as the angle of the vector for $H>1.0 * H_{s}$ at time, $t=50 T_{p}$. 


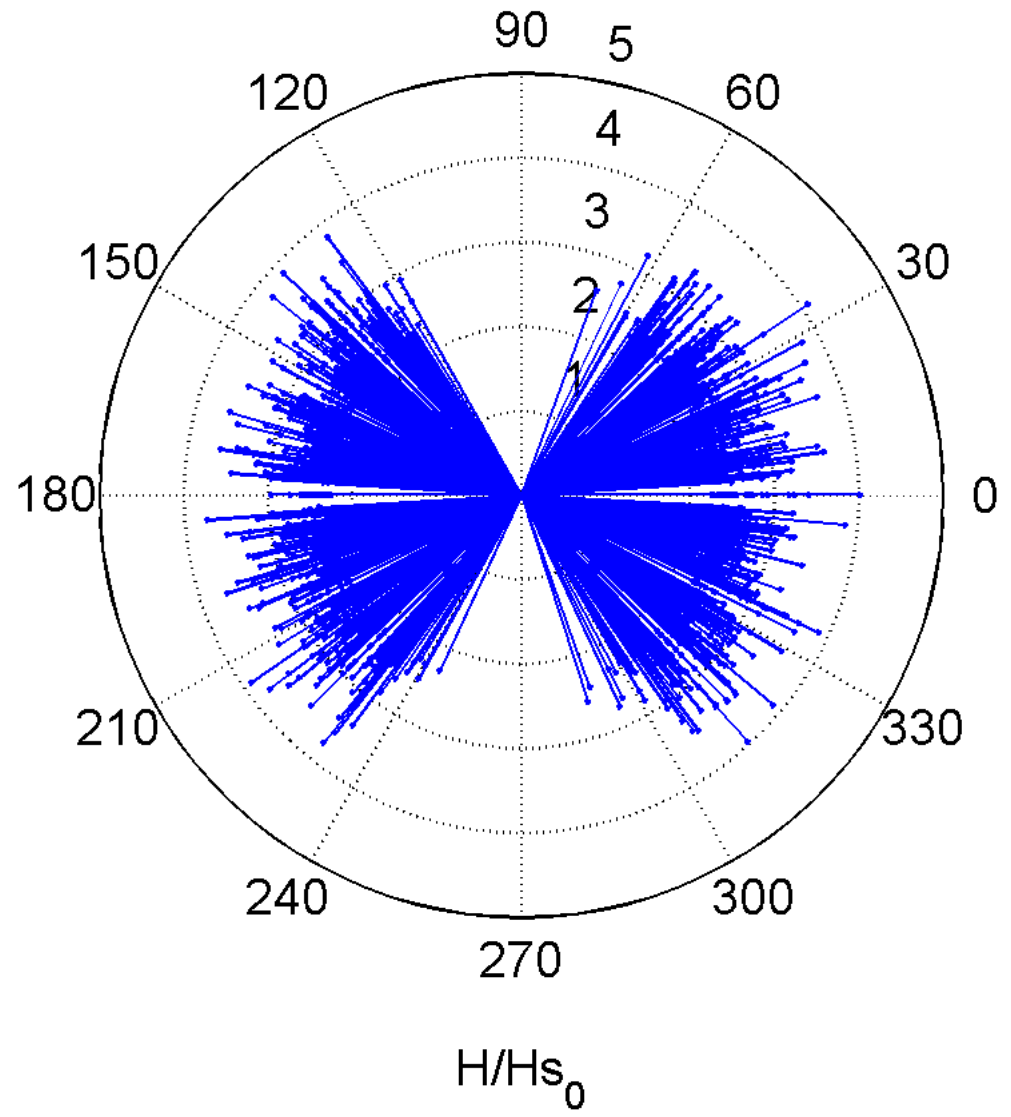

Figure 5-45: Wave height and travel direction in Case $\mathbf{E}$ (where $\alpha=0.0032$ ) at time, $t=100 T_{p}$. Graphics depict wave height $H / H_{r m s}$ as the length of the vector and the direction in which the wave travels $\Theta$ as the angle of the vector for $H>1.0 * H_{s}$ at time, $t=100 T_{p}$. 


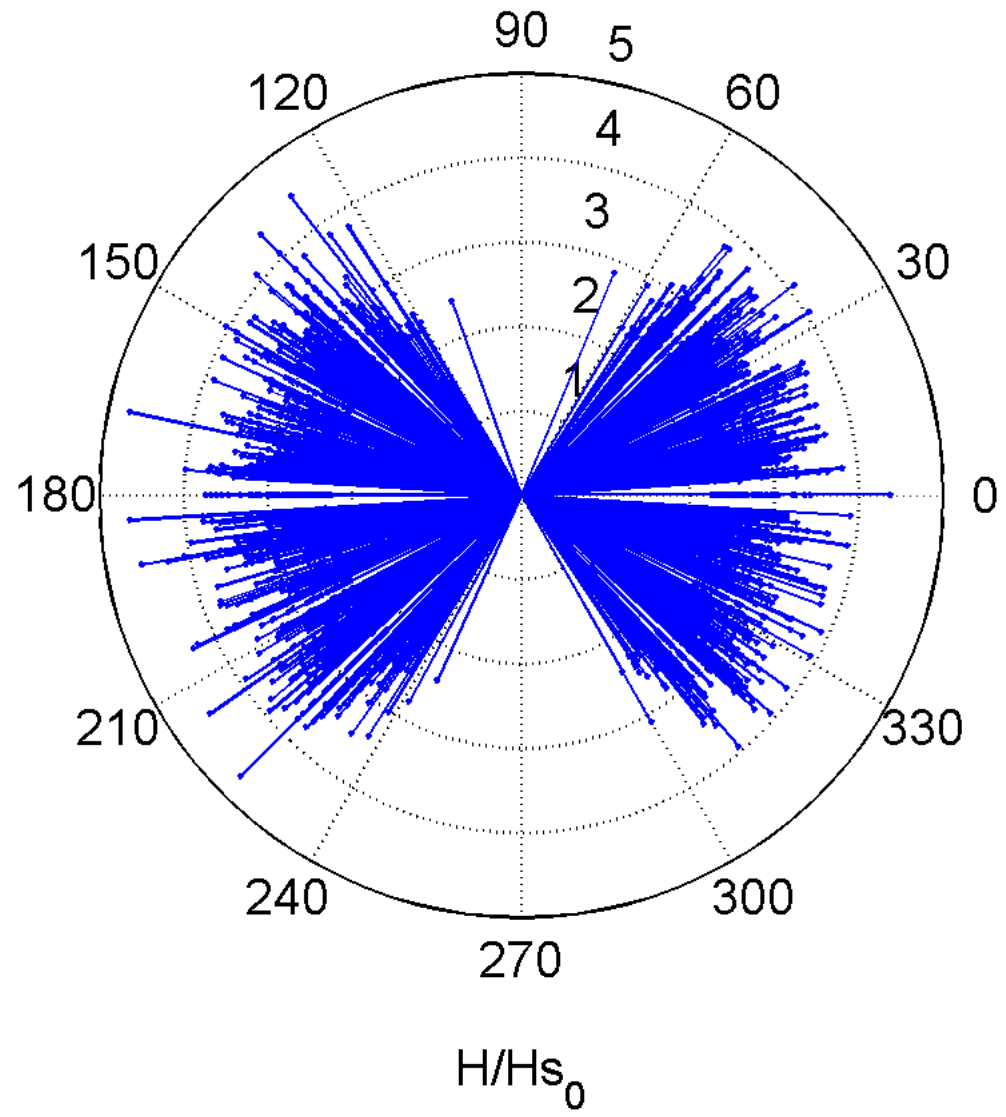

Figure 5-46: Wave height and travel direction in Case $\mathbf{B}$ (where $\alpha=0.0160$ ) at time, $t=100 T_{p}$. Graphics depict wave height $H / H_{r m s}$ as the length of the vector and the direction in which the wave travels $\Theta$ as the angle of the vector for $H>1.0 * H_{s}$ at time, $t=100 T_{p}$. 


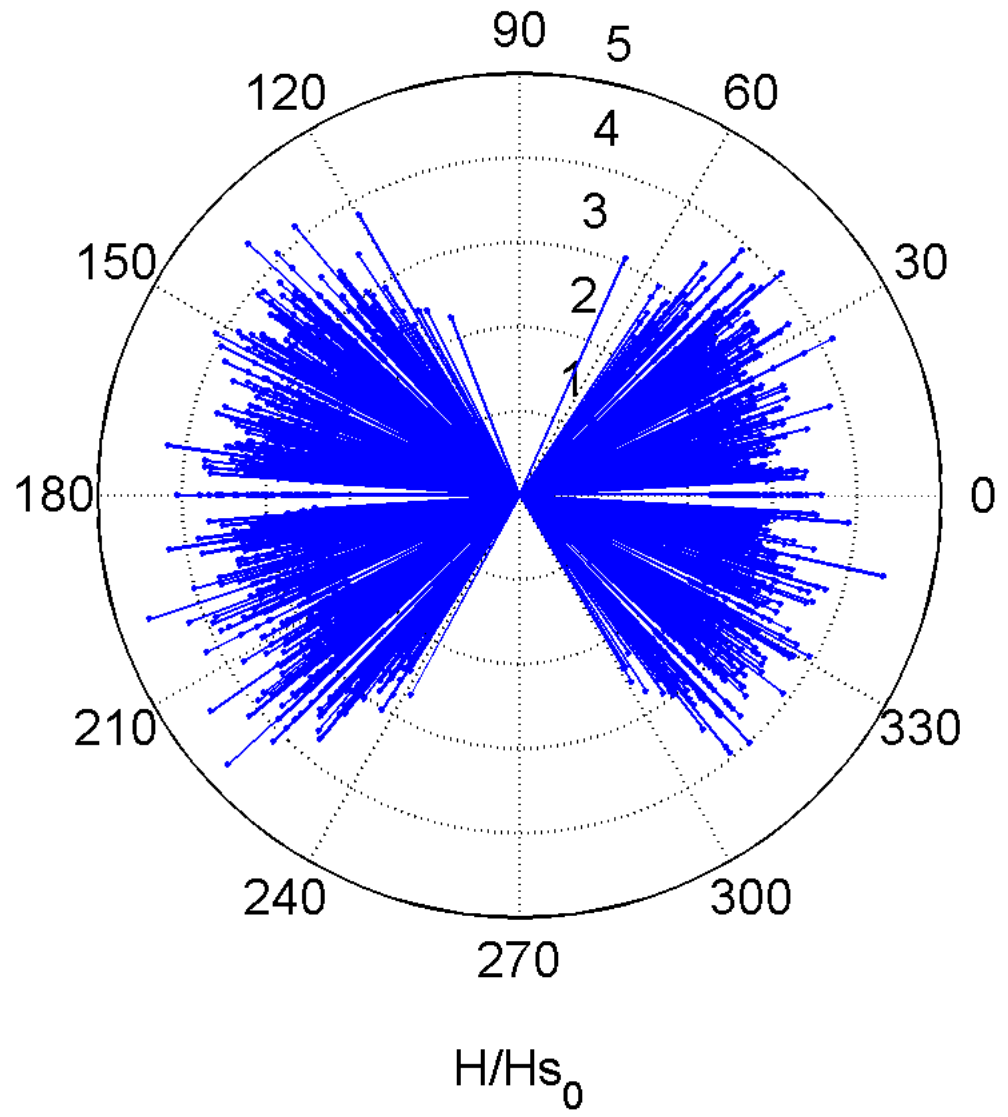

Figure 5-47: Wave height and travel direction in Case $\mathbf{F}$ (where $\alpha=0.0163$ ) at time, $t=100 T_{p}$. Graphics depict wave height $H / H_{r m s}$ as the length of the vector and the direction in which the wave travels $\Theta$ as the angle of the vector for $H>1.0 * H_{s}$ at time, $t=100 T_{p}$. 


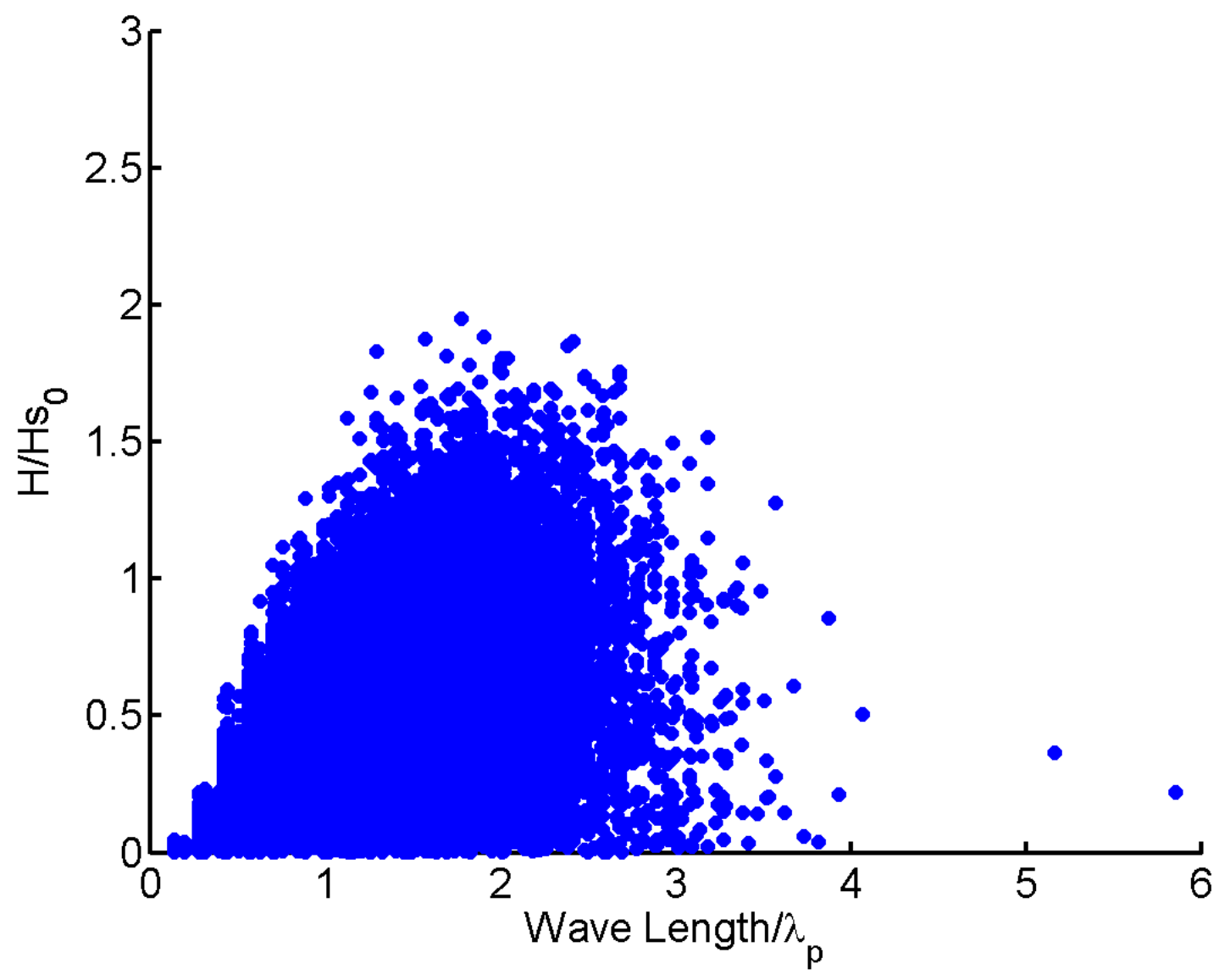

Figure 5-48: Scatter plot of dimensionless wave height vs. dimensionless wavelength in Case $\mathbf{E}$ (where $\alpha=0.0032$ ) at time, $t=50 T_{p}$. 


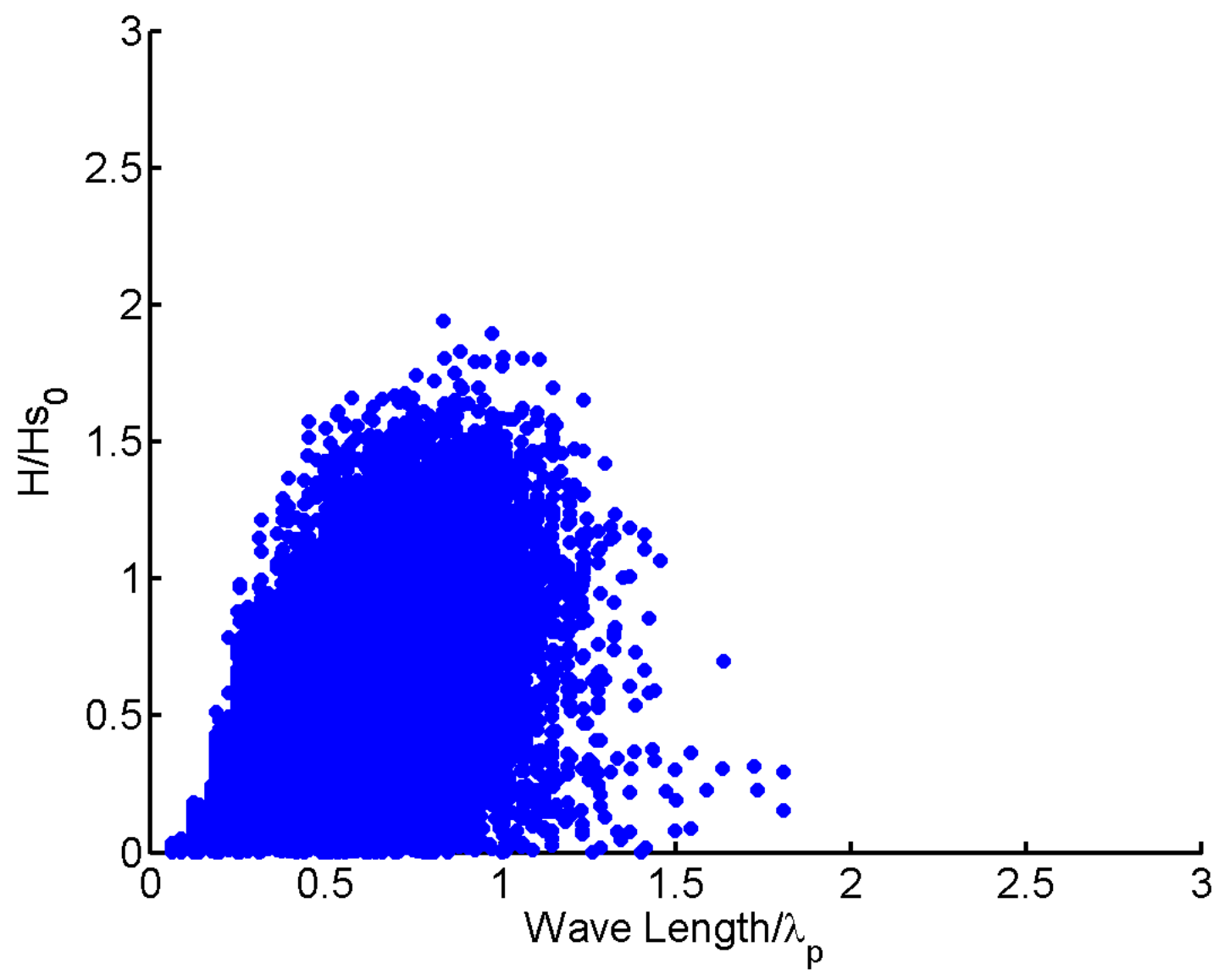

Figure 5-49: Scatter plot of dimensionless wave height vs. dimensionless wavelength in Case $\mathbf{B}$ (where $\alpha=0.0160$ ) at time, $t=50 T_{p}$. 


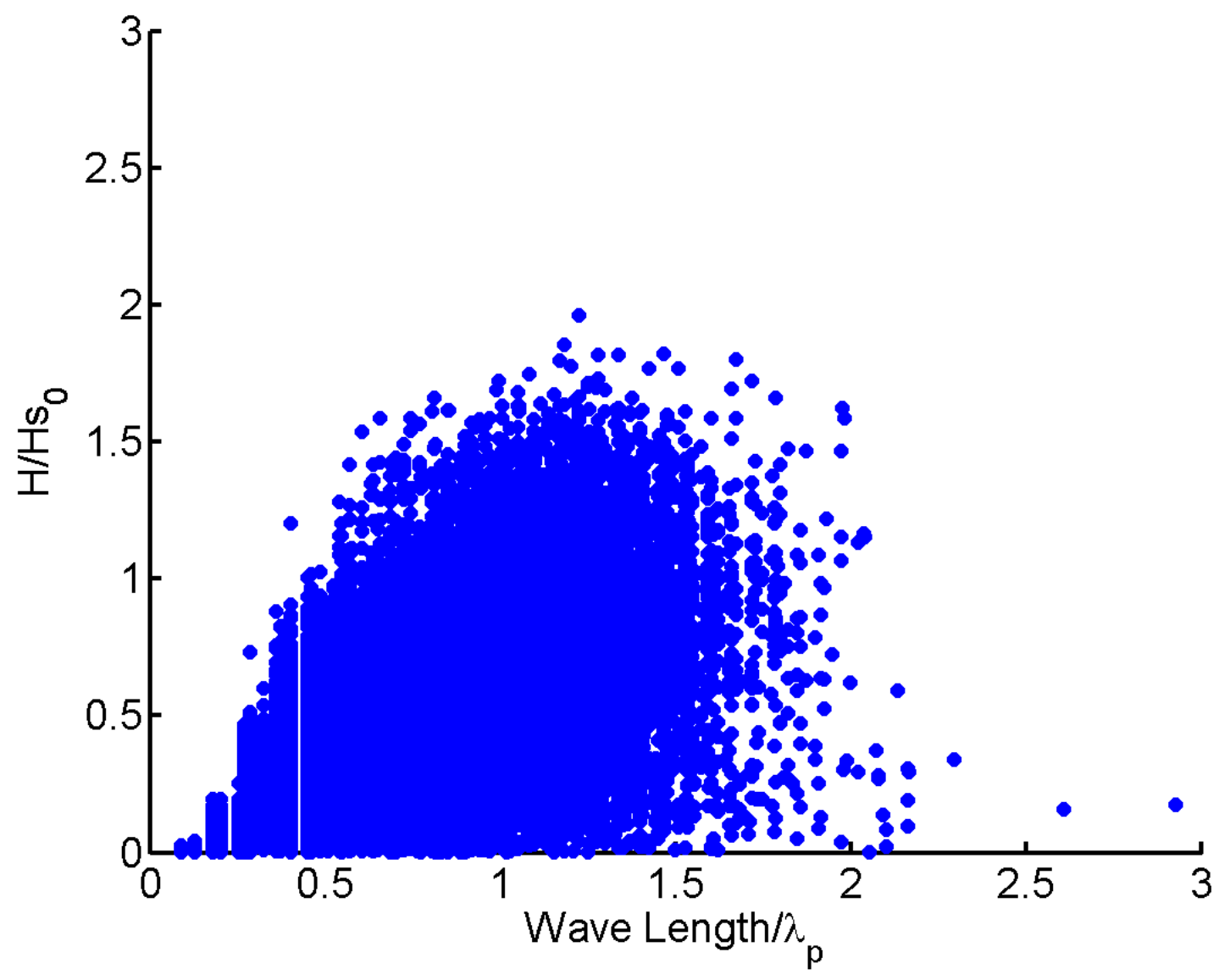

Figure 5-50: Scatter plot of dimensionless wave height vs. dimensionless wavelength in Case $\mathbf{F}$ (where $\alpha=0.0163$ ) at time, $t=50 T_{p}$. 


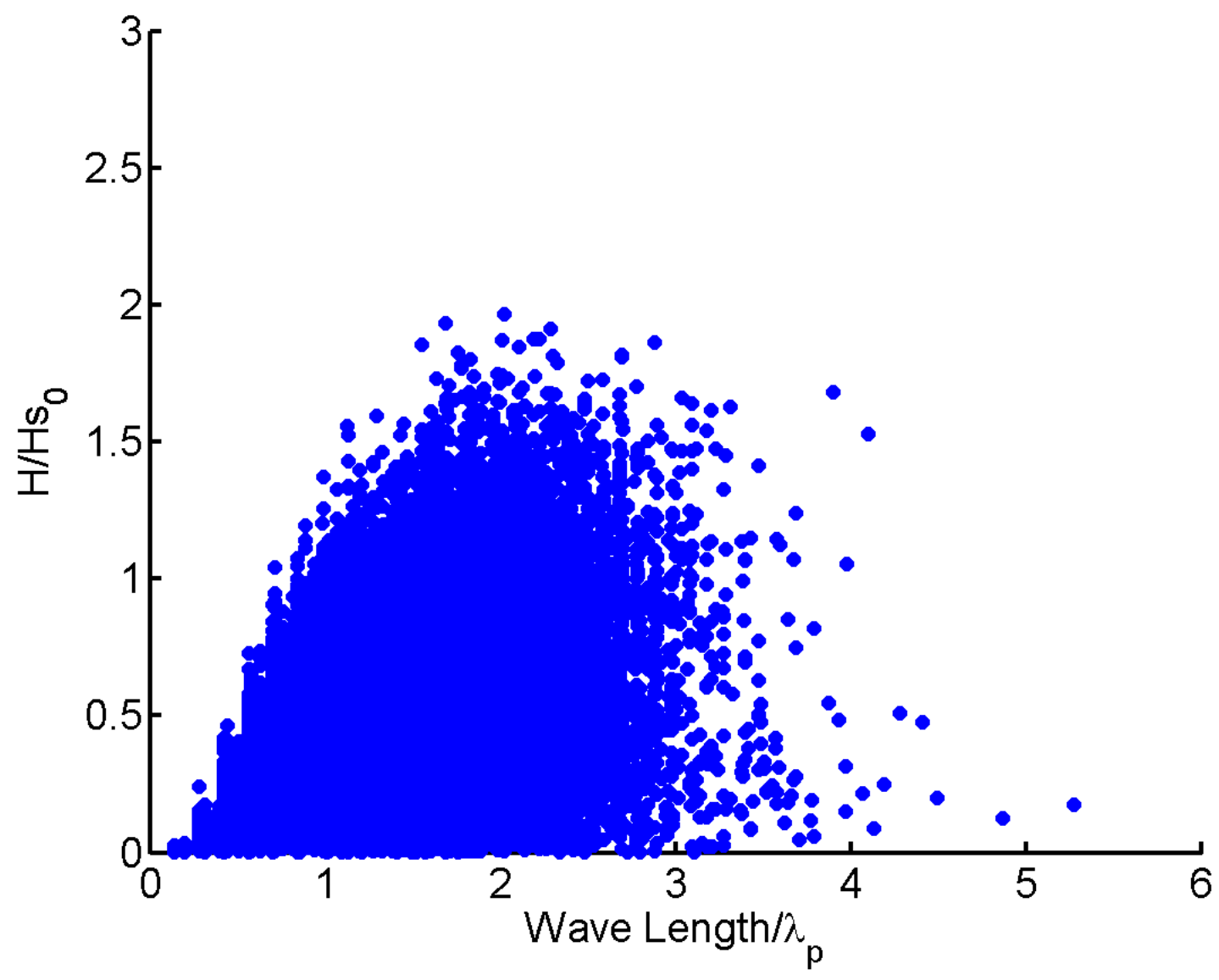

Figure 5-51: Scatter plot of dimensionless wave height vs. dimensionless wavelength in Case $\mathbf{E}$ (where $\alpha=0.0032$ ) at time, $t=100 T_{p}$. 


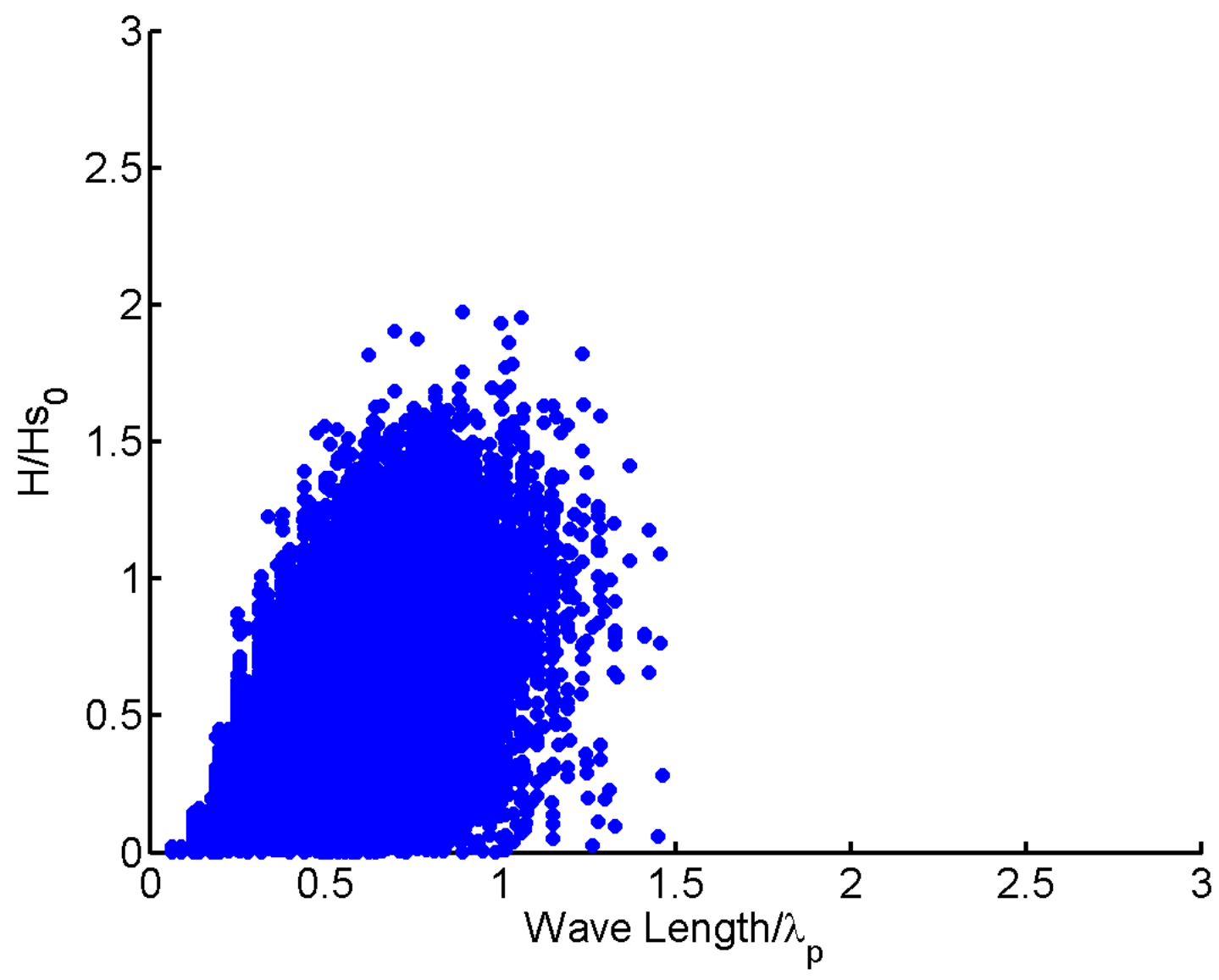

Figure 5-52: Scatter plot of dimensionless wave height vs. dimensionless wavelength in Case $\mathbf{B}$ (where $\alpha=0.0160$ ) at time, $t=100 T_{p}$. 


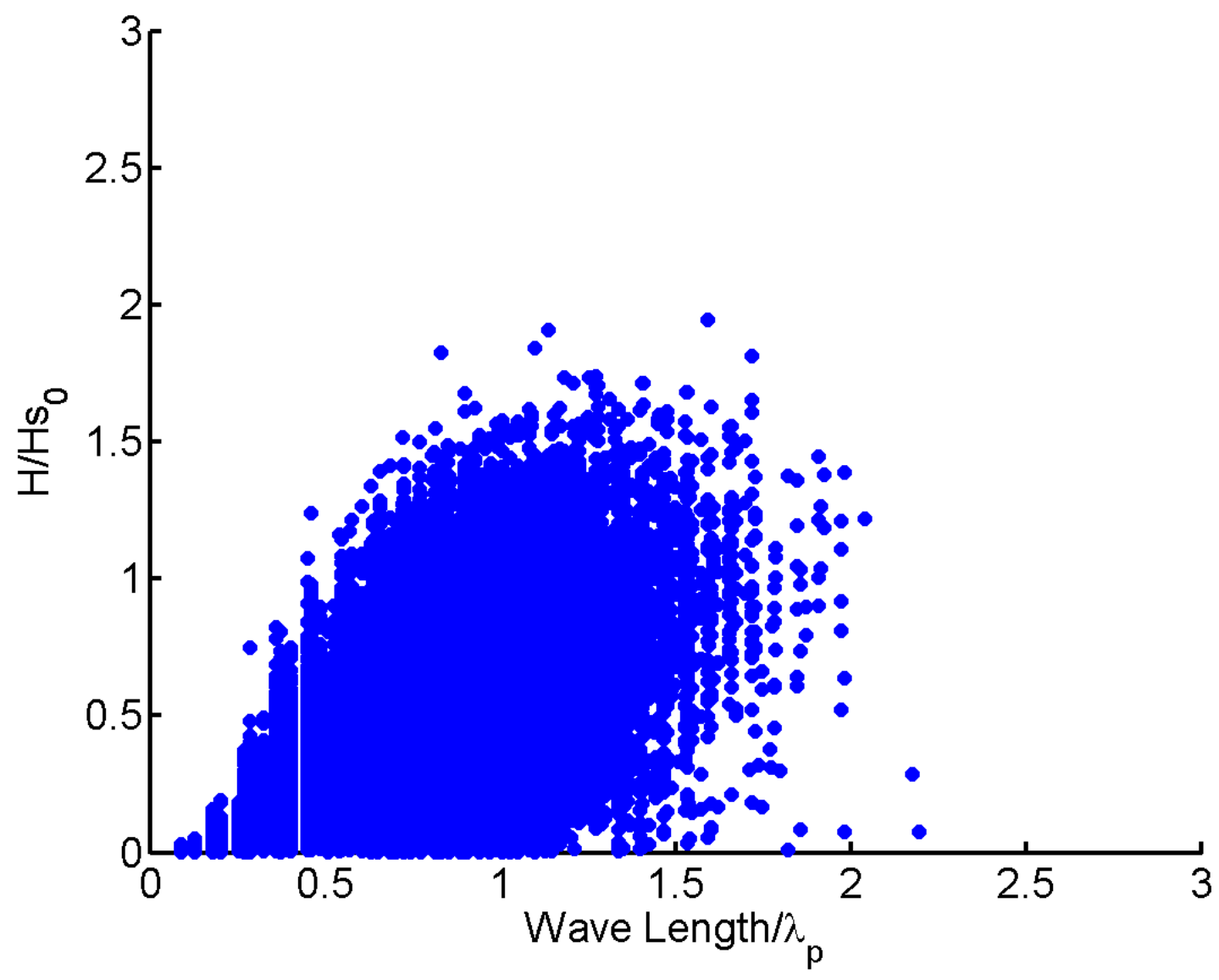

Figure 5-53: Scatter plot of dimensionless wave height vs. dimensionless wavelength in Case $\mathbf{F}$ (where $\alpha=0.0163$ ) at time, $t=100 T_{p}$. 


\subsubsection{Wavelength distribution in wavefields of various Phillips parameters}

Here we examine the wavelength distribution of Case $\mathbf{E}$, where $\alpha=0.0032$, in Figure 5-54, Case $\mathbf{B}$, where $\alpha=0.0160$ in Figure 5-55 and of Case $\mathbf{F}$, where $\alpha=0.0163$ in Figure 5-56. These distributions are all compared to the Rayleigh distribution and we find remarkably high correlations. In Figure 5-57 we see from the evolution of correlation that as time progresses, Cases $\mathbf{B}$ and $\mathbf{F}$ move further away from the Rayleigh Distribution, while Case $\mathbf{E}$ moves closer to the Rayleigh distribution.

\subsubsection{The spacing of large waves in wavefields of various Phillips parameters}

In Figures 5-58, 5-59 and 5-60 we show the spacing of large waves around the largest wave in the wavefield at a fixed time, in three separate cases. For such plots we use wave heights as defined in Section 4.3 as the narrow band of waves around the largest waves in a wavefield, as experienced by large vessels. We examine the effect of Phillips' parameter on the spacing of the large waves around the largest wave. From studying the result seen in these figures it appears that a very low Phillips' parameter produces greater spacing between the large waves in a wavefield. This is a sound conclusion, since we see in Figures 5-45, 5-46 and 5-47 that the same final number of large waves exists in all three wavefields.

\subsubsection{Conclusion: Phillips' parameter and wave height char- acteristics}

In this section we study the effect of Phillips' parameter, $\alpha$ on wave height characteristics, finding that:

- The value of Phillips' parameter influences how much wave height distribution correlates with linear, narrow-band theory. 

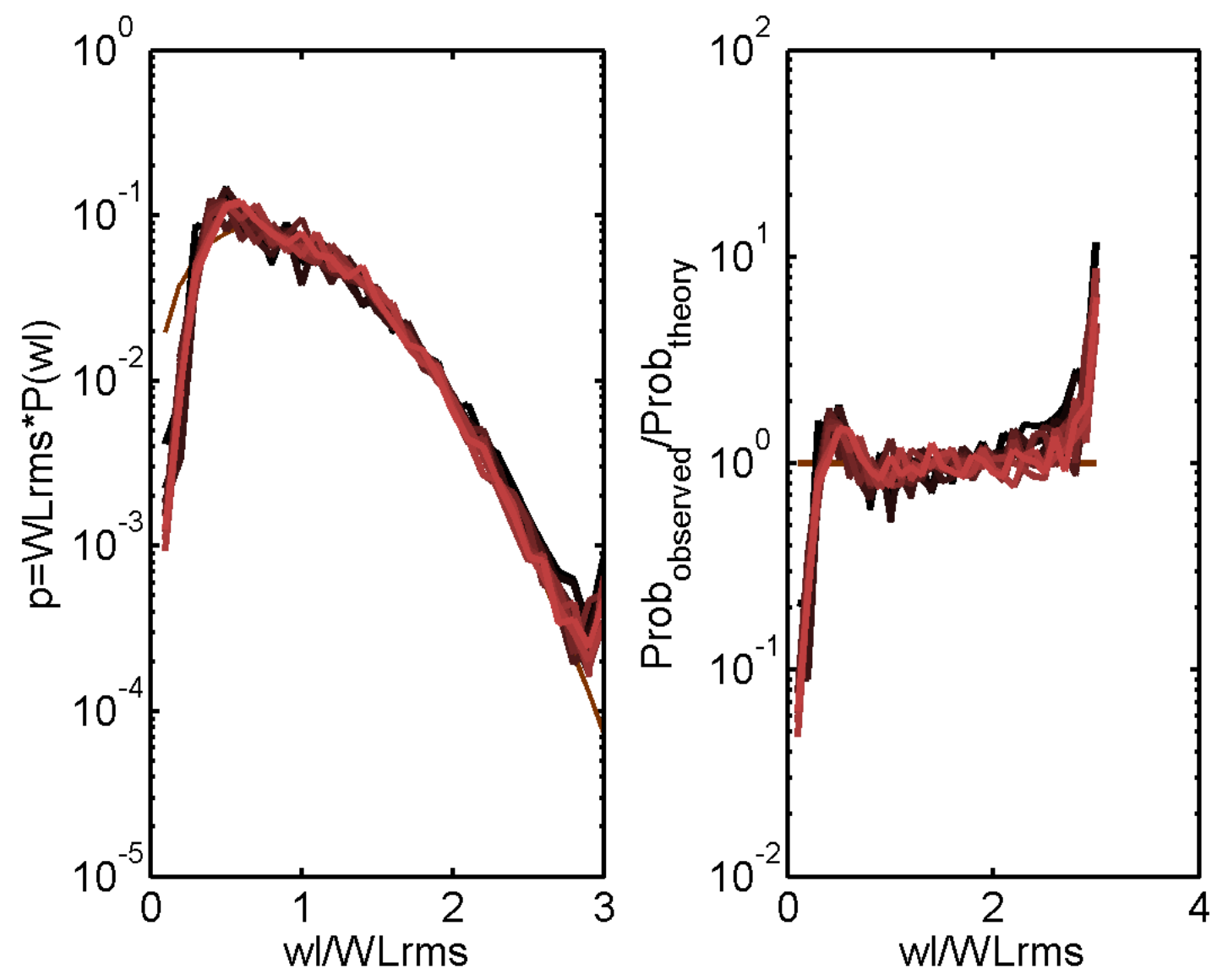

Figure 5-54: (Left) Horizontal axis shows non-dimensional wavelength and vertical axis shows the probability of occurrence of wavelength in the wavefield. Thick, brown line represents Rayleigh distribution. Other lines represent observed wavelength distribution of Case $\mathbf{E}$ where $\alpha=0.0032$, each such line representing all wavelengths in one time step. Earlier times (from $t=0 T_{p}$ ) are the darkest lines. Later times (up to $t=100 T_{p}$ ) are the brightest lines. (Right) Horizontal axis shows non-dimensional wavelength and vertical axis shows a probability ratio. Each line represents the ratio between a Case $\mathbf{E}$ time-step line and the Rayleigh distribution. 

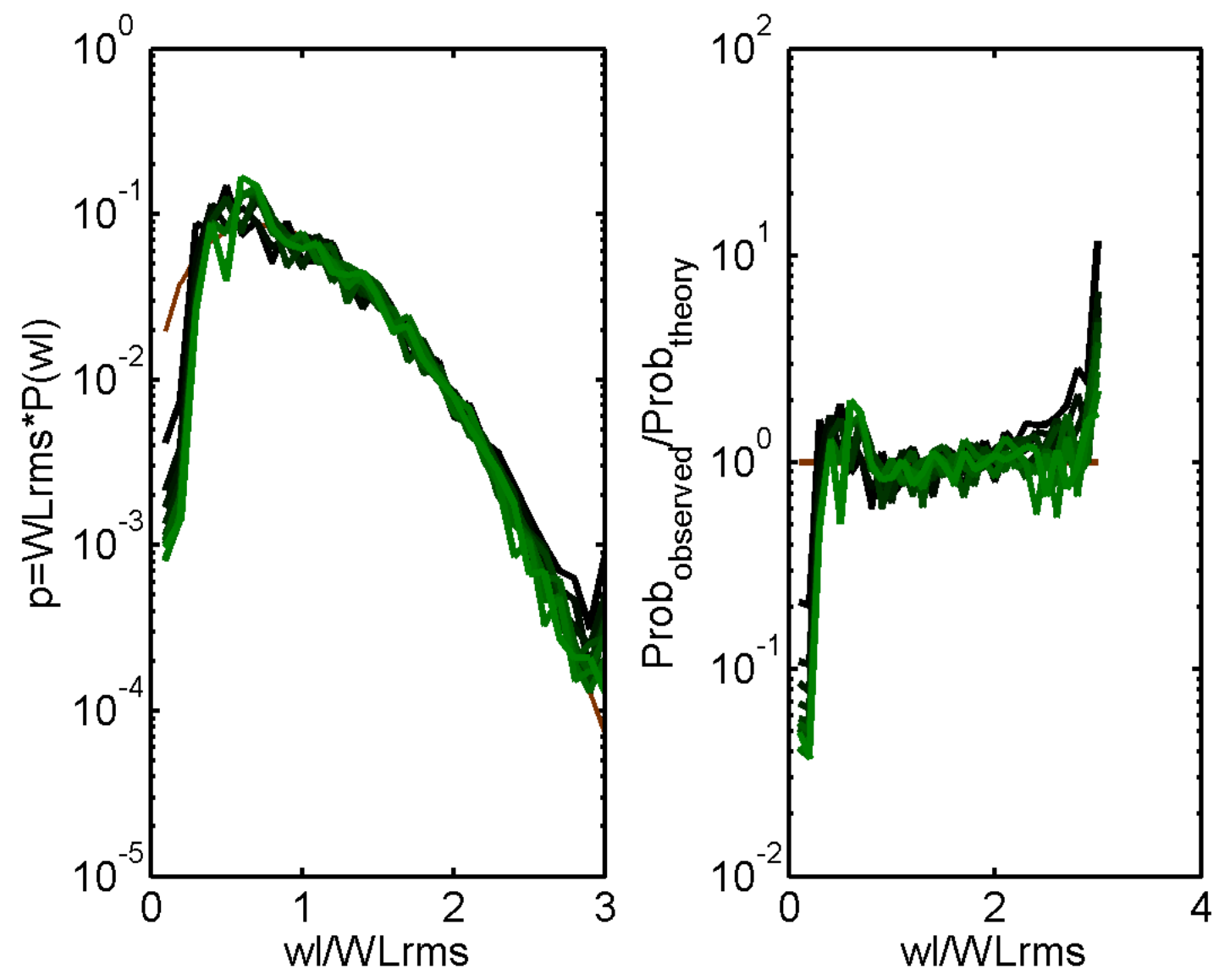

Figure 5-55: (Left) Horizontal axis shows non-dimensional wavelength and vertical axis shows the probability of occurrence of wavelength in the wavefield. Thick, brown line represents Rayleigh distribution. Other lines represent observed wavelength distribution of Case $\mathbf{B}$ where $\alpha=0.0160$, each such line representing all wavelengths in one time step. Earlier times (from $t=0 T_{p}$ ) are the darkest lines. Later times (up to $t=100 T_{p}$ ) are the brightest lines. (Right) Horizontal axis shows non-dimensional wavelength and vertical axis shows a probability ratio. Each line represents the ratio between a Case $\mathbf{B}$ time-step line and the Rayleigh distribution. 

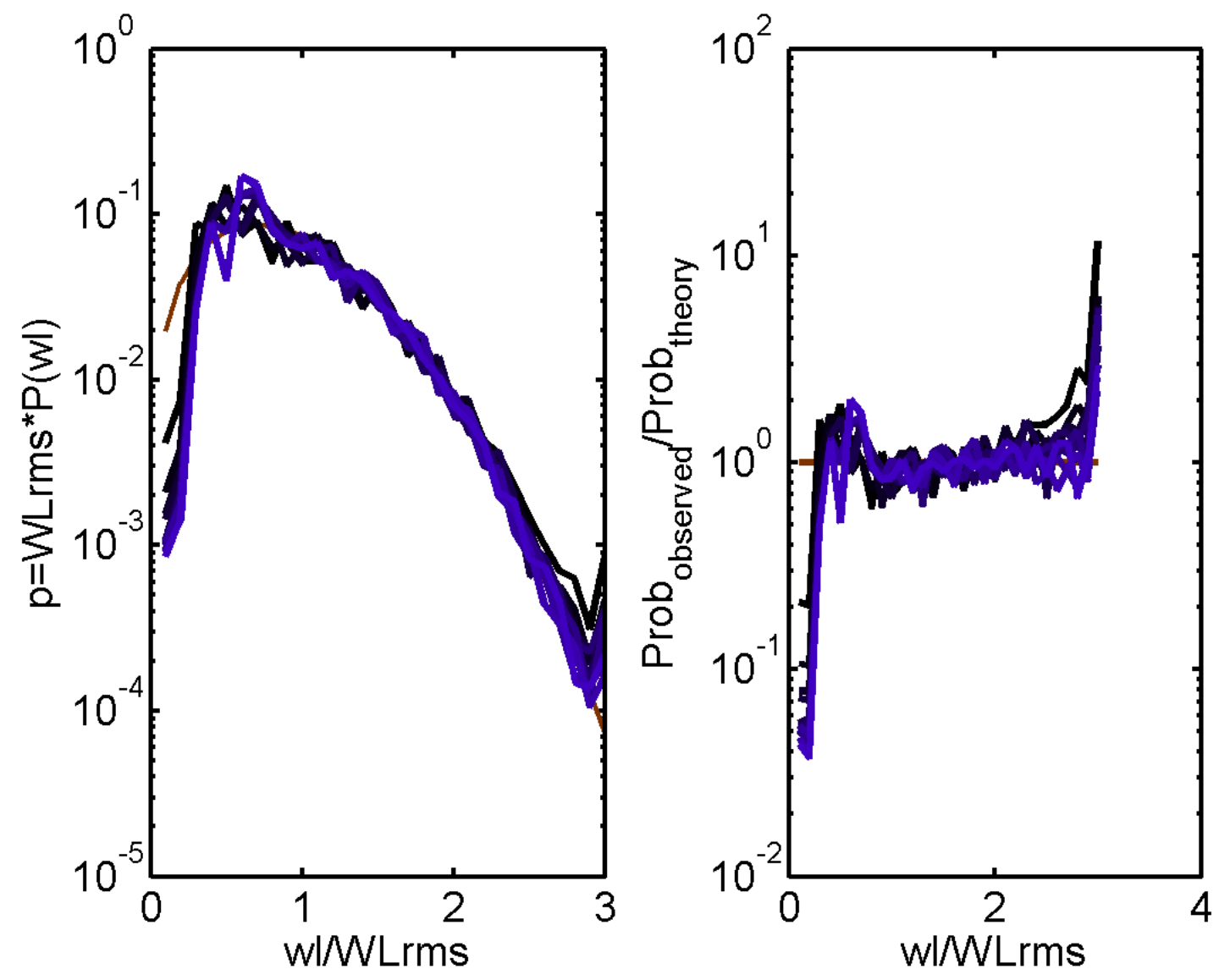

Figure 5-56: (Left) Horizontal axis shows non-dimensional wavelength and vertical axis shows the probability of occurrence of wavelength in the wavefield. Thick, brown line represents Rayleigh distribution. Other lines represent observed wavelength distribution of Case $\mathbf{F}$ where $\alpha=0.0163$, each such line representing all wavelengths in one time step. Earlier times (from $t=0 T_{p}$ ) are the darkest lines. Later times (up to $t=100 T_{p}$ ) are the brightest lines. (Right) Horizontal axis shows non-dimensional wavelength and vertical axis shows a probability ratio. Each line represents the ratio between a Case $\mathbf{F}$ time-step line and the Rayleigh distribution. 


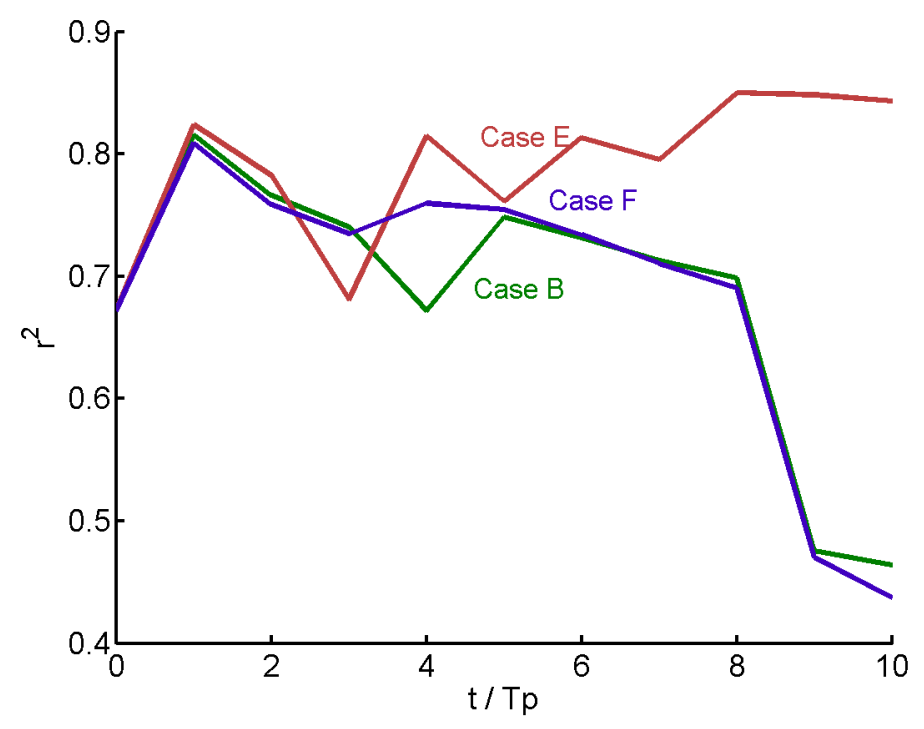

Figure 5-57: (In Color) Correlation coefficient, $r^{2}$-values between the simulated wavefields' wave length distribution and the Rayleigh distribution. Correlation coefficient, $r^{2}$ takes values between 0 and 1 where $r^{2}=1$ indicates that the simulated wavefields' wavelength distribution and the Rayleigh distribution are highly correlated and $r^{2}=0$ indicates that they are not correlated at all. We observe the time evolution of the correlation between wavelength distribution and the Rayleigh Distribution for three different non-linear simulated wavefields: (a)Case $\mathbf{E}, \alpha=0.0032$, (b)Case B, $\alpha=0.0160$ and (c) Case $\mathbf{F}, \alpha=0.0163$. 


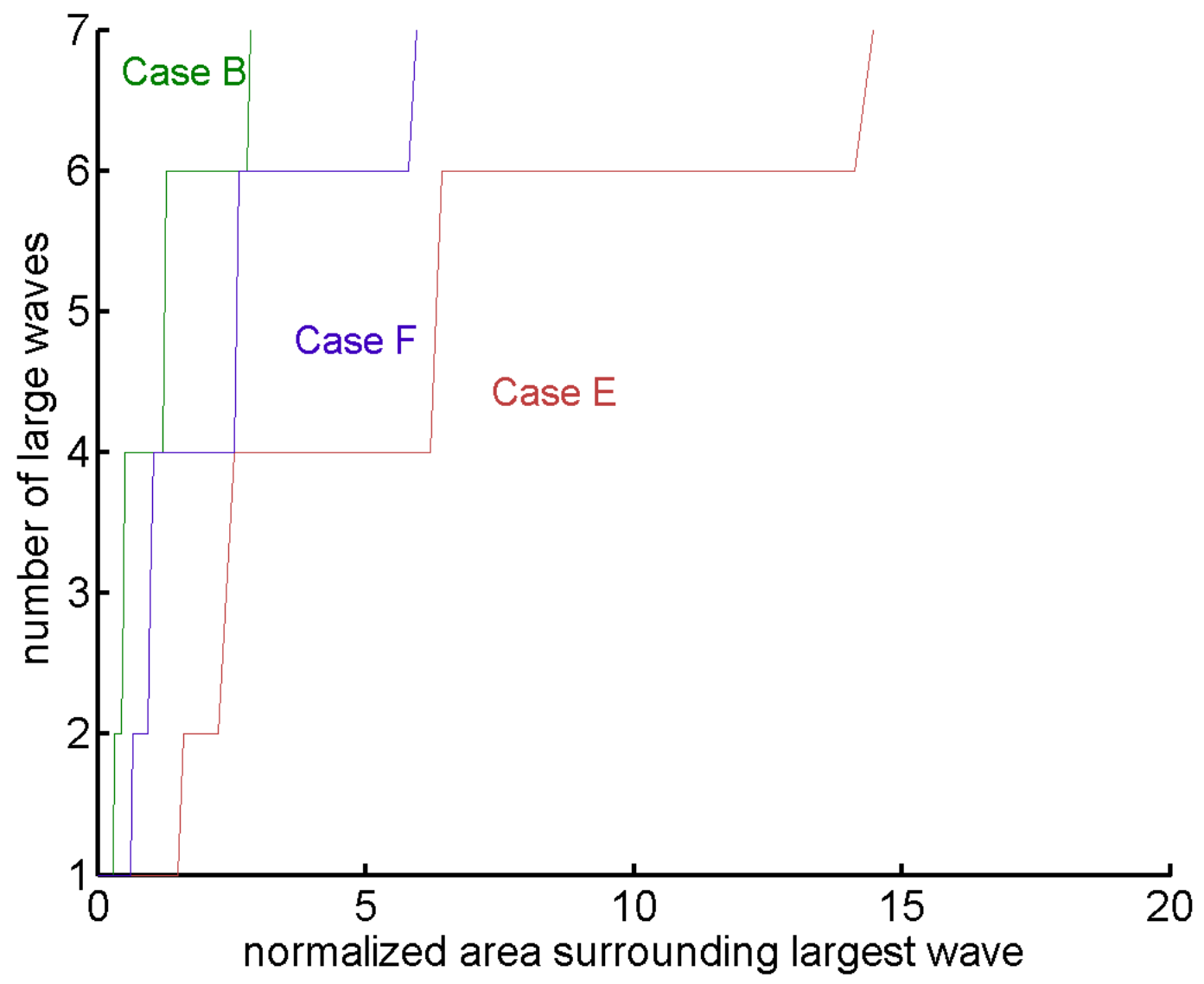

Figure 5-58: (In Color) Vertical axis depicts number of waves and horizontal axis depicts radius. Large wave spacing in Case $\mathbf{E}$ (where $\alpha=0.0032$ ), Case $\mathbf{B}$ (where $\alpha=0.0160$ ), and Case $\mathbf{F}$ (where $\alpha=0.0163$ ) at initial time, $t=0 T_{p}$. Spacing in waves with height $H>1.5 * H_{s}$ at times, $t=20 T_{p}, t=50 T_{p}$, and $t=100 T_{p}$. 


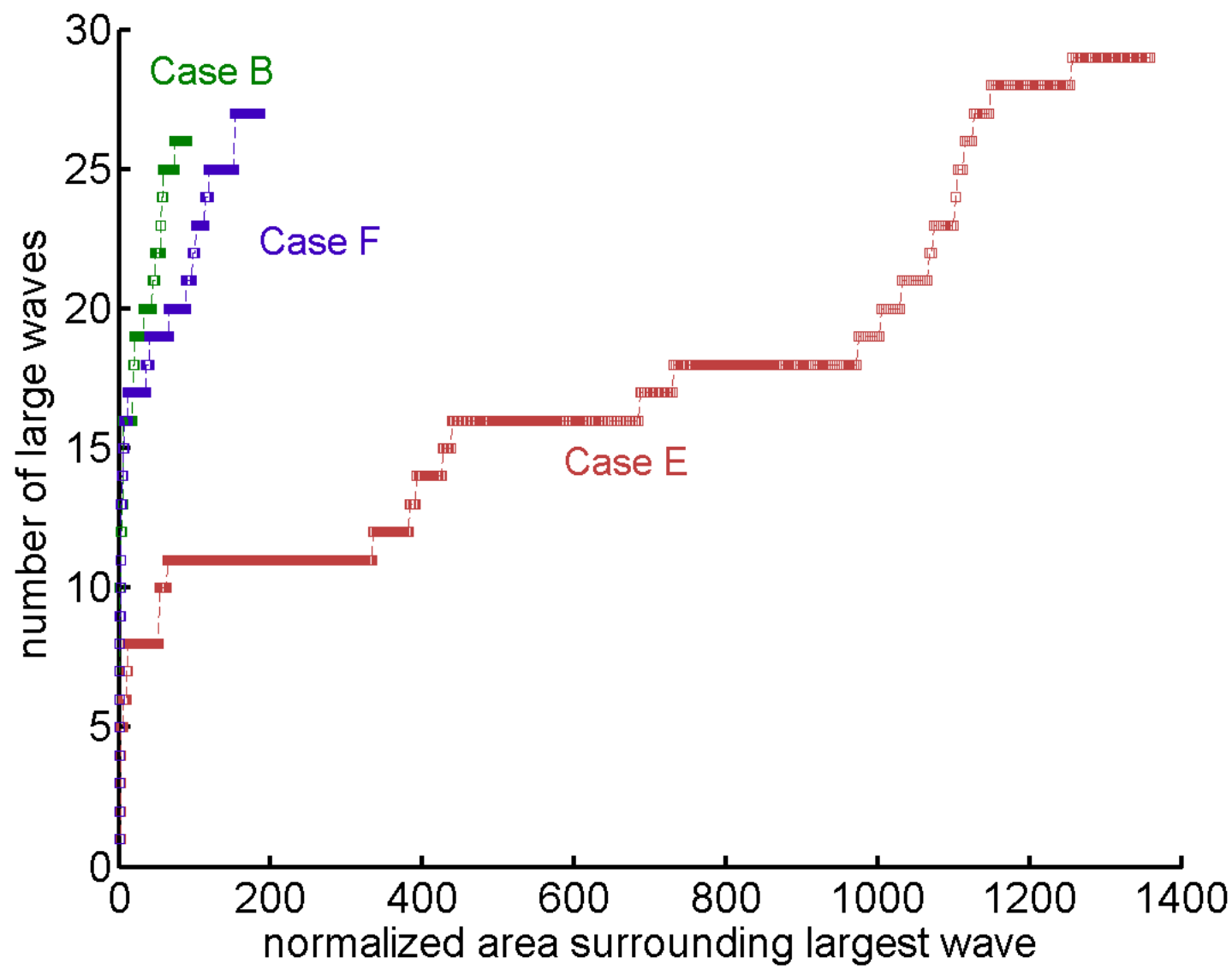

Figure 5-59: (In Color) Vertical axis depicts number of waves and horizontal axis depicts radius. Large wave spacing in Case $\mathbf{E}$ (where $\alpha=0.0032$ ), Case $\mathbf{B}$ (where $\alpha=0.0160$ ), and Case $\mathbf{F}$ (where $\alpha=0.0163$ ) at time, $t=50 T_{p}$. Spacing in waves with height $H>1.5 * H_{s}$ at times, $t=20 T_{p}, t=50 T_{p}$, and $t=100 T_{p}$. 


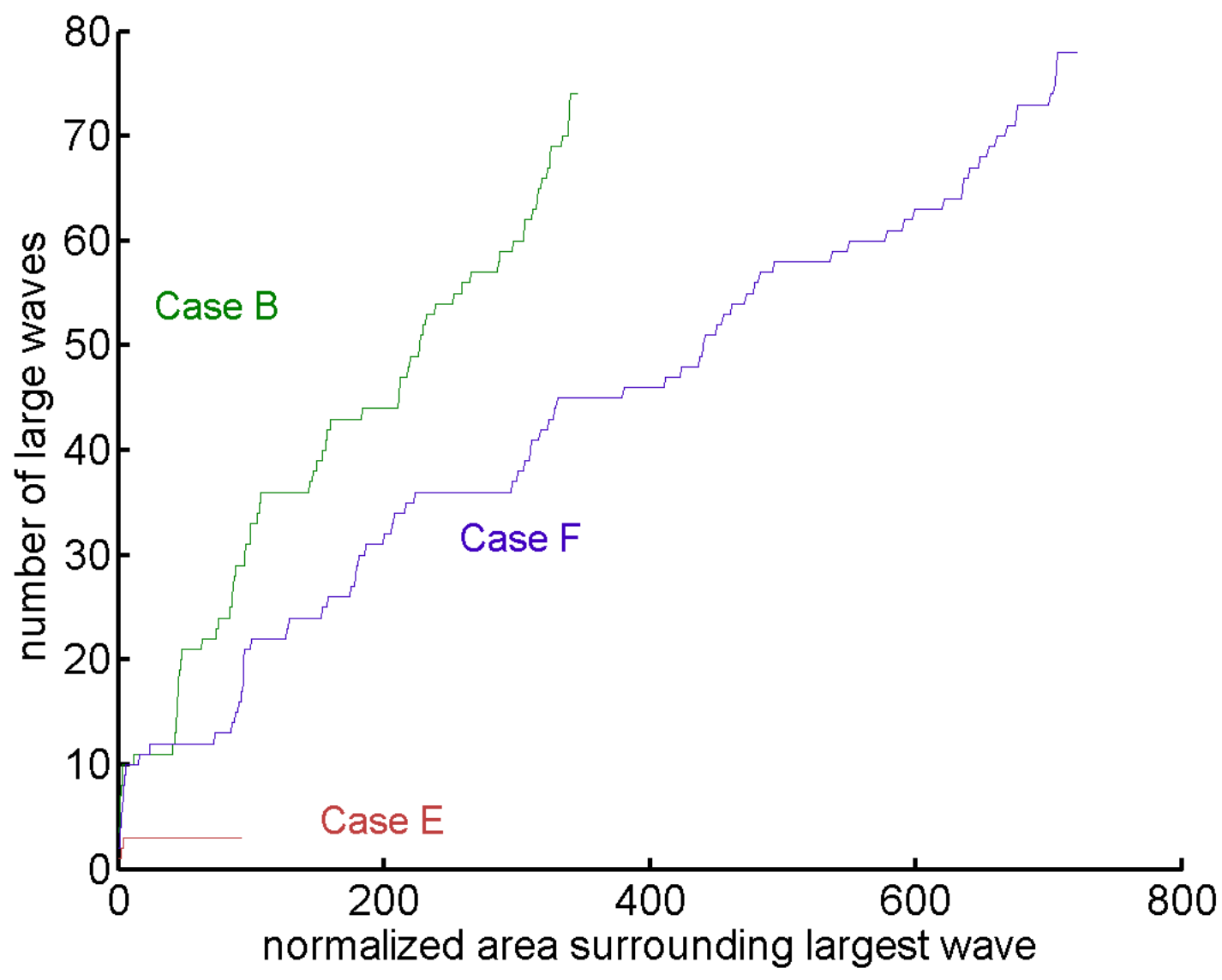

Figure 5-60: (In Color) Vertical axis depicts number of waves and horizontal axis depicts radius. Large wave spacing in Case $\mathbf{E}$ (where $\alpha=0.0032$ ), Case $\mathbf{B}$ (where $\alpha=0.0160$ ), and Case $\mathbf{F}$ (where $\alpha=0.0163$ ) at time, $t=100 T_{p}$. Spacing in waves with height $H>1.5 * H_{s}$ at times, $t=20 T_{p}, t=50 T_{p}$, and $t=100 T_{p}$. 
- Higher Phillips' parameter, $\alpha$ produces a greater occurrence of large rogue-like wave heights in the joint distribution between wave heights and wavelengths, and the lowest Phillips parameter produces the longest wavelengths.

- Lower values of Phillips' parameter produce greater adherence of wavelength distribution to the Rayleigh distribution, indicating that larger spectral width is associated with lower values of Phillips' parameter.

- A very low Phillips' parameter produces greater spacing between the large waves in a wavefield

- Phillips' parameter has a visible effect on the occurrence of large waves, but no visible effect on the direction in which these large waves move. 


\subsection{Wave height characteristics and input spectral spreading angle}

In this section we observe the distribution of wave heights, and the impact of the input spectral directional spreading function on the adherence of wave height distributions to linear theory, outlined in Appendix C. As in the previous two sections, we generally use wave height defined according to the local maximum corresponding with the nearest connected local minimum method in Section 4.4 unless otherwise stated.

\subsubsection{Wave height distribution and input spectral spreading angle}

We use a non-linear, non-narrow-band 3D analogue to the half-cycle excursion method to define wave heights. In figures 5-61, 5-62 and 5-63 we see that wave height distributions and distribution ratios are very similar across two regimes of spreading angle: A regime of strong spreading (where $\theta=80^{\circ}$ or $180^{\circ}$ ), and a regime of weak spreading (where $\theta=20^{\circ}$ ). Comparing the correlations for all the cases, we find that Figure 5-64 tells us that strong spreading produces a much better correlation between the Rayleigh distribution and the observed data than in the instance of weak spreading.

\subsubsection{Effect of input spectral directional spreading function on wave height exceedance probability}

In this section we observe the exceedance probability of wave heights, and the impact of the input spectral directional spreading function on the adherence of wave height exceedance probability to linear narrow band theory. The linear theory on wave height exceedance is equivalent to the integral of the Rayleigh distribution outlined in Appendix $\mathrm{C}$ and give a different perspective to the distributions we've already seen in the previous section. In Figures 5-65, 5-66 and 5-67 we see that exceedance probability distribution in Case $\mathbf{K}$, where $\theta=20^{\circ}$ moves closer to the linear narrow band theory as time proceeds whereas exceedance probability distributions in Case $\mathbf{J}$ 

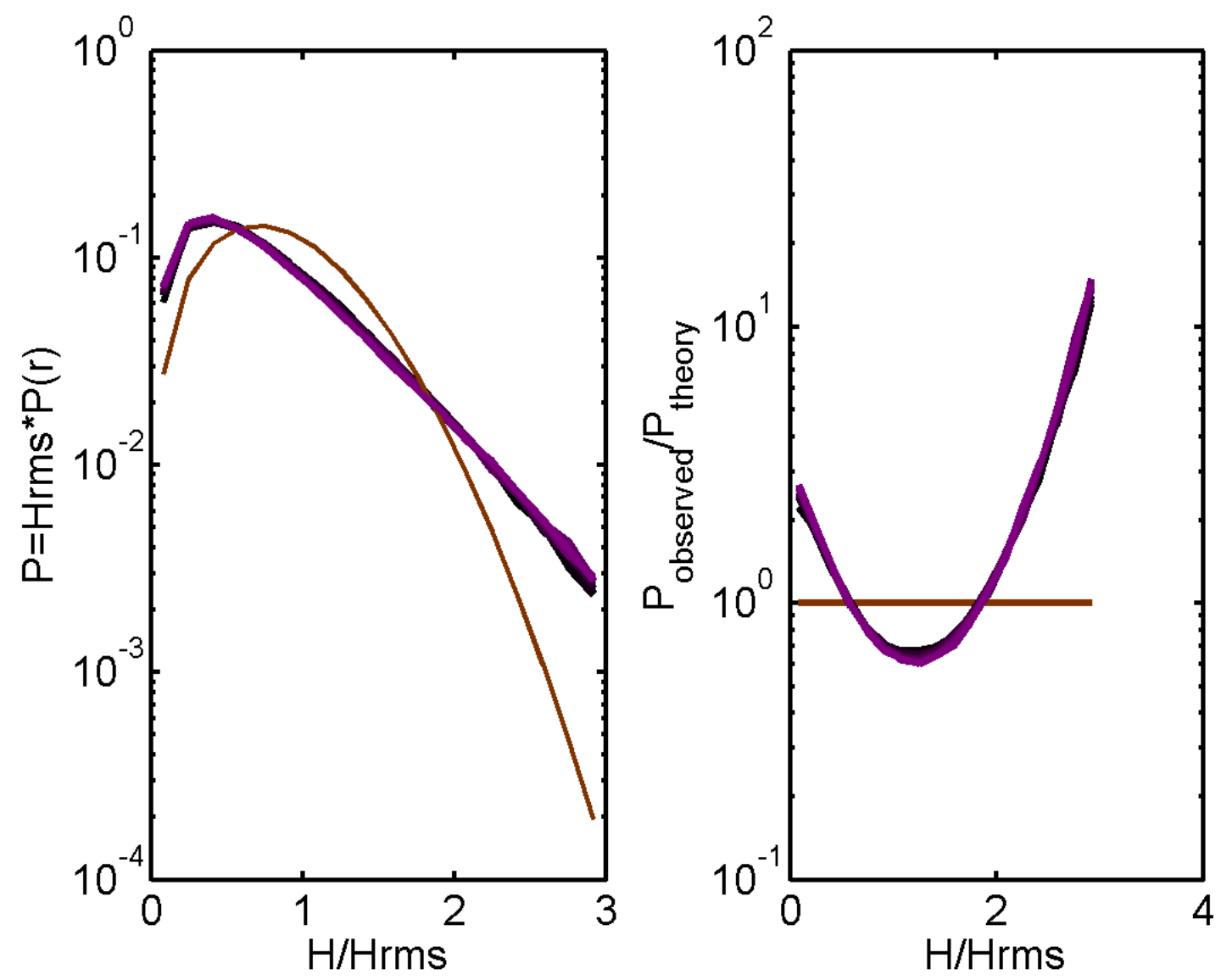

Figure 5-61: (In Color) (Left) Horizontal axis shows non-dimensional wave height and vertical axis shows the probability of occurrence of heights in the wavefield. Thick, brown line represents Rayleigh distribution. Other lines represent Case J (where $\theta=180^{\circ}$ ), each such line representing one time step. Earlier times (from $t=0 T_{p}$ ) are the darkest lines. Later times (up to $t=100 T_{p}$ ) are the brightest lines. (Right) Horizontal axis shows non-dimensional wave height and vertical axis shows a probability ratio. Each line represents the ratio between a Case $\mathbf{J}$ time-step line and the Rayleigh distribution. 

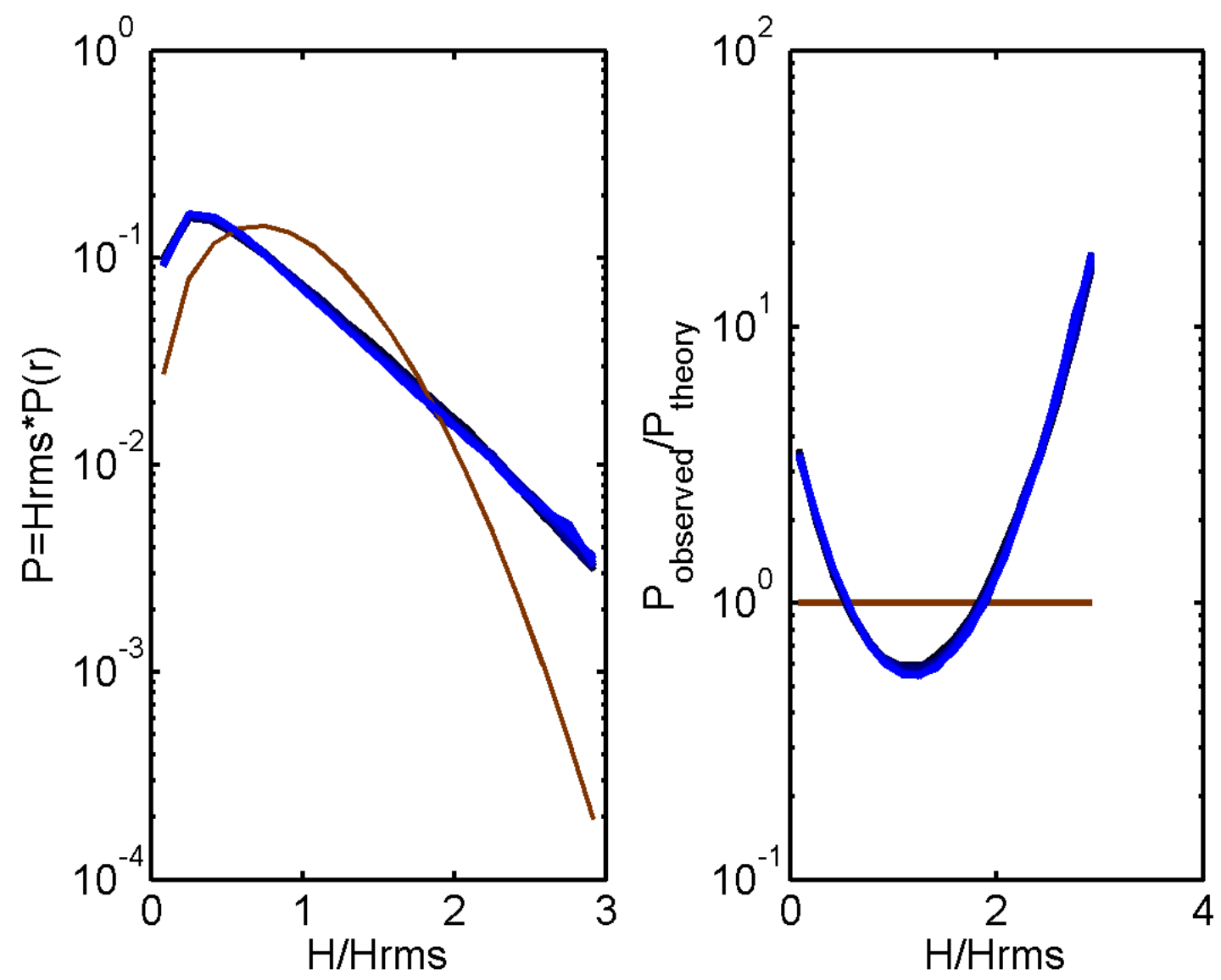

Figure 5-62: (In Color) (Left) Horizontal axis shows non-dimensional wave height and vertical axis shows the probability of occurrence of heights in the wavefield. Thick, brown line represents Rayleigh distribution. Other lines represent Case A (where $\theta=80^{\circ}$ ), each such line representing one time step. Earlier times (from $t=0 T_{p}$ ) are the darkest lines. Later times (up to $t=100 T_{p}$ ) are the brightest lines. (Right) Horizontal axis shows non-dimensional wave height and vertical axis shows a probability ratio. Each line represents the ratio between a Case $\mathbf{A}$ time-step line and the Rayleigh distribution. 

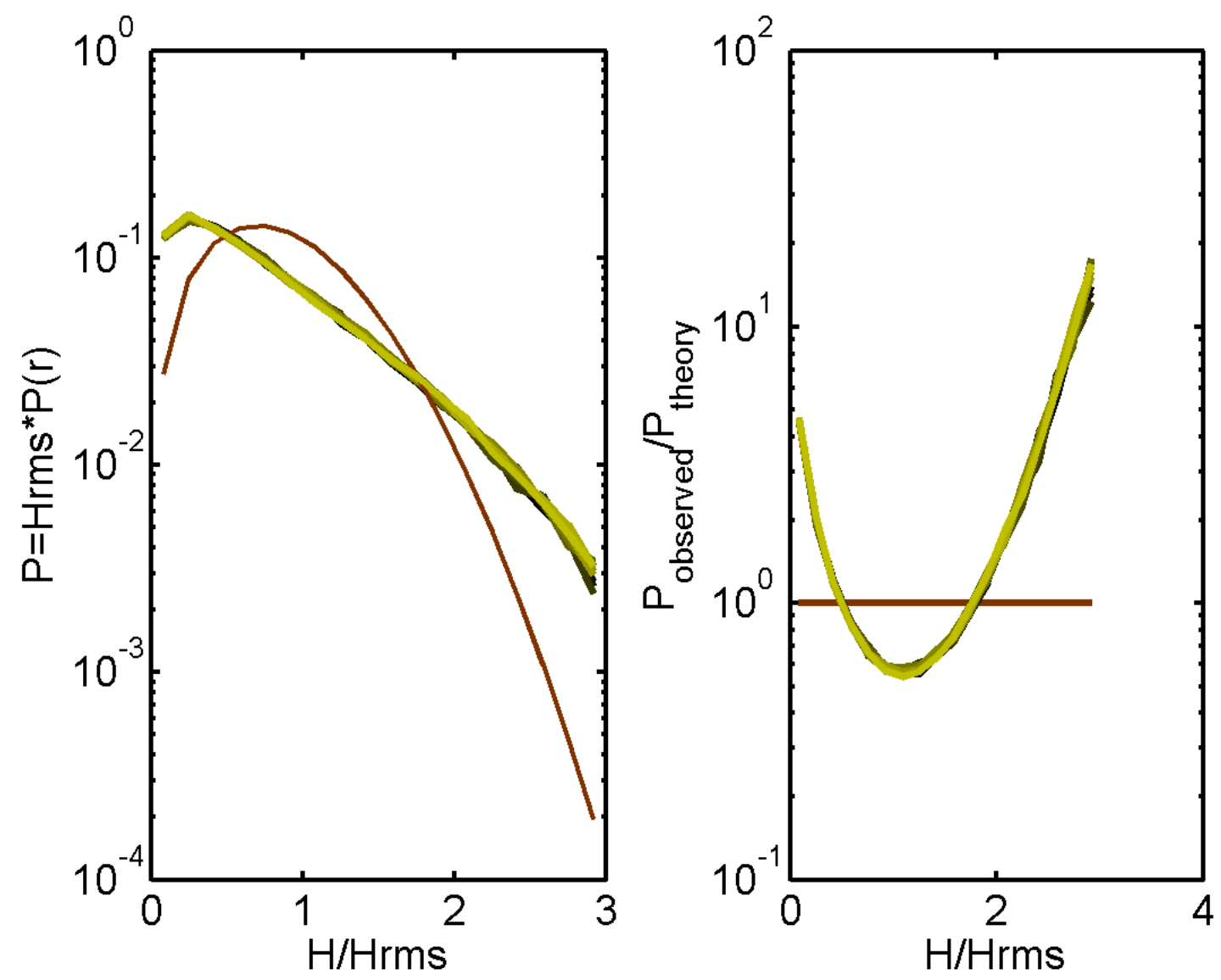

Figure 5-63: (In Color) (Left) Horizontal axis shows non-dimensional wave height and vertical axis shows the probability of occurrence of heights in the wavefield. Thick, brown line represents Rayleigh distribution. Other lines represent Case $\mathbf{K}$ (where $\theta=20^{\circ}$ ), each such line representing one time step. Earlier times (from $t=0 T_{p}$ ) are the darkest lines. Later times (up to $t=100 T_{p}$ ) are the brightest lines. (Right) Horizontal axis shows non-dimensional wave height and vertical axis shows a probability ratio. Each line represents the ratio between a Case $\mathbf{K}$ time-step line and the Rayleigh distribution. 


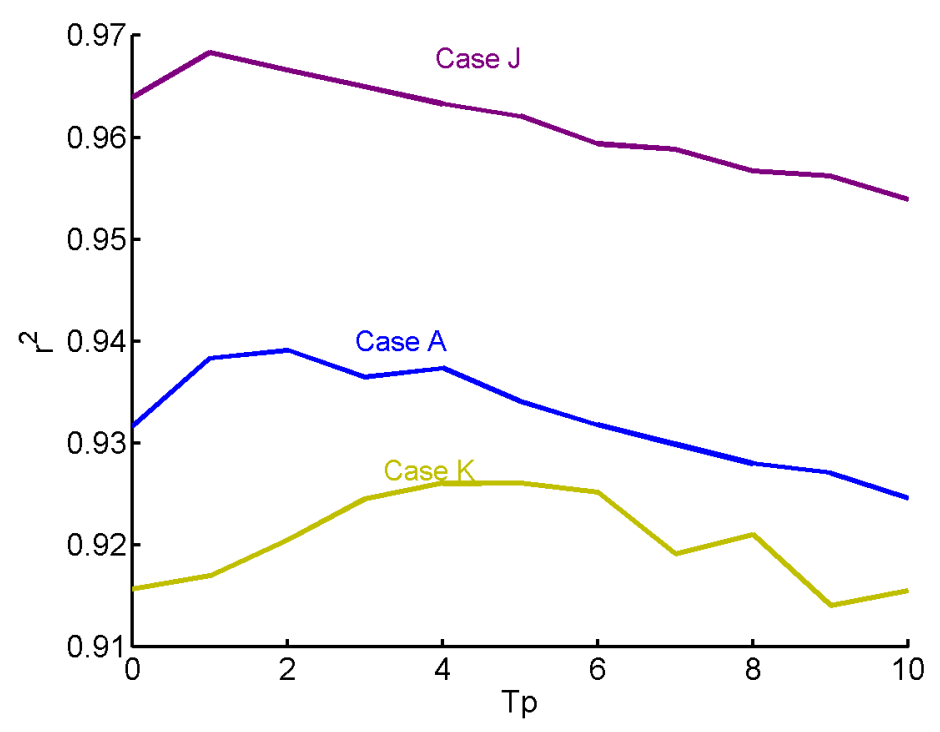

Figure 5-64: (In Color) Correlation coefficient, $r^{2}$-values between the simulated wavefields' wave height distribution and the Rayleigh distribution. Correlation coefficient, $r^{2}$ takes values between 0 and 1 where $r^{2}=1$ indicates that the simulated wavefields' wave height distribution and the Rayleigh distribution are highly correlated and $r^{2}=0$ indicates that they are not correlated at all. We observe wave height distribution correlation with the Rayleigh distribution in Case $\mathbf{J}$ (where $\theta=180^{\circ}$ ), Case $\mathbf{A}\left(\right.$ where $\theta=80^{\circ}$ ), Case $\mathbf{B}$ (where $\theta=40$ ), Case $\mathbf{M}$ (where $\theta=30$ ), and Case $\mathbf{K}\left(\right.$ where $\left.\theta=20^{\circ}\right)$. 
where $\theta=180^{\circ}$ and in Case $\mathbf{A}$ where $\theta=80^{\circ}$ vary less in time than in Case $\mathbf{K}$ but move further away from the linear narrow band theory as time proceeds.

\subsubsection{Effect of input spectral directional spreading function on the joint distribution between wave height and wave length}

In this section we study the effect of the input spectral directional spreading function on the joint distribution between wave height and wavelength. While the theoretical background of this result (discussed in Section 5.1.2) speaks only of the effect of spectral bandwidth on this distribution we see the directional spreading angle, $\theta$, producing enough of an effect to influence the spread of the scatter plots in Figures 5-68, 5-69, 5-70, 5-71, 5-72, 5-73, 5-74, 5-75, and 5-76. We see that throughout time, the greatest occurrence of rogue-like waves with height, $H / H_{s} \approx 2$ and the greatest occurrence of long waves are both associated with the lowest directional spreading angle in Case $\mathbf{K}$ where $\theta=20^{\circ}$.

\subsubsection{Effect of input spectral directional spreading function on wave length distribution}

In this section we observe how the distribution of wave lengths is impacted by input spectral directional spreading function. Section 5.1.3 outlines a theory for wavelength distribution which is quite similar to the Rayleigh distribution theory when spectral bandwidth is high enough. Our non-narrow-band definition of wavelengths (see Section 4.4) fits this category well. As we look at Figure 5-80, it can be concluded that, with all other input parameters equal, smaller spreading angles consistently result in wavelength distributions that are more correlated with the Rayleigh distribution theory.

We also see the wavelength distributions displayed in Figures 5-77, 5-78 and 579 where the Rayleigh distribution acts as an excellent theoretical match to these 

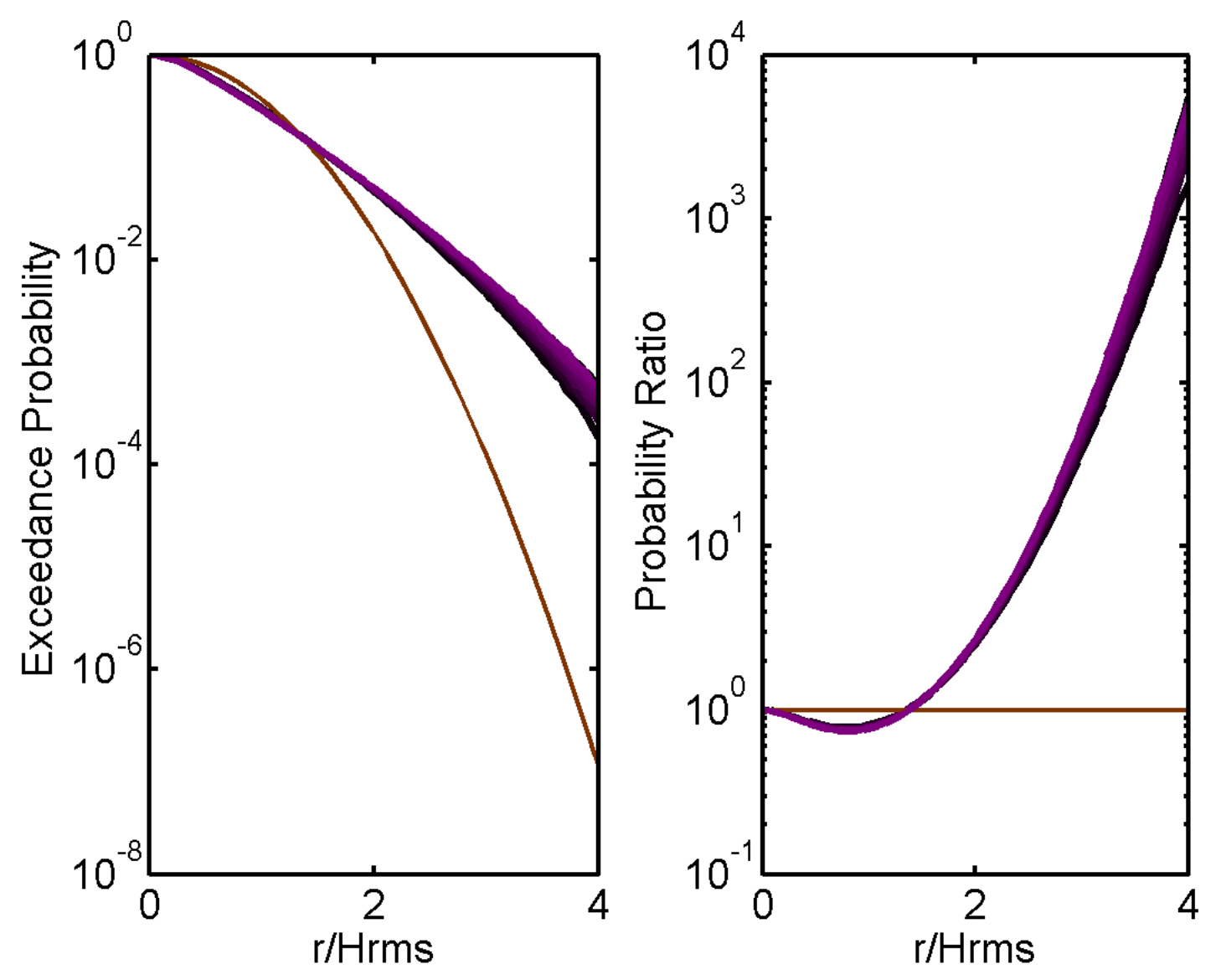

Figure 5-65: (In Color) Wave height exceedance probability in Case $\mathbf{J}$ (where $\theta=$ $180^{\circ}$ ) from times, $t=0 T_{p}$ (darkest line) to $t=100 T_{p}$ (brightest line) compared to the wave height exceedance probability in linear narrow-band theory (thick, red line). 

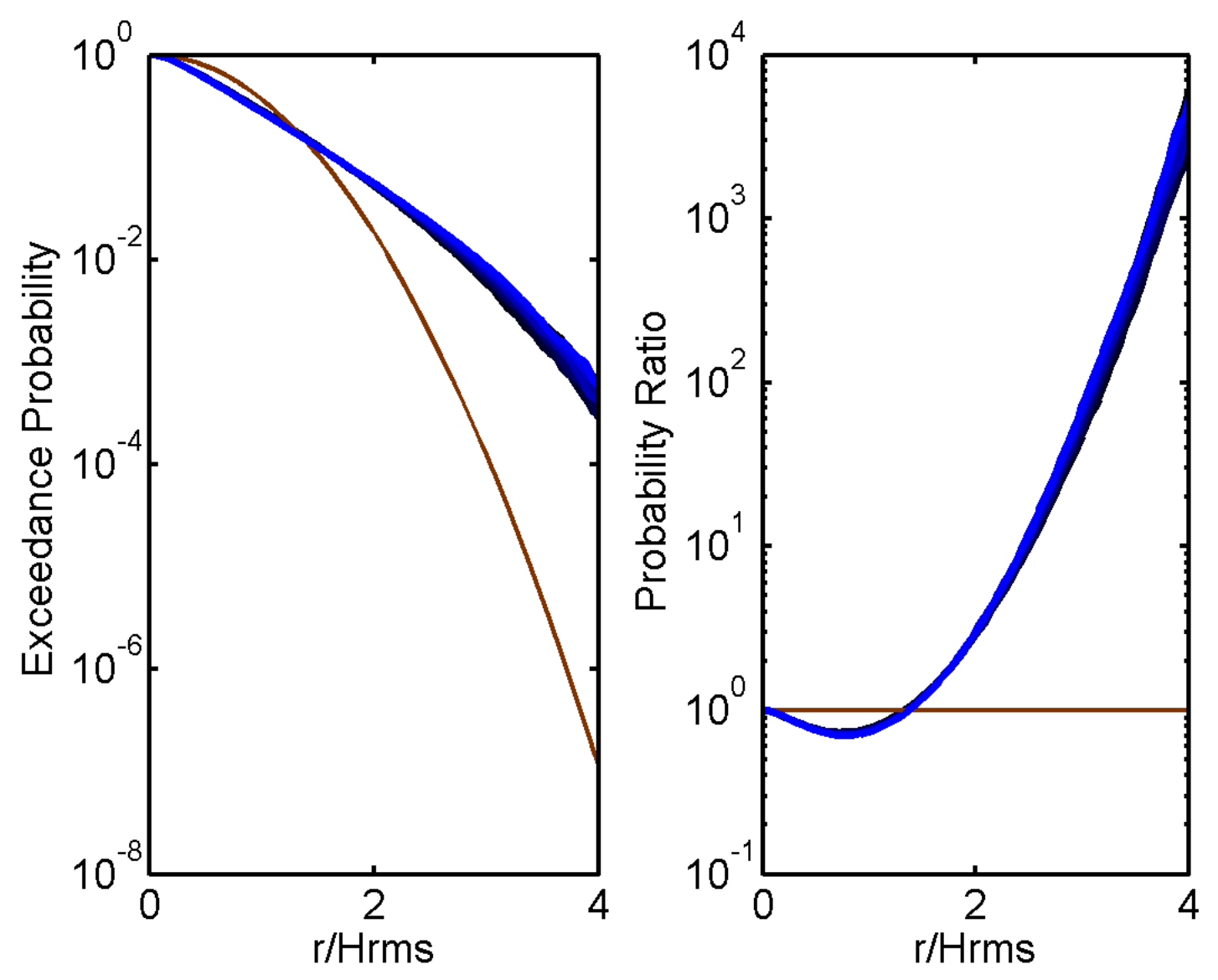

Figure 5-66: (In Color) Wave height exceedance probability in Case $\mathbf{A}$ (where $\theta=80^{\circ}$ ) from times, $t=0 T_{p}$ (darkest line) to $t=100 T_{p}$ (brightest line) compared to the wave height exceedance probability in linear narrow-band theory (thick, red line). 

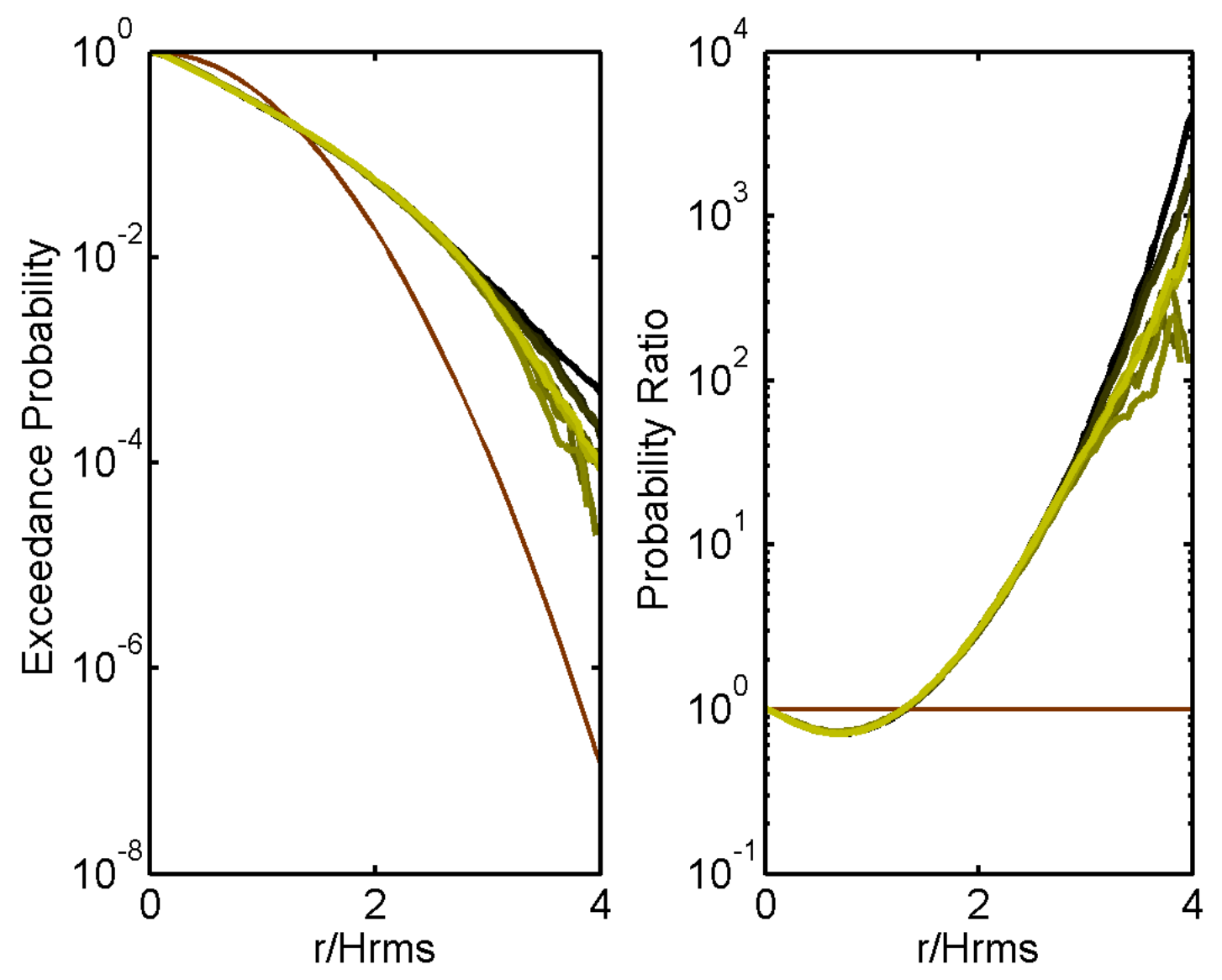

Figure 5-67: (In Color) Wave height exceedance probability in Case $\mathbf{K}$ (where $\theta=20^{\circ}$ ) from times, $t=0 T_{p}$ (darkest line) to $t=100 T_{p}$ (brightest line) compared to the wave height exceedance probability in linear narrow-band theory (thick, red line). 


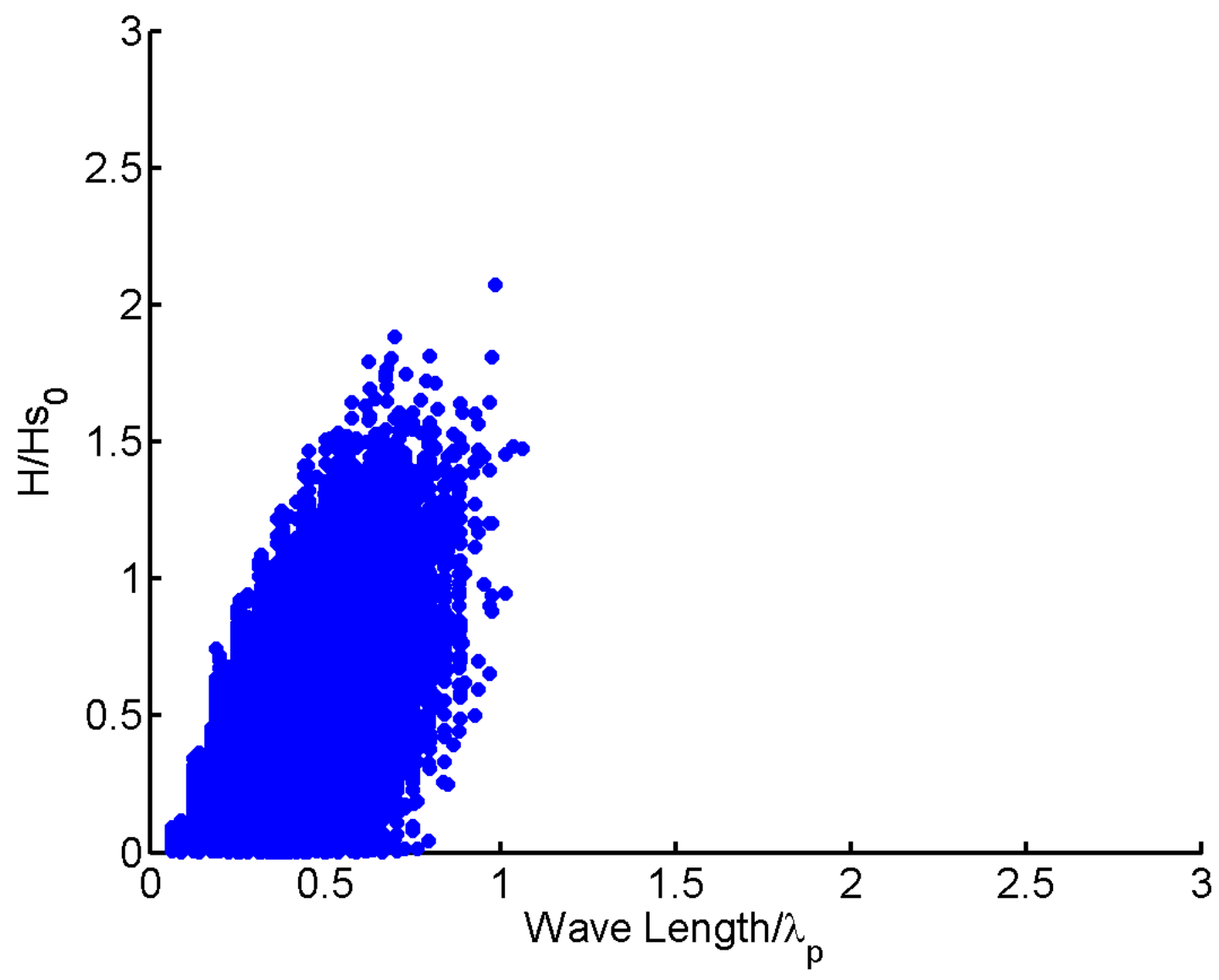

Figure 5-68: Scatter plot of dimensionless wave height vs. dimensionless wavelength in Case $\mathbf{J}$ (where $\theta=180^{\circ}$ ) at time, $t=0 T_{p}$. 


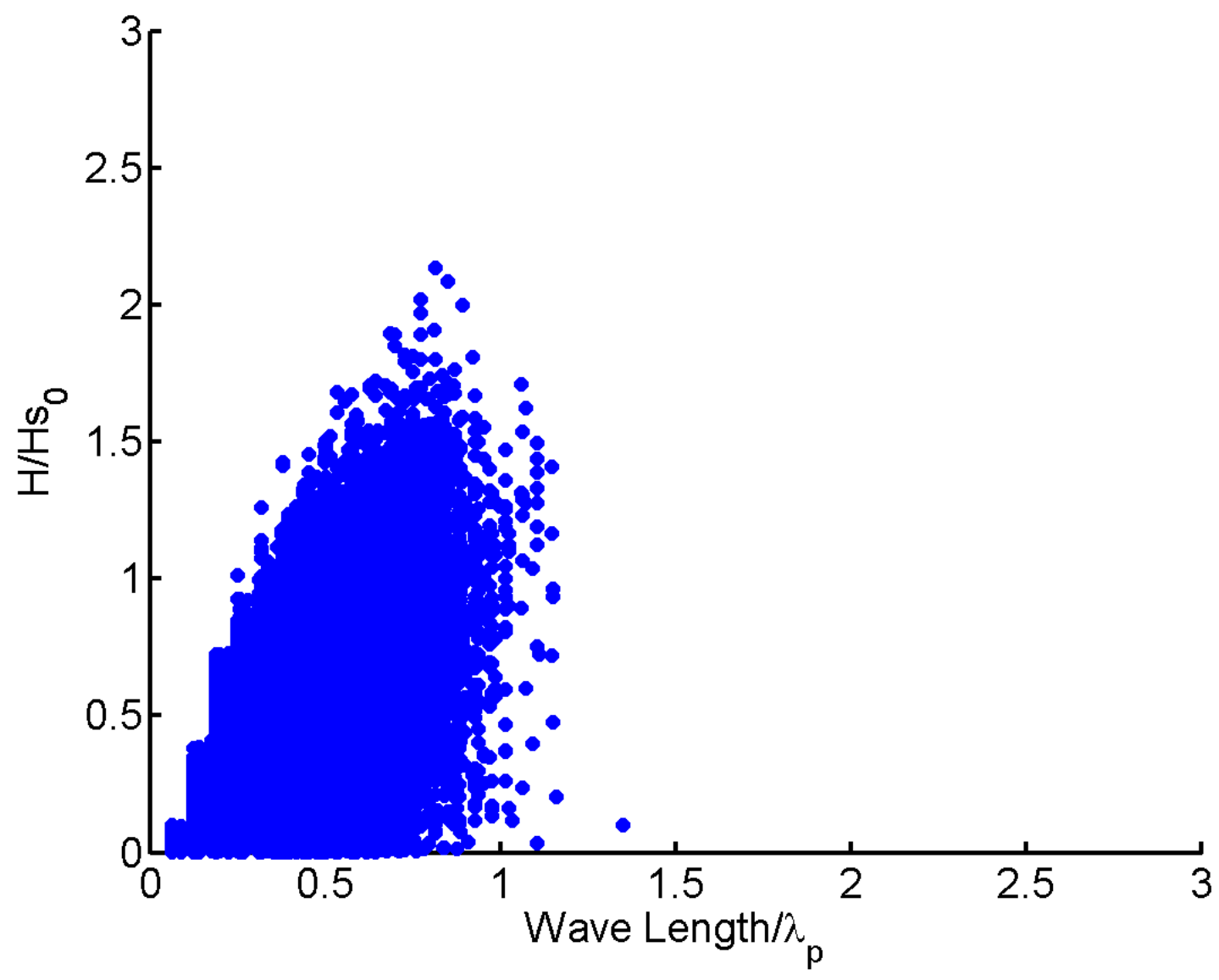

Figure 5-69: Scatter plot of dimensionless wave height vs. dimensionless wavelength in Case $\mathbf{A}$ (where $\theta=80^{\circ}$ ) at time, $t=0 T_{p}$. 


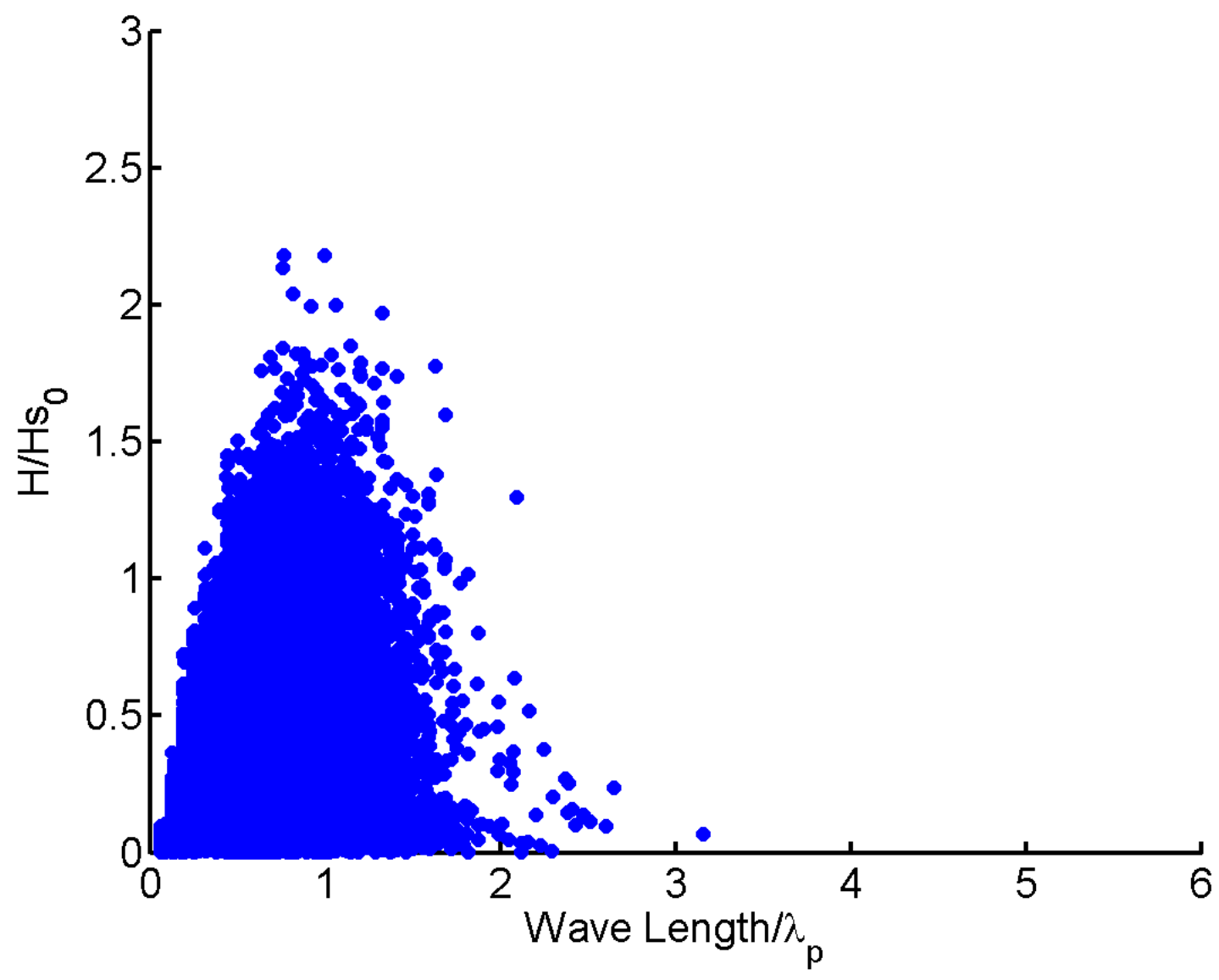

Figure 5-70: Scatter plot of dimensionless wave height vs. dimensionless wavelength in Case $\mathbf{K}$ (where $\theta=20^{\circ}$ ) at time, $t=0 T_{p}$. 


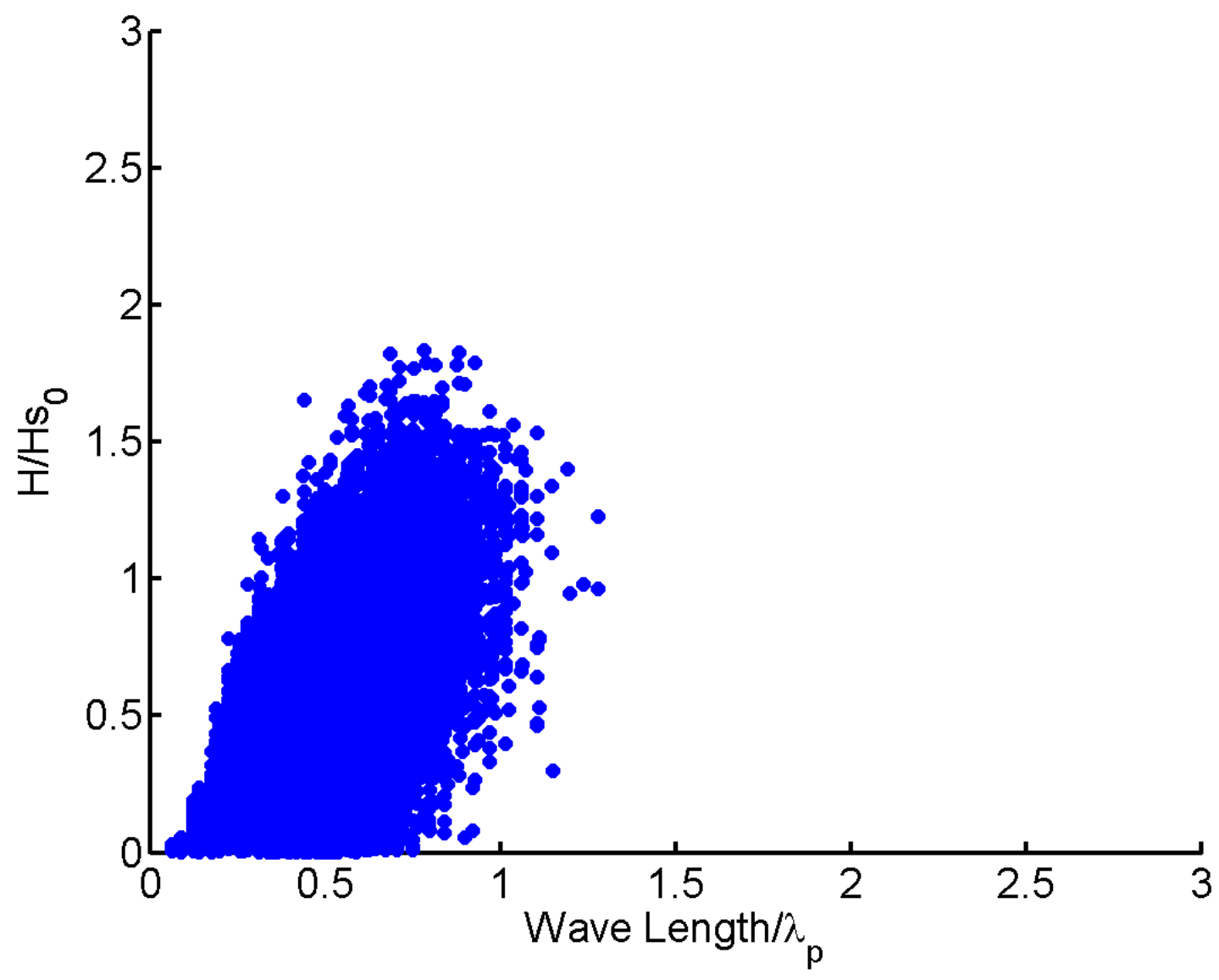

Figure 5-71: Scatter plot of dimensionless wave height vs. dimensionless wavelength in Case $\mathbf{J}$ (where $\theta=180^{\circ}$ ) at time, $t=50 T_{p}$. 


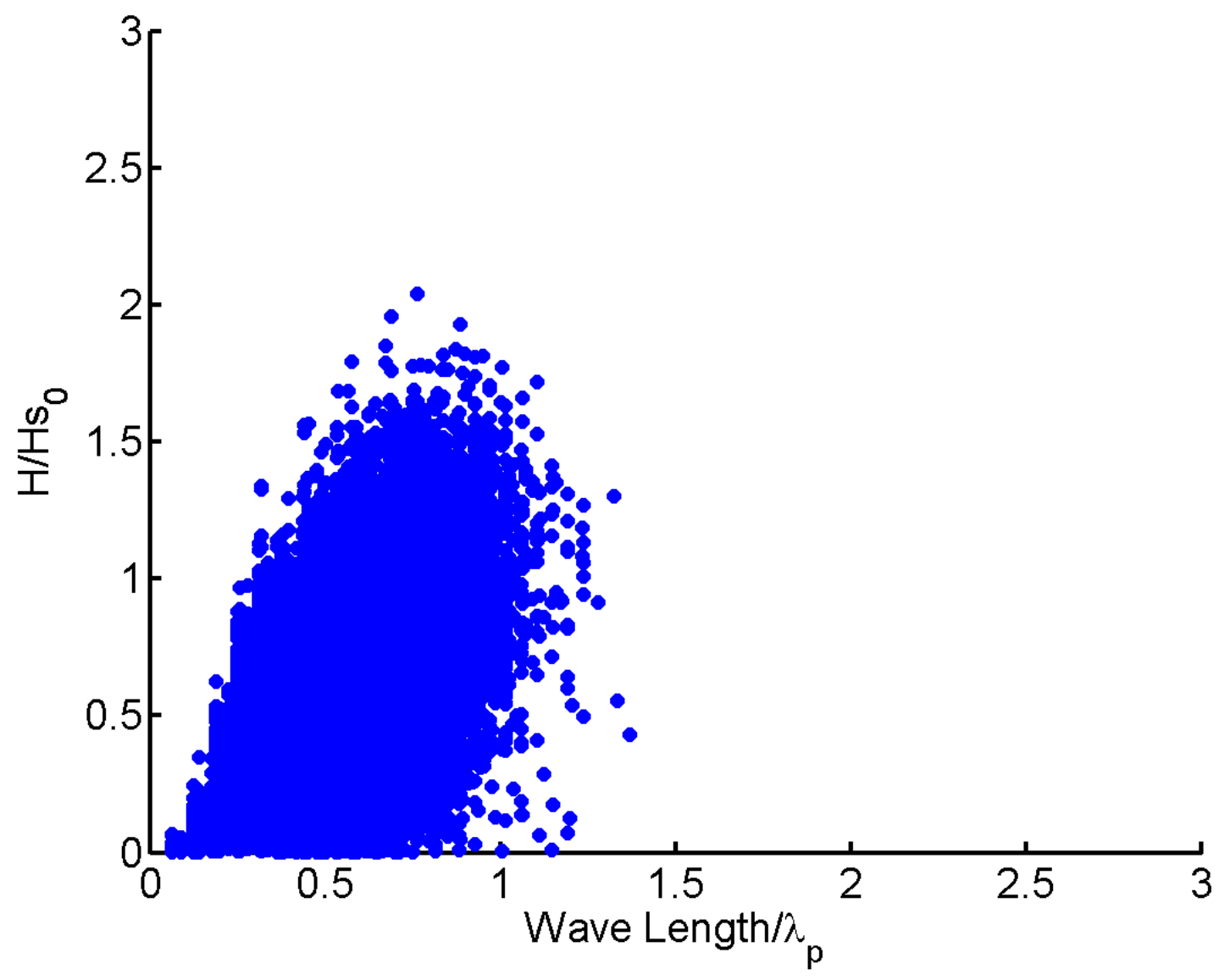

Figure 5-72: Scatter plot of dimensionless wave height vs. dimensionless wavelength in Case $\mathbf{A}$ (where $\theta=80^{\circ}$ ) at time, $t=50 T_{p}$. 


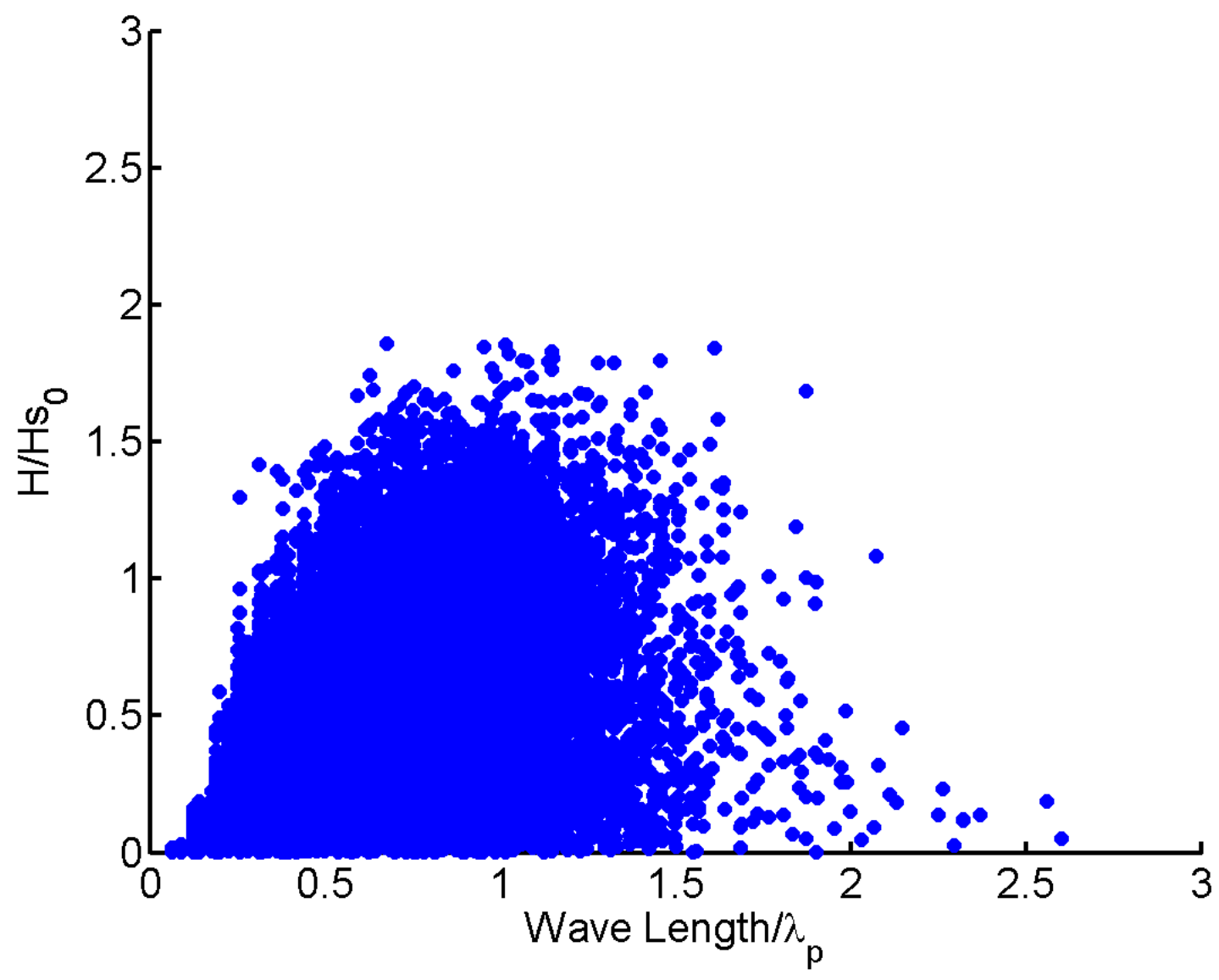

Figure 5-73: Scatter plot of dimensionless wave height vs. dimensionless wavelength in Case $\mathbf{K}$ (where $\theta=20^{\circ}$ ) at time, $t=50 T_{p}$. 


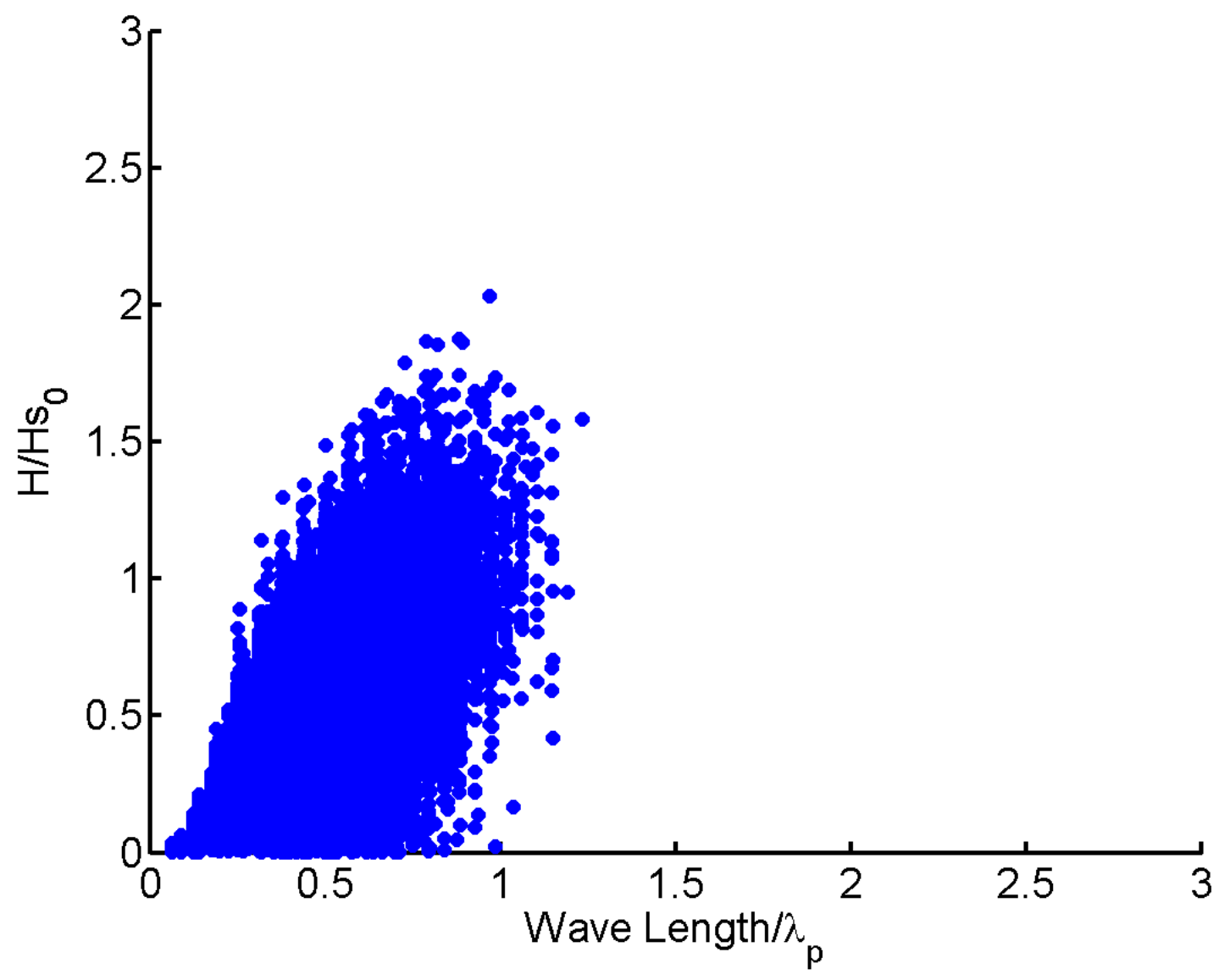

Figure 5-74: Scatter plot of dimensionless wave height vs. dimensionless wavelength in Case $\mathbf{J}$ (where $\theta=180^{\circ}$ ) at time, $t=100 T_{p}$. 


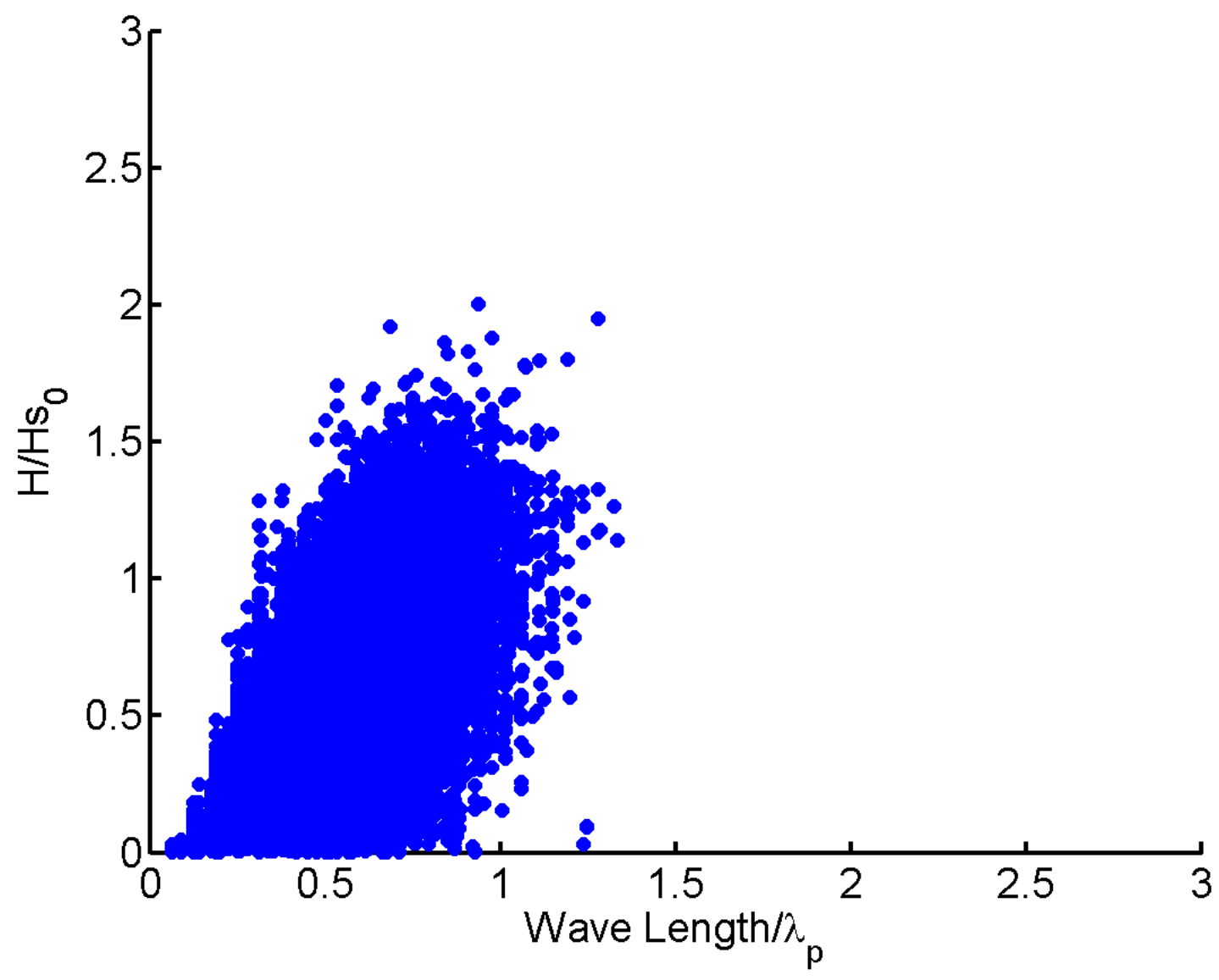

Figure 5-75: Scatter plot of dimensionless wave height vs. dimensionless wavelength in Case $\mathbf{A}$ (where $\theta=80^{\circ}$ ) at time, $t=100 T_{p}$. 


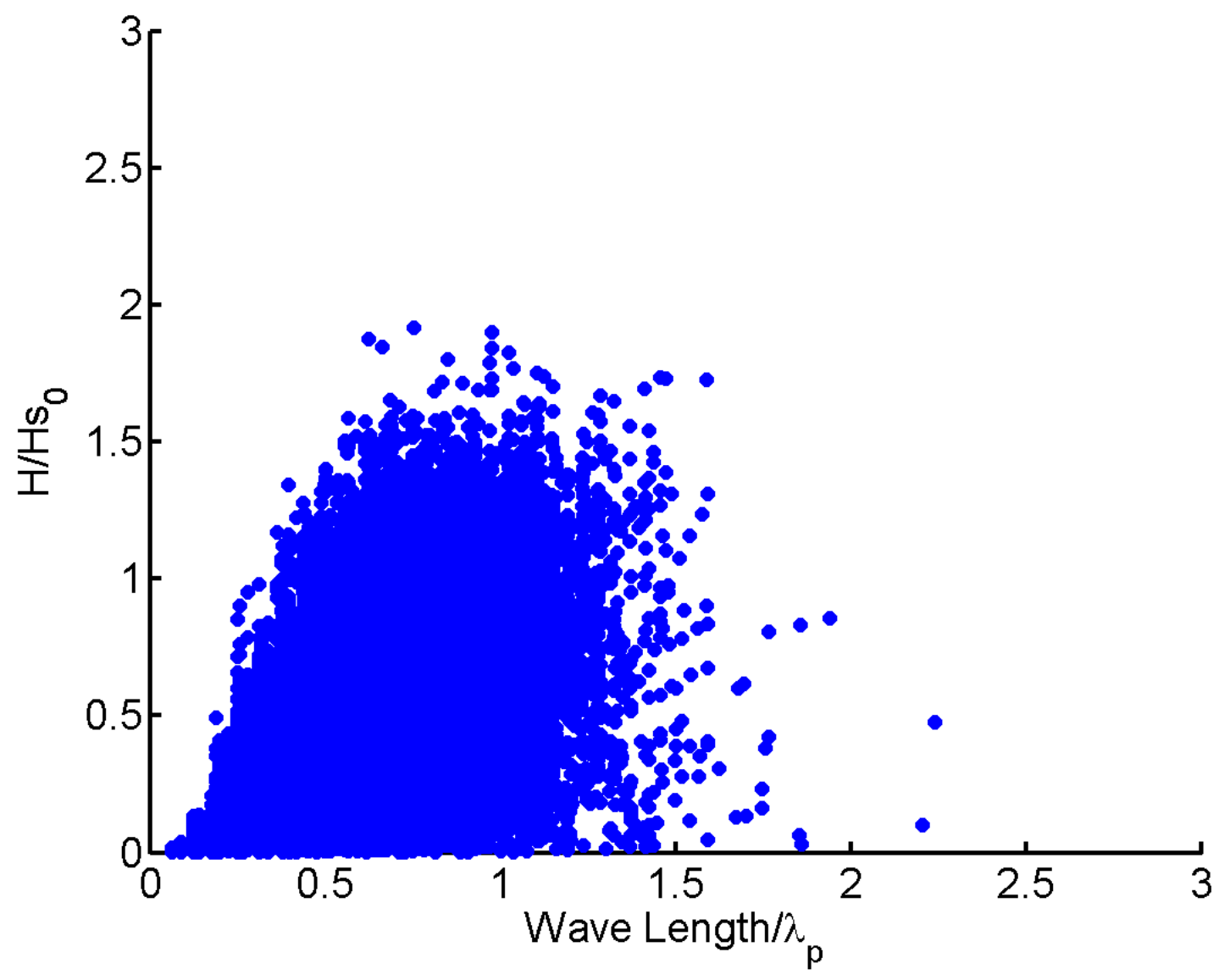

Figure 5-76: Scatter plot of dimensionless wave height vs. dimensionless wavelength in Case $\mathbf{K}\left(\right.$ where $\theta=20^{\circ}$ ) at time, $t=100 T_{p}$. 
non-narrow-band wavelength distributions, only breaking down near the longest or shortest waves in the wavefield.

\subsubsection{Effect of input spectral directional spreading function on large waves' spacing}

In this section we specifically observe waves as would be experienced by large vessels, defined by the local maximum connected to it's lowest local minimum, as outlined in Section 4.3. We investigate the impact of the input spectral directional spreading function on large waves' spacing. We use wavefields of various directional spreading angles and compare the occurrence of large waves (where $H / H_{s}>1.0$ ) in fixed areas of the wavefields. Our result is captured in Figures 5-81, 5-82 and 5-83. We conclude from this graphic that input spreading angles at initial and final times in our simulation do little to discriminate between large wave spreading distances around the largest wave. We see however, around $t=50 T_{p}$ when many of the wavefields show their largest waves, cases with $\theta=180^{\circ}$ and $\theta=80^{\circ}$ separate and fall far below the other wavefields, indicating either less total large waves, or greater spreading between large waves. We see wavefields with lower directional effects, i.e. $\theta=40^{\circ}, \quad \theta=30^{\circ}$ and $\theta=20^{\circ}$ having very similar space distributions and conclude that they form a common regime of large wave spreading. We observe up to 30 large waves within a radius of $15 \lambda_{p}^{\prime} s$ of the largest wave at this time step among these three wavefields.

We also observe, for cases with $\theta=180^{\circ}, \theta=80^{\circ}$, and $\theta=20^{\circ}$, a time evolution of large wave spacing, capturing this result in Figures 5-84, 5-85, and 5-86. We conclude from inspecting this graphic that there is a regime difference between the spreading of large waves around the largest wave under input spreading angle $\theta=20^{\circ}$ (where more large waves consistently appear closer to the largest wave) and the input spreading angles $\theta=180^{\circ}, \theta=80^{\circ}$ (where we see almost identical large waves grouping in a fixed radius around the largest wave). 

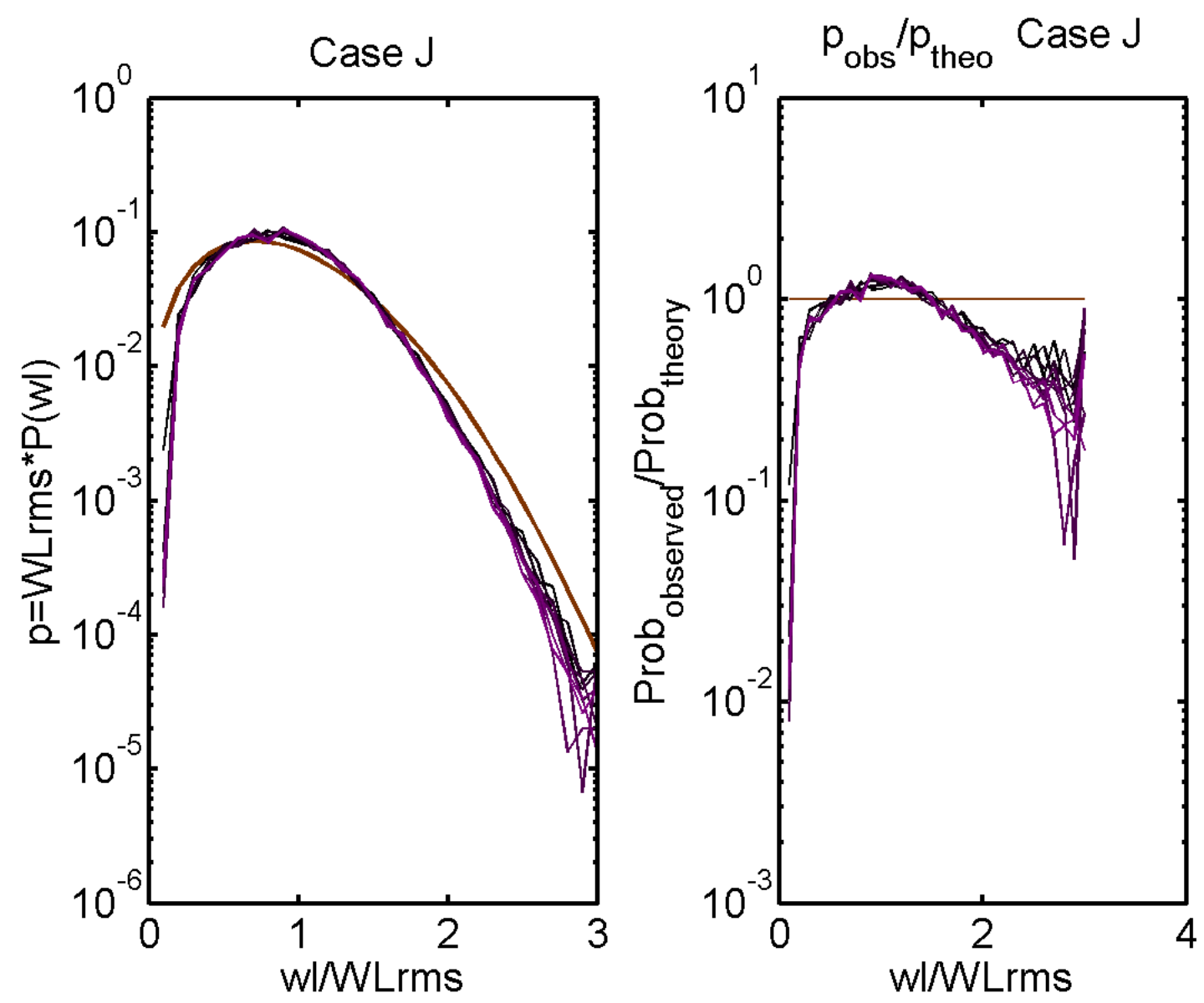

Figure 5-77: (In Color) Time evolution of the wave length distribution in Case $\mathbf{J}$ where $\theta=180^{\circ}$. Earlier times (from $t=0 T_{p}$ ) are the darkest lines. Later times (up to $t=100 T_{p}$ ) are the brightest lines, compared to Rayleigh distribution, (solid, red line). 

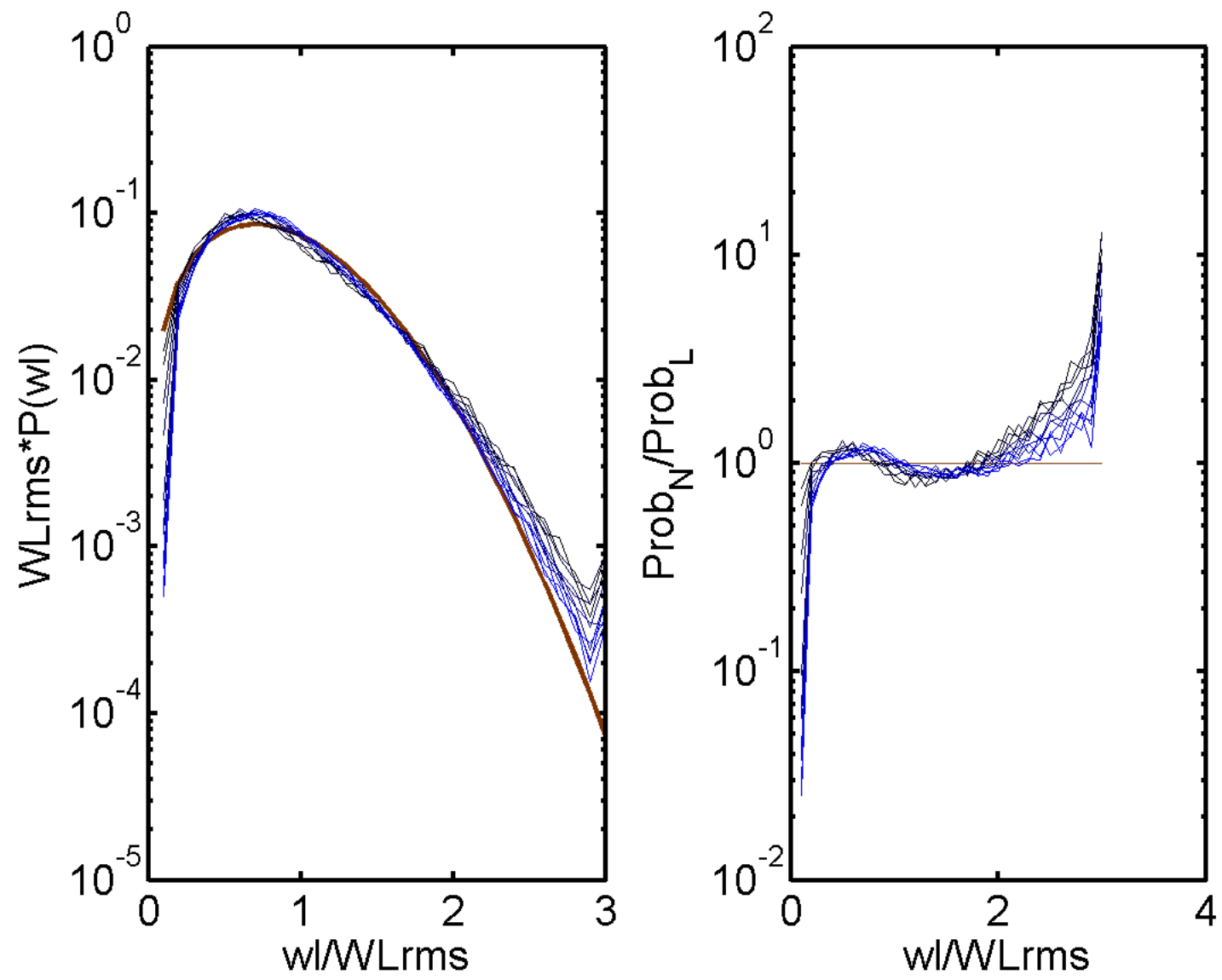

Figure 5-78: (In Color) Time evolution of the wave length distribution in Case A where $\theta=80^{\circ}$. Earlier times (from $t=0 T_{p}$ ) are the darkest lines. Later times (up to $t=100 T_{p}$ ) are the brightest lines, compared to Rayleigh distribution, (solid, red line). 


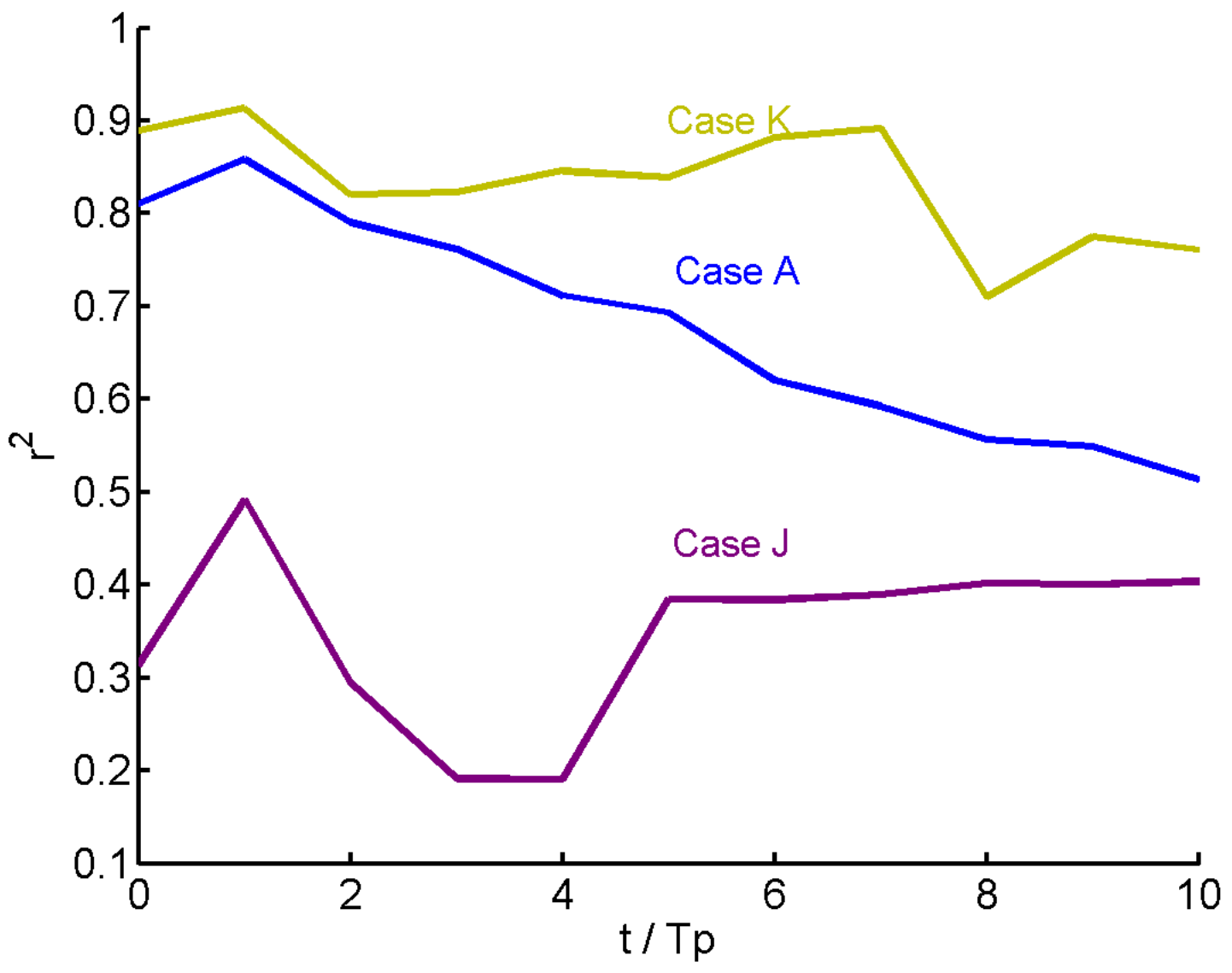

Figure 5-79: (In Color) Time evolution of the wave length distribution in Case $\mathbf{K}$ where $\theta=20^{\circ}$. Earlier times (from $t=0 T_{p}$ ) are the darkest lines. Later times (up to $t=100 T_{p}$ ) are the brightest lines, compared to Rayleigh distribution, (solid, red line). 


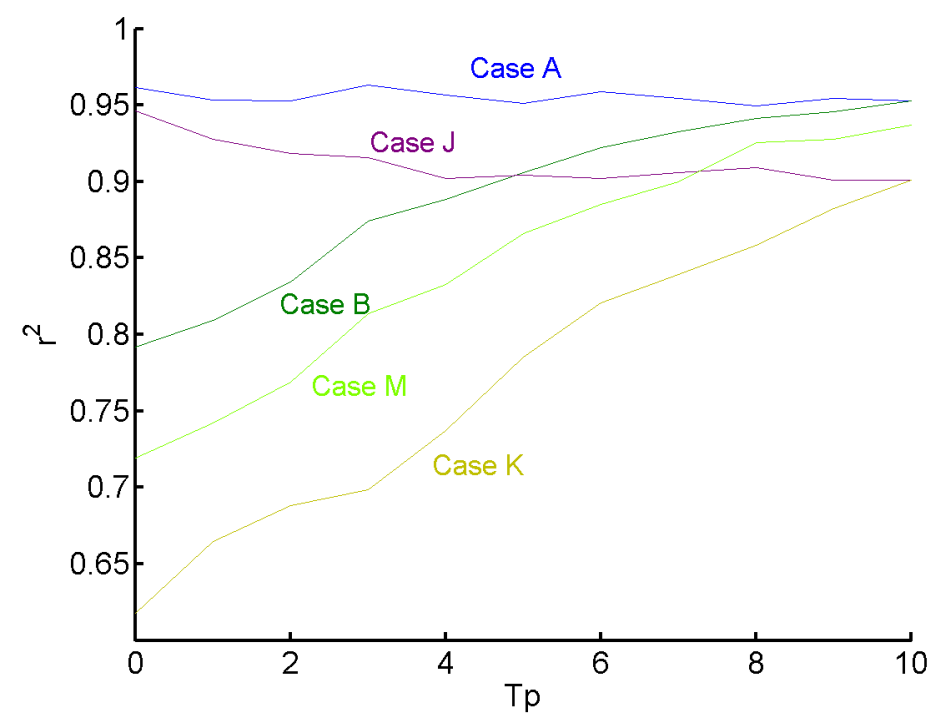

Figure 5-80: (In Color) Correlation coefficient, $r^{2}$-values between the simulated wavefields' wavelength distribution and the Rayleigh distribution. Correlation coefficient, $r^{2}$ takes values between 0 and 1 where $r^{2}=1$ indicates that the simulated wavefields' wavelength distribution and the Rayleigh distribution are highly correlated and $r^{2}=0$ indicates that they are not correlated at all. We observe wavelength distribution correlation with the Rayleigh distribution in three different nonlinear simulated wavefields: (a)Case $\mathbf{J}, \theta=180^{\circ}$, (b)Case $\mathbf{A}, \theta=80^{\circ}$ and (c)Case $\mathbf{K}, \theta=20^{\circ}$. 


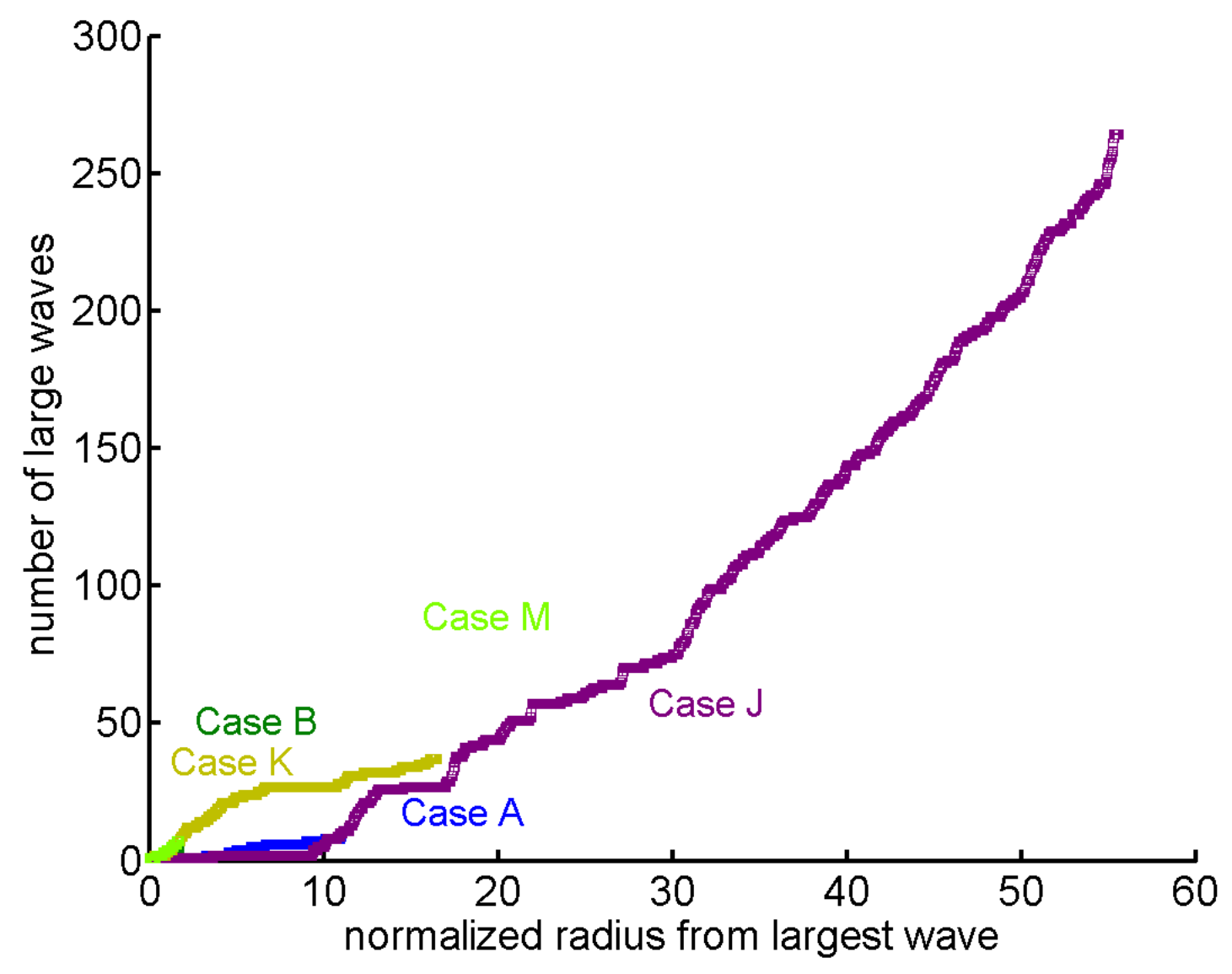

Figure 5-81: (In Color) Large wave spacing in Case $\mathbf{J}$ (where $\theta=180^{\circ}$ ), Case $\mathbf{A}$ (where $\theta=80^{\circ}$ ), Case $\mathbf{B}$ (where $\theta=40$ ), Case $\mathbf{M}$ (where $\theta=30$ ), and Case $\mathbf{K}$ (where $\theta=20^{\circ}$ ) at time, $t=20 T_{p}$. Spacing in waves with five different spreading angles with height $H>1.5 * H_{s}$ at time $t=20 T_{p}$. 


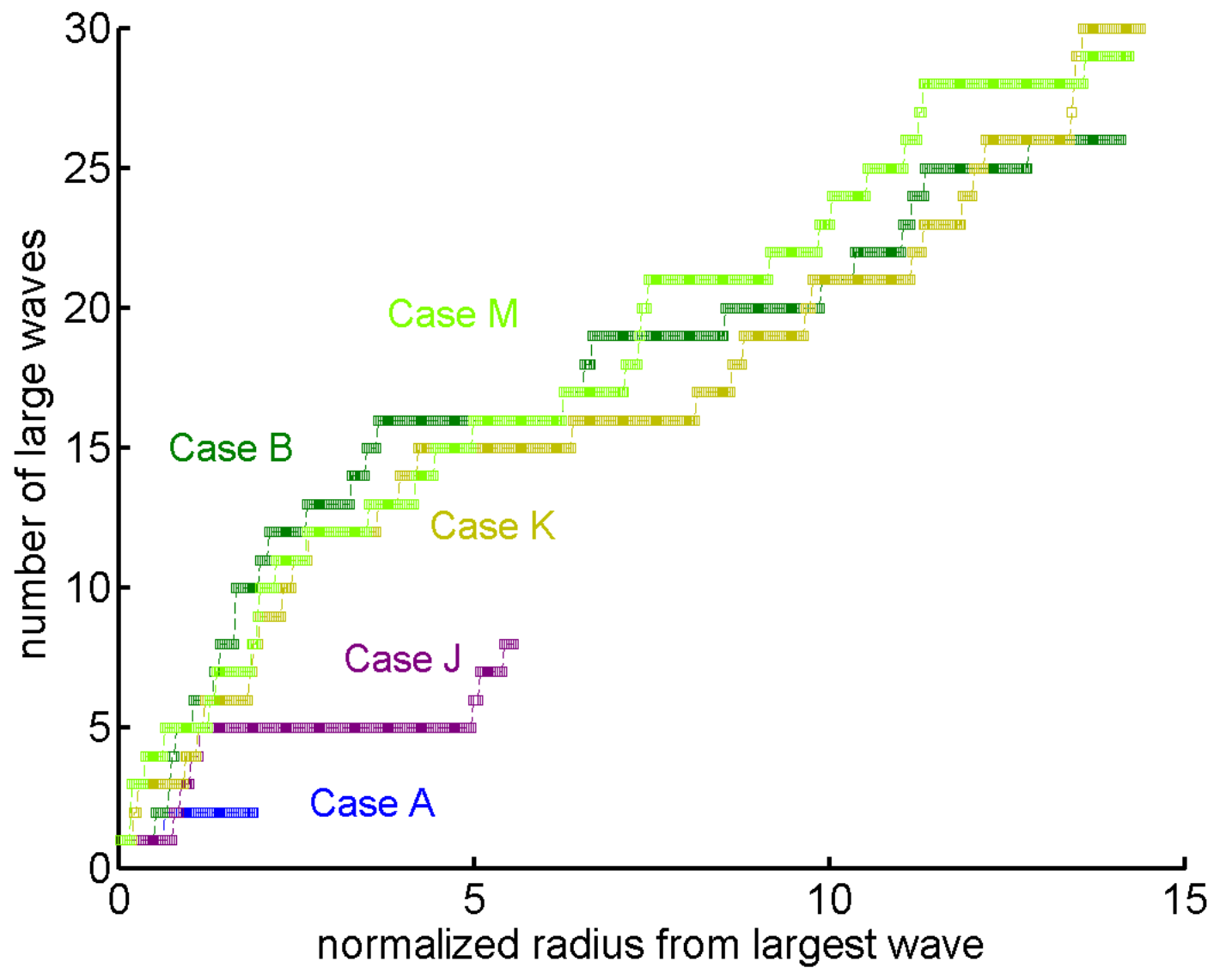

Figure 5-82: (In Color) Large wave spacing in Case $\mathbf{J}$ (where $\theta=180^{\circ}$ ), Case $\mathbf{A}$ (where $\theta=80^{\circ}$ ), Case $\mathbf{B}$ (where $\theta=40$ ), Case $\mathbf{M}$ (where $\theta=30$ ), and Case $\mathbf{K}$ (where $\theta=20^{\circ}$ ) at time, $t=50 T_{p}$. Spacing in waves with five different spreading angles with height $H>1.5 * H_{s}$ at time $t=50 T_{p}$. 


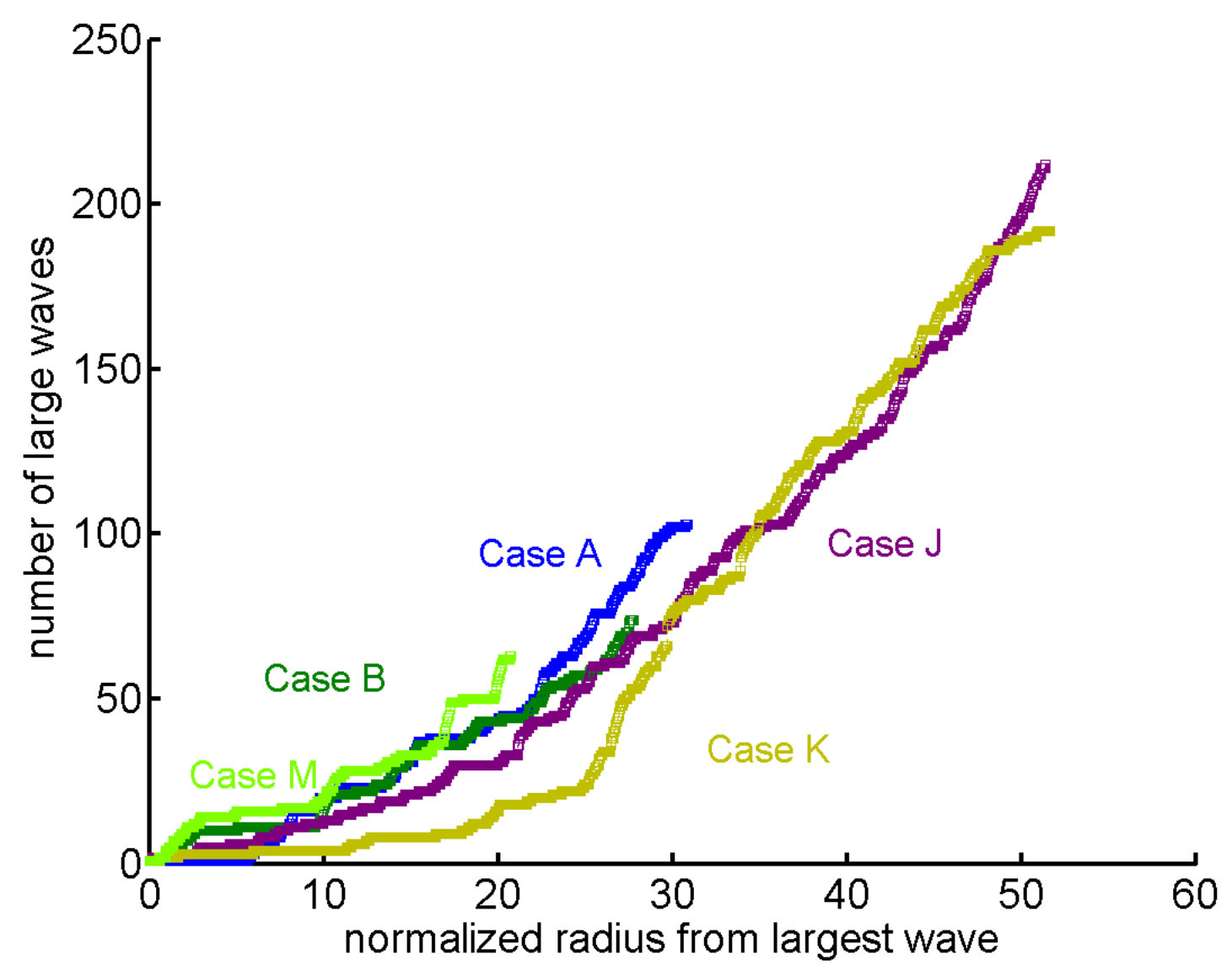

Figure 5-83: (In Color) Large wave spacing in Case $\mathbf{J}$ (where $\theta=180^{\circ}$ ), Case $\mathbf{A}$ (where $\theta=80^{\circ}$ ), Case $\mathbf{B}$ (where $\theta=40$ ), Case $\mathbf{M}$ (where $\theta=30$ ), and Case $\mathbf{K}$ (where $\left.\theta=20^{\circ}\right)$ at time, $t=100 T_{p}$. Spacing in waves with five different spreading angles with height $H>1.5 * H_{s}$ at time $t=100 T_{p}$. 


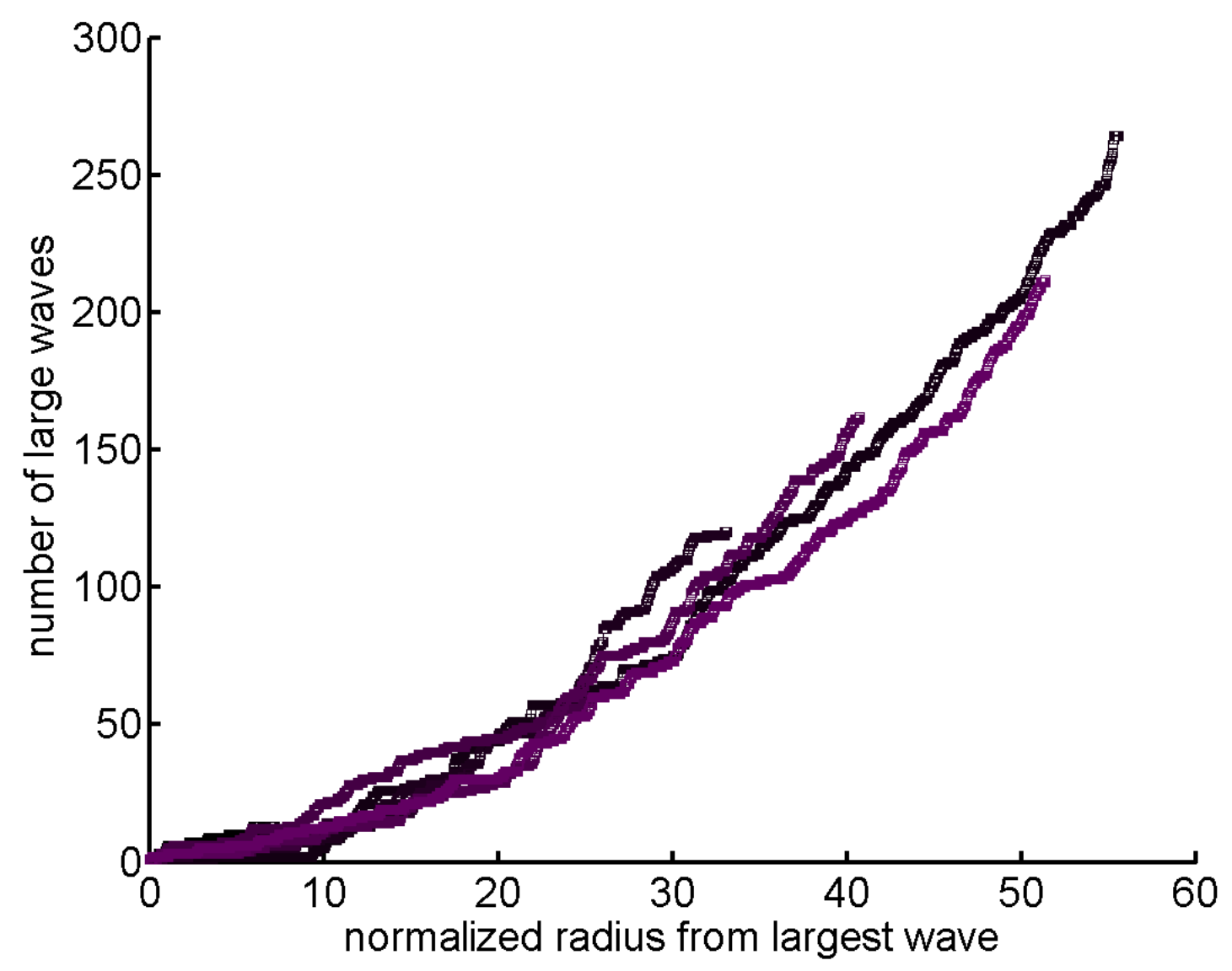

Figure 5-84: (In Color) Large wave spacing in Case $\mathbf{J}$ (where $\theta=180^{\circ}$ ) from times, $t=0 T_{p}$ (darkest line) to $t=100 T_{p}$ (brightest line). 


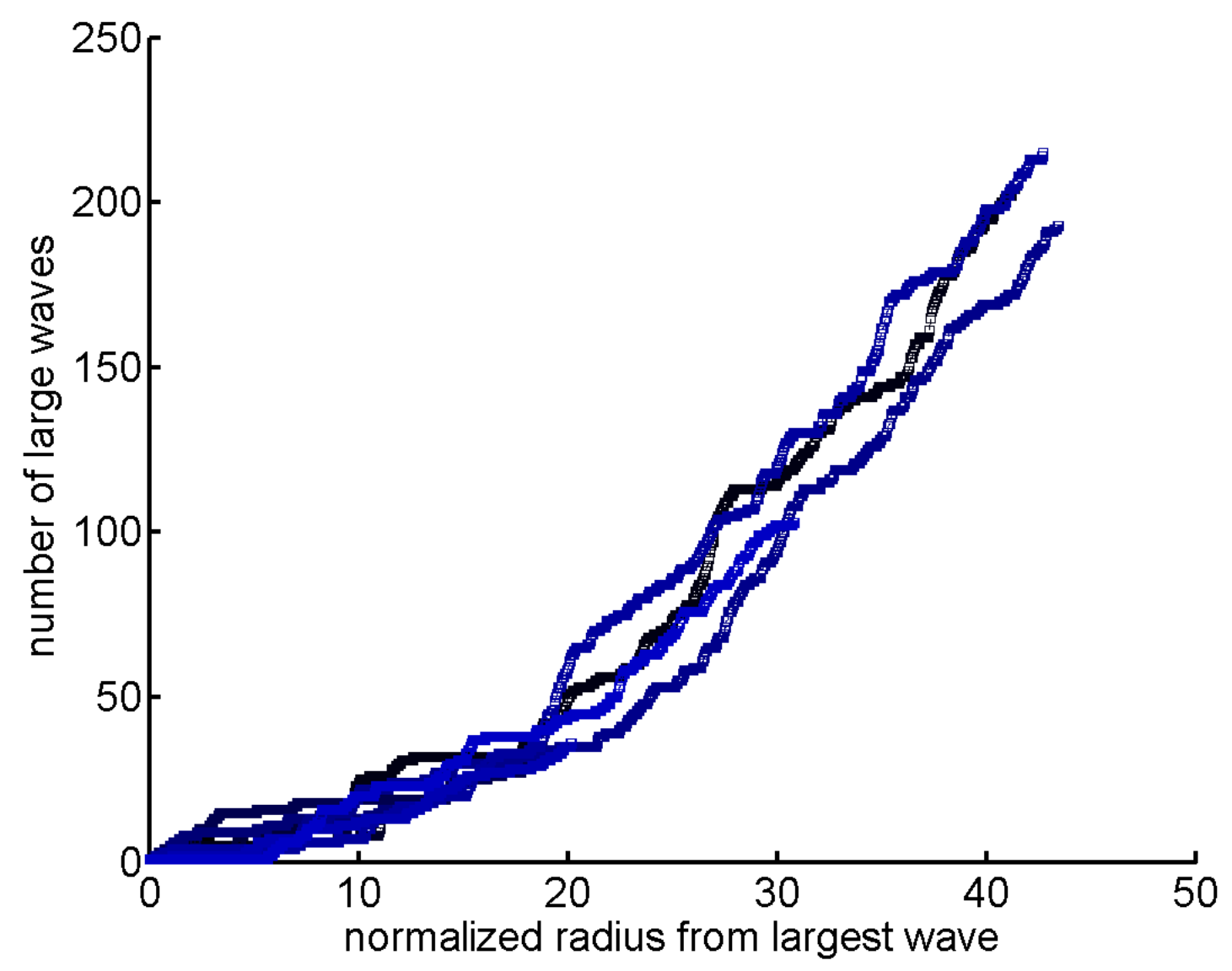

Figure 5-85: (In Color) Large wave spacing in Case $\mathbf{A}$ (where $\theta=80^{\circ}$ ) from times, $t=0 T_{p}$ (darkest line) to $t=100 T_{p}$ (brightest line). 


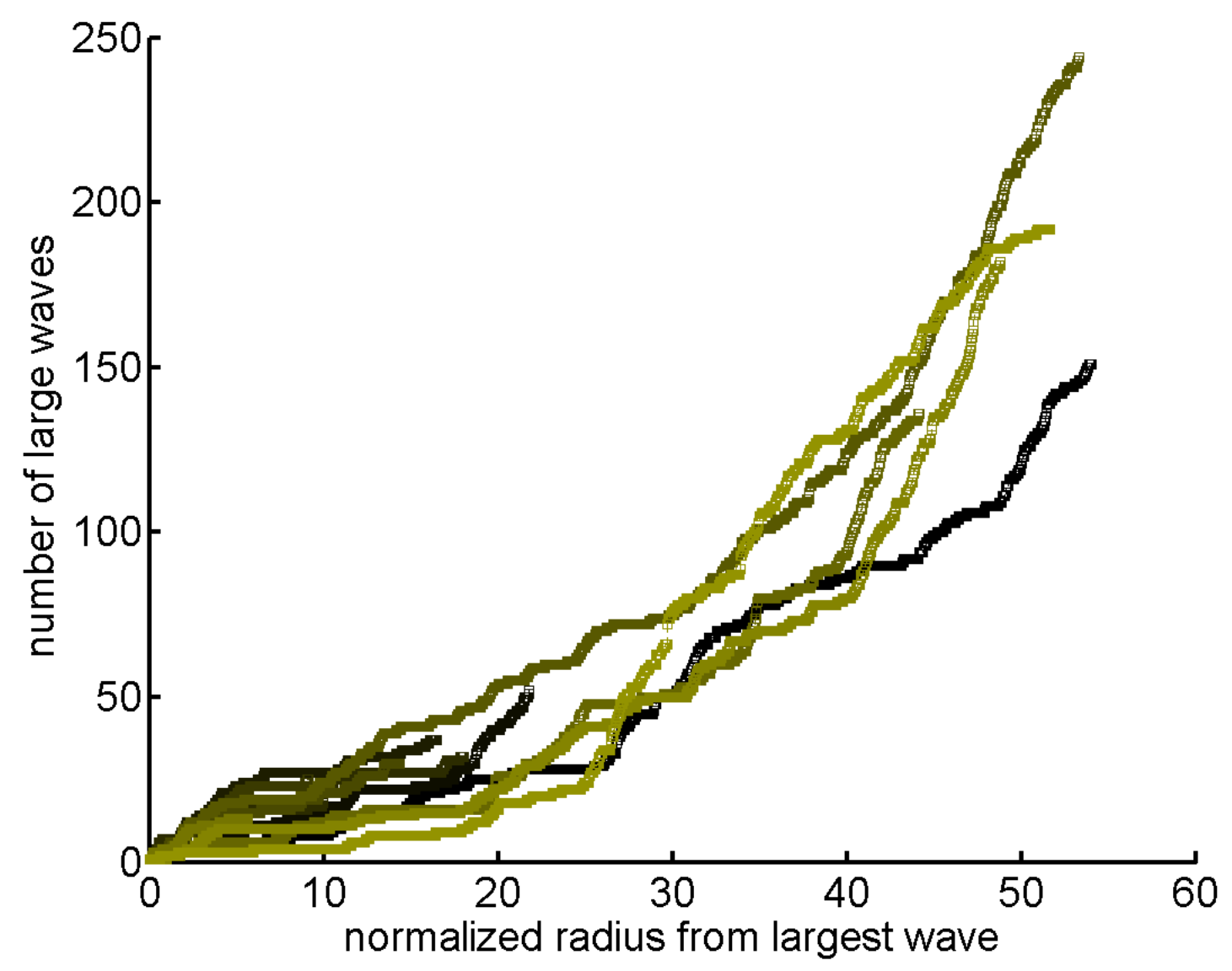

Figure 5-86: (In Color) Large wave spacing in Case $\mathbf{K}\left(\right.$ where $\theta=20^{\circ}$ ) from times, $t=0 T_{p}$ (darkest line) to $t=100 T_{p}$ (brightest line). 


\subsubsection{Input spectral directional spreading function and ob- served wave travel directions.}

In this section we observe the role of the input spectral directional spreading function in determining larger wave $\left(H / H_{s}>1.0\right)$ travel directions. We do so with the help of Figures 5-87, 5-88, and 5-89, and later in Figures 5-90, 5-91, and 5-92, where we plot wave height and wave direction in polar coordinates (wave travel direction coordinate, $\Theta$ and wave height coordinate, $h$ ). In these plots, the mean direction of travel of the wavefield is at polar angle, $\Theta=270^{\circ}$.

In Figures 5-87, 5-88, 5-89, 5-90, 5-91 and 5-92 we see that non-linear, nonnarrow-band, short waves produce higher wave heights in cases with larger directional spreading, and that input directional spreading plays a significant role in the travel directions of these waves.

From Figures 5-90, 5-91, and 5-92 we observe very similar directional effects in all three cases even after $t=100 T_{p}$ of time evolution have passed. However, we also see greater uniformity emerge and a much stronger proliferation of the largest waves in the case where $\theta=180^{\circ}$.

\subsubsection{Conclusion: Input spectral directional spreading and wave height characteristics}

In this section we study the effect of spreading angle, $\theta$ on wave height characteristics, finding that:

- Wavefields with the smallest directional spreading angles have the highest largewave probabilities among narrow-band, directional wavefields with low steepness

- Smaller spreading angles act to increase the probability of the largest and smallest wave lengths in the wave field

- There is a regime difference between the spreading of large waves around the largest wave under input spreading angle $\theta=20^{\circ}$, where more large waves 


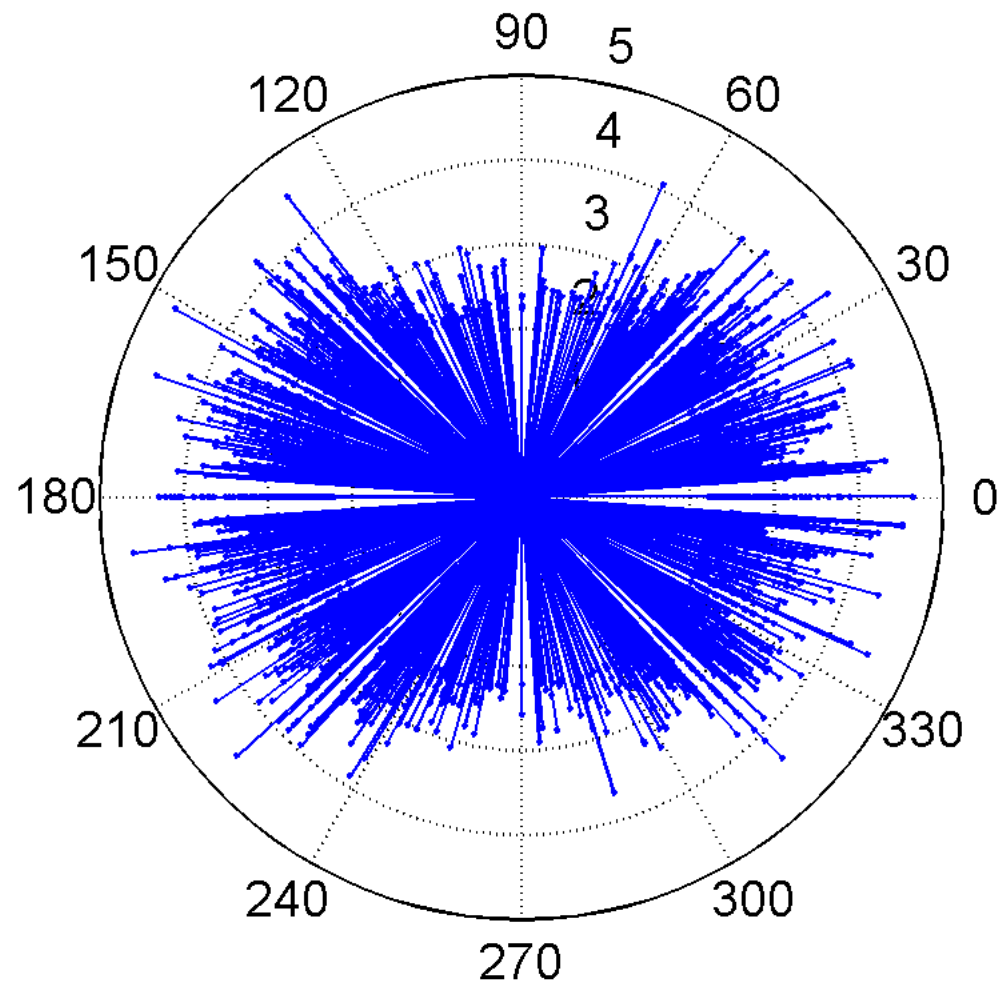

$\mathrm{H} / \mathrm{Hs}_{0}$

Figure 5-87: (In Color) Wave height and travel direction in Case $\mathbf{J}$ (where $\theta=180^{\circ}$ ) at time, $t=50 T_{p}$. Graphics depict wave height $H / H_{r m s}$ as the length of the vector and the direction in which the wave travels $\Theta$ as the angle of the vector for $H>1.0 * H_{s}$ at time, $t=50 T_{p}$. 


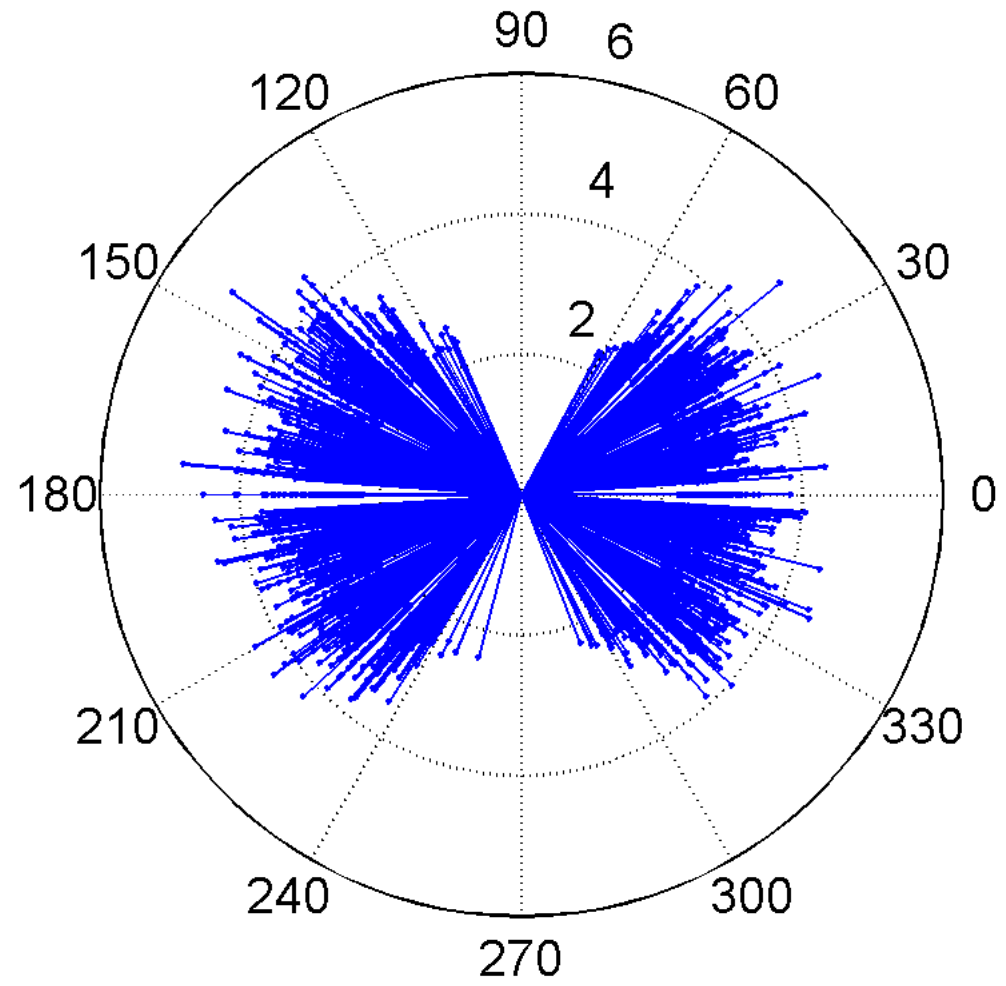

$\mathrm{H} / \mathrm{Hs}_{0}$

Figure 5-88: (In Color) Wave height and travel direction in Case $\mathbf{A}$ (where $\theta=80^{\circ}$ ) at time, $t=50 T_{p}$. Graphics depict wave height $H / H_{r m s}$ as the length of the vector and the direction in which the wave travels $\Theta$ as the angle of the vector for $H>1.0 * H_{s}$ at time, $t=50 T_{p}$. 


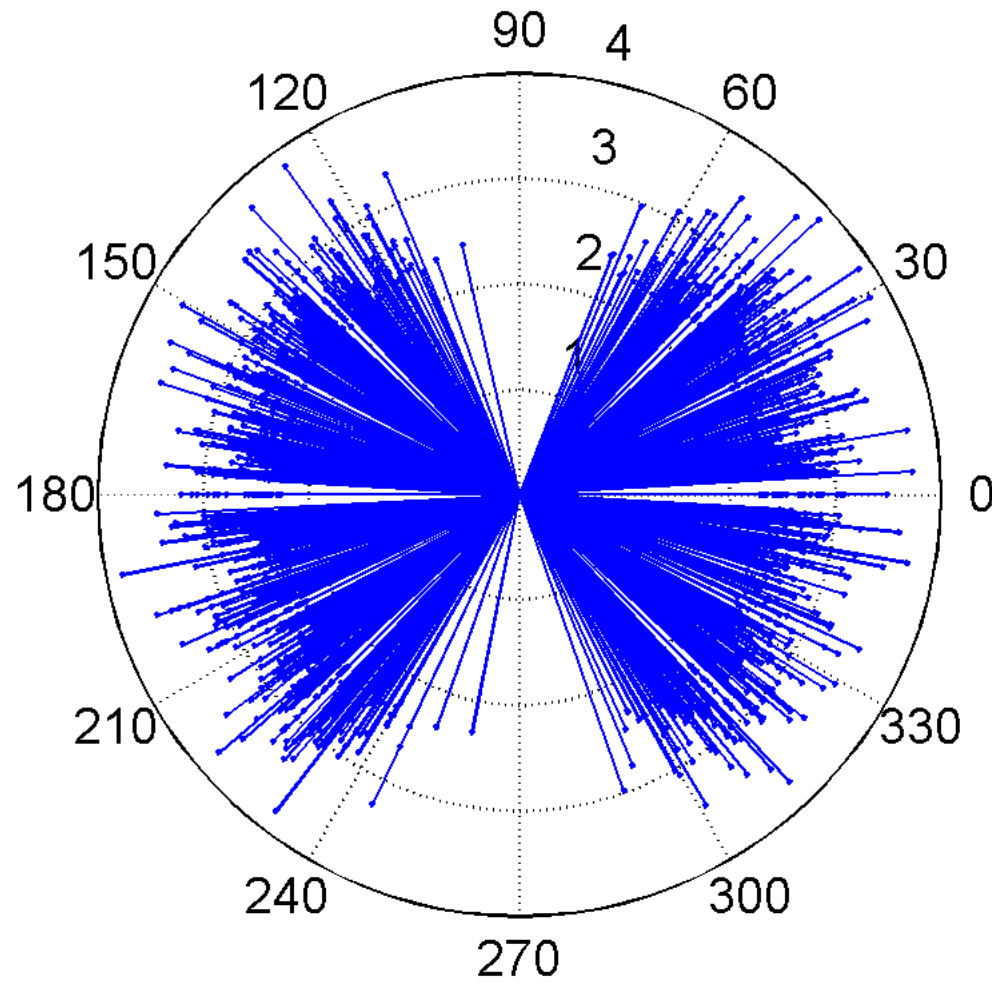

$\mathrm{H} / \mathrm{Hs}_{0}$

Figure 5-89: (In Color) Wave height and travel direction in Case $\mathbf{K}\left(\right.$ where $\theta=20^{\circ}$ ) at time, $t=50 T_{p}$. Graphics depict wave height $H / H_{r m s}$ as the length of the vector and the direction in which the wave travels $\Theta$ as the angle of the vector for $H>1.0 * H_{s}$ at time, $t=50 T_{p}$. 


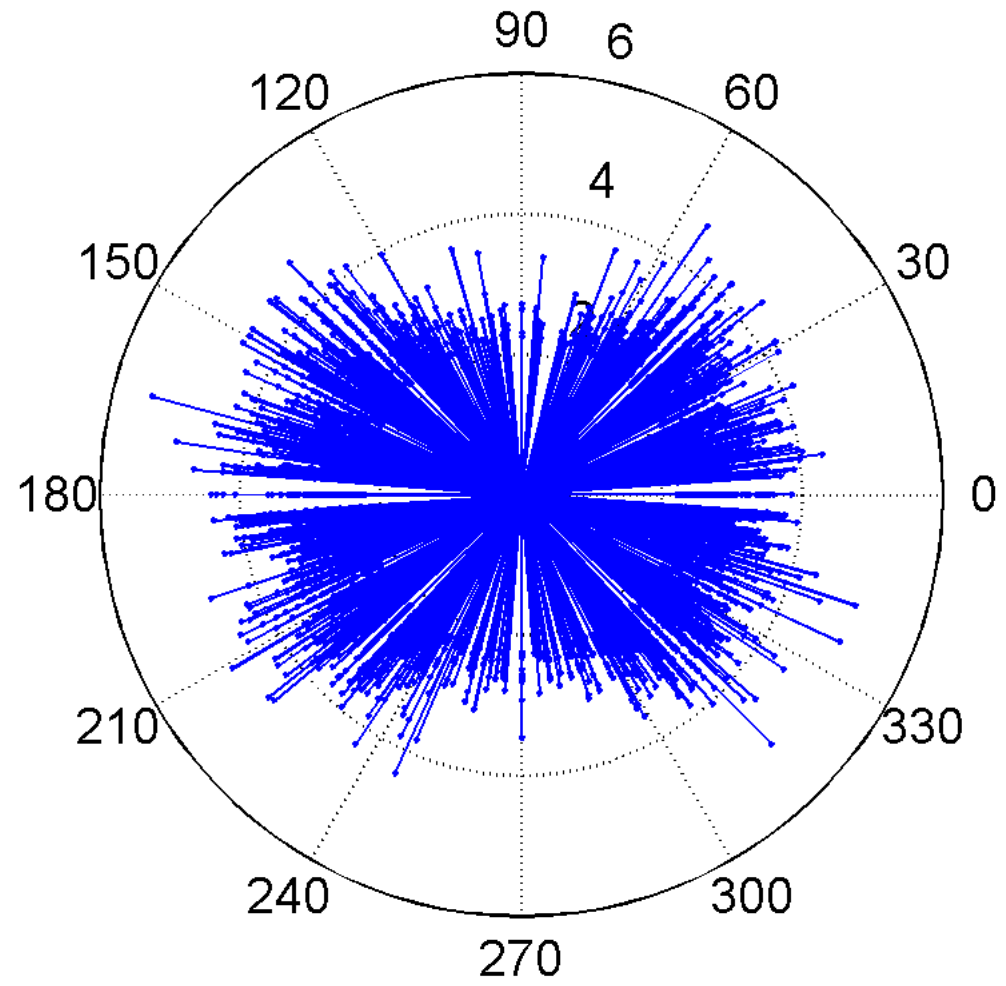

$\mathrm{H} / \mathrm{Hs}_{0}$

Figure 5-90: (In Color) Wave height and travel direction in Case $\mathbf{J}$ (where $\theta=180^{\circ}$ ) at time, $t=100 T_{p}$. Graphics depict wave height $H / H_{r m s}$ as the length of the vector and the direction in which the wave travels $\Theta$ as the angle of the vector for $H>1.0 * H_{s}$ at time, $t=100 T_{p}$. 


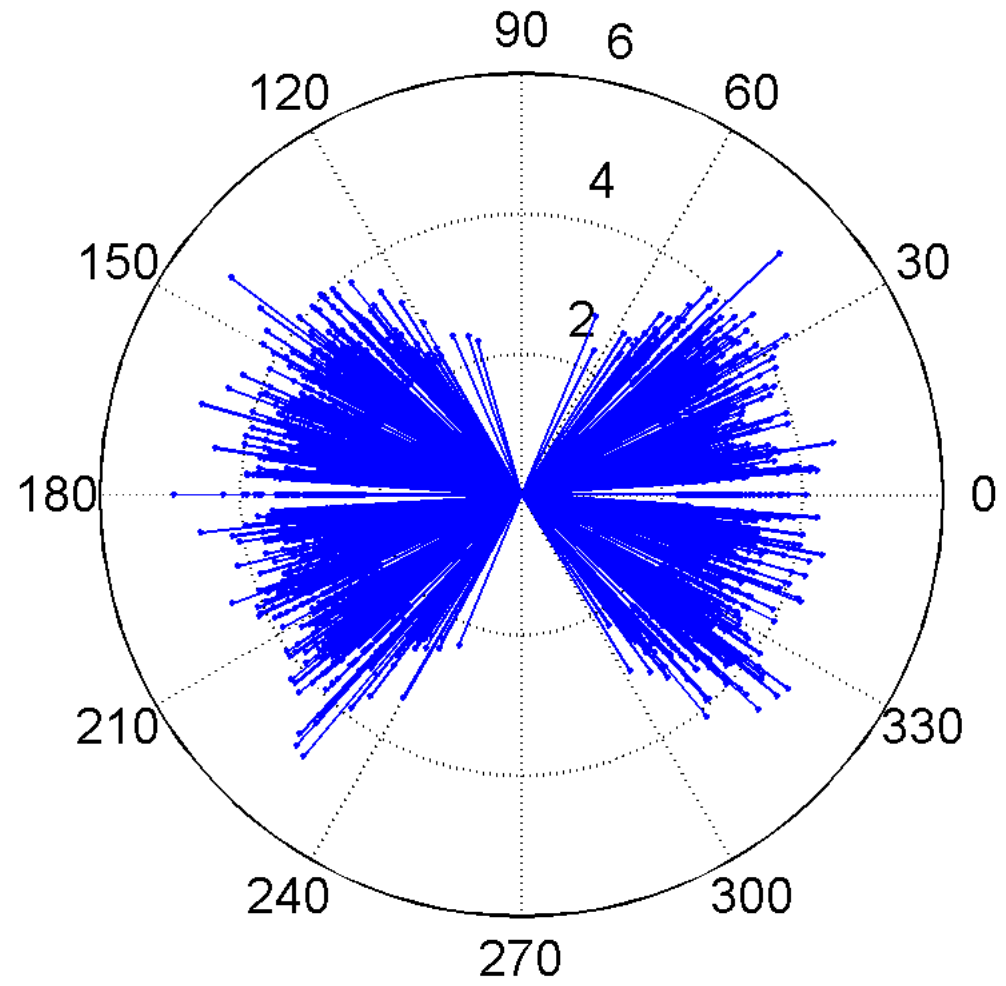

$\mathrm{H} / \mathrm{Hs}_{0}$

Figure 5-91: (In Color) Wave height and travel direction in Case $\mathbf{A}$ (where $\theta=80^{\circ}$ ) at time, $t=100 T_{p}$. Graphics depict wave height $H / H_{r m s}$ as the length of the vector and the direction in which the wave travels $\Theta$ as the angle of the vector for $H>1.0 * H_{s}$ at time, $t=100 T_{p}$. 


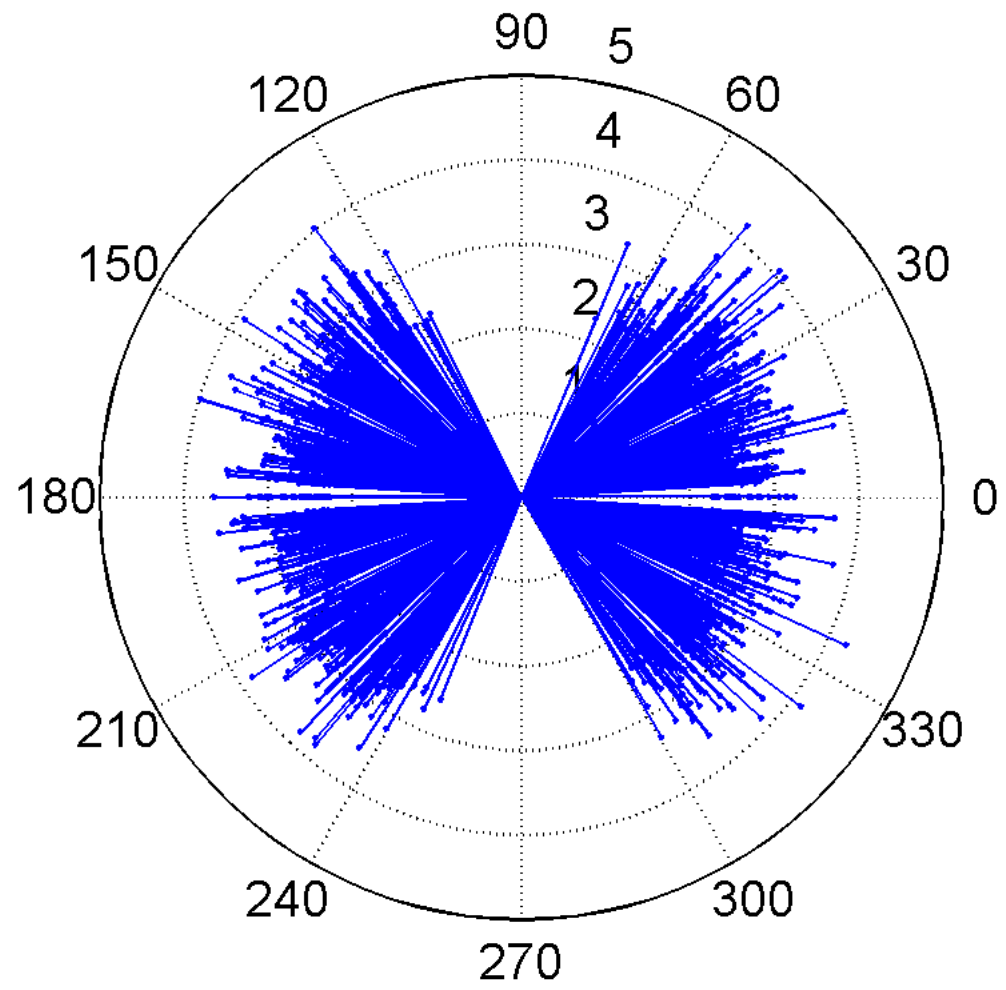

$\mathrm{H} / \mathrm{Hs}_{0}$

Figure 5-92: (In Color) Wave height and travel direction in Case $\mathbf{K}\left(\right.$ where $\theta=20^{\circ}$ ) at time, $t=100 T_{p}$. Graphics depict wave height $H / H_{r m s}$ as the length of the vector and the direction in which the wave travels $\Theta$ as the angle of the vector for $H>1.0 * H_{s}$ at time, $t=100 T_{p}$. 
consistently appear closer to the largest wave, and the input spreading angles $\theta=180^{\circ}, \theta=80^{\circ}$, where we see almost identical large waves grouping in a fixed radius around the largest wave.

- Input spreading angle determines the larger waves' travel directions in a wavefield among short, non-linear, non-narrow-band waves. 


\section{Chapter 6}

\section{Conclusion}

In the present work, we examine the statistical properties of the ocean surface elevation obtained from non-linear phase resolved simulations starting from prescribed JONSWAP spectra as out initial conditions. Our objective is to test the effect of input spectral peak shape parameter, $\gamma$, spectral Phillips' parameter, $\alpha$ and spectral angular spreading, $\theta$ on the statistical properties of the wavefield, including surface elevation distribution, wave height distributions, wavelength distributions, and the occurrence and spacing of large wave events.

\subsection{Contributions}

We find the following: Conclusion: Peak shape parameter and surface elevation characteristics In the present work we look at peak shape parameter, $\gamma$, and surface elevation characteristics, finding that:

- Higher peak shape parameter produces higher surface elevation kurtosis and variance but produces no effect on surface elevation skewness. We therefore conclude that $\gamma$ does not impact surface elevation skewness

- Observed surface elevation data distributions move further away from the first, second and third order theoretical statistical distributions in a nonlinear wave- 
field as time proceeds, due to its non-stationary statistical moments. We find a higher peak shape parameter produces higher even and this impacts surface elevation moments such that it moves the higher-order distributions apart similar to the spread seen in the time evolution of nonlinear wavefields.

- The highest elevations in a wavefield with $\gamma=3.3$ (which is the mean peak shape parameter of wave in a storm where peak shape parameter is normally distributed) are stabler than those in wavefields with $\gamma=1.0$, and 5.0 (which are usually the tails of the peak shape parameter distribution).

- The random rules in surface elevation kurtosis and surface slope kurtosis in linear wavefields. Kurtosis values are all very near the Gaussian value 3.0. We note that this is quite unlike what is seen in non-linear wavefields. We find that in non-linear wavefields, surface elevation kurtosis is much smaller than surface elevation slope kurtosis and we find that higher values of peak shape parameter, (see Figure 3-25) produce higher kurtosis of surface slope in the mean direction of propagation.

Conclusions: Phillips' parameter and surface elevation characteristics. In the present work we look at Phillips' parameter, $\alpha$, and surface elevation characteristics, finding that:

- A higher Phillips' parameter produces higher kurtosis, and higher skewness in nonlinear simulations of surface elevation, although the influence of Phillips' parameter on surface elevation variance is unapparent, if at all existent.

- The maximum spectral energy in the nonlinear wavefield's wavenumber spectrum where $\alpha=0.0032$, is much more stable than maximum energy in the wavenumber spectrum of cases where $\alpha=0.0160$ and $\alpha=0.0163$.

- With all other spectral parameters equal, Benjamin Feir Index is independent of Phillips' parameter. 
- Phillips' parameter is intimately connected to surface slope kurtosis values. A lower Phillips' parameter produces smaller deviations between the three kurtosis evolutions: surface elevation kurtosis, surface elevation slope in the $x$-direction kurtosis, and surface elevation slope in the $y$-direction kurtosis. If Phillips' parameter is small enough, it produces kurtosis evolutions that are so close that they resemble linear simulations.

- In a case with a very low Phillips' parameter, the observed effect of the nonlinearity order in the simulation is negligible. i.e. A low Phillips' parameter reduces the appearance of nonlinearity in the wavefield.

Conclusions: Directional spreading function and surface elevation characteristics In the present work we look at the effect of the directional spreading function on surface elevation characteristics during the first $100 T_{p}$ in surface evolution, finding that:

- Regimes are seen to form as a unidirectional wavefield has kurtosis, skewness and variance which compare unpredictably to those in a directional nonlinear wavefield. We see these regimes when we compare the order of magnitude of kurtosis and skewness and find their order of magnitude is much greater in a unidirectional wavefield than that of kurtosis and skewness in a directional wavefield.

- We see regime-type dependence of spectral stability on the values of directional spreading in wavefields. Unidirectional wavefields depict much lower stability for their highest spectral energy, compared to equivalent directional cases. Larger directional spreading is associated with more stable but slightly smaller maximum spectral energy.

- Directional spreading angle has regime-type effects on wavefield stability seen in the BFI. Uni-directional and near uni-directional cases experience a sudden spike in BFI value, and therefore a sudden decrease in wavefield stability after 
$t=50 T_{p}-60 T_{p}$. The directional cases huddle close in the first $t=100 T_{p}$ of time, showing very little difference or growth in their BFI values.

- Directional spreading angle has a regime-type impact on surface slope kurtosis. Smaller directional spreading, is associated with larger difference between the kurtosis of the surface elevation and the kurtosis of the surface elevation slopes in two perpendicular directions. Directional spreading angle has regime-type effects on the kurtosis of surface elevation $\mathrm{x}$-directional slope, surface elevation y-directional slope and surface elevation. For example, cases with greater directional effects show surface elevation $\mathrm{x}$-directional slope kurtosis less than surface elevation y-directional slope kurtosis.

- Directional spreading angle has regime-type effects on the spectral moments of surface elevation since we see one regime (cases with less directional effects) having growing spectral moments and the other regime (cases with strong directional effects) having falling spectral moments.

We present three solutions to the problem of defining waves on a non-linear, evolving free surface with a $2 \mathrm{D}$ wavenumber, $k=\left(k_{x}, k_{y}\right)$. Our study of wave height definition methods in a $3 \mathrm{D}$ wavefield with a $2 D$ wavenumber adds useful insight to common field practices. We study:

- the uni-directional, narrow-band zero-crossing method of wave-height definition

- a $3 D$ directional narrow-band approach to finding the distribution of the largest waves in the wavefield

- a $3 D$ analogue of the $2 D$ half-cycle excursion method

In so doing, we circumvent the bias of non-directional approaches to wave height values in directional wavefields, and the bias of narrow-band approaches to wave heights in non-narrow-band wavefields.

Conclusion: Peak shape parameter and wave height characteristics 
In the present work we study the effect of peak shape parameter, $\gamma$ on wave height characteristics, finding that:

- A higher peak shape parameter, $\gamma$ value moves the wave height distribution closer to the linear, narrow-band theory, and exceedance probability is sensitive to higher peak shape parameters.

- In the joint distribution between wave height and wave length we see a higher peak shape parameter, $\gamma$ value producing many more "rogue waves" with large height but short wavelength.

- A lower peak shape parameter $\gamma$ value moves wavelength distribution closer to the Rayleigh distribution which resembles Longuet-Higgins' [34] theoretical wavelength distribution for large spectral width.

- Peak shape parameter has no effect on the direction of travel of the largest waves in a wavefield, or the spacing of the large waves relative to each other in the wavefield

Conclusion: Phillips' parameter and wave height characteristics

In the present work we study the effect of Phillips' parameter, $\alpha$ on wave height characteristics, finding that:

- The value of Phillips' parameter influences how much wave height distribution correlates with linear, narrow-band theory.

- Higher Phillips' parameter, $\alpha$ produces a greater occurrence of large rogue-like wave heights in the joint distribution between wave heights and wavelengths, and the lowest Phillips parameter produces the longest wavelengths.

- Lower values of Phillips' parameter produce greater adherence of wavelength distribution to the Rayleigh distribution, indicating that larger spectral width is associated with lower values of Phillips' parameter. 
- A very low Phillips' parameter produces greater spacing between the large waves in a wavefield

- Phillips' parameter has a visible effect on the occurrence of large waves, but no visible effect on the direction in which these large waves move.

In the present work we study the effect of spreading angle, $\theta$ on wave height characteristics, finding that:

- Wavefields with the smallest directional spreading angles have the highest largewave probabilities among narrow-band, directional wavefields with low steepness

- Smaller spreading angles act to increase the probability of the largest and smallest wave lengths in the wave field

- There is a regime difference between the spreading of large waves around the largest wave under input spreading angle $\theta=20^{\circ}$, where more large waves consistently appear closer to the largest wave, and the input spreading angles $\theta=180^{\circ}, \theta=80^{\circ}$, where we see almost identical large waves grouping in a fixed radius around the largest wave.

- Input spreading angle determines the larger waves' travel directions in a wavefield among short, non-linear, non-narrow-band waves.

\subsection{Recommendations for further study}

This work is the beginning of a thorough investigation of the impact of spectral parameters on wavefield surface elevation characteristics and large wave occurrences. We study this question via direct numerical simulation (presented in this thesis). We anticipate the next step to involve an analytical investigation of the evolution of kurtosis in a nonlinear, random wavefield. Finally, experimental work will act as a validation mechanism, moving the findings in the entire investigation closer to reality, 
circumventing the inherent loss of insight due to simplifications in computational and analytical work.

\subsubsection{Analytical investigation of the evolution of kurtosis}

Direct numerical simulation (DNS) has shown the evolution of surface elevation kurtosis in nonlinear, non-stationary wavefields [2], [17], [65], [24], [45], [4], [29]. However, none has achieved the task of analytically capturing this phenomenon we see predictably and consistently in numerical solutions.

One approach to analytically capturing the evolution of kurtosis seen in DNS is to start with the third order non-linear Schrodinger equations (NLSE) and couple two individual nonlinear waves interacting with each other at a specified angle of approach. In this manner we can represent the entire system in an analytical form, in order to theoretically capture the effect of the wave-wave interaction on surface elevation kurtosis [17]. Gronlund et al represent the coupling of two individual nonlinear waves in the third-order term in the NLSE, using a coupling constant [48] which is dependent on the angle of approach of the two waves. In this way they relate directional spreading regimes to the evolution of surface elevation, using only two interacting wave trains. Such a vast simplification still holds true to the trends seen in kurtosis involving several interacting nonlinear waves in DNS methods. This simplification provides an avenue for analytically capturing kurtosis evolution.

Another approach to analytically capturing the evolution of kurtosis seen in DNS is to consider wavefield kurtosis in terms of canonical variables representing nonlinear normal modes in the evolution of the deep water Zakharov equations. In this context, kurtosis can be observed in separate modes, as kurtosis due to bound harmonics and kurtosis due to dynamic modes in our non-stationary wavefields. In the canonical transformation the bound harmonic terms can even be eliminated, allowing for the sole observation of dynamic kurtosis [25]. We expect in this case that field statistics remain Gaussian unless interactions between wave modes due to cubic nonlinearity are considered. These cubic interactions lead to the evolution of the wave spectrum and the departure of kurtosis from its Gaussian value [24]. Annenkov and Shrira [2] 
confirm that bound harmonic kurtosis is determined by only the current spectrum and is independent of wavefield history, unlike dynamic kurtosis. Annenkov and Shrira separate the dynamical and bound harmonic parts of kurtosis, finding that in a wavefield with narrow-banded spectrum, during its initial stages of wave development the dynamic kurtosis is dominant. However, as the waves become more developed in such a wavefield, kurtosis becomes entirely due to bound harmonics. We can take a similar approach, observing the kurtosis of SNOW wavefields in terms of canonical variables, so as to find an exact analytical connection between the evolving surface spectrum and wavefield kurtosis.

\subsubsection{Experimental investigations}

Field/experimental work can supplement this work as a validation mechanism, moving the findings in the entire investigation closer to reality, circumventing the inherent loss of insight due to simplifications in computational and analytical work. Many field investigations depict the effect of spectral parameters on the evolution of wavefield kurtosis and large wave occurrence [59], [46], [15], [68]. Modern technological capabilities allow for direct extraction of directional statistical data from the ocean surface [11], [15]. Field investigations of directional data can also be completed with movable arrays, gliders, and automated vehicles [3]. The capability to produce realistic, but equivalent wavefields in field-scale settings also exists, thanks to technological advances seen in large-scale basins such as the MARINTEK Norwegian Marine Technology Research Institute Ocean Basin Laboratory [72]. 
Table 6.1: Methods/approaches used in our work to build on what has been done by prior authors

\begin{tabular}{lllll}
\hline \hline Investigation & Onorato 2008 & Toffoli 2009 & Ducrozet 2007 & Henry 2009 \\
\hline Phase-resolved simulations & X & X & $\checkmark$ & $\checkmark$ \\
Spectral parameters -analysis & $\checkmark$ & $\checkmark$ & $\checkmark$ & $\checkmark$ \\
Controlled peak shape parameter & $\checkmark$ & X & X & $\checkmark$ \\
Controlled Phillips' parameter & $\checkmark$ & X & X & $\checkmark$ \\
Controlled Directionality & X & X & X & $\checkmark$ \\
Zero-crossing Wave Heights Analysis & $\checkmark$ & $\checkmark$ & $\checkmark$ & $\checkmark$ \\
Energy-carrying waves analysis, H & X & X & X & $\checkmark$ \\
Small amplitude waves analysis & X & X & X & $\checkmark$ \\
\hline
\end{tabular}




\section{Appendix A}

\section{Linear statistical theory of ocean surface elevation}

Table A.1: Symbols used in Appendix A

\begin{tabular}{lr}
\hline \hline Symbol & Meaning \\
\hline$\alpha$ & Spectral Phillips parameter. \\
$D(\theta)$ & Expected value of $\mathrm{x}$. \\
$\mathrm{E}(\mathrm{x})$ & Ocean surface elevation. \\
$\eta$ & Spectral directional spreading function. \\
$\gamma$ & mean of $\eta$. \\
$\mu$ & The number of sinusoidal wave components in the wavefield. \\
$\mathrm{n}$ & Probability density function of $\mathrm{Z}$. \\
$\mathrm{p}(\mathrm{Z})$ & Characteristic function of $\mathrm{Z}$. \\
$\phi_{z}$ & Variance of $X_{i}$. \\
$\sigma$ & Dummy variable in the characteristic function. \\
$\tau$ & Arbitrary random variable. \\
$\mathrm{x}$ & The $i^{\text {th }}$ sinusoidal wave component in a wavefield. \\
$X_{i}$ & Standard random variable with mean, 0 and variance, 1. \\
$\mathrm{Z}$ &
\end{tabular}

This appendix outlines the linear statistical theory of ocean surface elevation distribution. We use the linear statistical theory of ocean surface elevation along with its assumptions as a tool in studying the impact of $\gamma$ on ocean wave statistics. The main assumptions of linear theory are 1. that surface elevation is the sum of a large number of statistically independent, identically distributed, random wave components, 
and 2. that through the relatively small amplitude (and therefore small steepness) of each wave component, superposition by summation applies to all the components. Explicitly, the small amplitude approximation is the assumption that the wave components' amplitudes are so small that only the linear terms in their surface slopes are non-negligible, therefore allowing for linear superposition of signals via summation. This summation is consistent with the central limit theorem which leads to the Gaussian distribution: Hence the conclusion of the linear theory applied to ocean surface elevation that ocean surface elevation is Gaussian. Deviations from such a theory's statistical trends in simulated wavefields are observed in the present work. Such deviations from the linear theory speak of the wavefield's physical deviation from the 2 assumptions of linear theory.

Many early studies such as [57], [40], [51], [52], [62] and [33] start the movement towards examining statistical properties of the ocean against linear statistics, for insight about the physical properties of the ocean surface. First among these studies is Rudnick's analysis of ocean surface elevation data [57], which concludes that ocean surface elevation follows a Gaussian distribution, which we associate with linear theory. He successfully shows that his data, derived from pressure gauges on the bottom of the Pacific Ocean, conforms remarkably to the result of the following linear analysis:

Let $\eta(\mathrm{x})$, where $-\infty<x<\infty$, be the wave profile at a fixed time, $t$. $\eta$, having a small enough steepness, can be expressed as the sum or linear superposition of a large number of wave components $X_{i}$.

$$
\eta(x)=X_{1}+X_{2}+\ldots+X_{n}
$$

where each $X_{i}$ is a statistically independent, identical, random variable with mean, $\mu=0$, and variance, $\sigma^{2}=1$. Since the $X_{i}$ 's are independent, the probability distribution of $\eta$ has zero mean and variance equal to $n \sigma^{2}=n$ where $n$ is large. Let $Z$ have the same distribution as $\frac{\eta}{\sigma \sqrt{n}}$, such that $Z$ is classified as a standard random variable,

$$
Z=\sum_{j=1}^{n} \frac{X_{j}}{\sigma \sqrt{n}}
$$


Let the characteristic function of $X$ be $\phi_{x}(t)$ of unknown form. Then the characteristic function of the standardized random variable, $\frac{X_{i}}{\sigma \sqrt{n}}$ is $\phi_{x}\left(\frac{t}{\sigma \sqrt{n}}\right)$. Hence,

$$
\phi_{z}(\tau)=\left[\phi_{x}\left(\frac{\tau}{\sigma \sqrt{n}}\right)\right]^{n}
$$

The characteristic function can be expanded in general as follows:

$$
\phi_{x}(\tau)=1+i t E(x)-\frac{\tau^{2}}{2} E\left(x^{2}\right)+\ldots
$$

where $E(x)=0$ and $E\left(x^{2}\right)=1$.

$$
\begin{gathered}
\phi_{x}\left(\frac{\tau}{\sigma \sqrt{n}}\right)=1+i \tau(0)-\frac{\tau^{2}}{2 n}(1)+O\left(\frac{\tau^{2}}{n}\right)+\ldots \\
\phi_{z}(\tau)=1-\frac{\tau^{2}}{2 n}+O\left(\frac{\tau^{2}}{n}\right)^{n}
\end{gathered}
$$

Let $n \rightarrow \infty$. Then,

$$
\phi_{z}(\tau) \rightarrow e^{\left(-\tau^{2}\right) / 2}
$$

This characteristic function has the following statistical distribution function:

$$
p(Z)=\frac{1}{\sigma \sqrt{2 \pi}} \exp \left(-\frac{Z^{2}}{2}\right)
$$

This is the statistical distribution of $Z$, a large sum of independent identical distributions. This leads us to the conclusion that ocean surface elevation, $\eta$ of mild steepness, adhering to the form of equation (A.1) follows a probability distribution law of the form:

$$
p(\eta)=\frac{1}{\sigma \sqrt{2 \pi}} \exp \left(-\frac{(\eta-\mu)^{2}}{2 \sigma^{2}}\right)
$$

where $\sigma=1$ and $\mu=0$. This distribution is known as the Gaussian distribution, and the linear derivation leading to it [41] is a proof of the Central-limit theorem, which states that the distribution of any superposition of many identically distributed parameters 
is Gaussian.

Further, ocean surface elevation in very deep water, regardless of sea severity (i.e. in the presence of a a hurricane with extremely steep surface elevation or three hours after the hurricanes with smaller surface elevation steepness) is assumed to categorically follow the Gaussian distribution (see Equation (A.9)) based on empirical studies such as Borgman's 1973 work which concludes that the largest wave heights in hurricanes actually show statistical values similar to those derived via linear statistical theory [7]. The Gaussian or Normal distribution, is found to have a set skewness of 0, and a kurtosis of 3 (See Section 3.1.1). Ocean surface elevation data sets show that for most of the values of surface elevation, these values hold, and the Gaussian distribution is accurate, but for statistical distribution details at very low and very high surface elevations, this assumption of the Gaussian distribution is consistently inexact. This is a cause for further investigations, since safe engineering design is hinged on the statistical distribution details at very low and very high surface elevations. These distribution tails form the basis of the present work which investigates the impact of spectral parameters such as $\gamma$ on deviations in the distribution tails.

This appendix introduces a linear theoretical approach to the distribution of ocean surface elevation. 


\section{Appendix B}

\section{Non-linear statistical theory of}

\section{ocean surface elevation}

Table B.1: Symbols used in Appendix B

\begin{tabular}{|c|c|}
\hline Symbol & Meaning \\
\hline$a_{i}$ & Constant coefficient of the $i^{\text {th }}$ fundamental component of surface elevation. \\
\hline$\xi$ & The $i^{\text {th }}$ fundamental component of surface elevation. Random variable. \\
\hline $\mathrm{N}$ & The number of fundamental components of surface elevation being considered. \\
\hline$V_{i}$ & Variance of the distribution of random variable, $\xi$. \\
\hline $\bar{\xi}$ & Mean of the distribution of random variable, $\xi$. \\
\hline$\overline{\eta^{n}}$ & $n^{\text {th }}$ moment of surface elevation, $\eta$. \\
\hline $\bar{\omega}$ & A grouping of $A_{p}, A_{q}, \ldots, A_{s}$ into unordered sets containing $i, j, \ldots, l$ members. \\
\hline $\mathrm{r}$ & Number of suffices $i, j, \ldots, l$ in $a_{i j \ldots l}$. \\
\hline & Abbreviated form of $a_{i j \ldots l}=A_{r} ; r$ is the number of suffices $i, j, \ldots, l$. \\
\hline $\mathrm{C}(\mathrm{i}, \mathrm{j}, \ldots, \mathrm{l})$ & Number of ways of choosing unordered sets in $\bar{\omega}$. \\
\hline$K_{n}$ & The $n^{\text {th }}$ cumulant of the distribution of $\eta$. \\
\hline$\kappa_{n}$ & Coefficient of $(i \tau)^{n}$ in the second characteristic function. \\
\hline$\lambda_{n}$ & Scaled $n^{t h}$ moment of the distribution of $\eta$. \\
\hline$p(\eta)$ & Probability density function of $\eta$. \\
\hline & Dummy variable in the characteristic function for $\eta$. \\
\hline$\phi(i \tau)$ & Characteristic function for $\eta$. \\
\hline$\mu_{n}$ & The $n^{\text {th }}$ moment of the distribution of $\eta$. \\
\hline$\gamma$ & Peak shape parameter. \\
\hline & Phillips parameter. \\
\hline$D(\theta)$ & Directional spreading function. \\
\hline
\end{tabular}

This appendix introduces the concept of the weakly non-linear statistical distri- 
bution for ocean surface elevation, generated from using the linear theory as a first approximation and successively adding higher-order terms that are functions of the statistical moments.

As stated in equation (A.1), the linear representation of sea surface elevation, $\eta$ can be expressed as follows:

$$
\eta=\sum_{i=1}^{N} a_{i} \xi_{i}
$$

where the $a_{i}$ 's are constants and the $\xi_{i}$ 's are independent random variables symmetrically distributed about mean, $\bar{\xi}_{i}=0$ with variance, $V_{i}$. The convergence of $\mathrm{p}(\eta)$ to

$$
p(\eta)=\frac{1}{\sigma \sqrt{2 \pi}} \exp \left(-\frac{\left(\eta-\bar{\xi}_{i}\right)^{2}}{2 V_{i}^{2}}\right)
$$

is a case of the central limit theorem seen in the previous section. In order to capture surface elevation dynamics more exactly, higher order terms must be included in the expression for $\eta$, and [36] produces the following analysis:

$$
\eta=\sum_{i=1}^{N} a_{i} \xi_{i}+\sum_{i=1}^{N} \sum_{j=1}^{N} a_{i j} \xi_{i} \xi_{j}+\sum_{i=1}^{N} \sum_{j=1}^{N} \sum_{k=1}^{N} a_{i j k} \xi_{i} \xi_{j} \xi_{k}+\ldots
$$

where $a_{i}, a_{i j}, a_{i j k}, \ldots$, are all constants. For such a surface elevation, the probability density function is derived in [36], and it begins with a calculation of statistical moments.

Given that $\overline{\xi_{i}}=0$ we can conclude the following:

$$
\begin{array}{r}
\mu_{1}=\bar{\zeta}=a_{i} \overline{\xi_{i}}+a_{i j} \overline{\xi_{i} \xi_{j}}+a_{i j k} \overline{\xi_{i} \xi_{j} \xi_{k}} \\
\mu_{1}=a_{i i} V_{i}+3 a_{i i j j} V_{i} V_{j}+\ldots \\
\mu_{2}=\overline{\zeta^{2}}=a_{i} a_{i} V_{i}+\left(2 a_{i j} a_{j i}+a_{i i} a_{j j}\right) V_{i} V_{j}+6 a_{i} a_{i j j} V_{i} V_{j}+\ldots
\end{array}
$$

Further moments, such as $\mu_{3}=\overline{\zeta^{3}}$ can also be found similarly, but the algebra involved is complicated. A moment-generating function and some short cuts are appropriate to circumvent this complexity. Note that some of the terms in equation 
(B.4) can be factorized as such:

$$
a_{i i} a_{j j} V_{i} V_{j}=\left(a_{i i} V_{i}\right)\left(a_{j j} V_{j}\right)
$$

but other terms like $a_{i j} a_{j i} V_{i} V_{j}$ cannot be similarly factorized, and such terms, which can get very long and complicate the equations, can be expressed in an abbreviated form:

$$
a_{i j \ldots l}=A_{r}
$$

where $r$ represents the number of suffices $i, j, \ldots, l$. We can then denote the sum of all the non-factorizing terms in the mean product:

$$
\overline{\left(A_{p} \xi_{i 1} \ldots \xi_{i p}\right)\left(A_{q} \xi_{j 1} \ldots \xi_{j q}\right) \ldots\left(A_{s} \xi_{l 1} \ldots \xi_{l s}\right)}
$$

simply by

$$
\overline{\left(A_{p} A_{q} \ldots A_{s}\right)}
$$

Since $\overline{\xi_{i}}=0$, when $(\mathrm{p}+\mathrm{q}+\ldots+\mathrm{s})$ is odd, this expression vanishes.

The first few moments can now be written as:

$$
\begin{array}{r}
\bar{\eta}=\sum_{p} A_{p}, \\
\overline{\eta^{2}}=\sum_{p, q}\left[\left(A_{p} A_{q}\right)+\left(A_{p}\right)\left(A_{q}\right)\right], \\
\overline{\eta^{3}}=\sum_{p, q, r}\left[\left(A_{p} A_{q} A_{r}\right)+3\left(A_{p} A_{q}\right)\left(A_{r}\right)+\left(A_{p}\right)\left(A_{q}\right)\left(A_{r}\right)\right],
\end{array}
$$

where the summations are taken over all positive integral values of $p, q$ and $r$, including values when $p$ and/or $q$ and/or $r$ are equal. Following directly from this derivation, the general moment-generating function to calculate the $n^{\text {th }}$ moment of the distribution is as follows:

$$
\overline{\eta^{n}}=\sum_{p, q, s}[C(n) \bar{\omega}(n)+C(n-1,1) \bar{\omega}(n-1,1)+\ldots]
$$

where $\bar{\omega}(i, j, \ldots, l)$ denotes some grouping of $A_{p}, A_{q}, \ldots, A_{s}$ into unordered sets contain- 
ing $i, j, \ldots, l$ members and $C(i, j, \ldots, l)$ denotes the number of ways of choosing such sets. If $\mathrm{r}$ denotes the number of sets in $\bar{\omega}$, we have:

$$
C(i, j, \ldots l)=\frac{1}{r !} \frac{n !}{i ! j ! \ldots l !}
$$

$K_{n}$, the cumulants of the distribution, are defined by the cumulant-generating function, which is the logarithm of the moment generating function. In this case of a non-linear variable representing ocean surface elevation, the cumulants are particularly much simpler to define. The moments correspond to coefficients of $(i \tau)^{n}$ in the characteristic function for $\eta$,

$$
\phi(i \tau)=1+\frac{\mu_{1}}{1 !}(i \tau)+\frac{\mu_{2}}{2 !}(i \tau)^{2}+\ldots
$$

whereas the cumulants correspond to the coefficients of $(i t)^{n}$ in the second characteristic function,

$$
K(i t)=\log [\phi(i t)]=\frac{\kappa_{1}}{1 !}(i t)+\frac{\kappa_{2}}{2 !}(i t)^{2}+\ldots
$$

Equating the coefficients of $(i t)^{n}$ helps us to define:

$$
\begin{array}{r}
\kappa_{1}=\mu_{1} \\
\kappa_{2}=\mu_{2}-\mu_{1}^{2} \\
\kappa_{3}=\mu_{3}-3 \mu_{1} \mu_{2}+2 \mu_{1}^{3} \cdots
\end{array}
$$

This leads us from equation (B.9) to define:

$$
\begin{array}{r}
\kappa_{1}=\sum_{p} A_{p}, \\
\kappa_{2}=\sum_{p, q}\left[\left(A_{p} A_{q}\right)\right] \\
\kappa_{3}=\sum_{p, q, r}\left[\left(A_{p} A_{q} A_{r}\right)\right] \ldots
\end{array}
$$


suggesting that $\kappa_{n}=\sum_{p, q, \ldots, s}\left[\left(A_{p} A_{q} \ldots A_{s}\right)\right]$, where $s$ is the $n^{\text {th }}$ integer in the integers from $p$ to $s$. From the cumulants, we can define the coefficients of skewness and of kurtosis as follows:

$$
\begin{array}{r}
\lambda_{3}=\frac{\kappa_{3}}{\kappa_{2}^{3 / 2}} \\
\lambda_{4}=\frac{\kappa_{4}}{\kappa_{2}^{2}}
\end{array}
$$

More generally, $\operatorname{lambd} a_{n}=\kappa_{n} / \kappa_{2}^{n / 2}$. If $p(\eta)$ is uniquely defined by its moments, then by inverting the fourier transform of the characteristic equation, (B.12), it follows that:

$$
\begin{gathered}
p(\eta)=\frac{1}{2 \pi} \int_{-\infty}^{+\infty} \phi(i t) e^{-i t \eta} d t \\
p(\eta)=\frac{1}{2 \pi} \int_{-\infty}^{+\infty} e^{K(i t)-i t \eta} d t \\
p(\eta)=\frac{1}{2 \pi} \int_{-\infty}^{+\infty} \exp \left[\left(\kappa_{1}-\eta\right) i t+\frac{1}{2} \kappa_{2}(i t)^{2}+\frac{1}{6} \kappa_{3}(i t)^{3}+\ldots\right] d t
\end{gathered}
$$

Substituting $t=s / \kappa_{2}^{1 / 2}$ and $\left(\eta-\kappa_{1}\right)=f \kappa_{2}^{1 / 2}$, the pdf becomes:

$$
p(\eta)=\frac{1}{2 \pi \kappa_{2}^{1 / 2}} \int_{-\infty}^{+\infty} \exp \left[\frac{-1}{2}\left(s^{2}+2 i f s\right)+\frac{1}{3 !} \lambda_{3}(i s)^{3}+\frac{1}{4 !} \lambda_{4}(i s)^{4}+\ldots\right] d S
$$

Expanding, we get:

$$
p(\eta)=\frac{1}{2 \pi \kappa_{2}^{1 / 2}} \int_{-\infty}^{+\infty} \exp \left[\frac{-1}{2}\left(s^{2}+2 i f s\right)\right]\left[1+\frac{1}{6} \lambda_{3}(i s)^{3}+\left[\frac{1}{4 !} \lambda_{4}(i s)^{4}+\frac{1}{3 * 4 !} \lambda_{3}^{2}(i s)^{6}\right]+\ldots\right] d S
$$

But using the identity for the integral I,

$$
I=\frac{1}{(2 \pi)^{1 / 2}} \int_{-\infty}^{+\infty} \exp \left[\frac{-1}{2}\left(s^{2}+2 i f s\right)\right](i s)^{n} d S
$$


and therefore,

$$
I=\frac{(-1)^{n}}{(2 \pi)^{1 / 2}} \frac{d^{n}}{d f^{n}} \int_{-\infty}^{+\infty} e^{\frac{-1}{2}\left(s^{2}+2 i f s\right)} d S
$$

it follows that,

$$
I=(-1)^{n} \frac{d^{n}}{d f^{n}} e^{\frac{-1}{2} f^{2}}=e^{\frac{-1}{2} f^{2}} H_{n}(f)
$$

where $H_{n}(f)$ is defined as:

$$
H_{n}(f)=f^{n}-\frac{n(n-1)}{1 !} \frac{f^{n-2}}{2}+\frac{n(n-1)(n-2)(n-3)}{2 !} \frac{f^{n-4}}{2^{2}}-\ldots
$$

Plugging this into equation (B.21) we finally conclude, according to [36] that:

$$
p(\eta)=\left(2 \pi \kappa_{2}\right)^{\frac{-1}{2}} e^{\frac{-1}{2} f^{2}}\left[1+\frac{1}{3 !} \lambda_{3} H_{3}+\left(\frac{1}{4 !} \lambda_{4} H_{4}+\frac{1}{3 * 4 !} \lambda_{3}^{2} H_{6}\right)+\ldots\right]
$$

noting that in the leading order, neglecting second- and higher-order terms,

$$
p(\eta)=\frac{1}{\sqrt{2 \pi \kappa_{2}^{1 / 2}}} e^{\frac{-1}{2} f^{2}}
$$

which is exactly the same as derived in the linear case, seen in equation (A.9), where $f=\left(x-\kappa_{1}\right) /\left(\kappa_{2}+\kappa_{1}^{2}\right)$.

This appendix introduces Longuet-Higgins' 1963 approach to the weakly nonlinear statistical distribution for ocean surface elevation [36], generated from using the linear theory as a first approximation and successively adding higher-order terms that are functions of the statistical moments seen in Section 3.1.1. In Chapter 3, we examine the higher-order effects of spectral peak shape parameter, $\gamma$, spectral Phillips steepness parameter, $\alpha$ and directional spreading, $D(\theta)$ on wavefield statistical distributions by comparing the statistical distributions of surface elevation in various cases with their relevant weakly non-linear theoretical statistical distributions shown in Equation B.26. 


\section{Appendix C}

\section{Wave height distribution - linear statistical theory of wave height, $\mathrm{H}$ in a narrow band}

Table C.1: Symbols used in Appendix C

\begin{tabular}{lr}
\hline \hline Symbol & Meaning \\
\hline$\eta$ & Wavefield surface elevation. \\
$\sigma$ & Surface elevation frequency. \\
$\mathrm{B}(\mathrm{t})$ & Function which represents the envelope of the waves. \\
$b_{n}$ & Time. \\
$\mathrm{t}$ & Amplitude associated with frequency $\sigma$ in wavefield surface elevation. \\
$A(\sigma)$ & Wave height. \\
$\mathrm{H}$ & Probability density function of wave envelope. \\
$\mathrm{P}(\mathrm{r})$ & Probability density function of wave heights. \\
$\mathrm{P}(\mathrm{H})$ & \\
\hline
\end{tabular}

Narrow-band wavefields are simulated for use in this work, and in this Appendix we outline the existing theory for delineating the statistical distribution of wave heights in a narrow band wavefield [33].

In [33], M.S.Longuet-Higgins analytically derives the probability distribution of wave heights, $H_{i}$, (where $i=1, \ldots, n$ and $n$ is large) in a linear wavefield, having a narrow frequency band, where all wave heights are defined throughout the wavefield 
by some specified, consistent distance between two values of surface elevation, $\eta$. If surface elevation, $\eta$ can be represented as:

$$
\eta(\sigma, t)=\int_{-\infty}^{+\infty} A(\sigma) e^{i(\sigma) t} d \sigma
$$

then, a wave height can be derived from surface elevation (see an example of a surface elevation, $\zeta$ in Figure $\mathrm{C}-1$ ) as follows:

$$
H=\eta_{\text {maximum }}-\eta_{\text {minimum }}
$$

within one cycle $\sigma t=2 \pi$, where $\eta_{\text {maximum }}$ occurs when

$$
\frac{d \eta}{d t}=0
$$

and

$$
\frac{d^{2} \eta}{d t^{2}}<0
$$

and $\eta_{\text {minimum }}$ occurs when

$$
\frac{d \eta}{d t}=0
$$

and

$$
\frac{d^{2} \eta}{d t^{2}}>0
$$

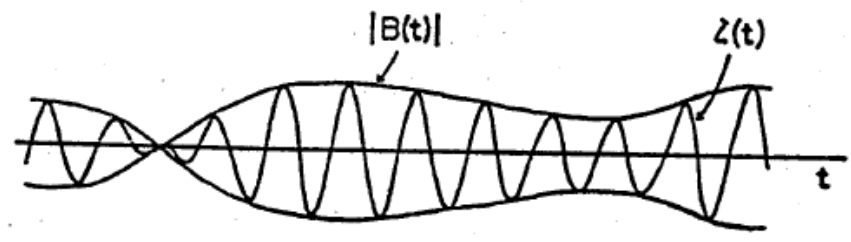

Figure C-1: This figure, taken from On the Statistical Distribution of the Heights of Sea Waves, M.S. Longuet-Higgins, 1952, shows a narrow frequency band disturbance, $\zeta(t)$, and its envelope $|B(t)|$. 
In this context, the wave spectrum consists of a single narrow frequency band of wavelength $2 \pi / \sigma_{0}$ so that the spectrum function $A(\sigma)$ is appreciable only for values of $\sigma$ near $\sigma_{0}$. In order to analytically represent the carrier wave, such as $\zeta(t)$ in Figure $\mathrm{C}-1$, separately from the wave envelope, such as $|B(t)|$ in Figure $\mathrm{C}-1$, we can say:

$$
\eta(\sigma, t)=e^{i\left(\sigma_{0}\right) t} \int_{-\infty}^{+\infty} A(\sigma) e^{i\left(\sigma-\sigma_{0}\right) t} d \sigma
$$

where $e^{i\left(\sigma_{0}\right) t}$ is the carrier wave of wavelength, $2 \pi / \sigma_{0}$ and the integral,

$$
B(t)=\int_{-\infty}^{+\infty} A(\sigma) e^{i\left(\sigma-\sigma_{0}\right) t} d \sigma
$$

is the function which represents the envelope of the waves, like $|B(t)|$ in Figure $\mathrm{C}-1$, slowly varying in time, due to the very small time-variation of $e^{i\left(\sigma-\sigma_{0}\right) t}$ when $\sigma-\sigma_{0}$ is small. Note that $|B(t)|$ is coincident on the maxima and minima of $\eta$, as illustrated in Figure C-1, where in this [33] illustration $\zeta$ represents surface elevation. This is a helpful form of representing the wavefield, since the probability distribution of wave heights is the same as the analytically tractable probability distribution of $2|B(t)|$. This analytical tractability is achieved in assuming that $B(t)$ is the sum of a very large number of small, independent components of random, independent phases in which case, the distribution of $B(t)$ is that of a random walk. Rayleigh [55] shows that if

$$
B(t)=\left|b_{1}\right|^{2}+\left|b_{2}\right|^{2}+\ldots\left|b_{M}\right|^{2}
$$

as in a random walk, within physical limits e.g. if $\left.\frac{d B}{d t}\right|_{\max }<0.14$ [28], then the probability that $|B(t)|$ lies between $r$ and $r+d r$ is given by,

$$
P(r) d r=e^{-r^{2} / B_{r m s}^{2}} \frac{2 r}{B_{r m s}^{2}} d r .
$$

So, we have a distribution for $|B(t)|$ which is exactly the same as the distribution for $H(t)$ and therefore $H(x)$, due to ergodicity assumptions. Hence, we have the probability distribution of wave heights as, 


$$
P(h) d h=e^{-h^{2} / H_{r m s}^{2}} \frac{2 h}{H_{r m s}^{2}} d h=-d e^{-r^{2} / H_{r m s}^{2}} .
$$

In this section we outlined the theory of wave height distributions in linear, narrowband wavefields. In the following sections, we will observe the comparison between this theoretical distribution and the statistics of wave heights in our simulated linear and non-linear wavefields. 


\section{Appendix D}

\section{Non-linear statistical theory of wave height, $H$}

Table D.1: Symbols used in Appendix D

\begin{tabular}{lr}
\hline \hline Symbol & Meaning \\
\hline$\eta$ & Wavefield surface elevation. \\
$\bar{\eta}$ & Hilbert transform of wavefield surface elevation. \\
$\xi$ & Wave envelope, $|B|$, scaled with $\mu_{2}$. \\
$\mathrm{t}$ & Time. \\
$\tau$ & Constant time lag. \\
$\mathrm{H}$ & Wave height. \\
$H_{j}$ & The $j^{\text {th }}$ order Hermite Polynomial. \\
$\kappa_{m n}$ & The $(m+n)^{t h}$ order normalized joint cumulant of $\eta$ and $\hat{\eta}$. \\
$\mu_{n}$ & The $n^{t h}$ moment of surface elevation distribution. \\
$\theta$ & Wave phase. \\
$|B|$ & Wave envelope. \\
$\Lambda$ & Total joint cumulant. \\
\hline
\end{tabular}

This appendix theoretically approaches the question of non-linearity in wave height distributions to account for data series' deviations from Longuet-Higgins' linear-narrow-band theory on wave height distributions. Linear theory on ocean wave statistics is justified by the small-amplitude assumption. If waves do not adhere to the small-amplitude description, as in many cases, this description visibly deviates from reality, as seen in the departure from the theoretical linear statistical distribution (as in (C.11)) seen in every simulated wavefield in the present work. 
Tayfun extends the existing theoretical work on wave height statistical distribution to include nonlinear distributions for wave height [64] since Longuet-Higgins only calculates this result for surface elevation [36]. There exists a model that represents approximations that arise from the Taylor series expansions of the characteristic function (see (A.3)) of random variables whose probability structure is not exactly known. For nonlinear wave envelopes with nonlinear phases, third order cumulants are of significant effect, have the same order of magnitude as $\mu_{1}$ and fourth order cumulants are less significant, having order $\mu_{1}^{2}$.

Let surface elevation, $\eta=\xi \cos \theta$ and the Hilbert transform of $\eta, \hat{\eta}=\xi \sin \theta$. Then, the wave envelope, $|B|$, scaled with $\mu_{2}$, can be represented by,

$$
\xi=\left(\eta^{2}+\hat{\eta}^{2}\right)
$$

and the wave phase, $\theta$, representing a non-uniform wave phase distribution over a $2 \pi$ interval is,

$$
\theta=\tan ^{-1}\left(\frac{\hat{\eta}}{\eta}\right)
$$

Assuming that $\eta$ is statistically homogeneous (this is valid for short-term statistics, but does not apply in long term statistics, which are outside the scope of this work), then $\eta$ and $\hat{\eta}$ are orthogonal, so that,

$$
\begin{gathered}
<\eta \hat{\eta}>=\frac{1}{\pi} \int_{-\infty}^{+\infty}<\eta(t) \eta(t-\tau)>\tau^{-1} d \tau \\
<\eta \hat{\eta}>=0,
\end{gathered}
$$

since the integral is odd.

This allows us to expand the joint pdf of $\eta$ and $\hat{\eta}$,

$$
<\eta \hat{\eta}>=\frac{1}{\pi}\left[1+\sum_{j=0}^{3} \frac{\kappa_{3-j, j}}{(3-j) ! j !} H_{3-j}(\eta) H_{j}(\hat{\eta})+\sum_{j=0}^{4} H_{4-j}(\eta) H_{j}(\hat{\eta})\right] \exp \left[\frac{-\left(\eta^{2}+\hat{\eta}^{2}\right)}{2}\right]
$$


where $\kappa_{m n}=<\eta^{m} \hat{\eta}^{n}>/ \mu_{2}^{m+n}$, the joint cumulant of $\eta$ and $\hat{\eta}$ (when $m+n=3$ we find the third order normalized joint cumulant of $\eta$ and $\hat{\eta}$, but when $m+n=4$, we have the fourth order normalized joint cumulant of $\eta$ and $\hat{\eta}$, with a slightly changed form of $\kappa$ which becomes $\left.\kappa_{m n}=<\eta^{m} \hat{\eta}^{n}>/ \mu_{2}^{m+n}+(-1)^{m / 2}(m-1)(n-1)\right), H_{j}$ is the $j^{\text {th }}$ order Hermite Polynomial, where,

$$
\begin{array}{r}
H_{0}=1 \\
H_{1}=x \\
H_{2}=x^{2}-1 \\
H_{3}=x^{3}-3 x \\
H_{4}=x^{4}-6 x^{2}+3
\end{array}
$$

The pdf of wave height, $\xi$, where $x=h / 2$ and $h$ is an arbitrary value of wave height, $H$,

$$
P(x)=\frac{x}{4} e^{-x^{2} / 8}\left[1+\frac{\Lambda}{1024}\left(x^{4}-32 x^{2}+128\right)\right]
$$

where,

$$
\Lambda=\kappa_{40}+2 \kappa_{22}+\kappa 04
$$

The pdf of wave height exceedance,

$$
P_{\text {exceedance }}(x)=e^{-x^{2} / 8}\left[1+\frac{\Lambda}{1024}\left(x^{2}\right)\left(x^{2}-16\right)\right] .
$$

Note: If $\Lambda$ is small enough, then this distribution becomes equivalent to LonguetHiggins' linear, narrow-band theory on wave height probability distribution. 


\section{Bibliography}

[1] I.E. Alber. The effects of randomness on the stability of two-dimensional surface wavetrains. Proceedings of the Royal Society, 363(1715):525-546, 1978.

[2] S.Y. Annenkov and V.I. Shrira. Evolution of kurtosis for wind waves. Geophysical Research Letters, 36(L13603):1-5, 2009.

[3] N.F. Barber. The directional resolving power of an array of wave detectors. Ocean Wave Spectra, Prentice-Hall, Englewood Cliffs, NJ, pages 137-150, 1961.

[4] Anastassia Baxevani and Igor Rychlik. Maxima for gaussian seas. Ocean Engineering, 33:895-911, 2006.

[5] T.B. Benjamin and J.E. Feir. The disintegration of wave trains on deep water. J. Fluid Mech., 27(3):417-430, 1967.

[6] E.M. Bitner. Non-linear effects of the statistical model of shallow-water wind waves. Appl. Ocean Res., 2:63-73, 1980.

[7] L.E. Borgman. Probabilities for highest wave in hurricane. J. Waterways, Harbours, and Coastal Engineering, 99:185-207, 1973.

[8] J. Dennis and G. Wolff. Waves. freak waves and rogues. World Wide Web electronic publication, 1996.

[9] M. W. Dingemans. A review of the physical formulations in swan, wl- delft hydraulics report h3306. Research report, Delft Hydraulics, the Netherlands, 1998. 
[10] L. Draper. Freak ocean waves. Oceanus, 10, 1964.

[11] W.M. Drennan, H.C. Graber, M.A. Donelan, and E.A. Terray. Directional wave measurements from the asis (air-sea interactionspar) buoy. OCEANS '98 Conference Proceedings, 1:414-418, 1998.

[12] G. Ducrozet, F. Bonnefoy, D. Le Touz, and P. Ferrant. 3-d hos simulations of extreme waves in open seas. Nat. Hazards Earth Syst. Sci., 7, 2007.

[13] O.M. Faltinsen, J.N. Newman, and E. Vinje. Nonlinear wave loads on a slender vertical cylinder. Jiurnal of Fluid Mechanics, (289):179-198, 1995.

[14] D. Faulkner. Rogue waves - defining their characterstics for marine design. In Olagnon M. and G.A. Athanassoulis, editors, Rogue Waves 2000, pages 3-18. Ifremer, France, 2001.

[15] Hans C. Graber, Eugene A. Terray, Mark A. Donelan, William M. Drennan, John C. Van Leer, and Donald B. Peters. Asisa new airsea interaction spar buoy: Design and performance at sea. Journal of Atmospheric and Oceanic Technology, 17(5):708-720, 2000.

[16] Odin Gramstad and Karsten Trulsen. Influence of crest and group length on the occurrence of freak waves. Journal of Fluid Mechanics, 582:463-472, 2007.

[17] A. Gronlund, B. Eliasson, and M. Marklund. Evolution of rogue waves in interacting wave systems. EPL Journal, 86(24001):p1-p5, 2009.

[18] Jim Gunson and Anne Karin Magnusson. Investigating conditions for rogue wave events from spectral wave observations and models. Research report, Met Office London Road, Bracknell, RG12 2SZ, United Kingdom, 2002.

[19] K. Hasselmann. Measurements of wind-wave growth and swell decay during the joint north sea wave project (jonswap). Research report, Dtsch. Hydrogr. Inst., Hamburg, 1973. 
[20] T. S. Hedges. Measurement and analysis of waves on currents. Proc. Symp. Mechanics of Wave-induced Forces on Cylinders, pages 249-259, 1979.

[21] Yelland M.J. Pascal R. et al Holliday, N.P. Were extreme waves in the rockall trough the largest ever recorded? Geophysical Research letters, 33: L05613, 2006.

[22] P. Janssen. In The Interaction of Ocean Waves and Wind, pages 74-83. Cambridge University Press, 2004.

[23] P.A.E.M. Janssen. On non-linear wave groups and consequences for spectral evolution, directional ocean wave spectra. The John Hopkins University, pages 46-52, 1991.

[24] P.A.E.M. Janssen. Nonlinear four-wave interactions and freak waves. J. Phys. Ocean., 33:863-883, 2003.

[25] Janssen2008. On some consequences of the canonical transformation in the hamiltonian theory of water waves. ECMWF Tech Memo, 579, 2008.

[26] H. Jeffreys. On the formation of water waves by wind. Proceedings of the Royal Society A, 107:189-206, 1925.

[27] C. Kharif, E. Pelinovsky, and A. Slunyaev. In Blair Kinsman, editor, Rogue Waves in the Ocean. Springer: Advances in Geophysical and Environmentl Mechanics and Mathematics, 2009.

[28] A. Ya. Khintchine. In Asymptotische Gesetz der Wahrscheinlichkeitsrechnung. Springer, Berlin, 1933.

[29] M. H. Kim, J. M. Niedzwecki, J. M. Roesset, J. C. Park, S. Y. Hong, and A. Tavassoli. Fully nonlinear multidirectional waves by a 3-d viscous numerical wave tank. Transactions of the ASME, 123:124-133, 2001. 
[30] Blair Kinsman. In Blair Kinsman, editor, Wind Waves, their generation and propagation on the ocean surface, pages 325-367. Dover Publications, Inc., NY, 1984.

[31] R.J. Lai, S.R. Long, and N.E. Huang. Laboratory studies of wave-current interaction: kinematics of the strong interaction. JOURNAL OF GEOPHYSICAL RESEARCH, 94(C11):201-214, 1989.

[32] MacHutchon K.R. Liu, P.C. Are there different kinds of rogue waves? In Proceedings of the 8th International Conference of Distributed Computing Systems, volume 92619, Hamburg, Germany.

[33] M. S. Longuet-Higgins. On the statistical distribution of the heights of sea waves. Journal of Marine Research, 11(3):245-266, 1952.

[34] M. S. Longuet-Higgins. On the joint distribution of wave periods and amplitudes in a random wave field. Proceedings of the Royal Society of London. Series A, Mathematical and Physical Sciences, 389(1797):241-258, 1983.

[35] M.S. Longuet-Higgins. Resonant interactions between two trains of gravity waves. Journal of Fluid Mechanica, 1962.

[36] M.S. Longuet-Higgins. The effect of non-linearities on statistical distributions in the theory of sea waves. Journal of Fluid Mechanics, 17(3):459-480, 1963.

[37] M.S. Longuet-Higgins, D.E. Cartwright, and N.D. Smith. Observations of the directional spectrum of sea waves using the motions of a floating body. Proceedings of the Conference on Ocean Wave Spectra, pages 111-132, 1961.

[38] V.K. Makin and V.N. Kudryavtsev. The effect of dominant wave breaking on the drag of the sea surface. Bdry-Layer Met., 103:83-99, 2002.

[39] W.K. Melville and P. Matusov. Distribution of breaking waves at the ocean surface. Nature, 417:58-62, 2002. 
[40] G. Neumann. On ocean wave spectra and a new method of forecasting windgenerated sea. Beach Erosion Board,U.S. Army Corps ofEngineers, 43, 1953.

[41] Michel K. Ochi. In Ocean Waves, The Stochastic Approach, pages 1-10. Cambridge University Press, 1998.

[42] M.K. Ochi. Observations of the directional spectrum of sea waves using the motions of a floating body. Proceedings of the International Conference on the Behaviors of Offshore Structures, pages 75-86, 1979.

[43] U. S. Navy Hydrographic Office. Techniques for forecasting wind waves and swell. U. S. Navy Hydrographic Office Publications, 604, 1951.

[44] Onorato, Osborne, and Serio. Extreme wave events in directional, random oceanic sea states. Physics of Fluids, 14:L25-L28, 2002.

[45] M. Onorato and et. al. Statistical properties of directional ocean waves: the role of modulational instability in the formation of extreme events. Physical Review Letters, 102(114502), 2009.

[46] M. Onorato, A. R. Osborne, M. Serio, L. Cavaleri, C. Brandini, and C. T. Stansberg. Observation of strongly non-gaussian statistics for random sea surface gravity waves in wave flume experiments. Physical Review E, 70(067302):1-4, 2004 .

[47] M. Onorato, A.R. Osborne, and S. Bertone. Freak waves in random oceanic sea states. Phys. Rev. Lett., 86:5831-5834, 2001.

[48] M. Onorato, A.R. Osborne, M. Serio, L. Cavaleri, C. Brandini, and C.T. Stansberg. Extreme waves, modulational instability and second order theory: wave flume experiments on irregular waves. European Journal of Mechanics - B/Fluids, 25(5):586 - 601, 2006. Rogue waves.

[49] O.M. Phillips. On the attenuation of long gravity waves by short breaking waves. J. Fluid Mech., 16:321-332, 1963. 
[50] O.M. Phillips. Spectral and statistical properties of the equilibrium range in wind-generated gravity waves. Journal of Fluid Mechanics, 156:505-531, 1985.

[51] W.J. Pierson. In A unified mathematical theory for the analysis of propagation and refraction of storm generated ocean surface waves - Parts I and II. New York University, College of Engineering, 1952.

[52] W.J. Pierson. In Wind generated gravity waves. Advances in Geophysics, 1955.

[53] W.J.JR. Pierson, G. Neumann, and R.W. James. Practical methods for observing and forecasting ocean waves by means of wave spectra and statistics. U.S. Navy Hydrographic Office, (603), 1955.

[54] R.C.T. Rainey. Slender-body expressions for the wave load on offshore structures. Proceedings of the Royal Society, London, 1995.

[55] Lord Rayleigh. On the resultant of a large number of vibrations of the same pitch and of arbitrary phase. Philos. Mag., 10:73-78, 1880.

[56] John A. Rice. In Cole Thomson, Brooks, editor, Mathematical statistics and data analysis, pages 53-57. Duxbury Press, 1944.

[57] P. Rudnick. Correlograms for pacific ocean waves. Proceedings of the 2nd Berkeley Symposium on Mathematical Statistics and Probability, 1951.

[58] John A. Scales and Roel Snieder. What is a wave? Nature, 401:739-740, 1999.

[59] Lev Shemer and Anna Sergeeva. An experimental study of spatial evolution of statistical parameters in a unidirectional narrow-banded random wavefield. Journal of Geophysical Research, 114(C1), 2009.

[60] R.J. Sobey. Wind-wave prediction. Annual Review of Fluid Mechanics, 1986.

[61] C.T. Stansberg, E. Huse, J.R. Krokstad, and E. Lehn. Experimental study of nonlinear loads on vertical cylinders in steep random waves. Proc. 5th ISOPE Conference, the Hague, the Netherlands, pages 75-82, 1995. 
[62] M. St.Denis and W.J. Pierson. On the motions of ships in confused seas. Trans. of the Society of Naval Architecture and Marine Engineering., 61:280-357, 1953.

[63] H. U. Sverdrup and W. H. Munk. Wind, sea and swell: theory of relations for forecasting. U. S. Navy Hydrographic Office Publications, 601, 1947.

[64] M. Aziz. Tayfun and Francesco Fedele. Wave-height distributions and nonlinear effects. Ocean Engineering, 34(11-12):1631 - 1649, 2007.

[65] A. Toffoli, E. Bitner-Gregerson, M. Onorato, and A.V. Babanin. Wave crest and trough distributions in a broad-banded directional wavefield. Ocean Engineering, 35:1784-1792, 2008.

[66] A. Toffoli, J.M. Lefevre, E. Bitner-Gregersen, and J. Monbaliu. Toward the identification of warning criteria: analysis of a ship accident database. Applied ocean Research, 27:281-291, 2005.

[67] A. Toffoli, M. Onorato, E. Bitner-Gregersen, A.R. Osborne, and A.V. Babanin. Surface gravity waves from direct numerical simulations of the euler equations: A comparison with second-order theory. Ocean Engineering, 35(3 - 4):367-379, 2007.

[68] T. Waseda, T. Kinoshita, and H. Tamura. Evolution of random directional wave and rogue wave occurence. Tenth International Worshop on Wave Hindcasting and Forecasting, 2008.

[69] D.J. Webb. Non-linear transfers between sea waves. Deep Sea Research, 1978.

[70] J.D. Wheeler. Method for calculating forces produced by irregular waves. Journal of Petroleum Technology, (249):359-367, 1970.

[71] Guangyu. Wu. Direct simulation and deterministic prediction of large-scale nonlinear ocean wavefield. PhD thesis, Massachusetts Institute of Technology, Dept. of Ocean Engineering, Cambridge, MA, 2004. 
[72] Marintek www.sintef.no. The ocean basin laboratory. World Wide Web electronic publication, 2005.

[73] Wenting Xiao, Legena Henry, Yuming Liu, Kelli Hendrickson, and Dick K.P. Yue. Ocean wave prediction using large-scale phase-resolved computations. HPCMP Users Group Conference 2008, pages 69-73, 2008.

[74] Zakharov. Stability of periodic waves of finite amplitude on the surface of a deep fluid. J. Appl. Mech. Tech. Phys., 9:190-194, 1968.

[75] Q. Zhu. Features of nonlinear wave-wave and wave-body interactions. PhD thesis, Massachusetts Institute of Technology, Dept. of Ocean Engineering, Cambridge, MA, 2000. 